Cochrane Database of Systematic Reviews

\title{
Interventions to improve disposal of child faeces for preventing diarrhoea and soil-transmitted helminth infection (Review)
}

Majorin F, Torondel B, Ka Seen Chan G, Clasen T

Majorin F, Torondel B, Ka Seen Chan G, Clasen T.

Interventions to improve disposal of child faeces for preventing diarrhoea and soil-transmitted helminth infection.

Cochrane Database of Systematic Reviews 2019, Issue 9. Art. No.: CD011055.

DOI: 10.1002/14651858.CD011055.pub2.

www.cochranelibrary.com

Interventions to improve disposal of child faeces for preventing diarrhoea and soil-transmitted helminth infection (Review) 
TABLE OF CONTENTS

HEADER

ABSTRACT

PLAIN LANGUAGE SUMMARY

SUMMARY OF FINDINGS

BACKGROUND

Figure 1.

OBJECTIVES

METHODS

RESULTS

Figure 2.

Figure 3.

DISCUSSION

AUTHORS' CONCLUSIONS

ACKNOWLEDGEMENTS

REFERENCES

CHARACTERISTICS OF STUDIES

DATA AND ANALYSES

Analysis 1.1. Comparison 1 Education and hygiene promotion interventions versus control, Outcome 1 Diarrhoea prevalence

- randomized controlled trials (RCTs).

Analysis 1.2. Comparison 1 Education and hygiene promotion interventions versus control, Outcome 2 Diarrhoea incidence RCTs.

Analysis 1.3. Comparison 1 Education and hygiene promotion interventions versus control, Outcome 3 Diarrhoea prevalence - controlled cohort studies: SHEWA-B versus control.

Analysis 1.4. Comparison 1 Education and hygiene promotion interventions versus control, Outcome 4 Diarrhoea prevalence controlled cross-sectional: HEP model households versus non-model.

Analysis 1.5. Comparison 1 Education and hygiene promotion interventions versus control, Outcome 5 Anthropometry - RCTs: height-for-age Z score (HAZ).

Analysis 1.6. Comparison 1 Education and hygiene promotion interventions versus control, Outcome 6 Behaviour change RCTs: latrine use by children.

Analysis 1.7. Comparison 1 Education and hygiene promotion interventions versus control, Outcome 7 Behaviour change RCTs: potty use by children.

Analysis 1.8. Comparison 1 Education and hygiene promotion interventions versus control, Outcome 8 Behaviour change RCTs: safe disposal of child faeces.

Analysis 1.9. Comparison 1 Education and hygiene promotion interventions versus control, Outcome 9 Behaviour change RCTs: appropriate disposal of child faeces.

Analysis 1.10. Comparison 1 Education and hygiene promotion interventions versus control, Outcome 10 Behaviour change RCTs: faeces not observed in yard/ $\mathrm{HH}$.

Analysis 1.11. Comparison 1 Education and hygiene promotion interventions versus control, Outcome 11 Behaviour change RCTs: faeces in compound.

Analysis 1.12. Comparison 1 Education and hygiene promotion interventions versus control, Outcome 12 Behaviour change controlled cohort studies: safe vs unsafe child faeces disposal.

Analysis 1.13. Comparison 1 Education and hygiene promotion interventions versus control, Outcome 13 Behaviour change controlled cross-sectional studies: safe vs unsafe child faeces disposal.

Analysis 2.1. Comparison 2 CLTS interventions plus adaptations, Outcome 1 Diarrhoea prevalence - randomized controlled trials (RCTs).

Analysis 2.2. Comparison 2 CLTS interventions plus adaptations, Outcome 2 Any helminth prevalence - RCTs. ...................... Analysis 2.3. Comparison 2 CLTS interventions plus adaptations, Outcome 3 Ascaris lumbricoides prevalence - RCTs. ............ Analysis 2.4. Comparison 2 CLTS interventions plus adaptations, Outcome 4 Dysentery - RCTs.

Analysis 2.5. Comparison 2 CLTS interventions plus adaptations, Outcome 5 Anthropometry: height-for-age Z score (HAZ) RCTs.

Analysis 2.6. Comparison 2 CLTS interventions plus adaptations, Outcome 6 Anthropometry: weight-for-age Z score (WAZ) RCTs.

Analysis 2.7. Comparison 2 CLTS interventions plus adaptations, Outcome 7 Behaviour change - RCTs: no open defecation by children aged $<5$ years. 
Analysis 2.8. Comparison 2 CLTS interventions plus adaptations, Outcome 8 Behaviour change - RCTs: safe disposal of child faeces.

Analysis 2.9. Comparison 2 CLTS interventions plus adaptations, Outcome 9 Behaviour change - RCTs: potty use by children. .. Analysis 3.1. Comparison 3 Sanitation hardware and behaviour change interventions, Outcome 1 Diarrhoea prevalence randomized controlled trials (RCTs).

Analysis 3.2. Comparison 3 Sanitation hardware and behaviour change interventions, Outcome 2 Anthropometry: height-forage $Z$ score (HAZ) - RCTs.

Analysis 3.3. Comparison 3 Sanitation hardware and behaviour change interventions, Outcome 3 Anthropometry: weight-forage $Z$ score (WAZ) - RCTs.

Analysis 3.4. Comparison 3 Sanitation hardware and behaviour change interventions, Outcome 4 Behaviour change - RCTs: safe disposal of child faeces.

Analysis 3.5. Comparison 3 Sanitation hardware and behaviour change interventions, Outcome 5 Behaviour change - RCTs: appropriate disposal of child faeces.

Analysis 3.6. Comparison 3 Sanitation hardware and behaviour change interventions, Outcome 6 Behaviour change - RCTs: potty use by children.

Analysis 3.7. Comparison 3 Sanitation hardware and behaviour change interventions, Outcome 7 Behaviour change - RCTs: faeces in compound.

Analysis 4.1. Comparison 4 WASH hardware and education interventions, Outcome 1 Diarrhoea prevalence - randomized controlled trials.

Analysis 4.2. Comparison 4 WASH hardware and education interventions, Outcome 2 Diarrhoea incidence - controlled beforeand-after studies.

Analysis 5.1. Comparison 5 Case-control studies: disposal of child faeces in latrine versus elsewhere, Outcome 1 Diarrhoea (including severe and cholera): subgrouped by age group.

Analysis 5.2. Comparison 5 Case-control studies: disposal of child faeces in latrine versus elsewhere, Outcome 2 Diarrhoea in all ages (including severe and cholera): subgrouped by country income level.

Analysis 5.3. Comparison 5 Case-control studies: disposal of child faeces in latrine versus elsewhere, Outcome 3 Diarrhoea in all ages (including severe and cholera): subgrouped by type of diarrhoea.

Analysis 5.4. Comparison 5 Case-control studies: disposal of child faeces in latrine versus elsewhere, Outcome 4 Diarrhoea in all ages (including severe and cholera): subgrouped by study quality.

Analysis 5.5. Comparison 5 Case-control studies: disposal of child faeces in latrine versus elsewhere, Outcome 5 Diarrhoea in all ages (including severe and cholera): subgrouped by setting.

Analysis 5.6. Comparison 5 Case-control studies: disposal of child faeces in latrine versus elsewhere, Outcome 6 Diarrhoea in all ages (including severe and cholera): subgrouped by method of data collection.

Analysis 6.1. Comparison 6 Case-control studies: defecation of children in latrine versus elsewhere, Outcome 1 Diarrhoea: casecontrol studies: subgrouped by age group.

Analysis 6.2. Comparison 6 Case-control studies: defecation of children in latrine versus elsewhere, Outcome 2 Diarrhoea in all ages: case-control studies: subgrouped by country income level.

Analysis 6.3. Comparison 6 Case-control studies: defecation of children in latrine versus elsewhere, Outcome 3 Diarrhoea in all ages: case-control studies: subgrouped by type of diarrhoea.

Analysis 6.4. Comparison 6 Case-control studies: defecation of children in latrine versus elsewhere, Outcome 4 Diarrhoea in all ages: case-control studies: subgrouped by study quality.

Analysis 6.5. Comparison 6 Case-control studies: defecation of children in latrine versus elsewhere, Outcome 5 Diarrhoea in all ages: case-control studies: subgrouped by setting.

Analysis 6.6. Comparison 6 Case-control studies: defecation of children in latrine versus elsewhere, Outcome 6 Diarrhoea in all ages: case-control studies: subgrouped by method of data collection.

ADDITIONAL TABLES

APPENDICES

CONTRIBUTIONS OF AUTHORS

DECLARATIONS OF INTEREST 
[Intervention Review]

\section{Interventions to improve disposal of child faeces for preventing diarrhoea and soil-transmitted helminth infection}

Fiona Majorin ${ }^{1}$, Belen Torondel ${ }^{1}$, Gabrielle Ka Seen Chan ${ }^{1}$, Thomas Clasen ${ }^{2}$

1Faculty of Infectious and Tropical Diseases, London School of Hygiene \& Tropical Medicine, London, UK. 2Department of Environmental Health, Rollins School of Public Health, Emory University, Atlanta, GA, USA

Contact address: Fiona Majorin, Faculty of Infectious and Tropical Diseases, London School of Hygiene \& Tropical Medicine, Keppel Street, London, WC1E 7HT, UK. fiona.majorin@lshtm.ac.uk.

Editorial group: Cochrane Infectious Diseases Group

Publication status and date: New, published in Issue 9, 2019.

Citation: Majorin F, Torondel B, Ka Seen Chan G, Clasen T. Interventions to improve disposal of child faeces for preventing diarrhoea and soil-transmitted helminth infection. Cochrane Database of Systematic Reviews 2019, Issue 9. Art. No.: CD011055. DOI: 10.1002/14651858.CD011055.pub2.

Copyright $\odot 2019$ The Authors. Cochrane Database of Systematic Reviews published by John Wiley \& Sons, Ltd. on behalf of The Cochrane Collaboration. This is an open access article under the terms of the Creative Commons Attribution-Non-Commercial Licence, which permits use, distribution and reproduction in any medium, provided the original work is properly cited and is not used for commercial purposes.

\section{A B S T R A C T}

\section{Background}

Diarrhoea and soil-transmitted helminth (STH) infections represent a large disease burden worldwide, particularly in low-income countries. As the aetiological agents associated with diarrhoea and STHs are transmitted through faeces, the safe containment and management of human excreta has the potential to reduce exposure and disease. Child faeces may be an important source of exposure even among households with improved sanitation.

\section{Objectives}

To assess the effectiveness of interventions to improve the disposal of child faeces for preventing diarrhoea and STH infections.

\section{Search methods}

We searched the Cochrane Infectious Diseases Group Specialized Register, CENTRAL, MEDLINE, Embase, and 10 other databases. We also searched relevant conference proceedings, contacted researchers, searched websites for organizations, and checked references from identified studies. The date of last search was 27 September 2018.

\section{Selection criteria}

We included randomized controlled trials (RCTs) and non-randomized controlled studies (NRS) that compared interventions aiming to improve the disposal of faeces of children aged below five years in order to decrease direct or indirect human contact with such faeces with no intervention or a different intervention in children and adults.

\section{Data collection and analysis}

Two review authors selected eligible studies, extracted data, and assessed the risk of bias. We used meta-analyses to estimate pooled measures of effect where appropriate, or described the study results narratively. We assessed the certainty of the evidence using the GRADE approach. 


\section{Main results}

Sixty-three studies covering more than 222,800 participants met the inclusion criteria. Twenty-two studies were cluster RCTs, four were controlled before-and-after studies (CBA), and 37 were NRS (27 case-control studies (one that included seven study sites), three controlled cohort studies, and seven controlled cross-sectional studies). Most study sites (56/69) were in low-or lower middle-income settings. Among studies using experimental study designs, most interventions included child faeces disposal messages along with other health education messages or other water, sanitation, and hygiene (WASH) hardware and software components. Among observational studies, the main risk factors relevant to this review were safe disposal of faeces in the latrine or defecation of children under five years of age in a latrine.

\section{Education and hygiene promotion interventions, including child faeces disposal messages (no hardware provision)}

Four RCTs found that diarrhoea incidence was lower, reducing the risk by an estimated $30 \%$ in children under six years old (rate ratio 0.71 , $95 \%$ confidence interval $(\mathrm{Cl}) 0.59$ to $0.86 ; 2$ trials, low-certainty evidence). Diarrhoea prevalence measured in two other RCTs in children under five years of age was lower, but evidence was low-certainty (risk ratio (RR) $0.93,95 \% \mathrm{Cl} 0.84$ to 1.04 ; low-certainty evidence).

Two controlled cohort studies that evaluated such an intervention in Bangladesh did not detect a difference on diarrhoea prevalence (RR $0.91,95 \%$ Cl 0.64 to 1.28; very low-certainty evidence). Two controlled cross-sectional studies that evaluated the Health Extension Package in Ethiopia were associated with a lower two-week diarrhoea prevalence in 'model' households than in 'non-model households' (odds ratio (OR) $0.26,95 \% \mathrm{Cl} 0.16$ to 0.42 ; very low-certainty evidence).

\section{Programmes to end open defecation by all (termed community-led total sanitation (CLTS) interventions plus adaptations)}

Four RCTs measured diarrhoea prevalence and did not detect an effect in children under five years of age (RR 0.92, 95\% Cl 0.79 to 1.07 ; moderate-certainty evidence). The analysis of two trials did not demonstrate an effect of the interventions on STH infection prevalence in children (pooled RR 1.03, 95\% Cl 0.64 to 1.65; low-certainty evidence).

One controlled cross-sectional study compared the prevalence of STH infection in open defecation-free (ODF) villages that had received a CLTS intervention with control villages and reported a higher level of STH infection in the intervention villages (RR $2.51,95 \% \mathrm{Cl} 1.74$ to 3.62; very low-certainty evidence).

\section{Sanitation hardware and behaviour change interventions, that included child faeces disposal hardware and messaging}

Two RCTs had mixed results, with no overall effect on diarrhoea prevalence demonstrated in the pooled analysis ( $\mathrm{RR} 0.79,95 \% \mathrm{Cl} 0.49$ to 1.26; very low-certainty evidence).

\section{WASH hardware and education/behaviour change interventions}

One RCT did not demonstrate an effect on diarrhoea prevalence (RR 1.15, 95\% Cl 0.93 to 1.41; very low-certainty evidence).

Two CBAs reported that the intervention reduced diarrhoea incidence by about a quarter in children under five years of age, but evidence was very low-certainty (rate ratio $0.77,95 \% \mathrm{Cl} 0.71$ to 0.84 ). Another CBA reported that the intervention reduced the prevalence of STH in an intervention village compared to a control village, again with GRADE assessed at very low-certainty $(\mathrm{OR} 0.17,95 \% \mathrm{Cl} 0.02$ to 0.73$)$.

\section{Case-control studies}

Pooled results from case-control studies that presented data for child faeces disposal indicated that disposal of faeces in the latrine was associated with lower odds of diarrhoea among all ages (OR $0.73,95 \% \mathrm{Cl}$ : 0.62 to $0.85 ; 23$ comparisons; very low-certainty evidence). Pooled results from case-control studies that presented data for children defecating in the latrine indicated that children using the latrine was associated with lower odds of diarrhoea in all ages (OR 0.54, 95\% $\mathrm{Cl} 0.33$ to $0.90 ; 7$ studies; very low-certainty evidence).

\section{Authors' conclusions}

Evidence suggests that the safe disposal of child faeces may be effective in preventing diarrhoea. However, the evidence is limited and of low certainty. The limited research on STH infections provides only low and very-low certainty evidence around effects, which means there is currently no reliable evidence that interventions to improve safe disposal of child faeces are effective in preventing such STH infections.

While child faeces may represent a source of exposure to young children, interventions generally only address it as part of a broader sanitation initiative. There is a need for RCTs and other rigorous studies to assess the effectiveness and sustainability of different hardware and software interventions to improve the safe disposal of faeces of children of different age groups.

23 September 2019

Up to date

All studies incorporated from most recent search

All eligible published studies found in the last search (27 Sep, 2018) were included and four ongoing studies identified 


\section{PLAIN LANGUAGE SUMMARY}

\section{Interventions to improve child faeces disposal and prevent diarrhoea and soil-transmitted helminths}

\section{What was the aim of this review?}

The aim of this Cochrane Review was to assess the impact of improved disposal of child faeces on diarrhoea and soil-transmitted helminth (STH) infection. We collected and analysed all relevant studies and found 63 studies covering over 222,800 participants.

\section{Key messages}

We found some evidence that interventions to promote safe disposal of child faeces were protective against diarrhoea. However, the evidence was mixed and its certainty was very low to moderate. We found no evidence that such interventions were protective against STH infections, but the evidence was very limited and the certainty was low to very low. More research is needed to study the health impact of different types of interventions to improve child faeces disposal.

\section{What was studied in this review?}

Diarrhoea and STH infections affect millions of people worldwide, particularly in low-income countries. Diarrhoea and STHs are transmitted through human faeces so the safe containment and management of human excreta has the potential to significantly reduce exposure and disease. An often-neglected source of exposure is from the unsafe disposal of child faeces. Research has shown that even in settings with improved sanitation, child faeces are thrown into refuse piles or elsewhere and not disposed of in latrines as considered safe by the World Health Organization (WHO) and United Nations Children's Fund (UNICEF).

We included 26 studies with experimental designs and 37 observational studies in this review. Most included studies were conducted in low- and middle-income countries.

\section{What were the main results of the review?}

Results from studies using experimental study designs suggest that:

Education and hygiene promotion interventions that included child faeces disposal messages may reduce diarrhoea incidence by about $30 \%$ but did not show an effect on diarrhoea prevalence (low-certainty evidence).

Evidence from interventions that addressed child faeces as part of a wider intervention aimed at ending open defecation by all household members did not detect an effect on diarrhoea prevalence (moderate-certainty evidence) or STH infection (low-certainty evidence).

Sanitation hardware (for example, faeces scoopers, potties) and behaviour change interventions (for example, to increase use of latrines) had mixed results on diarrhoea prevalence, but no effect was demonstrated in the combined analysis (very low-certainty evidence).

Interventions that addressed safe disposal of child faeces education as part of a wider water, sanitation, and hygiene hardware intervention did not demonstrate an effect on diarrhoea prevalence (one study; very low-certainty evidence). Although diarrhoea incidence (two studies) and STH prevalence (one study) were lower, the evidence was very low-certainty so we do not know if this is a true effect.

Results from observational studies (where researchers observe the effect of a treatment without trying to change who is or is not exposed to it) showed mixed results of education and hygiene promotion interventions, with two studies in Bangladesh showing no effect on diarrhoea prevalence (very low-certainty evidence) and two studies in Ethiopia reducing diarrhoea prevalence (very low-certainty evidence). One study evaluating an intervention aimed at ending open defecation found an increase in STH infection the intervention arm (very lowcertainty evidence). Pooled results from other studies that presented data for child faeces disposal indicate that disposal of faeces in the latrine may decrease the odds of diarrhoea by about a quarter among all ages (very low-certainty evidence). Children using the latrine to defecate may reduce the odds of diarrhoea by about half in all ages (very low-certainty evidence). However, given the very low-certainty evidence we are unsure about the effects of these risk factors on diarrhoea.

\section{How up to date was this review?}

We searched for available studies up to 27 September 2018. 


\section{SUMMARY OF FINDINGS}

\section{Summary of findings for the main comparison. Summary of findings table 1}

Education and hygiene promotion intervention compared with no intervention for preventing diarrhoea in low- and middle-income countries

Patient or population: adults and children

\section{Settings: LMICs}

Intervention: education and hygiene promotion intervention that includes promotion of safe child faeces disposal among other promoted behaviours

Comparison: no intervention

\begin{tabular}{|c|c|c|c|c|c|c|}
\hline \multirow[t]{2}{*}{ Outcomes } & \multicolumn{2}{|c|}{ Anticipated absolute effects $(95 \% \mathrm{CI})$} & \multirow{2}{*}{$\begin{array}{l}\text { Relative ef- } \\
\text { fect } \\
(95 \% \mathrm{Cl})\end{array}$} & \multirow{2}{*}{$\begin{array}{l}\text { No of partici- } \\
\text { pants } \\
\text { (studies) }\end{array}$} & \multirow{2}{*}{$\begin{array}{l}\text { Certainty of } \\
\text { the evidence } \\
\text { (GRADE) }^{\star \star}\end{array}$} & \multirow[t]{2}{*}{ Comments } \\
\hline & $\begin{array}{l}\text { Risk with no inter- } \\
\text { vention }\end{array}$ & Risk with intervention & & & & \\
\hline $\begin{array}{l}\text { Diarrhoea prevalence } \\
\text { Cluster RCTs }\end{array}$ & $\begin{array}{l}3 \text { episodes per per- } \\
\text { son per year }\end{array}$ & $\begin{array}{l}2.79 \text { episodes per person } \\
\text { per year ( } 2.52 \text { to } 3.12 \text { ) }\end{array}$ & $\begin{array}{l}\mathbf{R R} \mathbf{0 . 9 3}(0.84 \\
\text { to } 1.04)\end{array}$ & $\begin{array}{l}12,040(2 \\
\text { studies) }\end{array}$ & 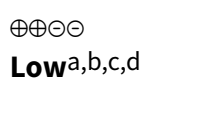 & $\begin{array}{l}\text { The intervention may make little or } \\
\text { no difference to diarrhoea preva- } \\
\text { lence. }\end{array}$ \\
\hline $\begin{array}{l}\text { Diarrhoea prevalence } \\
\text { Controlled cohort studies: } \\
\text { Sanitation Hygiene Educa- } \\
\text { tion and Water Supply in } \\
\text { Bangladesh (SHEWA-B) in- } \\
\text { tervention }\end{array}$ & $\begin{array}{l}3 \text { episodes per per- } \\
\text { son per year }\end{array}$ & $\begin{array}{l}2.73 \text { episodes per person } \\
\text { per year ( } 1.92 \text { to } 3.84)\end{array}$ & $\begin{array}{l}\mathbf{R R} \mathbf{0 . 9 1}(0.64 \\
\text { to } 1.28)\end{array}$ & $\begin{array}{l}\sim 2000 \text { (2 stud- } \\
\text { ies) }\end{array}$ & $\begin{array}{l}\oplus \ominus \odot \odot \\
\text { Very lowa,g,h,i }\end{array}$ & $\begin{array}{l}\text { We are uncertain whether or not } \\
\text { the intervention reduces diarrhoea } \\
\text { prevalence. }\end{array}$ \\
\hline $\begin{array}{l}\text { Diarrhoea prevalence } \\
\text { Controlled cross-section- } \\
\text { al studies: Health Exten- } \\
\text { sion Package intervention } \\
\text { (Ethiopia) }\end{array}$ & $\begin{array}{l}3 \text { episodes per per- } \\
\text { son per year }\end{array}$ & $\begin{array}{l}0.78 \text { episodes per person } \\
\text { per year ( } 0.48 \text { to } 1.26) j\end{array}$ & $\begin{array}{l}\text { OR } 0.26(0.16 \\
\text { to } 0.42)\end{array}$ & $\begin{array}{l}1660 \text { ( } 2 \text { stud- } \\
\text { ies) }\end{array}$ & $\begin{array}{l}\oplus \odot \Theta \odot \\
\text { Very } \\
\text { low }{ }^{a, b, d, k}\end{array}$ & $\begin{array}{l}\text { We are uncertain whether or not } \\
\text { the intervention reduces diarrhoea } \\
\text { prevalence. }\end{array}$ \\
\hline
\end{tabular}

*The assumed risk for diarrhoea is taken from Walker 2012 and represented an estimated mean for the incidence of diarrhoea in LMICs. The corresponding risk (and its $95 \% \mathrm{Cl}$ ) is based on the assumed risk in the comparison group and the relative effect of the intervention (and its $95 \% \mathrm{Cl}$ ).

${ }^{\star \star} \mathrm{RCTs}$ begin as high-certainty evidence and observational studies as low-certainty evidence (Guyatt 2008) 
GRADE Working Group grades of evidence

High certainty: further research is very unlikely to change our confidence in the estimate of effect.

Moderate certainty: further research is likely to have an important impact on our confidence in the estimate of effect and may change the estimate.

Low certainty: further research is very likely to have an important impact on our confidence in the estimate of effect and is likely to change the estimate.

Very low certainty: we are very uncertain about the estimate.

aDowngraded one level for serious risk of bias: the outcome was self-reported diarrhoea, and was susceptible to bias as all studies were unblinded

bNo serious inconsistency.

CDowngraded one level for indirectness: only two studies in low-income countries. Both conducted in rural settings, one in Rwanda and one in Democratic Republic of Congo.

Diarrhoea was only measured in children aged < 3 years in Haggerty 1994 DRC.

dNo serious imprecision.

eNo serious inconsistency: there was considerable statistical heterogeneity $\left(I^{2}=82 \%\right)$; however, there was consistency in the direction of the effect. Possible reasons for heterogeneity included the location of the studies; Stanton 1987 BGD was conducted in urban Bangladesh and Hashi 2017 ETH in rural Ethiopia. Furthermore, the studies used different definitions of diarrhoea and different age groups (aged less than six years for Stanton 1987 BGD and less than five years for Hashi 2017 ETH).

fDowngraded one level for indirectness: only two studies, one in an urban Asian setting (Bangladesh) and one in an African rural setting (Ethiopia).

gDowngraded one level for inconsistency: substantial statistical heterogeneity $\left(I^{2}=55 \%\right)$.

hDowngraded one level for indirectness: only two studies, both conducted in Bangladesh and evaluating the same intervention that was specifically tailored to Bangladesh

iDowngraded one level for imprecision: small sample size and large Cls which included important effects in both directions.

jCalculated using the OR as an approximation for RR.

kDowngraded one level for indirectness: only two studies, both conducted in rural Ethiopia and evaluating an intervention specifically designed for Ethiopia.

\section{Summary of findings 2 . Summary of findings table 2}

\section{CLTS or CLTS adaptation intervention compared with no intervention for preventing diarrhoea and STHs}

Patient or population: adults and children

\section{Settings: LMICS}

Intervention: CLTS or CLTS adaptation interventions, aiming to end open defecation by all

Comparison: no intervention

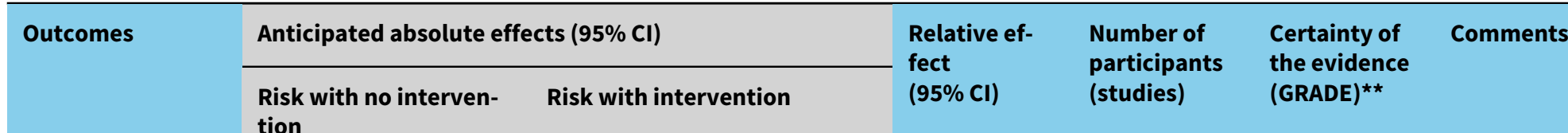

(95\% CI)

(studies)

(GRADE)** 


\begin{tabular}{|c|c|c|c|c|c|c|c|}
\hline 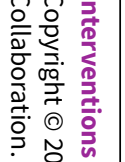 & $\begin{array}{l}\text { Diarrhoea preva- } \\
\text { lence } \\
\text { RCTs }\end{array}$ & $\begin{array}{l}3 \text { episodes per person } \\
\text { per year }\end{array}$ & $\begin{array}{l}2.76 \text { episodes per person per } \\
\text { year ( } 2.37 \text { to } 3.21 \text { ) }\end{array}$ & $\begin{array}{l}\mathbf{R R} 0.92(0.79 \\
\text { to } 1.07)\end{array}$ & $\begin{array}{l}16,033(4 \\
\text { studies) }\end{array}$ & $\begin{array}{l}\oplus \oplus \oplus \ominus \\
\text { Moder- } \\
\text { ate }^{a, b, c, d}\end{array}$ & $\begin{array}{l}\text { The intervention probably makes } \\
\text { little or no difference to diar- } \\
\text { rhoea prevalence. }\end{array}$ \\
\hline 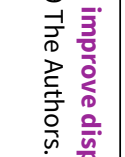 & $\begin{array}{l}\text { STH infection (any } \\
\text { helminth) } \\
\text { RCTs }\end{array}$ & $\begin{array}{l}4.8 \text { out of } 100 \text { people } \\
\text { with any helminths }\end{array}$ & $\begin{array}{l}4.9 \text { out of } 100 \text { people with any } \\
\text { helminths ( } 3.07 \text { to } 7.92)\end{array}$ & $\begin{array}{l}\mathbf{R R} \mathbf{1 . 0 3}(0.64 \\
\text { to } 1.65)\end{array}$ & $\begin{array}{l}3480 \text { ( } 2 \text { stud- } \\
\text { ies) }\end{array}$ & $\begin{array}{l}\oplus \oplus \ominus \ominus \\
\text { Low }^{b, e, f, g}\end{array}$ & $\begin{array}{l}\text { The intervention may make little } \\
\text { or no difference to STH infection. }\end{array}$ \\
\hline 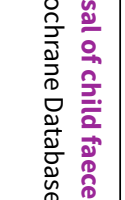 & $\begin{array}{l}\text { STH infection (any } \\
\text { helminth) } \\
\text { Controlled cross- } \\
\text { sectional study }\end{array}$ & $\begin{array}{l}4.8 \text { out of } 100 \text { people } \\
\text { with any helminths }\end{array}$ & $\begin{array}{l}12 \text { of } 100 \text { people with any } \\
\text { helminths ( } 8.4 \text { to } 17.4)\end{array}$ & $\begin{array}{l}\mathbf{R R} \mathbf{2 . 5 1}(1.74 \\
\text { to } 3.62)\end{array}$ & 341 (1 study) & $\begin{array}{l}\oplus \ominus \ominus \ominus \\
\text { Very low- } \\
\text { b,d,e,h }\end{array}$ & $\begin{array}{l}\text { We are uncertain whether or not } \\
\text { the intervention increases STH } \\
\text { infection. }\end{array}$ \\
\hline 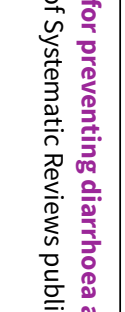 & $\begin{array}{l}{ }^{\star} \text { The assumed risk } \mathrm{f} \\
\text { in stool is an averag } \\
95 \% \mathrm{Cl}) \text { is based on } \\
{ }^{\star \star} \mathrm{RCTs} \text { begin as higl } \\
\mathrm{Cl} \text { : confidence inter } \\
\text { mitted helminth. }\end{array}$ & $\begin{array}{l}\text { diarrhoea is taken from } \\
\text { of the control group risk } \\
\text { le assumed risk in the co } \\
\text { certainty evidence and o } \\
\text { l; CLTS: community-led }\end{array}$ & $\begin{array}{l}\text { Iker } 2012 \text { and represented an es } \\
\text { Cameron } 2013 \text { INA (control grou } \\
\text { arison group and the relative ef } \\
\text { rvational studies as low-certain } \\
\text { al sanitation; LMICs: low- and m }\end{array}$ & $\begin{array}{l}\text { ated average fo } \\
\text { sk: } 3.9 \% \text { ) Patil } 2 \\
\text { of the interven } \\
\text { vidence (Guyatt } \\
\text { e-income count }\end{array}$ & $\begin{array}{l}\text { he incidence o } \\
14 \text { IND (control } \\
\text { on (and its 95\% } \\
\text { 008) } \\
\text { ies; RCT: rando }\end{array}$ & $\begin{array}{l}\text { iarrhoea in L } \\
\text { oup risk: } 5.6 \\
\text { I). } \\
\text { zed controll }\end{array}$ & $\begin{array}{l}\text { The assumed risk for any helminth } \\
\text { he corresponding risk (and its } \\
\text { al; RR: risk ratio; STH: soil-trans- }\end{array}$ \\
\hline 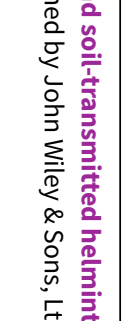 & $\begin{array}{l}\text { GRADE Working Gro } \\
\text { High certainty: furt } \\
\text { Moderate certaint } \\
\text { Low certainty: furt } \\
\text { Very low certainty: }\end{array}$ & $\begin{array}{l}\text { grades of evidence } \\
\text { er research is very unlike } \\
\text { further research is likely } \\
\text { ve research is very likely t } \\
\text { very uncertain abc }\end{array}$ & $\begin{array}{l}\text { o change our confidence in the } \\
\text { have an important impact on ou } \\
\text { ave an important impact on our } \\
\text { the estimate. }\end{array}$ & $\begin{array}{l}\text { nate of effect. } \\
\text { nfidence in the } \\
\text { fidence in the e }\end{array}$ & $\begin{array}{l}\text { timate of effec } \\
\text { imate of effect }\end{array}$ & $\begin{array}{l}\text { nd may chan } \\
\text { d is likely to }\end{array}$ & $\begin{array}{l}\text { he estimate. } \\
\text { nge the estimate. }\end{array}$ \\
\hline 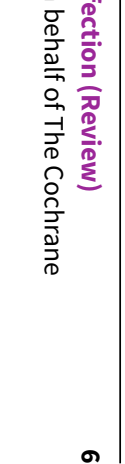 & $\begin{array}{l}\text { aDowngraded one lev } \\
\text { bNo serious inconsist } \\
\text { cNo serious indirectn } \\
\text { dNo serious imprecisi } \\
\text { eNo serious risk of bia } \\
\text { fDowngraded one lev } \\
\text { and India). } \\
\text { gDowngraded one lev } \\
\text { hDowngraded two ley } \\
\text { status of school-age a } \\
\text { izations to other setti }\end{array}$ & $\begin{array}{l}\text { I for serious risk of bias: } \\
\text { cy. } \\
\text { s: four studies, all condu } \\
\text { : although assessors and } \\
\text { for indirectness: only tw } \\
\text { I for imprecision: small s } \\
\text { ls for serious indirectnes } \\
\text { d preschool-age childre } \\
\text { s. }\end{array}$ & $\begin{array}{l}\text { outcome was measured as self-1 } \\
\text { d in rural settings of low- and lo } \\
\text { ticipants were not blinded to th } \\
\text { CTs assessed the impact of CLTS/ } \\
\text { ole size and large Cls which inclu } \\
\text { nly one small study conducted i } \\
\text { two open defecation-free villag }\end{array}$ & $\begin{array}{l}\text { rted diarrhoea, } \\
\text { middle-income } \\
\text { tervention, the } \\
\text { S adaptation int } \\
\text { important effec } \\
\text { ral Philippines. } \\
\text { nd two villages }\end{array}$ & $\begin{array}{l}\text { Ind is susceptib } \\
\text { ettings; two in } \\
\text { utcome was ob } \\
\text { ventions on ST } \\
\text { in both directi } \\
\text { his single contr } \\
\text { lat did not ben }\end{array}$ & $\begin{array}{l}\text { to bias as all } \\
\text { ica and two } \\
\text { tive. } \\
\text { Both studies } \\
\text { s. } \\
\text { ed cross-sec } \\
\text { from CLTS. I }\end{array}$ & $\begin{array}{l}\text { ies were unblinded. } \\
\text { ia. } \\
\text { conducted in rural Asia (in Indonesia } \\
\text { not possible to make broad general- }\end{array}$ \\
\hline
\end{tabular}




\section{Summary of findings 3 . Summary of findings table 3}

\section{Sanitation hardware and behaviour change intervention compared with no intervention for preventing diarrhoea}

Patient or population: adults and children

Settings: LMICS

Intervention: sanitation hardware and behaviour change interventions, which include child faeces management hardware and promotion

Comparison: no intervention

\begin{tabular}{|c|c|c|c|c|c|c|}
\hline \multirow[t]{2}{*}{ Outcomes } & \multicolumn{2}{|c|}{ Anticipated absolute effects $(95 \% \mathrm{Cl})$} & \multirow{2}{*}{$\begin{array}{l}\text { Relative effect } \\
(95 \% \mathrm{CI})\end{array}$} & \multirow{2}{*}{$\begin{array}{l}\text { Number of par- } \\
\text { ticipants } \\
\text { (studies) }\end{array}$} & \multirow{2}{*}{$\begin{array}{l}\text { Certainty of } \\
\text { the evidence } \\
\text { (GRADE) }^{\star \star}\end{array}$} & \multirow[t]{2}{*}{ Comments } \\
\hline & $\begin{array}{l}\text { Risk with no interven- } \\
\text { tion }\end{array}$ & Risk with intervention & & & & \\
\hline $\begin{array}{l}\text { Diarrhoea } \\
\text { prevalence }\end{array}$ & $\begin{array}{l}3 \text { episodes per person per } \\
\text { year }\end{array}$ & $\begin{array}{l}2.37 \text { episodes per person } \\
\text { per year ( } 1.47 \text { to } 3.78)\end{array}$ & $\begin{array}{l}\mathbf{R R} \mathbf{0 . 7 9} \text { (0.49 to } \\
1.26)\end{array}$ & 9558 ( 2 studies) & $\begin{array}{l}\oplus \ominus \odot \odot \\
\text { Very lowa,b,c,d }\end{array}$ & $\begin{array}{l}\text { We are uncertain whether or } \\
\text { not the intervention reduces di- } \\
\text { arrhoea prevalence. }\end{array}$ \\
\hline
\end{tabular}

*The assumed risk for diarrhoea is taken from Walker 2012 and represented an estimated mean for the incidence of diarrhoea in LMICs. The corresponding risk (and its $95 \% \mathrm{Cl}$ ) is based on the assumed risk in the comparison group and the relative effect of the intervention (and its $95 \% \mathrm{Cl}$ ).

${ }^{\star \star} \mathrm{RCTs}$ begin as high-certainty evidence and observational studies as low-certainty evidence (Guyatt 2008).

Cl: confidence interval; LMICs: low- and middle-income countries; RR: risk ratio.

GRADE Working Group grades of evidence

High certainty: further research is very unlikely to change our confidence in the estimate of effect.

Moderate certainty: further research is likely to have an important impact on our confidence in the estimate of effect and may change the estimate.

Low certainty: further research is very likely to have an important impact on our confidence in the estimate of effect and is likely to change the estimate.

Very low certainty: we are very uncertain about the estimate.

a Downgraded one level for serious risk of bias: the outcome was measured as self-reported diarrhoea, and was susceptible to bias as both studies were unblinded.

bDowngraded one level for serious inconsistency: considerable statistical heterogeneity $\left(l^{2}=90 \%\right)$. There were large effects in Bangladesh but not in Kenya.

cDowngraded one level for indirectness: only two studies, both conducted in rural areas, one in Bangladesh and one in Kenya.

dNo serious imprecision. The $95 \% \mathrm{Cl}$ of the pooled effect included important effects in both directions, but this imprecision was a result of the heterogeneity between studies. 
Summary of findings 4. Summary of findings table 4

WASH hardware and education/behaviour change interventions compared with no intervention for preventing diarrhoea and STHs

Patient or population: adults and children

Settings: LMICS

Intervention: WASH hardware interventions that included child faeces disposal messaging in their education or behaviour change component

Comparison: no intervention

\begin{tabular}{|c|c|c|c|c|c|c|}
\hline \multirow[t]{2}{*}{ Outcomes } & \multicolumn{2}{|c|}{ Anticipated absolute effects $(95 \% \mathrm{Cl})$} & \multirow{2}{*}{$\begin{array}{l}\text { Relative effect } \\
(95 \% \mathrm{CI})\end{array}$} & \multirow{2}{*}{$\begin{array}{l}\text { Number of } \\
\text { participants } \\
\text { (studies) }\end{array}$} & \multirow{2}{*}{$\begin{array}{l}\text { Certainty of } \\
\text { the evidence } \\
(\text { GRADE) }\end{array}$} & \multirow[t]{2}{*}{ Comments } \\
\hline & $\begin{array}{l}\text { Risk with no interven- } \\
\text { tion }\end{array}$ & Risk with intervention & & & & \\
\hline $\begin{array}{l}\text { Diarrhoea } \\
\text { prevalence } \\
\text { RCTs }\end{array}$ & $\begin{array}{l}3 \text { episodes per person } \\
\text { per year }\end{array}$ & $\begin{array}{l}3.45 \text { episodes per person per year } \\
\text { ( } 2.79 \text { to } 4.23 \text { ) }\end{array}$ & $\begin{array}{l}\text { RR } 1.15 \text { (0.93 to } \\
1.41)\end{array}$ & 3650 ( 1 study) & $\begin{array}{l}\oplus \odot \odot \odot \\
\text { Very } \\
\text { low } \\
\text { lo,b,c,d }\end{array}$ & $\begin{array}{l}\text { We are uncertain whether or not } \\
\text { the intervention reduces diarrhoea } \\
\text { prevalence. }\end{array}$ \\
\hline $\begin{array}{l}\text { Diarrhoea in- } \\
\text { cidence } \\
\text { CBAs }\end{array}$ & $\begin{array}{l}3 \text { episodes per person } \\
\text { per year }\end{array}$ & $\begin{array}{l}2.31 \text { episodes per person per year } \\
(2.13 \text { to } 2.52 \text { ) }\end{array}$ & $\begin{array}{l}\text { Rate ratio } 0.77 \\
(0.71 \text { to } 0.84)\end{array}$ & $\begin{array}{l}1028 \text { ( } 2 \text { stud- } \\
\text { ies) }\end{array}$ & $\begin{array}{l}\oplus \odot \Theta \odot \\
\text { Very } \\
\text { low }{ }^{a, b, d, e}\end{array}$ & $\begin{array}{l}\text { We are uncertain whether or not } \\
\text { the intervention reduces diarrhoea } \\
\text { incidence. }\end{array}$ \\
\hline $\begin{array}{l}\text { STH infec- } \\
\text { tion (any } \\
\text { helminth) }\end{array}$ & $\begin{array}{l}4.8 \text { out of } 100 \text { people } \\
\text { with any helminths }\end{array}$ & $\begin{array}{l}0.82 \text { of } 100 \text { people with any } \\
\text { helminths }(0.096 \text { to } 3.5)\end{array}$ & $\begin{array}{l}\text { OR } 0.17(0.02 \text { to } \\
0.73)^{f}\end{array}$ & 99 (1 study) & $\begin{array}{l}\oplus \odot \odot \odot \\
\text { Very low } b, g, h\end{array}$ & $\begin{array}{l}\text { We are uncertain whether or not } \\
\text { the intervention reduces STH infec- } \\
\text { tion. }\end{array}$ \\
\hline CBAs & & & & & & \\
\hline
\end{tabular}

*The assumed risk for diarrhoea is taken from Walker 2012 and represented an estimated mean for the incidence of diarrhoea in LMICs. The assumed risk for any helminth in stool was a mean of the control group risks of Cameron 2013 INA (control group risk: 3.9\%) and Patil 2014 IND (control group risk: 5.6\%). The corresponding risk (and its $95 \% \mathrm{Cl}$ ) is based on the assumed risk in the comparison group and the relative effect of the intervention (and its $95 \% \mathrm{Cl}$ ).

${ }^{\star *}$ RCTs begin as high-certainty evidence and observational studies as low-certainty evidence (Guyatt 2008).

CBA: controlled before-and-after study; CI: confidence interval; LMICs: low- and middle-income countries; OR: odds ratio; RCT: randomized controlled trial; RR: risk ratio; STH: soil-transmitted helminth; WASH: water, sanitation, and hygiene.

GRADE Working Group grades of evidence

High certainty: further research is very unlikely to change our confidence in the estimate of effect.

Moderate certainty: further research is likely to have an important impact on our confidence in the estimate of effect and may change the estimate.

Low certainty: further research is very likely to have an important impact on our confidence in the estimate of effect and is likely to change the estimate.

Very low certainty: we are very uncertain about the estimate. 
aDowngraded one level for serious risk of bias: the outcome was measured as self-reported diarrhoea, and was susceptible to bias as all studies were unblinded.

bNo serious inconsistency.

cDowngraded two levels for serious indirectness: this single RCT from Zimbabwe evaluated the provision of a WASH hardware and behaviour change intervention. It was not possible to make broad generalizations to other settings.

dNo serious imprecision.

eDowngraded one level for indirectness: only two studies, both in rural Bangladesh.

fCalculated using the OR as an approximation for RR.

gDowngraded two levels for serious indirectness: only one study that was conducted in rural Indonesia. This CBA study compared STH infection in one control village and one intervention village, where residents received a latrine constructed with local materials and health education. It was not possible to make broad generalizations to other settings.

howngraded one level for imprecision: small sample size and large $\mathrm{Cls}$.

\section{Summary of findings 5 . Summary of findings table 5}

\section{Disposal of child faeces in a latrine vs elsewhere for preventing diarrhoea (findings from case-control studies)}

Patient or population: adults and children

Settings: all settings

Intervention: child faeces disposal in latrine

Comparison: no intervention

\begin{tabular}{|c|c|c|c|c|c|c|}
\hline \multirow[t]{2}{*}{ Outcomes } & \multicolumn{2}{|c|}{ Anticipated absolute effects $(95 \% \mathrm{CI})$} & \multirow{2}{*}{$\begin{array}{l}\text { Relative ef- } \\
\text { fect } \\
(95 \% \mathrm{CI})\end{array}$} & \multirow{2}{*}{$\begin{array}{l}\text { Number of par- } \\
\text { ticipants } \\
\text { (studies) }\end{array}$} & \multirow{2}{*}{$\begin{array}{l}\text { Certainty of the } \\
\text { evidence } \\
\text { (GRADE) })^{\star \star}\end{array}$} & \multirow[t]{2}{*}{ Comments } \\
\hline & $\begin{array}{l}\text { Risk with no interven- } \\
\text { tion }\end{array}$ & Risk with intervention & & & & \\
\hline $\begin{array}{l}\text { Diarrhoea } \\
\text { Case-control studies: } \\
\text { child faeces disposal in } \\
\text { latrine }\end{array}$ & $\begin{array}{l}3 \text { episodes per person } \\
\text { per year }\end{array}$ & $\begin{array}{l}2.19 \text { episodes per person per } \\
\text { year ( } 1.86 \text { to } 2.55)^{a}\end{array}$ & $\begin{array}{l}\text { OR } 0.73(0.62 \\
\text { to } 0.85)\end{array}$ & $\begin{array}{l}\text { 32,957 (17 stud- } \\
\text { ies) }\end{array}$ & $\begin{array}{l}\oplus \ominus \ominus \ominus \\
\text { Very low } b, c, d\end{array}$ & $\begin{array}{l}\text { We are uncertain } \\
\text { whether or not the in- } \\
\text { tervention reduces diar- } \\
\text { rhoea. }\end{array}$ \\
\hline
\end{tabular}

*The assumed risk for diarrhoea was taken from Walker 2012 and represented an estimated mean for the incidence of diarrhoea in LMICs. The corresponding risk (and its $95 \% \mathrm{Cl}$ ) was based on the assumed risk in the comparison group and the relative effect of the intervention (and its $95 \% \mathrm{Cl}$ ).

${ }^{\star \star}$ RCTs begin as high-certainty evidence and observational studies as low-certainty evidence (Guyatt 2008).

CI: confidence interval; LMIC: low- and middle-income countries; OR: odds ratio.

GRADE Working Group grades of evidence

High certainty: further research is very unlikely to change our confidence in the estimate of effect.

Moderate certainty: further research is likely to have an important impact on our confidence in the estimate of effect and may change the estimate.

Low certainty: further research is very likely to have an important impact on our confidence in the estimate of effect and is likely to change the estimate. 
acalculated using the OR as an approximation for RR

bDowngraded one level for serious inconsistency: substantial statistical heterogeneity $\left(I^{2}=71 \%\right)$, which was not completely explained by the subgroup analyses.

cNo serious indirectness: these 17 studies were from a variety of low-, middle-, and high-income countries, in urban, rural, and periurban areas.

dNo serious imprecision.

Summary of findings 6 . Summary of findings table 6

Defecation of children in a latrine vs elsewhere for preventing diarrhoea (findings from case-control studies)

Patient or population: adults and children

\section{Settings: LMIC}

Intervention: defecation of children in latrine

Comparison: no intervention

\begin{tabular}{|c|c|c|c|c|c|c|}
\hline \multirow[t]{2}{*}{ Outcomes } & \multicolumn{2}{|c|}{ Anticipated absolute effects $(95 \% \mathrm{CI})$} & \multirow{2}{*}{$\begin{array}{l}\text { Relative ef- } \\
\text { fect } \\
(95 \% \mathrm{Cl})\end{array}$} & \multirow{2}{*}{$\begin{array}{l}\text { Number of par- } \\
\text { ticipants } \\
\text { (studies) }\end{array}$} & \multirow{2}{*}{$\begin{array}{l}\text { Certainty of the } \\
\text { evidence } \\
\text { (GRADE) }\end{array}$} & \multirow[t]{2}{*}{ Comments } \\
\hline & $\begin{array}{l}\text { Risk with no interven- } \\
\text { tion }\end{array}$ & Risk with intervention & & & & \\
\hline $\begin{array}{l}\text { Diarrhoea } \\
\text { Case-control studies: } \\
\text { defecation of children in } \\
\text { latrine }\end{array}$ & $\begin{array}{l}3 \text { episodes per person } \\
\text { per year }\end{array}$ & $\begin{array}{l}1.62 \text { episodes per person per } \\
\text { year ( } 0.99 \text { to } 2.70)^{a}\end{array}$ & $\begin{array}{l}\text { OR } 0.54(0.33 \\
\text { to } 0.90)\end{array}$ & 2996 (7 studies) & $\begin{array}{l}\oplus \ominus \ominus \ominus \\
\text { Very low } w^{b, c, d}\end{array}$ & $\begin{array}{l}\text { We are uncertain } \\
\text { whether or not the in- } \\
\text { tervention reduces diar- } \\
\text { rhoea. }\end{array}$ \\
\hline
\end{tabular}

*The assumed risk for diarrhoea was taken from Walker 2012 and represented an estimated mean for the incidence of diarrhoea in LMICs. The corresponding risk (and its $95 \% \mathrm{Cl}$ ) was based on the assumed risk in the comparison group and the relative effect of the intervention (and its $95 \% \mathrm{Cl}$ ).

${ }^{\star *}$ RCTs begin as high-certainty evidence and observational studies as low-certainty evidence (Guyatt 2008).

Cl: confidence interval; LMIC: low- and middle-income countries; OR: odds ratio.

GRADE Working Group grades of evidence

High certainty: further research is very unlikely to change our confidence in the estimate of effect.

Moderate certainty: further research is likely to have an important impact on our confidence in the estimate of effect and may change the estimate.

Low certainty: further research is very likely to have an important impact on our confidence in the estimate of effect and is likely to change the estimate.

Very low certainty: we are very uncertain about the estimate. 


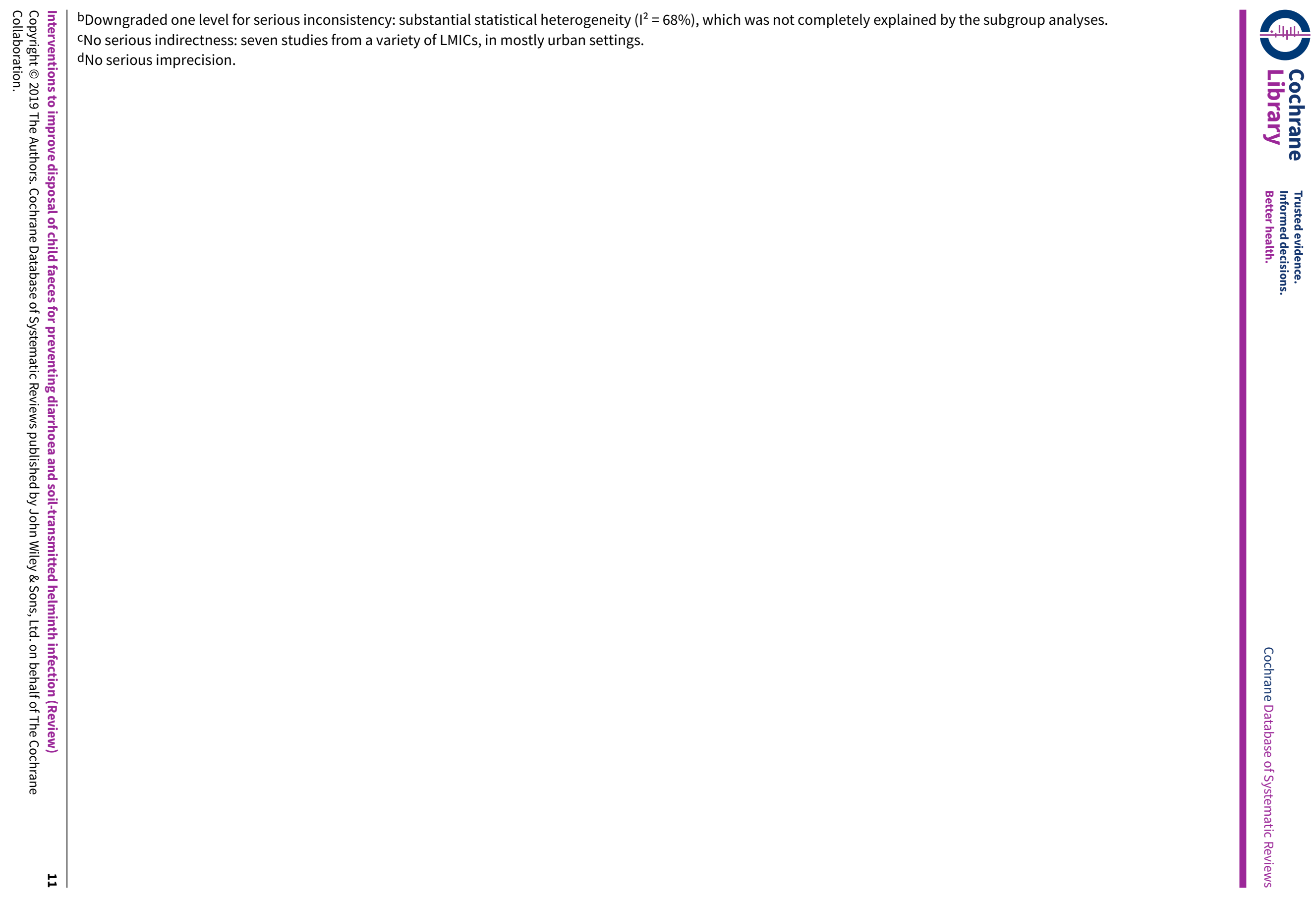




\section{B A C K G R O U N D}

\section{Epidemiology and transmission of diarrhoeal disease and soil-transmitted helminth infection}

Despite advances in prevention and treatment, diarrhoea and soiltransmitted helminth (STH) infections still represent a large disease burden, particularly in low-income countries. Diarrhoeal diseases account for an estimated 1.65 million deaths annually worldwide and rank eighth globally for leading causes of death among all ages (GBD 2018). Among children under the age of five years, diarrhoea kills more than 440,000 children annually, making it the fifth leading cause of death in that age group (GBD 2018). Over five billion people worldwide, including one billion school-aged children (aged five to 14 years), are at risk of infection with at least one STH species (Pullan 2012). The three STHs responsible for most infections are Ascaris lumbricoides, Trichuris trichiura, and hookworms (Ancylostoma duodenale or Necator americanus), with 819 million, 464.6 million, and 438.9 million people infected in 2010 , respectively (Pullan 2014).

The pathogens that cause diarrhoea are mainly transmitted via the faecal-oral route (Byers 2001). Pathogens from contaminated faeces can be passed on to a new susceptible host via contaminated hands, drinking water, soil, flies, or by ingesting contaminated food (Wagner 1958). The settings, pathogens, and their prevalence in different populations will determine the importance of each transmission route (Brown 2013). The symptoms of diarrhoea and course of disease vary with age, nutritional status, and immune status of the infected person, and the causative pathogens (Clasen 2010). The main characteristics of infection are changes in stool consistency, increases in volume or fluidity, and increased frequency of defecation (Thapar 2004). The three clinical presentations of diarrhoea are: acute watery diarrhoea lasting several hours or days; acute bloody diarrhoea (dysentery); and persistent diarrhoea lasting 14 days or more (Heymann 2008). The direct threat from acute watery diarrhoea is dehydration, and loss of fluids and electrolytes. Severe dehydration can result in death if untreated (Keusch 2006).

STHs are transmitted via ingestion of STH eggs (A lumbricoides and $T$ trichiura) or larvae (A duodenale), or via penetration of third-stage larvae (hookworms) (Bethony 2006). The larvae go through several developmental stages in the human host and, depending on the species, the adult parasites can settle in different parts of the gastrointestinal (Gl) tract, where they can live for several years, mating and producing eggs that are passed in the faeces (Bethony 2006). The eggs (A lumbricoides and $T$ trichiura) and larvae (hookworm) can survive in the soil for several months (eggs) or several weeks (larvae), depending on the environmental conditions, including humidity, soil moisture, and temperature (Brooker 2006). Morbidity caused by STHs is linked to the intensity of infection, which is the number of worms per human host measured by the number of eggs per gram of faeces (Bethony 2006). STHs infections can have several clinical features, which can be classified into acute manifestations linked to larval migrations through the skin and intestines, and acute and chronic manifestations associated with parasite presence in the GI tract (Bethony 2006).

An additional risk of contamination of the environment with faeces, including those of children, is that it may result in extended exposure of children to faecal pathogens which may lead to environmental enteric dysfunction (EED), a disorder of the small intestine that is characterized by villous atrophy, crypt hyperplasia, in- flammatory cell infiltrate, increased permeability, and malabsorption (Humphrey 2009; Mbuya 2016). EED is thought to lead to under nutrition and growth faltering (Humphrey 2009; Lin 2013; Mbuya 2016).

In addition to the direct health consequences of diarrhoeal diseases and STHs infections, they have longer-term impacts on human development due to malabsorption and malnutrition (resulting in stunting and chronic anaemia), and on capacity (via lower cognition, school absenteeism and inability to work), which in turn can have impacts on development and poverty (Harhay 2010). STHs are believed to be one of the main causes of physical and intellectual growth retardation in the world (Bethony 2006).

Furthermore, enteric infections or stunting can predispose to obesity and associated comorbidities (diabetes, hypertension, cardiovascular diseases), increasing healthcare costs which in turn contributes to poverty (Guerrant 2013).

\section{Sanitation and disposal of child faeces}

As the aetiological agents associated with diarrhoea and STHs are transmitted through faeces, the safe collection and disposal of human excreta has the potential to reduce exposure and disease. When $B M J$ readers were asked to vote on the "greatest medical advance" since 1840, they chose the sanitary revolution (the introduction of clean water and sewage disposal) over antibiotics, anaesthesia, vaccines, and germ theory (Ferriman 2007). Large-scale efforts have been made to increase coverage of improved sanitation, most recently as part of the Millennium Development Goal (MDG) sanitation target of halving the proportion of the population without access to basic sanitation by 2015 (UN 2013). However, this target was missed by almost 700 million people and 2.4 billion people were still without improved sanitation in 2015, including almost one billion people practicing open defecation (WHO/UNICEF 2015a). The post-2015 sustainable development goals (SDGs) include goal 6 "Ensure availability and sustainable management of water and sanitation for all" with target 6.2 aiming, by 2030, to "achieve access to adequate and equitable sanitation and hygiene for all and end open defecation, paying special attention to the needs of women and girls and those in vulnerable situations" (UN 2016).

A series of published systematic reviews has consistently concluded that sanitation interventions are effective in preventing diarrhoea and STH infections. Esrey 1991 reported a 22\% median reduction in diarrhoea from 11 observational studies and $36 \%$ reduction from five rigorous studies. They also reported reduction in Ascaris and hookworm from water supply and sanitation interventions, especially on the reduction in disease intensity (egg counts). Fewtrell 2005 reported a pooled risk ratio (RR) for diarrhoea of 0.68 (95\% confidence interval $(\mathrm{Cl}) 0.53$ to 0.87 ) from two intervention studies. Waddington 2009 reported a pooled RR for diarrhoea of 0.63 ( $95 \% \mathrm{Cl} 0.43$ to 0.93 ) from six controlled studies among children. Clasen 2010 found a consistent protective effect against diarrhoea among 13 intervention studies but noted that nearly all involved water or hygiene (various hygiene promotion, for example handwashing with soap, safe household water storage, etc.) interventions in addition to sanitation (interventions to introduce or expand the provision or use of facilities for excreta disposal). Norman 2010 reported that sewerage led to a $30 \%$ reduction in diarrhoea (RR $0.70,95 \% \mathrm{Cl} 0.58$ to 0.85 ) among 17 observational studies. Ziegelbauer 2012 reported that sanitation interventions were 
protective against Ascaris, Trichuris, and hookworm, while Strunz 2014 found that access to sanitation was associated with reduced odds of infection with any STH, Ascaris, and Trichuris but not hookworm. Freeman 2017 found that sanitation was associated with $12 \%$ lower odds of diarrhoea (OR $0.88,95 \% \mathrm{Cl} 0.83$ to $0.92 ; 27$ studies), when restricted to the 16 intervention studies, the protective effect doubled to $23 \%$ (OR $0.77,95 \% \mathrm{Cl} 0.66$ to 0.91 ). Freeman 2017 also found that sanitation was associated with lower odds of infection of Ascaris, Trichuris, hookworm, and Strongyloides stercoralis. Wolf 2018 found that sanitation interventions were associated with $25 \%$ reduction in diarrhoeal morbidity (RR $0.75,95 \% \mathrm{Cl} 0.63$ to 0.88 ; 22 studies).

However, these reviews focused on interventions to improve coverage, use, or functionality of sanitation facilities or services. Only one systematic review specifically addressed the disposal of child faeces, another source of exposure even among households with improved sanitation. The review, with different inclusion criteria to the current one, concluded that the health impact of improving child faeces disposal was inconclusive (Morita 2016). Our rationale for focusing on child faeces disposal was that the unsafe disposal of child faeces may represent a more important health risk to children, caregivers, and other community members than faeces of adults. This is because young children have the highest incidence of enteric infections (Walker 2012), and their faeces are most likely to contain infectious agents (Feachem 1983). Young children are more likely to defecate in places where susceptible children could be exposed (Lanata 1998). This exposure is worse for other young children due to the amount of time they spend on the ground and their exploratory behaviours, including putting fingers and fomites in their mouths, and common behaviours such as geophagia (intentional consumption of soil) (Moya 2004; Ngure 2013; Young 2011). Perhaps for these reasons, the World Health Organization (WHO) and the United Nations Children's Fund (UNICEF) Joint Monitoring Programme for Water Supply and Sanitation (JMP), which was charged with assessing progress toward the MDG sanitation targets, treated disposal of child faeces that were not deposited in a latrine or buried as unsanitary (WHO/UNICEF 2006). The JMP, which will also monitor progress towards SDGs, will classify the following methods for disposal of child stools as appropriate methods: the child using an improved toilet/latrine or the caretaker putting/rinsing stools into an improved toilet/latrine. Disposal with solid waste will only be considered appropriate if solid waste is stored, collected and disposed of in a sanitary manner (WHO/UNICEF 2018).

Only one recent peer-reviewed study has summarized the evidence on the impact of child faeces disposal on human health. However, it had different inclusion criteria to the current review, resulting in far fewer studies (eight) and included no quantitative analysis (Morita 2016). In an unpublished review and meta-analysis of 10 observational studies published between 1987 and 2001, Gil 2004 found that child faeces disposal behaviours considered risky (open defecation, stool disposal in the open, stools not removed from soil, stools seen in household soil, and children seen eating faeces) were associated with a $23 \%$ increase in risk of diarrhoeal diseases (RR $1.23,95 \% \mathrm{Cl} 1.15$ to 1.32 ); in contrast, behaviours considered safe (use of latrines, nappies, potties, toilets, washing nappies) were borderline protective (RR $0.93,95 \% \mathrm{Cl} 0.86$ to 1.00 ).

One observational study in rural Bangladesh found that disposal of child faeces in closed spaces, such as pit latrines, was associated with a $35 \%$ reduction in helminthiasis in children under two years of age compared with disposal in open spaces (Roy 2011). This indicated that safe disposal of child faeces may also play a role in the control of STH infections.

Furthermore, one study analysing Demographic and Health Surveys (DHS) data from 34 countries found that household child faeces disposal practices were strongly associated with child growth. The study found that improved child faeces disposal (child faeces disposed into improved latrine) practices were associated with reduced levels of child stunting and underweight and increases in height-for-age Z (HAZ) and weight-for-age Z (WAZ) scores (Bauza 2017), indicating that child faeces disposal may also be a determining factor for nutritional outcomes. Another cohort study in rural Bangladesh found that children from households that disposed of their children's faeces unsafely had higher scores of enteropathy and growth faltering, and greater odds of being wasted (George 2016), again supporting the possibly important role of safe child faeces disposal.

\section{Prevalence of safe child faeces disposal}

Safe disposal of child faeces has been defined in different ways, predominantly involving disposal of the faeces in a latrine (WHO/ UNICEF 2018; UNICEF 2012; WSP 2015), but also sometimes involving burying (WHO/UNICEF 2006). However, it was deemed that burying of faeces or throwing faeces in garbage should not be considered safe or improved disposal in an expert consultation (Bain 2015). Another definition of safe disposal of child faeces categorized safe disposal (disposal into any latrine) further into improved disposal if the latrine in which the faeces end up was considered improved (WSP 2015). In addition to disposal in an improved latrine, the JMP will consider disposal with solid waste as appropriate if the solid waste is stored, collected and disposed of in a sanitary manner (WHO/UNICEF 2018). None of these definitions are supported by high-quality evidence. The definitions of safe disposal of child faeces involve the child if the child defecates in a latrine directly or involves the caregiver disposing the faeces of the child safely into a latrine. The caregiver thus plays an important role, especially for younger children who are too young to be able to use a latrine, both to dispose of the faeces and also to train the child to use a latrine.

Data on child faeces disposal practices has been collected through DHS and Multiple Indicator Cluster surveys (MICS) since the start of these surveys in 1986 and 1995 (Bain 2015). The core question asked to caregivers of children under two (MICS) or under five (DHS) years of age is "The last time [name] passed stools, what was done to dispose of the stools?" (WHO/UNICEF 2006; WHO/UNICEF 2018).

Worldwide, safe disposal of child faeces is suboptimal. A report by the World Bank Water and Sanitation Program (WSP) presenting analysis from the latest available MICS/DHS surveys found that in 15 out of 26 locations more than $50 \%$ of households reported that the faeces of their youngest child under three years of age were disposed of unsafely (not into a latrine) (WSP 2015), and the percentage of faeces ending up in improved latrines was even lower. Worldwide, child faeces disposal was safer in urban settings, in households with improved sanitation, for older children, and in richer households (WSP 2015).

\section{Description of the intervention}

The interventions relevant to this Cochrane Review aim to improve the safe collection or disposal of faeces of children aged below five 
years in order to decrease direct or indirect human contact with such faeces. They may act by: improving the defecation site of the child, so the child defecates directly in the latrine; or improving collection and disposal of child faeces in a latrine (see Figure 1).

\section{Figure 1. Logic model. Abbreviations: HWWS: hand washing with soap; STH: soil-transmitted helminth.}

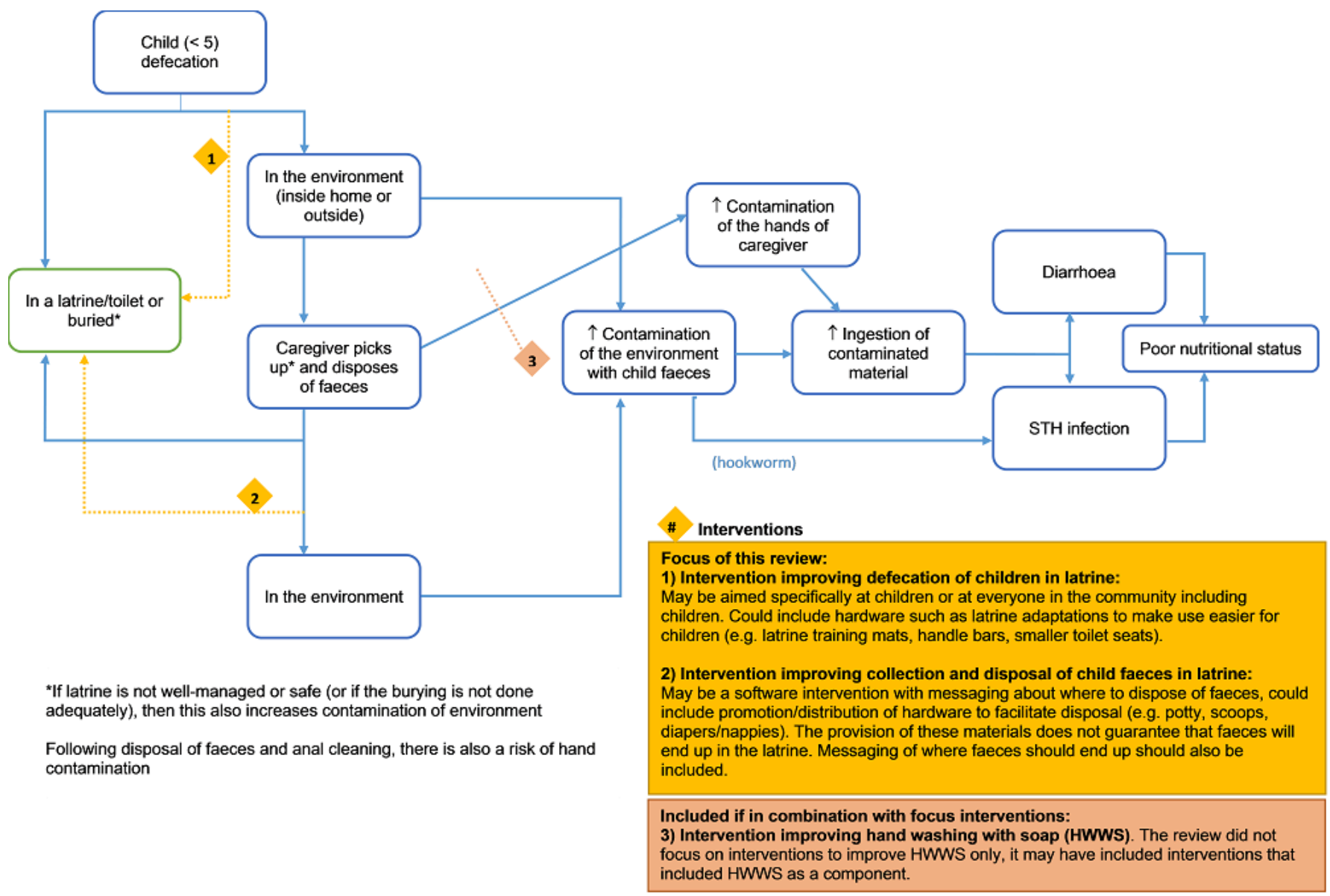

Interventions could include the provision of hardware (e.g. nappies, potties, faecal collection devices, cleaning products to remove faeces, child-friendly squatting slabs, or latrines used by children), software (e.g. promotion of safe disposal practices), or both. These interventions may be combined with or included in other interventions, such as hygiene promotion interventions (e.g. promotion of hand hygiene, food hygiene, etc.) or sanitation interventions (sanitation hardware provision or behaviour change messaging to end open defecation, or both).

It is important to note that these interventions may not completely reduce exposure to child faeces, as child faeces management involves a series of steps which present risks of exposure to pathogens in child faeces (Majorin 2017; Miller-Petrie 2016), including the defecation place of the child, where the faeces are disposed and how, and what hygiene behaviours are conducted. In addition, practices for child faeces disposal may differ depending on the caregiver, defecation place, or season. Furthermore, interventions seeking to improve child faeces disposal by providing hardware may not succeed in changing the behaviour of the caregivers, so the hardware (e.g. potties or scoops) may not be used or may not be used as intended, disposing of the child faeces in the open rather than in the latrine or toilet.
We categorized the results of this Cochrane Review into different types of intervention, in order to make them comparable to one another. The interventions were categorized as shown in Table 1 , and as described below.

\section{Education and hygiene promotion interventions}

These were software-only interventions that had no or limited (e.g. soap, chlorine, drinking container) hardware components. These interventions included safe child faeces disposal promotion, as their only promoted intervention or among other interventions (promotion of other WASH behaviours (e.g. hand washing with soap, safe water storage behaviours, use of latrines) or other public health behaviours (e.g. exclusive breastfeeding for children under six months of age, maternal nutrition during pregnancy, disposal of animal faeces, safe waste disposal, use of bed nets, immunizations)). While some of the interventions promoted child potties or dirt throwers/scoops, no child faeces disposal hardware was provided as part of the interventions. The intervention delivery method varied across all the interventions (e.g. education in health centres, mass-media campaigns, community-based volunteer groups, household visits). The messages on child faeces differed across interventions, but included one or more of the following messages. 
- Disposal of faeces in a latrine when available.

- Use of latrines by everyone, including children.

- Burying the faeces or constructing a specific pit to dispose of child faeces.

- Covering faeces with leaf or paper prior to burying them.

- Disposal of child faeces in a contained waste disposal sites, as opposed to uncollected waste.

- Use of chamber pots/potties.

- Use a dirt thrower/scoop to remove child faeces.

- Not letting dogs or pigs eat children's faeces.

- Discouraging children from defecating around households.

- Keeping the home environment free from faeces.

- Washing babies in a particular place after defecation.

\section{Community-led total sanitation interventions plus adaptations}

These interventions also had no hardware component, but their principal goal was to end open defecation by all household members (i.e. latrine use by all), with few other behaviours targeted for change. CLTS is an approach that aims to change behaviour in a community through stimulating a collective sense of disgust and shame that triggers the whole community to stop practicing open defecation; once communities succeed in ending open defecation, they are rewarded open defecation-free (ODF) certification (Kar 2008). CLTS does not encourage hardware subsidies; however, some of the included studies used CLTS techniques but also provided subsidies for building latrines and some included strengthening of the sanitation supply chain.

In this category of studies, it was not always clear whether children aged less than five years were specifically targeted in the triggering activities to end open defecation and none of the interventions included child faeces management hardware. A review of CLTS processes and protocols in Sub-Saharan Africa said that most countries' CLTS programmes require children's faeces to be safely disposed of. However, only two out of 15 countries reviewed had an indicator for child faeces disposal (Thomas 2013).

\section{Sanitation hardware and behaviour change interventions}

These interventions included a hardware and software component to improve the sanitation behaviours of everyone in the household. These interventions included providing child faeces management hardware, potties and sani-scoops (e.g. dustpans), as well as sanitation hardware (improvements to latrines or new latrines). The software component of these interventions included messages to encourage mothers to safely manage child faeces and to dispose of faeces in latrines.

\section{Water, Sanitation, and Hygiene hardware and education/ behaviour change interventions}

These were interventions that addressed child faeces disposal education as part of a wider water (e.g. building of hand pumps or provision of chlorine for water treatment) or sanitation (e.g. provision of latrines) or hygiene (handwashing facilities), or a combination of these, hardware intervention. The educational messages on child faeces disposal in different interventions included the following.
- Importance of everyone using latrines, including young children.

- Not disposing of used nappies in the garden or bushes or in waterways.

- Use handy tool (e.g. shovel) to collect and dispose of faeces and keep the tool clean.

- "Child faeces are more harmful than the adult."

- Wash hands after disposing of child faeces.

\section{Daycare centre-based hygiene hardware and education interventions}

These were studies conducted in the USA, which aimed to improve several hygiene behaviours in daycare centres. They also included some hygiene equipment, including nappy changing equipment and instructions on how to dispose of nappies.

\section{How the intervention might work}

The intervention might work through reducing exposure to child faeces, which are currently mostly ending up in the environment. This reduced exposure to faeces would reduce possible ingestion of faecal pathogens (bacteria, viruses, protozoa, and worm eggs) or penetration of hookworm larvae, leading to reduced diarrhoea and soil-transmitted infections, which in turn would improve nutritional status (see Figure 1).

\section{OB JECTIVES}

To assess the effectiveness of interventions to improve the disposal of child faeces for preventing diarrhoea and STH infections.

\section{METHODS}

\section{Criteria for considering studies for this review \\ Types of studies}

We included randomized controlled trials (RCTs) that were either individually-or cluster-randomized, and the following types of nonrandomized controlled studies (NRS): quasi-RCTs, non-RCTs, controlled before-and-after studies, interrupted time series studies, historically controlled studies, case-control studies, cohort studies, and cross-sectional studies (see definitions in Appendix 1). We included NRS as based on a previous review, Gil 2004, we assumed that there would be no or very few RCTs assessing the effect of improved disposal of child faeces for preventing diarrhoea and STH infection. Despite the risk of confounding, NRS studies contribute useful additional information to that provided by RCTs, as the interventions evaluated in the RCTs mostly evaluate interventions to improve WASH and other behaviours rather than just child faeces disposal and thus do not give measures of effect of improving child faeces disposal itself. We excluded non-controlled studies, such as case reports or case series, due to the importance of control groups to determine the effect of the intervention on the outcomes of interest.

\section{Types of participants}

Adults and children.

- Disposal of child's faeces soon after defecation. 


\section{Types of interventions}

\section{Intervention}

All interventions aiming to improve the safe collection or disposal of faeces of children aged below five years in order to decrease direct or indirect human contact with such faeces. For NRS, this extended to interventions that occurred in the course of usual healthcare or daily life, or those that were deliberately introduced. This included, but was not limited to, safe disposal practices as defined by the JMP, namely direct defecation into a latrine, disposal of stools in a latrine, or burying of stools (WHO/UNICEF 2006). Interventions could include the provision of hardware (e.g. nappies, potties, faecal collection devices, cleaning products to remove faeces, childfriendly squatting slabs, or latrines used by children), software (e.g. promotion of safe disposal practices), or both. We included interventions that combined the safe disposal of child faeces with other interventions, such as hygiene promotion interventions.

\section{Control}

Participants that continued their usual practices of child faeces disposal instead of the intervention, or who received a different type of intervention (e.g. a health promotion intervention).

\section{Types of outcome measures}

\section{Primary outcomes}

- Diarrhoea episodes among individuals, whether or not confirmed by microbiological examination. We defined an episode according to the case definitions used in each reviewed study. A third of the included studies used the WHO definition, which is the passage of three or more loose or liquid stools per day or more than usual for the individual (WHO 2013), while others used other definitions, which are defined in the results section. We treated this outcome as dichotomous, whether an individual had one or more episodes of diarrhoea.

- Infection with one or more of the following species of STHs: Ascaris lumbricoides (round worm), Trichuris trichiura (whip worm), Ancylostoma duodenale, orNecator americanus (hookworm). We defined infection as the presence of eggs, or juvenile nematodes, or both in the stools of the participants. We included any accepted diagnostic techniques.

\section{Secondary outcomes}

- Dysentery (bloody diarrhoea).

- Severe diarrhoea (clinical features associated with greater severity of diarrhoea illness include: high stool frequency or stool output and persistent diarrhoea (Bhandari 2002)).

- Persistent diarrhoea (diarrhoea lasting 14 days or longer).

- Clinical visits for diarrhoea.

- Intensity of STH infection (number of eggs per gram of stool).

- Presence of pathogenic microbes in stool assays.

- Anthropometry (weight-for-age and height-for-age).

- Serology.

- Other markers of infection and disease.

- Mortality.

- Use and adoption of the intervention (behaviour change).

- Adverse events.

\section{Search methods for identification of studies}

We attempted to identify all relevant studies regardless of language or publication status (published, unpublished, in press, and ongoing).

\section{Electronic searches}

The search terms are detailed in Appendix 2 and included terms for "faeces disposal" or "sanitation" and for "child". We did not include specific terms for study designs or outcomes to ensure relevant studies were not missed.

\section{We searched the following databases:}

- the Cochrane Infectious Diseases Group (CIDG) Specialized Register (27 September 2018);

- the Cochrane Central Register of Controlled Trials (CENTRAL), published in the Cochrane Library (27 September 2018);

- MEDLINE (27 September 2018);

- Embase (27 September 2018);

- Global Health (5 October 2018);

- Web of Science (27 September 2018);

- LILACS (27 September 2018);

- POPLINE (27 September 2018).

Also, we examined Chinese-language databases available in the China National Knowledge Infrastructure (25 January 2015) and the Wan Fang Portal (11 January 2015) using the search terms detailed in Appendix 2 or their Chinese language equivalents. We searched the metaRegister of Controlled Trials ( $m R C T)$, ClinicalTrials.gov (clinicaltrials.gov), and the WHO International Clinical Trials Registry Platform Search Portal (www.who.int/trialsearch) using "sanitation" and "hygiene" as search terms, as well as an index to theses in the UK (ethos.bl.uk) (27 September 2018). We searched the Open Grey (www.opengrey.eu) database for grey literature (27 September 2018).

\section{Searching other resources}

\section{Conference proceedings}

We searched the following organizations' conference proceedings: International Water Association and Water, Engineering and Development Centre, Loughborough University, UK.

\section{Researchers and organizations}

We contacted individuals working in the field, and contacted or searched websites of the following organizations for other potential published and unpublished studies:

- Water, Sanitation and Health Programme of the WHO;

- World Bank WSP;

- UNICEF Water, Environment and Sanitation;

- Environmental Health Project (US Agency for International Development (USAID));

- IRC International Water and Sanitation Centre;

- Global Water, Sanitation and Hygiene (Centers for Disease Control and Prevention);

- International Centre for Diarrhoeal Disease Research, Bangladesh (ICDDR,B);

- USAID; 
- UK Department for International Development (DFID);

- Asian Development Bank (ADB);

- WASHplus (www.washplus.org/);

- Sustainable Sanitation Alliance (www.susana.org/);

- community-led total sanitation (CLTS);

- the sanitation updates blog (sanitationupdates.wordpress.com/); and

- the STEPS Centre at the Institute of Development Studies University of Sussex (steps-centre.org).

\section{Reference lists}

We checked the reference lists of studies identified by the above methods.

\section{Data collection and analysis}

\section{Selection of studies}

One review author (FM) examined titles of all identified studies removing those that were clearly ineligible and off-topic. Two researchers (among FM, Lyndsey Gray (LG), BT, Christian Landon (CL), and Czarina Cooper (CC)) independently examined abstracts and selected all potentially eligible studies based on the inclusion criteria. If a title or abstract could not be rejected with certainty due to lack of information, we obtained the full-text article for further assessment. GC reviewed the results of the Chinese database search, undertaking the same process as FM, LG, BT, CL, and CC. We obtained full copies of all studies agreed by either reviewer to potentially fall within the inclusion criteria. Two researchers (FM and LG, $\mathrm{BT}, \mathrm{CL}$, or $\mathrm{CC}$ ) independently determined whether each study met the inclusion criteria using a form. When we agreed, we either included or excluded the study. If we were unable to agree, we consulted review author Thomas Clasen (TC) who made the final decision. One review author (FM) corresponded with authors in case data needed to assess eligibility was not obvious in the study or if data were missing from the report. Any studies that FM or the second reviewer ( $L G, B T, C L$, or CC) suggested to include but which was ultimately excluded through discussion or by a third review author (TC or FM) was presented with the reason for exclusion in the Characteristics of excluded studies table. We checked study reports to ensure that multiple publications of the same study were only included once.

\section{Data extraction and management}

Two review authors (FM and BT) independently extracted data from the included studies using a data extraction form after it was piloted on two included studies (items included in the form are presented in Appendix 3). In case of discrepancy, we discussed the data and consulted TC, if necessary, who made the final decision. One review author (FM) entered and analysed the agreed data in Review Manager 5 (Review Manager 2014), and a second review author (BT) independently cross-checked a sample of the data.

\section{Type of data extracted}

\section{Randomized controlled trials randomized by cluster}

For cluster RCTs, we extracted the number of participants enrolled and the number analysed in each treatment group for each outcome. We noted whether or not the authors reported adjusting for clustering in the analysis. We endeavoured to collect intracluster correlation coefficients (ICC) for cluster RCTs but only four of the trials reported this measure. In addition, we extracted data on the study setting, study design, study participants, details of the interventions and control groups and activities, details of outcomes measured in the study and their measures of effect, and when and how they were measured. When an RCT included several arms with a relevant intervention but only had one control group, we extracted data for the study arm most relevant to this review.

\section{Non-randomized studies}

For NRS, we extracted details on the features of the design, the confounding factors considered in the study, methods used to control for confounding, data on the risk of bias specific for NRS (see Assessment of risk of bias in included studies), the total numbers of participants included in the study and in each comparison group, and the measures of effect and Cls.

\section{Assessment of risk of bias in included studies}

Two review authors (BT and FM) independently applied the risk of bias criteria using an assessment form. In case of disagreement, we discussed the issue to make the final decision. For each study, we justified reasons for the level of risk of bias and included it in the 'Risk of bias' table.

For RCTs, we used the Cochrane tool to assess the risk of bias, which includes methods of random sequence generation; allocation concealment; blinding of participants, personnel, and outcome assessment; incomplete outcome data; and selective reporting (Higgins 2011a). For each domain, we followed the definitions of low risk, unclear risk, and high risk described in Higgins 2011a.

For cluster RCTs, we also assessed the risk of bias specific to this study design.

- Recruitment bias. We qualified the study at high risk of bias when the participants and staff were aware of which cluster the intervention or control was; unclear risk of bias when the information was not collected or reported; or low risk of bias if clusters were not known to be intervention or control during participant recruitment.

- Baseline imbalance. We assessed a study at high risk of bias when there were large differences in baseline characteristics and they were not adjusted for in the analysis; low risk of bias where statistical methods were used to match the clusters at the design stage or to adjust for imbalances in the analysis, or in case there were no substantial differences in baseline characteristics; or unclear risk of bias if it was not mentioned in the report.

- Loss of clusters. We qualified studies at high risk of bias where more than $10 \%$ of clusters were lost to follow-up; low risk of bias where less than $10 \%$ of clusters were lost to follow-up; or unclear risk of bias if loss to follow-up was not mentioned.

- Incorrect analyses. We assessed studies at high risk of bias if they did not analyse the data adjusting for clustering; low risk of bias where there were no unit-of analysis errors in the study and if clustering was adjusted for in the analysis; or unclear risk of bias if it was not reported in the study.

- Comparability with individually randomized RCTs. We analysed cluster-RCTs separately from other study designs.

For controlled before-and-after studies, controlled cohort studies, and cross-sectional studies, we used the EPOC criteria to assess the risk of bias (EPOC 2013). This tool includes random sequence gener- 
ation, allocation concealment, incomplete outcome data (less than $10 \%$ loss to follow-up or no difference between arms was considered low, more than $10 \%$ was considered high, and if it was not mentioned or reported, it was considered as unclear), selective outcome reporting, and other biases that were similar to the RCT 'Risk of bias' tool, as well as the following additional domains.

- Similarity of baseline characteristics. Important baseline characteristics for this study included: access and type of sanitation facilities, water access and quality, age, wealth, and hygiene practices. We qualified the studies as high risk of bias where there were substantial differences; low risk of bias if baseline characteristics were reported and there was no substantial difference; or unclear risk of bias if it was not reported or unknown.

- Similarity of baseline outcome measurements. We gave high risk of bias scores when large differences were present and they were not adjusted for in the analysis; low risk of bias scores to studies if participant outcomes were measured prior to the intervention and there were no substantial differences; or unclear risk of bias if it was not mentioned in the report.

- Adequate protection against contamination? We qualified a study as high risk of bias if it was likely that the control group received the intervention; low risk of bias if it was unlikely that the control group received the intervention; or unclear risk of bias in case it was possible contamination could have occurred.

- Adequate allocation of intervention concealment during the study. We qualified studies as high risk of bias if the outcomes were not assessed blindly; low risk of bias if the authors explicitly reported that the primary outcomes were assessed blindly or the outcomes were objective; or unclear risk of bias if it was not specified in the paper.

We also added a domain to assess whether the studies appropriately adjusted for confounders. The following confounders related to child faeces disposal and diarrhoea or STHs infections were considered important for this review: access to or ownership of a sanitation facility, type of sanitation facility (improved or unimproved according to the JMP classification (WHO/UNICEF 2014), use of sanitation facility, wealth, age, water access, season, water quality, animal ownership, household size, educational level, attendance to school or preschool by the children, shoe-wearing, and hygiene practices. We qualified studies as low risk of bias if they controlled for at least one of the listed confounders in the design (e.g. matching) or the analysis (e.g. multivariable statistical modelling). We qualified studies as high risk of bias if no adjustment for confounding variables was conducted and unclear risk of bias where it was not mentioned in the paper.

For case-control studies, we assessed the quality of the studies using the Newcastle Ottawa scale (NOS) (Wells 2013). The scale is divided into eight items grouped into three domains: selection, comparability, and ascertainment of exposure. For each item in the selection and exposure ascertainment domains a total of one 'star' can be awarded to a study; in the comparability domain two stars can be awarded. For one star in the comparability domain, the study had to control for access to or ownership of a sanitation facility. For two stars, the study had to control for at least one other important confounding variable, such as type of sanitation facility (improved or unimproved) use of sanitation facility, wealth, age, water access, season, water quality, animal ownership, household size, educational level, attendance to school or preschool by the children, shoe-wearing, and hygiene practices.

\section{Measures of treatment effect}

For RCTs with dichotomous outcomes, we calculated risk ratios (RR) with 95\% confidence intervals (Cls) where raw data were available. If not, we used the effect measures reported, along with the $95 \% \mathrm{Cl}$. For continuous variables, we extracted the mean differences (MD). We calculated or extracted standard errors and $95 \% \mathrm{Cl}$ from these studies.

For NRS, we reported measures of effect adjusted for confounders from the studies. If several adjusted estimates were reported, we used the estimate adjusting for the most confounders. We specified the confounders that were adjusted for in the study and whether it was done in the design or in the analysis. In case the effect measures extracted were expressed in different metrics, we converted them into a common measure, RR for controlled cohorts and cross-sectional studies and odds ratio (OR) for case-control studies; if they were all the same, we combined them using the effect measure used in the reports. If no adjusted measures could be obtained from the studies, we used unadjusted measures reported in the study or calculated RR or OR (for case-controls) and 95\% Cl from the raw data.

\section{Unit of analysis issues}

We searched for both individually and cluster-RCTs, however we identified no individually-RCTs that met our inclusion criteria. For cluster-RCTs, we assessed whether clustering was properly accounted for in the analysis and used the adjusted measure of effect reported. When the studies did not adjust for clustering or measures of effect needed to be calculated, we extracted or calculated unadjusted measures of effect and $\mathrm{Cls}$, the mean cluster sizes and calculated adjusted measures of effect that accounted for clustering using the inflating standard error method using ICC from other similar studies (Higgins 2011b). We added details of ICCs used in the footnotes of the forest plots.

\section{Dealing with missing data}

If studies had missing data needed for assessment of eligibility or analysis, one review author (FM) attempted to contact authors to obtain the data. We report the number of participants in each study and the number of participants who were lost to follow-up.

\section{Assessment of heterogeneity}

We assessed heterogeneity by visually examining the $\mathrm{Cls}$ in the forest plot and by using the $\mathrm{Chi}^{2}$ test and $\mathrm{I}^{2}$ statistic (Higgins 2003). We considered a significance level of $P$ less than 0.1 for the $\mathrm{Chi}^{2}$ test to be significant and indicate potential heterogeneity. To estimate the degree of heterogeneity, we classified an estimate of the $\mathrm{I}^{2}$ statistic greater than $50 \%$ to indicate substantial heterogeneity and greater than $75 \%$ to indicate considerable heterogeneity (Deeks 2011). We prespecified in the protocol that if there were sufficient studies (more than 10) and substantial heterogeneity, we would investigate causes of heterogeneity using subgroup analysis (Majorin 2014).

\section{Assessment of reporting biases}

We tried to minimize reporting bias by using a comprehensive search strategy including published and unpublished studies. We compared the outcomes listed in the methods and those reported in the results sections. We assessed the potential of publication bias using funnel plots of case-control studies included in the analy- 
sis of safe disposal of child faeces, as they were the only analysis that had sufficient studies (more than 10).

\section{Data synthesis}

We analysed the data using Review Manager 5 (Review Manager 2014). If there was more than one study with comparable participants, interventions, and outcomes, we conducted a meta-analysis to estimate a pooled measure of effect. We used random-effects models to pool the data. The comparisons made were between those with the intervention and those without or with a different intervention. Due to differences in potential risk of bias of different study designs (Reeves 2011), we only pooled results of similar study designs.

We stratified the case-control analyses according to the level of quality of the studies, according to the numbers of stars it received.

When there were not enough similar studies to pool them, we described them in the text organizing them by type of intervention, outcome, and study design.

\section{'Summary of findings' tables}

One review author (FM) assessed the methodological certainty of each outcome across the included studies using GRADE guidelines (Guyatt 2011). We summarized the methodological certainty in Summary of findings for the main comparison; Summary of findings 2; Summary of findings 3; Summary of findings 4; Summary of findings 5; Summary of findings 6.

The 'Summary of findings' tables present the following outcomes.

- Diarrhoea episodes.

- Infections with one or more species of STHs.

We used the following criteria to grade the certainty in the 'Summary of findings' tables.

- For study limitations: we downgraded studies one level for serious risk of bias if the outcome was self-reported or not objective and susceptible to bias due to the studies being unblinded. As most environmental interventions, including sanitation, are difficult or impossible to blind, studies that met other criteria for low risk of bias were nevertheless downgraded unless the outcome was objective.
- For inconsistency of results: we downgraded studies if there was substantial $\left(I^{2}\right.$ greater than $\left.50 \%\right)$ statistical heterogeneity and this could not be explained through subgroup analyses.

- For indirectness of evidence: we downgraded if there were limited populations or settings in the included the studies, which did not allow us to make generalizations about the findings to other settings relevant to this review.

- For imprecision: we downgraded if the studies had a small sample size and large $\mathrm{Cls}$ that included important effects in both directions

\section{Subgroup analysis and investigation of heterogeneity}

Only case-control studies had sufficient comparisons, as prespecified in our protocol (greater than 10), for subgroup analyses. In the case-control analyses, we conducted subgroup analyses to investigate the effects of:

- safe child faeces disposal on outcomes in different age groups, children aged under five years versus all ages;

- different case-definitions;

- intervention site (urban versus rural);

- intervention settings (low-, middle- or high-income country);

- different methods to ascertain child faeces disposal behaviour: observations versus survey questionnaire.

\section{Sensitivity analysis}

We conducted sensitivity analyses to check robustness of the choice of analysis method (random-effects model versus fixed-effect) for the main health outcomes.

\section{RES U L T S}

\section{Description of studies}

\section{Results of the search}

The searches identified 38,731 records (34,200 from English databases, 3613 from Chinese databases, and 918 from other sources). We screened the titles and abstracts and obtained 935 full texts, of which 78 reports of 63 studies met the inclusion criteria (see Figure 2). 
2017 ETH also provided WASH kits or soap and Sarrassat 2018 BUR was a mass radio campaign.

Five studies focused on ending open defecation throughout the target community using either CLTS (Pickering 2015 MLI) or TSSM, which included CLTS-messaging and sanitation marketing (Briceño 2015 TAN; Cameron 2013 INA), or India's Total Sanitation Campaign (TSC), which included subsidies and latrine promotion (Dickinson 2015 IND and Patil 2014 IND, which also included additional TSSM support including CLTS messaging).

Four studies evaluated sanitation hardware and behaviour change interventions, which included the provision of child sanitation hardware (potties and sani-scoops) and behaviour messaging (Caruso 2019 IND; Christensen 2015a KEN; Luby 2018 BGD; Null 2018 KEN). Three of these trials were from WASH Benefits (WASH B) study, one from the pilot in Kenya (Christensen 2015a KEN), and on the main outcomes from Kenya (Null 2018 KEN) and Bangladesh (Luby 2018 BGD). The WASH B studies included several study arms, for this review we included only the sanitation versus control results as they were most relevant.

One study, the Sanitation Hygiene Infant Nutrition Efficacy (SHINE) trial, evaluated a WASH hardware and behaviour change intervention (Humphrey 2019 ZIM).

Two studies included child faeces disposal in their multicomponent interventions in daycare centres (Butz 1990 USA; Kotch 2007 USA).

\section{Controlled before-and-after studies}

Ahmed 1993 BGD consisted of an education intervention on sanitation, food, and personal hygiene.

The other three CBAs were WASH hardware and education interventions that included instructions for children to use toilets constructed in its WASH intervention (Aziz 1990 BGD), or included child faeces disposal messaging in their health education component along with providing hand pumps (Alam 1989 BGD), or providing latrines (Park 2016 INA).

\section{Non-randomized studies}

\section{Controlled cohort studies}

Two controlled cohort studies were education and hygiene promotion interventions that evaluated the Sanitation Hygiene Education and Water Supply in Bangladesh (SHEWA-B) intervention in Bangladesh. The intervention included child faeces disposal in its hygiene education component (Huda 2012 BGD; Luby 2014 BGD). The third controlled cohort was a WASH hardware and education interventions that compared wards that received a community-based health project and WASH-focused activities, which included messages about child faeces disposal in its mothers' groups and children's club meetings, with wards that only received the community-based health project (Hoq 2016 BGD).

\section{Controlled cross-sectional studies}

Six controlled cross-sectional studies were education and hygiene promotion interventions (Berhe 2014 ETH; Fisher 2011 BGD; Gebru 2014 ETH; Mathew 2004 ZIM; Oguro 2016 MYA; Waterkeyn 2005 ZIM). Two cross-sectional studies compared "model" and "non-model" families from the Ethiopian Health Extension Package (HEP) (Berhe 2014 ETH; Gebru 2014 ETH). Model families were those that fully implemented the HEP, whereas non-model families did not fully im- plement the HEP. The HEP consisted of health promotion in four main categories: family health services, infectious disease prevention and control, hygiene and environmental sanitation, and health education and communication. The maternal and child health package (in the family health services category) included messaging about safe child stool disposal (the stool should be cleared and disposed of in a pit latrine, or should be covered with a leaf or paper and be buried) (HEP 2003).

Two studied the behaviour change as a result of community health clubs, which provided participatory health education classes on various health topics (Mathew 2004 ZIM; Waterkeyn 2005 ZIM). One of the lessons included child faeces disposal in a latrine. One study investigated the behaviour change and health effect of the BRAC WASH programme (a WASH programme of BRAC, which is a nongovernmental development organization based in Bangladesh) (Fisher 2011 BGD), which provided hygiene education including child faeces disposal in a latrine in its sanitation messaging. One study compared behaviour change in two villages that received a Women's Health Volunteer Group (WVG) intervention with two villages that did not (Oguro 2016 MYA).

One controlled cross-sectional study evaluated a CLTS intervention by comparing the parasitology and nutritional status of children in two villages that benefited from CLTS and attained ODF status with two other villages that did not benefit from CLTS (Belizario 2015 $\mathrm{PHI})$.

\section{Case-control studies}

In the case-control studies, three studies included two risk factors related to child faeces disposal, and one study had seven different study sites (Baker 2016 BGD), thus making a total of 29 comparisons. Six studies could not be included in the analyses as they either had insufficient or no data or could not be compared to the other case-control studies (Arvelo 2009 USA; Bassal 2016 ISR; Chiang 2005 TWN; Daniels 1990 LES; Menon 1990 USA; Nanan 2003 PAK).

\section{Study participants and settings}

\section{Randomized controlled trials}

Most RCTs (19/22) were conducted in low- or lower middle-income settings, apart from Butz 1990 USA and Kotch 2007 USA, which were conducted in daycare centres in the USA and Yeager 2002 PER, which was conducted in urban Peru.

Stanton 1987 BGD was conducted in urban Bangladesh; Barrios 2008 PHI in rural Philippines; Cameron 2013 INA in rural Indonesia; Caruso 2019 IND, Dickinson 2015 IND, Nair 2017 IND, and Patil 2014 IND in rural India; and Luby 2018 BGD in rural Bangladesh. Altmann 2018 TCD was conducted in Chad, Briceño 2015 TAN in rural Tanzania, Christensen 2015a KEN and Null 2018 KEN in rural Kenya, Haggerty 1994 DRC in rural Democratic Republic of Congo (DRC), Hashi 2017 ETH in rural Ethiopia, Jinadu 2007 NGR in rural Nigeria, Pickering 2015 MLI in rural Mali, Sarrassat 2018 BUR in rural Burkina Faso, Sinharoy 2017 RWA in rural Rwanda, and Humphrey 2019 ZIM in rural Zimbabwe.

Apart from Stanton 1987 BGD, which collected diarrhoea morbidity data in children aged less than six years, Jinadu 2007 NGR, which collected data on children aged five year or less and Butz 1990 USA, which included children aged between one month and seven years in daycare centres, all other studies collected data for children aged less than five years. 


\section{Controlled before-and-after studies}

Three studies were conducted in rural Bangladesh and collected data for children aged less than five years (Aziz 1990 BGD), less than 23 months (Alam 1989 BGD), and less than 19 months (Ahmed 1993 BGD). Park 2016 INA was conducted in rural Indonesia and collected data on STH in children aged between three and 13 years.

\section{Non-randomized studies}

\section{Controlled cohort studies}

The three cohort studies were conducted in Bangladesh. Huda 2012 BGD included only rural populations, while Luby 2014 BGD included both urban and rural areas and Hoq 2016 BGD was in periurban areas. Huda 2012 BGD and Luby 2014 BGD studied outcomes in children aged below five years, while Hoq 2016 BGD measured outcomes in children aged below two years.

\section{Controlled cross-sectional studies}

Berhe 2014 ETH and Gebru 2014 ETH were conducted in rural Ethiopia and measured outcomes in children aged less than five years. Mathew 2004 ZIM and Waterkeyn 2005 ZIM were conducted in rural Zimbabwe and did not specify the age of the children whose defecation or faeces disposal behaviour were collected. Fisher 2011 BGD covered children aged less than five years in rural Bangladesh. Belizario 2015 PHI was conducted in rural Philippines and measured STH prevalence in children that were aged between two and 15 years. Oguro 2016 MYA was conducted in Myanmar and measured behaviour change reported by caregivers of children aged less than five years.

\section{Case-control studies}

Most of the case-control study sites (23/33) occurred in low- or lower middle-income countries apart from Chompook 2006 THA; Genthe 1997 SAF; Heller 2003 BRA; Knight 1992 MAL; and Strina 2012 BRA, which were in upper middle-income countries, and Abalkhail 1995 KSA; Arvelo 2009 USA; Bassal 2016 ISR Chiang 2005 TWN; and Menon 1990 USA, which were in high-income countries.

In general, included studies considered cases and controls only aged less than five years or younger age groups. The exceptions were Arvelo 2009 USA, which did not specify the age of the children in the daycare centres; Chompook 2006 THA, which included all ages (median age: five years in cases and controls); Clemens 1987 BGD included children aged less than six years; Cummings 2012 UGA, which only collected data on cases and controls aged more than 10 years (median age in cases: 26 years, in controls: 33 years); Genthe 1997 SAF, which included preschool children (age range 0.2 to 67.2 months); Nanan 2003 PAK, who considered cases and controls aged between four and 71 months; Oketcho 2012 TAN aged between six and 60 months; and Strina 2012 BRA aged less than 10 years.

Most of the case-control studies (11 studies) recruited cases from healthcare settings and controls from the community (of those Menon 1990 USA; Mertens 1992 SRI; and Traoré 1994b BUR had both community and hospital controls), eight recruited cases and controls from healthcare settings, seven recruited cases and controls from the community, and Arvelo 2009 USA recruited cases and controls from among licensed daycare centres.

\section{Interventions}

\section{Education and hygiene promotion interventions}

A summary of the study designs, settings, and outcome measures of the education and hygiene promotion interventions is presented in Table 2.

\section{Randomized controlled trials}

The 10 education and hygiene promotion interventions included different messages on child faeces disposal (Characteristics of included studies table).

Yeager 2002 PER focused on promoting the use of a potty for children aged 15 to 47 months and to keep the home environment free of faeces through the routine health service. Although the intervention described what messages were promoted to train children to defecate in potties, there were no details in the report as to where potties should have been emptied.

Altmann 2018 TCD evaluated a WASH package given alongside routine Outpatient Therapeutic feeding Program (OTP) for severe acute malnutrition. The WASH package consisted of a WASH kit (safe drinking water storage container, water disinfection tablets, soap bars, and a plastic cup with handle) and promotion, which included messaging to bury children's stools.

Barrios $2008 \mathrm{PHI}$ focused its intervention messages on hand washing and stool disposal aiming to ensure the sanitary disposal of faeces in a latrine or burying in case no latrine was available, regardless of where the child defecated.

Haggerty 1994 DRC promoted the disposal of animal faeces, hand washing at different key moments, and disposal of children's faeces, emphasizing digging or improving pit latrines.

Hashi 2017 ETH provided health education and soap (white bars). The health education consisted of 12 sessions on key WASH messages (hand washing with soap, water storage behaviour, latrine availability and use, safe waste disposal including child faeces disposal) and demonstration of hand washing with soap.

Jinadu 2007 NGR promoted the hygienic disposal of children's faeces by educating mothers to use chamber pots for disposal (although no details on final disposal site are provided in the paper), discouraging children from defecating around households, and also promoting the construction of ventilated improved pit (VIP) latrines and educating mothers to wash their hands after using the toilet and cleaning up children's faeces.

Nair 2017 IND used community-based female workers (Suposhan Karyakarta, or SPK) to conduct home visits with individual families and participatory meetings with groups of women, to improve health and nutrition in the first 1000 days of life. This included advising caregivers to place the child's faeces in a pit latrine or if no latrines were available to bury them in a shallow hole away from their living area and any waterway rather than disposing of them in the open field or the household compound.

Sarrassat 2018 BUR evaluated a mass radio campaign targeted at women of reproductive age and caregivers of children aged less than five years, on 17 childcare behaviours, including safe child faeces disposal (using latrines or using potties for young children or burying the stools outside the house/compound). 
Sinharoy 2017 RWA evaluated two versions of the Community-Based Environmental Health Promotion Programme ('lite' and 'classic'), which involved community health clubs that promoted healthy behaviours. The lite version included eight topics, and the classic version included 20 topics. Topics range from handwashing, diarrhoea, water sources, and sanitation to specific diseases. In the sanitation topic (included in both the lite and classic version), it promoted that children should defecate into chamber pots, that their faeces should be buried if there is no latrine (cat sanitation), and that one should never let the dog or pig eat children's faeces after defecation.

Stanton 1987 BGD promoted proper hand washing before food preparation, defecation away from the house and in a proper site, and suitable disposal of waste and faeces. The final disposal site for child faeces was not specified in the paper.

\section{Controlled before-and-after studies}

Ahmed 1993 BGD generated the intervention messages through participation with the community and thus contained a large amount of target behaviours, including the use of a dirt thrower to immediately remove child or animal faeces from the compound and to construct a pit to dispose of faeces and other dirty material from the compound.

\section{Controlled cohort studies}

The SHEWA-B programme promoted the disposal of children's faeces into hygienic latrines and the importance of everyone in the household, including children, using the latrine, among other messages in their educational component (Huda 2012 BGD; Luby 2014 BGD).

\section{Controlled cross-sectional studies}

In the HEP programme in Ethiopia (Berhe 2014 ETH; Gebru 2014 $E T H)$, education on child faeces disposal was included in the maternal and child health package, emphasizing cleaning faeces and disposing of them in a pit latrine or burying the faeces (HEP 2003). The HEP includes health promotion and education on 16 packages in four main categories: family health services, disease prevention and control, hygiene and environmental sanitation, and health education and communication.

The CHC (Mathew 2004 ZIM; Waterkeyn 2005 ZIM), and BRAC WASH (Fisher 2011 BGD), programmes promoted the disposal of children's faeces into hygienic latrines, among other messages in their educational component.

In Oguro 2016 MYA, as part of the sanitation education, the WVG encouraged latrine use by children aged less than five years to villagers and promoted appropriate disposal (flushing in a latrine) of child faeces.

\section{Community-led total sanitation interventions plus adaptations}

A summary of the interventions, settings and outcome measures of the CLTS interventions plus adaptations is presented in Table 3.

\section{Randomized controlled trials}

Briceño 2015 TAN; Cameron 2013 INA; Dickinson 2015 IND; Patil 2014 IND; and Pickering 2015 MLI focused on ending open defecation, including by children in their intervention using CLTS messaging. CLTS aimed to change the behaviour in a community through stimulating a collective sense of disgust and shame that triggered the whole community to stop practicing open defecation; once communities succeeded in ending open defecation, they were rewarded ODF certification (Kar 2008). Briceño 2015 TAN; Cameron 2013 INA; Dickinson 2015 IND; and Patil 2014 IND also had other components to increase demand for sanitation as part of the TSSM project (Briceño 2015 TAN; Cameron 2013 INA; Patil 2014 IND), and in India the TSC also included subsidies for latrine construction (Dickinson 2015 IND; Patil 2014 IND). In the criteria for ODF certification in Mali, among other indicators was that "all family members must use the latrine or a child potty" (Pickering 2015 MLI).

\section{Controlled cross-sectional studies}

In the CLTS intervention in the Philipines (Belizario 2015 PHI), community leaders and volunteers delivered the following key messages to households: 1 . the shame of having open defecation in the village and the importance of attaining ODF status in the village; 2. the importance for each household to possess its own sanitary toilet; and 3. the need for households to ensure solid waste management and disposal, as well as maintain sanitary conditions in animal facilities in the backyard (e.g. pig pens). Messages about child faeces disposal and use of toilets by children were also included.

\section{Sanitation hardware and behaviour change interventions}

A summary of the interventions, settings, and outcome measures of the sanitation hardware and behaviour change interventions is presented in Table 4.

In the WASH Benefits trials (Luby 2018 BGD; Null 2018 KEN), and the pilot study in Kenya (Christensen 2015a KEN; Christensen 2015b KEN), the sanitation arm included the provision of hardware (faeces disposal sani-scooper, a plastic child potty, and improvements to their existing latrine or construction of a new latrine if they had none). In addition, there was behaviour change communication, which emphasized preventing faecal contamination of the environment and safe removal of faeces (human and animal) from the environment facilitated by the potty, sani-scooper, and latrine.

Caruso 2019 IND evaluated a multilevel behaviour change intervention the "Sundara Grama", which aimed to increase latrine use and safe disposal of child faeces. The intervention included activities at the community level (a traditional folk dance, a transect walk, community meeting, recognition of positive deviants, village map painting), group level (mother's group meeting), and household level (household visits and latrine repairs). The mother's group meeting was for mothers and caregivers of children aged under five years, to provide action knowledge and hardware (potties and scoops) to enable the safe disposal of child faeces. The importance of child faeces disposal was also mentioned during the folk dance performance and other activities.

\section{WASH hardware and education/behaviour change interventions}

A summary of the interventions, settings, and outcome measures of the WASH hardware and education/behaviour change interventions is presented in Table 5.

\section{Randomized controlled trials}

In the WASH arm of the SHINE study, households were provided with VIP latrines, two handwashing stations, a plastic mat and play yard, and monthly deliveries of soap and chlorine (Humphrey 2019 ZIM). Behaviour change modules were delivered by village health 
workers, in the WASH group the messages included information about child faeces disposal, hand washing with soap at key times, protection of infants from geophagia and ingestion of animal faeces, chlorination of drinking water (especially for infants), and hygienic preparation of complementary food.

\section{Controlled before-and-after studies}

Aziz 1990 BGD included the provision of water and sanitation infrastructure as well as hygiene education, which included the need for children to use the toilets constructed.

Alam 1989 BGD provided hand pumps to communities as well as health education on use of hand pump water, improvement of water handling and storage practices, disposal of child's faeces soon after defecation (with no details on how or where), and washing hands before handling food.

Park 2016 INA provided simple squat latrines with a septic tank or pit to households and gave health education regarding hygiene, sanitation, and prevention of STH infections. The health education component consisted of many messages, including hand washing, boiling water, food hygiene, and sanitation. The messages included not disposing of used nappies in the garden, bushes or waterways (if the nappies were not disposable) and for children to stay away from any faeces around their home.

\section{Controlled cohort studies}

Hoq 2016 BGD included several messages regarding child faeces disposal in both the intervention and control wards in the mass awareness behaviour change campaign. However, in the intervention wards this was done in additional mediums including mother's group meetings and child clubs. The child faeces disposal messages were: 1 . throw the child faeces in the latrine immediately after defecation; 2. use handy tool (shovel, etc.) to collect and dispose the faeces. Keep the tool clean; 3. encourage the children and start practicing defecation in the latrine instead of defecating on yard; 4. "child faeces are more harmful than the adult" as the mothers believed that children faeces were less harmful; and 5. wash hands after dispose of child faeces.

\section{Interventions in daycare centres}

Of the two studies in daycare centres in the USA, Butz 1990 USA included advice on handwashing and nappy-changing practices and instructions to dispose of gloves, disposable pads, and nappies in plastic bags and centres were given supplies (gloves, nappy changing pads, hand rinse solution). Kotch 2007 USA provided nappy changing, handwashing, and food-preparation equipment with impermeable, seamless surfacing and automatic faucets and foot-activated, roll-out waste bins for nappy disposal. A summary of the interventions, settings, and outcome measures of the interventions in daycare centres is presented in Table 6.

\section{Case-control studies}

Among the case-control studies, child faeces disposal variables were categorized into safe and unsafe disposal differently (Characteristics of included studies table). The most common categorization of child faeces disposal was disposal into a latrine versus elsewhere (10 comparisons of which one included both disposal in a latrine after defecation elsewhere and defecation in a latrine). In some studies, the authors classifies the defecation in a latrine as well as disposal in a latrine as safe in the same variable, whereas other studies presented separate variables for disposal in a latrine and defecation in a latrine. Thus, we pooled studies that had variables of safe disposal into a latrine (which in some cases included defecation into a latrine) and separately pooled studies with variables of defecation into a latrine.

Some definitions of safe disposal were more specific, including only certain disposal places as safe, such as Baker 2016 BGD only considered certain types of latrines in which the faeces were disposed of as safe: hanging latrines and bucket latrines were considered open disposal. Baltazar $1989 \mathrm{PHI}$ defined sanitary disposal as child defecated in a nappy and faeces were thrown away in washing, child used chamber pot/piece of paper and faecal matter was thrown in the toilet or child used the toilet, whereas unsanitary was when the faeces were deposited elsewhere than latrine or the child defecated outside (regardless of where faecal matter was finally thrown away). Mertens 1992 SRI defined unsanitary stool disposal as stools passed, or disposed of, in or out of the yard without being disposed within one day in a latrine or in a covered rubbish pit, while proper disposal was stools passed in a potty and later disposed of in a latrine or in a covered pit.

Asfaha 2018 ETH did not specify what they considered to be "safe" disposal. Ghosh 1994 IND and Ghosh 1997 IND did not define what they considered indiscriminate disposal of stools. Strina 2012 BRA did not define what they considered to be inadequate/adequate disposal of excreta of children.

In the studies with variables including defecation in a latrine, Chompook 2006 THA categorized data into children always using latrines versus not/sometimes using latrines. Clemens 1987 BGD considered the latrine or some other specially designated place versus open defecation. Knight 1992 MAL grouped defecation in a nappy and latrine as safe, whereas Maung 1992a MYA and Traoré 1994b BUR categorized data into defecation in pots and latrines versus elsewhere. Mediratta 2010b ETH and Oketcho 2012 TAN categorized defecation into the latrine or elsewhere.

In Arvelo 2009 USA, the risk factor relevant to this review was whether daycare centres had lined, lidded bins for nappy disposal (the unit of analysis was the daycare centre). In Bassal 2016 ISR, the risk factor relevant to this review was children who were not toilet trained and used nappies versus children who were toilet trained and did not use nappies. In Chiang 2005 TWN, the risk factor relevant to the review was open defecation of children aged less than five years but the reference category was not provided. Daniels 1990 LES collected data on disposal of child faeces in latrines in cases and controls but did not provide data separately for both groups. In Menon 1990 USA, the risk factor of interest was whether households had dirty nappies in the yard. Nanan 2003 PAK studied whether cases and controls were from Water and Sanitation Extension Programme (WASEP) villages, which included in its intervention education on the safe disposal of faeces (adult, child, and household animals). Thus, these six studies could not be compared with the other case-control studies and were excluded from the analyses.

\section{Primary outcome measures}

\section{Diarrhoea}

For the 50 studies that measured diarrhoea as an outcome, 18 used the WHO's definition (passage of three or more loose or liquid stools per day or more than usual for the individual) for the case definition 
of diarrhoea (Characteristics of included studies table). Other studies defined diarrhoea as: softer than usual, one to five stools per day; watery, one to five stools per day; softer than usual, five to 10 stools per day; watery, five to 10 stools per day; watery more than 10 stools per day; or dysentery (Ahmed 1993 BGD), three or more soft liquid stools within 12 hours or a single soft or liquid stool with blood, pus, or mucous (Abalkhail $1995 \mathrm{KSA}$ ), three or more loose/ watery stools in a 24-hour period or having a stool with blood or mucous (Briceño 2015 TAN; Cameron 2013 INA; Mertens 1992 SRI; Patil 2014 IND), at least three loose or watery stools within 24 hours or at least one stool with blood (Luby 2018 BGD; Null 2018 KEN), the passage of three or more liquid or semi-liquid stools in a 24-hour period or the passage of at least one liquid or semi-liquid stool with blood or mucous (Hashi 2017 ETH), occurrence of loose, unformed bowel movements at twice the normal frequency (infants, one to two stools per day; and older children, one stool per day) (Butz 1990 USA), passage of at least three liquid, watery mucoid stools with or without blood during the past 24 hours. For infants aged up to three months, an increase in the frequency and a change in the consistency of stools which was of concern to mothers (Ghosh 1997 IND), mother's own definition using local term to describe diarrhoea (Haggerty 1994 DRC), any loose, watery stool that if contained would assume the shape of the container (Kotch 2007 USA), caretaker reported increase in the stool fluidity and frequency of passing stool for at least two days (Oketcho 2012 TAN) or as reported by the mother and examined by a doctor (Traoré 1994a BUR).

Baker 2016 BGD included criteria qualifying the episode to be moderate or severe. Cummings 2012 UGA used acute watery diarrhoea in an area with laboratory-confirmed cholera cases.

Other definitions required laboratory testing to confirm shigella (Arvelo 2009 USA; Chiang 2005 TWN; Chompook 2006 THA), rotavirus (Menon 1990 USA; Strina 2012 BRA), or campylobacter (Bassal 2016 ISR). Maung 1992a MYA used persistent diarrhoea and protein energy malnutrition.

Eight studies did not provide a case definition for diarrhoea (Baltazar 1989 PHI; Berhe 2014 ETH; Dikassa 1993 DRC; Dickinson 2015 IND; Gebru 2014 ETH; Ghosh 1994 IND; Godana 2013 ETH; Heller 2003 BRA).

\section{Soil-transmitted infections}

Belizario $2015 \mathrm{PHI}$ and Patil 2014 IND both assessed the presence of STH in stool samples using the Kato-Katz technique. Park 2016 INA used the Impankaew faecal flotation technique. Cameron 2013 INA did not specify STH diagnosis technique.

\section{Excluded studies}

The 44 studies that were discussed but subsequently excluded are described in the Characteristics of excluded studies table. The other studies that were excluded without requiring discussion have reasons summarized in Figure 2.

\section{Ongoing studies}

Four studies appeared to meet our inclusion criteria but are still ongoing are presented in the Characteristics of ongoing studies table.

\section{Risk of bias in included studies}

The risk of bias of trials and non-randomized studies apart from case-control studies are summarized in Table 7, Table 8 and in the Characteristics of included studies table.

\section{Allocation (selection bias)}

Random sequence generation was at low risk of selection bias in 16 of the cluster RCTs and unclear risk in the other six. Concealment was at low risk in fourteen studies and unclear risk in eight. All CBAs, cohort, and cross-sectional studies were at high risk.

\section{Blinding (performance bias and detection bias)}

All cluster RCTs were at high risk for blinding participants and personnel. Apart from one study at unclear risk (Kotch 2007 USA) and one study at low risk (Nair 2017 IND), all other cluster RCTs were at high risk for blinding of outcome assessment.

\section{Incomplete outcome data (attrition bias)}

Barrios $2008 \mathrm{PHI}$ and Christensen 2015a KEN were at high risk for incomplete outcome data, five RCTs were at unclear risk (Butz 1990 USA; Caruso 2019 IND; Jinadu 2007 NGR; Stanton 1987 BGD; Yeager 2002 PER), and the remaining 15 at low risk.

Two CBAs were at unclear risk (Ahmed 1993 BGD; Aziz 1990 BGD), and two at low risk (Alam 1989 BGD; Park 2016 INA). The three cohort studies were at unclear risk. Of the cross-sectional studies, two were at unclear risk (Mathew 2004 ZIM; Oguro 2016 MYA), and five at low risk.

\section{Selective reporting (reporting bias)}

Three RCTs were at high risk of selective reporting (Barrios 2008 PHI; Christensen 2015a KEN; Haggerty 1994 DRC), three were at unclear risk (Humphrey 2019 ZIM; Luby 2018 BGD; Null 2018 KEN), while the other 16 RCTs were at low risk.

All CBAs, cohorts, and cross-sectional studies were at low risk apart from Mathew 2004 ZIM and Oguro 2016 MYA, which were at unclear risk.

\section{Risk of bias specific to cluster-randomized controlled trials}

Fourteen cluster-RCTs were at high risk and the remaining eight at low risk for recruitment bias. For baseline imbalance, three CRCTs were at high risk, Jinadu 2007 NGR at unclear risk, and the rest at low risk. For loss of clusters, two studies were at unclear risk (Stanton 1987 BGD; Yeager 2002 PER), and all other cluster-RCTs were at low risk. For incorrect analysis, five cluster-RCTs were at high risk, while the remaining 17 were at low risk.

\section{Risk of bias specific to non-randomized studies (except case- control studies)}

For similarity of baseline outcome measurements, Ahmed 1993 BGD was at high risk, Alam 1989 BGD at unclear risk, and Aziz 1990 BGD and Park 2016 INA at low risk. The cohort and cross-sectional studies were at unclear risk apart from Hoq 2016 BGD, which was at low risk. For similarity of baseline characteristics, Ahmed 1993 BGD was at high risk while the three other CBAs were at unclear risk. In the cohort studies, Huda 2012 BGD and Luby 2014 BGD were at low risk and Hoq 2016 BGD at high risk. The seven cross-sectional studies were at unclear risk. For adequate allocation of intervention concealment, all CBAs apart from Park 2016 INA and the 
three cohorts were at high risk. Three of the cross-sectional studies were at high risk, Gebru 2014 ETH and Oguro 2016 MYA were at unclear, and Berhe 2014 ETH and Belizario 2015 PHI at low risk. For adequate protection against contamination, Alam 1989 BGD was at high risk while the three other CBAs were at low risk. Hoq 2016 BGD was at unclear risk and the two other cohorts studies were at low risk. Berhe 2014 ETH and Gebru 2014 ETH were at high risk, while Fisher 2011 BGD; Mathew 2004 ZIM; and Oguro 2016 MYA were at unclear risk and Belizario $2015 \mathrm{PHI}$ and Waterkeyn 2005 ZIM at low risk. For adequate adjustment for confounders, the four CBAs, the cohort studies, and four cross-sectional studies were at high risk.
Berhe 2014 ETH; Gebru 2014 ETH; and Oguro 2016 MYA were at low risk.

\section{Risk of bias of the case-control studies}

The case-control studies risk of bias are presented in Table 9. In addition a funnel plot investigating the potential publication bias of case-control studies was conducted (Figure 3). The funnel plot appeared to be fairly symmetrical, indicating a low risk of publication bias. However, given the studies were observational, and the investigators may have collected data on many risk factors, they may not always present the results of the effect of child faeces disposal if it was not an important risk factor.

Figure 3. Funnel plot of case-control studies that included the disposal of child faeces in latrine versus elsewhere as a risk factor for diarrhoea (including severe and cholera).

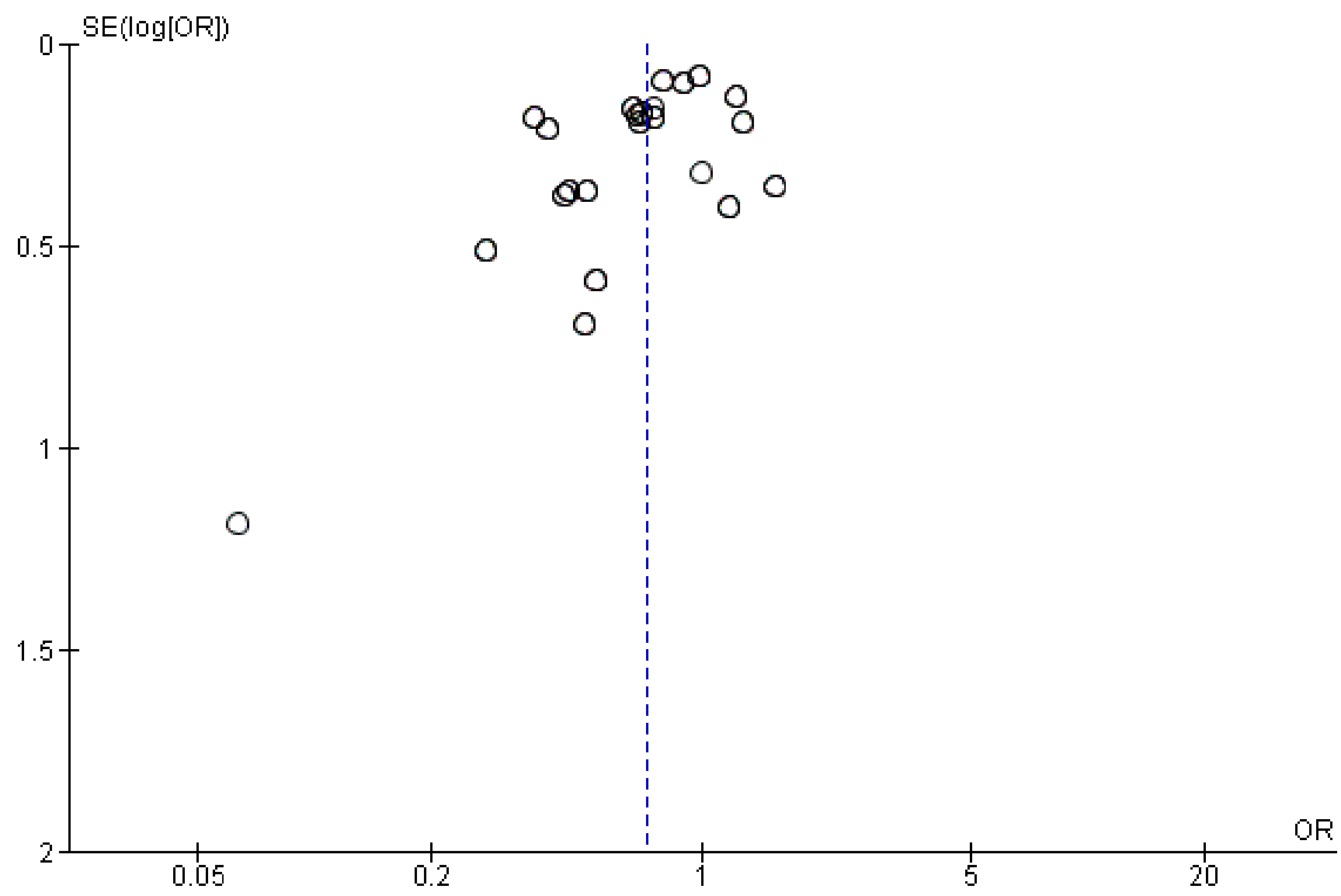

\section{Effects of interventions}

See: Summary of findings for the main comparison Summary of findings table 1; Summary of findings 2 Summary of findings table 2; Summary of findings 3 Summary of findings table 3; Summary of findings 4 Summary of findings table 4; Summary of findings 5 Summary of findings table 5; Summary of findings 6 Summary of findings table 6

\section{Education and hygiene promotion interventions \\ Randomized controlled trials}

Barrios 2008 PHI; Jinadu 2007 NGR; and Yeager 2002 PER did not measure health impacts of the interventions. Nair 2017 IND did not measure the impact of the intervention on diarrhoea, the primary outcome was children's length-for-age $Z$ score at 18 months of age. Sarrassat 2018 BUR did not measure the impact of the intervention on diarrhoea, rather on all-cause postneonatal mortality in children aged under five years and all-cause mortality in children aged under five years. None of the education and hygiene promotion interventions measured STH outcomes. 


\section{Non-randomized studies}

Mathew 2004 ZIM; Oguro 2016 MYA; and Waterkeyn 2005 ZIM did not measure health impacts of their programmes.

\section{Diarrhoea}

\section{Randomized controlled trials}

Five RCTs evaluated the impact of education and hygiene promotion interventions on diarrhoea. Two studies showed no effect on diarrhoea prevalence (RR $0.93,95 \% \mathrm{Cl} 0.84$ to 1.04 ; Analysis 1.1). Haggerty 1994 DRC found the intervention reduced the risk of children aged three to 35 months with one or more episodes of diarrhoea at any time during the surveillance period by $11 \%$ but it was not statistically demonstrated at P 0.05 level when adjusted for clustering using the inflating standard errors method. Sinharoy 2017 RWA found no effect of the 'lite' or 'classic' community health club intervention on diarrhoea in the previous seven days in children aged less than five years.

Two studies reduced diarrhoea incidence by about 30\% (RR 0.71 $95 \% \mathrm{Cl} 0.59$ to 0.86 ; Analysis 1.2). Hashi 2017 ETH reduced diarrhoea incidence by $35 \%$ (rate ratio $0.65,95 \% \mathrm{Cl} 0.57$ to 0.73 ) and Stanton 1987 BGD reduced diarrhoea incidence by $22 \%$ (rate ratio 0.78 , $95 \% \mathrm{Cl} 0.71$ to 0.86 ). There was high heterogeneity in Analysis 1.2, which could not be further investigated through subgroup analyses as there are only two studies. Possible reasons for heterogeneity included the location of the studies; Stanton 1987 BGD was conducted in urban Bangladesh and Hashi 2017 ETH in rural Ethiopia. Furthermore, the studies used different definitions of diarrhoea and different age groups (aged less than six years for Stanton 1987 BGD and aged less than five years for Hashi 2017 ETH).

Altmann 2018 TCD measured longitudinal prevalence of diarrhoea as a secondary outcome and did not detect a difference between the intervention and control groups (absolute difference -1.7, 95\% $\mathrm{Cl}-4.5$ to 1.0). The data were not in a format that could be pooled with the other studies.

\section{Controlled before-and-after studies}

Ahmed 1993 BGD only presented trends in daily diarrhoea prevalence in the education intervention and control groups in graphs. It seemed that, although for a portion of the intervention the prevalence of diarrhoea was lower than the control group, by the end of the study the prevalence was similar between groups.

\section{Controlled-cohort studies}

The SHEWA-B evaluation did not demonstrate a difference in diarrhoea prevalence in children aged less than five years (recall two days) in intervention and control groups during the first 24 months of the evaluation $(10.5 \%$ with intervention versus $10.3 \%$ with control; P = 0.67) (Luby 2014 BGD). In the last 18 months of the evaluation, they found that children in the intervention group had less diarrhoea in rural areas ( $9 \%$ with intervention versus $12 \%$ with control; RR 0.80; $P=0.033$ ); however, the evaluation found no impact in the urban slums exposed to the intervention compared to control slums ( $7 \%$ with intervention versus $6 \%$ with control; RR 1.12 $\mathrm{P}=0.348$ ). The pooled effect showed no difference in diarrhoea between intervention and control areas (RR $0.91,95 \% \mathrm{Cl} 0.64$ to 1.28 ; Analysis 1.3).

\section{Controlled cross-sectional studies}

Fisher 2011 BGD found that among households in the BRAC villages, five children had diarrhoea during the month preceding data collection compared to six in the control village, which was reported as less, significant at the $\mathrm{P} 0.05$ level, but provided no additional data $(P=0.027)$.

Berhe 2014 ETH and Gebru 2014 ETH studied the difference in twoweek diarrhoea prevalence in model and non-model households of the HEP and found that being a model family decreased the odds of having diarrhoea by about three-quarters (OR $0.26,95 \% \mathrm{Cl} 0.16$ to 0.42 ; Analysis 1.4).

\section{Severe diarrhoea}

\section{Controlled before-and-after studies}

Ahmed 1993 BGD only presented trends in daily severe diarrhoea prevalence in the intervention and control sites and it seems that although for a portion of the intervention the prevalence of severe diarrhoea was lower, by the end of the study the prevalence was similar between groups.

\section{Anthropometry \\ Randomized controlled trials}

Stanton 1987 BGD and Sinharoy 2017 RWA reported no differences in the intervention and control groups on anthropometry. Nair 2017 IND found that fewer children were underweight at 18 months in the intervention than the control arm (OR $0.81,95 \% \mathrm{Cl} 0.66$ to 0.99). However, the intervention did not have any impact on other child anthropometry measures (length-for-age $Z$ score, weight-forheight Z score, WAZ score, mid-upper arm circumference, stunting, or wasting). Analysis 1.5 shows the pooled effects of Nair 2017 IND and Sinharoy 2017 RWA on height/length-for-age.

Altmann 2018 TCD found no differences in the relapse rates to SAM at two months (absolute difference $-0.4 \%, 95 \% \mathrm{Cl}-7.2$ to 6.4 ) and six months $(-1.0 \%, 95 \% \mathrm{Cl}-4.0$ to 2.0$)$.

\section{Controlled before-and-after studies}

Ahmed 1993 BGD reported that percentages of severely malnourished children (-3 SD WAZ) reduced over time in the intervention compared to the control site (at end of the study the percentage of children -3 SD WAZ score was approximately $21.5 \%$ in the intervention group and $35.5 \%$ in the control group; $\mathrm{P}<0.0001$ ).

\section{Cohort studies}

Luby 2014 BGD did not detect a difference in nutritional status in HAZ score, WAZ, or weight-for-height Z (WHZ) score in the intervention and control groups.

\section{Mortality \\ Randomized controlled trials}

Stanton 1987 BGD reported that rates of child and infant death were similar in the intervention and control groups. Nair 2017 IND found that fewer infants died in the intervention than the control (OR 0.63, $95 \% \mathrm{Cl} 0.39$ to 1.00 ). Altmann $2018 \mathrm{TCD}$ did not detect a difference in death rate between the intervention and control groups. Sarrassat 2018 BUR did not detect an intervention effect on all-cause postneonatal mortality in children aged under five years or all-cause mortality in children aged under five years. 


\section{Behaviour change}

\section{Randomized controlled trials}

Six RCTs reported behavioural outcomes after the education and hygiene promotion intervention. For three of the studies, this was the main outcome (Barrios 2008 PHI; Jinadu 2007 NGR; Yeager 2002 PER), while for the other three studies it was as intermediate outcome (Sarrassat 2018 BUR; Sinharoy 2017 RWA; Stanton 1987 BGD). Different behaviours related to child faeces disposal were measured in the different interventions.

Analysis 1.6 shows the impact of the interventions on latrine use by children aged less than five years. Jinadu 2007 NGR reported an increase in latrine use by children aged 25 to 60 months, while Yeager 2002 PER observed no effect of the intervention on latrine use by children aged 15 to 47 months. Stanton 1987 BGD found no decrease in open defecation in the living area by ambulatory children (67\% in the intervention group versus $63 \%$ in control group).

Analysis 1.7 presents data on potty use of children after the intervention, which was higher in households in the intervention arm in Jinadu 2007 NGR compared to the control arm, but did not show a difference between intervention and control households in Yeager 2002 PER.

Analysis 1.8 shows the impacts of interventions on child faeces disposal behaviours. Safe child faeces disposal practices were not different between intervention and control arms in Sarrassat 2018 BUR or Yeager 2002 PER. Sinharoy 2017 RWA also found no impact of the 'classic' or 'lite' intervention on safe disposal (Analysis 1.9).

Analysis 1.10 shows the impact of interventions on faeces observed in the yard. Barrios $2008 \mathrm{PHI}$ found no effect on faeces visible in the yard, Jinadu 2007 NGR reported an increase in no child faeces observed in the yard. There was no obvious difference between study arms, in either intervention ('classic' and 'lite') in faeces observed in compounds in Sinharoy 2017 RWA (Analysis 1.11). It is important to note that studies observing fewer faeces in the yard, might not necessarily be an indicator of increased safe disposal as the child faeces may not have been disposed of in a latrine but rather been thrown elsewhere.

\section{Controlled-cohort studies}

Huda 2012 BGD and Luby 2014 BGD found no impact of the SHEWA-B intervention on child faeces disposal behaviour at mid-study and end of the study compared to controls (Analysis 1.12).

\section{Controlled cross-sectional studies}

Berhe 2014 ETH; Fisher 2011 BGD; and Gebru 2014 ETH found an increase in safe disposal of child faeces in the intervention areas compared to the control areas (Analysis 1.13). Although Gebru 2014 ETH did not specify what they considered to be safe disposal, it was assumed that their definition included burying of faeces as well as disposal in the latrines as that is what is promoted in the HEP. Thus when calculating the risk of safe disposal for Berhe 2014 ETH, the same classification of safe disposal was used, although restricting the definition of safe disposal to just defecation in a latrine and disposal in a latrine; it also showed that intervention increased safe disposal. Oguro 2016 MYA found that the presence of a WVG did not have a significant effect on the proportion of appropriately disposed faeces compared to the control villages (OR 3.57, 95\% Cl 0.53 to 23.65).
Mathew 2004 ZIM found that in CHC areas, a lower percentage of children were not using a latrine compared to control areas (approximately $54 \%$ in $\mathrm{CHC}$ area versus $83 \%$ in control areas); however, no statistical analysis was presented and insufficient data were provided to perform an analysis. In Waterkeyn $2005 \mathrm{ZIM}$, there was no difference detected in observing child faeces in the yard in $\mathrm{CHC}$ households versus control households (in Tsolotsho: $4 \%$ in $\mathrm{CHC}$ households versus $0 \%$ in control households; $\mathrm{P}=0.0807$; in Makoni: $16 \%$ in $\mathrm{CHC}$ households versus $23 \%$ in control household; $\mathrm{P}=0.0972$.

\section{Community-led total sanitation interventions plus adaptations}

\section{Diarrhoea}

\section{Randomized controlled trials}

The pooled effect of the CLTS interventions plus adaptations revealed no effect on diarrhoea prevalence (RR $0.92,95 \% \mathrm{Cl} 0.79$ to 1.07; Analysis 2.1). Pickering $2015 \mathrm{MLI}$ did not find a difference in child diarrhoea prevalence between intervention and control groups with either a two-day (22.5\% with intervention versus $24.1 \%$ with control; $P=0.486)$ or two-week recall period ( $31.2 \%$ with intervention versus $32.0 \%$ with control; $P=0.787$ ). Patil 2014 IND did not find a difference in diarrhoea prevalence (seven-day recall) between the intervention and control ( $7.4 \%$ with intervention versus $7.7 \%$ with control; $P=0.687$ ). Briceño 2015 TAN found no decrease in diarrhoea prevalence between the sanitation arm and the control arm, but found a decrease in diarrhoea in the sanitation and handwashing combined arm (12.5\% with sanitation and handwashing versus $16.8 \%$ with control for 14 -day recall). Diarrhoea symptoms in the past seven days did not show a difference between either treatment (TSSM and HWWS combined or TSSM alone) and control groups. Cameron 2013 INA found that the intervention group had lower diarrhoea prevalence compared to control children $(2.4 \%$ with intervention versus $3.8 \%$ with control: $P=0.07$ for seven-day recall and $1.6 \%$ with intervention versus $3.1 \%$ with control; $P=0.025$ for two-day recall). Dickinson 2015 IND did not present data in a way that could be pooled with the other studies, but found that the TSC was associated with decreased diarrhoea rates (point estimate -0.21 ); however, these effects were not statistically significant at the $\mathrm{P} 0.05$ level.

\section{Soil-transmitted helminth}

\section{Randomized controlled trials}

Two of the CLTS RCTs reported on the impact of the interventions on STHs and found no effect on any STH infection (RR 1.03, 95\% $\mathrm{Cl} 0.64$ to 1.65; Analysis 2.2) or on A lumbricoides (RR $1.01,95 \% \mathrm{Cl}$ 0.60 to 1.71; Analysis 2.3). Patil 2014 IND did not find a difference in helminth prevalence between intervention and control groups (any helminth: 5.9\% with intervention versus 5.6\% with control; A lumbricoides: $4.3 \%$ with intervention versus $4.4 \%$ with control). Cameron 2013 INA did not detect a difference in the probability of having any helminth between the children in the treatment and control groups ( $4.0 \%$ with intervention versus $3.9 \%$ with control; $\mathrm{P}$ $=0.889$ ), A lumbricoides (3.4\% with intervention versus $3.3 \%$ with control; $\mathrm{P}=0.881), T$ Trichuris $(0 \%$ with intervention versus $0.1 \%$ with control; $P=0.319)$, or hookworm $(0.6 \%$ with intervention versus $0.5 \%$ with control; $\mathrm{P}=0.733$ ). 


\section{Controlled cross-sectional studies}

Belizario $2015 \mathrm{PHI}$ found that in villages with CLTS, the prevalence of STH was $42 \%$ (67.4\% in Buenavista and $4.9 \%$ in Caubang), whereas in villages without CLTS, the prevalence of STH was $16.8 \%(16.7 \%$ in Bitoon and $16.8 \%$ in Saub). Prevalence in CLTS versus non-CLTS villages of Ascaris was $22 \%$ versus $11 \%$, for Trichiuris was $34 \%$ versus $8.9 \%$, and for hookworm was $4 \%$ versus $0 \%$.

\section{Dysentery}

\section{Randomized controlled trials}

Pickering $2015 \mathrm{MLI}$ did not detect a difference in prevalence of blood in stools between intervention and control groups using a two-day recall period ( $1.2 \%$ with intervention versus $1.4 \%$ with control; $\mathrm{P}=0.481$ ), but the two-week prevalence was lower in the intervention than control villages (prevalence ratio (PR): $0.68,95 \% \mathrm{Cl}$ 0.48 to $0.97 ; P=0.031$ ). Cameron 2013 INA found lower prevalence of mucous or blood in stools (seven-day prevalence) in intervention versus control $(0.8 \%$ with intervention versus $2 \%$ with control; $\mathrm{P}=$ 0.034). Overall the pooled effect showed no effect of the interventions (RR $0.69,95 \% \mathrm{Cl} 0.35$ to 1.34; Analysis 2.4).

\section{Intensity of soil-transmitted helminth infection (number of eggs per gram of stool)}

\section{Randomized controlled trials}

Cameron 2013 INA did not detect a difference in infection intensity between intervention and control groups.

\section{Controlled cross-sectional studies}

Belizario $2015 \mathrm{PHI}$ found that the prevalence of moderate-heavy intensity infections was $14.5 \%$ in CLTS villages compared to $2.8 \%$ in non-CLTS villages.

\section{Presence of pathogenic microbes in stool assays}

\section{Randomized controlled trials}

Patil 2014 IND did not detect a difference in prevalence of any protozoan present in intervention and control $(21.7 \%$ with intervention versus $25.7 \%$ with control) or entamoeba histolytica (3.3\% with intervention versus $2.5 \%$ with control). They found lower prevalence of Giardia Lamblia ( $18.4 \%$ with intervention versus $23.2 \%$ with control; MD 4.8\%; $\mathrm{P}=0.047)$.

\section{Anthropometry}

\section{Randomized controlled trials}

Patil 2014 IND and Cameron 2013 INA reported finding no differences in the intervention and controls groups on anthropometry. Pickering $2015 \mathrm{MLI}$ found that children aged less than five years in intervention villages were taller than those in control villages by a mean of 0.17 in $\mathrm{HAZ}$ score $(95 \% \mathrm{Cl} 0.04$ to 0.31$)$ and did not find a difference in WAZ scores (mean $0.09 \mathrm{WAZ}$ score, $95 \% \mathrm{Cl}-0.03$ to 0.20 ), when restricting the analysis to younger children a larger effect was found on HAZ. Briceño 2015 TAN did not find a difference between the sanitation only arm and the control group (there was a decrease in weight for age by 0.075 SDs off a mean WAZ score of $1.03(\mathrm{P}<0.05)$ and weight-for-height by 0.097 SDs from a mean WHZ score of $0.055(P<0.05)$ in the combined arm of the intervention (hand washing with soap and sanitation) compared to the control group). The pooled effect on HAZ (MD $0.06,95 \% \mathrm{Cl}-0.07$ to $0.19 ; 3$ studies with usable data) and WAZ scores (MD 0.04, 95\% Cl -0.04 to 0.11) did not demonstrate an effect (Analysis 2.5; Analysis 2.6). Dickinson 2015 IND could not be pooled due to the analysis presented in the paper. The study reported that mid-upper-arm-circumference (MUAC) $Z$ scores were 0.20 to 0.30 SDs higher in treatment villages relative to controls after the sanitation campaign. HAZ had increased by about 0.37 to 0.52 SDs $(P<0.01)$ and WAZ increased by 0.26 to $0.31 \mathrm{SDs}(P<0.05)$ in treatment villages relative to controls after the sanitation campaign.

\section{Cross-sectional studies}

Belizario $2015 \mathrm{PHI}$ examined the nutritional status of subgroups of children (weight for age and height for age for two- to five-year olds and six- to nine-year olds and BMI for age and height for age for 10to 15-year olds). The study did not identify a difference between CLTS villages and non-CLTS villages apart from BMI for age (10- to 15 -year olds, $n=120$ ). About $2.5 \%$ of children in CLTS villages were stunted compared to $21.3 \%$ in the non-CLTS villages.

\section{Mortality}

\section{Randomized controlled trials}

Pickering 2015 MLI did not find a difference in all-cause mortality between intervention and control groups but fewer households in the intervention group reported to have had a diarrhoeal-related death ( 16 total diarrhoeal deaths with intervention versus 34 with control; PR $0.46,95 \% \mathrm{Cl} 0.26$ to 0.83 ) and child diarrhoeal deaths (11 child diarrhoea deaths in intervention versus 23 in control; PR $0.47,95 \% \mathrm{Cl} 0.23$ to 0.98 ) than controls.

Briceño 2015 TAN did not find a difference in the mortality of children aged less than five years in control and intervention groups.

\section{Behaviour change}

\section{Randomized controlled trials}

All the CLTS intervention studies reported on behavioural outcomes as intermediate outcomes of their intervention.

Analysis 2.7 shows the effects of the CLTS interventions on open defecation by children aged less than five years. Cameron 2013 INA; Patil 2014 IND; and Pickering 2015 MLI reported a significant difference in no open defecation by children aged less than five years in intervention arms compared to control.

Analysis 2.8 shows the impacts of CLTS interventions on child faeces disposal behaviours (the data for Cameron 2013 INA were not in a usable format). Safe child faeces disposal practices was higher in the intervention than control arms in Briceño 2015 TAN (safe disposal also improved in the hand washing and sanitation combination arm) and Patil 2014 IND.

Pickering $2015 \mathrm{MLI}$ found that potty use of children after the intervention was higher in intervention arms compared to control arms (Analysis 2.9).

Dickinson 2015 IND measured the reported time spent walking to defecation sites and found that children (aged less than five years) in the intervention arm experienced time savings of about $2.2 \mathrm{~min}-$ utes per defecation trip.

\section{Sanitation hardware and behaviour change interventions}

The WASH-B studies measured effects of the interventions on health outcomes, including diarrhoea and anthropometry (Luby 
2018 BGD; Null 2018 KEN). The pilot study of the WASH-B intervention, Christensen 2015a KEN, and the Sundara Grama intervention, Caruso 2019 IND, only measured the effect of their intervention on behaviour change.

\section{Diarrhoea}

\section{Randomized controlled trials}

Pooled results from the WASH-B sanitation arms showed no effect on diarrhoea prevalence (RR $0.79,95 \% \mathrm{Cl} 0.49$ to 1.26; Analysis 3.1). However, the two trials had disparate effects on diarrhoea. In Bangladesh, Luby 2018 BGD found that the seven-day diarrhoea prevalence was lower among index children and children aged under three years at enrolment who received the sanitation intervention compared to the control arm (PR $0.61,95 \% \mathrm{Cl} 0.46$ to 0.81 ). In Kenya, however, Null 2018 KEN found no effect of the sanitation intervention on diarrhoea prevalence.

\section{Soil-transmitted helminth}

\section{Randomized controlled trials}

The WASH-B studies also assessed the impact of the intervention on STH prevalence in a subset of children (results pending publication) and on STH presence in household soil. In Kenya, the authors found that the combined sanitation intervention group (sanitation and WASH households) had no impact on STH prevalence in household soil ( $17.0 \%$ with intervention versus $18.9 \%$ with control), concentration of STH eggs in soil or single STH species or viable eggs (Steinbaum 2017 (see under Null 2018 KEN)).

\section{Anthropometry}

\section{Randomized controlled trials}

The WASH-B sanitation arms had no effects on anthropometry outcomes. Luby 2018 BGD and Null 2018 KEN did not find a difference in length-for-age $Z$ scores (pooled MD $-0.04,95 \% \mathrm{Cl}-0.12$ to 0.04 ; Analysis 3.2) or in WAZ scores in children in sanitation intervention arms (pooled MD $-0.04,95 \% \mathrm{Cl}-0.11$ to 0.04 ; Analysis 3.3). Both studies also found no impact of the sanitation arms on other anthropometry outcomes (weight-for-length Z scores, head circumference, stunting, severe stunting, wasting, and underweight).

\section{Mortality}

\section{Randomized controlled trials}

The sanitation arms of the WASH-B studies had no impact on allcause mortality (Luby 2018 BGD; Null 2018 KEN).

\section{Behaviour change}

All sanitation hardware and behaviour change RCTs reported on behavioural outcomes. For Christensen 2015a KEN and Caruso 2019 IND it was the main outcome. While for Luby 2018 BGD and Null 2018 KEN it was as intermediate outcomes of their intervention.

Analysis 3.4 shows the impacts of interventions on child faeces disposal behaviours. Safe child faeces disposal practices were higher in the intervention than control arms in Luby 2018 BGD (although adjusted risk difference (RD) did not show a difference, RD 20, 95\% $\mathrm{Cl}-11$ to 51) and Null 2018 KEN. In Null 2018 KEN, safe child faeces disposal improved from baseline (19\% safely disposed) more in year one $(77 \%)$ than at the end of the study $(37 \%)$ in the sanitation arm. In both pilot RCTs in Kenya, child faeces disposal was higher in the intervention arm compared to the control arm (Analysis 3.5).
In the sanitation only arm, a difference was not detected (RD 0.10, $95 \% \mathrm{Cl}-0.21$ to 0.42 ; Christensen 2015a KEN), whereas in the combined WASH arm, appropriate child faeces disposal was 47.8 percentage points higher than in the control arm (RD $0.47,95 \% \mathrm{Cl} 0.372$ to 0.571 ; Christensen 2015b KEN). The intention-to-treat difference analysis in Caruso 2019 IND found an increase in reported safe disposal of child faeces of $20.4 \%(95 \% \mathrm{Cl} 11.7 \%$ to $29.2 \%$; $\mathrm{P}<0.001)$ in the intervention group at the end of the study after accounting for the increase in safe disposal of child faeces observed in the control group.

Luby 2018 BGD found that potty use of children after the intervention was higher in intervention arms compared to control arms (although adjusted RD in the sanitation arm did not demonstrate an effect, RD 22, $95 \% \mathrm{Cl}-18$ to 61 ; Analysis 3.6). Luby 2018 BGD also observed some use of the sani-scoops for cleaning human faeces in the sanitation (27\%) and combined WASH arms (25\% in the WASH arm, $38 \%$ in the WASH-nutrition arm) of the study, while this behaviour was not observed in the control arm.

Christensen 2015a KEN and Christensen 2015b KEN observed human faeces in the compound was lower in the intervention arms compared to the control arms (Analysis 3.7); however, this was only statistically significant at the 0.05 level in the combined WASH trial. Luby 2018 BGD also observed fewer human faeces in the compounds in the sanitation intervention arm compared to the control; however, this was not reported as statistically significant at the 0.05 level.

\section{WASH hardware and education/behaviour change interventions}

\section{Diarrhoea}

\section{Randomized controlled trials}

Humphrey 2019 ZIM found that at 12 and 18 months, the prevalence of diarrhoea was not different between WASH and non-WASH groups(Analysis 4.1).

\section{Controlled before-and-after studies}

The pooled effect of the CBAs evaluating WASH hardware and education interventions reduced diarrhoea incidence by about a quarter (rate ratio $0.77,95 \% \mathrm{Cl} 0.71$ to 0.84 ; two studies; Analysis 4.2 ).

\section{Soil-transmitted helminth}

\section{Controlled before-and-after studies}

Park 2016 INA found that the odds of STH reinfection (participants in both arms were given albendazole if found to be infected at baseline) were lower in the intervention village compared to the control (OR $0.17,95 \% \mathrm{Cl} 0.02$ to $0.73, \mathrm{P}=0.014$ ).

\section{Dysentery and persistent diarrhoea}

\section{Controlled before-and-after studies}

Aziz 1990 BGD found that children had 27\% less dysentery (incidence density ratio (IDR) $0.73,95 \% \mathrm{Cl} 0.61$ to 0.88 ) and $40 \%$ less persistent diarrhoea (IDR $0.58,95 \% \mathrm{Cl} 0.52$ to 0.65 ) in the intervention than controls. 


\section{Anthropometry}

\section{Randomized controlled trials}

Humphrey 2019 ZIM found that WASH interventions had no effect on the mean infant length-for-age $Z$ score or any other growth measurements except for mean head-circumference-for-age $Z$ scores in adjusted analyses ( $Z$ score difference $0.08,95 \% \mathrm{Cl} 0.01$ to 0.15 ); however, this effect was driven entirely by the infant and young child feeding plus WASH group.

\section{Controlled before-and-after studies}

Aziz 1990 BGD did not find a difference in nutritional status in the intervention and control groups.

\section{Cohort studies}

Hoq 2016 BGD did not detect a difference in the rate of change in underweight children (WAZ < 2) over time. However, the study found a difference in the rate of change in acute malnutrition (MUAC < $125 \mathrm{~mm}$ ), which was significantly higher in the integrated WASH intervention site $(0.02 \%, 95 \% \mathrm{Cl} 0.014 \%$ to $0.026 \%)$ compared to the comparison site $(0.006 \%, 95 \% \mathrm{Cl} 0.002 \%$ to $0.010 \%)\left(\mathrm{Chi}^{2}\right.$ test $=20$, $\mathrm{P}=0.0001)$.

\section{Mortality}

\section{Randomized controlled trials}

Humphrey 2019 ZIM found that cumulative mortality at 18 months was similar between WASH and non-WASH groups (adjusted PR $0.96,95 \% \mathrm{Cl} 0.72$ to 1.30$)$.

\section{Behaviour change}

\section{Randomized controlled trials}

Humphrey 2019 ZIM found that $77 \%$ of mothers in the WASH groups reported to dispose of water from cleaning infant nappies with faeces in a latrine compared with $32 \%$ in non-WASH groups.

\section{Daycare centre-based hygiene hardware and education interventions}

\section{Diarrhoea}

Two interventions were conducted in daycare centres in the USA Butz 1990 USA found that symptoms of diarrhoea were lower in intervention daycare centres (OR $0.715,95 \% \mathrm{Cl} 0.54$ to 0.72 ). Kotch 2007 USA found that children in the intervention daycare centres had fewer episodes of diarrhoea compared to the control group (0.90 diarrhoea illnesses per 100 child-days with intervention versus 1.58 diarrhoea illnesses per 100 child-days with control; $\mathrm{P}<$ $0.001)$.

\section{Case-control studies: disposal of child faeces in the latrine versus elsewhere}

\section{Diarrhoea}

Pooled results from case-control studies that presented data for child faeces disposal indicated that disposal of faeces in the latrine decreased the odds of diarrhoea by about a quarter among all ages (OR $0.73,95 \% \mathrm{Cl} 0.62$ to $0.85 ; 23$ comparisons) and children aged less than five years (OR $0.72,95 \% \mathrm{Cl} 0.61$ to $0.85 ; 20$ comparisons) (Analysis 5.1). See Table 10 for more information on those studies. In subgroup analyses, it seemed the effect of disposal of faeces in a latrine differed according to the type of diarrhoea, with a larger re- duction in acute (possibly bloody) diarrhoea than moderate-to-severe diarrhoea (Analysis 5.2; Analysis 5.3; Analysis 5.4; Analysis 5.5; Analysis 5.6). Although studies with no specified case definition also had a lower OR. The quality of the studies, as indicated by the number of stars obtained when applying the NOS risk of bias criteria, also seemed to differ among groups, with higher quality subgroups having lower ORs. The effect of safe disposal on diarrhoea did not seem to differ according to the data collection method, income level of the country, or setting where the study was conducted (Analysis 5.2; Analysis 5.5; Analysis 5.6).

Case-control studies: defecation of children in the latrine versus elsewhere

\section{Diarrhoea}

Pooled results from case-control studies that presented data for children defecating in the latrine indicated that children using the latrine reduced the odds of diarrhoea by about half in all ages (OR $0.54,95 \% \mathrm{Cl} 0.33$ to $0.90 ; 7$ studies); the corresponding pooled point estimate for children aged less than five years was similar, although the confidence intervals were wide $(\mathrm{OR} 0.54,95 \% \mathrm{Cl} 0.28$ to 1.07 ; 5 studies) (Analysis 6.1). See Table 11 for more information about these studies. In subgroup analyses, persistent diarrhoea had lower ORs than acute and acute watery diarrhoea (Analysis 6.2; Analysis 6.3; Analysis 6.4; Analysis 6.5; Analysis 6.6). The quality of the studies also seemed to change the observed association between children defecating in a latrine and diarrhoea. As the quality of the studies improved, as indicated by the number of stars, the association became closer to null. The effect of child defecation in the latrine on diarrhoea also seemed to differ according to the data collection method and country income level.

\section{Case-control studies: other interventions}

Arvelo 2009 USA did not show a difference in lidded bins for nappy disposal between case and control licensed daycare centres (OR $2.0,95 \% \mathrm{Cl} 0.5$ to 8.1 ). Bassal 2016 ISR found that the odds for infection with campylobacter among children who were not toilet trained and used nappies were higher than among those who did not use nappies (OR $7.36,95 \% \mathrm{Cl} 1.66$ to 32.70 ; $\mathrm{P}<0.01$ ). Chiang 2005 TWN found that open defecation of children increased the odds of being a case (OR 6.32, 95\% $\mathrm{Cl} 0.7$ to 54.5 , adjusted for ethnicity and living residence). Daniels 1990 LES found that among both the cases and controls, $50 \%$ of latrine owners reported that they disposed of the child's stools in the latrine; however, this was not shown separately for cases and controls. Menon 1990 USA did not find a difference in the number of dirty nappies in the yards of case households compared to controls (OR 3.5, $95 \% \mathrm{Cl} 0.88$ to 13.93). Nanan 2003 PAK found that cases were more likely to come from non-WASEP villages than controls (OR $1.33,95 \% \mathrm{Cl} 1.0$ to 1.8 ).

\section{Clinical visits for diarrhoea, serology, and other markers of infection and disease}

No included study reported on these outcomes.

\section{Adverse events}

No study reported adverse events related to the child faeces disposal components of the interventions. 


\section{Sensitivity analyses}

The fixed-effect and random-effects analyses were similar and did not change the conclusions of the analyses. The random-effects method measures were more conservative, having larger Cls.

\section{DISCUSSION}

See Summary of findings for the main comparison; Summary of findings 2; Summary of findings 3; Summary of findings 4; Summary of findings 5; Summary of findings 6 .

\section{Summary of main results}

While numerous studies met the review's inclusion criteria, we consider the evidence linking the safe disposal of child faeces with diarrhoea or STH infection to be limited. Few studies focused solely on interventions aimed at improving the collection or disposal of child faeces. Of the 22 RCTs, only one focused exclusively on improving child faeces disposal behaviour, and that study only measured behaviour change. Nine other RCTs included child faeces disposal as one of the messages in their education and hygiene promotion intervention, only seven of those included health outcomes. Of the other RCTs, five measured the health impacts of their intervention to end open defecation of the whole community including children as well as indicators of child faeces disposal behaviour change, one evaluated a WASH hardware and behaviour change intervention, four included child faeces disposal hardware (potties and saniscoops) within its sanitation intervention, and two were based in daycare facilities. Of the four CBAs, one included child faeces disposal as part of several messages in its education and hygiene promotion intervention, while the other three provided WASH hardware along with education that included child sanitation messages. The health impacts of the child faeces disposal component of these interventions can thus not be measured.

The three cohort studies and four of the seven cross-sectional studies included in the review also measured the health effect of combined interventions, while three only measured the behaviour change after the $\mathrm{CHC}$ or women volunteer group intervention.

The most direct evidence supporting the protective effect from safe child faeces disposal in a latrine on diarrhoea came from the casecontrol studies. Twenty-seven case-control studies were included, with 21 of them being used in the quantitative analyses. The evidence from these studies suggested that disposing of child faeces in a latrine was associated with reduced odds of diarrhoea (OR 0.73 , $95 \% \mathrm{Cl} 0.62$ to 0.85 ; very low-certainty evidence). These studies also suggested that children defecating in a latrine rather than elsewhere was associated with reduced odds of diarrhoea (OR 0.54 , $95 \% \mathrm{Cl} 0.33$ to 0.90 ; very low-certainty evidence). It is important to note that we classified safe child faeces disposal as disposal into any latrine or as defined by the study authors. It is unclear from current evidence whether there is a difference in effect between disposing of faeces in improved versus unimproved latrines.

Only four studies (two RCTs, one CBA, and one cross-sectional) reported impacts of their intervention on STH infection. Both RCTs were interventions aiming to stop open defecation generally (not safe disposal of child faeces specifically) and neither study found a health impact on helminth infection. Both RCTs reported reduction in open defecation of children and Patil 2014 IND reported improved disposal of child faeces in the intervention arm. However, Patil 2014 IND found that the intervention led to a small in- crease in latrine construction accompanied with a small decrease of open defecation and that these improvements were not sufficient to see an improvement in health outcomes (both diarrhoea and STH). In Cameron 2013 INA, the intervention led to a moderate increase in toilet construction, with associated decreases in open defecation in households that did not have access to sanitation at baseline, which suggested an improvement in behaviour due to the toilet construction. While, the intervention was associated with lower diarrhoea prevalence in the intervention communities, there was no effect on STH infection. This could be because diarrhoea prevalence was measured through self-reports, which could have been biased due to non-blinding while the STH infections were diagnosed from stools, thus a more objective measure. Alternatively, as STH eggs can survive longer in the environment than diarrhoea-causing pathogens, it may take longer to observe an impact on STH. The CBA study, Park 2016 INA, found that providing simple pit latrines and hygiene education reduced the prevalence of STH in the intervention village compared to the control village. It should be noted that this study had a small sample size and no intermediary outcomes, such as behaviour change, were measured to support the conclusions of the study. In the cross-sectional study, which compared STH prevalence and nutritional outcomes in two villages that were ODF after a CLTS campaign with control villages, found that STH prevalence was higher in the CLTS villages (Belizario $2015 \mathrm{PHI}$ ). Again, this study did not report indicators of the campaign success, such as indicators of latrine use or child sanitation, which could have explained the findings.

\section{Overall completeness and applicability of evidence}

Most of the included studies were conducted in low- or lower middle-income countries, while some were in upper middle- or highincome countries. Most study sites were in rural areas (64\%).

Few studies investigated specific hardware for safe child faeces disposal. Two studies promoted potties (Jinadu 2007 NGR; Yeager 2002 PER), and potties were one of the criteria of the ODF certification in CLTS in Mali (all family members had to use the latrine or a child potty) (Pickering 2015 MLI). However, it was unclear how much focus there was on safe disposal of child faeces as part of the triggering of activities in the paper. The sanitation hardware and behaviour change studies provided potties and sani-scoops (Caruso 2019 IND; Christensen 2015a KEN; Christensen 2015b KEN; Luby 2018 BGD; Null 2018 KEN). The studies did find improvements in child faeces disposal at follow-up and some use of the hardware. Ahmed 1993 BGD included messaging to use a dirt thrower to dispose of child faeces. Butz 1990 USA and Kotch 2007 USA included some nappy changing equipment in their intervention and instructions to dispose of nappies in plastic bags (Butz 1990 USA), and rollout waste bins for nappy disposal (Kotch 2007 USA). No other included study had a hardware component and none encompassed different hardware solutions for different age groups (e.g. nappies for babies, latrine slabs for latrine training).

Few studies included details of the behaviour change messaging that was provided and only a few based their interventions on theory and behavioural frameworks and developed them through formative research (Caruso 2019 IND; Humphrey 2019 ZIM; Luby 2018 BGD; Null 2018 KEN; Yeager 2002 PER). 


\section{Certainty of the evidence}

The certainty of evidence of the RCTs was very low, low, or moderate due to the risk of bias, the indirectness of the evidence, heterogeneity, and imprecision. The CBAs, cohort studies, and crosssectional studies were all very low-certainty evidence due to risk of bias, heterogeneity, indirectness, and imprecision. The certainty of evidence for case-control studies was very low due to heterogeneity (Summary of findings for the main comparison; Summary of findings 2; Summary of findings 3; Summary of findings 4; Summary of findings 5 ; Summary of findings 6 ).

\section{Potential biases in the review process}

We endeavoured to identify all eligible studies by conducting searches with no time or language restrictions. The high number of studies resulting from the search criteria meant that it was not possible for two review authors to check the titles, so only one author went through all titles excluding those that were clearly irrelevant. This could have biased the findings as some relevant findings could have been missed by the single author. However, with knowledge of this risk, we sought only to exclude titles that were clearly irrelevant (e.g. dental hygiene; chemical pollution; non-relevant infectious diseases such as tuberculosis, malaria, or dengue; surgery; pharmacology; etc.) and kept anything that was unclear or possibly relevant for abstract screening, which two review authors conducted.

\section{Agreements and disagreements with other studies or reviews}

There are only two previous reviews on the safe disposal of child faeces. Gil 2004 was conducted in the early 2000s, and included 10 observational studies and no intervention studies. It reported that child faeces disposal behaviours considered risky (open defecation, stool disposal in the open, stools not removed from soil, stools seen in household soil, and children seen eating faeces) were associated with a $23 \%$ increase in risk of diarrhoea (RR $1.23,95 \% \mathrm{Cl} 1.15$ to 1.32 ); however, behaviours considered safe (use of latrines, nappies, potties, toilets, washing nappies) were borderline protective (RR $0.93,95 \% \mathrm{Cl} 0.86$ to 1.00 ). An unpublished update of that systematic review, Scott 2008, found a further four papers. Two papers found that unsafe disposal of child faeces (not in a latrine) increased the risk of diarrhoea (Heller 2003 BRA; Tumwine 2002), while two papers did not demonstrate an association between presence of human faeces in the compound and bloody diarrhoea (Brooks 2003), and between potty use and typhoid fever (Ram 2007). Although we identified and included substantially more studies in our review, the results were not inconsistent with this previous research. Both found safe disposal of child faeces to be protective against diarrhoea.

The second review was published in 2016 (Morita 2016). This review differed from ours in that it used different inclusion criteria, resulting in far fewer studies (eight) compared to our 63 studies. Both reviews agreed that none of the included studies that reported health outcomes focused exclusively on improving child faeces disposal and that there is a need for RCTs to evaluate the health impact of safe child faeces disposal interventions.

Our results are also generally consistent with recent reviews of the effects of sanitation generally against diarrhoeal disease. Freeman and colleagues reported improved sanitation to reduce the odds of diarrhoeal disease by $12 \%$ compared to unimproved sanitation
(OR $0.88,95 \% \mathrm{Cl} 0.83$ to $0.92 ; 27$ studies), when restricted to 16 intervention studies, the protective effect doubled to $23 \%$ (OR 0.77 , $95 \% \mathrm{Cl} 0.66$ to 0.91 ) (Freeman 2017).

\section{AUTHORS' CONCLUSIONS}

\section{Implications for practice}

While child faeces may represent an important source of pathogen exposure, there is little research on the health effects of interventions to improve the safe disposal of child faeces, except as part of a larger sanitation initiative. The available evidence suggests that children should be encouraged to use latrines and that child faeces should be disposed of in a latrine. However, the evidence is of very low certainty, thus we are unsure about the effect of these interventions.

\section{Implications for research}

Randomized controlled trials (RCTs) that study the health impact of different hardware and software interventions aimed specifically at improving the safe disposal of child faeces of different age groups will help to clarify the potential for child faeces management to prevent diarrhoea and soil-transmitted helminth (STH) infections. These studies should be conducted in different settings to improve external validity. Additionally, since these studies cannot normally be blinded, measuring effects using objective outcomes, such as pathogens in stools or anthropometry, will also reduce potential risk of bias associated with reported diarrhoea. The RCTs should include intermediate measures to study the impact of the intervention on possible transmission routes, such as contamination of water, soil, and hands, to increase the plausibility of the findings. Additionally, the studies should measure behaviour change over longer periods and within entire communities.

Future studies should consider the various steps involved in the management of child faeces, as there are several points which may cause exposure, including the place of defecation, cleaning practices, place of disposal, and subsequent handwashing.

Some studies did not explain their definition of safe disposal. Water, sanitation, and hygiene (WASH) interventions should be more explicit about their 'hygiene' or 'sanitation' education interventions to outline what messages were included and how these were developed. Additionally community-led total sanitation (CLTS) studies should be more clear on whether children were specifically included in their efforts to end open defecation. None of the five interventions aiming to eliminate open defecation explicitly described the messages that were given to the communities about child faeces disposal or the use of latrines by children. We would recommend that interventions that use CLTS messaging to eliminate open defecation be more explicit about their contents and how they address the needs of different age groups, including children. In addition, CLTS interventions should include child faeces disposal in their manuals and in the indicators that are measured for communities to be considered open defecation-free, as this is not consistently done.

\section{ACKNOWLEDGEMENTS}

The editorial base of the Cochrane Infectious Diseases Group is funded by UK aid from the UK government for the benefit of lowand middle-income countries (project number 300342-104). The 
views expressed do not necessarily reflect the UK government's official policies.
We thank Aurelie Jeandron for assessing the eligibility of the Russian literature; Lyndsey Gray, Christian Landon, and Czarina Cooper for doing abstract and full-text eligibility assessments; and Christianne Esparza and her colleagues for assistance in finding articles. 


\section{R E F E R E N C E S}

\section{References to studies included in this review}

Abalkhail 1995 KSA \{published data only\}

Abalkhail B, Bahnassy AA, Al Abasi H. Maternal knowledge, attitudes, and practices as predictors of diarrhoeal disease in Saudi children. Journal of the Egyptian Public Health Association 1995;70(5-6):559-77.

\section{Ahmed 1993 BGD \{published data only\}}

Ahmed NU, Zeitlin MF, Beiser AS, Super CM, Gershoff SN. A longitudinal study of the impact of behavioural change intervention on cleanliness, diarrhoeal morbidity and growth of children in rural Bangladesh. Social Science \& Medicine 1993;37(2):159-71.

\section{Alam 1989 BGD \{published data only\}}

Alam N, Wojtyniak B, Henry FJ, Rahaman MM. Mothers' personal and domestic hygiene and diarrhoea incidence in young children in rural Bangladesh. International Journal of Epidemiology 1989;18(1):242-7.

\section{Altmann 2018 TCD \{published data only\}}

Altmann M, Altare C, van der Spek N, Barbiche JC, Dodos J, Bechir M, et al. Effectiveness of a household water, sanitation and hygiene package on an outpatient program for severe acute malnutrition: a pragmatic cluster-randomized controlled trial in Chad. American Journal of Tropical Medicine and Hygiene 2018;98(4):1005-12.

\section{Arvelo 2009 USA \{published data only\}}

Arvelo W, Hinkle CJ, Nguyen TA, Weiser T, Steinmuller N, Khan F, et al. Transmission risk factors and treatment of pediatric shigellosis during a large daycare center-associated outbreak of multidrug resistant Shigella sonnei: implications for the management of shigellosis outbreaks among children. Pediatric Infectious Disease Journal 2009;28(11):976-80.

\section{Asfaha 2018 ETH \{published data only\}}

Asfaha KF, Tesfamichael FA, Fisseha GK, Misgina KH, Weldu MG, Welehaweria NB, et al. Determinants of childhood diarrhea in Medebay Zana District, Northwest Tigray, Ethiopia: a community based unmatched case-control study. BMC Pediatrics 2018;18(1):120.

\section{Aziz 1990 BGD \{published data only\}}

* Aziz KM, Hoque BA, Hasan KZ, Patwary MY, Huttly SR, Rahaman MM, et al. Reduction in diarrhoeal diseases in children in rural Bangladesh by environmental and behavioural modifications. Transactions of the Royal Society of Tropical Medicine \& Hygiene 1990;84(3):433-8.

Hasan KZ, Briend A, Aziz KM, Hoque BA, Patwary MY, Huttly SR. Lack of impact of a water and sanitation intervention on the nutritional status of children in rural Bangladesh. European Journal of Clinical Nutrition 1989;43(12):837-43.

Baker 2016 BGD \{published data only\}

* Baker KK, O'Reilly CE, Levine MM, Kotloff KL, Nataro JP, Ayers TL, et al. Sanitation and hygiene-specific risk factors for moderate-to-severe diarrhea in young children in the Global Enteric Multicenter Study, 2007-2011: case-control study. PLOS Medicine 2016;13(5):e1002010.

Kotloff KL, Blackwelder WC, Nasrin D, Nataro JP, Farag TH, van Eijk A, et al. The Global Enteric Multicenter Study (GEMS) of diarrheal disease in infants and young children in developing countries: epidemiologic and clinical methods of the case/ control study. Clinical Infectious Diseases 2012;55(S4):S232-45.

Kotloff KL, Nataro JP, Blackwelder WC, Nasrin D, Farag TH, Panchalingam S, et al. Burden and aetiology of diarrhoeal disease in infants and young children in developing countries (the Global Enteric Multicenter Study, GEMS): a prospective, case-control study. Lancet 2013;382(9888):209-22.

Long KZ, Faruque AS, Ahmed T, Gunanti IR, Hoque ME, Das SK, et al. Household sanitation and hygiene indicators of enteric pathogen transmission and childhood diarrheal exposure risk in Mirzapur, Bangladesh. Tropical Medicine \& International Health 2015:437.

\section{Baker 2016 GMB $\{$ published data only\}}

Baker KK, O'Reilly CE, Levine MM, Kotloff KL, Nataro JP, Ayers TL, et al. Sanitation and hygiene-specific risk factors for moderate-to-severe diarrhea in young children in the Global Enteric Multicenter Study, 2007-2011: case-control study. PLOS Medicine 2016;13(5):e1002010.

\section{Baker 2016 IND \{published data only\}}

Baker KK, O'Reilly CE, Levine MM, Kotloff KL, Nataro JP, Ayers TL, et al. Sanitation and hygiene-specific risk factors for moderate-to-severe diarrhea in young children in the Global Enteric Multicenter Study, 2007-2011: case-control study. PLOS Medicine 2016;13(5):e1002010.

\section{Baker 2016 KEN \{published data only\}}

Baker KK, O'Reilly CE, Levine MM, Kotloff KL, Nataro JP, Ayers TL, et al. Sanitation and hygiene-specific risk factors for moderate-to-severe diarrhea in young children in the Global Enteric Multicenter Study, 2007-2011: case-control study. PLOS Medicine 2016;13(5):e1002010.

\section{Baker 2016 MLI \{published data only\}}

Baker KK, O'Reilly CE, Levine MM, Kotloff KL, Nataro JP, Ayers TL, et al. Sanitation and hygiene-specific risk factors for moderate-to-severe diarrhea in young children in the Global Enteric Multicenter Study, 2007-2011: case-control study. PLOS Medicine 2016;13(5):e1002010.

\section{Baker 2016 MOZ \{published data only\}}

* Baker KK, O'Reilly CE, Levine MM, Kotloff KL, Nataro JP, Ayers TL, et al. Sanitation and hygiene-specific risk factors for moderate-to-severe diarrhea in young children in the Global Enteric Multicenter Study, 2007-2011: case-control study. PLOS Medicine 2016;13(5):e1002010.

Nhampossa T, Mandomando I, Acacio S, Quintó L, Vubil D, Ruiz J, et al. Diarrheal disease in rural Mozambique: burden, risk factors and etiology of diarrheal disease among children 
aged 0-59 months seeking care at health facilities. PLOS One 2015;10(5):e0119824.

\section{Baker 2016 PAK \{published data only\}}

Baker KK, O'Reilly CE, Levine MM, Kotloff KL, Nataro JP, Ayers TL, et al. Sanitation and hygiene-specific risk factors for moderate-to-severe diarrhea in young children in the Global Enteric Multicenter Study, 2007-2011: case-control study. PLOS Medicine 2016;13(5):e1002010.

\section{Baltazar 1989 PHI \{published data only\}}

Baltazar JC, Solon FS. Disposal of faeces of children under two years old and diarrhoea incidence: a case-control study. International Journal of Epidemiology 1989;18(4 Suppl 2):S16-9.

\section{Barrios 2008 PHI \{unpublished data only\}}

Barrios M. Behavioral Determinants of Diarrhea Among Children Under Five Years of Age in the Rural Philippines: Hand Washing, Stool Disposal, and the Effects of Demographic, Psychosocial, and Social Ecological Factors [PhD thesis]. Los Angeles (CA): University of California Los Angeles, 2008.

\section{Bassal 2016 ISR \{published data only\}}

Bassal R, Ovadia A, Bromberg M, Stein M, Shainberg B, Loewenthal S, et al. Risk factors for sporadic infection with Campylobacter Spp. among children in Israel: a case-control study. Pediatric Infectious Disease Journal 2016;35(3):249-52.

\section{Belizario 2015 PHI \{published data only\}}

Belizario VY Jr, Liwanag HJ, Naig JR, Chua PL, Madamba MI, Dahildahil RO. Parasitological and nutritional status of schoolage and preschool-age children in four villages in Southern Leyte, Philippines: lessons for monitoring the outcome of community-led total sanitation. Acta Tropica 2015;141(Pt A):16-24.

\section{Berhe 2014 ETH $\{$ published data only\}}

Berhe F, Berhane Y. Under five diarrhea among model household and non model households in Hawassa, South Ethiopia: a comparative cross-sectional community based survey. BMC Public Health 2014;14:187.

\section{Briceño 2015 TAN \{published and unpublished data\}}

Briceño B, Coville A, Gertler P, Martinez S. Are there synergies from combining hygiene and sanitation promotion campaigns: evidence from a large-scale cluster-randomized trial in rural Tanzania. PLOS One 2017;12(11):e0186228.

* Briceño B, Coville A, Martinez S. Promoting handwashing and sanitation: evidence from a large-scale randomized trial in rural Tanzania. World Bank, Policy Research Working Paper 7164. 2015. documents.worldbank.org/curated/ en/545961468165561161/pdf/WPS7164.pdf (accessed prior to 9 August 2019).

\section{Butz 1990 USA \{published data only\}}

Butz AM, Larson E, Fosarelli P, Yolken R. Occurrence of infectious symptoms in children in day care homes. American Journal of Infection Control 1990;18(6):347-53.

\section{Cameron 2013 INA \{unpublished data only\}}

Borja-Vega C. The effects of the Total Sanitation and Sanitation Marketing programme on gender and ethnic groups in Indonesia. Waterlines 2014;33(1):55-70.

${ }^{*}$ Cameron L, Shah M, Olivia S. Impact evaluation of a largescale rural sanitation project in Indonesia. World Bank, Policy research working paper. Impact evaluation series 83. 2013. documents.worldbank.org/curated/en/783781468044120966/ pdf/wps6360.pdf (accessed prior to 9 August 2019).

\section{Caruso 2019 IND \{unpublished data only\}}

Caruso BA, Sclar GD, Routray P, Majorin F, Nagel C, Clasen T. A cluster-randomized multi-level intervention to increase latrine use and safe disposal of child feces in rural Odisha, India: the Sundara Grama research protocol. BMC Public Health 2019;19:322.

\section{Chiang 2005 TWN \{published data only\}}

Chiang TY, Jiang DD, Shaw CK. A matched case-control study of shigellosis in children under the age of five in Hualien County. Taiwan Journal of Public Health 2005;24(5):431-9.

\section{Chompook 2006 THA \{published and unpublished data\}}

Chompook P. The Epidemiology of Shigellosis in Thailand [PhD thesis]. London (UK): University of London, 2004.

* Chompook P, Todd J, Wheeler JG, von Seidlein L, Clemens J, Chaicumpa W. Risk factors for shigellosis in Thailand. International Journal of Infectious Diseases 2006;10(6):425-33.

\section{Christensen 2015a KEN \{published data only\}}

Christensen G, Dentz HN, Pickering AJ, Bourdier T, Arnold BF, Colford JM Jr, et al. Pilot cluster randomized controlled trials to evaluate adoption of water, sanitation, and hygiene interventions and their combination in rural western Kenya. American Journal of Tropical Medicine and Hygiene 2015;92(2):437-47.

\section{Christensen 2015b KEN \{published data only\}}

Christensen G, Dentz HN, Pickering AJ, Bourdier T, Arnold BF, Colford JM Jr, et al. Pilot cluster randomized controlled trials to evaluate adoption of water, sanitation, and hygiene interventions and their combination in rural western Kenya. American Journal of Tropical Medicine and Hygiene 2015;92(2):437-47.

\section{Clemens 1987 BGD \{published data only\}}

Clemens JD, Stanton BF. An educational intervention for altering water-sanitation behaviors to reduce childhood diarrhea in urban Bangladesh. I. Application of the case-control method for development of an intervention. American Journal of Epidemiology 1987;125(2):284-91.

\section{Cummings 2012 UGA $\{$ published data only\}}

Cummings MJ, Wamala JF, Eyura M, Malimbo M, Omeke ME, Mayer D, et al. A cholera outbreak among semi-nomadic pastoralists in northeastern Uganda: epidemiology and interventions. Epidemiology and Infection 2012;140(8):1376-85. 
Daniels 1990 LES \{published data only\}

Daniels DL, Cousens SN, Makoae LN, Feachem RG. A casecontrol study of the impact of improved sanitation on diarrhoea morbidity in Lesotho. Bulletin of the World Health Organization 1990;68(4):455-63.

\section{Dickinson 2015 IND \{published data only\}}

Dickinson KL, Patil SR, Pattanayak SK, Poulos C, Yang JH. Nature's call: impacts of sanitation choices in Orissa, India. Economic Development and Cultural Change 2015;64(1):1-29.

\section{Dikassa 1993 DRC \{published data only\}}

Dikassa L, Mock N, Magnani R, Rice J, Abdoh A, Mercer D, et al. Maternal behavioural risk factors for severe childhood diarrhoeal disease in Kinshasa, Zaire. International Journal of Epidemiology 1993;22(2):327-33.

\section{Fisher 2011 BGD $\{$ published data only\}}

Fisher S, Kabir B, Lahiff E, MacLachlan M. Knowledge, attitudes, practices and implications of safe water management and good hygiene in rural Bangladesh: assessing the impact and scope of the BRAC WASH programme. Journal of Water and Health 2011;9(1):80-93.

\section{Gebru 2014 ETH \{published data only\}}

Gebru T, Taha M, Kassahun W. Risk factors of diarrhoeal disease in under-five children among health extension model and nonmodel families in Sheko district rural community, Southwest Ethiopia: comparative cross-sectional study. BMC Public Health 2014;14:395

\section{Genthe 1997 SAF \{published data only\}}

Genthe B, Strauss N, Vundule C, Maforah F, Seager J. The effect of water supply, handling and usage on water quality in relation to health indices in developing communities. Urbanisation and Health Newsletter. WRC Report No. 562/1/96, 1997. www.ircwash.org/sites/default/files/Genthe-1996-Effect.pdf (accessed prior to 9 August 2019).

\section{Ghosh 1994 IND \{published data only\}}

Ghosh S, Sengupta PG, Mandal SK, Manna B, Sikder SN, Sirkar BK. Maternal behaviour and feeding practices as determinants of childhood diarrhoea: some observations amongst rural Bengalee mothers. Indian Journal of Public Health 1994;38(2):77-80.

\section{Ghosh 1997 IND \{published data only\}}

Ghosh S, Sengupta PG, Gupta DN, Mondal SK, Goswami M, Bhattacharya SK, et al. Maternal knowledge on risk behavioural practices and it's association with diarrhoea in a rural community of West Bengal, India. Journal of Communicable Diseases 1998;30(4):251-5.

* Ghosh S, Sengupta PG, Mondal SK, Banu MK, Gupta DN, Sircar BK. Risk behavioural practices of rural mothers as determinants of childhood diarrhoea. Journal of Communicable Diseases 1997;29(1):7-14.

\section{Godana 2013 ETH \{published data only\}}

Godana W, Mengistie B. Determinants of acute diarrhoea among children under five years of age in Derashe District, Southern Ethiopia. Rural and Remote Health 2013;13(3):2329.

\section{Haggerty 1994 DRC \{published data only\}}

Haggerty PA, Manunebo MN, Ashworth A, Muladi K, Kirkwood BR. Methodological approaches in a baseline study of diarrhoeal morbidity in weaning-age children in rural Zaire. International Journal of Epidemiology 1994;23(5):1040-9.

* Haggerty PA, Muladi K, Kirkwood BR, Ashworth A, Manunebo M. Community-based hygiene education to reduce diarrhoeal disease in rural Zaire: impact of the intervention on diarrhoeal morbidity. International Journal of Epidemiology 1994;23(5):1050-9.

\section{Hashi 2017 ETH \{published data only\}}

Hashi A, Kumie A, Gasana J. Hand washing with soap and WASH educational intervention reduces under-five childhood diarrhoea incidence in Jigjiga District, Eastern Ethiopia: a community-based cluster randomized controlled trial. Preventive Medicine Reports 2017;6:361-8.

\section{Heller 2003 BRA \{published data only\}}

* Heller L, Colosimo EA, Antunes CM. Environmental sanitation conditions and health impact: a case-control study. Revista Da Sociedade Brasileira de Medicina Tropical 2003;36(1):41-50.

Heller L, Colosimo EA, Antunes CM. Setting priorities for environmental sanitation interventions based on epidemiological criteria: a Brazilian study. Journal of Water and Health 2005;3(3):271-81.

\section{Hoq 2016 BGD \{published data only\}}

Hoq M, Brogan J. Evaluation of an integrated health-nutritionWASH project to reduce malnutrition prevalence in children under two in Bangladesh. Field Exchange Emergency Nutrition Network ENN, January 2016. www.ennonline.net/fex/51/ healthnutwashbangladesh (accessed prior to 9 August 2019).

\section{Huda 2012 BGD \{published data only\}}

Huda TM, Unicomb L, Johnston RB, Halder AK, Yushuf Sharker MA, Luby SP. Interim evaluation of a large scale sanitation, hygiene and water improvement programme on childhood diarrhea and respiratory disease in rural Bangladesh. Social Science \& Medicine 2012;75(4):604-11.

\section{Humphrey 2019 ZIM \{published data only\}}

* Humphrey JH, Mbuya MN, Ntozini R, Moulton LH, Stoltzfus RJ, Tavengwa NV, et al. Independent and combined effects of improved water, sanitation, and hygiene, and improved complementary feeding, on child stunting and anaemia in rural Zimbabwe: a cluster-randomised trial. Lancet Global Health 2019;7(1):e132-47.

Sanitation Hygiene Infant Nutrition Efficacy (SHINE) Trial Team, Humphrey JH, Jones AD, Manges A, Mangwadu G, Maluccio JA, et al. The Sanitation Hygiene Infant Nutrition Efficacy (SHINE) trial: rationale, design, and methods. Clinical Infectious Diseases 2015;61(Suppl 7):S685-702. 


\section{Jinadu 2007 NGR \{published data only\}}

Jinadu MK, Adegbenro CA, Esmai AO, Ojo AA, Oyeleye BA. Health promotion intervention for hygienic disposal of children's faeces in a rural area of Nigeria. Health Education Journal 2007;66(3):222-8.

\section{Knight 1992 MAL \{published data only\}}

Knight SM, Toodayan W, Caique WC, Kyi W, Barnes A, Desmarchelier P. Risk factors for the transmission of diarrhoea in children: a case-control study in rural Malaysia. International Journal of Epidemiology 1992;21(4):812-8.

\section{Kotch 2007 USA \{published data only\}}

Kotch JB, Isbell P, Weber DJ, Nguyen V, Savage E, Gunn E, et al. Hand-washing and diapering equipment reduces disease among children in out-of-home child care centers. Pediatrics 2007;120(1):e29-36.

\section{Luby 2014 BGD \{unpublished data only\}}

Luby SP, Unicomb L, Halder AK, Nurul Huda TM, Sultana S, Bulbul T, et al (SHEWA-B evaluation team). SHEWA-B Health Impact Study Report. September 2014. www.unicef.org/evaldatabase/files/WASH_SHEWABangladesh_health_impact_eval.pdf (accessed 18 April 2016).

\section{Luby 2018 BGD \{published data only\}}

* Luby SP, Rahman M, Arnold BF, Unicomb L, Ashraf S, Winch PJ, et al. Effects of water quality, sanitation, handwashing, and nutritional interventions on diarrhoea and child growth in rural Bangladesh: a cluster randomised controlled trial. Lancet Global Health 2018;6(3):e302-15.

Parvez SM, Azad R, Rahman M, Unicomb L, Ram PK, Naser AM, et al. Achieving optimal technology and behavioral uptake of single and combined interventions of water, sanitation hygiene and nutrition, in an efficacy trial (WASH benefits) in rural Bangladesh. Trials 2018;19:358.

Rahman M, Ashraf S, Unicomb L, Mainuddin AK, Parvez SM, Begum F, et al. WASH Benefits Bangladesh trial: system for monitoring coverage and quality in an efficacy trial. Trials 2018;19:360.

Unicomb L, Begum F, Leontsini E, Rahman M, Ashraf S, Naser AM, et al. WASH Benefits Bangladesh trial: management structure for achieving high coverage in an efficacy trial. Trials 2018;19:359.

\section{Mathew 2004 ZIM \{unpublished data only\}}

Mathew B. Ensuring Sustained Beneficial Outcomes for Water and Sanitation (WATSAN) Programmes in the Developing World [PhD thesis]. Cranfield (UK): Institute of Water and Environment, Cranfield University, 2004.

\section{Maung 1992a MYA \{published data only\}}

Maung K, Khin M, Wai NN, Hman NW, Myint TT, Butler T. Risk factors for the development of persistent diarrhoea and malnutrition in Burmese children. International Journal of Epidemiology 1992;21(5):1021-9.
Maung 1992b MYA \{published data only\}

Maung K, Khin M, Wai NN, Hman NW, Myint TT, Butler T. Risk factors for the development of persistent diarrhoea and malnutrition in Burmese children. International Journal of Epidemiology 1992;21(5):1021-9.

\section{Mediratta 2010a ETH \{published data only\}}

Mediratta RP, Feleke A, Moulton LH, Yifru S, Sack RB. Risk factors and case management of acute diarrhoea in North Gondar Zone, Ethiopia. Journal of Health, Population \& Nutrition 2010;28(3):253-63.

\section{Mediratta 2010b ETH \{published data only\}}

Mediratta RP, Feleke A, Moulton LH, Yifru S, Sack RB. Risk factors and case management of acute diarrhoea in North Gondar Zone, Ethiopia. Journal of Health, Population, and Nutrition 2010;28(3):253-63.

\section{Menon 1990 USA \{published data only\}}

Menon S, Santosham M, Reid R, Almeido-Hill J, Sack RB, Comstock GW. Rotavirus diarrhoea in Apache children: a case-control study. International Journal of Epidemiology 1990;19(3):715-21.

\section{Mertens 1992 SRI \{published data only\}}

Mertens TE, Jaffar S, Fernando MA, Cousens SN, Feachem RG. Excreta disposal behaviour and latrine ownership in relation to the risk of childhood diarrhoea in Sri Lanka. International Journal of Epidemiology 1992;21(6):1157-64.

Nair 2017 IND \{published data only\}

Nair N, Tripathy P, Sachdev HS, Pradhan H, Bhattacharyya S, Gope R, et al. Effect of participatory women's groups and counselling through home visits on children's linear growth in rural eastern India (CARING trial): a cluster-randomised controlled trial. Lancet Global Health 2017;5(10):e1004-16.

\section{Nanan 2003 PAK \{published data only\}}

Nanan D, White F, Azam I, Afsar H, Hozhabri S. Evaluation of a water, sanitation, and hygiene education intervention on diarrhoea in northern Pakistan. Bulletin of the World Health Organization 2003;81(3):160-5.

\section{Null 2018 KEN \{published data only\}}

* Null C, Stewart CP, Pickering AJ, Dentz HN, Arnold BF, Arnold CD, et al. Effects of water quality, sanitation, handwashing, and nutritional interventions on diarrhoea and child growth in rural Kenya: a cluster-randomised controlled trial. Lancet. Global Health 2018;6(3):e316-29.

Steinbaum L, Mboya J, Mahoney R, Otuke J, Njenga S, Null C, et al. Effect of a sanitation intervention on soil transmitted helminth prevalence and concentration in household soil: a cluster randomized controlled trial. American Journal of Tropical Medicine and Hygiene 2017;97(5 Suppl 1):25-6.

Stewart CP, Kariger P, Fernald L, Pickering AJ, Arnold CD, Arnold BF, et al. Effects of water quality, sanitation, handwashing, and nutritional interventions on child development in rural Kenya (WASH Benefits Kenya): a cluster- 
randomised controlled trial. Lancet. Child and Adolescent Health 2018;2(4):269-80.

\section{Oguro 2016 MYA \{published data only\}}

Oguro M, Horiuchi S. A cross-sectional study of communitybased maternal and child health interventions involving women's health volunteer groups in rural Myanmar. Public Health Nursing 2016;33(5):449-59.

\section{Oketcho 2012 TAN \{published data only\}}

Oketcho R, Nyaruhucha CN, Taybali S, Karimuribo ED. Influence of enteric bacteria, parasite infections and nutritional status on diarrhoea occurrence among 6-60 months old children admitted at a Regional Hospital in Morogoro, Tanzania. Tanzania Journal of Health Research 2012;14(2):104-14.

Park 2016 INA \{published data only\} Park MJ, Laksono B, Clements A, Sadler R, Stewart D. Wormfree children: an integrated approach to reduction of soiltransmitted helminth infections in Central Java. Reviews on Environmental Health 2016;31(1):111-3.

\section{Patil 2014 IND \{published data only\}}

Patil SR, Arnold BF, Salvatore AL, Briceno B, Ganguly S, Colford JM Jr, et al. The effect of India's total sanitation campaign on defecation behaviors and child health in rural Madhya Pradesh: a cluster randomized controlled trial. PLOS Medicine 2014;11(8):e1001709.

\section{Pickering 2015 MLI \{published data only\}}

Pickering AJ. Effect of a community-led sanitation intervention on child diarrhoea and child growth in rural Mali. Field Exchange Emergency Nutrition Network ENN. 2016. www.ennonline.net/ fex/52/sanitationchildgrowth (accessed prior to 9 August 2019).

Pickering AJ, Alzua ML, Djebbari H. Impact of a communityled total sanitation intervention on child health in rural Mali: evidence from a cluster randomized controlled trial. American Journal of Tropical Medicine and Hygiene 2014; Vol. 5, issue Suppl 1.

* Pickering AJ, Djebbari H, Lopez C, Coulibaly M, Alzua ML. Effect of a community-led sanitation intervention on child diarrhoea and child growth in rural Mali: a cluster-randomised controlled trial. Lancet Global Health 2015;3(11):e701-11.

\section{Sarrassat 2018 BUR \{published data only\}}

Sarrassat S, Meda N, Badolo H, Ouedraogo M, Some H, Bambara $\mathrm{R}$, et al. Effect of a mass radio campaign on family behaviours and child survival in Burkina Faso: a repeated cross-sectional, cluster-randomised trial. Lancet Global Health 2018;6(3):e330-41.

\section{Sinharoy 2017 RWA \{published data only\}}

Sinharoy SS, Schmidt WP, Wendt R, Mfura L, Crossett E, Grépin KA, et al. Effect of community health clubs on child diarrhoea in western Rwanda: cluster-randomised controlled trial. Lancet Global Health 2017;5(7):e699-709.

\section{Stanton 1987 BGD \{published data only\}}

* Stanton BF, Clemens JD. An educational intervention for altering water-sanitation behaviors to reduce childhood diarrhea in urban Bangladesh. II. A randomized trial to assess the impact of the intervention on hygienic behaviors and rates of diarrhea. American Journal of Epidemiology 1987;125(2):292-301.

Stanton BF, Clemens JD, Khair T. Educational intervention for altering water-sanitation behavior to reduce childhood diarrhea in urban Bangladesh: impact on nutritional status. American Journal of Clinical Nutrition 1988;48(5):1166-72.

\section{Strina 2012 BRA \{published data only\}}

Strina A, Rodrigues LC, Cairncross S, Ferrer SR, Fialho AM, Leite JPG, et al. Factors associated with rotavirus diarrhoea in children living in a socially diverse urban centre in Brazil. Transactions of the Royal Society of Tropical Medicine and Hygiene 2012;106(7):445-51.

\section{Traoré 1994a BUR \{published data only\}}

Traoré E, Cousens S, Curtis V, Mertens T, Tall F, Traoré A, et al. Child defecation behaviour, stool disposal practices, and childhood diarrhoea in Burkina Faso: results from a casecontrol study. Journal of Epidemiology and Community Health 1994;48(3):270-5.

\section{Traoré 1994b BUR \{published data only\}}

Traoré E, Cousens S, Curtis V, Mertens T, Tall F, Traoré A, et al. Child defecation behaviour, stool disposal practices, and childhood diarrhoea in Burkina Faso: results from a casecontrol study. Journal of Epidemiology and Community Health 1994;48(3):270-5.

\section{Waterkeyn 2005 ZIM \{published and unpublished data\}} Waterkeyn J. Cost-effective Health Promotion and Hygiene Behaviour Change through Community Health Clubs in Zimbabwe [PhD thesis]. London (UK): London School of Hygiene \& Tropical Medicine, 2006.

* Waterkeyn J, Cairncross S. Creating demand for sanitation and hygiene through Community Health Clubs: a cost-effective intervention in two districts in Zimbabwe. Social Science \& Medicine 2005;61(9):1958-70.

\section{Wijewardene 1992 SRI \{published data only\}}

Wijewardene K, Fonseka P, Wijayasiri WA. Risk factors contributing to acute diarrhoeal disease in children below five years. Ceylon Medical Journal 1992;37(4):116-9.

\section{Yeager 2002 PER \{published data only\}}

Yeager BA, Huttly SR, Diaz J, Bartolini R, Marin M, Lanata CF. An intervention for the promotion of hygienic feces disposal behaviors in a shanty town of Lima, Peru. Health Education Research 2002;17(6):761-73.

\section{References to studies excluded from this review}

\section{Assefa 2010 \{unpublished data only\}}

Assefa B. Impacts of WASH on Community-Managed Acute Child Malnutrition in Ethiopia [MSc thesis]. Loughborough (UK): Loughborough University, 2010. 


\section{Babu 2015 \{published data only\}}

Babu MC, Tandur B, Sharma D, Murki S. Disposable diapers decrease the incidence of neonatal infections compared to cloth diapers in a level II neonatal intensive care unit. Journal of Tropical Pediatrics 2015;61(4):250-4.

Ban 2015 \{published data only\}

Ban HQ, Li T, Shen J, Li J, Peng PZ, Ye HP, et al. Effects of multiple cleaning and disinfection interventions on infectious diseases in children: a group randomized trial in China. Biomedical \& Environmental Sciences 2015;28(11):779-87.

\section{Blum 1990 \{published data only\}}

Blum D, Emeh RN, Huttly SR, Dosunmu-Ogunbi O, Okeke N, Ajala M, et al. The Imo State (Nigeria) Drinking Water Supply and Sanitation Project, 1. Description of the project, evaluation methods, and impact on intervening variables. Transactions of the Royal Society of Tropical Medicine and Hygiene 1990;84(2):309-15.

\section{Boehm 2016 \{published data only\}}

Boehm AB, Wang D, Ercumen A, Shea M, Harris AR, Shanks OC, et al. Occurrence of host-associated fecal markers on child hands, household soil, and drinking water in rural Bangladeshi households. Environmental Science \& Technology Letters 2016;3(11):393-8.

\section{Bohnert 2016 \{published data only\}}

Bohnert K, Chard AN, Mwaki A, Kirby AE, Muga R, Nagel CL, et al. Comparing sanitation delivery modalities in urban informal settlement schools: a randomized trial in Nairobi, Kenya. International Journal of Environmental Research \& Public Health 2016;13(12):E1189.

\section{Carnell 2014 \{published data only\}}

Carnell MA, Dougherty L, Pomeroy AM, Karim AM, Mekonnen YM, Mulligan BE. Effectiveness of scaling up the 'three pillars' approach to accelerating MDG 4 progress in Ethiopia. Journal of Health, Population, and Nutrition 2014;32(4):549-63.

\section{Clarke 2016 \{published data only\}}

Clarke NE, Clements AC, Amaral S, Richardson A, McCarthy JS, McGown J, et al. (S)WASH-D for Worms: a pilot study investigating the differential impact of school- versus community-based integrated control programs for soiltransmitted helminths. PLOS Neglected Tropical Diseases 2018;12(5):e0006389.

Clarke NE, Clements AC, Bryan S, McGown J, Gray D, Nery SV. Investigating the differential impact of school and communitybased integrated control programmes for soil-transmitted helminths in Timor-Leste: the (S)WASH-D for Worms pilot study protocol. Pilot Feasibility Study 2016;2:69.

\section{Clasen 2015 \{published data only\}}

Clasen T, Boisson S, Routray P, Torondel B, Bell M, Cumming O, et al. Effectiveness of a rural sanitation programme on diarrhoea, soil-transmitted helminth infection, and child malnutrition in Odisha, India: a cluster-randomised trial. Lancet Global Health 2015;2(11):e645-53.

\section{Ditai 2016 \{published data only\}}

Ditai J, Abeso J, Mudoola M, Faragher B, Adengo M, Dusabe Richards J, et al. A pilot cluster randomised trial of alcoholbased hand rub to prevent community neonatal sepsis in rural Uganda. RCOG World Congress; 2016 Jun 20-22; Birmingham (UK).

Dumba 2013 \{published data only\}

Dumba R, Kaddu JB, Wabwire-Mangen F. Design and implementation of participatory hygiene and sanitation transformation (PHAST) as a strategy to control soil-transmitted helminth infections in Luweero, Uganda. African Health Sciences 2013;13(2):512-7.

\section{Erismann 2017 \{published data only\}}

Erismann S, Diagbouga S, Schindler C, Odermatt P, Knoblauch AM, Gerold J, et al. School children's intestinal parasite and nutritional status one year after complementary school garden, nutrition, water, sanitation, and hygiene interventions in Burkina Faso. American Journal of Tropical Medicine and Hygiene 2017;97(3):904-13.

\section{Francis 2016 \{published data only\}}

Francis MR, Sarkar R, Roy S, Jaffar S, Mohan VR, Kang G, et al. Effectiveness of membrane filtration to improve drinking water: a quasi-experimental study from rural Southern India. American Journal of Tropical Medicine and Hygiene 2016;95(5):1192-200.

\section{Freeman 2015 \{published data only\}}

Freeman MC, Majorin F, Boisson S, Routray P, Torondel B, Clasen T. The impact of a rural sanitation programme on safe disposal of child faeces: a cluster randomised trial in Odisha, India. Transactions of the Royal Society of Tropical Medicine and Hygiene 2015;110(7):386-92.

\section{Galiani 2016 \{published data only\}}

Galiani S, Gertler P, Ajzenman N, Orsola-Vidal A. Promoting handwashing behavior: the effects of large-scale community and school-level interventions. Health Economics 2016;25(12):1545-59.

\section{Garn 2016 \{published data only\}}

Garn JV, Brumback BA, Drews-Botsch CD, Lash TL, Kramer MR, Freeman MC. Estimating the effect of school water, sanitation, and hygiene improvements on pupil health outcomes. Epidemiology 2016;27(5):752-60.

\section{Gelaye 2014 \{published data only\}}

Gelaye B, Kumie A, Aboset N, Berhane Y, Williams MA. Schoolbased intervention: evaluating the role of water, latrines and hygiene education on trachoma and intestinal parasitic infections in Ethiopia. Journal of Water, Sanitation and Hygiene for Development 2014;4(1):120-30.

\section{Gorter 1998 \{published data only\}}

Gorter AC, Sandiford P, Pauw J, Morales P, Pérez RM, Alberts H. Hygiene behaviour in rural Nicaragua in relation to diarrhoea. International Journal of Epidemiology 1998;27(6):1090-100. 


\section{Greenland 2016 \{published data only\}}

Greenland K, de Witt Huberts J, Wright RL, Hawkes L, Ekor C, Biran A. A cross-sectional survey to assess household sanitation practices associated with uptake of "Clean Team" serviced home toilets in Kumasi, Ghana. Environment and Urbanization 2016;28(2):583-98.

\section{Gungoren 2007 \{published data only\}}

Gungoren B, Latipov R, Regallet G, Musabaev E. Effect of hygiene promotion on the risk of reinfection rate of intestinal parasites in children in rural Uzbekistan. Transactions of the Royal Society of Tropical Medicine and Hygiene 2007;101(6):564-9.

\section{Hartinger 2016 \{published data only\}}

Hartinger SM, Lanata CF, Hattendorf J, Verastegui H, Gil Al, Wolf J, et al. Improving household air, drinking water and hygiene in rural Peru: a community-randomized-controlled trial of an integrated environmental home-based intervention package to improve child health. International Journal of Epidemiology 2016;45(6):2089-99.

\section{Hunter 2004 \{published data only\}}

Hunter PR, Hughes S, Woodhouse S, Syed Q, Verlander NQ, Chalmers RM, et al. Sporadic cryptosporidiosis casecontrol study with genotyping. Emerging Infectious Diseases 2004;10(7):1241-9.

\section{Hürlimann 2018 \{published data only\}}

Hürlimann E, Silué KD, Zouzou F, Ouattara M, Schmidlin T, Yapi RB, et al. Effect of an integrated intervention package of preventive chemotherapy, community-led total sanitation and health education on the prevalence of helminth and intestinal protozoa infections in Cote d'Ivoire. Parasites and Vectors 2018;11(1):115.

\section{IOB/UNICEF 2011 \{unpublished data only\}}

Policy and Operations Evaluation Department (IOB) of the Netherlands Ministry of Foreign Affairs, UNICEF Evaluation Office, UNICEF Mozambique. Impact evaluation of drinking water supply and sanitation interventions in rural Mozambique. 2011. www.unicef.org/evaldatabase/ files/283729_B85_IOB_360_BW_WEB_Mozambique-Final.pdf (accessed 18 April 2016).

\section{Islam 2018 \{published data only\}}

Islam M, Ercumen A, Ashraf S, Rahman M, Shoab AK, Luby SP, et al. Unsafe disposal of feces of children $<3$ years among households with latrine access in rural Bangladesh: association with household characteristics, fly presence and child diarrhea. PLOS One 2018;13(4):e0195218.

\section{JDC/IHI 2012 \{unpublished data only\}}

Jimat Development Consultants, Ifakara Health Institute. Government of Tanzania/UNICEF 7 Learning Districts Strategy (2007-2011). www.unicef.org/evaldatabase/files/ TNZ-2011-004-1.pdf (accessed 18 April 2016).

\section{Kaatano 2015 \{published data only\}}

Kaatano GM, Siza JE, Mwanga JR, Min DY, Yong TS, Chai JY, et al. Integrated schistosomiasis and soil-transmitted helminthiasis control over five years on Kome Island, Tanzania. Korean Journal of Parasitology 2015;53(5):535-43.

Lamichhane 2018 \{published data only\}

Lamichhane P, Sharma A, Mahal A. Does safe disposal of child faeces matter? An assessment of access to improved sanitation and child faeces disposal behaviour and diarrhoea in rural Nepal. International Health 2018;10(4):277-84.

Law 2016 \{published data only\}

Law E, Yang JH, Coit MH, Chan E. Toilet school for children with failure to toilet train: comparing a group therapy model with individual treatment. Journal of Developmental \& Behavioral Pediatrics 2016;37(3):223-30.

\section{Liu 2017 \{published data only\}}

Liu C, Lu L, Zhang L, Luo R, Sylvia S, Medina A, et al. Effect of deworming on indices of health, cognition, and education among schoolchildren in rural China: a cluster-randomized controlled trial. American Journal of Tropical Medicine and Hygiene 2017;96(6):1478-89.

\section{Messou 1997 \{published data only\}}

Messou E, Sangaré SV, Josseran R, Le Corre C, Guelain J. Effect of improvement of sanitary conditions and of domestic hygiene on incidence of enteric for ascaridiasis and ankylostomiasis of children aged 2 to 4 years in rural area of Côte d'Ivoire [Impact de l'assainissement et de l'hygiène domestique sur l'incidence de l'ascaridiose et de l'ankylostomose chez les enfants de 2 à 4 ans dans les zones rurales de Côte d'Ivoire]. Bulletin de la Société de Pathologie Exotique 1997;90(1):48-50.

Nerkar 2015 \{published data only\}

Nerkar SS, Pathak A, Lundborg CS, Tamhankar AJ. Can integrated watershed management contribute to improvement of public health? A cross-sectional study from Hilly Tribal Villages in India. International Journal of Environmental Research and Public Health 2015;12(3):2653-69.

\section{Njuguna 2016 \{published data only\}}

Njuguna C, Njeru I, Mgamb E, Langat D, Makokha A, Ongore D, et al. Enteric pathogens and factors associated with acute bloody diarrhoea, Kenya. BMC Infectious Diseases 2016;16:477.

\section{Olayo 2014 \{published data only\}}

Olayo R, Wafula C, Aseyo E, Loum C, Kaseje D. A quasiexperimental assessment of the effectiveness of the Community Health Strategy on health outcomes in Kenya. BMC Health Services Research 2014;14(Suppl 1):S3.

\section{Park 2018 \{published data only\}}

Park MJ, Jung HS, Stewart DE. Reducing the exposure of children in rural Indonesia to environmental contamination by human waste a pilot study. Therapeutic Research 2018;39(6):565-70.

\section{Raso 2018 \{published data only\}}

Raso G, Essé C, Dongo K, Ouattara M, Zouzou F, Hürlimann E, et al. An integrated approach to control soil-transmitted helminthiasis, schistosomiasis, intestinal protozoa infection, 
and diarrhea: protocol for a cluster randomized trial. JMIR Research Protocols 2018;7(6):e145.

\section{Reese 2017 \{published data only\}}

Reese H, Routray P, Sinharoy S, Torondel B, Chang H, Clasen T. Effectiveness of a combined household-level piped water and sanitation intervention in rural Odisha, India on health: a matched cohort study. American Journal of Tropical Medicine and Hygiene 2017;97(5 Suppl 1):25.

\section{Sarkar 2014 \{published data only\}}

Sarkar R, Kattula D, Francis MR, Ajjampur SS, Prabakaran AD, Jayavelu N, et al. Risk factors for cryptosporidiosis among children in a semi urban slum in southern India: a nested case-control study. American Journal of Tropical Medicine and Hygiene 2014;91(6):1128-37.

\section{Slayton 2016 \{published data only\}}

Slayton RB, Murphy JL, Morris J, Faith SH, Oremo J, Odhiambo A, et al. A cluster randomized controlled evaluation of the health impact of a novel antimicrobial hand towel on the health of children under 2 years old in rural communities in Nyanza Province, Kenya. American Journal of Tropical Medicine and Hygiene 2016;94(2):437-44.

Taha 2000 \{published data only\}

Taha AZ, Sebai ZA, Shahidullah M, Hanif M, Ahmed HO. Assessment of water use and sanitation behavior in a rural area of Bangladesh. Archives of Environmental Health 2000;55(1):51-7.

\section{Trinies 2016 \{published data only\}}

Trinies V, Garn JV, Chang HH, Freeman MC. The impact of a school-based water, sanitation, and hygiene program on absenteeism, diarrhea, and respiratory infection: a matchedcontrol trial in Mali. American Journal of Tropical Medicine and Hygiene 2016;94(6):1418-25.

\section{Yeasmin 2017 \{published data only\}}

Yeasmin F, Luby SP, Saxton RE, Nizame FA, Alam MU, Dutta NC, et al. Piloting a low-cost hardware intervention to reduce improper disposal of solid waste in communal toilets in lowincome settlements in Dhaka, Bangladesh. BMC Public Health 2017;17:682.

\section{Yentur 2015 \{published data only\}}

Yentur Doni N, Yildiz Zeyrek F, Simsek Z, Gurses G, Sahin I. Risk factors and relationship between intestinal parasites and the growth retardation and psychomotor development delays of children in Sanliurfa, Turkey. Turkiye Parazitolojii Dergisi 2015;39(4):270-6.

\section{Zomer 2015 \{published data only\}}

Zomer TP, Erasmus V, Looman CW, Tjon-A-Tsien A, Van Beeck EF, De Graaf JM, et al. A hand hygiene intervention to reduce infections in child daycare: a randomized controlled trial. Epidemiology and Infection 2015;143(12):2494-502.

\section{References to ongoing studies}

\section{ACTRN12613000523707 \{unpublished data only\}}

ACTRN12613000523707. The effectiveness and acceptability of the 'BALatrine': a culturally acceptable latrine intervention in resource limited environments. www.anzctr.org.au/Trial/ Registration/TrialReview.aspx?ACTRN=12613000523707 (first received 10 May 2013).

\section{ISRCTN10419317 \{published data only\}}

Cha S, Lee J, Seo D, Park BM, Mansiangi P, Bernard K, et al. Effects of improved sanitation on diarrheal reduction for children under five in Idiofa, DR Congo: a cluster randomized trial. Infectious Diseases of Poverty 2017;6(1):137.

* ISRCTN10419317. The impact of improved sanitation on the diarrhoeal reduction of under-five children in Democratic Republic of Congo. www.isrctn.com/ISRCTN10419317 (first received 13 March 2015).

\section{ISRCTN16961836 \{published data only\}}

ISRCTN16961836. Efficacy of a behavioural intervention based on food consumption, nutritional state and micronutrient deficiency in under five children, Angola. www.isrctn.com/ ISRCTN16961836 (first received 5 August 2016).

\section{NCT02754583 \{published data only\}}

NCT02754583. Sanitation, Water, and Instruction in Facewashing for Trachoma (SWIFT). clinicaltrials.gov/ct2/show/ NCT02754583 (first posted 28 April 2016).

\section{Additional references}

\section{Bain 2015}

Bain R, Luyendijk R. Are burial or disposal with garbage safe forms of child faeces disposal? An expert consultation. Waterlines 2015;34(3):241-54.

\section{Bauza 2017}

Bauza V, Guest JS. The effect of young children's faeces disposal practices on child growth: evidence from 34 countries. Tropical Medicine \& International Health 2017;22(10):1233-48.

\section{Bethony 2006}

Bethony J, Brooker S, Albonico M, Geiger SM, Loukas A, Diemert $\mathrm{D}$, et al. Soil-transmitted helminth infections: ascariasis, trichuriasis, and hookworm. Lancet 2006;367(9521):1521-32.

\section{Bhandari 2002}

Bhandari N, Bahl R, Taneja S, Strand T, Mølbak K, Ulvik RJ, et al. Substantial reduction in severe diarrheal morbidity by daily zinc supplementation in young north Indian children. Pediatrics 2002;109(6):e86.

\section{Brooker 2006}

Brooker S, Clements AC, Bundy DA. Global epidemiology, ecology and control of soil-transmitted helminth infections. Advances in Parasitology 2006;62:221-61. 


\section{Brooks 2003}

Brooks JT, Shapiro RL, Kumar L, Wells JG, Phillips-Howard PA, Shi YP, et al. Epidemiology of sporadic bloody diarrhea in rural Western Kenya. American Journal of Tropical Medicine and Hygiene 2003;68(6):671-7.

\section{Brown 2013}

Brown J, Cairncross S, Ensink JH. Water, sanitation, hygiene and enteric infections in children. Archives of Disease in Childhood 2013;98(8):629-34

\section{Byers 2001}

Byers KE, Guerrant RL, Farr BM. Faecal-oral transmission. In: Thomas JC, Webber DJ editor(s). Epidemiological Methods for the Study of Infectious Diseases. Oxford (UK): Oxford University Press, 2001:228-48.

\section{Clasen 2010}

Clasen TF, Bostoen K, Schmidt WP, Boisson S, Fung IC, Jenkins MW, et al. Interventions to improve disposal of human excreta for preventing diarrhoea. Cochrane Database of Systematic Reviews 2010, Issue 6. [DOI: 10.1002/14651858.CD007180.pub2]

\section{Deeks 2011}

Deeks JJ, Higgins JP, Altman DG. Chapter 9: Analysing data and undertaking meta-analyses. In: Higgins JP, Green S, editor(s). Cochrane Handbook for Systematic Reviews of Interventions Version 5.1.0 (updated March 2011). The Cochrane Collaboration, 2011. Available from handbook.cochrane.org.

\section{EPOC 2013}

Cochrane Effective Practice, Organisation of Care. Suggested risk of bias criteria for EPOC reviews. epoc.cochrane.org (accessed 31 August 2013).

\section{Esrey 1991}

Esrey SA, Potash JB, Roberts L, Shiff C. Effects of improved water supply and sanitation on ascariasis, diarrhoea, dracunculiasis, hookworm infection, schistosomiasis, and trachoma. Bulletin of the World Health Organization 1991;69:609-21.

\section{Feachem 1983}

Feachem RG, Bradley DJ, Garelick H, Mara DD. Sanitation and Disease: Health Aspects of Wastewater and Excreta Management. Chichester (UK): John Wiley \& Sons, 1983.

\section{Ferriman 2007}

Ferriman A. BMJ readers choose the "sanitary revolution" as greatest medical advance since 1840. BMJ 2007;334:111.

\section{Fewtrell 2005}

Fewtrell L, Kaufmann RB, Kay D, Enanoria W, Haller L, Colford JM Jr. Water, sanitation, and hygiene interventions to reduce diarrhoea in less developed countries: a systematic review and meta-analysis. Lancet Infectious Diseases 2005;5(1):42-52.

\section{Freeman 2017}

Freeman MC, Garn JV, Sclar GD, Boisson S, Medlicott K, Alexander KT, et al. The impact of sanitation on infectious disease and nutritional status: a systematic review and metaanalysis. International Journal of Hygiene and Environmental Health 2017;220(6):928-49.

\section{GBD 2018}

GBD 2016 Diarrhoeal Disease Collaborators. Estimates of the global, regional, and national morbidity, mortality, and aetiologies of diarrhoea in 195 countries: a systematic analysis for the Global Burden of Disease Study 2016. Lancet Infectious Diseases 2018;18:1211-28.

\section{George 2016}

George CM, Oldja L, Biswas S, Perin J, Sack RB, Ahmed S, et al. Unsafe child feces disposal is associated with environmental enteropathy and impaired growth. Journal of Pediatrics 2016;176:43-9.

\section{Gil 2004}

Gil A, Lanata C, Kleinau E, Penny M. Children's faeces disposal practices in developing countries and interventions to prevent diarrhoeal diseases. 2004. www.ehproject.org/PDF/ Strategic_papers/SR11-Child\%20Excreta\%20Format.pdf (accessed prior to 9 August 2019).

\section{Guerrant 2013}

Guerrant RL, DeBoer MD, Moore SR, Scharf RJ, Lima AA. The impoverished gut - a triple burden of diarrhoea, stunting and chronic disease. Nature Reviews Gastroenterology and Hepatology 2013;10:220-9.

\section{Guyatt 2008}

Guyatt GH, Oxman AD, Vist GE, Kunz R, Falck-Ytter Y, Schünemann HJ. GRADE: what is "quality of evidence" and why is it important to clinicians?. BMJ 2008;336:995-998.

\section{Guyatt 2011}

Guyatt G, Oxman AD, Akl EA, Kunz R, Vist G, Brozek J, et al. GRADE guidelines: 1 . Introduction - GRADE evidence profiles and summary of findings tables. Journal of Clinical Epidemiology 2011;64(4):383-94.

\section{Harhay 2010}

Harhay MO, Horton J, Olliaro PL. Epidemiology and control of human gastrointestinal parasites in children. Expert Review of Anti-infective Therapy 2010;8(2):219-34.

\section{HEP 2003}

Federal Democratic Republic of Ethiopia, Ministry of Health. HEP training materials, 2003. cnhde.ei.columbia.edu/training/ documents/Maternal_and_Child_Health.pdf (accessed 20 April 2016).

\section{Heymann 2008}

Heymann D, editor. Control of Communicable Diseases Manual. Washington (DC): American Public Health Association, 2008. 


\section{Higgins 2003}

Higgins JP, Thompson SG, Deeks JJ, Altman DG. Measuring inconsistency in meta-analyses. BMJ 2003;327(7414):557-60.

\section{Higgins 2011a}

Higgins JP, Altman DG, Sterne JA. Chapter 8: Assessing risk of bias in included studies. In: Higgins JP, Green S, editor(s). Cochrane Handbook for Systematic Reviews of Interventions Version 5.1.0 (updated March 2011). The Cochrane Collaboration, 2011. Available from handbook.cochrane.org. The Cochrane Collaboration.

\section{Higgins 2011b}

Higgins JP, Deeks JJ, Altman DG. Chapter 16: Special topics in statistics. In: Higgins JP, Green S, editor(s). Cochrane Handbook for Systematic Reviews of Interventions Version 5.1.0 (updated March 2011). The Cochrane Collaboration, 2011. Available from handbook.cochrane.org.

\section{Higgins 2011c}

Higgins JP, Deeks JJ, Altman DG. Cochrane Handbook for Systematic Reviews of Interventions Version 5.1.0 (updated March 2011). The Cochrane Collaboration, 2011. Available from handbook.cochrane.org.

\section{Humphrey 2009}

Humphrey JH. Child undernutrition, tropical enteropathy, toilets, and handwashing. Lancet 2009;374(9694):1032-5.

\section{Kar 2008}

Kar K, Chambers R. Handbook on community-led total sanitation. 2008. www.communityledtotalsanitation.org/ sites/communityledtotalsanitation.org/files/media/ cltshandbook.pdf (accessed 9 May 2016).

\section{Keusch 2006}

Keusch GT, Fontaine O, Bhargava A, Boschi-Pinto C, Bhutta ZA, Gotuzzo E, et al. Chapter 19. Diarrheal Diseases. In: Jamison DT, Breman JG, Measham AR, Alleyne G, Claeson M, Evans DB, et al. editor(s). Disease Control Priorities in Developing Countries. 2nd Edition. Washington (DC): World Bank, 2006.

\section{Lanata 1998}

Lanata CF, Huttly SR, Yeager BA. Diarrhea: whose feces matter? Reflections from studies in a Peruvian shanty town. Pediatric Infectious Disease Journal 1998;17(1):7-9.

\section{Lin 2013}

Lin A, Arnold BF, Afreen S, Goto R, Huda TM, Haque R, et al. Household environmental conditions are associated with enteropathy and impaired growth in rural Bangladesh. American Journal of Tropical Medicine and Hygiene 2013;89(1):130-7.

\section{Luby 2006}

Luby SP, Agboatwalla M, Painter J, Altaf A, Billhimer W, Keswick $B$, et al. Combining drinking water treatment and hand washing for diarrhoea prevention, a cluster randomised controlled trial. Tropical Medicine \& International Health 2006;11(4):479-89.

\section{Majorin 2017}

Majorin F, Torondel B, Routray P, Rout M, Clasen T. Identifying potential sources of exposure along the child feces management pathway: a cross-sectional study among urban slums in Odisha, India. American Journal of Tropical Medicine and Hygiene 2017;97(3):861-9.

\section{Mbuya 2016}

Mbuya MNN, Humphrey JH. Preventing environmental enteric dysfunction through improved water, sanitation and hygiene: an opportunity for stunting reduction in developing countries. Maternal \& Child Nutrition 2016;12(Suppl 1):106-20.

\section{Miller-Petrie 2016}

Miller-Petrie MK, Voigt L, McLennan L, Cairncross S, Jenkins MW. Infant and young child feces management and enabling products for their hygienic collection, transport, and disposal in Cambodia. American Journal of Tropical Medicine and Hygiene 2016;94(2):456-65.

\section{Morita 2016}

Morita T, Godfrey S, George CM. Systematic review of evidence on the effectiveness of safe child faeces disposal interventions. Tropical Medicine \& International Health 2016;21(11):1403-19.

\section{Moya 2004}

Moya J, Bearer CF, Etzel RA. Children's behavior and physiology and how it affects exposure to environmental contaminants. Pediatrics 2004;113(4 Suppl):996-1006.

\section{Nery 2015}

Nery SV, McCarthy JS, Traub R, Andrews RM, Black J, Gray D, et al. A cluster-randomised controlled trial integrating a community-based water, sanitation and hygiene programme, with mass distribution of albendazole to reduce intestinal parasites in Timor-Leste: the WASH for WORMS research protocol. BMJ Open 2015;5(12):e009293.

\section{Ngure 2013}

Ngure FM, Humphrey JH, Mbuya MN, Majo F, Mutasa K, Govha M, et al. Formative research on hygiene behaviors and geophagy among infants and young children and implications of exposure to fecal bacteria. American Journal of Tropical Medicine and Hygiene 2013;89(4):709-16.

\section{Norman 2010}

Norman G, Pedley S, Takkouche B. Effects of sewerage on diarrhoea and enteric infections: a systematic review and metaanalysis. Lancet Infectious Diseases 2010;10(8):536-44.

\section{Pullan 2012}

Pullan RL, Brooker SJ. The global limits and population at risk of soil-transmitted helminth infections in 2010. Parasites and Vectors 2012;5:81.

\section{Pullan 2014}

Pullan RL, Smith JL, Jasrasaria R, Brooker SJ. Global numbers of infection and disease burden of soil transmitted helminth infections in 2010. Parasites and Vectors 2014;7:37. 


\section{Ram 2007}

Ram PK, Naheed A, Brooks WA, Hossain MA, Mintz ED, Breiman RF, et al. Risk factors for typhoid fever in a slum in Dhaka, Bangladesh. Epidemiology and Infection 2007;135:458-65.

\section{Reeves 2011}

Reeves BC, Deeks JJ, Higgins JP, Wells GA. Chapter 13: Including non-randomized studies. In: Higgins JP, Green S, editor(s), Cochrane Handbook for Systematic Reviews of Interventions Version 5.1.0 (updated March 2011). The Cochrane Collaboration, 2011. Available from handbook.cochrane.org.

\section{Review Manager 2014 [Computer program]}

Nordic Cochrane Centre, The Cochrane Collaboration. Review Manager 5 (RevMan 5). Version 5.3. Copenhagen: Nordic Cochrane Centre, The Cochrane Collaboration, 2014.

\section{Roy 2011}

Roy E, Hasan KZ, Haque R, Fazlul Haque AKM, Siddique AK, Bradley Sack R. Patterns and risk factors for helminthiasis in rural children aged under 2 in Bangladesh. South African Journal of Child Health 2011;5(3):78-84.

\section{Scott 2008}

Scott B. Children's stool disposal - a review of prevalence of practice and its relationship with health, and recommendations for filling the evidence gaps (as supplied in 2008). Review on file.

\section{Strunz 2014}

Strunz EC, Addiss DG, Stocks ME, Ogden S, Utzinger J, Freeman MC. Water, sanitation, hygiene, and soil-transmitted helminth infection: a systematic review and meta-analysis. PLOS Medicine 2014;11(3):e1001620.

\section{Thapar 2004}

Thapar N, Sanderson IR. Diarrhoea in children: an interface between developing and developed countries. Lancet 2004;363(9409):641-53.

\section{Thomas 2013}

Thomas A, Bevan J. Developing and monitoring protocol for the elimination of open defecation in Sub-Saharan Africa. 2013. www.ircwash.org/sites/default/files/ thomas_and_bevan_elimination_of_open_defecation_in_subsaharan_africa.pdf (accessed prior to 9 August 2019).

\section{Tumwine 2002}

Tumwine JK, Thompson J, Katua-Katua M, Mujwajuzi M, Johnstone N, Wood E, Porras I. Diarrhoea and effects of different water sources, sanitation and hygiene behaviour in East Africa. Tropical Medicine and International Health 2002;7(9):750-6.

\section{UN 2013}

United Nations. Millenium Development Goals. www.un.org/ millenniumgoals/environ.shtml (accessed 13 March 2014).

\section{UN 2016}

United Nations. Sustainable Development Goals. sustainabledevelopment.un.org/sdg6 (accessed 18 March 2016).

\section{UNICEF 2012}

UNICEF. Pneumonia and diarrhoea: tackling the deadliest diseases for the world's poorest children. 2012. www.unicef.org/ publications/index_65491.html (accessed prior to 9 August 2019).

\section{Waddington 2009}

Waddington $\mathrm{H}$, Snilstveit B, White H, Fewtrell L. Water, sanitation and hygiene interventions to combat childhood diarrhoea in developing countries. International Initiative for Impact Evaluation 2009;1:1-119.

\section{Wagner 1958}

Wagner EG, Lanoix JN. Excreta disposal for rural areas and small communities. 9th Edition. Geneva: World Health Organization, 1958.

\section{Walker 2012}

Walker CL, Perin J, Aryee MJ, Boschi-Pinto C, Black RE. Diarrhea incidence in low- and middle-income countries in 1990 and 2010: a systematic review. BMC Public Health 2012;12:220.

\section{Wells 2013}

Wells GA, Shea B, O'Connell D, Peterson J, Welch V, Losos M, et al. The Newcastle-Ottawa Scale (NOS) for assessing the quality of non randomised studies in meta-analyses. www.ohri.ca/ programs/clinical_epidemiology/oxford.asp (accessed 9 August 2013).

\section{WHO 2013}

World Health Organization. Diarrhoeal disease. www.who.int/ mediacentre/factsheets/fs330/en/index.html (accessed 9 August 2013).

\section{WHO/UNICEF 2006}

World Health Organization, United Nations Children's Fund. Core questions on drinking water and sanitation for household surveys. www.who.int/water_sanitation_health/monitoring/ oms_brochure_core_questionsfinal24608.pdf. Geneva: World Health Organization, (accessed 13 March 2014).

\section{WHO/UNICEF 2014}

World Health Organization, United Nations Children's Fund. Types of drinking-water sources and sanitation. 2014. www.wssinfo.org/definitions-methods/watsan-categories/ (accessed 12 January 2014).

\section{WHO/UNICEF 2015a}

World Health Organization, United Nations Children's Fund. Joint Monitoring Programme for Water Supply and Sanitation. Progress on sanitation and drinking water - 2015 update and MDG assessment. World Health Organization and United Nations Children's Fund (accessed prior to 9 August 2019).

\section{WHO/UNICEF 2015b}

World Health Organization, United Nations Children's Fund. JMP Green Paper: Global monitoring of water, sanitation and hygiene post-2015 Draft. Updated October 2015. www.wssinfo.org/fileadmin/user_upload/resources/JMPGreen-Paper-15-Oct-2015.pdf (accessed 10 May 2016). 


\section{WHO/UNICEF 2018}

World Health Organization, United Nations Children's Fund. Core questions on drinking water, sanitation and hygiene for household surveys: 2018 update. New York: United Nations Children's Fund (UNICEF) and World Health Organization 2018.

\section{Wolf 2018}

Wolf J, Hunter PR, Freeman MC, Cumming O, Clasen T, Bartram J, et al. Impact of drinking water, sanitation and handwashing with soap on childhood diarrhoeal disease: updated meta-analysis and meta-regression. Tropical Medicine \& International Health 2018;23(5):508-25.

\section{WSP 2015}

Water and Sanitation Program. Management of child feces: current disposal practices. 2015. www.wsp.org/sites/wsp.org/ files/publications/WSP-CFD-Summary-Brief.pdf (accessed 18 March 2016).

\section{Young 2011}

Young SL, Sherman PW, Lucks JB, Pelto GH. Why on earth?: evaluating hypotheses about the physiological

\section{CHARACTERISTICS OF STUDIES}

Characteristics of included studies [ordered by study ID] functions of human geophagy. Quarterly Review of Biology 2011;86(2):97-120.

\section{Ziegelbauer 2012}

Ziegelbauer K, Speich B, Mäusezahl D, Bos R, Keiser J, Utzinger J. Effect of sanitation on soil-transmitted helminth infection: systematic review and meta-analysis. PLOS Medicine 2012;9(1):e1001162.

\section{References to other published versions of this review \\ Majorin 2014}

Majorin F, Torondel B, Ka Seen Chan G, Clasen T. Interventions to improve disposal of child faeces for preventing diarrhoea and soil-transmitted helminth infection. Cochrane Database of Systematic Reviews 2014, Issue 4. [DOI: 10.1002/14651858.CD011055]

* Indicates the major publication for the study

\section{Abalkhail 1995 KSA}

\begin{tabular}{ll}
\hline Methods & Case-control study \\
\hline Participants & $\begin{array}{l}\text { Cases: children aged }<3 \text { years admitted to } 20 \text { primary HCs for primary diagnosis of diarrhoea with in- } \\
\text { fectious origin, } n=319 \text { (after excluding 3), mean age } 13.1 \text { months, } 45.3 \% \text { girls. } \\
\text { Controls: children aged }<3 \text { years with no history of hospitalization for diarrhoeal diseases, selected } \\
\text { randomly from the nearest residential neighbours, } n=312 \text { (after excluding 13). mean age 19.2 months, } \\
52.6 \% \text { girls. }\end{array}$ \\
\hline Interventions & Risk factor of interest: \\
\hline Outcomes & disposal of child faeces elsewhere vs in the latrine. \\
\hline cous) & Location: urban Makkah area, 20 primary HCs, Saudi Arabia \\
& Length of recruitment: 3 months (October 1994 to January 1995) \\
Publication status: journal
\end{tabular}

\section{Risk of bias}

\begin{tabular}{lll}
\hline Bias & Authors' judgement & Support for judgement \\
\hline $\begin{array}{l}\text { Random sequence genera- } \\
\text { tion (selection bias) }\end{array}$ & Unclear risk & NA \\
\hline $\begin{array}{l}\text { Allocation concealment } \\
\text { (selection bias) }\end{array}$ & Unclear risk & NA \\
\hline
\end{tabular}


Abalkhail 1995 KSA (Continued)

\begin{tabular}{|c|c|c|}
\hline $\begin{array}{l}\text { Blinding of participants } \\
\text { and personnel (perfor- } \\
\text { mance bias) } \\
\text { All outcomes }\end{array}$ & Unclear risk & NA \\
\hline $\begin{array}{l}\text { Blinding of outcome as- } \\
\text { sessment (detection bias) } \\
\text { All outcomes }\end{array}$ & Unclear risk & NA \\
\hline $\begin{array}{l}\text { Incomplete outcome data } \\
\text { (attrition bias) } \\
\text { All outcomes }\end{array}$ & Unclear risk & NA \\
\hline $\begin{array}{l}\text { Selective reporting (re- } \\
\text { porting bias) }\end{array}$ & Unclear risk & NA \\
\hline Other bias & Unclear risk & NA \\
\hline $\begin{array}{l}\text { Similarity of baseline out- } \\
\text { come measurements }\end{array}$ & Unclear risk & NA \\
\hline $\begin{array}{l}\text { Similarity of baseline char- } \\
\text { acteristics }\end{array}$ & Unclear risk & NA \\
\hline $\begin{array}{l}\text { Adequate allocation of in- } \\
\text { tervention concealment } \\
\text { during the study }\end{array}$ & Unclear risk & NA \\
\hline $\begin{array}{l}\text { Adequate protection } \\
\text { against contamination }\end{array}$ & Unclear risk & NA \\
\hline $\begin{array}{l}\text { Confounders adequate- } \\
\text { ly adjusted for in analy- } \\
\text { sis/design }\end{array}$ & Unclear risk & NA \\
\hline Recruitment bias & Unclear risk & NA \\
\hline Baseline imbalance & Unclear risk & NA \\
\hline Loss of clusters & Unclear risk & NA \\
\hline Incorrect analysis & Unclear risk & NA \\
\hline
\end{tabular}

Ahmed 1993 BGD

\begin{tabular}{ll}
\hline Methods & CBA study \\
\hline Participants & Number: 370 families (after lost $17: 9$ deaths and 8 left the study area) \\
& Inclusion criteria: families with a child aged < 19 months \\
& Intervention group: mean age of children 8.8 months and $51 \%$ girls. Control group: mean age 8.9 \\
& months and 56\% girls \\
\hline Interventions & $\begin{array}{l}1 \text { intervention site (5 contiguous villages): participatory behaviour change intervention, campaign } \\
\text { called "Porichchhanna Jibon" (clean life). The campaign was developed in partnership with the com- }\end{array}$ \\
\hline
\end{tabular}


munity. The intervention involved teaching the germ theory of disease then encouraging mothers to identify their problems and to find solutions through group participation and discussion. Interventions were developed, implemented, and adopted by community.

- Theme l: ground sanitation - keeping babies from touching and eating disease-causing matter on the dirt surface of the compound.

* Sweep the baby's play area 4 times a day.

* Use a dirt thrower (similar to a flat garden trowel provided by the project at USD 0.30) to immediately remove the baby's or animal faeces from the compound surface, so that the crawling baby could not be contaminated by faeces from the ground.

* Construct a faeces pit to dispose of faeces and other filthy matter from the compound. The faeces pit was about 2 feet deep, with a narrow neck.

* Wash babies in a particular place after defecation so that germ-contaminated water did not spread everywhere.

* Keep crawling babies in a playpen (locally constructed, provided by the project at a cost of USD 1.0) instead of permitting them to crawl in the dirt.

- Theme II: personal hygiene - reducing the transmission of germs from defecation and other personal hygiene behaviours (hand washing with ashes or soap, anal cleaning, clean baby after defecation, cut nails, clean rag to dry hands, clean baby rug/mat).

- Theme III: food hygiene - reducing the transmission of germs during supplementary and bottle feeding (do not use any feeding bottle if possible, clean bottle, prepare small amount, use tube well water for drinking and baby food, wash hands before eating, cover food, do not eat leftovers, store plates and pans upside down, cover water pitchers).

1 control site (5 contiguous villages) where a structured observation study was taking place.

Outcomes

Diarrhoea daily prevalence and severe diarrhoea daily prevalence. Mothers were asked to recall the presence or absence of diarrhoea according to their own perceptions day-by-day. If diarrhoea was reported, the mother was asked if the stool was: softer than usual, 1-5 stools; watery, 1-5 stools; softer than usual, 5-10 stools; watery, 5-10 stools; > 10 watery stools per day; or dysentery. Diarrhoea was recategorized into 2 levels: any diarrhoea and severe diarrhoea (all reported watery stools and dysentery). Severe diarrhoea $=$ all reported watery stools and dysentery. Daily prevalence $=$ number of children sick with diarrhoea over total children observed.

Anthropometry (weight for age)

Awareness, understanding, and adoption of each message

Cleanliness observations

Notes
Lecation: 10 rural villages, Bangladesh
Length of study: 9 months (October 1985 to July 1986)
Publication status: journal

\section{Risk of bias}

\begin{tabular}{lll}
\hline Bias & Authors' judgement & Support for judgement \\
\hline $\begin{array}{l}\text { Random sequence genera- } \\
\text { tion (selection bias) }\end{array}$ & High risk & $\begin{array}{l}\text { No randomization, researchers chose the community for intervention as the } \\
\text { poorer, less hygienic site. }\end{array}$ \\
\hline $\begin{array}{l}\text { Allocation concealment } \\
\text { (selection bias) }\end{array}$ & High risk & Investigators could foresee assignment. \\
\hline $\begin{array}{l}\text { Blinding of participants } \\
\text { and personnel (perfor- } \\
\text { mance bias) } \\
\text { All outcomes }\end{array}$ & Unclear risk & $\mathrm{NA}$ \\
\hline \hline
\end{tabular}


Ahmed 1993 BGD (Continued)
Blinding of outcome as-
Unclear risk
NA sessment (detection bias)
All outcomes

\begin{tabular}{lll}
\hline $\begin{array}{l}\text { Incomplete outcome data } \\
\text { (attrition bias) } \\
\text { All outcomes }\end{array}$ & Unclear risk & Not specified how many child days are missing in analysis. \\
\hline $\begin{array}{l}\text { Selective reporting (re- } \\
\text { porting bias) }\end{array}$ & Low risk & Report on all outcomes specified in methods. \\
\hline Other bias & Unclear risk & - \\
\hline $\begin{array}{l}\text { Similarity of baseline out- } \\
\text { come measurements }\end{array}$ & High risk & $\begin{array}{l}\text { There were baseline imbalances in all outcomes and the study did not adjust } \\
\text { for it in analysis. }\end{array}$ \\
\hline $\begin{array}{l}\text { Similarity of baseline char- } \\
\text { acteristics }\end{array}$ & High risk & $\begin{array}{l}\text { There were baseline imbalances in crowding, mother and father education, fa- } \\
\text { ther occupation, land and animal ownership and the study did not adjust for it } \\
\text { in analysis. }\end{array}$
\end{tabular}

Adequate allocation of in- High risk Outcomes were not assessed blindly.

tervention concealment

during the study

\begin{tabular}{ll}
\hline $\begin{array}{l}\text { Adequate protection } \\
\text { against contamination }\end{array}$ & Low risk \\
$\begin{array}{l}\text { Quote: "The intervention site was } 5 \mathrm{~km} \text { away from the control site and accessi- } \\
\text { ble by a 2-hr boat ride most of the year, and by foot over narrow foot paths in } \\
\text { about } 1.5 \mathrm{hr} \text { during the driest months." }\end{array}$ \\
$\begin{array}{l}\text { Comment: the intervention was delivered by members of the community so } \\
\text { likely they would know participants. }\end{array}$
\end{tabular}

\begin{tabular}{lll}
\hline $\begin{array}{l}\text { Confounders adequate- } \\
\text { ly adjusted for in analy- } \\
\text { sis/design }\end{array}$ & High risk & No adjustments for any confounders. \\
\hline Recruitment bias & Unclear risk & NA \\
\hline Baseline imbalance & Unclear risk & NA \\
\hline Loss of clusters & Unclear risk & NA \\
\hline Incorrect analysis & Unclear risk & NA \\
\hline
\end{tabular}

\section{Alam 1989 BGD}

\begin{tabular}{ll}
\hline Methods & CBA study \\
\hline Participants & Number: 623 children (after excluded 27 in intervention group and 50 in control group) \\
& Inclusion criteria: $\mathrm{HHs}$ with children aged $6-23$ months, with $>6$ months' observations per year \\
\hline Interventions & $\begin{array}{l}\text { Intervention site ( } 3 \text { subunits): hand pumps were provided with a ratio of } 4-6 \mathrm{HHs} \text { ( } 3 \text { times more than } \\
\text { control) + health education (main objectives: promotion of consistent and exclusive use of hand pump }\end{array}$
\end{tabular}


Alam 1989 BGD (Continued)

water, improvement of water handling and storage practices, disposal of child's faeces soon after defecation, washing hands before handling food and rubbing hands in ash or using soap after defecation).

Control site (2 subunits): no project input.

Outcomes

Incidence of diarrhoea among children aged 6-23 months. Diarrhoea: $\geq 3$ loose motions in 24-hour period whether or not blood was present. An episode was considered new if there was an interval of $\geq 48$ hours between symptoms (recall $=7$ days).

Observed sources of water, faeces visible in the yard, handwashing before food and after defecation

Notes

Location: 5 subunits (paras) in a village in rural Bangladesh

Length: 3 years (July 1980 to June 1983)

Publication status: journal

\section{Risk of bias}

\begin{tabular}{lll}
\hline Bias & Authors' judgement & Support for judgement \\
\hline $\begin{array}{l}\text { Random sequence genera- } \\
\text { tion (selection bias) }\end{array}$ & High risk & Allocation not random. \\
\hline $\begin{array}{l}\text { Allocation concealment } \\
\text { (selection bias) }\end{array}$ & High risk & No allocation concealment. \\
\hline $\begin{array}{l}\text { Blinding of participants } \\
\text { and personnel (perfor- } \\
\text { mance bias) } \\
\text { All outcomes }\end{array}$ & Unclear risk & NA \\
\hline
\end{tabular}

Blinding of outcome as-
sessment (detection bias)

\begin{tabular}{ll}
\hline Incomplete outcome data & Low risk \\
(attrition bias) & Similar number of child-periods excluded in the analysis in both groups (54 vs \\
All outcomes &
\end{tabular}

Selective reporting (re- Low risk Report on outcomes prespecified in methods.
porting bias)

\begin{tabular}{lll}
\hline Other bias & Unclear risk & - \\
\hline $\begin{array}{l}\text { Similarity of baseline out- } \\
\text { come measurements }\end{array}$ & Unclear risk & No mention of baseline risk. \\
\hline $\begin{array}{l}\text { Similarity of baseline char- } \\
\text { acteristics }\end{array}$ & Unclear risk & $\begin{array}{l}\text { The intervention and control "populations were comparable in terms of edu- } \\
\text { cation, HH size and sanitation conditions". but no data presented). }\end{array}$ \\
\hline $\begin{array}{l}\text { Adequate allocation of in- } \\
\text { tervention concealment } \\
\text { during the study }\end{array}$ & High risk & Quote: "Workers' knowledge of which area was intervention and control." \\
\hline $\begin{array}{l}\text { Adequate protection } \\
\text { against contamination }\end{array}$ & High risk & $\begin{array}{l}\text { Allocation by community - adjacent paras and in the control group some HHs } \\
\text { installed hand pumps. }\end{array}$
\end{tabular}




\begin{tabular}{lll}
\hline $\begin{array}{l}\text { Confounders adequate- } \\
\text { ly adjusted for in analy- } \\
\text { sis/design }\end{array}$ & High risk & No analysis adjusting for confounders. \\
\hline Recruitment bias & Unclear risk & NA \\
\hline Baseline imbalance & Unclear risk & NA \\
\hline Loss of clusters & Unclear risk & NA \\
\hline Incorrect analysis & Unclear risk & NA \\
\hline
\end{tabular}

\section{Altmann 2018 TCD}

\begin{tabular}{ll}
\hline Methods $\quad$ Cluster RCT \\
\hline
\end{tabular}

Participants Number: $20 \mathrm{HCs}$ (1626 children aged 6 to 59 months)

Inclusion criteria: all new admissions of children aged 6 to 59 months to the HCs for OTP. Routine criteria for OTP admission included children aged 6 to 59 months with a WHZ score $<-3$ or a MUAC $<115$ $\mathrm{mm}$ or the presence of mild or moderate bilateral oedema, or a combination of these.

Interventions

Intervention (10 HCs, 850 children): WASH kit plus promotion, which included messaging to bury children's stool. The HH WASH kit given at admission contained a safe drinking water storage container with a lid, water disinfection consumables ( 180 chlorine tablets), 12 bars of soap for hand washing, a plastic cup with handle (to be reserved for the child to facilitate safe drinking water practice), and a laminated leaflet with pictures representing the main hygiene messages. They also received a promotion session on the kit use at each weekly visit to the $\mathrm{HC}$ and 2 extra home visits for assessing and reinforcing adherence. Promotion at $\mathrm{HC}$ included key messages on:

- a protected space for children to play;

- washing the child with soap;

- cleaning and rapid burial of children's stools;

- hand washing at key times;

- safe storage of water;

- exclusive breastfeeding of children before 6 months; and

- water treatment and food hygiene.

The HH WASH kit was designed to last for 3 months ( 2 months during treatment in the OTP and 1 month after the end of the treatment). The intervention group also received the routine OTP services (as the control group).

Control (10 HCs, 776 children): routine OTP services (implemented as per the national guideline for nutrition rehabilitation) and basic hygiene education and care practice sessions during $\mathrm{HC}$ visits.

\section{Outcomes}

Primary outcome:

- relapse rates to SAM at 2 and 6 months postrecovery

Secondary outcomes:

- recovery rate from SAM

- time-to-recovery

- weight gain 
Tertiary outcomes:

- defaulter rate

- internal transfer rate

- death rate

- non-responder rate

- vomiting

- cough

- fever

- total morbidity

Notes

Location: $20 \mathrm{HCs}$, in Mondo and Mao districts, Kanem region, Chad

Length of study: recruitment: April to December 2015, 6-month follow-up phase finished May 2016

Publication status: report

\section{Risk of bias}

\begin{tabular}{lll}
\hline Bias & Authors' judgement & Support for judgement \\
\hline $\begin{array}{l}\text { Random sequence genera- } \\
\text { tion (selection bias) }\end{array}$ & Low risk & $\begin{array}{l}\text { Quote: "We randomly extracted one letter of the alphabet and we assigned } \\
\text { within each pair the intervention to the HC with the first letter of its name clos- } \\
\text { est to this letter." }\end{array}$ \\
\hline $\begin{array}{l}\text { Allocation concealment } \\
\text { (selection bias) }\end{array}$ & Low risk & $\begin{array}{l}\text { Participants and investigators could not foresee assignment due to random } \\
\text { selection. }\end{array}$ \\
\hline $\begin{array}{l}\text { Blinding of participants } \\
\begin{array}{l}\text { and personnel (perfor- } \\
\text { mance bias) } \\
\text { All outcomes }\end{array}\end{array}$ & High risk & $\begin{array}{l}\text { Quotes: "Masking of participants was not possible because of the nature of the } \\
\text { intervention." "It was not possible to blind research staff, but they rotated so } \\
\text { they covered different groups." }\end{array}$
\end{tabular}

\begin{tabular}{lll}
\hline $\begin{array}{l}\text { Blinding of outcome as- } \\
\text { sessment (detection bias) } \\
\text { All outcomes }\end{array}$ & High risk & $\begin{array}{l}\text { Quote: "It was not possible to blind research staff, but they rotated so they } \\
\text { covered different groups." }\end{array}$ \\
\hline $\begin{array}{l}\text { Incomplete outcome data } \\
\text { (attrition bias) } \\
\text { All outcomes }\end{array}$ & Low risk & Similar loss to follow-up in the 2 arms. \\
\hline $\begin{array}{l}\text { Selective reporting (re- } \\
\text { porting bias) }\end{array}$ & Low risk & Reported on main outcomes. \\
\hline $\begin{array}{l}\text { Other bias } \\
\text { Similarity of baseline out- } \\
\text { come measurements }\end{array}$ & Unclear risk & - \\
\hline $\begin{array}{l}\text { Similarity of baseline char- } \\
\text { acteristics }\end{array}$ & Unclear risk & - \\
\hline $\begin{array}{l}\text { Adequate allocation of in- } \\
\text { tervention concealment } \\
\text { during the study }\end{array}$ & Unclear risk \\
\hline
\end{tabular}


Altmann 2018 TCD (Continued)

\begin{tabular}{ll}
$\begin{array}{l}\text { Adequate protection } \\
\text { against contamination }\end{array}$ & Unclear risk \\
\hline $\begin{array}{l}\text { Confounders adequate- } \\
\text { ly adjusted for in analy- } \\
\text { sis/design }\end{array}$ & Unclear risk
\end{tabular}

\begin{tabular}{lll}
\hline Recruitment bias & High risk & $\begin{array}{l}\text { Quote: "Recruitment started } 1 \text { month after allocation of each HC to either } \\
\text { group." }\end{array}$ \\
\hline Baseline imbalance & Low risk & $\begin{array}{l}\text { Quote: "Health centers were stratified in pairs (intervention and control) ac- } \\
\text { cording to the monthly number of SAM admissions (historic data from the year } \\
\text { 2013) to obtain a balanced number of enrolments in the two arms." }\end{array}$ \\
\hline Loss of clusters & Low risk & No loss of clusters. \\
\hline Incorrect analysis & Low risk & Analysis adjusted for clustering. \\
\hline
\end{tabular}

\section{Arvelo 2009 USA}

\begin{tabular}{|c|c|}
\hline Methods & Case-control study \\
\hline Participants & $\begin{array}{l}\text { Case LDC: LDC with a secondary attack rate of shigellosis } \geq 2 \% \text { (median } 5 \% \text {; range } 2-25 \% \text { ), } n=18 \\
\text { Control LDCs: LDC with a secondary attack rate }<2 \% \text { (median 0; range } 0-1.2 \% \text { ), } n=21\end{array}$ \\
\hline Interventions & $\begin{array}{l}\text { Risk factor of interest: } \\
\text { - no lined, lidded bins for nappy disposal vs lined, lidded bins }\end{array}$ \\
\hline Outcomes & $\begin{array}{l}\text { Daycare centre with a secondary attack rate of shigellosis (shigellosis case was defined as a person with } \\
\text { any Shigella species isolated from stool) } \geq 2 \% \text {. }\end{array}$ \\
\hline Notes & $\begin{array}{l}\text { Location: } 39 \text { LDCs in Kansas City metropolitan area, USA } \\
\text { Length: } 2 \text { months (October to November 2005) } \\
\text { Publication status: journal }\end{array}$ \\
\hline
\end{tabular}

\section{Risk of bias}

\begin{tabular}{|c|c|c|}
\hline Bias & Authors' judgement & Support for judgement \\
\hline $\begin{array}{l}\text { Random sequence genera- } \\
\text { tion (selection bias) }\end{array}$ & Unclear risk & NA \\
\hline $\begin{array}{l}\text { Allocation concealment } \\
\text { (selection bias) }\end{array}$ & Unclear risk & NA \\
\hline $\begin{array}{l}\text { Blinding of participants } \\
\text { and personnel (perfor- } \\
\text { mance bias) } \\
\text { All outcomes }\end{array}$ & Unclear risk & NA \\
\hline $\begin{array}{l}\text { Blinding of outcome as- } \\
\text { sessment (detection bias) }\end{array}$ & Unclear risk & NA \\
\hline
\end{tabular}


Arvelo 2009 USA (Continued)

All outcomes

\begin{tabular}{|c|c|c|}
\hline $\begin{array}{l}\text { Incomplete outcome data } \\
\text { (attrition bias) } \\
\text { All outcomes }\end{array}$ & Unclear risk & NA \\
\hline $\begin{array}{l}\text { Selective reporting (re- } \\
\text { porting bias) }\end{array}$ & Unclear risk & NA \\
\hline Other bias & Unclear risk & NA \\
\hline $\begin{array}{l}\text { Similarity of baseline out- } \\
\text { come measurements }\end{array}$ & Unclear risk & NA \\
\hline $\begin{array}{l}\text { Similarity of baseline char- } \\
\text { acteristics }\end{array}$ & Unclear risk & NA \\
\hline $\begin{array}{l}\text { Adequate allocation of in- } \\
\text { tervention concealment } \\
\text { during the study }\end{array}$ & Unclear risk & NA \\
\hline $\begin{array}{l}\text { Adequate protection } \\
\text { against contamination }\end{array}$ & Unclear risk & NA \\
\hline $\begin{array}{l}\text { Confounders adequate- } \\
\text { ly adjusted for in analy- } \\
\text { sis/design }\end{array}$ & Unclear risk & NA \\
\hline Recruitment bias & Unclear risk & NA \\
\hline Baseline imbalance & Unclear risk & NA \\
\hline Loss of clusters & Unclear risk & NA \\
\hline Incorrect analysis & Unclear risk & NA \\
\hline
\end{tabular}

\section{Asfaha 2018 ETH}

\begin{tabular}{ll}
\hline Methods & Case-control study (community-based, not-matched) \\
\hline Participants & $\begin{array}{l}\text { Case: } 0-59 \text { months children with diarrhoea in the preceding of } 2 \text { weeks during a house-to-house survey, } \\
\mathrm{n}=199(0.5 \% \text { non-response) } \\
\text { Control: } 0-59 \text { months children without diarrhoea in the preceding of } 2 \text { weeks during a house to house } \\
\text { survey, } \mathrm{n}=398 \text { (0.5\% non-response) }\end{array}$ \\
\hline Interventions & Risk factor of interest: \\
\hline Outcomes & Dafe vs not safe child stool disposal (no definition of safe/unsafe) \\
the child.
\end{tabular}

Notes

Location: Medebay Zana district, northwest Tigray, Ethiopia

Length: 1.5 months (1 October 2015 to 15 November 2015) 
Asfaha 2018 ETH (Continued)

Publication status: journal

\section{Risk of bias}

\begin{tabular}{|c|c|c|}
\hline Bias & Authors' judgement & Support for judgement \\
\hline $\begin{array}{l}\text { Random sequence genera- } \\
\text { tion (selection bias) }\end{array}$ & Unclear risk & NA \\
\hline $\begin{array}{l}\text { Allocation concealment } \\
\text { (selection bias) }\end{array}$ & Unclear risk & NA \\
\hline $\begin{array}{l}\text { Blinding of participants } \\
\text { and personnel (perfor- } \\
\text { mance bias) } \\
\text { All outcomes }\end{array}$ & Unclear risk & NA \\
\hline $\begin{array}{l}\text { Blinding of outcome as- } \\
\text { sessment (detection bias) } \\
\text { All outcomes }\end{array}$ & Unclear risk & NA \\
\hline $\begin{array}{l}\text { Incomplete outcome data } \\
\text { (attrition bias) } \\
\text { All outcomes }\end{array}$ & Unclear risk & NA \\
\hline $\begin{array}{l}\text { Selective reporting (re- } \\
\text { porting bias) }\end{array}$ & Unclear risk & NA \\
\hline Other bias & Unclear risk & NA \\
\hline $\begin{array}{l}\text { Similarity of baseline out- } \\
\text { come measurements }\end{array}$ & Unclear risk & NA \\
\hline
\end{tabular}

Similarity of baseline char- Unclear risk NA

acteristics

$\begin{array}{ll}\text { Adequate allocation of in- } & \text { Unclear risk } \\ \text { tervention concealment } & \end{array}$

during the study

\begin{tabular}{lll}
\hline $\begin{array}{l}\text { Adequate protection } \\
\text { against contamination }\end{array}$ & Unclear risk & NA \\
\hline $\begin{array}{l}\text { Confounders adequate- } \\
\text { ly adjusted for in analy- } \\
\text { sis/design }\end{array}$ & Unclear risk & NA \\
\hline Recruitment bias & Unclear risk & NA \\
\hline Baseline imbalance & Unclear risk & NA \\
\hline Loss of clusters & Unclear risk & NA \\
\hline Incorrect analysis & Unclear risk & NA \\
\hline
\end{tabular}


Aziz 1990 BGD

\begin{tabular}{|c|c|}
\hline Methods & CBA study \\
\hline Participants & $\begin{array}{l}\text { Number: exact numbers not presented, on average complete data available for } 405 \text { children } \\
\text { Inclusion criteria: HHs with children aged < } 5 \text { years }\end{array}$ \\
\hline Interventions & $\begin{array}{l}\text { Intervention ( } 2 \text { villages): } 148 \text { new hand pumps (1 pump: } 30 \text { people on average) + free maintenance, } \\
92 \% \text { of } \mathrm{HHs} \text { received a double pit water sealed latrine, hygiene education emphasising exclusive use of } \\
\text { the pump water for all personal and domestic use and the need for all members of the } \mathrm{HH} \text {, including } \\
\text { young children to use the latrines. } \\
\text { Control ( } 3 \text { villages): no intervention provided. ORS was given to sick children + referral to hospital if } \\
\text { sick. }\end{array}$ \\
\hline \multirow[t]{6}{*}{ Outcomes } & $\begin{array}{l}\text { Diarrhoea incidence, case definition: } \geq 3 \text { loose motions in a } 24 \text {-hour period. Recall period } 7 \text { days, an } \\
\text { episode was considered complete after } 2 \text { diarrhoea-free days. }\end{array}$ \\
\hline & Dysentery incidence, case definition: blood was present in the stools. \\
\hline & Persistent diarrhoea incidence, case definition: episodes of duration $>14$ days \\
\hline & Days of diarrhoea \\
\hline & $\begin{array}{l}\text { Anthropometry (weight for age, height for age, weight for height) (Hasan 1989; reference is listed under } \\
\text { Aziz } 1990 \text { BGD)) }\end{array}$ \\
\hline & $\begin{array}{l}\text { Hand pump distance and use, defecation of children or disposal of their faeces in latrine (only reported } \\
\text { in intervention arm) }\end{array}$ \\
\hline \multirow[t]{3}{*}{ Notes } & Location: 5 villages in rural Bangladesh \\
\hline & Length: 3 years (January 1984 to December 1987) \\
\hline & Publication status: journal \\
\hline
\end{tabular}

\section{Risk of bias}

\begin{tabular}{lll}
\hline Bias & Authors' judgement & Support for judgement \\
\hline $\begin{array}{l}\text { Random sequence genera- } \\
\text { tion (selection bias) }\end{array}$ & High risk & No randomized allocation.
\end{tabular}

Allocation concealment High risk No allocation concealment.

(selection bias)

Blinding of participants Unclear risk
and personnel (perfor-
mance bias)
All outcomes

Blinding of outcome as-
sessment (detection bias) $\quad$ Unclear risk NA

\begin{tabular}{lll}
\hline $\begin{array}{l}\text { Incomplete outcome data } \\
\text { (attrition bias) }\end{array}$ & Unclear risk & Total number of children or loss to follow-up not reported. \\
All outcomes & \\
\hline $\begin{array}{l}\text { Selective reporting (re- } \\
\text { porting bias) }\end{array}$ & Low risk & Report on outcomes prespecified in methods. \\
\hline
\end{tabular}


Aziz 1990 BGD (Continued)

Similarity of baseline out- Low risk_ Diarrhoea and anthropometry measures were similar at baseline. come measurements

Similarity of baseline char- Unclear risk acteristics

Quote: "The two areas were comparable with respect to most sociodemographic and economic characteristics although the control area was slightly better off in terms of female education and socio-economic level."

Comment: however, no data presented.

\begin{tabular}{lll}
$\begin{array}{l}\text { Adequate allocation of in- } \\
\text { tervention concealment } \\
\text { during the study }\end{array}$ & High risk & $\begin{array}{l}\text { Quote: "Project staff and the community under investigation knew that the } \\
\text { aim of the study was to decrease the diarrhoea incidence." }\end{array}$ \\
\hline $\begin{array}{l}\text { Adequate protection } \\
\text { against contamination }\end{array}$ & Low risk & The 2 areas were 5 km apart. \\
\hline $\begin{array}{l}\text { Confounders adequate- } \\
\text { ly adjusted for in analy- } \\
\text { sis/design }\end{array}$ & High risk & No adjustments in the analysis. \\
\hline $\begin{array}{l}\text { Recruitment bias } \\
\text { Baseline imbalance }\end{array}$ & Unclear risk & NA \\
\hline Loss of clusters & Unclear risk & NA \\
\hline $\begin{array}{l}\text { Incorrect analysis } \\
\text { Unclear risk }\end{array}$ & NA \\
\hline
\end{tabular}

Baker 2016 BGD

\begin{tabular}{|c|c|}
\hline Methods & Case-control study (prospective, age-stratified, matched) \\
\hline Participants & $\begin{array}{l}\text { Case: children aged } 0-59 \text { months belonging to the demographic surveillance system population at the } \\
\text { site, not currently enrolled as a case (previously enrolled and pending } 60 \text {-day visit) seeking care at HC } \\
\text { with moderate-to-severe diarrhoea, } n=1374 \text { ( } 1.4 \% \text { LTFU compared to all cases enrolled at site) }\end{array}$ \\
\hline & $\begin{array}{l}\text { Control: child with no diarrhoea in the previous } 7 \text { days, residing in demographic surveillance system } \\
\text { area, matched to the case for age (SD } 2 \text { months for } 0-11 \text { and } 12-23 \text { months, SD } 4 \text { months for } 24-59 \\
\text { months, not exceeding the stratum boundaries of the case), sex, residence (lives in the same or nearby } \\
\text { village/neighbourhood as the case), and time (enrolled within } 14 \text { days of presentation of the case), } n= \\
2428 \text { (1.5\% LTFU compared to all controls enrolled at site) }\end{array}$ \\
\hline
\end{tabular}

Interventions

Risk factor of interest:

- disposal of child faeces in the open vs disposal in any type of latrine with a pit or sewer. Hanging latrines and bucket latrines were considered open disposal.

Outcomes

Diarrhoea: $\geq 3$ abnormally loose stools in the previous 24 hours. Diarrhoea episode had to be acute (onset within 7 days of study enrolment) and be a new episode (onset after $\geq 7$ diarrhoea-free days).

Moderate-to-severe: child met $\geq 1$ of the following criteria:

- sunken eyes, confirmed by parent/primary caretaker as more than normal

- loss of skin turgor (determined by abdominal skin pinch (slow return ( $\leq 2$ seconds) or very slow return (>2 seconds))

- intravenous rehydration administered or prescribed

- dysentery (visible blood in a loose stool) 
Baker 2016 BGD (Continued)

- hospitalized with diarrhoea or dysentery

Notes Location: 1 rural sentinel HC, Mirzapur, Bangladesh

Length: 3 years (1 December 2007 to 3 March 2011)

Publication status: journal

\section{Risk of bias}

\begin{tabular}{lll}
\hline Bias & Authors' judgement & Support for judgement \\
\hline $\begin{array}{l}\text { Random sequence genera- } \\
\text { tion (selection bias) }\end{array}$ & Unclear risk & NA \\
\hline $\begin{array}{l}\text { Allocation concealment } \\
\text { (selection bias) }\end{array}$ & Unclear risk & NA \\
\hline $\begin{array}{l}\text { Blinding of participants } \\
\text { and personnel (perfor- } \\
\text { mance bias) } \\
\text { All outcomes }\end{array}$ & Unclear risk & NA \\
\hline
\end{tabular}

$\begin{array}{ll}\text { Blinding of outcome as- } & \text { Unclear risk }\end{array}$

All outcomes

Incomplete outcome data Unclear risk NA

(attrition bias)

NA

All outcomes

\begin{tabular}{|c|c|c|}
\hline $\begin{array}{l}\text { Selective reporting (re- } \\
\text { porting bias) }\end{array}$ & Unclear risk & NA \\
\hline Other bias & Unclear risk & NA \\
\hline $\begin{array}{l}\text { Similarity of baseline out- } \\
\text { come measurements }\end{array}$ & Unclear risk & NA \\
\hline $\begin{array}{l}\text { Similarity of baseline char- } \\
\text { acteristics }\end{array}$ & Unclear risk & NA \\
\hline $\begin{array}{l}\text { Adequate allocation of in- } \\
\text { tervention concealment } \\
\text { during the study }\end{array}$ & Unclear risk & NA \\
\hline $\begin{array}{l}\text { Adequate protection } \\
\text { against contamination }\end{array}$ & Unclear risk & NA \\
\hline $\begin{array}{l}\text { Confounders adequate- } \\
\text { ly adjusted for in analy- } \\
\text { sis/design }\end{array}$ & Unclear risk & NA \\
\hline Recruitment bias & Unclear risk & NA \\
\hline Baseline imbalance & Unclear risk & NA \\
\hline Loss of clusters & Unclear risk & NA \\
\hline
\end{tabular}

Interventions to improve disposal of child faeces for preventing diarrhoea and soil-transmitted helminth infection (Review) 
Baker 2016 BGD (Continued)
Incorrect analysis
Unclear risk
NA

Baker 2016 GMB

\begin{tabular}{ll}
\hline Methods & Case-control study (prospective, age-stratified, matched) \\
\hline Participants & $\begin{array}{l}\text { Case and control definitions were the same as Baker 2016 BGD. Cases n }=910 \text { (11.6\% LTFU), controls } n \\
=1456(7.2 \% \text { LTFU). }\end{array}$ \\
\hline Interventions & Same as Baker 2016 BGD \\
\hline Outcomes & Same as Baker 2016 BGD \\
\hline Notes & Location: 5 rural sentinel HCs, Basse, The Gambia \\
& Length: 3 years (1 December 2007 to 3 March 2011) \\
& Publication status: journal
\end{tabular}

\section{Risk of bias}

\section{Bias}

Random sequence genera-

tion (selection bias)

Allocation concealment $\quad$ Unclear risk
(selection bias)

\section{Authors' judgement Support for judgement}

NA

NA

NA

and personnel (perfor-

mance bias)

All outcomes

\begin{tabular}{ll}
\hline Blinding of outcome as- & Unclear risk
\end{tabular}

\begin{tabular}{lll}
\hline $\begin{array}{l}\text { Incomplete outcome data } \\
\text { (attrition bias) } \\
\text { All outcomes }\end{array}$ & Unclear risk \\
\hline $\begin{array}{l}\text { Selective reporting (re- } \\
\text { porting bias) }\end{array}$ & Unclear risk & NA \\
\hline Other bias & Unclear risk & NA \\
\hline $\begin{array}{l}\text { Similarity of baseline out- } \\
\text { come measurements }\end{array}$ & Unclear risk & NA \\
\hline $\begin{array}{l}\text { Similarity of baseline char- } \\
\text { acteristics }\end{array}$ & Unclear risk & NA \\
\hline
\end{tabular}


Baker 2016 GMB (Continued)
Adequate allocation of in-
Unclear risk
NA tervention concealment during the study

\begin{tabular}{lll}
\hline $\begin{array}{l}\text { Adequate protection } \\
\text { against contamination }\end{array}$ & Unclear risk & NA \\
\hline $\begin{array}{l}\text { Confounders adequate- } \\
\text { ly adjusted for in analy- } \\
\text { sis/design }\end{array}$ & Unclear risk & NA \\
\hline Recruitment bias & Unclear risk & NA \\
\hline Baseline imbalance & Unclear risk & NA \\
\hline Loss of clusters & Unclear risk & NA \\
\hline Incorrect analysis & Unclear risk & NA \\
\hline
\end{tabular}

\section{Baker 2016 IND}

\begin{tabular}{ll}
\hline Methods & Case-control study (prospective, age-stratified, matched) \\
\hline Participants & $\begin{array}{l}\text { Case and control definitions were the same as Baker 2016 BGD. Cases n = 1505 (4\% LTFU), controls n }= \\
1967(2.3 \% \text { LTFU). }\end{array}$ \\
\hline Interventions & Same as Baker 2016 BGD \\
\hline Outcomes & Same as Baker 2016 BGD \\
\hline Notes & Location: 2 urban sentinel HCs, Kolkata, West Bengal, India \\
& Length: 3 years (1 December 2007 to 3 March 2011) \\
& Publication status: journal
\end{tabular}

\section{Risk of bias}

\begin{tabular}{lll}
\hline Bias & Authors' judgement & Support for judgement \\
\hline $\begin{array}{l}\text { Random sequence genera- } \\
\text { tion (selection bias) }\end{array}$ & Unclear risk & NA \\
\hline $\begin{array}{l}\text { Allocation concealment } \\
\text { (selection bias) }\end{array}$ & Unclear risk & NA \\
\hline $\begin{array}{l}\text { Blinding of participants } \\
\text { and personnel (perfor- } \\
\text { mance bias) }\end{array}$ & Unclear risk & NA \\
All outcomes & \\
\hline $\begin{array}{l}\text { Blinding of outcome as- } \\
\text { sessment (detection bias) } \\
\text { All outcomes }\end{array}$ & Unclear risk & NA \\
\hline
\end{tabular}


Baker 2016 IND (Continued)

Incomplete outcome data Unclear risk NA
(attrition bias)

All outcomes

A

outcomes

Selective reporting (re- Unclear risk NA
porting bias)

NA

\begin{tabular}{lll}
\hline Other bias & Unclear risk & NA \\
\hline $\begin{array}{l}\text { Similarity of baseline out- } \\
\text { come measurements }\end{array}$ & Unclear risk & NA \\
\hline $\begin{array}{l}\text { Similarity of baseline char- } \\
\text { acteristics }\end{array}$ & Unclear risk & NA
\end{tabular}

\begin{tabular}{|c|c|c|}
\hline $\begin{array}{l}\text { Adequate allocation of in- } \\
\text { tervention concealment } \\
\text { during the study }\end{array}$ & Unclear risk & NA \\
\hline $\begin{array}{l}\text { Adequate protection } \\
\text { against contamination }\end{array}$ & Unclear risk & NA \\
\hline $\begin{array}{l}\text { Confounders adequate- } \\
\text { ly adjusted for in analy- } \\
\text { sis/design }\end{array}$ & Unclear risk & NA \\
\hline Recruitment bias & Unclear risk & NA \\
\hline Baseline imbalance & Unclear risk & NA \\
\hline Loss of clusters & Unclear risk & NA \\
\hline Incorrect analysis & Unclear risk & NA \\
\hline
\end{tabular}

\title{
Baker 2016 KEN
}

\begin{tabular}{ll}
\hline Methods & Case-control study (prospective, age-stratified, matched) \\
\hline Participants & $\begin{array}{l}\text { Case and control definitions were the same as Baker 2016 BGD. Cases n = 1419 (3.9\% LTFU), controls n } \\
=1841(2.2 \% \text { LTFU). }\end{array}$ \\
\hline Interventions & Same as Baker 2016 BGD \\
\hline Outcomes & Same as Baker 2016 BGD \\
\hline Notes & Location: 11 rural sentinel HCs, Nyanza Province, Kenya \\
& Length: 3 years (1 December 2007 to 3 March 2011) \\
& Publication status: journal \\
\hline
\end{tabular}

\section{Risk of bias}

\section{Bias}

\author{
Authors' judgement Support for judgement
}


Baker 2016 KEN (Continued)

\begin{tabular}{lll}
$\begin{array}{l}\text { Random sequence genera- } \\
\text { tion (selection bias) }\end{array}$ & Unclear risk & NA \\
\hline $\begin{array}{l}\text { Allocation concealment } \\
\text { (selection bias) }\end{array}$ & Unclear risk & NA \\
\hline $\begin{array}{l}\text { Blinding of participants } \\
\text { and personnel (perfor- } \\
\text { mance bias) }\end{array}$ & Unclear risk & NA \\
All outcomes &
\end{tabular}

Blinding of outcome as-
sessment (detection bias) $\quad$ Unclear risk $\quad N A$

All outcomes

Incomplete outcome data Unclear risk NA
(attrition bias)

All outcomes

NA

NA

\begin{tabular}{|c|c|c|}
\hline $\begin{array}{l}\text { Selective reporting (re- } \\
\text { porting bias) }\end{array}$ & Unclear risk & NA \\
\hline Other bias & Unclear risk & NA \\
\hline $\begin{array}{l}\text { Similarity of baseline out- } \\
\text { come measurements }\end{array}$ & Unclear risk & NA \\
\hline $\begin{array}{l}\text { Similarity of baseline char- } \\
\text { acteristics }\end{array}$ & Unclear risk & NA \\
\hline $\begin{array}{l}\text { Adequate allocation of in- } \\
\text { tervention concealment } \\
\text { during the study }\end{array}$ & Unclear risk & NA \\
\hline $\begin{array}{l}\text { Adequate protection } \\
\text { against contamination }\end{array}$ & Unclear risk & NA \\
\hline $\begin{array}{l}\text { Confounders adequate- } \\
\text { ly adjusted for in analy- } \\
\text { sis/design }\end{array}$ & Unclear risk & NA \\
\hline Recruitment bias & Unclear risk & NA \\
\hline Baseline imbalance & Unclear risk & NA \\
\hline Loss of clusters & Unclear risk & NA \\
\hline Incorrect analysis & Unclear risk & NA \\
\hline
\end{tabular}

\section{Baker 2016 MLI}

\begin{tabular}{ll}
\hline Methods & Case-control study (prospective, age-stratified, matched) \\
\hline Participants & $\begin{array}{l}\text { Case and control definitions were the same as Baker } 2016 \text { BGD. Cases } n=1786 \text { (12.1\% LTFU), controls n } \\
=1891(8.4 \% \text { LTFU). }\end{array}$ \\
\hline
\end{tabular}


Baker 2016 MLI (Continued)

\begin{tabular}{ll} 
Interventions & Same as Baker 2016 BGD \\
\hline Outcomes & Same as Baker 2016 BGD \\
\hline Notes & Location: 9 urban sentinel HCs, Bamako, Mali \\
& Length: 3 years (1 December 2007 to 3 March 2011) \\
& Publication status: journal
\end{tabular}

\section{Risk of bias}

\begin{tabular}{|c|c|c|}
\hline Bias & Authors' judgement & Support for judgement \\
\hline $\begin{array}{l}\text { Random sequence genera- } \\
\text { tion (selection bias) }\end{array}$ & Unclear risk & NA \\
\hline $\begin{array}{l}\text { Allocation concealment } \\
\text { (selection bias) }\end{array}$ & Unclear risk & NA \\
\hline $\begin{array}{l}\text { Blinding of participants } \\
\text { and personnel (perfor- } \\
\text { mance bias) } \\
\text { All outcomes }\end{array}$ & Unclear risk & NA \\
\hline $\begin{array}{l}\text { Blinding of outcome as- } \\
\text { sessment (detection bias) } \\
\text { All outcomes }\end{array}$ & Unclear risk & NA \\
\hline $\begin{array}{l}\text { Incomplete outcome data } \\
\text { (attrition bias) } \\
\text { All outcomes }\end{array}$ & Unclear risk & NA \\
\hline $\begin{array}{l}\text { Selective reporting (re- } \\
\text { porting bias) }\end{array}$ & Unclear risk & NA \\
\hline Other bias & Unclear risk & NA \\
\hline $\begin{array}{l}\text { Similarity of baseline out- } \\
\text { come measurements }\end{array}$ & Unclear risk & NA \\
\hline $\begin{array}{l}\text { Similarity of baseline char- } \\
\text { acteristics }\end{array}$ & Unclear risk & NA \\
\hline $\begin{array}{l}\text { Adequate allocation of in- } \\
\text { tervention concealment } \\
\text { during the study }\end{array}$ & Unclear risk & NA \\
\hline $\begin{array}{l}\text { Adequate protection } \\
\text { against contamination }\end{array}$ & Unclear risk & NA \\
\hline $\begin{array}{l}\text { Confounders adequate- } \\
\text { ly adjusted for in analy- } \\
\text { sis/design }\end{array}$ & Unclear risk & NA \\
\hline Recruitment bias & Unclear risk & NA \\
\hline Baseline imbalance & Unclear risk & NA \\
\hline
\end{tabular}

Interventions to improve disposal of child faeces for preventing diarrhoea and soil-transmitted helminth infection (Review) 
Baker 2016 MLI (Continued)

\begin{tabular}{lll} 
Loss of clusters & Unclear risk & NA \\
\hline Incorrect analysis & Unclear risk & NA \\
\hline
\end{tabular}

Baker $2016 \mathrm{MOZ}$

\begin{tabular}{|c|c|}
\hline Methods & Case-control study (prospective, age-stratified, matched) \\
\hline Participants & $\begin{array}{l}\text { Case and control definitions were the same as Baker } 2016 \text { BGD. Cases } n=602(11.6 \%) \text {, controls } n=1182 \\
(8.8 \% \text { LTFU). }\end{array}$ \\
\hline Interventions & Same as Baker 2016 BGD \\
\hline Outcomes & Same as Baker 2016 BGD \\
\hline \multirow[t]{3}{*}{ Notes } & Location: 5 rural sentinel HCs, Manhiça, Mozambique \\
\hline & Length: 3 years (1 December 2007 to 3 March 2011) \\
\hline & Publication status: journal \\
\hline
\end{tabular}

\section{Risk of bias}

\begin{tabular}{|c|c|c|}
\hline Bias & Authors' judgement & Support for judgement \\
\hline $\begin{array}{l}\text { Random sequence genera- } \\
\text { tion (selection bias) }\end{array}$ & Unclear risk & NA \\
\hline $\begin{array}{l}\text { Allocation concealment } \\
\text { (selection bias) }\end{array}$ & Unclear risk & NA \\
\hline $\begin{array}{l}\text { Blinding of participants } \\
\text { and personnel (perfor- } \\
\text { mance bias) } \\
\text { All outcomes }\end{array}$ & Unclear risk & NA \\
\hline $\begin{array}{l}\text { Blinding of outcome as- } \\
\text { sessment (detection bias) } \\
\text { All outcomes }\end{array}$ & Unclear risk & NA \\
\hline $\begin{array}{l}\text { Incomplete outcome data } \\
\text { (attrition bias) } \\
\text { All outcomes }\end{array}$ & Unclear risk & NA \\
\hline $\begin{array}{l}\text { Selective reporting (re- } \\
\text { porting bias) }\end{array}$ & Unclear risk & NA \\
\hline Other bias & Unclear risk & NA \\
\hline $\begin{array}{l}\text { Similarity of baseline out- } \\
\text { come measurements }\end{array}$ & Unclear risk & NA \\
\hline $\begin{array}{l}\text { Similarity of baseline char- } \\
\text { acteristics }\end{array}$ & Unclear risk & NA \\
\hline
\end{tabular}


Baker 2016 MOZ (Continued)
Adequate allocation of in-
Unclear risk
NA tervention concealment during the study

\begin{tabular}{lll}
\hline $\begin{array}{l}\text { Adequate protection } \\
\text { against contamination }\end{array}$ & Unclear risk & NA \\
\hline $\begin{array}{l}\text { Confounders adequate- } \\
\text { ly adjusted for in analy- } \\
\text { sis/design }\end{array}$ & Unclear risk & NA \\
\hline Recruitment bias & Unclear risk & NA \\
\hline Baseline imbalance & Unclear risk & NA \\
\hline Loss of clusters & Unclear risk & NA \\
\hline Incorrect analysis & Unclear risk & NA \\
\hline
\end{tabular}

\section{Baker 2016 PAK}

\begin{tabular}{ll}
\hline Methods & Case-control study (prospective, age-stratified, matched) \\
\hline Participants & $\begin{array}{l}\text { Case and control definitions were the same as Baker 2016 BGD. Cases n }=996 \text { (20.8\% LTFU), controls n } \\
=1625(11.6 \% \text { LTFU). }\end{array}$ \\
\hline Interventions & Same as Baker 2016 BGD \\
\hline Outcomes & Same as Baker 2016 BGD \\
\hline Notes & Location: 7 periurban sentinel HCs, Karachi (Bin Qasim Town), Pakistan \\
& Length: 3 years (1 December 2007 to 3 March 2011) \\
Publication status: journal
\end{tabular}

\section{Risk of bias}

\begin{tabular}{lll}
\hline Bias & Authors' judgement & Support for judgement \\
\hline $\begin{array}{l}\text { Random sequence genera- } \\
\text { tion (selection bias) }\end{array}$ & Unclear risk & NA \\
\hline $\begin{array}{l}\text { Allocation concealment } \\
\text { (selection bias) }\end{array}$ & Unclear risk & NA \\
\hline $\begin{array}{l}\text { Blinding of participants } \\
\text { and personnel (perfor- } \\
\text { mance bias) }\end{array}$ & Unclear risk & NA \\
All outcomes & \\
\hline $\begin{array}{l}\text { Blinding of outcome as- } \\
\text { sessment (detection bias) } \\
\text { All outcomes }\end{array}$ & Unclear risk & NA \\
\hline
\end{tabular}


Baker 2016 PAK (Continued)

Incomplete outcome data Unclear risk NA
(attrition bias)

All outcomes

A

Selective reporting (re- Unclear risk
porting bias)

\begin{tabular}{lll}
\hline Other bias & Unclear risk & NA \\
\hline $\begin{array}{l}\text { Similarity of baseline out- } \\
\text { come measurements }\end{array}$ & Unclear risk & NA \\
\hline $\begin{array}{l}\text { Similarity of baseline char- } \\
\text { acteristics }\end{array}$ & Unclear risk & NA \\
\hline
\end{tabular}

\begin{tabular}{lll}
\hline $\begin{array}{l}\text { Adequate allocation of in- } \\
\text { tervention concealment } \\
\text { during the study }\end{array}$ & Unclear risk & NA \\
\hline $\begin{array}{l}\text { Adequate protection } \\
\text { against contamination }\end{array}$ & Unclear risk & NA \\
\hline $\begin{array}{l}\text { Confounders adequate- } \\
\text { ly adjusted for in analy- } \\
\text { sis/design }\end{array}$ & Unclear risk & NA \\
\hline $\begin{array}{l}\text { Recruitment bias } \\
\text { Baseline imbalance }\end{array}$ & Unclear risk & NA \\
\hline Loss of clusters & Unclear risk & NA \\
\hline \begin{tabular}{l} 
Incorrect analysis \\
\hline
\end{tabular} & Unclear risk & NA \\
\hline
\end{tabular}

\section{Baltazar 1989 PHI}

\begin{tabular}{|c|c|}
\hline Methods & Case-control study \\
\hline \multirow[t]{2}{*}{ Participants } & $\begin{array}{l}\text { Cases: children aged }<2 \text { years brought to clinic for diarrhoea, } n=275 \text { (after excluding 6), 68\% aged }<1 \\
\text { year }\end{array}$ \\
\hline & $\begin{array}{l}\text { Controls: children aged }<2 \text { years brought to clinic for ARI without diarrhoea in past } 24 \text { hours, } n=381 \\
\text { (after excluding 3), } 73 \% \text { aged }<1 \text { year }\end{array}$ \\
\hline \multirow[t]{3}{*}{ Interventions } & Risk factor of interest: \\
\hline & $\begin{array}{l}\text { - unsanitary vs sanitary disposal of stools: } \\
\text { * sanitary: child defecated in a nappy and faeces were thrown away in washing, child used pot- } \\
\text { ty/piece of paper and faecal matter was thrown in the toilet or child used the toilet; }\end{array}$ \\
\hline & $\begin{array}{l}\text { * unsanitary: faecal matter was deposited elsewhere than latrine/child defecated outside (regard- } \\
\text { less of where faecal matter was finally thrown away). }\end{array}$ \\
\hline \multirow[t]{2}{*}{ Outcomes } & Diarrhoea (no case definition) \\
\hline & $\begin{array}{l}\text { Rectal swabs for diagnosis of diarrhoea pathogens and carried out a subgroup analysis for laborato- } \\
\text { ry-confirmed cases. }\end{array}$ \\
\hline
\end{tabular}


Baltazar 1989 PHI (Continued)

Notes

Location: 16 clinics, Cebu area (urban and rural), Phillippines

Length of recruitment: 5 months (June-October 1985)

Publication status: journal

\section{Risk of bias}

\begin{tabular}{|c|c|c|}
\hline Bias & Authors' judgement & Support for judgement \\
\hline $\begin{array}{l}\text { Random sequence genera- } \\
\text { tion (selection bias) }\end{array}$ & Unclear risk & NA \\
\hline $\begin{array}{l}\text { Allocation concealment } \\
\text { (selection bias) }\end{array}$ & Unclear risk & NA \\
\hline $\begin{array}{l}\text { Blinding of participants } \\
\text { and personnel (perfor- } \\
\text { mance bias) } \\
\text { All outcomes }\end{array}$ & Unclear risk & NA \\
\hline $\begin{array}{l}\text { Blinding of outcome as- } \\
\text { sessment (detection bias) } \\
\text { All outcomes }\end{array}$ & Unclear risk & NA \\
\hline $\begin{array}{l}\text { Incomplete outcome data } \\
\text { (attrition bias) } \\
\text { All outcomes }\end{array}$ & Unclear risk & NA \\
\hline $\begin{array}{l}\text { Selective reporting (re- } \\
\text { porting bias) }\end{array}$ & Unclear risk & NA \\
\hline Other bias & Unclear risk & NA \\
\hline $\begin{array}{l}\text { Similarity of baseline out- } \\
\text { come measurements }\end{array}$ & Unclear risk & NA \\
\hline $\begin{array}{l}\text { Similarity of baseline char- } \\
\text { acteristics }\end{array}$ & Unclear risk & NA \\
\hline $\begin{array}{l}\text { Adequate allocation of in- } \\
\text { tervention concealment } \\
\text { during the study }\end{array}$ & Unclear risk & NA \\
\hline $\begin{array}{l}\text { Adequate protection } \\
\text { against contamination }\end{array}$ & Unclear risk & NA \\
\hline $\begin{array}{l}\text { Confounders adequate- } \\
\text { ly adjusted for in analy- } \\
\text { sis/design }\end{array}$ & Unclear risk & NA \\
\hline Recruitment bias & Unclear risk & NA \\
\hline Baseline imbalance & Unclear risk & NA \\
\hline Loss of clusters & Unclear risk & NA \\
\hline Incorrect analysis & Unclear risk & NA \\
\hline
\end{tabular}

Interventions to improve disposal of child faeces for preventing diarrhoea and soil-transmitted helminth infection (Review) 
Barrios 2008 PHI

\begin{tabular}{ll}
\hline Methods & Cluster RCT \\
\hline Participants & Number: 495 respondents (enrolment rate 90\%) \\
& Inclusion criteria: HHs with children aged $<5$ years \\
\hline
\end{tabular}

Interventions

Interventions ( 2 barangays (smallest local government unit)): hygiene promotion programme that focused on improving hand washing and stool disposal behaviours. Midwives and barangay health workers delivered the educational sessions in small group meetings and in home visits. Activities to promote the behaviours included demonstrations of proper hand washing, a drawing activity with a brief story-board of the negative effects of improper stool disposal. For the disposal of child faeces, caretakers were encouraged to use toilets (any type) as the final site of faeces disposal. When a toilet was not available, burying faeces $\geq 10 \mathrm{~m}$ away from water sources and living areas was discussed. The main message was the sanitary disposal of faeces, regardless of where a child defecated.

Control intervention (2 barangays): caregivers received education on signs and symptoms of dehydration and the importance of oral rehydration during diarrhoea.

Control with no contact (2 barangays): no contact, no treatment

\begin{tabular}{ll}
\hline Outcomes & Diarrhoea (measured but not reported on) \\
& Handwashing behaviour \\
& Stool disposal behaviour: observed faeces in the yard \\
& Knowledge, attitudes, beliefs on hand washing and stool disposal \\
\hline Notes & Location: 6 rural barangays in Basista, Philippines \\
& Length of study: 2 months \\
& Publication status: PhD thesis
\end{tabular}

\section{Risk of bias}

\begin{tabular}{lll}
\hline Bias & Authors' judgement & Support for judgement \\
\hline $\begin{array}{l}\text { Random sequence genera- } \\
\text { tion (selection bias) }\end{array}$ & Low risk & $\begin{array}{l}\text { Quote: "Random assignment to one of three experimental conditions was } \\
\text { achieved by a simple sample draw with replacement." }\end{array}$ \\
\hline $\begin{array}{l}\text { Allocation concealment } \\
\text { (selection bias) }\end{array}$ & Unclear risk & No details \\
\hline $\begin{array}{l}\text { Blinding of participants } \\
\text { and personnel (perfor- } \\
\text { mance bias) }\end{array}$ & High risk & $\begin{array}{l}\text { Not possible to blind to the intervention although 1 of the control groups had } \\
\text { all outcomes } \\
\text { received no intervention. }\end{array}$ \\
\hline $\begin{array}{l}\text { Blinding of outcome as- } \\
\text { sessment (detection bias) } \\
\text { All outcomes }\end{array}$ & High risk & \begin{tabular}{l} 
Midwives who delivered the intervention also collected data on outcomes \\
\hline $\begin{array}{l}\text { Incomplete outcome data } \\
\text { (attrition bias) } \\
\text { All outcomes }\end{array}$
\end{tabular} \\
\hline
\end{tabular}


Barrios 2008 PHI (Continued)

Selective reporting (re- High risk Collected data on diarrhoea but no results presented. porting bias)

\begin{tabular}{|c|c|c|}
\hline Other bias & Unclear risk & - \\
\hline $\begin{array}{l}\text { Similarity of baseline out- } \\
\text { come measurements }\end{array}$ & Unclear risk & NA \\
\hline $\begin{array}{l}\text { Similarity of baseline char- } \\
\text { acteristics }\end{array}$ & Unclear risk & NA \\
\hline $\begin{array}{l}\text { Adequate allocation of in- } \\
\text { tervention concealment } \\
\text { during the study }\end{array}$ & Unclear risk & NA \\
\hline $\begin{array}{l}\text { Adequate protection } \\
\text { against contamination }\end{array}$ & Unclear risk & NA \\
\hline $\begin{array}{l}\text { Confounders adequate- } \\
\text { ly adjusted for in analy- } \\
\text { sis/design }\end{array}$ & Unclear risk & NA \\
\hline Recruitment bias & High risk & Participants were recruited once the clusters had been randomly allocated. \\
\hline Baseline imbalance & High risk & Only 3 demographic variables presented. \\
\hline Loss of clusters & Low risk & No mention of loss of barangays. \\
\hline Incorrect analysis & High risk & No adjustments for clustering. \\
\hline
\end{tabular}

Bassal 2016 ISR

\begin{tabular}{|c|c|}
\hline Methods & Case-control study (matched) \\
\hline \multirow[t]{2}{*}{ Participants } & $\begin{array}{l}\text { Cases: children aged } 1-5 \text { years, living in central Israel, having diarrhoea, and a positive stool culture for } \\
\text { Campylobacter. Cases diagnosed in the community and reported to the Israel Center for Disease Con- } \\
\text { trol between August } 2009 \text { and April } 2010 \text { were identified. } n=113 \text {, mean age } 2.5 \text { (SD 1.3), } 40.7 \% \text { girls. }\end{array}$ \\
\hline & $\begin{array}{l}\text { Control: healthy children with no history of diarrhoea } 2 \text { weeks before the interview (each case was } \\
\text { matched by gender, age (SD } 3 \text { months), and neighbourhood (the streets surrounding the case house, in } \\
\text { which a control was available, from Israeli Population Register), } n=113 \text {, mean age } 2.5 \text { (SD 1.3), } 40.7 \% \\
\text { girls. }\end{array}$ \\
\hline \multirow[t]{2}{*}{ Interventions } & Risk factor of interest: \\
\hline & $\begin{array}{l}\text { - children who were not toilet trained and used nappies vs children who were toilet trained and did not } \\
\text { use nappies. }\end{array}$ \\
\hline Outcomes & Campylobacter diarrhoea: diarrhoea with positive stool culture for Campylobacter \\
\hline \multirow[t]{3}{*}{ Notes } & Location: Central Israel (Ashdod to Hadera) \\
\hline & Length of recruitment: August 2009 to April 2010 \\
\hline & Publication status: journal \\
\hline
\end{tabular}


Bassal 2016 ISR (Continued)

Risk of bias

\begin{tabular}{|c|c|c|}
\hline Bias & Authors' judgement & Support for judgement \\
\hline $\begin{array}{l}\text { Random sequence genera- } \\
\text { tion (selection bias) }\end{array}$ & Unclear risk & NA \\
\hline $\begin{array}{l}\text { Allocation concealment } \\
\text { (selection bias) }\end{array}$ & Unclear risk & NA \\
\hline $\begin{array}{l}\text { Blinding of participants } \\
\text { and personnel (perfor- } \\
\text { mance bias) } \\
\text { All outcomes }\end{array}$ & Unclear risk & NA \\
\hline $\begin{array}{l}\text { Blinding of outcome as- } \\
\text { sessment (detection bias) } \\
\text { All outcomes }\end{array}$ & Unclear risk & NA \\
\hline $\begin{array}{l}\text { Incomplete outcome data } \\
\text { (attrition bias) } \\
\text { All outcomes }\end{array}$ & Unclear risk & NA \\
\hline $\begin{array}{l}\text { Selective reporting (re- } \\
\text { porting bias) }\end{array}$ & Unclear risk & NA \\
\hline Other bias & Unclear risk & NA \\
\hline $\begin{array}{l}\text { Similarity of baseline out- } \\
\text { come measurements }\end{array}$ & Unclear risk & NA \\
\hline $\begin{array}{l}\text { Similarity of baseline char- } \\
\text { acteristics }\end{array}$ & Unclear risk & NA \\
\hline $\begin{array}{l}\text { Adequate allocation of in- } \\
\text { tervention concealment } \\
\text { during the study }\end{array}$ & Unclear risk & NA \\
\hline $\begin{array}{l}\text { Adequate protection } \\
\text { against contamination }\end{array}$ & Unclear risk & NA \\
\hline $\begin{array}{l}\text { Confounders adequate- } \\
\text { ly adjusted for in analy- } \\
\text { sis/design }\end{array}$ & Unclear risk & NA \\
\hline Recruitment bias & Unclear risk & NA \\
\hline Baseline imbalance & Unclear risk & NA \\
\hline Loss of clusters & Unclear risk & NA \\
\hline Incorrect analysis & Unclear risk & NA \\
\hline
\end{tabular}


Belizario 2015 PHI

\begin{tabular}{ll}
\hline Methods & Controlled cross-sectional study \\
\hline Participants & Number: 341 respondents \\
& $\begin{array}{l}\text { Inclusion criteria: school-aged children }(6-15 \text { years old) and preschool aged children }(2-5 \text { years old) en- } \\
\text { rolled in public elementary schools and daycare centres and residing in the selected villages }\end{array}$
\end{tabular}

Interventions

Intervention (150 respondents): 2 villages with CLTS + mass drug administration. Key messages delivered by the community leaders and volunteers to HHs included:

- the shame of having open defecation in the village and the importance of attaining ODF status in the village;

- the importance for each $\mathrm{HH}$ to possess its own sanitary toilet; and

- the need for HHs to ensure solid waste management and disposal, as well as maintain sanitary conditions in animal facilities in the backyard (e.g. pig pens).

The criteria for declaring ODF status included the following:

- no signs of open defecation observed during transect walks and $\mathrm{HH}$ visits;

- $100 \%$ of HHs possessed sanitary toilets;

- enactment of local legislation at the village level supporting CLTS activities; and

- implementation of other local government activities that supported the maintenance of ODF status (e.g. village "clean and green" programme).

Messages about child faeces disposal and use of toilets by children were included during the CLTS activities in the villages.

Control (191 respondents): 2 villages with no CLTS + mass drug administration

STH prevalence (stool samples were processed using the Kato-Katz technique)
STH intensity
Prevalence of Ascaris
Intensity of Ascaris
Prevalence of Trichuris
Intensity of Trichuris
Prevalence of hookworm
Intensity of hookworm
Weight for age
Height for age
BMI for age (10-15 years old)
Haemoglobin status (anaemia)

Notes Location: 4 villages in the province of Southern Leyte in Eastern Province, Philippines

Length of study: 1 month (August 2013)

Publication status: journal

\section{Risk of bias}


Belizario 2015 PHI (Continued)

Random sequence genera- High risk No random allocation to intervention/control.
tion (selection bias)

$\begin{aligned} & \text { Allocation concealment } \\ & \text { (selection bias) }\end{aligned}$
High risk No allocation concealment/NA to the study design.

(selection bias)

Blinding of participants - Unclear risk
and personnel (perfor-
mance bias)
All outcomes

Blinding of outcome as-
sessment (detection bias)

\begin{tabular}{|c|c|c|}
\hline $\begin{array}{l}\text { Incomplete outcome data } \\
\text { (attrition bias) } \\
\text { All outcomes }\end{array}$ & Low risk & $\begin{array}{l}\text { Proportion of missing data was similar across intervention (9.6\%) and control } \\
\text { villages }(11.6 \%) \text {. }\end{array}$ \\
\hline
\end{tabular}

\begin{tabular}{lll}
\hline $\begin{array}{l}\text { Selective reporting (re- } \\
\text { porting bias) }\end{array}$ & Low risk & All important outcomes specified in methods were reported on. \\
\hline Other bias & High risk & $\begin{array}{l}\text { No adjustments for clustering. Did not measure use of sanitation facilities at } \\
\text { the time of the study. }\end{array}$ \\
\hline $\begin{array}{l}\text { Similarity of baseline out- } \\
\text { come measurements }\end{array}$ & High risk & NA, not relevant to design. \\
\hline
\end{tabular}

Similarity of baseline char- High risk NA, not relevant to design.

acteristics

Adequate allocation of in- Low risk Outcome measures were objective (STH in stool/anthropometry).
tervention concealment
during the study

\begin{tabular}{lll}
\hline $\begin{array}{l}\text { Adequate protection } \\
\text { against contamination }\end{array}$ & Low risk & CLTS and non-CLTS villages were far from one another. \\
\hline $\begin{array}{l}\text { Confounders adequate- } \\
\text { ly adjusted for in analy- } \\
\text { sis/design }\end{array}$ & High risk & $\begin{array}{l}\text { No analysis adjusting of confounders. They said the villages were selected to } \\
\text { be similar (in number of school-aged children and preschool-aged children, } \\
\text { number of HHs, presence of elementary schools and daycare centres, sources } \\
\text { of livelihood, security, accessibility, and willingness of community leaders to } \\
\text { collaborate) }\end{array}$ \\
\hline
\end{tabular}

\begin{tabular}{lll}
\hline Recruitment bias & Unclear risk & - \\
\hline Baseline imbalance & Unclear risk & - \\
\hline Loss of clusters & Unclear risk & - \\
\hline Incorrect analysis & Unclear risk & - \\
\hline
\end{tabular}

\section{Berhe 2014 ETH}

Methods Controlled cross-sectional study


Berhe 2014 ETH (Continued)

Participants Number: $650 \mathrm{HHs}$ (866 children aged $<5$ years) (model HHs had 1\% non-response)

Inclusion criteria: HHs that had $\geq 1$ child aged $<5$ years in 12 gotts. For model families (intervention): $\mathrm{HHs}$ that fully implemented the HEP. For non-model families (control): HHs that did not fully implement the HEP.

Interventions

Intervention (327 respondents): HHs who had implemented the HEP packages fully. The HEP was implemented by full-time female health extension workers, who trained HHs to implement packages. The packages included interventions in 4 main categories: family health services, infectious disease prevention and control, hygiene and environmental sanitation, and health education and communication. The maternal and child health package (in the family health services category) includes safe child stool disposal (the stool should be cleaned and disposed in a pit latrine, or shall be covered with a leaf or paper and be buried) (HEP 2003).

Control (323 respondents): non-model families

\begin{tabular}{ll}
\hline Outcomes & $\begin{array}{l}\text { 2-week diarrhoea prevalence (having diarrhoea in the } 2 \text { weeks prior to the interview, no additional de- } \\
\text { tails on case definition) } \\
\text { WASH and nutritional behaviours including child stool disposal method }\end{array}$ \\
\hline Notes & Location: 12 gotts, Tula subcity, Ethiopia \\
& Length of study: 1 month (January 2012) \\
& Publication status: journal
\end{tabular}

\section{Risk of bias}

\begin{tabular}{|c|c|c|}
\hline Bias & Authors' judgement & Support for judgement \\
\hline $\begin{array}{l}\text { Random sequence genera- } \\
\text { tion (selection bias) }\end{array}$ & High risk & Non-random allocation to model or non-model HHs. \\
\hline $\begin{array}{l}\text { Allocation concealment } \\
\text { (selection bias) }\end{array}$ & High risk & Non-random allocation to model or non-model HHs. \\
\hline $\begin{array}{l}\text { Blinding of participants } \\
\text { and personnel (perfor- } \\
\text { mance bias) } \\
\text { All outcomes }\end{array}$ & Unclear risk & NA \\
\hline $\begin{array}{l}\text { Blinding of outcome as- } \\
\text { sessment (detection bias) } \\
\text { All outcomes }\end{array}$ & Unclear risk & NA \\
\hline $\begin{array}{l}\text { Incomplete outcome data } \\
\text { (attrition bias) } \\
\text { All outcomes }\end{array}$ & Low risk & $99 \%$ response rate in model $\mathrm{HHs}$ and $100 \%$ in non-model $\mathrm{HHs}$. \\
\hline $\begin{array}{l}\text { Selective reporting (re- } \\
\text { porting bias) }\end{array}$ & Low risk & Report on main outcomes specified in methods. \\
\hline Other bias & Unclear risk & - \\
\hline $\begin{array}{l}\text { Similarity of baseline out- } \\
\text { come measurements }\end{array}$ & Unclear risk & NA, no baseline \\
\hline
\end{tabular}


Berhe 2014 ETH (Continued)

Similarity of baseline char- Unclear risk NA, no baseline. acteristics

Adequate allocation of in- Low risk tervention concealment during the study

$\begin{array}{ll}\text { Adequate protection } & \text { High risk } \\ \text { against contamination } & \end{array}$

Quote: "Data collectors were blinded regarding whether each $\mathrm{HH}$ was model or non-model in order to reduce interviewer bias."

Quote: "The absence of clear demarcation between model and non-model with reference to distance (closeness of model and non-model) may have created information contamination as well as diarrhoeal disease transmission to the model $\mathrm{HH}$ members and vice versa."

Confounders adequate- Low risk Multivariate analysis.

ly adjusted for in analysis/design

\begin{tabular}{lll}
\hline Recruitment bias & Unclear risk & NA \\
\hline Baseline imbalance & Unclear risk & NA \\
\hline Loss of clusters & Unclear risk & NA \\
\hline Incorrect analysis & Unclear risk & NA \\
\hline
\end{tabular}

\section{Briceño 2015 TAN}

\begin{tabular}{ll}
\hline Methods & Cluster RCT (factorial design) \\
\hline Participants & Number: $3619 \mathrm{HHs}$ ( 5768 children aged < 5 years) $(97.2 \%$ response rate) \\
& $\begin{array}{l}\text { Inclusion criteria: } \mathrm{HH} \text { was present during the period of listing; had been living in the village since the } \\
\text { beginning of } 2009 \text { or earlier; and had } \geq 1 \text { child under the age of } 5 \text { years. }\end{array}$
\end{tabular}

Interventions Interventions: 3 arms (TSSM only, hand-washing promotion only, combined TSSM and HWWS)

- TSSM (43 wards): uses CLTS (triggering of community to increase demand for improved sanitation and promote ODF communities) and sanitation marketing to increase demand for improved sanitation. Also strengthens the supply of sanitation goods and services to local markets to make these products more affordable and accessible. Sanitation marketing messages concentrated on positive aspirational messages rather than shame tactics. No subsidies were used.

- TSSM and HWWS (47 wards): TSSM intervention + provision of intensive social marketing interventions and technical assistance to build handwashing stations with local materials (tippy tap).

Control (46 wards): no intervention

Outcomes Access to an improved latrine and open defecation practice

Caregiver handwashing practices

Diarrhoea (7- and 14-day recall): $\geq 3$ loose/watery stools in a 24-hour period or having a stool with blood or mucous

Anaemia

Anthropometry (weight for age, height for age, weight for height, head circumference)

Abrasions, bruising, scrapes 
Briceño 2015 TAN (Continued)

Notes

Location: 181 rural wards, in 10 districts, Tanzania

Length of study: 46 months (February 2009 to December 2012)

Publication status: report

\section{Risk of bias}

\begin{tabular}{|c|c|c|}
\hline Bias & Authors' judgement & Support for judgement \\
\hline $\begin{array}{l}\text { Random sequence genera- } \\
\text { tion (selection bias) }\end{array}$ & Unclear risk & No details apart from "randomly assigned." \\
\hline $\begin{array}{l}\text { Allocation concealment } \\
\text { (selection bias) }\end{array}$ & Unclear risk & No details \\
\hline $\begin{array}{l}\text { Blinding of participants } \\
\text { and personnel (perfor- } \\
\text { mance bias) } \\
\text { All outcomes }\end{array}$ & High risk & No blinding \\
\hline $\begin{array}{l}\text { Blinding of outcome as- } \\
\text { sessment (detection bias) } \\
\text { All outcomes }\end{array}$ & High risk & $\begin{array}{l}\text { Quote: "Ensured interviewers were blinded to the intervention status of each } \\
\text { village." } \\
\text { Comment: however, not possible to completely blind. }\end{array}$ \\
\hline $\begin{array}{l}\text { Incomplete outcome data } \\
\text { (attrition bias) } \\
\text { All outcomes }\end{array}$ & Low risk & $\begin{array}{l}\text { Quote: "3,619 completed interviews from 3,724 attempted ( } 97.2 \% \text { response } \\
\text { rate)." }\end{array}$ \\
\hline $\begin{array}{l}\text { Selective reporting (re- } \\
\text { porting bias) }\end{array}$ & Low risk & Report on prespecified outcomes in methods. \\
\hline Other bias & Unclear risk & - \\
\hline $\begin{array}{l}\text { Similarity of baseline out- } \\
\text { come measurements }\end{array}$ & Unclear risk & NA \\
\hline
\end{tabular}

Similarity of baseline char- Unclear risk NA

acteristics

\begin{tabular}{lll}
\hline $\begin{array}{l}\text { Adequate allocation of in- } \\
\text { tervention concealment } \\
\text { during the study }\end{array}$ & Unclear risk & NA \\
\hline $\begin{array}{l}\text { Adequate protection } \\
\text { against contamination }\end{array}$ & Unclear risk & $N A$ \\
\hline $\begin{array}{l}\text { Confounders adequate- } \\
\text { ly adjusted for in analy- } \\
\text { sis/design }\end{array}$ & Unclear risk & $N A$ \\
\end{tabular}

\begin{tabular}{lll}
\hline Recruitment bias & High risk & $\begin{array}{l}\text { Recruited participants after their villages had received intervention/not (no } \\
\text { baseline). }\end{array}$ \\
\hline Baseline imbalance & High risk & No baseline \\
\hline Loss of clusters & Low risk & 9 wards $(<10 \%)$ were reassigned and lost after they were randomized. \\
\hline
\end{tabular}


Briceño 2015 TAN (Continued)

Incorrect analysis Low risk Q Quote: "Standard errors are clustered at the ward level."

Butz 1990 USA

\begin{tabular}{|c|c|}
\hline Methods & Cluster RCT \\
\hline \multirow[t]{3}{*}{ Participants } & Number: 114 children (aged 1 month to 7 years) attending 24 FDCHs \\
\hline & Inclusion criteria: all children attending FDCHs \\
\hline & $\begin{array}{l}\text { Intervention group: } 69 \% \text { aged } \leq 36 \text { months and } 57 \% \text { girls; control group: } 62 \% \text { aged } \leq 36 \text { months and } \\
42 \% \text { girls. }\end{array}$ \\
\hline \multirow[t]{2}{*}{ Interventions } & $\begin{array}{l}\text { Intervention ( } 12 \mathrm{FDCHs} \text { ): instruction to daycare providers on modes of transmission of pathogens, in- } \\
\text { structions of handwashing, use of vinyl gloves and disposable nappy changing pads at each nappy } \\
\text { change. Providers were instructed to dispose of gloves, disposable pads, and nappies in plastic bags } \\
\text { and given supplies (gloves, nappy changing pads, hand rinse solution). }\end{array}$ \\
\hline & Control (12 FDCHs): no education but received biweekly nurse visits for symptom data collection. \\
\hline \multirow[t]{3}{*}{ Outcomes } & $\begin{array}{l}\text { Diarrhoea longitudinal prevalence (diarrhoea symptom days/childcare days). Diarrhoea: occurrence } \\
\text { of loose, unformed bowel movements at twice the normal frequency (infants: } 1-2 \text { stools per day; older } \\
\text { children: } 1 \text { stool per day). Symptoms recorded daily }\end{array}$ \\
\hline & Longitudinal prevalence of vomiting and runny nose \\
\hline & Absence from daycare home (reasons for absenteeism not recorded) \\
\hline \multirow[t]{3}{*}{ Notes } & Location: 24 FDCHs in urban Baltimore, USA \\
\hline & Length of study: 12 months (4 January 1988 to 31 December 1988) \\
\hline & Publication status: journal \\
\hline
\end{tabular}

\section{Risk of bias}

\begin{tabular}{|c|c|c|}
\hline Bias & Authors' judgement & Support for judgement \\
\hline $\begin{array}{l}\text { Random sequence genera- } \\
\text { tion (selection bias) }\end{array}$ & Unclear risk & Quote: "FDCHs were randomly assigned to control or intervention group." \\
\hline $\begin{array}{l}\text { Allocation concealment } \\
\text { (selection bias) }\end{array}$ & Unclear risk & No details \\
\hline $\begin{array}{l}\text { Blinding of participants } \\
\text { and personnel (perfor- } \\
\text { mance bias) } \\
\text { All outcomes }\end{array}$ & High risk & $\begin{array}{l}\text { Daycare providers were aware that the intervention programme was being } \\
\text { tested in certain homes. }\end{array}$ \\
\hline $\begin{array}{l}\text { Blinding of outcome as- } \\
\text { sessment (detection bias) } \\
\text { All outcomes }\end{array}$ & High risk & $\begin{array}{l}\text { Quote: "Daycare providers recorded the symptoms." } \\
\text { Comment: daycare providers not blinded. }\end{array}$ \\
\hline $\begin{array}{l}\text { Incomplete outcome data } \\
\text { (attrition bias) } \\
\text { All outcomes }\end{array}$ & Unclear risk & $\begin{array}{l}10.6 \% \text { of missing/absent days excluded in analysis, with no information on } \\
\text { whether they were from intervention or control FDCHs. }\end{array}$ \\
\hline
\end{tabular}


Butz 1990 USA (Continued)

Selective reporting (re- Low risk $\quad$ Reported main outcomes.
porting bias)

\begin{tabular}{|c|c|c|}
\hline Other bias & Unclear risk & - \\
\hline $\begin{array}{l}\text { Similarity of baseline out- } \\
\text { come measurements }\end{array}$ & Unclear risk & NA \\
\hline $\begin{array}{l}\text { Similarity of baseline char- } \\
\text { acteristics }\end{array}$ & Unclear risk & NA \\
\hline $\begin{array}{l}\text { Adequate allocation of in- } \\
\text { tervention concealment } \\
\text { during the study }\end{array}$ & Unclear risk & NA \\
\hline $\begin{array}{l}\text { Adequate protection } \\
\text { against contamination }\end{array}$ & Unclear risk & NA \\
\hline $\begin{array}{l}\text { Confounders adequate- } \\
\text { ly adjusted for in analy- } \\
\text { sis/design }\end{array}$ & Unclear risk & NA \\
\hline Recruitment bias & High risk & Staff were aware of which cluster were intervention and control. \\
\hline Baseline imbalance & Low risk & No significant baseline imbalances. \\
\hline Loss of clusters & Low risk & Only 2 clusters lost $(1$ control and 1 intervention $)=8.3 \%$. \\
\hline Incorrect analysis & High risk & Not adjusted for clustering in analyses. \\
\hline
\end{tabular}

Cameron 2013 INA

\begin{tabular}{ll}
\hline Methods & Cluster RCT \\
\hline Participants & $\begin{array}{l}\text { Number: } 2500 \mathrm{HH} \text { at end of study } \\
\text { Inclusion criteria: HHs with children aged }<2 \text { years (and HH with children aged }<5 \text { years where too few } \\
\text { HH with aged }<2 \text { years found) }\end{array}$ \\
\hline $\begin{array}{l}\text { Intervention ( } 80 \text { subvillages): TSSM which included CLTS to stop open defecation, social sanitation mar- } \\
\text { keting to increase availability of products and services and strengthening the enabling environment at } \\
\text { policy and institutional levels. } \\
\text { Control (80 subvillages): no intervention }\end{array}$ \\
\hline
\end{tabular}

\title{
Outcomes
}

\author{
Changes in perceptions of consequences of poor sanitation \\ Sanitation improvements (toilet construction and access to improved sanitation) \\ Open defecation practices
}

Diarrhoea prevalence (2-, 7-, or 14-day recall): $\geq 3$ stools per day and the stools were loose or watery, or blood or mucous (or both) visible in stool

Symptoms: nausea, vomiting, water or soft stools, mucous or blood in stool, refusal to eat, bruising, abrasion, itchy skin or scalp 
Intestinal parasite infections (Ascaris, Trichuris, hookworm infections)

Anthropometry (stunting and wasting)

Iron-deficiency anaemia

Cognitive and motor development (communication skills, mobility skills, and social-personal skills for age)

\section{Water source}

Handwashing practices

ARIS

\begin{tabular}{|c|c|}
\hline \multirow[t]{3}{*}{ Notes } & Location: 160 rural subvillages, East Java, Indonesia \\
\hline & Length of study: 30 months (August 2008 to February 2011) \\
\hline & Publication status: report \\
\hline
\end{tabular}

\section{Risk of bias}

\begin{tabular}{lll}
\hline Bias & Authors' judgement & Support for judgement \\
\hline $\begin{array}{l}\text { Random sequence genera- } \\
\text { tion (selection bias) }\end{array}$ & Low risk & $\begin{array}{l}\text { Quote: "Using a random number generator in STATA, the IE [impact evalua- } \\
\text { tion] team randomly selected } 10 \text { treatment and } 10 \text { control villages in each dis- } \\
\text { trict." }\end{array}$ \\
\hline $\begin{array}{l}\text { Allocation concealment } \\
\text { (selection bias) }\end{array}$ & Low risk & $\begin{array}{l}\text { Quote: "Once the IE team received the sub-village lists from the district offices } \\
\text { for all } 20 \text { villages, they told district offices which villages were in the treatment } \\
\text { group and which were in the control group." }\end{array}$ \\
\hline $\begin{array}{l}\text { Blinding of participants } \\
\text { and personnel (perfor- } \\
\text { mance bias) }\end{array}$ & High risk & No blinding. \\
All outcomes &
\end{tabular}

Blinding of outcome as-

High risk

No blinding.

sessment (detection bias)

All outcomes

\begin{tabular}{|c|c|c|}
\hline $\begin{array}{l}\text { Incomplete outcome data } \\
\text { (attrition bias) }\end{array}$ & Low risk & $\begin{array}{l}\text { Quote: " } 179 \text { could not be contacted ( } 86 \text { households in the control group and } \\
93 \text { households in the treatment group)" ( } 8.5 \% \text { LTFU). }\end{array}$ \\
\hline
\end{tabular}

All outcomes

Selective reporting (re- Low risk $\quad$ Reported all outcomes specified in methods.
porting bias)

\begin{tabular}{lll}
\hline Other bias & Unclear risk & - \\
\hline $\begin{array}{l}\text { Similarity of baseline out- } \\
\text { come measurements }\end{array}$ & Unclear risk & NA \\
\hline $\begin{array}{l}\text { Similarity of baseline char- } \\
\text { acteristics }\end{array}$ & Unclear risk & NA \\
\hline
\end{tabular}

Adequate allocation of in- Unclear risk NA
tervention concealment
during the study


Cameron 2013 INA (Continued)

\begin{tabular}{lll}
$\begin{array}{l}\text { Adequate protection } \\
\text { against contamination }\end{array}$ & Unclear risk & NA \\
\hline $\begin{array}{l}\text { Confounders adequate- } \\
\text { ly adjusted for in analy- } \\
\text { sis/design }\end{array}$ & Unclear risk & NA \\
\hline
\end{tabular}

\begin{tabular}{lll}
\hline Recruitment bias & High risk & $\begin{array}{l}\text { Seemed the baseline data collection occurred after assignment to intervention } \\
\text { and control. }\end{array}$ \\
\hline Baseline imbalance & Low risk & $\begin{array}{l}\text { Quotes: "For the key outcome variables (household water and sanitation con- } \\
\text { dition, as well as children's health variables), balance is achieved." "demo- } \\
\text { graphic and socio-economic characteristics are also similar across treatment } \\
\text { and control groups." }\end{array}$ \\
\hline Loss of clusters & Low risk & No loss of clusters reported. \\
\hline Incorrect analysis & Low risk & In multivariate analysis adjust for clustering. \\
\hline
\end{tabular}

Caruso 2019 IND

\begin{tabular}{ll}
\hline Methods & Cluster RCT \\
\hline Participants & Number: HHs in 66 villages (406 HHs with children aged $<5$ years) \\
& Inclusion criteria: villages that had not been declared ODF by the Government of India, had $50-150$ \\
& HHs, and minimum $60 \%$ latrine coverage. \\
& In selected villages, all HHs that owned latrines (regardless of functionality) were eligible for inclusion.
\end{tabular}

Interventions Intervention (33 villages): "Sundara Grama," a multilevel behaviour change intervention that included the following activities.

- Community-level activities:

* a Palla, a folk dance performance common in Odisha, that communicated messages about latrine use, health, child faeces disposal, and the importance of overall village cleanliness;

* a transect walk that went around the village and marked piles of faeces with coloured powder;

* a community meeting to discuss the village state and create a plan for its cleanliness;

* the recognition of $\mathrm{HH}$ s whose members all used the latrine all the time, with a banner hung in front of their house;

* a village map painting of all HHs, with special recognition of those using the latrines at all times and a description of the community action plan decided in the meeting.

- HH-level activities:

* a targeted visit for latrine owners, reiterated messages from the other activities, and elicited commitment from the $\mathrm{HH}$ members to use the latrine to keep the village clean and beautiful;

* latrine repairs were carried out to provide minor repairs to those latrines that were not functional and to doors to all latrines that did not have one or had one that was broken.

- A mother's group meeting for mothers and caregivers of children aged $<5$ years, regardless of their $\mathrm{HH}$ latrine status to provide action knowledge and hardware to enable the safe disposal of child faeces.

Control (33 villages): no intervention

Primary outcome:
- latrine use, including use for the safe disposal of child faeces
Secondary outcomes:


Caruso 2019 IND (Continued)

- latrine coverage

- determinants of latrine use and child faeces disposal

\begin{tabular}{|c|c|}
\hline \multirow[t]{3}{*}{ Notes } & Location: 66 villages in Puri district, Odisha, India \\
\hline & Length of study: trial: 14 months (October 2017 to March 2019) \\
\hline & Publication status: unpublished report \\
\hline
\end{tabular}

\section{Risk of bias}

\begin{tabular}{|c|c|c|}
\hline Bias & Authors' judgement & Support for judgement \\
\hline $\begin{array}{l}\text { Random sequence genera- } \\
\text { tion (selection bias) }\end{array}$ & Low risk & $\begin{array}{l}\text { Quote: "Village allocation was performed by a study investigator using a com- } \\
\text { puter-generated randomization sequence generated in Stata v.14." }\end{array}$ \\
\hline $\begin{array}{l}\text { Allocation concealment } \\
\text { (selection bias) }\end{array}$ & Low risk & $\begin{array}{l}\text { Randomization was done using a computer-generated randomization se- } \\
\text { quence at the start of the study. }\end{array}$ \\
\hline $\begin{array}{l}\text { Blinding of participants } \\
\text { and personnel (perfor- } \\
\text { mance bias) } \\
\text { All outcomes }\end{array}$ & High risk & $\begin{array}{l}\text { Quote: "Due to the nature of the intervention, neither participants nor study } \\
\text { investigators will be blinded to treatment assignment." }\end{array}$ \\
\hline $\begin{array}{l}\text { Blinding of outcome as- } \\
\text { sessment (detection bias) } \\
\text { All outcomes }\end{array}$ & High risk & $\begin{array}{l}\text { Quote: "Due to the nature of the intervention, neither participants nor study } \\
\text { investigators will be blinded to treatment assignment." }\end{array}$ \\
\hline $\begin{array}{l}\text { Incomplete outcome data } \\
\text { (attrition bias) } \\
\text { All outcomes }\end{array}$ & Unclear risk & Only preliminary unpublished data thus far. \\
\hline $\begin{array}{l}\text { Selective reporting (re- } \\
\text { porting bias) }\end{array}$ & Low risk & Reported on main outcomes. \\
\hline Other bias & Unclear risk & - \\
\hline $\begin{array}{l}\text { Similarity of baseline out- } \\
\text { come measurements }\end{array}$ & Unclear risk & - \\
\hline $\begin{array}{l}\text { Similarity of baseline char- } \\
\text { acteristics }\end{array}$ & Unclear risk & - \\
\hline $\begin{array}{l}\text { Adequate allocation of in- } \\
\text { tervention concealment } \\
\text { during the study }\end{array}$ & Unclear risk & - \\
\hline $\begin{array}{l}\text { Adequate protection } \\
\text { against contamination }\end{array}$ & Unclear risk & - \\
\hline $\begin{array}{l}\text { Confounders adequate- } \\
\text { ly adjusted for in analy- } \\
\text { sis/design }\end{array}$ & Unclear risk & - \\
\hline
\end{tabular}

\begin{tabular}{|c|c|c|}
\hline Recruitment bias & High risk & $\begin{array}{l}\text { Randomization was conducted prior to baseline. However, all eligible partici- } \\
\text { pants were recruited so unlikely to have affected recruitment. }\end{array}$ \\
\hline
\end{tabular}

Baseline imbalance Low risk No baseline imbalance.


Caruso 2019 IND (Continued)

\begin{tabular}{lll} 
Loss of clusters & Low risk & No loss of clusters. \\
\hline Incorrect analysis & Low risk & Analysis accounted for clustering.
\end{tabular}

\section{Chiang 2005 TWN}

\begin{tabular}{ll}
\hline Methods & Case-control study \\
\hline Participants & $\begin{array}{l}\text { Cases: children aged }<5 \text { years in Hualian County with shigellosis (confirmed by laboratory test) from } \\
\text { hospitals and clinics. } n=46,50 \% \text { girls } \\
\text { Controls: children aged }<5 \text { years who visited the same hospitals/clinics } \pm 10 \text { days of the cases, for vac- } \\
\text { cination (excluding those with diarrhoea symptoms or fever within } 10 \text { days of house visit/survey), } \\
\text { matched for age group (0-1, 1-3, 3-5 years). } n=92,41.3 \% \text { girls }\end{array}$ \\
\hline Interventions & Risk factor of interest: \\
\hline Outcomes & open defecation of children (no definition of comparison). \\
\hline Shigella: symptoms of diarrhoea, abdominal pain, fever, nausea, mucous stool, tenesmus etc, and test- \\
ed positive for Group B or D Shigella
\end{tabular}

\section{Risk of bias}

\begin{tabular}{lll}
\hline Bias & Authors' judgement & Support for judgement \\
\hline $\begin{array}{l}\text { Random sequence genera- } \\
\text { tion (selection bias) }\end{array}$ & Unclear risk & NA \\
\hline $\begin{array}{l}\text { Allocation concealment } \\
\text { (selection bias) }\end{array}$ & Unclear risk & NA \\
\hline $\begin{array}{l}\text { Blinding of participants } \\
\text { and personnel (perfor- } \\
\text { mance bias) } \\
\text { All outcomes }\end{array}$ & Unclear risk & NA \\
\hline $\begin{array}{l}\text { Blinding of outcome as- } \\
\text { sessment (detection bias) } \\
\text { All outcomes }\end{array}$ & Unclear risk & NA \\
\hline $\begin{array}{l}\text { Incomplete outcome data } \\
\text { (attrition bias) } \\
\text { All outcomes }\end{array}$ & Unclear risk & NA \\
\hline $\begin{array}{l}\text { Selective reporting (re- } \\
\text { porting bias) }\end{array}$ & Unclear risk & NA \\
\hline \begin{tabular}{l} 
Other bias \\
\hline
\end{tabular} & Unclear risk & NA \\
\hline
\end{tabular}


Chiang 2005 TWN (Continued)

\begin{tabular}{lll}
$\begin{array}{l}\text { Similarity of baseline out- } \\
\text { come measurements }\end{array}$ & Unclear risk & NA \\
\hline $\begin{array}{l}\text { Similarity of baseline char- } \\
\text { acteristics }\end{array}$ & Unclear risk & NA \\
\hline $\begin{array}{l}\text { Adequate allocation of in- } \\
\text { tervention concealment } \\
\text { during the study }\end{array}$ & Unclear risk & NA \\
\hline $\begin{array}{l}\text { Adequate protection } \\
\text { against contamination }\end{array}$ & Unclear risk & NA \\
\hline $\begin{array}{l}\text { Confounders adequate- } \\
\text { ly adjusted for in analy- } \\
\text { sis/design }\end{array}$ & Unclear risk & \\
\hline $\begin{array}{l}\text { Recruitment bias } \\
\text { Baseline imbalance }\end{array}$ & Unclear risk & NA \\
\hline $\begin{array}{l}\text { Loss of clusters } \\
\text { Incorrect analysis }\end{array}$ & Unclear risk & NA \\
\hline
\end{tabular}

\section{Chompook 2006 THA}

\begin{tabular}{ll}
\hline Methods & Case-control study (matched) \\
\hline Participants & $\begin{array}{l}\text { Cases: attended health facility with diarrhoea and shigella isolated from rectal swab, } \mathrm{n}=139 \text { (after } 53 \\
\text { not enrolled: not resident, not found, moved away, died, or time-constraints), median age } 5 \text { years, } 57 \% \\
\text { girls. } \\
\text { Controls: individuals free from diarrhoea or dysentery during the } 4 \text { weeks prior to recruitment, } \\
\text { matched for sex and age with the cases (within } 3 \text { months for children aged }<2 \text { years; within } 6 \text { months } \\
\text { for children aged }<5 \text { years; within } 12 \text { months for children aged }<16 \text { years old; and within } 5 \text { years for } \\
\text { people aged } \geq 16 \text { years), randomly selected from the population list of the HC where the case resided. } \mathrm{n} \\
=264 \text { (after } 7 \text { moved and } 2 \text { refused), median age } 5 \text { years, 58\% girls. }\end{array}$ \\
\hline Risk factors of interest: \\
- children not/sometimes using latrine vs always using latrine; \\
child excreta disposal method (no data presented).
\end{tabular}

\section{Risk of bias}

Bias Authors' judgement Support for judgement

Interventions to improve disposal of child faeces for preventing diarrhoea and soil-transmitted helminth infection (Review) 
Chompook 2006 THA (Continued)

\begin{tabular}{lll}
$\begin{array}{l}\text { Random sequence genera- } \\
\text { tion (selection bias) }\end{array}$ & Unclear risk & NA \\
\hline $\begin{array}{l}\text { Allocation concealment } \\
\text { (selection bias) }\end{array}$ & Unclear risk & NA \\
\hline $\begin{array}{l}\text { Blinding of participants } \\
\text { and personnel (perfor- } \\
\text { mance bias) }\end{array}$ & Unclear risk & NA \\
All outcomes & & \\
\hline
\end{tabular}

Blinding of outcome as-
sessment (detection bias) $\quad$ Unclear risk $\quad$ NA

All outcomes

\begin{tabular}{ll}
\hline $\begin{array}{l}\text { Incomplete outcome data } \\
\text { (attrition bias) }\end{array}$ & Unclear risk
\end{tabular}

All outcomes

\begin{tabular}{|c|c|c|}
\hline $\begin{array}{l}\text { Selective reporting (re- } \\
\text { porting bias) }\end{array}$ & Unclear risk & NA \\
\hline Other bias & Unclear risk & NA \\
\hline $\begin{array}{l}\text { Similarity of baseline out- } \\
\text { come measurements }\end{array}$ & Unclear risk & NA \\
\hline $\begin{array}{l}\text { Similarity of baseline char- } \\
\text { acteristics }\end{array}$ & Unclear risk & NA \\
\hline $\begin{array}{l}\text { Adequate allocation of in- } \\
\text { tervention concealment } \\
\text { during the study }\end{array}$ & Unclear risk & NA \\
\hline $\begin{array}{l}\text { Adequate protection } \\
\text { against contamination }\end{array}$ & Unclear risk & NA \\
\hline $\begin{array}{l}\text { Confounders adequate- } \\
\text { ly adjusted for in analy- } \\
\text { sis/design }\end{array}$ & Unclear risk & NA \\
\hline Recruitment bias & Unclear risk & NA \\
\hline Baseline imbalance & Unclear risk & NA \\
\hline Loss of clusters & Unclear risk & NA \\
\hline Incorrect analysis & Unclear risk & NA \\
\hline
\end{tabular}

\section{Christensen 2015a KEN}

\begin{tabular}{ll}
\hline Methods & Cluster RCT \\
\hline Participants & Number: 113 HHs at end of study (after 14.4\% LTFU from baseline)
\end{tabular}


Christensen 2015a KEN (Continued) Inclusion criteria: pregnant women in their second/third trimester and caregivers of children aged $<3$ months

Interventions
Interventions ( 3 arms)

- Water (baseline: $38 \mathrm{HHs}$, end of study: $36 \mathrm{HHs}, 9$ villages): installing chlorine dispensers at respondents' reported water sources within the village (usually a protected spring, well, or other source of groundwater) and behaviour change messaging focused on treatment of drinking water with chlorine at all times and storage in a covered container and emphasis of convenience of use at the point of collection and the prevention of recontamination by chlorination.

- Sanitation (baseline: $31 \mathrm{HHs}$, end of study: $25 \mathrm{HHs}, 8$ villages) (arm relevant to this review and used in analysis): Hardware: sanitation compounds received a faeces disposal sani-scooper tool similar to a dustpan with a metal paddle (1 for each $\mathrm{HH}$ in the compound, cost approximately USD 2.25), a plastic child's potty ( 1 for each $\mathrm{HH}$ in the compound with a child aged $<3$ years, cost approximately USD 1.07), and improvements to their existing latrine (consisting of a plastic latrine slab with a builtin drop-hole cover if the latrine floor was not concrete and simple mud walls, roof, and door if not present) or construction of a new latrine if they had none (which cost approximately USD 21.88 for the slab and up to approximately USD 237.50 for a new latrine). In addition there were monthly HH visits for behaviour change communication, including: songs, interactive games, and visual aids (calendars, cue cards, picture sheets). The sanitation intervention's primary behaviour change messages emphasized preventing faecal contamination of the environment and safe removal of faeces (human and animal) from the environment facilitated by the potty, sani-scooper, and latrine. The sanitation behaviour change messages also focused on contamination pathways, behaviours that could lead to exposure, and motivators and barriers of the targeted behaviours.

- Hygiene: (baseline: $33 \mathrm{HHs}$, end of study: $24 \mathrm{HHs}$, 8 villages): $\mathrm{HHs}$ received 2 locally manufactured dual tippy-tap handwashing stations ( 2 separate pedal-controlled jugs: 1 with soapy water and 1 with plain water): 1 for near their latrine and 1 for their cooking area, and behaviour change messaging emphasized HWWS at critical times defined as after faecal contact (e.g. after defecation and after cleaning a child who has defecated) and before handling food (e.g. before preparing food, eating, or feeding a child).

Control (baseline: $30 \mathrm{HHs}$, end of study: $24 \mathrm{HHs}, 9$ villages): no intervention

Uptake of interventions:

- presence of total and free chlorine in water

- use of the chlorine dispenser

- what the respondent had done, if anything, to dispose of the most recent child defecation (all children aged $<3$ years in $\mathrm{HH}$ ). Appropriate disposal: the child defecated directly in the latrine, or the child defecated in a nappy or potty and the parent immediately dumped the faeces into the latrine.

- observation of faeces in compound

- use of the sani-scooper

- handwashing frequency

- observed cleanliness of hands

Location: 34 rural villages near Bungoma, Western Kenya

Length of study: 6 months (November 2011 to May 2012)

Publication status: journal

\section{Risk of bias}

Bias Authors' judgement Support for judgement

Random sequence genera- Low risk tion (selection bias)
Each village was assigned a randomly generated number using Stata, and intervention assignments were made to villages in ascending numerical order. 
Christensen 2015a KEN (Continued)

Allocation concealment Low risk $\quad$ Centrally allocated.
(selection bias)

Blinding of participants High risk No blinding.
and personnel (perfor-
mance bias)
All outcomes

Blinding of outcome as- High risk No blinding and some of the outcomes were self-reported.

sessment (detection bias)

All outcomes

\begin{tabular}{lll}
\hline $\begin{array}{l}\text { Incomplete outcome data } \\
\text { (attrition bias) } \\
\text { All outcomes }\end{array}$ & High risk & LTFU was high and different across arms. \\
\hline $\begin{array}{l}\text { Selective reporting (re- } \\
\text { porting bias) }\end{array}$ & High risk & $\begin{array}{l}\text { Did not report on some of the measures collected. However, authors stated } \\
\text { that the conclusions were not affected. }\end{array}$ \\
\hline
\end{tabular}

\begin{tabular}{|c|c|c|}
\hline Other bias & Unclear risk & - \\
\hline $\begin{array}{l}\text { Similarity of baseline out- } \\
\text { come measurements }\end{array}$ & Unclear risk & - \\
\hline $\begin{array}{l}\text { Similarity of baseline char- } \\
\text { acteristics }\end{array}$ & Unclear risk & - \\
\hline $\begin{array}{l}\text { Adequate allocation of in- } \\
\text { tervention concealment } \\
\text { during the study }\end{array}$ & Unclear risk & - \\
\hline $\begin{array}{l}\text { Adequate protection } \\
\text { against contamination }\end{array}$ & Unclear risk & - \\
\hline $\begin{array}{l}\text { Confounders adequate- } \\
\text { ly adjusted for in analy- } \\
\text { sis/design }\end{array}$ & Unclear risk & - \\
\hline Recruitment bias & Low risk & $\begin{array}{l}\text { Assignment of individuals to clusters was done before randomization by hav- } \\
\text { ing village elders define the boundaries of their village and specify in which vil- } \\
\text { lage all potentially eligible respondents lived. }\end{array}$ \\
\hline Baseline imbalance & Low risk & $\begin{array}{l}\text { There were some significant baseline imbalances in child faeces disposal prac- } \\
\text { tices. The authors adjusted for baseline imbalance (presented in supplemen- } \\
\text { tary table 3), which did not change the conclusions. }\end{array}$ \\
\hline Loss of clusters & Low risk & No loss of clusters \\
\hline Incorrect analysis & Low risk & Used robust standard errors \\
\hline
\end{tabular}

\section{Christensen 2015b KEN}

\begin{tabular}{ll}
\hline Methods & Cluster RCT \\
\hline Participants & Number: $323 \mathrm{HHs}$ at end of study (after 12\% LTFU from baseline)
\end{tabular}


Christensen 2015b KEN (Continued)

Inclusion criteria: caregivers of children aged 4-16 months

Interventions Interventions (3 arms):

- WASH (baseline: $90 \mathrm{HHs}$, end of study: $78 \mathrm{HHs}, 9$ villages): combination of water arm (chlorine dispensers) + sanitation arm (potty, scoop, latrine improvement/new latrine) + hygiene arm (2 dual tippy taps - 1 for near latrine, 1 for cooking area) + behaviour changes messaging which emphasized synergistic nature of the interventions.

- WASH+ (baseline: $87 \mathrm{HHs}$, end of study: $74 \mathrm{HHs}, 10$ villages): included WASH + nutrient supplement (LNSs) + behaviour changes messaging which emphasized synergistic nature of the interventions.

- nutrition (baseline: $89 \mathrm{HHs}$, end of study: $77 \mathrm{HHs}, 9$ villages): respondents were provided with $2 \times 10$ $\mathrm{g}$ sachets of LNS per day for each of their children aged 6-24 months.

Control (baseline: $101 \mathrm{HHs}$, end of study: $94 \mathrm{HHs}, 10$ villages): no intervention

Child illness and growth (not sufficiently powered for it)
Uptake of interventions:
- $\quad$ presence of total and free chlorine in water
- use of the chlorine dispenser
- what the respondent had done, if anything, to dispose of the most recent child defecation
- use of the sani-scooper
- handwashing frequency
- observed cleanliness of hands

Notes

Location: 28 rural villages near Kakamega, in Western Kenya

Length of study: 6 months (November 2011 to May 2012)

Publication status: journal

\section{Risk of bias}

\begin{tabular}{lll}
\hline Bias & Authors' judgement & Support for judgement \\
\hline $\begin{array}{l}\text { Random sequence genera- } \\
\text { tion (selection bias) }\end{array}$ & Low risk & $\begin{array}{l}\text { Each village was assigned a randomly generated number using STATA, and in- } \\
\text { tervention assignments were made to villages in ascending numerical order. }\end{array}$ \\
\hline $\begin{array}{l}\text { Allocation concealment } \\
\text { (selection bias) }\end{array}$ & Low risk & Centrally allocated. \\
\hline $\begin{array}{l}\text { Blinding of participants } \\
\begin{array}{l}\text { and personnel (perfor- } \\
\text { mance bias) } \\
\text { All outcomes }\end{array}\end{array}$ & High risk & No blinding. \\
\end{tabular}

Blinding of outcome as-

High risk

No blinding and some of the outcomes were self-reported.

sessment (detection bias)

All outcomes

\begin{tabular}{lll}
\hline $\begin{array}{l}\text { Incomplete outcome data } \\
\text { (attrition bias) } \\
\text { All outcomes }\end{array}$ & High risk & LTFU was high and different across arms. \\
$\begin{array}{l}\text { Selective reporting (re- } \\
\text { porting bias) }\end{array}$ & High risk & $\begin{array}{l}\text { Did not report on some of the measures collected. However, authors stated } \\
\text { that the conclusions were not affected. }\end{array}$ \\
\hline
\end{tabular}


Christensen 2015b KEN (Continued)

\begin{tabular}{lll} 
Other bias & Unclear risk & - \\
\hline Similarity of baseline out- & Unclear risk
\end{tabular}

come measurements

Similarity of baseline char- Unclear risk -
acteristics

\begin{tabular}{ll}
\hline $\begin{array}{l}\text { Adequate allocation of in- } \\
\text { tervention concealment } \\
\text { during the study }\end{array}$ & Unclear risk \\
\hline $\begin{array}{l}\text { Adequate protection } \\
\text { against contamination }\end{array}$ & Unclear risk \\
\hline $\begin{array}{l}\text { Confounders adequate- } \\
\text { ly adjusted for in analy- } \\
\text { sis/design }\end{array}$ & Unclear risk \\
\end{tabular}

\begin{tabular}{ll}
\hline Recruitment bias & Low risk \\
& $\begin{array}{l}\text { Assignment of individuals to clusters was done before randomization by hav- } \\
\text { ing village elders define the boundaries of their village and specify in which vil- } \\
\text { lage all potentially eligible respondents lived. }\end{array}$
\end{tabular}

Baseline imbalance Low risk There were some significant baseline imbalances in child faeces disposal practices. The authors adjusted for baseline imbalance (presented in supplementary table 3), which did not change the conclusions.

\begin{tabular}{lll}
\hline Loss of clusters & Low risk & No loss of clusters. \\
\hline Incorrect analysis & Low risk & Used robust standard errors. \\
\hline
\end{tabular}

\section{Clemens 1987 BGD}

\begin{tabular}{ll}
\hline Methods & $\begin{array}{l}\text { Case-control study (community-based, cases and control selected from families in diarrhoea surveil- } \\
\text { lance) }\end{array}$ \\
\hline Participants & $\begin{array}{l}\text { Case families: sentinel families with diarrhoea rate } 1.7 \text { times expected rate for similar aged children } \\
\text { during 3-month observation, } n=45 \\
\text { Control families: sentinel families without any episodes of childhood diarrhoea during the 3-month pe- } \\
\text { riod of observation, } n=53\end{array}$ \\
\hline Interventions & $\begin{array}{l}\text { Risk factor of interest: } \\
\text { open defecation of ambulatory children (aged < } 6 \text { years) in family living area vs in latrine or specially } \\
\text { designated place. }\end{array}$ \\
\hline Outcomes & $\begin{array}{l}\text { Diarrhoea: } \geq 3 \text { unformed stools in any 24-hour period during the 2-week interval. Stipulated that a child } \\
\text { without diarrhoea (or in the first round) and ended with the next diarrhoea-free round (data collected } \\
\text { fortnightly). }\end{array}$ \\
\hline Notes & $\begin{array}{l}\text { Location: Dhaka slums, Bangladesh } \\
\text { Length of recruitment: } 3 \text { months' fortnightly histories of diarrhoea + observations in sentinel families } \\
\text { (October } 1984 \text { to January 1985) }\end{array}$
\end{tabular}


Clemens 1987 BGD (Continued)

Publication status: journal

\section{Risk of bias}

\section{Bias}

\section{Random sequence genera-} tion (selection bias)

$\begin{aligned} & \text { Allocation concealment } \\ & \text { (selection bias) }\end{aligned}$
Unclear risk NA

Blinding of participants Unclear risk NA
and personnel (perfor-
mance bias)
All outcomes

NA

NA

NA

\section{Authors' judgement Support for judgement}

Blinding of outcome as-
sessment (detection bias) $\quad$ Unclear risk $\quad$ NA

All outcomes
Incomplete outcome data Unclear risk NA

(attrition bias)

All outcomes

Selective reporting (re- Unclear risk NA

porting bias)

\begin{tabular}{lll}
\hline Other bias & Unclear risk & NA \\
\hline $\begin{array}{l}\text { Similarity of baseline out- } \\
\text { come measurements }\end{array}$ & Unclear risk & NA \\
\hline
\end{tabular}

Similarity of baseline char- Unclear risk NA

acteristics

Adequate allocation of in- Unclear risk NA
tervention concealment
during the study

during the study

\begin{tabular}{lll}
\hline $\begin{array}{l}\text { Adequate protection } \\
\text { against contamination }\end{array}$ & Unclear risk & NA \\
\hline $\begin{array}{l}\text { Confounders adequate- } \\
\text { ly adjusted for in analy- } \\
\text { sis/design }\end{array}$ & Unclear risk & NA \\
\hline Recruitment bias & Unclear risk & NA \\
\hline Baseline imbalance & Unclear risk & NA \\
\hline Loss of clusters & Unclear risk & NA \\
\hline Incorrect analysis & Unclear risk & NA \\
\hline
\end{tabular}


Cummings 2012 UGA

\begin{tabular}{|c|c|}
\hline Methods & Case-control study (unmatched) \\
\hline \multirow[t]{2}{*}{ Participants } & $\begin{array}{l}\text { Cases: people aged > } 10 \text { years who met the } \mathrm{UMOH} \text { 's outbreak case definition admitted to a cholera } \\
\text { treatment centre in Moroto during April-June } 2010 \text {; and resided in } 1 / 15 \text { selected villages in Nadunget, } n \\
=99 \text {, median age } 26 \text { years, } 64.6 \% \text { female. }\end{array}$ \\
\hline & $\begin{array}{l}\text { Controls: people aged > } 10 \text { years who had not experienced any form of diarrhoea from April } 2010 \text { to the } \\
\text { time of investigation, resided in } 1 / 15 \text { selected villages in Nadunget, } n=99 \text {, median age } 33 \text { years, } 51.5 \% \\
\text { female. }\end{array}$ \\
\hline
\end{tabular}

Risk factor of interest:
$\begin{aligned} & \text { Interventions } \\ & \text { not disposing of child faeces in latrine vs using latrine to dispose of faeces (unclear what the age of } \\ & \text { the children whose faeces were disposed, referred to as younger children in the } \mathrm{HH} \text { ). }\end{aligned}$

\begin{tabular}{ll}
\hline Outcomes & Cholera: acute watery diarrhoea in an area with laboratory-confirmed cholera cases \\
\hline Notes & Location: rural Karamoja subregion, north-east Uganda \\
& Length of recruitment: 3 months (April-June 2010) \\
& Publication status: journal
\end{tabular}

\section{Risk of bias}

\begin{tabular}{|c|c|c|}
\hline Bias & Authors' judgement & Support for judgement \\
\hline $\begin{array}{l}\text { Random sequence genera- } \\
\text { tion (selection bias) }\end{array}$ & Unclear risk & NA \\
\hline $\begin{array}{l}\text { Allocation concealment } \\
\text { (selection bias) }\end{array}$ & Unclear risk & NA \\
\hline $\begin{array}{l}\text { Blinding of participants } \\
\text { and personnel (perfor- } \\
\text { mance bias) } \\
\text { All outcomes }\end{array}$ & Unclear risk & NA \\
\hline $\begin{array}{l}\text { Blinding of outcome as- } \\
\text { sessment (detection bias) } \\
\text { All outcomes }\end{array}$ & Unclear risk & NA \\
\hline $\begin{array}{l}\text { Incomplete outcome data } \\
\text { (attrition bias) } \\
\text { All outcomes }\end{array}$ & Unclear risk & NA \\
\hline $\begin{array}{l}\text { Selective reporting (re- } \\
\text { porting bias) }\end{array}$ & Unclear risk & NA \\
\hline Other bias & Unclear risk & NA \\
\hline $\begin{array}{l}\text { Similarity of baseline out- } \\
\text { come measurements }\end{array}$ & Unclear risk & NA \\
\hline $\begin{array}{l}\text { Similarity of baseline char- } \\
\text { acteristics }\end{array}$ & Unclear risk & NA \\
\hline
\end{tabular}


Cummings 2012 UGA (Continued)

\begin{tabular}{|c|c|c|}
\hline $\begin{array}{l}\text { Adequate allocation of in- } \\
\text { tervention concealment } \\
\text { during the study }\end{array}$ & Unclear risk & NA \\
\hline $\begin{array}{l}\text { Adequate protection } \\
\text { against contamination }\end{array}$ & Unclear risk & NA \\
\hline $\begin{array}{l}\text { Confounders adequate- } \\
\text { ly adjusted for in analy- } \\
\text { sis/design }\end{array}$ & Unclear risk & NA \\
\hline Recruitment bias & Unclear risk & NA \\
\hline Baseline imbalance & Unclear risk & NA \\
\hline Loss of clusters & Unclear risk & NA \\
\hline Incorrect analysis & Unclear risk & NA \\
\hline
\end{tabular}

Daniels 1990 LES

\begin{tabular}{ll}
\hline Methods & Case-control study (clinic-based) \\
\hline Participants & $\begin{array}{l}\text { Cases: children aged }<5 \text { years who presented to the participating health facilities with diarrhoea, } \mathrm{n}= \\
803 \text { (after excluding 3), } 43.5 \% \text { aged }<12 \text { months, } 48.8 \% \text { girls. } \\
\text { Controls: the same age range who reported with either respiratory infections or trauma, but without di- } \\
\text { arhoea. Children also had to: be accompanied by a parent or guardian who had been responsible for } \\
\text { the child for the previous } 3 \text { months, be living in a HH within Mohale's Hoek district, have no congenital } \\
\text { abnormality or chronic illness, and the accompanying adult had to consent to his or her child's inclu- } \\
\text { sion in the study. } \mathrm{n}=810 \text { (after excluding } 4 \text { ). } 54.6 \%<12 \text { months, } 52.4 \% \text { girls. }\end{array}$
\end{tabular}

Interventions Risk factor of interest:

- child faeces disposed in latrine vs not (no usable data, data reported for cases and controls jointly).

\begin{tabular}{ll}
\hline Outcomes & Diarrhoea: as defined by the mother, with $\geq 3$ loose or watery stools in previous 24 hours \\
\hline Notes & Location: 4 health facilities in rural Mohale's Hoek district, Lesotho \\
& Length of recruitment: 6 months (8 December 1987 to 6 June 1988) \\
& Publication status: journal \\
\hline
\end{tabular}

\section{Risk of bias}

\begin{tabular}{lll}
\hline Bias & Authors' judgement & Support for judgement \\
\hline $\begin{array}{l}\text { Random sequence genera- } \\
\text { tion (selection bias) }\end{array}$ & Unclear risk & NA \\
\hline $\begin{array}{l}\text { Allocation concealment } \\
\text { (selection bias) }\end{array}$ & Unclear risk & NA \\
\hline $\begin{array}{l}\text { Blinding of participants } \\
\text { and personnel (perfor- } \\
\text { mance bias) }\end{array}$ & Unclear risk & NA \\
\hline
\end{tabular}


Daniels 1990 LES (Continued)

All outcomes

\begin{tabular}{ll}
$\begin{array}{l}\text { Blinding of outcome as- } \\
\text { sessment (detection bias) } \\
\text { All outcomes }\end{array}$ & Unclear risk \\
\hline $\begin{array}{l}\text { Incomplete outcome data } \\
\text { (attrition bias) }\end{array}$ & Unclear risk
\end{tabular}

All outcomes

\begin{tabular}{|c|c|c|}
\hline $\begin{array}{l}\text { Selective reporting (re- } \\
\text { porting bias) }\end{array}$ & Unclear risk & NA \\
\hline Other bias & Unclear risk & NA \\
\hline $\begin{array}{l}\text { Similarity of baseline out- } \\
\text { come measurements }\end{array}$ & Unclear risk & NA \\
\hline $\begin{array}{l}\text { Similarity of baseline char- } \\
\text { acteristics }\end{array}$ & Unclear risk & NA \\
\hline $\begin{array}{l}\text { Adequate allocation of in- } \\
\text { tervention concealment } \\
\text { during the study }\end{array}$ & Unclear risk & NA \\
\hline $\begin{array}{l}\text { Adequate protection } \\
\text { against contamination }\end{array}$ & Unclear risk & NA \\
\hline $\begin{array}{l}\text { Confounders adequate- } \\
\text { ly adjusted for in analy- } \\
\text { sis/design }\end{array}$ & Unclear risk & NA \\
\hline Recruitment bias & Unclear risk & NA \\
\hline Baseline imbalance & Unclear risk & NA \\
\hline Loss of clusters & Unclear risk & NA \\
\hline Incorrect analysis & Unclear risk & NA \\
\hline
\end{tabular}

\section{Dickinson 2015 IND}

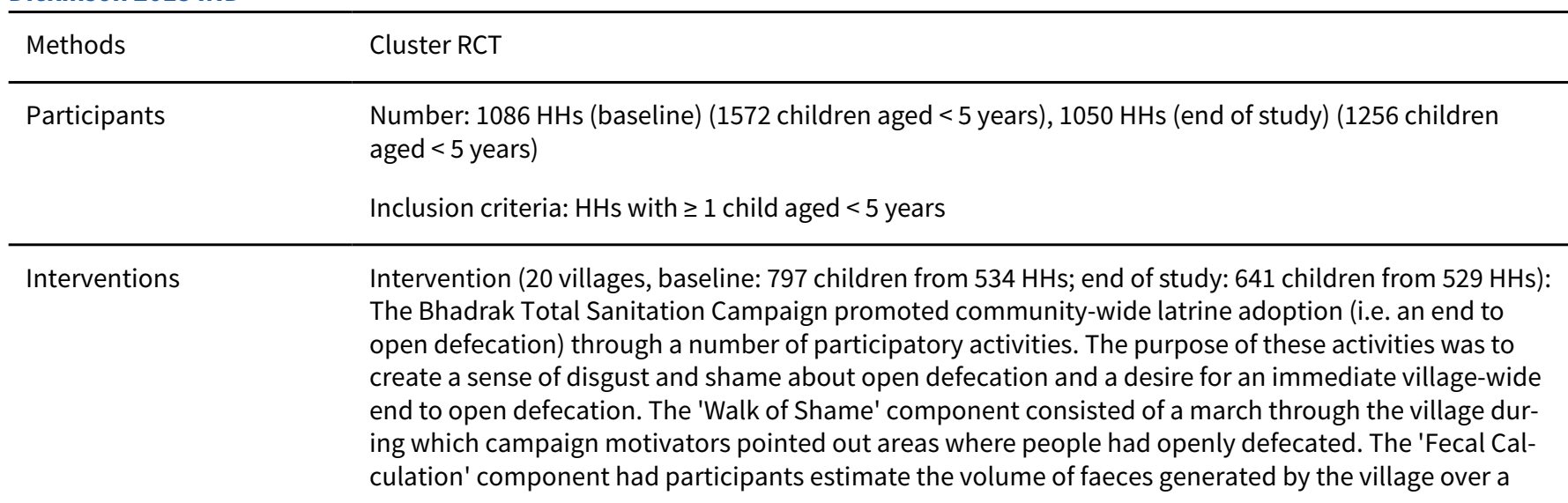


Dickinson 2015 IND (Continued)

given period of time. The 'Spatial Mapping' activity had participants examine the spatial distribution of houses, open defecation hot spots, and drinking water sources to understand community exposure. These activities were meant to call attention to the level of contamination in the village and the collective nature of the problem: unless everyone stopped open defecation by using latrines, everyone would continue to be exposed to faecal matter. The goal was to induce entire villages to commit to becoming ODF by a collectively agreed-on date. The campaign subsidized materials and labour for latrine construction for HHs eligible for government of India subsidies (i.e. below poverty line HHs). The intervention also supplied masons to guide $\mathrm{HHs}$ and organized sanitation marts (production centres) operated by local non-governmental organizations in each village. Messages were also given on the benefits of latrines, both health and non-health (convenience of time-saving, privacy, dignity). Messages to improve child faeces disposal practices where included (according to personal communication).

Control (20 villages, baseline: 775 children from $552 \mathrm{HHs}$; end of study: 615 children from $521 \mathrm{HHs}$ ): no intervention.

\begin{tabular}{ll}
\hline Outcomes & Diarrhoea (in past 2 weeks) \\
MUAC & Height for age \\
& Weight for age \\
& Walking time to defecation sites (women, men, children aged < 5 years) \\
& Satisfaction with sanitation facilities \\
Notes & Location: 40 rural villages in Bhadrak district in Orissa, India \\
& Length of study: 14 months (August 2005 to September 2006) \\
& Publication status: journal
\end{tabular}

\section{Risk of bias}

\begin{tabular}{lll}
\hline Bias & Authors' judgement & Support for judgement \\
\hline $\begin{array}{l}\text { Random sequence genera- } \\
\text { tion (selection bias) }\end{array}$ & Low risk & Used a public lottery. \\
\hline $\begin{array}{l}\text { Allocation concealment } \\
\text { (selection bias) }\end{array}$ & Low risk & Participants and investigators could not foresee assignment due to lottery. \\
\hline $\begin{array}{l}\text { Blinding of participants } \\
\begin{array}{l}\text { and personnel (perfor- } \\
\text { mance bias) }\end{array}\end{array}$ & High risk \\
All outcomes & No blinding. \\
\hline
\end{tabular}

\begin{tabular}{|c|c|c|}
\hline $\begin{array}{l}\text { Blinding of outcome as- } \\
\text { sessment (detection bias) } \\
\text { All outcomes }\end{array}$ & High risk & No blinding and some outcomes were self-reported. \\
\hline $\begin{array}{l}\text { Incomplete outcome data } \\
\text { (attrition bias) } \\
\text { All outcomes }\end{array}$ & Low risk & $\begin{array}{l}\text { The } \% \text { of attrition was similar in intervention ( } 20 \%) \text { and control ( } 21 \%) \text { groups, } \\
\text { mostly due to children turning } 5 \text { years of age between baseline and end of } \\
\text { study. }\end{array}$ \\
\hline $\begin{array}{l}\text { Selective reporting (re- } \\
\text { porting bias) }\end{array}$ & Low risk & Report on all outcomes listed in methods. \\
\hline Other bias & Unclear risk & - \\
\hline
\end{tabular}


Dickinson 2015 IND (Continued)

\begin{tabular}{lll}
$\begin{array}{l}\text { Similarity of baseline out- } \\
\text { come measurements }\end{array}$ & Unclear risk \\
\hline $\begin{array}{l}\text { Similarity of baseline char- } \\
\text { acteristics }\end{array}$ & Unclear risk & - \\
\hline
\end{tabular}
acteristics

\begin{tabular}{ll}
\hline $\begin{array}{l}\text { Adequate allocation of in- } \\
\text { tervention concealment } \\
\text { during the study }\end{array}$ & Unclear risk \\
\hline $\begin{array}{l}\text { Adequate protection } \\
\text { against contamination }\end{array}$ & Unclear risk \\
\hline $\begin{array}{l}\text { Confounders adequate- } \\
\text { ly adjusted for in analy- } \\
\text { sis/design }\end{array}$ & Unclear risk \\
\hline
\end{tabular}

\begin{tabular}{lll}
\hline Recruitment bias & Low risk & $\begin{array}{l}\text { Participants were recruited prior to random allocation of village to interven- } \\
\text { tion. }\end{array}$ \\
\hline Baseline imbalance & Low risk & $\begin{array}{l}\text { No substantial differences in baseline characteristics were observed in gener- } \\
\text { al, treatment and control villages are similar, with few significant differences } \\
\text { in observable covariates (treatment villages had lower population density, } \\
\text { televisions, and latrines) and baseline characteristics were included in analy- } \\
\text { sis. }\end{array}$ \\
\hline Loss of clusters & Low risk & No loss of clusters reported. \\
\hline Incorrect analysis & Low risk & $\begin{array}{l}\text { Reported measures are adjusted for clustering at village level. However, it was } \\
\text { unclear whether it was also adjusted at HH level if }>\text { than } 1 \text { child. }\end{array}$ \\
\hline
\end{tabular}

\section{Dikassa 1993 DRC}

\begin{tabular}{ll}
\hline Methods & Case-control study (matched) \\
\hline Participants & $\begin{array}{l}\text { Cases: children aged }<3 \text { years admitted to hospital and admission for primary diagnosis of diarrhoea of } \\
\text { infectious origin, } n=107 \text { (after excluding } 6 \text { ), mean age } 11.9 \text { months, 39.3\% girls } \\
\text { Controls: age-matched children who were the nearest residential neighbours of the cases recruited for } \\
\text { the study and who had no history of hospitalization for diarrhoeal disease, } n=107 \text { (after excluding 6), } \\
\text { mean age } 10.5 \text { months, } 41.1 \% \text { girls }\end{array}$ \\
\hline Interventions & $\begin{array}{l}\text { Risk factor of interest: } \\
\text { not disposing of child faeces in latrine vs using latrine for disposal. }\end{array}$ \\
\hline Severe diarrhoea, all cases were identified by the first author (no case definition). The severity of diar- \\
rhoea was assessed based on evident dehydration of the child requiring hospitalization.
\end{tabular}

\section{Risk of bias}

Interventions to improve disposal of child faeces for preventing diarrhoea and soil-transmitted helminth infection (Review) 
Dikassa 1993 DRC (Continued)

Bias

Authors' judgement Support for judgement

Random sequence genera- Unclear risk NA

tion (selection bias)

Allocation concealment Unclear risk NA

(selection bias)

Blinding of participants $\quad$ Unclear risk NA
and personnel (perfor-
mance bias)
All outcomes

Blinding of outcome as- Unclear risk NA

sessment (detection bias)

All outcomes

Incomplete outcome data Unclear risk
(attrition bias)

All outcomes

\begin{tabular}{ll}
\hline $\begin{array}{l}\text { Selective reporting (re- } \\
\text { porting bias) }\end{array}$ & Unclear risk
\end{tabular}

\begin{tabular}{lll}
\hline Other bias & Unclear risk & NA \\
\hline $\begin{array}{l}\text { Similarity of baseline out- } \\
\text { come measurements }\end{array}$ & Unclear risk & NA \\
\hline $\begin{array}{l}\text { Similarity of baseline char- } \\
\text { acteristics }\end{array}$ & Unclear risk & NA \\
\hline
\end{tabular}

\begin{tabular}{|c|c|c|}
\hline $\begin{array}{l}\text { Adequate allocation of in- } \\
\text { tervention concealment } \\
\text { during the study }\end{array}$ & Unclear risk & NA \\
\hline $\begin{array}{l}\text { Adequate protection } \\
\text { against contamination }\end{array}$ & Unclear risk & NA \\
\hline $\begin{array}{l}\text { Confounders adequate- } \\
\text { ly adjusted for in analy- } \\
\text { sis/design }\end{array}$ & Unclear risk & NA \\
\hline Recruitment bias & Unclear risk & NA \\
\hline Baseline imbalance & Unclear risk & NA \\
\hline Loss of clusters & Unclear risk & NA \\
\hline Incorrect analysis & Unclear risk & NA \\
\hline
\end{tabular}

Fisher 2011 BGD

Methods Controlled cross-sectional study


Fisher 2011 BGD (Continued)
Participants
Number: 107 respondents (1.8\% non-response)
Inclusion criteria: caregivers of a child aged $<5$ years

Interventions

Intervention (2 villages, 80 respondents): BRAC hygiene education intervention; trained field workers provided water, sanitation, and hygiene education to separate clusters of men, women, adolescents, and children at least once every 3 months. The education used pictorial flip chart with a total of 39 messages covering multiple aspects of cleanliness, clean water, and sanitation. Villagers are also encouraged to learn the '19 Messages to Remember', concerning hand washing, sanitation (included child faeces disposal in latrine), and safe water.

Control (1 village, 27 respondents): no BRAC intervention.

\begin{tabular}{ll}
\hline Outcomes & Diarrhoea in previous month: $\geq 3$ loose or watery stools within a 24-hour period (WHO definition) \\
& $\begin{array}{l}\text { Behaviour change: comparison between disposal of child faeces in latrine (child used latrine + faeces } \\
\text { disposed in latrine) vs elsewhere for the last time the child defecated } \\
\text { Knowledge and practices covered in BRAC }\end{array}$ \\
\hline Notes & Location: 3 rural villages, Mymensingh District, Bangladesh \\
& Length of study: not specified \\
& Publication status: journal
\end{tabular}

\section{Risk of bias}

\begin{tabular}{|c|c|c|}
\hline Bias & Authors' judgement & Support for judgement \\
\hline $\begin{array}{l}\text { Random sequence genera- } \\
\text { tion (selection bias) }\end{array}$ & High risk & Intervention not allocated randomly. \\
\hline $\begin{array}{l}\text { Allocation concealment } \\
\text { (selection bias) }\end{array}$ & High risk & No details on concealment. \\
\hline $\begin{array}{l}\text { Blinding of participants } \\
\text { and personnel (perfor- } \\
\text { mance bias) } \\
\text { All outcomes }\end{array}$ & Unclear risk & NA \\
\hline $\begin{array}{l}\text { Blinding of outcome as- } \\
\text { sessment (detection bias) } \\
\text { All outcomes }\end{array}$ & Unclear risk & NA \\
\hline $\begin{array}{l}\text { Incomplete outcome data } \\
\text { (attrition bias) } \\
\text { All outcomes }\end{array}$ & Low risk & Only $1.8 \%$ non-response. \\
\hline $\begin{array}{l}\text { Selective reporting (re- } \\
\text { porting bias) }\end{array}$ & Low risk & Report on outcomes from methods. \\
\hline Other bias & High risk & Small sample size with only 1 control village. \\
\hline $\begin{array}{l}\text { Similarity of baseline out- } \\
\text { come measurements }\end{array}$ & Unclear risk & NA, no baseline. \\
\hline $\begin{array}{l}\text { Similarity of baseline char- } \\
\text { acteristics }\end{array}$ & Unclear risk & NA, no baseline. \\
\hline
\end{tabular}


Fisher 2011 BGD (Continued)

\begin{tabular}{|c|c|c|}
\hline $\begin{array}{l}\text { Adequate allocation of in- } \\
\text { tervention concealment } \\
\text { during the study }\end{array}$ & High risk & $\begin{array}{l}\text { Allocation to intervention occurred prior to study and the interviews were con- } \\
\text { ducted by BRAC field workers (presumably aware of allocation of interven- } \\
\text { tion). }\end{array}$ \\
\hline
\end{tabular}

\begin{tabular}{lll}
\hline $\begin{array}{l}\text { Adequate protection } \\
\text { against contamination }\end{array}$ & Unclear risk & $\begin{array}{l}\text { Control village was } 7 \mathrm{~km} \text { away from the other 2 villages but unclear whether it } \\
\text { was nearby to another BRAC village. }\end{array}$ \\
\hline $\begin{array}{l}\text { Confounders adequate- } \\
\text { ly adjusted for in analy- } \\
\text { sis/design }\end{array}$ & High risk & No analysis controlling for confounders. \\
\hline Recruitment bias & Unclear risk & NA \\
\hline Baseline imbalance & Unclear risk & NA \\
\hline Loss of clusters & Unclear risk & NA \\
\hline Incorrect analysis & Unclear risk & NA \\
\hline
\end{tabular}

\section{Gebru 2014 ETH}

\begin{tabular}{|c|c|}
\hline Methods & Controlled cross-sectional study \\
\hline \multirow[t]{2}{*}{ Participants } & Number: 794 respondents ( $96.2 \%$ response rate) \\
\hline & $\begin{array}{l}\text { Inclusion criteria: } \mathrm{HHs} \text { with } \geq 1 \text { child aged }<5 \text { years in } 11 \text { randomly selected kebeles. For model families } \\
\text { (intervention), all HHs graduated (trained) health extension programme (HEP). For non-model families } \\
\text { (control), all non-graduated HHs. }\end{array}$ \\
\hline \multirow[t]{2}{*}{ Interventions } & $\begin{array}{l}\text { Intervention ( } 265 \text { respondents): health promotion and education. Female and male HH heads who had } \\
\text { graduated as model families after being given basic training on the } 16 \text { HEP packages for } 96 \text { hours (ma- } \\
\text { ternal and child health package included safe child stool disposal HEP 2003). }\end{array}$ \\
\hline & Control (529 respondents): non-model-families. \\
\hline \multirow[t]{2}{*}{ Outcomes } & 2-week diarrhoea prevalence (adapted WHO questionnaire but no additional details on case definition) \\
\hline & $\begin{array}{l}\text { Possible environmental and behavioural risk factors for diarrhoea, including proper vs improver child } \\
\text { stool disposal method (no definition of proper disposal) }\end{array}$ \\
\hline \multirow[t]{3}{*}{ Notes } & Location: 11 rural kebeles, Sheko district, South West Ethiopia \\
\hline & Length of study: 1 month (31 January to 29 February 2012) \\
\hline & Publication status: journal \\
\hline
\end{tabular}

\section{Risk of bias}

\begin{tabular}{lll}
\hline Bias & Authors' judgement & Support for judgement \\
\hline $\begin{array}{l}\text { Random sequence genera- } \\
\text { tion (selection bias) }\end{array}$ & High risk & The model HHs were not allocated to the intervention at random. \\
\hline $\begin{array}{l}\text { Allocation concealment } \\
\text { (selection bias) }\end{array}$ & High risk & No allocation concealment. \\
\hline
\end{tabular}


Gebru 2014 ETH (Continued)

\begin{tabular}{lll}
$\begin{array}{l}\text { Blinding of participants } \\
\text { and personnel (perfor- } \\
\text { mance bias) } \\
\text { All outcomes }\end{array}$ & Unclear risk & \\
\hline $\begin{array}{l}\text { Blinding of outcome as- } \\
\text { sessment (detection bias) } \\
\text { All outcomes }\end{array}$ & Unclear risk & NA \\
\hline $\begin{array}{l}\text { Incomplete outcome data } \\
\text { (attrition bias) } \\
\text { All outcomes }\end{array}$ & Low risk & $96.2 \%$ response rate. \\
\hline $\begin{array}{l}\text { Selective reporting (re- } \\
\text { porting bias) }\end{array}$ & Low risk & Report on outcomes from methods. \\
\hline $\begin{array}{l}\text { Other bias } \\
\text { Similarity of baseline out- } \\
\text { come measurements }\end{array}$ & Unclear risk & NA, no baseline. \\
\hline $\begin{array}{l}\text { Similarity of baseline char- } \\
\text { acteristics }\end{array}$ & Unclear risk & NA, no baseline. \\
\hline
\end{tabular}

\begin{tabular}{|c|c|c|}
\hline $\begin{array}{l}\text { Adequate allocation of in- } \\
\text { tervention concealment } \\
\text { during the study }\end{array}$ & Unclear risk & $\begin{array}{l}\text { Allocation to intervention occurred prior to study but no mention of whether } \\
\text { data collectors were blind to whether } \mathrm{HH} \text { was model/non-model. }\end{array}$ \\
\hline
\end{tabular}

\begin{tabular}{lll}
\hline $\begin{array}{l}\text { Adequate protection } \\
\text { against contamination }\end{array}$ & High risk & $\begin{array}{l}\text { No specification about whether the model and non-model HHs were in the } \\
\text { same kebeles. }\end{array}$ \\
\hline $\begin{array}{l}\text { Confounders adequate- } \\
\text { ly adjusted for in analy- } \\
\text { sis/design }\end{array}$ & Low risk & $\begin{array}{l}\text { Analysis of diarrhoea risk factors controls for wealth, education, and hand- } \\
\text { washing. }\end{array}$ \\
\hline Recruitment bias & Unclear risk & NA \\
\hline Baseline imbalance & Unclear risk & NA \\
\hline Loss of clusters & Unclear risk & NA \\
\hline Incorrect analysis & Unclear risk & NA \\
\hline
\end{tabular}

Genthe 1997 SAF

\begin{tabular}{|c|c|}
\hline Methods & Case-control study \\
\hline Participants & $\begin{array}{l}\text { Cases: a sample was drawn from preschool children who were brought to the day hospitals with diar- } \\
\text { rhoea, } n=169 \text {, median age } 12 \text { months, } 50.6 \% \text { girls. }\end{array}$ \\
\hline & $\begin{array}{l}\text { Controls: selected according to age ( } \pm 6 \text { months) and type of water supply from the immediate neigh- } \\
\text { bourhood of the case and who had not had diarrhoea during the preceding } 14 \text { days of the visit. Controls } \\
\text { were matched for the time of occurrence of the case as well as the dates for interviews and observa- } \\
\text { tional studies, } n=166 \text {. median age } 18 \text { months, } 47.3 \% \text { girls. }\end{array}$ \\
\hline
\end{tabular}


Genthe 1997 SAF (Continued)

- open disposal of stools vs disposal of stools into any form of sanitation system (private or communal toilet).

\begin{tabular}{ll}
\hline Outcomes & Diarrhoea: $\geq 3$ loose or watery stools in a period of 24 hours (WHO definition) \\
\hline Notes & Location: 2 day hospitals, urban townships, Kliayelitsha, Cape Flats, South Africa \\
& Length of recruitment: $2 \times 3$-month periods (wet and dry seasons) in 1993-1994 \\
& Publication status: report \\
\hline
\end{tabular}

\section{Risk of bias}

\begin{tabular}{|c|c|c|}
\hline Bias & Authors' judgement & Support for judgement \\
\hline $\begin{array}{l}\text { Random sequence genera- } \\
\text { tion (selection bias) }\end{array}$ & Unclear risk & NA \\
\hline $\begin{array}{l}\text { Allocation concealment } \\
\text { (selection bias) }\end{array}$ & Unclear risk & NA \\
\hline $\begin{array}{l}\text { Blinding of participants } \\
\text { and personnel (perfor- } \\
\text { mance bias) } \\
\text { All outcomes }\end{array}$ & Unclear risk & NA \\
\hline $\begin{array}{l}\text { Blinding of outcome as- } \\
\text { sessment (detection bias) } \\
\text { All outcomes }\end{array}$ & Unclear risk & NA \\
\hline $\begin{array}{l}\text { Incomplete outcome data } \\
\text { (attrition bias) } \\
\text { All outcomes }\end{array}$ & Unclear risk & NA \\
\hline $\begin{array}{l}\text { Selective reporting (re- } \\
\text { porting bias) }\end{array}$ & Unclear risk & NA \\
\hline Other bias & Unclear risk & NA \\
\hline $\begin{array}{l}\text { Similarity of baseline out- } \\
\text { come measurements }\end{array}$ & Unclear risk & NA \\
\hline $\begin{array}{l}\text { Similarity of baseline char- } \\
\text { acteristics }\end{array}$ & Unclear risk & NA \\
\hline $\begin{array}{l}\text { Adequate allocation of in- } \\
\text { tervention concealment } \\
\text { during the study }\end{array}$ & Unclear risk & NA \\
\hline $\begin{array}{l}\text { Adequate protection } \\
\text { against contamination }\end{array}$ & Unclear risk & NA \\
\hline $\begin{array}{l}\text { Confounders adequate- } \\
\text { ly adjusted for in analy- } \\
\text { sis/design }\end{array}$ & Unclear risk & NA \\
\hline Recruitment bias & Unclear risk & NA \\
\hline
\end{tabular}


Genthe 1997 SAF (Continued)

\begin{tabular}{lll} 
Baseline imbalance & Unclear risk & NA \\
\hline Loss of clusters & Unclear risk & NA \\
\hline Incorrect analysis & Unclear risk & NA \\
\hline
\end{tabular}

Ghosh 1994 IND

Case-control study (nested in a community longitudinal study following up of children aged $<3$ years
with twice a week active surveillance for diarrhoea)

\begin{tabular}{ll}
\hline Participants & $\begin{array}{l}\text { Cases: families with a child aged }<3 \text { years with diarrhoea, } \mathrm{n}=105 \text { (initially } 76 \text { but } 29 \text { controls developed } \\
\text { diarrhoea and became a case). } \\
\text { Controls: families with an age-matched child aged }<3 \text { years without diarrhoea in neighbourhood, } \mathrm{n}=47 \\
\text { (initially } 76 \text { but } 29 \text { controls developed diarrhoea and became a case). }\end{array}$ \\
\hline Interventions & Risk factor of interest: \\
\hline indiscriminate child stool disposal (no definition of indiscriminate). \\
\hline Outcomes & Diarrhoea (no case definition), data collected twice per week \\
\hline Lotes & Lengtion: rural West Bengal, India \\
Publication status: journal
\end{tabular}

\section{Risk of bias}

\begin{tabular}{|c|c|c|}
\hline Bias & Authors' judgement & Support for judgement \\
\hline $\begin{array}{l}\text { Random sequence genera- } \\
\text { tion (selection bias) }\end{array}$ & Unclear risk & NA \\
\hline $\begin{array}{l}\text { Allocation concealment } \\
\text { (selection bias) }\end{array}$ & Unclear risk & NA \\
\hline $\begin{array}{l}\text { Blinding of participants } \\
\text { and personnel (perfor- } \\
\text { mance bias) } \\
\text { All outcomes }\end{array}$ & Unclear risk & NA \\
\hline $\begin{array}{l}\text { Blinding of outcome as- } \\
\text { sessment (detection bias) } \\
\text { All outcomes }\end{array}$ & Unclear risk & NA \\
\hline $\begin{array}{l}\text { Incomplete outcome data } \\
\text { (attrition bias) } \\
\text { All outcomes }\end{array}$ & Unclear risk & NA \\
\hline $\begin{array}{l}\text { Selective reporting (re- } \\
\text { porting bias) }\end{array}$ & Unclear risk & NA \\
\hline Other bias & Unclear risk & NA \\
\hline
\end{tabular}


Ghosh 1994 IND (Continued)

\begin{tabular}{lll}
$\begin{array}{l}\text { Similarity of baseline out- } \\
\text { come measurements }\end{array}$ & Unclear risk & NA \\
\hline $\begin{array}{l}\text { Similarity of baseline char- } \\
\text { acteristics }\end{array}$ & Unclear risk & NA \\
\hline $\begin{array}{l}\text { Adequate allocation of in- } \\
\text { tervention concealment } \\
\text { during the study }\end{array}$ & Unclear risk & NA \\
\hline $\begin{array}{l}\text { Adequate protection } \\
\text { against contamination }\end{array}$ & Unclear risk & NA \\
\hline $\begin{array}{l}\text { Confounders adequate- } \\
\text { ly adjusted for in analy- } \\
\text { sis/design }\end{array}$ & Unclear risk & \\
\hline $\begin{array}{l}\text { Recruitment bias } \\
\text { Baseline imbalance }\end{array}$ & Unclear risk & NA \\
\hline $\begin{array}{l}\text { Loss of clusters } \\
\text { Incorrect analysis }\end{array}$ & Unclear risk & NA \\
\hline
\end{tabular}

\section{Ghosh 1997 IND}

$\begin{array}{ll}\text { Methods } & \text { Case-control study (nested in a community longitudinal study following up of children aged }<4 \text { years } \\ \text { with twice a week active surveillance for diarrhoea) }\end{array}$
with twice a week active surveillance for diarrhoea)

\section{Participants}

Cases: families with a child aged $<4$ years with diarrhoea, $\mathrm{n}=108$ (initially 90 but 18 control families became cases).

Controls: neighbourhood families with a study child of similar age but without diarrhoea within preceding 7 months (if control family developed diarrhoea in following 6 months it became a case family instead of a control family), $n=72$ (initially 90 but 18 control families became cases).

Risk factors of interest:
$\begin{aligned} & \text { Interventions } \\ & \text { - }\end{aligned}$
mothers who dispose of child faeces indiscriminately without knowledge compared to mothers who
have knowledge of risk of indiscriminate child faeces disposal and do not practice indiscriminate child
faeces disposal (no definition of indiscriminate disposal) (Ghosh 1998).

\section{Outcomes}

Diarrhoea: passage of $\geq 3$ liquid, watery mucoid stools with or without blood during the past 24 hours. For infants aged up to 3 months, an increase in the frequency and a change in the consistency of stools which was of concern to mothers.

Lotes
Lengtion: 3 rural villages in West Bengal, India
Publication status: journal

\section{Risk of bias}


Ghosh 1997 IND (Continued)

\begin{tabular}{llc} 
Bias & Authors' judgement & Supp \\
\hline $\begin{array}{l}\text { Random sequence genera- } \\
\text { tion (selection bias) }\end{array}$ & Unclear risk & NA \\
\hline $\begin{array}{l}\text { Allocation concealment } \\
\text { (selection bias) }\end{array}$ & Unclear risk & NA \\
\hline $\begin{array}{l}\text { Blinding of participants } \\
\text { and personnel (perfor- } \\
\text { mance bias) } \\
\text { All outcomes }\end{array}$ & Unclear risk & NA \\
\hline
\end{tabular}

$\begin{array}{lll}\text { Blinding of outcome as- } & \text { Unclear risk }\end{array}$

sessment (detection bias)

All outcomes

\begin{tabular}{l}
\hline Incomplete outcome data Unclear risk NA \\
(attrition bias) \\
All outcomes
\end{tabular}

Selective reporting (re- Unclear risk
porting bias)

\begin{tabular}{lll}
\hline Other bias & Unclear risk & NA \\
\hline $\begin{array}{l}\text { Similarity of baseline out- } \\
\text { come measurements }\end{array}$ & Unclear risk & NA \\
\hline $\begin{array}{l}\text { Similarity of baseline char- } \\
\text { acteristics }\end{array}$ & Unclear risk & NA \\
\hline
\end{tabular}

\begin{tabular}{|c|c|c|}
\hline $\begin{array}{l}\text { Adequate allocation of in- } \\
\text { tervention concealment } \\
\text { during the study }\end{array}$ & Unclear risk & NA \\
\hline $\begin{array}{l}\text { Adequate protection } \\
\text { against contamination }\end{array}$ & Unclear risk & NA \\
\hline $\begin{array}{l}\text { Confounders adequate- } \\
\text { ly adjusted for in analy- } \\
\text { sis/design }\end{array}$ & Unclear risk & NA \\
\hline Recruitment bias & Unclear risk & NA \\
\hline Baseline imbalance & Unclear risk & NA \\
\hline Loss of clusters & Unclear risk & NA \\
\hline Incorrect analysis & Unclear risk & NA \\
\hline
\end{tabular}

Methods Case-control study (community-based, unmatched)


Godana 2013 ETH (Continued)

Participants

Cases: children aged $<5$ years, resident in a Derashe rural area, with a report of diarrhoea by mother or caretaker in the 2 weeks preceding the survey, $\mathrm{n}=199$ (after 5 non-responders), $57.8 \%<12$ months

Controls: children aged $<5$ years without diarrhoea in the preceding 2 weeks, randomly chosen from the resident population in the rural kebele, $\mathrm{n}=393$ (after 15 non-responders), $57.5 \%<12$ months
Risk factor of interest:

- disposal of infant faeces elsewhere vs in latrine.

\section{Outcomes}

Diarrhoea: report of diarrhoea by mother or caretaker in the 2 weeks preceding survey

$\begin{array}{ll}\text { Notes } & \text { Location: } 5 \text { rural kebeles, Derashe District, Southern Nations Nationalities and Peoples Region, } \\ \text { Ethiopia }\end{array}$

Length of recruitment: 2 months (January and February 2012)

Publication status: journal

\section{Risk of bias}

\section{Bias}

Authors' judgement Support for judgement

Random sequence genera- Unclear risk NA

tion (selection bias)

Allocation concealment $\quad$ Unclear risk
(selection bias)

Blinding of participants $\quad$ Unclear risk
and personnel (perfor-
mance bias)
All outcomes

Blinding of outcome as-
sessment (detection bias)

\begin{tabular}{lll}
\hline $\begin{array}{l}\text { Incomplete outcome data } \\
\text { (attrition bias) } \\
\text { All outcomes }\end{array}$ & Unclear risk \\
\hline $\begin{array}{l}\text { Selective reporting (re- } \\
\text { porting bias) }\end{array}$ & Unclear risk & NA \\
\hline Other bias & Unclear risk & NA \\
\hline $\begin{array}{l}\text { Similarity of baseline out- } \\
\text { come measurements }\end{array}$ & Unclear risk & NA \\
\hline $\begin{array}{l}\text { Similarity of baseline char- } \\
\text { acteristics }\end{array}$ & Unclear risk & NA \\
\hline $\begin{array}{l}\text { Adequate allocation of in- } \\
\text { tervention concealment } \\
\text { during the study }\end{array}$ & Unclear risk & NA \\
\hline
\end{tabular}


Godana 2013 ETH (Continued)

\begin{tabular}{lll}
$\begin{array}{l}\text { Adequate protection } \\
\text { against contamination }\end{array}$ & Unclear risk & NA \\
\hline $\begin{array}{l}\text { Confounders adequate- } \\
\text { ly adjusted for in analy- } \\
\text { sis/design }\end{array}$ & Unclear risk & NA \\
\hline Recruitment bias & Unclear risk & NA \\
\hline Baseline imbalance & Unclear risk & NA \\
\hline Loss of clusters & Unclear risk & NA \\
\hline Incorrect analysis & Unclear risk & NA \\
\hline
\end{tabular}

\section{Haggerty 1994 DRC}

\begin{tabular}{|c|c|}
\hline Methods & Cluster RCT \\
\hline \multirow[t]{2}{*}{ Participants } & Number: 1764 (after excluding 190 children with < 9 weeks' diarrhoea morbidity data) \\
\hline & Inclusion criteria: children aged 3-35 months \\
\hline \multirow[t]{2}{*}{ Interventions } & $\begin{array}{l}\text { Intervention (9 villages): education intervention to improve personal and domestic hygiene behav- } \\
\text { iour including: disposal of animal faeces; hand washing before meal preparation and after defeca- } \\
\text { tion/washing hands and buttocks of young children after defecation; disposal of children's faeces (em- } \\
\text { phasized digging or improving pit latrines). The messages were delivered by female community volun- } \\
\text { teers in village-wide meetings and small group discussions. }\end{array}$ \\
\hline & $\begin{array}{l}\text { Control ( } 9 \text { villages): education to continue breastfeeding and give rice water during diarrhoea by com- } \\
\text { munity volunteers selected and trained in the same way as intervention. }\end{array}$ \\
\hline
\end{tabular}

Outcomes Diarrhoea incidence, duration of diarrhoeal episodes, number of diarrhoea days. Weekly visit (7-day recall). The mother's own definition of diarrhoea was used, employing the local word ("pulu-pulu") to describe diarrhoea. For each day that diarrhoea occurred, the mother was asked if the child was febrile, whether there was blood in the stool and what (if any) treatment was used. A gap of $\geq 2$ diarrhoea-free days was used to define a new episode of diarrhoea.

Observed hygiene practices (data not presented)

Child growth (data not presented)

Notes Location: 18 rural villages, in Bandundu province, Democratic Republic of the Congo

Length of study: 14 months (October 1987 to December 1988)

Publication status: journal

\section{Risk of bias}

Bias Authors' judgement Support for judgement

Random sequence genera- Unclear risk tion (selection bias)

Quote: "Following the baseline diarrhoeal and observational studies, all sites were ranked from lowest to highest according to age-adjusted mean days of diarrhoea [...] and then one in each pair was chosen at random to receive the intervention, the other to serve as a control." 
Haggerty 1994 DRC (Continued)

\begin{tabular}{lll}
$\begin{array}{l}\text { Allocation concealment } \\
\text { (selection bias) }\end{array}$ & Unclear risk & No details \\
\hline $\begin{array}{l}\text { Blinding of participants } \\
\begin{array}{l}\text { and personnel (perfor- } \\
\text { mance bias) }\end{array}\end{array}$ & High risk & $\begin{array}{l}\text { Control sites also received a placebo intervention but the intervention was } \\
\text { clearly different. }\end{array}$ \\
Alloutcomes & &
\end{tabular}

mance bias)

All outcomes

$\begin{array}{lll}\text { Blinding of outcome as- } & \text { High risk } & \text { Not specified. } \\ \text { sessment (detection bias) } & & \end{array}$

(detection bias)

All outcomes

Incomplete outcome data Low risk $\quad<10 \%$ had $<9$ complete weeks of diarrhoea data.
(attrition bias)

All outcomes

\begin{tabular}{|c|c|c|}
\hline $\begin{array}{l}\text { Selective reporting (re- } \\
\text { porting bias) }\end{array}$ & High risk & $\begin{array}{l}\text { Did not report on behaviour change in the study although it was specified in } \\
\text { methods. }\end{array}$ \\
\hline
\end{tabular}

\begin{tabular}{|c|c|c|}
\hline Other bias & Unclear risk & - \\
\hline $\begin{array}{l}\text { Similarity of baseline out- } \\
\text { come measurements }\end{array}$ & Unclear risk & NA \\
\hline $\begin{array}{l}\text { Similarity of baseline char- } \\
\text { acteristics }\end{array}$ & Unclear risk & NA \\
\hline $\begin{array}{l}\text { Adequate allocation of in- } \\
\text { tervention concealment } \\
\text { during the study }\end{array}$ & Unclear risk & NA \\
\hline $\begin{array}{l}\text { Adequate protection } \\
\text { against contamination }\end{array}$ & Unclear risk & NA \\
\hline $\begin{array}{l}\text { Confounders adequate- } \\
\text { ly adjusted for in analy- } \\
\text { sis/design }\end{array}$ & Unclear risk & NA \\
\hline Recruitment bias & Low risk & $\begin{array}{l}\text { Clusters were not known to be intervention or control during participant re- } \\
\text { cruitment. }\end{array}$ \\
\hline Baseline imbalance & Low risk & Matched clusters according to mean days of diarrhoea. \\
\hline Loss of clusters & Low risk & No reported loss of clusters. \\
\hline Incorrect analysis & Low risk & Clusters were adjusted for in analysis. \\
\hline
\end{tabular}

Hashi 2017 ETH

\begin{tabular}{ll}
\hline Methods & Cluster RCT \\
\hline Participants & Number: 1199 children after excluding 25 children who had migrated \\
& $\begin{array}{l}\text { Inclusion criteria: a HH was considered eligible for the study if they had } \geq 1 \text { child aged 1-59 months liv- } \\
\text { ing in the home and were not a model health extension HH. }\end{array}$
\end{tabular}


Hashi 2017 ETH (Continued)

Interventions

Intervention (12 subkebelles): health education and provision of soap (white bars). The health education was provided by clinical nurse professionals (field workers) and consisted of 12 sessions on key WASH messages and demonstration of HWWS.

Primary caretakers were instructed to keep their water storage container clean and covered, to have a latrine and utilize it properly, and to wash their hands and children's hands ideally with soap after defecation, and before meal preparation and eating. Caregivers received the message to dispose of their children's waste properly via demonstrations and instructions. The messages were to dispose of child waste properly in the waste disposal site (in a waste container at the corner/back of the house) as opposed to the garbage (uncollected waste) and in a latrine (if they had 1) but never in the open field, garbage, or around utensils and kitchen.

Control (12 subkebelles): no intervention

Outcomes Diarrhoea incidence (diarrhoea defined as passage of $\geq 3$ liquid or semi-liquid stool or the passage of $\geq$ 1 liquid or semi-liquid stool with blood or mucous) at 2-week recall

Bacteriological quality of drinking water at $\mathrm{HH}$ level

Lotes
Length of study: 6 months (1 February to 30 July 2015)
Publication status: journal

\section{Risk of bias}

\begin{tabular}{lll}
\hline Bias & Authors' judgement & Support for judgement \\
\hline $\begin{array}{ll}\text { Random sequence genera- } \\
\text { tion (selection bias) }\end{array}$ & Low risk & $\begin{array}{l}\text { Used lottery to allocate the } 2 \text { kebelles groups to intervention or control and } \\
\text { then used computer-generated numbers to pick the subkebelles within the ke- } \\
\text { belles. }\end{array}$
\end{tabular}

\begin{tabular}{|c|c|c|}
\hline $\begin{array}{l}\text { Allocation concealment } \\
\text { (selection bias) }\end{array}$ & Low risk & $\begin{array}{l}\text { Used public lottery to allocate the kebelles but then the assignment was al- } \\
\text { ready known when randomly selecting the subkebelles. }\end{array}$ \\
\hline
\end{tabular}

\begin{tabular}{|c|c|c|}
\hline $\begin{array}{l}\text { Blinding of participants } \\
\text { and personnel (perfor- }\end{array}$ & High risk & $\begin{array}{l}\text { Participants were not blind to the intervention and personnel could have in- } \\
\text { ferred it. }\end{array}$ \\
\hline
\end{tabular}
mance bias)

All outcomes

$\begin{array}{lll}\text { Blinding of outcome as- } & \text { High risk } & \text { Outcome assessors could have inferred intervention allocation. } \\ \text { sessment (detection bias) } & \end{array}$

All outcomes

\begin{tabular}{lll}
\hline $\begin{array}{l}\text { Incomplete outcome data } \\
\text { (attrition bias) } \\
\text { All outcomes }\end{array}$ & Low risk & Few losses to follow-up in both groups. \\
\hline $\begin{array}{l}\text { Selective reporting (re- } \\
\text { porting bias) }\end{array}$ & Low risk & Reported on both prespecified outcomes. \\
\hline $\begin{array}{l}\text { Other bias } \\
\text { Similarity of baseline out- }\end{array}$ & Unclear risk & - \\
\hline come measurements & & - \\
\hline $\begin{array}{l}\text { Similarity of baseline char- } \\
\text { acteristics }\end{array}$ & Unclear risk & - \\
\hline
\end{tabular}


Hashi 2017 ETH (Continued)

\begin{abstract}
Adequate allocation of intervention concealment during the study
\end{abstract}

\begin{tabular}{|c|c|c|}
\hline $\begin{array}{l}\text { Adequate protection } \\
\text { against contamination }\end{array}$ & Unclear risk & - \\
\hline $\begin{array}{l}\text { Confounders adequate- } \\
\text { ly adjusted for in analy- } \\
\text { sis/design }\end{array}$ & Unclear risk & - \\
\hline Recruitment bias & High risk & The subkebelles were assigned to intervention group prior to recruitment. \\
\hline Baseline imbalance & Low risk & $\begin{array}{l}\text { No apparent imbalance in baseline characteristics and analysis controlled for } \\
\text { possible confounders. }\end{array}$ \\
\hline Loss of clusters & Low risk & No loss of clusters reported. \\
\hline Incorrect analysis & Low risk & $\begin{array}{l}\text { Quote: "Generalized estimating equation with log link Poisson distribution } \\
\text { family was used to compute adjusted incidence rate ratio and the correspond- } \\
\text { ing } 95 \% \text { confidence interval of the dependent variable (longitudinal incidence } \\
\text { of diarrhoea) and covariates." }\end{array}$ \\
\hline
\end{tabular}

Heller 2003 BRA

\begin{tabular}{ll}
\hline Methods & Case-control study \\
\hline Participants & Cases: children aged $<5$ years resident in Betim area attending a HC for diarrhoea, $\mathrm{n}=997$, mean age \\
1.72 years, $47.1 \%$ girls \\
Controls: children aged $<5$ years resident in Betim area chosen randomly from a register (used by mu- \\
nicipality with purpose of housing taxes), $\mathrm{n}=999$, mean age 2.63 years, $49.8 \%$ girls
\end{tabular}

\begin{tabular}{ll}
\hline Interventions & Risk factors of interest: \\
- faeces disposal from swaddle disposed elsewhere vs in toilet/latrine.
\end{tabular}

\begin{tabular}{ll}
\hline Outcomes & Diarrhoea: the attendant physician diagnosis of diarrhoea was assumed as the case definition. \\
\hline Notes & Location: 29 HCs in urban area of Betim in Minais Gerais State in South-East Brazil \\
& Length of recruitment: 5 months (November 1993 to April 1994) \\
& Publication status: journal \\
\hline
\end{tabular}

\section{Risk of bias}

\begin{tabular}{lll}
\hline Bias & Authors' judgement & Support for judgement \\
\hline $\begin{array}{l}\text { Random sequence genera- } \\
\text { tion (selection bias) }\end{array}$ & Unclear risk & NA \\
\hline $\begin{array}{l}\text { Allocation concealment } \\
\text { (selection bias) }\end{array}$ & Unclear risk & NA \\
\hline
\end{tabular}


Heller 2003 BRA (Continued)

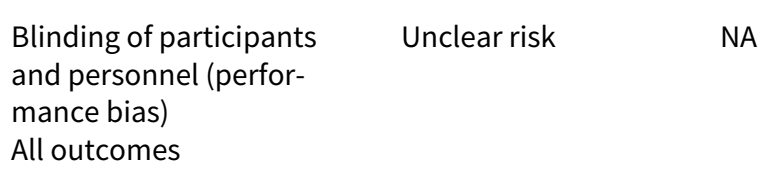

Unclear risk NA

\begin{tabular}{ll}
\hline Blinding of outcome as- & Unclear risk \\
sessment (detection bias) &
\end{tabular}

All outcomes

Incomplete outcome data Unclear risk
(attrition bias)

(attrition bias)

NA

All outcomes

\begin{tabular}{|c|c|c|}
\hline $\begin{array}{l}\text { Selective reporting (re- } \\
\text { porting bias) }\end{array}$ & Unclear risk & NA \\
\hline Other bias & Unclear risk & NA \\
\hline $\begin{array}{l}\text { Similarity of baseline out- } \\
\text { come measurements }\end{array}$ & Unclear risk & NA \\
\hline $\begin{array}{l}\text { Similarity of baseline char- } \\
\text { acteristics }\end{array}$ & Unclear risk & NA \\
\hline $\begin{array}{l}\text { Adequate allocation of in- } \\
\text { tervention concealment } \\
\text { during the study }\end{array}$ & Unclear risk & NA \\
\hline $\begin{array}{l}\text { Adequate protection } \\
\text { against contamination }\end{array}$ & Unclear risk & NA \\
\hline $\begin{array}{l}\text { Confounders adequate- } \\
\text { ly adjusted for in analy- } \\
\text { sis/design }\end{array}$ & Unclear risk & NA \\
\hline Recruitment bias & Unclear risk & NA \\
\hline Baseline imbalance & Unclear risk & NA \\
\hline Loss of clusters & Unclear risk & NA \\
\hline Incorrect analysis & Unclear risk & NA \\
\hline
\end{tabular}

\section{Hoq 2016 BGD}

\begin{tabular}{ll}
\hline Methods & Controlled cohort study \\
\hline Participants & Number: 2037 (total number of children registered in study period) \\
& Inclusion criteria: children aged $<2$ years \\
\hline
\end{tabular}

Interventions Intervention (2 wards): community-based health project + WASH-focused activities.

Community-based health project included: 
- growth monitoring of children aged $<2$ years (quarterly for children aged 0-23 months and biannually for children aged 24-59 months);

- community management of acute malnutrition of children aged < 5 years;

- facility-based Integrated Management of Childhood Illness;

- management of complicated cases of SAM;

- identification of pregnant and lactating women and referral for antenatal and postnatal care;

- behaviour change communication on IYCF practices and reproductive health-related issues, including early marriage and family planning. This messaging included child faeces disposal messages.

WASH-focused activities included:

- construction of 119 community-managed, deep-tube wells and $1280 \mathrm{HH}$ pour-flush twin pit latrines (offset and direct-drop pits according to space constraints). Well and latrine structures were raised to reduce the risk of inaccessibility during flooding;

- monthly neighbourhood WASH committee meetings;

- toilet maintenance promotion;

- monthly children's club meetings for hygiene: included messages about child faeces disposal;

- mother's group meetings: 20 mothers in a session, monthly basis. Discussion point-hygienic latrine, use, operation and maintenance of latrine, safe drinking water, child faeces disposal, hand washing, etc.

Child faeces disposal messages (included in both intervention and control but this was done in more detail in the children's clubs and mother's group meetings):

- throw the child faeces in the latrine immediately after defecation;

- use handy tool (shovel, etc.) to collect and dispose the faeces. Keep the tool clean;

- encourage the children and start practicing defecation in the latrine instead of defecating in yard;

- "child faeces are more harmful than the adult" as the mothers believed that children faeces were less harmful;

- wash hands after dispose of child faeces.

Control (7 wards): community-based health project activities only (same as intervention, included same child faeces disposal messages in mass awareness behaviour change communication campaign).

\begin{tabular}{ll} 
Outcomes & Weight for age (underweight defined as WAZ $<-2$ ) \\
& MUAC (acute malnutrition defined as $M U A C<125 \mathrm{~mm}$ ) \\
\hline Notes & Location: 9 wards in periurban area of Kurigam Municipality, Bangladesh \\
& Length of study: 3 years (November 2011 to December 2014) \\
& Publication status: report
\end{tabular}

\section{Risk of bias}

Bias Authors' judgement Support for judgement

\begin{tabular}{lll}
\hline $\begin{array}{l}\text { Random sequence genera- } \\
\text { tion (selection bias) }\end{array}$ & High risk & $\begin{array}{l}\text { Not random allocation. Out of 9 wards, chose the 2 with highest prevalence of } \\
\text { acute malnutrition to do the WASH intervention. }\end{array}$ \\
\hline $\begin{array}{l}\text { Allocation concealment } \\
\text { (selection bias) }\end{array}$ & High risk & No concealment. \\
\hline $\begin{array}{l}\text { Blinding of participants } \\
\text { and personnel (perfor- } \\
\text { mance bias) } \\
\text { All outcomes }\end{array}$ & Unclear risk
\end{tabular}


Hoq 2016 BGD (Continued)

Blinding of outcome assessment (detection bias)

All outcomes

\section{Unclear risk}

Incomplete outcome data Unclear risk No details of LTFU.
(attrition bias)
All outcomes

\begin{tabular}{ll}
\hline $\begin{array}{l}\text { Selective reporting (re- } \\
\text { porting bias) }\end{array}$ Low risk & Report on nutritional outcomes specified in methods.
\end{tabular}

porting bias)

\begin{tabular}{lll}
\hline Other bias & Unclear risk & - \\
\hline $\begin{array}{l}\text { Similarity of baseline out- } \\
\text { come measurements }\end{array}$ & Low risk & $\begin{array}{l}\text { Prevalence in the outcomes was different. Measured rate of change in out- } \\
\text { come so accounted for it in analysis. }\end{array}$ \\
\hline $\begin{array}{l}\text { Similarity of baseline char- } \\
\text { acteristics }\end{array}$ & High risk & $\begin{array}{l}\text { No baseline measures, although authors stated that the comparison site had } \\
\text { similar ecological and demographic characteristics, childcare practices, and } \\
\text { hygiene behaviour and sanitation coverage and then they confirmed at the } \\
\text { end of the first year of the project. }\end{array}$
\end{tabular}

\begin{tabular}{|c|c|c|}
\hline $\begin{array}{l}\text { Adequate allocation of in- } \\
\text { tervention concealment }\end{array}$ & High risk & $\begin{array}{l}\text { Outcomes were assessed in centres in the wards, they would have known the } \\
\text { allocation of the wards (same implementers). }\end{array}$ \\
\hline
\end{tabular}
during the study

\begin{tabular}{lll}
\hline $\begin{array}{l}\text { Adequate protection } \\
\text { against contamination }\end{array}$ & Unclear risk & No details of how far the intervention and control wards were. \\
\hline $\begin{array}{l}\text { Confounders adequate- } \\
\text { ly adjusted for in analy- } \\
\text { sis/design }\end{array}$ & High risk & No confounders included in the analyses. \\
\hline Recruitment bias & Unclear risk & - \\
\hline Baseline imbalance & Unclear risk & - \\
\hline Loss of clusters & Unclear risk & - \\
\hline Incorrect analysis & Unclear risk & - \\
\hline
\end{tabular}

Huda 2012 BGD

\begin{tabular}{|c|c|}
\hline Methods & Controlled cohort study \\
\hline Participants & $\begin{array}{l}\text { Number: } 1699 \mathrm{HHs} \text { for structured observations and } 1000 \mathrm{HHs} \text { for diarrhoea surveillance. } \\
\text { Inclusion criteria: } \mathrm{HH} \text { with a child aged < } 5 \text { years and a guardian of the child agreed to participate in the } \\
\text { study. }\end{array}$ \\
\hline Interventions & $\begin{array}{l}\text { Intervention ( } 50 \text { communities): SHEWA-B, a large-scale hygiene promotion intervention which engaged } \\
\text { local residents to develop their own community action plans, including targets for improvements in la- } \\
\text { trine coverage and use, access to arsenic-free water, and improved hygiene practices. Community hy- } \\
\text { giene promoters were trained to deliver } 11 \text { key messages including "use hygienic latrine by all family } \\
\text { members including children" and "dispose of children's faeces into hygienic latrines" using HH visits, } \\
\text { courtyard meetings, and different activities, e.g. hygiene fairs, village theatre, group discussions in tea } \\
\text { stalls. Promoters used flip charts and flash cards. }\end{array}$ \\
\hline
\end{tabular}


Huda 2012 BGD (Continued)

Control (50 communities): no major water, sanitation, hygiene programme ongoing.

Outcomes Diarrhoea prevalence. Diarrhoea: passage of $\geq 3$ loose or watery stools in 24-hour period. Monthly visits to ask about episodes of diarrhoea in previous 2 days.

Acute respiratory illness

Observed hygiene behaviours including child faeces disposal, considered appropriate if faeces were observed to be disposed in a toilet or in a specific pit.

\begin{tabular}{l} 
Location: 100 rural villages across Bangladesh \\
Length of study: 24 months (October 2007 to September 2009) \\
Publication status: journal \\
\hline
\end{tabular}

\section{Risk of bias}

\begin{tabular}{lll}
\hline Bias & Authors' judgement & Support for judgement \\
\hline $\begin{array}{l}\text { Random sequence genera- } \\
\text { tion (selection bias) }\end{array}$ & High risk & Intervention not randomly allocated. \\
\hline $\begin{array}{l}\text { Allocation concealment } \\
\text { (selection bias) }\end{array}$ & High risk & Intervention communities were allocated prior to enrolment. \\
\hline $\begin{array}{l}\text { Blinding of participants } \\
\text { and personnel (perfor- } \\
\text { mance bias) } \\
\text { All outcomes }\end{array}$ & Unclear risk & NA \\
\hline
\end{tabular}

\begin{tabular}{lll}
\hline Blinding of outcome as- & Unclear risk & NA \\
sessment (detection bias) & &
\end{tabular}

All outcomes

Incomplete outcome data Unclear risk $\quad$ Numbers of respondents not reported for the health outcomes.
(attrition bias)

All outcomes

\begin{tabular}{lll}
\hline $\begin{array}{l}\text { Selective reporting (re- } \\
\text { porting bias) }\end{array}$ & Low risk & Reported all outcomes prespecified in the methods. \\
\hline Other bias & Unclear risk & - \\
\hline $\begin{array}{l}\text { Similarity of baseline out- } \\
\text { come measurements }\end{array}$ & Unclear risk & $\begin{array}{l}\text { From figure it appears the baseline diarrhoea prevalence was slightly different } \\
\text { but no data presented. }\end{array}$ \\
\hline
\end{tabular}

Similarity of baseline char- Low risk No major differences at baseline.

acteristics

\begin{tabular}{lll}
\hline $\begin{array}{l}\text { Adequate allocation of in- } \\
\text { tervention concealment } \\
\text { during the study }\end{array}$ & High risk & $\begin{array}{l}\text { Although the community monitors were not aware of the hypothesis, they } \\
\text { were aware of allocation to intervention/control group. }\end{array}$ \\
\hline $\begin{array}{l}\text { Adequate protection } \\
\text { against contamination }\end{array}$ & Low risk & $\begin{array}{l}\text { Selected subdistricts in which (quote) "Department of Public Health Engineer- } \\
\text { ing of the Government of Bangladesh, who were responsible for implementing } \\
\text { SHEWA-B and confirmed that there was no similar intervention ongoing." }\end{array}$ \\
\hline
\end{tabular}


Huda 2012 BGD (Continued)

Confounders adequately adjusted for in analy-

High risk No confounders adjusted for in analyses. sis/design

\begin{tabular}{lll}
\hline Recruitment bias & Unclear risk & NA \\
\hline Baseline imbalance & Unclear risk & NA \\
\hline Loss of clusters & Unclear risk & NA \\
\hline Incorrect analysis & Unclear risk & NA \\
\hline
\end{tabular}

Humphrey 2019 ZIM

Methods Cluster RCT

Participants Number: 5280 pregnant women in 211 clusters

Inclusion criteria: women were eligible if they permanently resided in a study cluster and were confirmed pregnant.

Interventions Intervention: 3 arms

- WASH (53 clusters): standard of care messages plus information about safe disposal of faeces in a latrine, HWWS at key times, protection of infants from geophagia and ingestion of animal faeces, chlorination of drinking water (especially for infants), and hygienic preparation of complementary food. Provision of $\mathrm{HH}$ ventilated improved pit latrines, chlorine for water treatment, 2 handwashing facilities, soap and a plastic mat, and play space for infants.

- IYCF (53 clusters): standard care messages plus information about the importance of nutrition for infant health, growth and development, feeding nutrient-dense food and $20 \mathrm{~g}$ of LNS (Nutriset) daily from 6 to 18 months, processing foods, feeding during illness, and dietary diversity. Monthly provision of LNS sachets.

- WASH and IYCF combined (53 clusters): standard of care messages, WASH and IYCF interventions.

Control (52 clusters): standard of care messages, which consisted of village health workers promoting exclusive breastfeeding to 6 months of age, advised on neonatal care, and promoted uptake of Ministry of Health and Child Care services, including antenatal care, immunizations, and family planning.

Putcomes
- mean LAZ score at 18 months
- haemoglobin concentration at 18 months
Secondary outcomes:
- mean WAZ scores
- WHZ scores
- MUAC-for-age Z scores
- head circumference-for-age Z scores
- stunting (LAZ score $<-2$ )
- anaerely stunted (LAZ score $<-3$ )
- severely anaemogic (haemogin concentration $<105 \mathrm{~g} / \mathrm{L}$ )
- underweight (i.e. WAZ scores $<-2$ )
- wasted (WHZ scores $<-2$ )


Humphrey 2019 ZIM (Continued)

- mean prevalence of diarrhoea (based on 7-day maternal history of infant aged 12 months and 18 months)

- mean prevalence of dysentery

- mean prevalence of ARI

- cumulative mortality up to age 18 months

- infant environmental enteric dysfunction

- process and intermediate outcomes

Notes

Location: rural districts of Chirumanzu and Shurugwi, Zimbabwe

Length of study: 18 months' follow-up (recruitment: November 2012 to March 2015, end of follow-up: July 2017)

Publication status: journal

\section{Risk of bias}

\begin{tabular}{|c|c|c|}
\hline Bias & Authors' judgement & Support for judgement \\
\hline $\begin{array}{l}\text { Random sequence genera- } \\
\text { tion (selection bias) }\end{array}$ & Low risk & Allocation was random. \\
\hline $\begin{array}{l}\text { Allocation concealment } \\
\text { (selection bias) }\end{array}$ & Low risk & $\begin{array}{l}\text { Quote: "the final allocation was selected at a public randomization event at- } \\
\text { tended by elected representatives of the study districts." }\end{array}$ \\
\hline $\begin{array}{l}\text { Blinding of participants } \\
\text { and personnel (perfor- } \\
\text { mance bias) } \\
\text { All outcomes }\end{array}$ & High risk & $\begin{array}{l}\text { Quote: "Masking of participants and fieldworkers was not possible because of } \\
\text { the obvious visual differences between interventions, but investigators were } \\
\text { blinded to treatment groups until the final analysis of each prespecified out- } \\
\text { come." }\end{array}$ \\
\hline $\begin{array}{l}\text { Blinding of outcome as- } \\
\text { sessment (detection bias) } \\
\text { All outcomes }\end{array}$ & High risk & $\begin{array}{l}\text { Quote: "Masking of participants and fieldworkers was not possible because of } \\
\text { the obvious visual differences between interventions, but investigators were } \\
\text { blinded to treatment groups until the final analysis of each prespecified out- } \\
\text { come." }\end{array}$ \\
\hline $\begin{array}{l}\text { Incomplete outcome data } \\
\text { (attrition bias) } \\
\text { All outcomes }\end{array}$ & Low risk & Similar missing data across groups. \\
\hline $\begin{array}{l}\text { Selective reporting (re- } \\
\text { porting bias) }\end{array}$ & Unclear risk & $\begin{array}{l}\text { Reported on primary outcomes but future publications will cover additional } \\
\text { prespecified outcomes. }\end{array}$ \\
\hline Other bias & Unclear risk & - \\
\hline $\begin{array}{l}\text { Similarity of baseline out- } \\
\text { come measurements }\end{array}$ & Unclear risk & - \\
\hline $\begin{array}{l}\text { Similarity of baseline char- } \\
\text { acteristics }\end{array}$ & Unclear risk & - \\
\hline $\begin{array}{l}\text { Adequate allocation of in- } \\
\text { tervention concealment } \\
\text { during the study }\end{array}$ & Unclear risk & - \\
\hline $\begin{array}{l}\text { Adequate protection } \\
\text { against contamination }\end{array}$ & Unclear risk & - \\
\hline
\end{tabular}


Humphrey 2019 ZIM (Continued)

\begin{tabular}{lll}
$\begin{array}{l}\text { Confounders adequate- } \\
\text { ly adjusted for in analy- } \\
\text { sis/design }\end{array}$ & Unclear risk & - \\
\hline Recruitment bias & High risk & $\begin{array}{l}\text { Participants were prospectively enrolled into the study once the clusters had } \\
\text { already been randomized to intervention groups. }\end{array}$ \\
\hline Baseline imbalance & Low risk & Most baseline characteristics of enrolled HHs were similar across groups. \\
\hline Loss of clusters & Low risk & $<10 \%$ (only 2 clusters lost in the IYCF group) clusters LTFU. \\
\hline Incorrect analysis & Low risk & Accounted for clustering.
\end{tabular}

Jinadu 2007 NGR

\begin{tabular}{ll}
\hline Methods & Cluster RCT \\
\hline Participants & Number: 514 \\
& Inclusion criteria: mothers of children aged $<5$ years \\
& Intervention group: $65.8 \%$ aged $\leq 12$ months; control group: $65.9 \%$ aged $\leq 12$ months \\
\hline Interventions & Intervention ( 5 villages): educational intervention programme to promote the hygienic disposal of chil- \\
dren's faeces: & educating mothers about the hygienic use of potties for the disposal of children's faeces; \\
- & discouraging children from defecating around HHs; \\
& latrines by members of the communities; \\
- educating mothers to HWWS and water after going to toilet and after cleaning up children's faeces. \\
Control (5 villages): no health promotion activities
\end{tabular}

Outcomes Hygienic behaviours: child defecation pattern, $\mathrm{HHs}$ with sanitary latrines, $\mathrm{HH}$ using potties, $\mathrm{HH}$ where mothers HWWS after cleaning child faeces and defecation, $\mathrm{HH}$ with no children's faeces lying around.

Notes

Location: 10 rural villages in Osun State, Nigeria

Length of study: 12 months

Publication status: journal

\section{Risk of bias}

\begin{tabular}{lll}
\hline Bias & Authors' judgement & Support for judgement \\
\hline $\begin{array}{l}\text { Random sequence genera- } \\
\text { tion (selection bias) }\end{array}$ & Unclear risk & No description. \\
\hline $\begin{array}{l}\text { Allocation concealment } \\
\text { (selection bias) }\end{array}$ & Unclear risk & No description. \\
\hline
\end{tabular}

Blinding of participants High risk No mention of blinding.
and personnel (perfor-
mance bias)


Jinadu 2007 NGR (Continued)

All outcomes

\begin{tabular}{|c|c|c|}
\hline $\begin{array}{l}\text { Blinding of outcome as- } \\
\text { sessment (detection bias) }\end{array}$ & High risk & $\begin{array}{l}\text { No mention of blinding and no mention of relation of interviewers in relation } \\
\text { to trial. }\end{array}$ \\
\hline
\end{tabular}

All outcomes

Incomplete outcome data Unclear risk No data on loss to follow-up.
(attrition bias)

All outcomes

Selective reporting (re- Low risk Present outcomes prespecified in the methods.
porting bias)

porting bias)

\begin{tabular}{|c|c|c|}
\hline Other bias & Unclear risk & - \\
\hline $\begin{array}{l}\text { Similarity of baseline out- } \\
\text { come measurements }\end{array}$ & Unclear risk & NA \\
\hline $\begin{array}{l}\text { Similarity of baseline char- } \\
\text { acteristics }\end{array}$ & Unclear risk & NA \\
\hline $\begin{array}{l}\text { Adequate allocation of in- } \\
\text { tervention concealment } \\
\text { during the study }\end{array}$ & Unclear risk & NA \\
\hline $\begin{array}{l}\text { Adequate protection } \\
\text { against contamination }\end{array}$ & Unclear risk & NA \\
\hline $\begin{array}{l}\text { Confounders adequate- } \\
\text { ly adjusted for in analy- } \\
\text { sis/design }\end{array}$ & Unclear risk & NA \\
\hline Recruitment bias & High risk & $\begin{array}{l}\text { Clusters were known to be intervention or control during participant recruit- } \\
\text { ment (only selected participants to measure outcomes after intervention had } \\
\text { been implemented). }\end{array}$ \\
\hline Baseline imbalance & Unclear risk & No baseline. \\
\hline Loss of clusters & Low risk & No reported loss of villages. \\
\hline Incorrect analysis & High risk & No correction for clustering. \\
\hline
\end{tabular}

Knight 1992 MAL

\begin{tabular}{ll}
\hline Methods & Case-control study (matched) \\
\hline Participants & $\begin{array}{l}\text { Cases: child aged } 4-59 \text { months resident in Tumpat, Malaysia, who presented at a HC with } \geq 3 \text { loose } \\
\text { stools in } 24 \text { hours and duration of diarrhoea }<2 \text { weeks (and without measles, malaria, urinary tract in- } \\
\text { fection, ARI, acute otitis media, or antibiotics use in the previous } 2 \text { weeks), } \mathrm{n}=98 \text { (after } 2 \text { left area). }\end{array}$ \\
& $\begin{array}{l}\text { Controls: randomly selected from children resident in Tumpat, Malaysia, registered at a HC usually } \\
\text { within } 1 \text { week of their respective case child, with a condition other than diarrhoea, and age ( } \pm 6 \text { weeks } \\
\text { for children aged }<1 \text { year, } \pm 3 \text { months for children aged } 1 \text { year, } \pm 6 \text { months for children aged } \geq 2 \text { years) } \\
\text { and sex matched to case child and who did not have skin infection, conjunctivitis, or worm infestation } \\
\text { as their provisional diagnosis, } \mathrm{n}=98 .\end{array}$ \\
\end{tabular}


Knight 1992 MAL (Continued)

Interventions

Risk factor of interest:

- indiscriminate child defecation (anywhere other than a toilet or nappy).

\begin{tabular}{ll}
\hline Outcomes & Diarrhoea: $\geq 3$ loose stools in 24 hours \\
\hline Notes & Location: 5 HCs, Tumpat rural district, Malaysia \\
& Length of recruitment: 2 months (February and March 1989) \\
& Publication status: journal
\end{tabular}

\section{Risk of bias}

\begin{tabular}{lll}
\hline Bias & Authors' judgement & Support for judgement \\
\hline $\begin{array}{l}\text { Random sequence genera- } \\
\text { tion (selection bias) }\end{array}$ & Unclear risk & NA \\
\hline $\begin{array}{l}\text { Allocation concealment } \\
\text { (selection bias) }\end{array}$ & Unclear risk & NA \\
\hline $\begin{array}{l}\text { Blinding of participants } \\
\text { and personnel (perfor- } \\
\text { mance bias) } \\
\text { All outcomes }\end{array}$ & Unclear risk & NA \\
\hline
\end{tabular}

\begin{tabular}{lll}
\hline Blinding of outcome as- & Unclear risk & NA \\
sessment (detection bias) &
\end{tabular}

All outcomes

Incomplete outcome data Unclear risk
(attrition bias)
All outcomes

\begin{tabular}{ll} 
All outcomes & \\
\hline $\begin{array}{l}\text { Selective reporting (re- } \\
\text { porting bias) }\end{array}$ & Unclear risk
\end{tabular}

\begin{tabular}{lll}
\hline Other bias & Unclear risk & NA \\
\hline $\begin{array}{l}\text { Similarity of baseline out- } \\
\text { come measurements }\end{array}$ & Unclear risk & NA \\
\hline $\begin{array}{l}\text { Similarity of baseline char- } \\
\text { acteristics }\end{array}$ & Unclear risk & NA \\
\hline $\begin{array}{l}\text { Adequate allocation of in- } \\
\text { tervention concealment } \\
\text { during the study }\end{array}$ & Unclear risk & NA \\
\hline $\begin{array}{l}\text { Adequate protection } \\
\text { against contamination }\end{array}$ & Unclear risk & NA \\
\hline $\begin{array}{l}\text { Confounders adequate- } \\
\text { ly adjusted for in analy- } \\
\text { sis/design }\end{array}$ & Unclear risk & NA \\
\hline \begin{tabular}{l} 
Recruitment bias \\
\hline
\end{tabular} & Unclear risk & NA \\
\hline
\end{tabular}


Knight 1992 MAL (Continued)

\begin{tabular}{lll} 
Baseline imbalance & Unclear risk & NA \\
\hline Loss of clusters & Unclear risk & NA \\
\hline Incorrect analysis & Unclear risk & NA \\
\hline
\end{tabular}

\section{Kotch 2007 USA}

\begin{tabular}{|c|c|}
\hline Methods & Cluster RCT \\
\hline \multirow[t]{3}{*}{ Participants } & Number: 388 children \\
\hline & $\begin{array}{l}\text { Inclusion criteria: children were expected to remain assigned to the same classroom throughout the } 7 \text { - } \\
\text { month study period and be } 36 \text { months of age at the end of data collection and that } \geq 1 \text { family contact } \\
\text { could participate in a telephone survey in English. Siblings were allowed to participate when they also } \\
\text { attended the study centre and met the eligibility criteria. }\end{array}$ \\
\hline & $\begin{array}{l}\text { Intervention group: mean age of children }=21.26 \text { months and } 6.39 \text { boys per class. Control group: mean } \\
\text { age }=21.41 \text { months and } 3.61 \text { boys per class. }\end{array}$ \\
\hline \multirow[t]{2}{*}{ Interventions } & $\begin{array}{l}\text { Intervention ( } 23 \text { childcare centres): staff were trained using the 'Keep It Clean' training module to im- } \\
\text { prove and standardize the handwashing, sanitation, nappy changing, and food-preparation proce- } \\
\text { dures. Nappy changing, handwashing, and food-preparation equipment with impermeable, seamless } \\
\text { surfacing were provided. In addition, automatic faucets and foot-activated, roll-out waste bins for nap- } \\
\text { py disposal were provided. }\end{array}$ \\
\hline & $\begin{array}{l}\text { Control ( } 23 \text { childcare centres): staff were trained using the 'Keep It Clean' training module but received } \\
\text { no equipment. }\end{array}$ \\
\hline
\end{tabular}

Sutcomes
Severe diarrhoea incidence: any loose, watery stool that if conta
every 2 weeks.
Number of days sick
Number of days child absent for centre because of illness
Number of days parents missed work because of child illness
Sick days of caregivers in centres
Nappy and food preparations practices

Notes Location: 46 childcare centres in 21 counties, NC, USA

Length of study: 7 months' follow-up (December 2002 to July 2003)

Publication status: journal

\section{Risk of bias}

\begin{tabular}{lll}
\hline Bias & Authors' judgement & Support for judgement \\
\hline $\begin{array}{ll}\text { Random sequence genera- } \\
\text { tion (selection bias) }\end{array}$ & Unclear risk & Comment: no details. \\
& & $\begin{array}{l}\text { Quote: "from each pair 1 centre was randomly selected as intervention cen- } \\
\text { tre." }\end{array}$ \\
\hline
\end{tabular}


Kotch 2007 USA (Continued)

\begin{tabular}{lll}
$\begin{array}{l}\text { Allocation concealment } \\
\text { (selection bias) }\end{array}$ & Unclear risk & No details. \\
\hline $\begin{array}{l}\text { Blinding of participants } \\
\text { and personnel (perfor- } \\
\text { mance bias) }\end{array}$ & High risk & No blinding. \\
All outcomes & &
\end{tabular}

\begin{tabular}{|c|c|c|}
\hline $\begin{array}{l}\text { Blinding of outcome as- } \\
\text { sessment (detection bias) }\end{array}$ & Unclear risk & $\begin{array}{l}\text { No blinding specified although as the outcome was assessed by telephone by } \\
\text { the survey research unit at UNC, it could have been blinded. }\end{array}$ \\
\hline
\end{tabular}

All outcomes

$\begin{array}{ll}\text { Incomplete outcome data } & \text { Low risk } \\ \text { (attrition bias) } & 121 \text { children LTFU from } 388 \text { children in total ( } 31 \% \text { LTFU) but the numbers were } \\ \text { All outcomes } & \text { similar in intervention and control groups ( } 59 \text { control and } 62 \text { intervention LT- } \\ & \text { FU, not significant). }\end{array}$

Selective reporting (re- Low risk Report on prespecified outcomes in paper.

porting bias)

\begin{tabular}{|c|c|c|}
\hline Other bias & Unclear risk & - \\
\hline $\begin{array}{l}\text { Similarity of baseline out- } \\
\text { come measurements }\end{array}$ & Unclear risk & NA \\
\hline $\begin{array}{l}\text { Similarity of baseline char- } \\
\text { acteristics }\end{array}$ & Unclear risk & NA \\
\hline $\begin{array}{l}\text { Adequate allocation of in- } \\
\text { tervention concealment } \\
\text { during the study }\end{array}$ & Unclear risk & NA \\
\hline $\begin{array}{l}\text { Adequate protection } \\
\text { against contamination }\end{array}$ & Unclear risk & NA \\
\hline $\begin{array}{l}\text { Confounders adequate- } \\
\text { ly adjusted for in analy- } \\
\text { sis/design }\end{array}$ & Unclear risk & NA \\
\hline Recruitment bias & High risk & $\begin{array}{l}\text { Appeared the directors recruiting the children were aware of which cluster the } \\
\text { centre was in. }\end{array}$ \\
\hline Baseline imbalance & High risk & $\begin{array}{l}\text { Baseline imbalances in mean classroom enrolment, mean number of children } \\
\text { participating in the study per classroom, mean number of boys enrolled in the } \\
\text { classroom, and mean number of boys participating in the study per classroom. } \\
\text { Because the direction of the differences, more boys and more total children in } \\
\text { intervention classrooms and did not adjust in analysis. }\end{array}$ \\
\hline Loss of clusters & Low risk & No loss of centres reported. \\
\hline Incorrect analysis & Low risk & Adjusted for clustering at class level by adding random effect. \\
\hline
\end{tabular}

Luby 2014 BGD

\begin{tabular}{ll}
\hline Methods Controlled cohort study \\
\hline
\end{tabular}


Luby 2014 BGD (Continued)

Participants
Number: 1000 urban $\mathrm{HH}$ and 1000 rural HHs for diarrhoea surveillance, $1000 \mathrm{HHs}$ for anthropometry and $1000 \mathrm{HHs}$ for structured observations

Inclusion criteria: $\mathrm{HH}$ with a child aged $<5$ years and a guardian of the child agreed to participate in the study.

\begin{abstract}
Interventions
Intervention: SHEWA-B, improved from findings in Huda 2012 BGD. Changes in the intervention included a mass media campaign including radio spots across 6 regional channels from November 2011 to February 2012 encouraging HWWS before food, after defecation, and after cleaning a child and video spots on 5 television stations (November 2011 to February 2012) encouraging HWWS, using sanitary latrines for defecation and discarding child faeces and keeping latrines clean to reduce bad smells and flies. A second series of videos encouraged testing tube-wells for arsenic and using arsenic-free water for cooking and drinking. The intervention target population also expanded to include urban $\mathrm{HHS}$.

Control: no major water, sanitation, hygiene programme ongoing.
\end{abstract}

Outcomes
Monthly visits to ask about episodes of diarrhoea in previous 2 days.
Acute respiratory illness
Anthropometry
Observed hygiene and sanitation behaviours including child faeces disposal, considered appropriate if
faeces were observed to be disposed in a toilet or in a specific pit.
Water quality
Location: rural villages and urban slums across Bangladesh
Length of study: 60 months in total (October 2007 to September 2012). This study reported from 2011
to 2012.
Publication status: report

\title{
Risk of bias
}

\begin{tabular}{lll}
\hline Bias & Authors' judgement & Support for judgement \\
\hline $\begin{array}{l}\text { Random sequence genera- } \\
\text { tion (selection bias) }\end{array}$ & High risk & Intervention not randomly allocated. \\
\hline $\begin{array}{l}\text { Allocation concealment } \\
\text { (selection bias) }\end{array}$ & High risk & Intervention communities were allocated prior to enrolment. \\
\hline $\begin{array}{l}\text { Blinding of participants } \\
\begin{array}{l}\text { and personnel (perfor- } \\
\text { mance bias) } \\
\text { All outcomes }\end{array}\end{array}$ & Unclear risk & \\
\hline
\end{tabular}

Blinding of outcome as-
sessment (detection bias) Unclear risk NA

Incomplete outcome data Unclear risk Numbers of respondents is not reported for the health outcomes.
(attrition bias)

All outcomes

Selective reporting (re- Low risk Report on all outcomes prespecified in methods. porting bias) 
Luby 2014 BGD (Continued)

\begin{tabular}{lll} 
Other bias & Unclear risk & - \\
\hline $\begin{array}{l}\text { Similarity of baseline out- } \\
\text { come measurements }\end{array}$ & Unclear risk & $\begin{array}{l}\text { In figure it looks like the baseline diarrhoea prevalence was different but no } \\
\text { data presented. }\end{array}$ \\
\hline $\begin{array}{l}\text { Similarity of baseline char- } \\
\text { acteristics }\end{array}$ & Low risk & $\begin{array}{l}\text { No major differences at baseline; however, the control and intervention HHs at } \\
\text { follow-up were different. }\end{array}$
\end{tabular}

\begin{tabular}{lll}
\hline $\begin{array}{l}\text { Adequate allocation of in- } \\
\text { tervention concealment } \\
\text { during the study }\end{array}$ & High risk & Were aware of allocation to intervention/control group. \\
\hline $\begin{array}{l}\text { Adequate protection } \\
\text { against contamination }\end{array}$ & Low risk & $\begin{array}{l}\text { Selected subdistricts in which "Department of Public Health Engineering of the } \\
\text { Government of Bangladesh, who were responsible for implementing SHEWA-B } \\
\text { and confirmed that there was no similar intervention ongoing." }\end{array}$
\end{tabular}

Confounders adequate- High risk $\quad$ No confounders adjusted for in analyses.
ly adjusted for in analy-
or in analysis/design

\begin{tabular}{lll}
\hline Recruitment bias & Unclear risk & NA \\
\hline Baseline imbalance & Unclear risk & NA \\
\hline Loss of clusters & Unclear risk & NA \\
\hline Incorrect analysis & Unclear risk & NA \\
\hline
\end{tabular}

\section{Luby 2018 BGD}

\begin{tabular}{|c|c|}
\hline Methods & Cluster RCT \\
\hline \multirow[t]{2}{*}{ Participants } & $\begin{array}{l}\text { Number: } 14,425 \text { children ( } 7331 \text { in year } 1,7094 \text { in year } 2 \text { ) with diarrhoea data at year } 1 \text { or } 2 \text { in all arms. } \\
\text { Children who were in utero or aged }<3 \text { years at enrolment }\end{array}$ \\
\hline & $\begin{array}{l}\text { Inclusion criteria: children of enrolled pregnant women (index children) were eligible for inclusion if } \\
\text { their mother was planning to live in the study village for the next } 2 \text { years, regardless of where she gave } \\
\text { birth. Only } 1 \text { pregnant woman (in the first } 2 \text { trimesters of her pregnancy) was enrolled per compound, } \\
\text { but if she gave birth to twins, both children were enrolled. Children aged < } 3 \text { years at enrolment and } \\
\text { lived in the compound were included in diarrhoea measurements. }\end{array}$ \\
\hline
\end{tabular}

Interventions Intervention: 6 intervention arms

- Water quality (90 clusters, each consisting of 8 compounds): chlorine tablets (Aquatabs; NaDCC) and a safe storage vessel to treat and store drinking water. Behaviour change messaging to treat drinking water for all children aged $<36$ months.

- Sanitation (90 clusters, each consisting of 8 compounds): provision of free child potties, sani-scoop hoes to remove faeces from $\mathrm{HH}$ environments, and latrine upgrades or construction if the compound did not have one. For promotion, local promoters visited study compounds to deliver behaviour change messages on the use of latrines for defecation and the removal of human and animal faeces from the compound.

- Hand washing (90 clusters, each consisting of 8 compounds): handwashing stations, soapy water bottles, detergent soap to supply soapy water. Behaviour change messages focused on HWWS at critical times around food preparation, defecation, and contact with faeces.

- Combined WASH (90 clusters, each consisting of 8 compounds): water quality, sanitation, and handwashing components. 
- Nutrition (90 clusters, each consisting of 8 compounds): LNS given twice daily for children aged 6-24 months. The key behavioural recommendations were: exclusive breastfeeding up to 180 days, introducing diverse complementary food at 6 months, feed LNS from 6-24 months.

- Nutrition + combined WASH (90 clusters, each consisting of 8 compounds).

Control (180 clusters, each consisting of 8 compounds): no intervention

Primary outcomes:
- LAZ-scores (measured 24 months after intervention)
- $\quad$ diarrhoea prevalence ( defined as $\geq 3$ loose or watery stools in 24 hours or $\geq 1$ stools with blood in 24
hours. Diarrhoea was measured in interviews using caregiver-reported symptoms with 7 -day recall,
measured 12 and 24 months after intervention).
Secondary outcomes:
- LAZ scores
- weight-for-length Z score
- WAZ score
- head circumference-for-age Z score
- prevalence of moderate stunting (LAZ score <-2)
- severe stunting (LAZ score <-3)
- underweight (WAZ score <-2)
- wasting (WAZ score <-2)
- enteropathy biomarkers (measured 12 and 24 months after intervention)
- Ages and Stages Questionnaire Child Development Scores (measured 24 months after intervention)
Tertiary outcome:
- all-cause mortality among index children

Notes Location: rural villages, Gazipur, Kishoreganj, Mymensingh, and Tangail districts, Bangladesh

Length of study: 37 months (recruitment 31 May 2012 to 7 July 2013 followed by 2 years' follow-up)

Publication status: journal

\section{Risk of bias}

\begin{tabular}{|c|c|c|}
\hline Bias & Authors' judgement & Support for judgement \\
\hline $\begin{array}{l}\text { Random sequence genera- } \\
\text { tion (selection bias) }\end{array}$ & Low risk & $\begin{array}{l}\text { Quote: "Clusters were randomly allocated to treatment using a random num- } \\
\text { ber generator by a coinvestigator at University of California, Berkeley." }\end{array}$ \\
\hline $\begin{array}{l}\text { Allocation concealment } \\
\text { (selection bias) }\end{array}$ & Low risk & $\begin{array}{l}\text { Quote: "Clusters were randomly allocated to treatment using a random num- } \\
\text { ber generator by a coinvestigator at University of California, Berkeley." }\end{array}$ \\
\hline $\begin{array}{l}\text { Blinding of participants } \\
\text { and personnel (perfor- } \\
\text { mance bias) } \\
\text { All outcomes }\end{array}$ & High risk & $\begin{array}{l}\text { Quote: "Interventions included distinct visible components so neither partici- } \\
\text { pants nor data collectors were masked to intervention assignment, although } \\
\text { the data collection and intervention teams were different individuals." }\end{array}$ \\
\hline $\begin{array}{l}\text { Blinding of outcome as- } \\
\text { sessment (detection bias) } \\
\text { All outcomes }\end{array}$ & High risk & $\begin{array}{l}\text { Quote: "Interventions included distinct visible components so neither partici- } \\
\text { pants nor data collectors were masked to intervention assignment, although } \\
\text { the data collection and intervention teams were different individuals." }\end{array}$ \\
\hline $\begin{array}{l}\text { Incomplete outcome data } \\
\text { (attrition bias) } \\
\text { All outcomes }\end{array}$ & Low risk & Loss to follow-up was fairly balanced across groups. \\
\hline
\end{tabular}


Luby 2018 BGD (Continued)

\begin{tabular}{|c|c|c|}
\hline $\begin{array}{l}\text { Selective reporting (re- } \\
\text { porting bias) }\end{array}$ & Unclear risk & $\begin{array}{l}\text { Reported on primary outcomes but future publications will cover additional } \\
\text { prespecified outcomes. }\end{array}$ \\
\hline
\end{tabular}
porting bias) prespecified outcomes.

\begin{tabular}{|c|c|c|}
\hline Other bias & Unclear risk & - \\
\hline $\begin{array}{l}\text { Similarity of baseline out- } \\
\text { come measurements }\end{array}$ & Unclear risk & - \\
\hline $\begin{array}{l}\text { Similarity of baseline char- } \\
\text { acteristics }\end{array}$ & Unclear risk & - \\
\hline $\begin{array}{l}\text { Adequate allocation of in- } \\
\text { tervention concealment } \\
\text { during the study }\end{array}$ & Unclear risk & - \\
\hline $\begin{array}{l}\text { Adequate protection } \\
\text { against contamination }\end{array}$ & Unclear risk & - \\
\hline $\begin{array}{l}\text { Confounders adequate- } \\
\text { ly adjusted for in analy- } \\
\text { sis/design }\end{array}$ & Unclear risk & - \\
\hline Recruitment bias & Low risk & Participants were enrolled prior to knowing allocation of intervention. \\
\hline Baseline imbalance & Low risk & Baseline characteristics of enrolled HHs were similar across group. \\
\hline Loss of clusters & Low risk & No reported loss of cluster. \\
\hline Incorrect analysis & Low risk & Low, accounted for clustering. \\
\hline
\end{tabular}

Mathew 2004 ZIM

\begin{tabular}{ll}
\hline Methods & Controlled cross-sectional study \\
\hline Participants & Number: 115 respondents \\
& Inclusion criteria: no details \\
\hline Interventions & $\begin{array}{l}\text { Intervention (2 villages): CHCs: structured weekly course of participatory health education classes. } 15 \\
\text { health topics covered using PHAST techniques, within the hygiene lesson cover disposal of toddler's } \\
\text { faeces in a latrine. }\end{array}$ \\
& Control (2 villages): no CHCs \\
\hline Outcomes & $\begin{array}{l}\text { Knowledge of risks and practices including: percentage of children aged }<5 \text { years present at the time of } \\
\text { observations not using a latrine. }\end{array}$ \\
\hline Notes & Location: 4 rural villages, Bikita district, Zimbabwe \\
& $\begin{array}{l}\text { Length of study: not specified } \\
\text { Publication status: PhD thesis }\end{array}$
\end{tabular}

\section{Risk of bias}

Bias Authors' judgement Support for judgement


Mathew 2004 ZIM (Continued)

Random sequence genera- High risk Intervention not randomly allocated. tion (selection bias)

Allocation concealment High risk $\quad$ Allocation not concealed.
(selection bias)

(selection bias)

Blinding of participants $\quad$ Unclear risk NA
and personnel (perfor-
mance bias)
All outcomes

$\begin{array}{ll}\text { Blinding of outcome as- } & \text { Unclear risk }\end{array}$

All outcomes

Incomplete outcome data Unclear risk Non-response data not reported.
(attrition bias)

All outcomes

\begin{tabular}{|c|c|c|}
\hline $\begin{array}{l}\text { Selective reporting (re- } \\
\text { porting bias) }\end{array}$ & Unclear risk & Tool for observations not available. \\
\hline Other bias & Unclear risk & - \\
\hline $\begin{array}{l}\text { Similarity of baseline out- } \\
\text { come measurements }\end{array}$ & Unclear risk & NA, not relevant to design. \\
\hline $\begin{array}{l}\text { Similarity of baseline char- } \\
\text { acteristics }\end{array}$ & Unclear risk & NA, not relevant to design. \\
\hline $\begin{array}{l}\text { Adequate allocation of in- } \\
\text { tervention concealment } \\
\text { during the study }\end{array}$ & High risk & No blinding. \\
\hline $\begin{array}{l}\text { Adequate protection } \\
\text { against contamination }\end{array}$ & Unclear risk & No details about distance or possibility for contamination. \\
\hline $\begin{array}{l}\text { Confounders adequate- } \\
\text { ly adjusted for in analy- } \\
\text { sis/design }\end{array}$ & High risk & No adjustments for any confounders. \\
\hline Recruitment bias & Unclear risk & NA \\
\hline Baseline imbalance & Unclear risk & NA \\
\hline Loss of clusters & Unclear risk & NA \\
\hline Incorrect analysis & Unclear risk & NA \\
\hline
\end{tabular}

\section{Maung 1992a MYA}

\begin{tabular}{ll}
\hline Methods & Case-control study \\
\hline Participants & $\begin{array}{l}\text { Cases: children aged } 1-59 \text { months admitted to paediatric wards of the North Okkalapa General Hospi- } \\
\text { tal, or presented at the urban HC or at the emergency department of the North Okkalapa General Hos- } \\
\text { pital, for persistent diarrhoea and PEM, } \mathrm{n}=67 .\end{array}$ \\
\hline
\end{tabular}


Maung 1992a MYA (Continued)

Controls: age- and sex-matched apparently healthy children within the neighbourhood of the case children (usually within the same street, selected from houses with structural appearances similar to that of the cases). The control children had no diarrhoea or PEM in the last 2 months, $n=67$.

\begin{tabular}{ll}
\hline Interventions & $\begin{array}{l}\text { Risk factor of interest: } \\
\text { - faeces were disposed around house vs latrine (assumed this was reporting data on child faeces dis- } \\
\text { posal as the risk factors were all related to child defecation but it was not stated in the paper). }\end{array}$ \\
\hline Outcomes & $\begin{array}{l}\text { Persistent diarrhoea: passage of watery or loose stools (with or without mucous) }>3 \text { times/day on most } \\
\text { days lasting } \geq 14 \text { days during the last } 2 \text { months, with an interval of } \leq 6 \text { days during which loose motions } \\
\text { were }<3 \text { times/day. }\end{array}$ \\
& $\begin{array}{l}\text { PEM: children with kwashiorkor, marasmic kwashiorkor or marasmus, or children with weight-for-age }< \\
2 \text { SD below the median National Centre for Health Statistics reference. }\end{array}$ \\
\hline Notes & Location: town hospital and urban HC, Yangon region, Myanmar \\
& Length of recruitment: not specified \\
Publication status: journal
\end{tabular}

\section{Risk of bias}

\section{Bias}

Random sequence genera- Unclear risk

tion (selection bias)

\begin{tabular}{lll}
\hline $\begin{array}{l}\text { Allocation concealment } \\
\text { (selection bias) }\end{array}$ & Unclear risk
\end{tabular}

Blinding of participants Unclear risk
and personnel (perfor-
mance bias)
All outcomes

NA

NA

NA

\section{Authors' judgement Support for judgement}

NA

$\begin{array}{lll}\text { Blinding of outcome as- } & \text { Unclear risk }\end{array}$

sessment (detection bias)

All outcomes

Incomplete outcome data Unclear risk NA

(attrition bias)

All outcomes

Selective reporting (re- Unclear risk
porting bias)

\begin{tabular}{lll}
\hline Other bias & Unclear risk & NA \\
\hline $\begin{array}{l}\text { Similarity of baseline out- } \\
\text { come measurements }\end{array}$ & Unclear risk & $N A$ \\
\hline $\begin{array}{l}\text { Similarity of baseline char- } \\
\text { acteristics }\end{array}$ & Unclear risk & NA \\
\hline
\end{tabular}

Adequate allocation of in- Unclear risk NA
tervention concealment
during the study

Interventions to improve disposal of child faeces for preventing diarrhoea and soil-transmitted helminth infection (Review)

Collaboration. 
Maung 1992a MYA (Continued)

\begin{tabular}{lll}
$\begin{array}{l}\text { Adequate protection } \\
\text { against contamination }\end{array}$ & Unclear risk & NA \\
\hline $\begin{array}{l}\text { Confounders adequate- } \\
\text { ly adjusted for in analy- } \\
\text { sis/design }\end{array}$ & Unclear risk & NA \\
\hline Recruitment bias & Unclear risk & NA \\
\hline Baseline imbalance & Unclear risk & NA \\
\hline Loss of clusters & Unclear risk & NA \\
\hline Incorrect analysis & Unclear risk & NA \\
\hline
\end{tabular}

\section{Maung 1992b MYA}

\begin{tabular}{ll}
\hline Methods & Case-control study \\
\hline Participants & $\begin{array}{l}\text { Cases: children aged 1-59 months admitted to paediatric wards of the North Okkalapa General Hospi- } \\
\text { tal, or presented at the urban HC or at the emergency department of the North Okkalapa General Hos- } \\
\text { pital, for persistent diarrhoea and PEM, } \mathrm{n}=67 .\end{array}$ \\
& $\begin{array}{l}\text { Controls: age- and sex-matched apparently healthy children within the neighbourhood of the case chil- } \\
\text { dren (usually within the same street, selected from houses with structural appearances similar to that } \\
\text { of the cases). The control children had no diarrhoea or PEM in the last } 2 \text { months, } \mathrm{n}=67 .\end{array}$ \\
\hline
\end{tabular}

Interventions

Risk factor of interest:

- child defecated on the floor vs in pot/latrine

Outcomes

Persistent diarrhoea: passage of watery or loose stools (with or without mucous) $>3$ times/day on most days lasting $\geq 14$ days during the last 2 months, with an interval of not more than 6 days during which loose motions were $<3$ times/day

PEM: children with kwashiorkor, marasmic kwashiorkor or marasmus, or children with weight-for-age < 2 SD below the median National Centre for Health Statistics reference

Lotes
Length of recruitment: not specified
Publication status: journal

\section{Risk of bias}

\begin{tabular}{lll}
\hline Bias & Authors' judgement & Support for judgement \\
\hline $\begin{array}{l}\text { Random sequence genera- } \\
\text { tion (selection bias) }\end{array}$ & Unclear risk & NA \\
\hline $\begin{array}{l}\text { Allocation concealment } \\
\text { (selection bias) }\end{array}$ & Unclear risk & NA \\
\hline $\begin{array}{l}\text { Blinding of participants } \\
\text { and personnel (perfor- } \\
\text { mance bias) }\end{array}$ & Unclear risk & NA \\
\hline
\end{tabular}


Maung 1992b MYA (Continued)

All outcomes

\begin{tabular}{|c|c|c|}
\hline $\begin{array}{l}\text { Blinding of outcome as- } \\
\text { sessment (detection bias) } \\
\text { All outcomes }\end{array}$ & Unclear risk & NA \\
\hline $\begin{array}{l}\text { Incomplete outcome data } \\
\text { (attrition bias) } \\
\text { All outcomes }\end{array}$ & Unclear risk & NA \\
\hline $\begin{array}{l}\text { Selective reporting (re- } \\
\text { porting bias) }\end{array}$ & Unclear risk & NA \\
\hline Other bias & Unclear risk & NA \\
\hline $\begin{array}{l}\text { Similarity of baseline out- } \\
\text { come measurements }\end{array}$ & Unclear risk & NA \\
\hline $\begin{array}{l}\text { Similarity of baseline char- } \\
\text { acteristics }\end{array}$ & Unclear risk & NA \\
\hline $\begin{array}{l}\text { Adequate allocation of in- } \\
\text { tervention concealment } \\
\text { during the study }\end{array}$ & Unclear risk & NA \\
\hline $\begin{array}{l}\text { Adequate protection } \\
\text { against contamination }\end{array}$ & Unclear risk & NA \\
\hline $\begin{array}{l}\text { Confounders adequate- } \\
\text { ly adjusted for in analy- } \\
\text { sis/design }\end{array}$ & Unclear risk & NA \\
\hline Recruitment bias & Unclear risk & NA \\
\hline Baseline imbalance & Unclear risk & NA \\
\hline Loss of clusters & Unclear risk & NA \\
\hline Incorrect analysis & Unclear risk & NA \\
\hline
\end{tabular}

\section{Mediratta 2010a ETH}

\begin{tabular}{ll}
\hline Methods & Case-control study (clinic based) \\
\hline Participants & $\begin{array}{l}\text { Cases: children aged }<5 \text { years with acute diarrhoea were consecutively enrolled from the outpatient } \\
\text { department and inpatient paediatric ward, } n=220, \text { mean age } 1.57 \text { years, } 35 \% \text { girls }\end{array}$ \\
& $\begin{array}{l}\text { Controls: selected from children with other conditions who did not present with acute diarrhoea for } \geq \\
14 \text { days before the date of interview. Match the cases with } 1: 1 \text { ratio for age (within } 6 \text { months), sex, with- } \\
\text { in } 2 \text { weeks from the date of the case visit and the same ward, } n=220, \text { mean age } 1.51 \text { years, 35\% girls }\end{array}$ \\
\hline Interventions & Risk factors of interest: \\
& disposal of stools elsewhere (thrown in garbage, buried, left on ground) vs in latrine (child used latrine \\
\hline Outcomes & Diarrhoea: $\geq 3$ liquid stools within a 24-hour period \\
\hline
\end{tabular}


Mediratta 2010a ETH (Continued)

$$
\text { Acute diarrhoea: having diarrhoea for }<14 \text { days }
$$

Notes Location: University of Gondar Referral and Teaching Hospital in the North Gondar Zone, Ethiopia

Length of recruitment: 6 months (July 2007 to January 2008)

Publication status: journal

\section{Risk of bias}

\begin{tabular}{|c|c|c|}
\hline Bias & Authors' judgement & Support for judgement \\
\hline $\begin{array}{l}\text { Random sequence genera- } \\
\text { tion (selection bias) }\end{array}$ & Unclear risk & NA \\
\hline $\begin{array}{l}\text { Allocation concealment } \\
\text { (selection bias) }\end{array}$ & Unclear risk & NA \\
\hline $\begin{array}{l}\text { Blinding of participants } \\
\text { and personnel (perfor- } \\
\text { mance bias) } \\
\text { All outcomes }\end{array}$ & Unclear risk & NA \\
\hline $\begin{array}{l}\text { Blinding of outcome as- } \\
\text { sessment (detection bias) } \\
\text { All outcomes }\end{array}$ & Unclear risk & NA \\
\hline $\begin{array}{l}\text { Incomplete outcome data } \\
\text { (attrition bias) } \\
\text { All outcomes }\end{array}$ & Unclear risk & NA \\
\hline $\begin{array}{l}\text { Selective reporting (re- } \\
\text { porting bias) }\end{array}$ & Unclear risk & NA \\
\hline Other bias & Unclear risk & NA \\
\hline $\begin{array}{l}\text { Similarity of baseline out- } \\
\text { come measurements }\end{array}$ & Unclear risk & NA \\
\hline $\begin{array}{l}\text { Similarity of baseline char- } \\
\text { acteristics }\end{array}$ & Unclear risk & NA \\
\hline $\begin{array}{l}\text { Adequate allocation of in- } \\
\text { tervention concealment } \\
\text { during the study }\end{array}$ & Unclear risk & NA \\
\hline $\begin{array}{l}\text { Adequate protection } \\
\text { against contamination }\end{array}$ & Unclear risk & NA \\
\hline $\begin{array}{l}\text { Confounders adequate- } \\
\text { ly adjusted for in analy- } \\
\text { sis/design }\end{array}$ & Unclear risk & NA \\
\hline Recruitment bias & Unclear risk & NA \\
\hline Baseline imbalance & Unclear risk & NA \\
\hline Loss of clusters & Unclear risk & NA \\
\hline
\end{tabular}


Mediratta 2010a ETH (Continued)

Incorrect analysis Unclear risk NA

Mediratta 2010b ETH

\begin{tabular}{ll}
\hline Methods & Case-control study (clinic based) \\
\hline Participants & $\begin{array}{l}\text { Cases: children aged }<5 \text { years with acute diarrhoea were consecutively enrolled from the outpatient } \\
\text { department and inpatient paediatric ward, } n=220, \text { mean age } 1.57 \text { years, } 35 \% \text { girls } \\
\text { Controls: selected from children with other conditions who did not present with acute diarrhoea for } \geq \\
14 \text { days before the date of interview. Match the cases with } 1: 1 \text { ratio for age (within } 6 \text { months), sex, with- } \\
\text { in } 2 \text { weeks from the date of the case visit and the same ward, } n=220 . \text { mean age } 1.51 \text { years, 35\% girls }\end{array}$ \\
\hline Interventions & Risk factors of interest: \\
\hline Outcomes & place of child's last defecation was elsewhere (ground, small bucket (popo), underclothes) vs latrine. \\
& Diarrhoea: $\geq 3$ liquid stools within a $24-h o u r$ period \\
& Acute diarrhoea: having diarrhoea for $<14$ days \\
\hline Notes & Location: University of Gondar Referral and Teaching Hospital in the North Gondar Zone, Ethiopia \\
& Length of recruitment: 6 months (July 2007 to January 2008) \\
Publication status: journal
\end{tabular}

\section{Risk of bias}

\begin{tabular}{|c|c|c|}
\hline Bias & Authors' judgement & Support for judgement \\
\hline $\begin{array}{l}\text { Random sequence genera- } \\
\text { tion (selection bias) }\end{array}$ & Unclear risk & NA \\
\hline $\begin{array}{l}\text { Allocation concealment } \\
\text { (selection bias) }\end{array}$ & Unclear risk & NA \\
\hline $\begin{array}{l}\text { Blinding of participants } \\
\text { and personnel (perfor- } \\
\text { mance bias) } \\
\text { All outcomes }\end{array}$ & Unclear risk & NA \\
\hline $\begin{array}{l}\text { Blinding of outcome as- } \\
\text { sessment (detection bias) } \\
\text { All outcomes }\end{array}$ & Unclear risk & NA \\
\hline $\begin{array}{l}\text { Incomplete outcome data } \\
\text { (attrition bias) } \\
\text { All outcomes }\end{array}$ & Unclear risk & NA \\
\hline $\begin{array}{l}\text { Selective reporting (re- } \\
\text { porting bias) }\end{array}$ & Unclear risk & NA \\
\hline Other bias & Unclear risk & NA \\
\hline
\end{tabular}


Mediratta 2010b ETH (Continued)

\begin{tabular}{lll}
$\begin{array}{l}\text { Similarity of baseline out- } \\
\text { come measurements }\end{array}$ & Unclear risk & NA \\
\hline $\begin{array}{l}\text { Similarity of baseline char- } \\
\text { acteristics }\end{array}$ & Unclear risk & NA \\
\hline $\begin{array}{l}\text { Adequate allocation of in- } \\
\text { tervention concealment } \\
\text { during the study }\end{array}$ & Unclear risk & NA \\
\hline $\begin{array}{l}\text { Adequate protection } \\
\text { against contamination }\end{array}$ & Unclear risk & NA \\
\hline $\begin{array}{l}\text { Confounders adequate- } \\
\text { ly adjusted for in analy- } \\
\text { sis/design }\end{array}$ & Unclear risk & \\
\hline $\begin{array}{l}\text { Recruitment bias } \\
\text { Baseline imbalance }\end{array}$ & Unclear risk & NA \\
\hline $\begin{array}{l}\text { Loss of clusters } \\
\text { Incorrect analysis }\end{array}$ & Unclear risk & NA \\
\hline
\end{tabular}

\section{Menon 1990 USA}

\begin{tabular}{|c|c|}
\hline Methods & Case-control study \\
\hline \multirow[t]{3}{*}{ Participants } & $\begin{array}{l}\text { Cases: Apache children aged }<2 \text { years residing on the White Mountain reservation, seen at the Whiteriv- } \\
\text { er Indian Hospital with rotavirus diarrhoea, } n=45 \text { (after } 1 \text { refused, } 27 \text { respondents were not available } \\
\text { and } 5 \text { cases were dropped as had no matched control). }\end{array}$ \\
\hline & $\begin{array}{l}\text { Hospital controls: children aged }<2 \text { years residing on the White Mountain reservation, matched for sex } \\
\text { and age within } 2 \text { months, chosen from outpatient and inpatient records for a variety of other non-diar- } \\
\text { rhoeal illnesses, and visited the hospital within } 2 \text { weeks of the date of diagnosis of the case, } n=45 \text {. }\end{array}$ \\
\hline & $\begin{array}{l}\text { Neighbourhood controls: children aged }<2 \text { years within same age group, same sex, and neighbourhood } \\
\text { (area served by same water supply system), } n=24 \text {. }\end{array}$ \\
\hline Interventions & Risk factor of interest: \\
\hline Outcomes & $\begin{array}{l}\text { Rotavirus diarrhoea: } \geq 3 \text { loose or watery stools during the previous } 24 \text { hours which tested positive ( } 2+) \\
\text { for rotavirus antigen using the enzyme-linked immunosorbent assay. }\end{array}$ \\
\hline \multirow[t]{3}{*}{ Notes } & Location: 1 hospital on White Mountain reservation in east-central Arizona, USA \\
\hline & Length of recruitment: 7 months (1 May to 15 December 1985) \\
\hline & Publication status: journal \\
\hline
\end{tabular}

\section{Risk of bias}

Bias Authors' judgement Support for judgement


Menon 1990 USA (Continued)

\begin{tabular}{lll}
$\begin{array}{l}\text { Random sequence genera- } \\
\text { tion (selection bias) }\end{array}$ & Unclear risk & NA \\
\hline $\begin{array}{l}\text { Allocation concealment } \\
\text { (selection bias) }\end{array}$ & Unclear risk & NA \\
\hline $\begin{array}{l}\text { Blinding of participants } \\
\text { and personnel (perfor- } \\
\text { mance bias) }\end{array}$ & Unclear risk & NA \\
All outcomes & &
\end{tabular}

$\begin{array}{ll}\text { Blinding of outcome as- } & \text { Unclear risk } \\ \text { sessment (detection bias) } & \text { NA }\end{array}$

All outcomes

Incomplete outcome data Unclear risk NA
(attrition bias)

All outcomes

\begin{tabular}{|c|c|c|}
\hline $\begin{array}{l}\text { Selective reporting (re- } \\
\text { porting bias) }\end{array}$ & Unclear risk & NA \\
\hline Other bias & Unclear risk & NA \\
\hline $\begin{array}{l}\text { Similarity of baseline out- } \\
\text { come measurements }\end{array}$ & Unclear risk & NA \\
\hline $\begin{array}{l}\text { Similarity of baseline char- } \\
\text { acteristics }\end{array}$ & Unclear risk & NA \\
\hline $\begin{array}{l}\text { Adequate allocation of in- } \\
\text { tervention concealment } \\
\text { during the study }\end{array}$ & Unclear risk & NA \\
\hline $\begin{array}{l}\text { Adequate protection } \\
\text { against contamination }\end{array}$ & Unclear risk & NA \\
\hline $\begin{array}{l}\text { Confounders adequate- } \\
\text { ly adjusted for in analy- } \\
\text { sis/design }\end{array}$ & Unclear risk & NA \\
\hline Recruitment bias & Unclear risk & NA \\
\hline Baseline imbalance & Unclear risk & NA \\
\hline Loss of clusters & Unclear risk & NA \\
\hline Incorrect analysis & Unclear risk & NA \\
\hline
\end{tabular}

\section{Mertens 1992 SRI}

\begin{tabular}{ll}
\hline Methods & Case-control study \\
\hline Participants & Cases: all children aged $<5$ years presenting with diarrhoea to 1 of 5 hospitals, $n=2458$ (only visited \\
& 1415), mean age 20.6 months, $45.6 \%$ girls.
\end{tabular}


Mertens 1992 SRI (Continued)

Hospital controls: children with a control disease, frequency matched for age with the cases (within a range of 5 months), $\mathrm{n}=4140$ (only visited $\mathrm{HH}$ of 2279), mean age 23.3 months, $48.8 \%$ girls.

Community controls: a random sample of children aged $<5$ years was recruited from the community in the catchment areas of the hospitals, using multistage sampling, and applying the same exclusion criteria as the clinic controls, $n=1659$, mean age 25.8 months, $47.6 \%$ girls.

$\begin{array}{ll}\text { Interventions } & \text { Risk factor of interest: } \\ & \text { unsanitary disposal (stools passed, or disposed of, in or out of the yard without being later (within } 1 \\ \text { day) disposed of in a latrine or in a covered rubbish pit) vs sanitary disposal (stools passed in a potty } \\ \text { and later disposed of in a latrine or in a covered pit). }\end{array}$

Outcomes

Diarrhoea defined as $\geq 3$ loose or watery stools in the previous 24 hours, or as stools with blood or mucous

\begin{tabular}{l} 
Location: 5 rural hospitals and community, district of Kurunegala, Sri Lanka \\
Length of recruitment: 14 months (January 1987 to March 1988) \\
Publication status: journal \\
\hline
\end{tabular}

\section{Risk of bias}

\begin{tabular}{|c|c|c|}
\hline Bias & Authors' judgement & Support for judgement \\
\hline $\begin{array}{l}\text { Random sequence genera- } \\
\text { tion (selection bias) }\end{array}$ & Unclear risk & NA \\
\hline $\begin{array}{l}\text { Allocation concealment } \\
\text { (selection bias) }\end{array}$ & Unclear risk & NA \\
\hline $\begin{array}{l}\text { Blinding of participants } \\
\text { and personnel (perfor- } \\
\text { mance bias) } \\
\text { All outcomes }\end{array}$ & Unclear risk & NA \\
\hline $\begin{array}{l}\text { Blinding of outcome as- } \\
\text { sessment (detection bias) } \\
\text { All outcomes }\end{array}$ & Unclear risk & NA \\
\hline $\begin{array}{l}\text { Incomplete outcome data } \\
\text { (attrition bias) } \\
\text { All outcomes }\end{array}$ & Unclear risk & NA \\
\hline $\begin{array}{l}\text { Selective reporting (re- } \\
\text { porting bias) }\end{array}$ & Unclear risk & NA \\
\hline Other bias & Unclear risk & NA \\
\hline $\begin{array}{l}\text { Similarity of baseline out- } \\
\text { come measurements }\end{array}$ & Unclear risk & NA \\
\hline $\begin{array}{l}\text { Similarity of baseline char- } \\
\text { acteristics }\end{array}$ & Unclear risk & NA \\
\hline $\begin{array}{l}\text { Adequate allocation of in- } \\
\text { tervention concealment } \\
\text { during the study }\end{array}$ & Unclear risk & NA \\
\hline
\end{tabular}


Mertens 1992 SRI (Continued)

\begin{tabular}{lll}
$\begin{array}{l}\text { Adequate protection } \\
\text { against contamination }\end{array}$ & Unclear risk & NA \\
\hline $\begin{array}{l}\text { Confounders adequate- } \\
\text { ly adjusted for in analy- } \\
\text { sis/design }\end{array}$ & Unclear risk & NA \\
\hline Recruitment bias & Unclear risk & NA \\
\hline Baseline imbalance & Unclear risk & NA \\
\hline Loss of clusters & Unclear risk & NA \\
\hline Incorrect analysis & Unclear risk & NA
\end{tabular}

\section{Nair 2017 IND}

\begin{tabular}{ll}
\hline Methods & Cluster RCT \\
\hline Participants & Number: 2633 children found at 18 months \\
& $\begin{array}{l}\text { Inclusion criteria: pregnant women in their third trimester. Their children were followed up to } 18 \\
\text { months of age. }\end{array}$ \\
& $\begin{array}{l}\text { Exclusion criteria: stillbirths and neonatal deaths; infants whose mothers died; those with congenital } \\
\text { abnormalities, multiple births, and mother and infant pairs who migrated out of the study area perma- } \\
\text { nently during the trial period. }\end{array}$
\end{tabular}

Interventions

Intervention (60 clusters, each consisted of a geographical cluster with a population of around 1000 people each to approximate the catchment area of an Anganwadi worker):

- community-based female worker (Suposhan Karyakarta, or SPK) carrying home visits with individual families and participatory meetings with groups of women, to improve health and nutrition in the first 1000 days of life. The SPK was responsible for 2 main activities:

* conducting a single home visit to each pregnant woman in the third trimester of pregnancy with counselling on maternal nutrition, followed by monthly home visits to all children aged $<2$ years, with counselling for growth promotion. The training to prepare SPK to home visits included: advising caregivers to place the child's faeces in a pit latrine, or if no latrines were available (the case for $>90 \%$ of $\mathrm{HH}$ in the trial areas), to bury them in a shallow hole away from their living area and any waterway rather than disposing of them in the open field or the $\mathrm{HH}$ compound;

* a monthly participatory meeting with a local women's group. The SPK uses a problem-solving approach in both of these activities.

In addition, 5 participatory meetings were held in both the intervention and control arm, with village health sanitation and nutrition committees to strengthen the capacity of the committees to assess community health needs, prepare and implement village health plans, and monitor the provision of local health and nutrition services.

Control (60 clusters): only the participatory meetings with the village health sanitation and nutrition committees.

Outcomes

Primary outcome:

- LAZ at 18 months

Secondary outcomes:

- wasting 
Nair 2017 IND (Continued)

- underweight

- birth weight

- growth velocity

- feeding, hygiene, and care practices

Notes

Location: 120 clusters, West Singhbhum and Kendujhar, 2 adjoining rural districts of Jharkhand and Odisha in eastern India

Length of study: 27 months (randomization: July 2013; recruitment and data collection: 1 October 2013 to 31 December 2015)

Publication status: journal

\section{Risk of bias}

\begin{tabular}{|c|c|c|}
\hline Bias & Authors' judgement & Support for judgement \\
\hline $\begin{array}{l}\text { Random sequence genera- } \\
\text { tion (selection bias) }\end{array}$ & Low risk & $\begin{array}{l}\text { Clusters were randomly allocated to treatment or control using lottery } \\
\text { method. }\end{array}$ \\
\hline $\begin{array}{l}\text { Allocation concealment } \\
\text { (selection bias) }\end{array}$ & Low risk & $\begin{array}{l}\text { Clusters were randomly allocated to treatment or control using lottery } \\
\text { method. }\end{array}$ \\
\hline $\begin{array}{l}\text { Blinding of participants } \\
\text { and personnel (perfor- } \\
\text { mance bias) } \\
\text { All outcomes }\end{array}$ & High risk & Participants and the intervention team were not blinded to allocation. \\
\hline $\begin{array}{l}\text { Blinding of outcome as- } \\
\text { sessment (detection bias) } \\
\text { All outcomes }\end{array}$ & Low risk & $\begin{array}{l}\text { Quote: "the data collection team and data manager were masked to alloca- } \\
\text { tion." }\end{array}$ \\
\hline $\begin{array}{l}\text { Incomplete outcome data } \\
\text { (attrition bias) } \\
\text { All outcomes }\end{array}$ & Low risk & Incomplete data similar across arms. \\
\hline $\begin{array}{l}\text { Selective reporting (re- } \\
\text { porting bias) }\end{array}$ & Low risk & $\begin{array}{l}\text { Reported on all outcomes in methods. Although the outcomes were changed } \\
\text { in the trial registration this was prior to data collection. }\end{array}$ \\
\hline Other bias & Unclear risk & - \\
\hline $\begin{array}{l}\text { Similarity of baseline out- } \\
\text { come measurements }\end{array}$ & Unclear risk & - \\
\hline $\begin{array}{l}\text { Similarity of baseline char- } \\
\text { acteristics }\end{array}$ & Unclear risk & - \\
\hline $\begin{array}{l}\text { Adequate allocation of in- } \\
\text { tervention concealment } \\
\text { during the study }\end{array}$ & Unclear risk & - \\
\hline $\begin{array}{l}\text { Adequate protection } \\
\text { against contamination }\end{array}$ & Unclear risk & - \\
\hline $\begin{array}{l}\text { Confounders adequate- } \\
\text { ly adjusted for in analy- } \\
\text { sis/design }\end{array}$ & Unclear risk & - \\
\hline
\end{tabular}


Nair 2017 IND (Continued)

\begin{tabular}{lll} 
Recruitment bias & High risk & Clusters were randomized prior to recruitment of participants. \\
\hline Baseline imbalance & Low risk & No baseline imbalance. \\
\hline Loss of clusters & Low risk & No loss of clusters. \\
\hline Incorrect analysis & Low risk & Adjusted for clustering using random effects.
\end{tabular}

\section{Nanan 2003 PAK}

\begin{tabular}{|c|c|}
\hline Methods & Case-control study (clinic based) \\
\hline \multirow[t]{2}{*}{ Participants } & $\begin{array}{l}\text { Cases: children aged } 4-71 \text { months with diarrhoea (episode-based) that attended the recruitment cen- } \\
\text { tres during the study period, had been resident in the same village for the previous } 2 \text { weeks, and were } \\
\text { accompanied by a parent or guardian who was willing to participate in the study, } n=454 \text { (after exclud- } \\
\text { ing } 54 \text { ), } 63 \% \text { aged }<24 \text { months, } 45 \% \text { girls. }\end{array}$ \\
\hline & $\begin{array}{l}\text { Controls: children aged } 4-71 \text { months with any complaint other than diarrhoea and without a skin con- } \\
\text { dition or worm infestation that attended the recruitment centres during the study period, had been } \\
\text { resident in the same village for the previous } 2 \text { weeks, and were accompanied by a parent or guardian } \\
\text { who was willing to participate in the study, frequency matched on the HC of recruitment and time of di- } \\
\text { agnosis (selected within } 24 \text { hours of a case), } n=349 \text { (after excluding 125), } 49 \% \text { aged }<24 \text { months, } 38 \% \\
\text { girls. }\end{array}$ \\
\hline
\end{tabular}

Interventions $\quad$ Risk factor of interest:

- non-WASEP village vs WASEP village. WASEP (Aga Khan Development Network) aimed to improve potable water supply at village and $\mathrm{HH}$ levels, sanitation facilities and their use, and awareness and practices about hygiene behaviour. WASEP delivered water supply, water quality, drainage, sanitation, and school- and community-based hygiene education. The hygiene education contained information on safe disposal of faeces (adult, child, and $\mathrm{HH}$ animals), and use and maintenance of a latrine (if the $\mathrm{HH}$ possessed a latrine).

\begin{tabular}{ll}
\hline Outcomes & Diarrhoea: $\geq 3$ loose, watery stools in the last 24 hours \\
\hline Notes & Location: 6 Aga Khan Health services, Pakistan (AKHS,P) centres, Ghizer and Gilgit districts, Pakistan \\
& Length of recruitment: 2 months (July-September 2001) \\
& Publication status: journal \\
\hline
\end{tabular}

\section{Risk of bias}

\begin{tabular}{lll}
\hline Bias & Authors' judgement & Support for judgement \\
\hline $\begin{array}{l}\text { Random sequence genera- } \\
\text { tion (selection bias) }\end{array}$ & Unclear risk & NA \\
\hline $\begin{array}{l}\text { Allocation concealment } \\
\text { (selection bias) }\end{array}$ & Unclear risk \\
\hline $\begin{array}{l}\text { Blinding of participants } \\
\text { and personnel (perfor- } \\
\text { mance bias) } \\
\begin{array}{l}\text { All outcomes } \\
\text { Unclear risk }\end{array}\end{array}$ & NA \\
\end{tabular}


Nanan 2003 PAK (Continued)

$\begin{array}{lll}\text { Blinding of outcome as- } & \text { Unclear risk } & \text { NA } \\ \text { sessment (detection bias) } & \end{array}$

All outcomes

\begin{tabular}{|c|c|c|}
\hline $\begin{array}{l}\text { Incomplete outcome data } \\
\text { (attrition bias) } \\
\text { All outcomes }\end{array}$ & Unclear risk & NA \\
\hline $\begin{array}{l}\text { Selective reporting (re- } \\
\text { porting bias) }\end{array}$ & Unclear risk & NA \\
\hline Other bias & Unclear risk & NA \\
\hline $\begin{array}{l}\text { Similarity of baseline out- } \\
\text { come measurements }\end{array}$ & Unclear risk & NA \\
\hline $\begin{array}{l}\text { Similarity of baseline char- } \\
\text { acteristics }\end{array}$ & Unclear risk & NA \\
\hline $\begin{array}{l}\text { Adequate allocation of in- } \\
\text { tervention concealment } \\
\text { during the study }\end{array}$ & Unclear risk & NA \\
\hline $\begin{array}{l}\text { Adequate protection } \\
\text { against contamination }\end{array}$ & Unclear risk & NA \\
\hline $\begin{array}{l}\text { Confounders adequate- } \\
\text { ly adjusted for in analy- } \\
\text { sis/design }\end{array}$ & Unclear risk & NA \\
\hline Recruitment bias & Unclear risk & NA \\
\hline Baseline imbalance & Unclear risk & NA \\
\hline Loss of clusters & Unclear risk & NA \\
\hline Incorrect analysis & Unclear risk & NA \\
\hline
\end{tabular}

\section{Null 2018 KEN}

\begin{tabular}{ll}
\hline Methods & Cluster RCT \\
\hline Participants & $\begin{array}{l}\text { Number: } 6494 \text { children with diarrhoea data at year } 1 \text { or } 2 \text { in all arms. Children who were in utero or } \\
\text { aged }<3 \text { years at enrolment } \\
\text { Inclusion criteria: children of enrolled pregnant women (index children) were eligible for inclusion if } \\
\text { their mother was planning to live in the study village for the next } 2 \text { years, regardless of where she gave } \\
\text { birth. Only } 1 \text { pregnant woman (in the first } 2 \text { trimesters of her pregnancy) was enrolled per compound, } \\
\text { but if she gave birth to twins, both children were enrolled. Children aged < 3 years at enrolment and } \\
\text { lived in the compound were included in diarrhoea measurements. }\end{array}$ \\
\hline
\end{tabular}

Interventions Intervention : 6 intervention arms

- Water quality (77 clusters, each consisting of $1-3$ neighbouring villages to have $\geq 6$ pregnant women per cluster): chlorine tablets (Aquatabs; $\mathrm{NaDCC}$ ) and a safe storage vessel to treat and store drinking water. Behaviour change messaging to treat drinking water for all children aged $<36$ months. 
Null 2018 KEN (Continued)

- Sanitation (77 clusters): provision of free child potties, sani-scoop to remove faeces from HH environments, and latrine upgrades or construction of latrine if did not own 1. For promotion, local promoters visited study compounds to deliver behaviour change messages on the use of latrines for defecation and the removal of human and animal faeces from the compound.

- Hand washing (77 clusters): handwashing stations, soapy water bottles, detergent soap to supply soapy water. Behaviour change messages focused on HWWS at critical times around food preparation, defecation, and contact with faeces.

- Combined WASH (76 clusters): water quality, sanitation, and handwashing components.

- Nutrition (78 clusters): LNS given twice daily for children 6-24 months. The key recommendations for nutrition were: dietary diversity during pregnancy and lactation, early initiation of breastfeeding, exclusive breastfeeding until 6 months, introduction of appropriate and diverse complementary foods at 6 months, and continued breastfeeding through 24 months.

- Nutrition + combined WASH (79 clusters).

Control (158 clusters): no intervention, monthly visits by community-based health promoter to measure the child's MUAC.

Passive control (80 clusters): no activity apart from data collection.
Primary outcomes:

- LAZ scores (measured 24 months after intervention)

- diarrhoea prevalence (defined as $\geq 3$ loose or watery stools in 24 hours or $\geq 1$ stools with blood in 24 hours. Diarrhoea was measured in interviews using caregiver-reported symptoms with 7-day recall, measured 12 and 24 months after intervention)

Secondary outcomes:

- LAZ scores

- weight for length $Z$ score

- WAZ score

- head circumference-for-age Z score

- prevalence of moderate stunting (LAZ score $<-2)$

- severe stunting (LAZ score $<-3)$

- underweight (WAZ score $<-2$ )

- wasting (WAZ score $<-2$ )

- enteropathy biomarkers (measured 12 and 24 months after intervention)

- Ages and Stages Questionnaire Child Development Scores (measured 24 months after intervention)

Tertiary outcome:

- all-cause mortality among index children

Notes Location: rural villages in Bungoma, Kakamega, and Vihiga counties in Kenya's western region

Length of study: 42 months (recruitment: 27 November 2012 to 21 May 2014 with 2 years' follow-up)

Publication status: journal

\section{Risk of bias}

\begin{tabular}{lll}
\hline Bias & Authors' judgement & Support for judgement \\
\hline $\begin{array}{l}\text { Random sequence genera- } \\
\text { tion (selection bias) }\end{array}$ & Low risk & $\begin{array}{l}\text { Quote: "Clusters were randomly allocated to treatment using a random num- } \\
\text { ber generator with reproducible seed at the University of California, Berkeley." }\end{array}$ \\
\hline $\begin{array}{l}\text { Allocation concealment } \\
\text { (selection bias) }\end{array}$ & Low risk & $\begin{array}{l}\text { Quote: "Clusters were randomly allocated to treatment using a random num- } \\
\text { ber generator with reproducible seed at the University of California, Berkeley." }\end{array}$ \\
\hline
\end{tabular}


Null 2018 KEN (Continued)

Blinding of participants and personnel (performance bias)

All outcomes
High risk

Quote: "Blinding of participants was not possible. Participants were informed of their treatment assignment after baseline data collection and might have known the treatment assignment of nearby villages."

\section{Blinding of outcome as- High risk sessment (detection bias) \\ All outcomes \\ Quote: "The health promoters and staff who delivered the interventions were not involved in data collection, but the data collection team could have in- ferred treatment status if they saw intervention materials in study communi- ties."}

Incomplete outcome data Low risk Loss to follow-up fairly balanced across groups.

(attrition bias)

All outcomes

$\begin{array}{lll}\begin{array}{l}\text { Selective reporting (re- } \\ \text { porting bias) }\end{array} & \text { Unclear risk } & \begin{array}{l}\text { Reported on primary outcomes but future publications will cover additional } \\ \text { prespecified outcomes. }\end{array}\end{array}$

\begin{tabular}{|c|c|c|}
\hline Other bias & Unclear risk & - \\
\hline $\begin{array}{l}\text { Similarity of baseline out- } \\
\text { come measurements }\end{array}$ & Unclear risk & - \\
\hline $\begin{array}{l}\text { Similarity of baseline char- } \\
\text { acteristics }\end{array}$ & Unclear risk & - \\
\hline $\begin{array}{l}\text { Adequate allocation of in- } \\
\text { tervention concealment } \\
\text { during the study }\end{array}$ & Unclear risk & - \\
\hline $\begin{array}{l}\text { Adequate protection } \\
\text { against contamination }\end{array}$ & Unclear risk & - \\
\hline $\begin{array}{l}\text { Confounders adequate- } \\
\text { ly adjusted for in analy- } \\
\text { sis/design }\end{array}$ & Unclear risk & - \\
\hline Recruitment bias & Low risk & Participants were enrolled prior to knowing allocation of intervention. \\
\hline Baseline imbalance & Low risk & Baseline characteristics of enrolled HHs were similar across groups. \\
\hline Loss of clusters & Low risk & No reported loss of cluster. \\
\hline Incorrect analysis & Low risk & Accounted for clustering in analysis. \\
\hline
\end{tabular}

Oguro 2016 MYA

\begin{tabular}{ll}
\hline Methods & Controlled cross-sectional study \\
\hline Participants & Number: 188 respondents \\
& $\begin{array}{l}\text { Inclusion criteria: aged } 15-49 \text { years, living in the experimental or control villages, } \geq 1 \text { child aged } \leq 5 \\
\text { years, able to communicate in the Myanmar language, and no serious mental illness. }\end{array}$
\end{tabular}

Interventions

Intervention (2 villages): WVGs were established by organizing women and training them using a participatory approach. The activities of the WVGs after 3 years of being established included: 
- educating pregnant women and mothers regarding the necessity of health checks and immunizations and helping them attend these appointments;

- early detection of abnormal signs and symptoms during the perinatal period;

- managing the family planning fund, which allowed women who could not afford contraception to borrow money at no interest;

- providing first aid to injured people (e.g. for injuries that were sustained during agricultural work); and

- educating women regarding appropriate sanitation and malaria prevention. The WVG encouraged latrine use by children (aged $<5$ years) to villagers as part of a programme to promote sanitation education and promoted appropriate disposal (flushing in a latrine) of child faeces.

Control (2 villages): no WVGs

\begin{tabular}{|c|c|c|}
\hline \multirow[t]{6}{*}{ Outcomes } & \multicolumn{2}{|c|}{$\begin{array}{l}\text { Appropriate disposal of child stool (flushed in latrine) vs inappropriate (left in the open, thrown in } \\
\text { garbage) }\end{array}$} \\
\hline & \multicolumn{2}{|l|}{ Any antenatal care } \\
\hline & \multicolumn{2}{|c|}{ Knowledge of danger signs } \\
\hline & \multicolumn{2}{|c|}{ Knowledge of modern contraceptive methods } \\
\hline & \multicolumn{2}{|l|}{ Acceptable first aid } \\
\hline & \multicolumn{2}{|c|}{ Knowledge of malaria prevention } \\
\hline \multirow[t]{3}{*}{ Notes } & \multicolumn{2}{|c|}{ Location: 4 villages in Meiktila Township, Mandalay Division } \\
\hline & \multicolumn{2}{|c|}{ Length of study: 2 months (February-March 2007) } \\
\hline & \multicolumn{2}{|c|}{ Publication status: journal } \\
\hline \multicolumn{3}{|l|}{ Risk of bias } \\
\hline Bias & Authors' judgement & Support for judgement \\
\hline $\begin{array}{l}\text { Random sequence genera- } \\
\text { tion (selection bias) }\end{array}$ & High risk & No random allocation. \\
\hline $\begin{array}{l}\text { Allocation concealment } \\
\text { (selection bias) }\end{array}$ & High risk & No allocation concealment. \\
\hline $\begin{array}{l}\text { Blinding of participants } \\
\text { and personnel (perfor- } \\
\text { mance bias) } \\
\text { All outcomes }\end{array}$ & Unclear risk & NA \\
\hline $\begin{array}{l}\text { Blinding of outcome as- } \\
\text { sessment (detection bias) } \\
\text { All outcomes }\end{array}$ & Unclear risk & NA \\
\hline $\begin{array}{l}\text { Incomplete outcome data } \\
\text { (attrition bias) } \\
\text { All outcomes }\end{array}$ & Unclear risk & $\begin{array}{l}\text { Not all respondents reported on child faeces disposal and it was unclear why } \\
\text { they were missing. }\end{array}$ \\
\hline $\begin{array}{l}\text { Selective reporting (re- } \\
\text { porting bias) }\end{array}$ & Unclear risk & $\begin{array}{l}\text { Authors did not specify what the main outcomes were. They stated there were } \\
102 \text { questions in questionnaire but only presented } 6 \text { outcome measures. }\end{array}$ \\
\hline Other bias & Unclear risk & - \\
\hline
\end{tabular}


Oguro 2016 MYA (Continued)

\begin{tabular}{lll}
$\begin{array}{l}\text { Similarity of baseline out- } \\
\text { come measurements }\end{array}$ & Unclear risk & NA, not relevant to design. \\
\hline $\begin{array}{l}\text { Similarity of baseline char- } \\
\text { acteristics }\end{array}$ & Unclear risk & -
\end{tabular}

istics

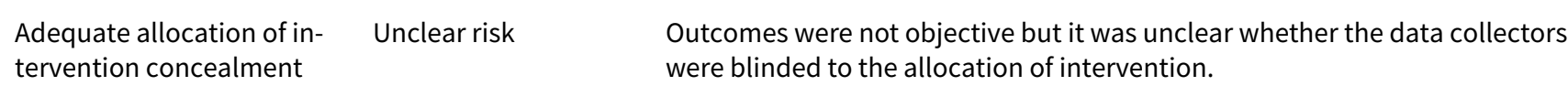

during the study

\begin{tabular}{lll}
\hline $\begin{array}{l}\text { Adequate protection } \\
\text { against contamination }\end{array}$ & Unclear risk & No details of how far the villages were to one another. \\
\hline $\begin{array}{l}\text { Confounders adequate- } \\
\text { ly adjusted for in analy- } \\
\text { sis/design }\end{array}$ & Low risk & Adjusted for wealth in the logistic regression. \\
\hline Recruitment bias & Unclear risk & - \\
\hline Baseline imbalance & Unclear risk & - \\
\hline Loss of clusters & Unclear risk & - \\
\hline Incorrect analysis & Unclear risk & - \\
\hline
\end{tabular}

\section{Oketcho 2012 TAN}

\begin{tabular}{ll}
\hline Methods & Case-control study (clinic-based) \\
\hline Participants & $\begin{array}{l}\text { Cases: children aged 6-60 months admitted to the paediatric infectious diseases ward and the caretak- } \\
\text { er reported increase in the stool fluidity and frequency of passing stool for } \geq 2 \text { days, } \mathrm{n}=151 .\end{array}$ \\
& $\begin{array}{l}\text { Controls: children aged 6-60 months admitted to the ward for management of non-infectious diseases, } \\
\text { without diarrhoea within the previous } 2 \text { weeks. All children meeting the case and control criteria admit- } \\
\text { ted at the same time of the same age group and residing in Morogoro region were included in the study, } \\
\mathrm{n}=152 .\end{array}$ \\
\hline
\end{tabular}

Interventions Risk factor of interest:

- child used toilet vs defecated elsewhere.

\begin{tabular}{ll}
\hline Outcomes & Diarrhoea: caretaker reported increase in the stool fluidity and frequency of passing stool for $\geq 2$ days \\
\hline Notes & Location: urban, Morogoro Regional Hospital, Tanzania \\
& Length of recruitment: 8 months (January-September 2011) \\
& Publication status: journal
\end{tabular}

\section{Risk of bias}

\begin{tabular}{lll}
\hline Bias & Authors' judgement & Support for judgement \\
\hline $\begin{array}{l}\text { Random sequence genera- } \\
\text { tion (selection bias) }\end{array}$ & Unclear risk & NA \\
\hline
\end{tabular}


Oketcho 2012 TAN (Continued)

\begin{tabular}{lll}
$\begin{array}{l}\text { Allocation concealment } \\
\text { (selection bias) }\end{array}$ & Unclear risk & NA \\
\hline $\begin{array}{l}\text { Blinding of participants } \\
\text { and personnel (perfor- } \\
\text { mance bias) }\end{array}$ & Unclear risk & \\
All outcomes &
\end{tabular}

\begin{tabular}{lll}
\hline Blinding of outcome as- & Unclear risk & NA \\
sessment (detection bias) & &
\end{tabular}

All outcomes

\begin{tabular}{|c|c|c|}
\hline $\begin{array}{l}\text { Incomplete outcome data } \\
\text { (attrition bias) } \\
\text { All outcomes }\end{array}$ & Unclear risk & NA \\
\hline $\begin{array}{l}\text { Selective reporting (re- } \\
\text { porting bias) }\end{array}$ & Unclear risk & NA \\
\hline Other bias & Unclear risk & NA \\
\hline $\begin{array}{l}\text { Similarity of baseline out- } \\
\text { come measurements }\end{array}$ & Unclear risk & NA \\
\hline $\begin{array}{l}\text { Similarity of baseline char- } \\
\text { acteristics }\end{array}$ & Unclear risk & NA \\
\hline $\begin{array}{l}\text { Adequate allocation of in- } \\
\text { tervention concealment } \\
\text { during the study }\end{array}$ & Unclear risk & NA \\
\hline $\begin{array}{l}\text { Adequate protection } \\
\text { against contamination }\end{array}$ & Unclear risk & NA \\
\hline $\begin{array}{l}\text { Confounders adequate- } \\
\text { ly adjusted for in analy- } \\
\text { sis/design }\end{array}$ & Unclear risk & NA \\
\hline Recruitment bias & Unclear risk & NA \\
\hline Baseline imbalance & Unclear risk & NA \\
\hline Loss of clusters & Unclear risk & NA \\
\hline Incorrect analysis & Unclear risk & NA \\
\hline
\end{tabular}

\section{Park 2016 INA}

\begin{tabular}{ll}
\hline Methods & CBA study \\
\hline Participants & Number: 99 children \\
& Inclusion criteria: children aged 3-13 years \\
& $\begin{array}{l}\text { Mean age: intervention group 7.1 (SD 3.2, range 3-13) years; control group 8.4 (SD 3, range 4-13). Inter- } \\
\text { vention group 54\% girls; control group 65.7\% girls }\end{array}$
\end{tabular}


Park 2016 INA (Continued) Interventions
Intervention site (1 village, 50 children): Budi's Amphibious Latrine (BALatrines) (simple squat latrines with a septic tank or pit) were constructed and all residents were given health education regarding hygiene, sanitation, and prevention of STH infections. The health education included many messages about preventing soil-transmitted helminthiases: appropriate hand washing; boiling water before home use; not drinking river water; peeling fruit; cooking vegetables; avoiding street food; not defecating in waterways, paddy fields, or gardens; keeping domestic animals in cages not close to waterways; etc. Regarding latrines, the messages mentioned that they should be $\geq 10 \mathrm{~m}$ away from wells, and that it is best for them to flush and cover after use. Messages about recognizing signs and symptoms of soiltransmitted helminthiases were also included.

For mothers of small children, the messages included not disposing of used nappies in the garden or bush or in waterways.

2 messages directed specifically at children were that they should stay away from any faeces they might find around their home, and that they should report any symptoms (diarrhoea, fever, etc.) to a parent or teacher. All children who were found to have STH infection at a baseline were treated with albendazole $400 \mathrm{mg}$.

Control site (1 village, 49 children): no intervention. All children who were found to have STH infection at a baseline were treated with albendazole $400 \mathrm{mg}$.

Outcomes STH infection (presence of helminth eggs in stool, diagnosed using Impankaew method (simple faecal flotation))

Notes Location: 2 villages in the Gunungpati subdistrict, Semarang, Central Java, Indonesia

Length: 8 months (no specific dates)

Publication status: journal

\section{Risk of bias}

\begin{tabular}{lll}
\hline Bias & Authors' judgement & Support for judgement \\
\hline $\begin{array}{l}\text { Random sequence genera- } \\
\text { tion (selection bias) }\end{array}$ & High risk & No random allocation. \\
\hline $\begin{array}{l}\text { Allocation concealment } \\
\text { (selection bias) }\end{array}$ & High risk & No concealment. \\
\hline $\begin{array}{l}\text { Blinding of participants } \\
\text { and personnel (perfor- } \\
\text { mance bias) }\end{array}$ & Unclear risk & NA \\
All outcomes & \\
\hline
\end{tabular}

\begin{tabular}{|c|c|c|}
\hline $\begin{array}{l}\text { Blinding of outcome as- } \\
\text { sessment (detection bias) } \\
\text { All outcomes }\end{array}$ & Unclear risk & NA \\
\hline $\begin{array}{l}\text { Incomplete outcome data } \\
\text { (attrition bias) } \\
\text { All outcomes }\end{array}$ & Low risk & $\begin{array}{l}\text { It seemed there was no LTFU. However, there was no information about how } \\
\text { many non-respondents were at baseline/recruitment. }\end{array}$ \\
\hline $\begin{array}{l}\text { Selective reporting (re- } \\
\text { porting bias) }\end{array}$ & Low risk & Reported outcomes outlined in the methods. \\
\hline Other bias & Unclear risk & - \\
\hline
\end{tabular}


Park 2016 INA (Continued)

Similarity of baseline out- Low risk Prevalence of STH infection was statistically not different at baseline. come measurements

Similarity of baseline char- Unclear risk acteristics
No baseline characteristics apart from child age and sex. However, sanitation coverage and other measures would be important.

\title{
Adequate allocation of in- Low risk \\ Outcome measures were objective (STH in stool).
} tervention concealment during the study

\begin{tabular}{|c|c|c|}
\hline $\begin{array}{l}\text { Adequate protection } \\
\text { against contamination }\end{array}$ & Low risk & $\begin{array}{l}\text { Quote: "Although they are in the same sub-district, the two villages are not in } \\
\text { close proximity to each other." }\end{array}$ \\
\hline
\end{tabular}

\begin{tabular}{lll}
\hline $\begin{array}{l}\text { Confounders adequate- } \\
\text { ly adjusted for in analy- } \\
\text { sis/design }\end{array}$ & High risk & No confounders adjusted for. \\
\hline Recruitment bias & Unclear risk & NA \\
\hline Baseline imbalance & Unclear risk & NA \\
\hline Loss of clusters & Unclear risk & NA \\
\hline Incorrect analysis & Unclear risk & NA
\end{tabular}

\section{Patil 2014 IND}

\begin{tabular}{ll}
\hline Methods Cluster RCT \\
\hline
\end{tabular}

Participants Number: $3039 \mathrm{HHs}$ ( 5209 children aged < 5 years) (after $15.3 \%$ LTFU)

Inclusion criteria: $\mathrm{HH}$ with $\geq 1$ child aged $<24$ months at enrolment. For follow-up, the HH had to have $\geq$ 1 child aged 21-45 months and were living in the village at the time of baseline.

Mean age: intervention group 21.9 months; control group 22.1 months

Interventions Intervention (40 villages): India Total Sanitation Campaign (subsidies and promotion of individual HH latrines) and Nirmal Vatika (additional subsidies) and support from WSP through TSSM project, which included creation of enabling environment + capacity building to implement CLTS-based behaviour change methods.

Control (40 villages): no intervention.

\section{Outcomes}

\author{
Toilet coverage, defecation behaviours (including daily open defecation by children (aged $<5$ years), \\ hygienic child faeces disposal)
}

Diarrhoea: $\geq 3$ loose or watery stools in 24 hours or a single stool with blood/mucous. 7 -day recall in questionnaire at baseline and at end of study.

Highly credible gastrointestinal illness

Acute lower respiratory illness

Bruising/abrasions and itchy skin/scalp (negative control outcomes)

Anthropometry (weight for age, height for age, weight for height, MUAC)

Anaemia 
Patil 2014 IND (Continued)

Water quality

Child stool parasitology (including helminth present in stool,Ascaris lumbricoides present in stool)

Location: 80 rural villages in 2 neighbouring districts in Madhya Pradesh, India
Length of study: 23 months (25 May 2009 to 25 April 2011)
Publication status: journal

\section{Risk of bias}

\begin{tabular}{|c|c|c|}
\hline Bias & Authors' judgement & Support for judgement \\
\hline $\begin{array}{l}\text { Random sequence genera- } \\
\text { tion (selection bias) }\end{array}$ & Low risk & Used public lottery to assign villages to arms. \\
\hline $\begin{array}{l}\text { Allocation concealment } \\
\text { (selection bias) }\end{array}$ & Low risk & Used public lottery to assign villages to arms. \\
\hline $\begin{array}{l}\text { Blinding of participants } \\
\text { and personnel (perfor- } \\
\text { mance bias) } \\
\text { All outcomes }\end{array}$ & High risk & $\begin{array}{l}\text { No blinding of participants possible but outcomes were self-reported so could } \\
\text { have been affected by lack of blinding. }\end{array}$ \\
\hline \multirow[t]{2}{*}{$\begin{array}{l}\text { Blinding of outcome as- } \\
\text { sessment (detection bias) } \\
\text { All outcomes }\end{array}$} & High risk & $\begin{array}{l}\text { Quote: "Field interviewers were not informed of group assignment, but it was } \\
\text { possible for them to identify intervention villages during interviews of Block } \\
\text { officers or the village secretary." }\end{array}$ \\
\hline & & Comment: incomplete blinding. \\
\hline
\end{tabular}

\begin{tabular}{|c|c|c|}
\hline $\begin{array}{l}\text { Incomplete outcome data } \\
\text { (attrition bias) }\end{array}$ & Low risk & $\begin{array}{l}\text { Attrition was not differential by randomized group and no missing values for } \\
\text { main outcomes. }\end{array}$ \\
\hline
\end{tabular}

All outcomes

Attrition was not differential by randomized group and no missing values for

Selective reporting (re- Low risk $\quad$ Report on main outcomes.
porting bias)

\begin{tabular}{lll}
\hline Other bias & Unclear risk & - \\
\hline $\begin{array}{l}\text { Similarity of baseline out- } \\
\text { come measurements }\end{array}$ & Unclear risk & NA \\
\hline $\begin{array}{l}\text { Similarity of baseline char- } \\
\text { acteristics }\end{array}$ & Unclear risk & NA \\
\hline $\begin{array}{l}\text { Adequate allocation of in- } \\
\text { tervention concealment } \\
\text { during the study }\end{array}$ & Unclear risk & NA \\
\hline $\begin{array}{l}\text { Adequate protection } \\
\text { against contamination }\end{array}$ & Unclear risk & NA \\
\hline $\begin{array}{l}\text { Confounders adequate- } \\
\text { ly adjusted for in analy- } \\
\text { sis/design }\end{array}$ & Unclear risk & NA \\
\hline
\end{tabular}

\begin{tabular}{ll}
\hline Recruitment bias $\quad$ High risk & $\begin{array}{l}\text { Follow-up data which were the data used for analysis were measured in newly } \\
\text { recruited HHs that belonged to either intervention or control arms. }\end{array}$
\end{tabular}


Patil 2014 IND (Continued)

$\begin{array}{ll}\text { Baseline imbalance } \quad \text { Low risk } & \begin{array}{l}\text { No major imbalance and the analysis adjusted for the } 3 \text { characteristics that } \\ \text { had slight imbalance between groups. }\end{array}\end{array}$

\begin{tabular}{lll}
\hline Loss of clusters & Low risk & No loss of clusters. \\
\hline Incorrect analysis & Low risk & Adjusted for clustering in the analyses.
\end{tabular}

\section{Pickering 2015 MLI}

\begin{tabular}{ll}
\hline Methods & Cluster RCT \\
\hline Participants & Number: 6319 children aged < 5 years at end of study ( $4031 \mathrm{HHs})$ (after 11.1\% LTFU) \\
& Inclusion criteria: HHs with $\geq 1$ child aged < 10 years \\
\hline Interventions & $\begin{array}{l}\text { Interventions ( } 60 \text { villages, } 2365 \mathrm{HHs}): \text { CLTS which used participatory methods to eliminate the practice } \\
\text { of open defecation in rural HHs and promote building of toilets. No hardware or subsidies was provid- } \\
\text { ed to HHs. } \\
\text { Control ( } 61 \text { villages, } 2167 \mathrm{HHs} \text { ): no intervention }\end{array}$ \\
\hline
\end{tabular}

Outcomes

Diarrhoea (2-day and 2-week prevalence): $\geq 3$ loose or watery stools per 24 hours

Symptoms: loose stool by chart, blood in stool, vomit, fever, cough, congestion, difficulty breathing, earache, and bruising (negative controls)

Anthropometry (height for age, weight for age)

Self-reported all-cause and cause-specific mortality

Sanitation access and defecation behaviours (including open defecation by children and use of potty)

Drinking water quality

Hand hygiene

Lotes
Length of study: 24 months (April 2011 to May 2013)
Publication status: published

\section{Risk of bias}

\begin{tabular}{lll}
\hline Bias & Authors' judgement & Support for judgement \\
\hline $\begin{array}{l}\text { Random sequence genera- } \\
\text { tion (selection bias) }\end{array}$ & Low risk & $\begin{array}{l}\text { Quote: "One of the study investigators (MLA) used a computer-generated algo- } \\
\text { rithm that randomly assigned villages (1:1) to treatment and control groups." }\end{array}$ \\
\hline $\begin{array}{l}\text { Allocation concealment } \\
\text { (selection bias) }\end{array}$ & Low risk & $\begin{array}{l}\text { Quote: "The algorithm generated a random number for each village, which } \\
\text { was then used to sort villages and assigned the first } 60 \text { to the intervention } \\
\text { group and the remaining } 61 \text { to the control group." }\end{array}$ \\
\hline $\begin{array}{l}\text { Blinding of participants } \\
\text { and personnel (perfor- } \\
\text { mance bias) }\end{array}$ & High risk & $\begin{array}{l}\text { Quote: "Masking of participants was not possible because of the nature of the } \\
\text { intervention." }\end{array}$ \\
\hline
\end{tabular}


Pickering 2015 MLI (Continued)

$\begin{array}{lll}\text { Blinding of outcome as- } & \text { High risk } & \text { Quote: "Field staff were not informed of village treatment status, but could } \\ \text { sessment (detection bias) } & \text { have inferred this during the follow-up from the presence of signage showing } \\ \text { All outcomes } & \text { village certification of an open defecation free status." }\end{array}$

\begin{tabular}{|c|c|c|}
\hline $\begin{array}{l}\text { Incomplete outcome data } \\
\text { (attrition bias) } \\
\text { All outcomes }\end{array}$ & Low risk & $\begin{array}{l}\text { Similar percentage LTFU ( } 11.8 \% \text { of HHs in control group and } 10.4 \% \text { in interven- } \\
\text { tion group). }\end{array}$ \\
\hline $\begin{array}{l}\text { Selective reporting (re- } \\
\text { porting bias) }\end{array}$ & Low risk & All outcomes prespecified in methods were reported. \\
\hline Other bias & Unclear risk & - \\
\hline $\begin{array}{l}\text { Similarity of baseline out- } \\
\text { come measurements }\end{array}$ & Unclear risk & NA \\
\hline $\begin{array}{l}\text { Similarity of baseline char- } \\
\text { acteristics }\end{array}$ & Unclear risk & NA \\
\hline $\begin{array}{l}\text { Adequate allocation of in- } \\
\text { tervention concealment } \\
\text { during the study }\end{array}$ & Unclear risk & NA \\
\hline $\begin{array}{l}\text { Adequate protection } \\
\text { against contamination }\end{array}$ & Unclear risk & NA \\
\hline $\begin{array}{l}\text { Confounders adequate- } \\
\text { ly adjusted for in analy- } \\
\text { sis/design }\end{array}$ & Unclear risk & NA \\
\hline Recruitment bias & Low risk & $\begin{array}{l}\text { The participants were unaware whether they were randomized to CLTS or con- } \\
\text { trol villages. }\end{array}$ \\
\hline Baseline imbalance & Low risk & No substantial differences in baseline characteristics were observed. \\
\hline & & $\begin{array}{l}\text { Quote: "access to sanitation and an improved water source were similar across } \\
\text { groups. Baseline diarrhoeal and respiratory illness symptoms were at higher } \\
\text { prevalence in villages assigned to the CLTS intervention." }\end{array}$ \\
\hline Loss of clusters & Low risk & No loss of villages reported. \\
\hline Incorrect analysis & Low risk & $\begin{array}{l}\text { In the analysis used (quote) "robust standard errors (the Huber-White Sand- } \\
\text { wich estimator) to account for correlated outcomes at the village level." }\end{array}$ \\
\hline
\end{tabular}

Sarrassat 2018 BUR

\begin{tabular}{|c|c|}
\hline Methods & Cluster RCT \\
\hline \multirow[t]{4}{*}{ Participants } & $\begin{array}{l}\text { Number: pregnancy histories were completed for } 102,684 \text { women at end of study. At baseline, } 5043 \\
\text { mothers completed the behavioural questionnaire and } 5670 \text { mothers at end of study. }\end{array}$ \\
\hline & Inclusion criteria: \\
\hline & $\begin{array}{l}\text { - for the mortality outcomes: all women aged } 15-49 \text { years were included in the survey on full pregnancy } \\
\text { histories; }\end{array}$ \\
\hline & - for the intermediate outcomes: mothers with $\geq 1$ child aged $<5$ years living with them. \\
\hline
\end{tabular}


Sarrassat 2018 BUR (Continued)

Interventions

Intervention (7 geographical areas): mass radio campaign targeted at women of reproductive age and caregivers of children aged < 5 years, on 17 childcare behaviours, including safe child faeces disposal. The radio campaign included short broadcasts (1-minute duration, broadcast approximately 10 times per day) and interactive long-format programmes (2-hour duration, broadcast 5 days per week, followed by phone-ins to allow listeners to comment). All materials were produced in the predominant local languages of each intervention cluster. Behaviours covered by broadcasts changed weekly. The long-format programme covered 2 behaviours per day and changed daily. Safe child stool disposal was covered in 3 weeks of broadcasts and 94 long-format modules.

Control (7 geographical areas): no radio campaign

Primary outcome:
Outcomes
Secondary outcome:
- all-cause mortality in children aged $<5$ years
Intermediate outcomes:
- coverage of the campaign (proportion of mothers who reported listening to the campaign)
- family behaviours targeted by the campaign (proportion of mothers who reported a given behaviour
during interviews and the number of attendances at primary health facilities)

Notes

Location: 14 distinct geographical areas centred around a community FM radio station across Burkina Faso. Each clusters included about 40,000 inhabitants.

Length of study: December 2011 to March 2015. Baseline (December 2011 to February 2012); end of study (November 2014 to March 2015). Intervention ran from March 2012 to January 2015.

Publication status: journal

\section{Risk of bias}

\begin{tabular}{lll}
\hline Bias & Authors' judgement & Support for judgement \\
\hline $\begin{array}{l}\text { Random sequence genera- } \\
\text { tion (selection bias) }\end{array}$ & Low risk & Quote: "Computer generated random numbers." \\
\hline $\begin{array}{l}\text { Allocation concealment } \\
\text { (selection bias) }\end{array}$ & Low risk & $\begin{array}{l}\text { Participants and investigators could not foresee assignment due to random al- } \\
\text { location. }\end{array}$ \\
\hline $\begin{array}{l}\text { Blinding of participants } \\
\begin{array}{l}\text { land personnel (perfor- } \\
\text { mance bias) }\end{array}\end{array}$ & High risk & $\begin{array}{l}\text { Quote: "The nature of the intervention precluded formal masking of respon- } \\
\text { dents and interviewers." }\end{array}$ \\
\hline $\begin{array}{l}\text { All outcomes } \\
\end{array}$ &
\end{tabular}

\begin{tabular}{lll}
\hline Blinding of outcome as- & High risk & $\begin{array}{l}\text { Quote: "The nature of the intervention precluded formal masking of respon- } \\
\text { dents and interviewers." }\end{array}$
\end{tabular}

All outcomes

\begin{tabular}{lll}
\hline $\begin{array}{l}\text { Incomplete outcome data } \\
\text { (attrition bias) }\end{array}$ & Low risk & No difference across arms. \\
All outcomes & \\
\hline $\begin{array}{l}\text { Selective reporting (re- } \\
\text { porting bias) }\end{array}$ & Low risk & Reported on all outcomes.
\end{tabular}

Other bias Unclear risk -


Sarrassat 2018 BUR (Continued)

Similarity of baseline out- Unclear risk come measurements

Similarity of baseline char- Unclear risk acteristics

\begin{tabular}{lll}
\hline $\begin{array}{l}\text { Adequate allocation of in- } \\
\text { tervention concealment } \\
\text { during the study }\end{array}$ & Unclear risk & - \\
\hline $\begin{array}{l}\text { Adequate protection } \\
\text { against contamination }\end{array}$ & Unclear risk & - \\
\hline $\begin{array}{l}\text { Confounders adequate- } \\
\text { ly adjusted for in analy- } \\
\text { sis/design }\end{array}$ & Unclear risk & - \\
\hline $\begin{array}{l}\text { Recruitment bias } \\
\text { Baseline imbalance }\end{array}$ & High risk & Quote: "Randomisation was done before baseline survey." \\
\hline Loss of clusters & Low risk & $\begin{array}{l}\text { Quote: "Pair matched randomisation based on geography and radio listener- } \\
\text { ship" and then analysis included adjusting for a confounder score. }\end{array}$ \\
\hline \begin{tabular}{l} 
Incorrect analysis \\
\hline
\end{tabular} & Low risk & No loss of clusters. \\
\hline
\end{tabular}

Sinharoy 2017 RWA

\begin{tabular}{ll}
\hline Methods & Cluster RCT \\
\hline Participants & Number: 10,793 children aged $<5$ years at end of study $(7934 \mathrm{HHs})($ after $18.6 \%$ of children $<5$ years LT- \\
FU) & Inclusion criteria: all HHs with a child aged $<5$ years in the study area
\end{tabular}

Interventions, 2 arms testing 2 different versions of the CBEHPP, which used the $\mathrm{CHC}$ approach to promote healthy practices.

- Lite intervention (50 villages), baseline: $2773 \mathrm{HHs}$ ( 4171 children aged < 5 years), end of study: $2482 \mathrm{HHs}$ (3369 children aged $<5$ years): lite intervention held 8 sessions on village mapping, personal hygiene, handwashing, diarrhoea, water sources, safe storage of drinking water, treatment of drinking water, and sanitation.

- Classic intervention (50 villages), baseline: $3013 \mathrm{HHs}$ (4558 children aged < 5 years), end of study: 2729 $\mathrm{HHs}$ ( 3642 children aged $<5$ years): included 20 sessions, consisting of all the lite sessions plus common diseases, skin diseases, infant care (weaning and immunization), worms and intestinal parasites, food hygiene, nutrition, food safety and food security, the model home, good parenting, respiratory disease, malaria, bilharzia, and HIV/AIDS. Facilitators had a training manual and visual aids. CHCs in villages allocated the classic intervention also had attendance cards and organized graduation ceremonies, at which participants received certificates.

Both the lite and classic intervention included messages on child sanitation under the topic of sanitation (zero open defecation). The participants were mainly recommended the following:

- children should defecate into chamber-pot;

- children faeces should be buried if there is no latrine (cat sanitation) - but always emphasize in throwing the faeces in the latrine;

- never let the dog or pig eat children's faeces after defecation. 
Sinharoy 2017 RWA (Continued)

Control (50 villages), baseline: 2948 HHs ( 4523 children aged < 5 years); end of study: 2723 HHs (3782 children aged $<5$ years): no intervention

Diarrhoea (7-day recall)
Height-for-age or LAZ score
WHZ or weight-for-length Z score
Colony-forming units of thermotolerant (faecal) coliforms per $100 \mathrm{~mL}$ water
Intermediary outcomes:
- improved drinking water source
- HH water treatment (boiling, filtration, chlorination, or solar disinfection)
- presence of improved sanitation facility
- into toilet/latrine/buried, child used potty/nappies and thrown in the latrine immediately after
- the structure of sanitation facility (presence of floors, walls, and a roof)
- presence of faeces (human, animal, or both) in the HH courtyard
- exclusive breastfeeding for infants aged < 6 months
- dietary diversity for children aged 6 -23 months
- HH food security
- clinical data for diarrhoea and malaria and data for infant and child mortality (these outcomes will
be reported elsewhere)

Notes

Location: 150 villages in Rusizi district, Western Rwanda

Length of study: 32 months (May 2013 to December 2015)

Publication status: published

\section{Risk of bias}

\section{Bias}

Random sequence genera- Low risk tion (selection bias)

\section{Authors' judgement Support for judgement}

Quote: "We assessed villages for eligibility then randomly selected 150 [villages] for the study using a simple random sampling routine in STATA. We stratified villages by wealth index and by the proportion of children younger than 2 years with caregiver-reported diarrhoea within the past 7 days. We randomly allocated these villages to three study groups: no intervention (control; $\mathrm{n}=50$ ), eight community health club sessions (Lite intervention; $\mathrm{n}=50$ ), or 20 community health club sessions (Classic intervention; $n=50) . "$

$\begin{array}{ll}\begin{array}{l}\text { Allocation concealment } \\ \text { (selection bias) }\end{array} & \text { Low risk }\end{array} \quad \begin{aligned} & \text { Quote: "used Stata to randomly order the villages and divide them into three } \\ & \text { groups with approximately the same number of villages in each group." }\end{aligned}$

Blinding of participants High risk No blinding.
and personnel (performance bias)

All outcomes

$\begin{array}{lll}\text { Blinding of outcome as- } & \text { High risk } & \text { No blinding and some outcomes were self-reported. } \\ \text { sessment (detection bias) }\end{array}$

All outcomes

Incomplete outcome data Low risk (attrition bias) 
Sinharoy 2017 RWA (Continued)

All outcomes

Selective reporting (re- Low risk porting bias)
Report on outcomes specified in methods apart from clinical data for diarrhoea and malaria and data for infant and child mortality, but authors stated that these outcomes will be reported elsewhere.

\begin{tabular}{|c|c|c|}
\hline Other bias & Unclear risk & - \\
\hline $\begin{array}{l}\text { Similarity of baseline out- } \\
\text { come measurements }\end{array}$ & Unclear risk & - \\
\hline $\begin{array}{l}\text { Similarity of baseline char- } \\
\text { acteristics }\end{array}$ & Unclear risk & - \\
\hline $\begin{array}{l}\text { Adequate allocation of in- } \\
\text { tervention concealment } \\
\text { during the study }\end{array}$ & Unclear risk & - \\
\hline $\begin{array}{l}\text { Adequate protection } \\
\text { against contamination }\end{array}$ & Unclear risk & - \\
\hline $\begin{array}{l}\text { Confounders adequate- } \\
\text { ly adjusted for in analy- } \\
\text { sis/design }\end{array}$ & Unclear risk & - \\
\hline Recruitment bias & Low risk & Conducted baseline first then allocated villages to intervention arms. \\
\hline Baseline imbalance & Low risk & $\begin{array}{l}\text { Conducted stratification on average fraction of children aged }<2 \text { years with } \\
\text { caregiver-reported diarrhoea in the previous } 7 \text { days; and mean wealth index. }\end{array}$ \\
\hline Loss of clusters & Low risk & No loss of clusters. \\
\hline Incorrect analysis & Low risk & Used generalized estimating equations to account for village-level clustering. \\
\hline
\end{tabular}

Stanton 1987 BGD

Methods Cluster RCT

Participants

Number: 1923 families, 1350 with children aged < 6 years (after $0.8 \%$ emigrated)

Inclusion criteria: families with children aged $<6$ years

Interventions

Intervention ( 25 slums): educational intervention emphasizing 3 messages: proper hand washing before food preparation, defecation away from the house and in a proper site, and suitable disposal of waste and faeces. The intervention was delivered in the community over 8 weeks through small group discussions, larger demonstrations, community wide planning and action meeting, posters, games, pictorial stories, flexi flans (flannel board with movable characters).

Control (26 slums): community health workers continued to provide the primary healthcare services.

Outcomes Diarrhoea incidence in 6 months following intervention and 1 year following intervention. Diarrhoea: $\geq$ 3 unformed stools in any 24-hour period during the 2-week interval. stipulated that a child could have a maximum of 1 episode in any 1 recall period, and that a new episode began only after a round without diarrhoea (or in the first round) and ended with the next diarrhoea-free round.

Nutritional status (weight for age, height for age, weight for height) (Stanton 1988) 
Stanton 1987 BGD (Continued)

Hygiene behaviour change: hand washing before serving food, child defecate in living area, garbage and faeces seen in living area, child observed to put garbage in mouth.

Lotes
Length of study: 18 months (October 1984 to March 1986).
Publication status: journal

\section{Risk of bias}

\begin{tabular}{lll}
\hline Bias & Authors' judgement & Support for judgement \\
\hline $\begin{array}{l}\text { Random sequence genera- } \\
\text { tion (selection bias) }\end{array}$ & Low risk & Use of a random number table.
\end{tabular}

tion (selection bias)

$\begin{aligned} & \text { Allocation concealment } \\ & \text { (selection bias) }\end{aligned} \quad$ Unclear risk $\quad$ No detail on how allocation was concealed.

Blinding of participants $\quad$ High risk Quote: "This study was not performed in a double-blinded fashion."
and personnel (perfor-
mance bias)
All outcomes

\begin{tabular}{lll}
\hline $\begin{array}{l}\text { Blinding of outcome as- } \\
\text { sessment (detection bias) } \\
\text { All outcomes }\end{array}$ & High risk & Quote: "This study was not performed in a double-blinded fashion." \\
\hline $\begin{array}{l}\text { Incomplete outcome data } \\
\text { (attrition bias) }\end{array}$ & Unclear risk & Similar attrition in both groups. \\
All outcomes & $\begin{array}{l}\text { Quote: "equivalent percentages of intervention and control communities im- } \\
\text { migrated (19\% in intervention vs. } 23 \% \text { in control) or emigrated ( } 38 \% \text { in inter- } \\
\text { vention vs. } 37 \% \text { in control)" but unclear number of children who provided full } \\
\text { histories of diarrhoea. }\end{array}$
\end{tabular}

\begin{tabular}{lll}
\hline $\begin{array}{l}\text { Selective reporting (re- } \\
\text { porting bias) }\end{array}$ & Low risk & Report on all outcomes. \\
\hline Other bias & Unclear risk & NA \\
\hline $\begin{array}{l}\text { Similarity of baseline out- } \\
\text { come measurements }\end{array}$ & Unclear risk & NA \\
\hline $\begin{array}{l}\text { Similarity of baseline char- } \\
\text { acteristics }\end{array}$ & Unclear risk & NA \\
\hline $\begin{array}{l}\text { Adequate allocation of in- } \\
\text { tervention concealment } \\
\text { during the study }\end{array}$ & Unclear risk & \\
\hline $\begin{array}{l}\text { Adequate protection } \\
\text { against contamination }\end{array}$ & Unclear risk & NA \\
\hline $\begin{array}{l}\text { Confounders adequate- } \\
\text { ly adjusted for in analy- } \\
\text { sis/design }\end{array}$ & Unclear risk & NA \\
\hline \begin{tabular}{l} 
Recruitment bias \\
\hline
\end{tabular} & Low risk & Participants were recruited in clusters prior to randomization. \\
\hline
\end{tabular}


Stanton 1987 BGD (Continued)

Baseline imbalance Low risk Similar baseline characteristics and matched at design stage.

Quote: "grouped the ordered communities into 25 adjacent pairs and one remaining community....within each stratum (pair), one community was assigned to intervention and one to control."

$\begin{array}{ll}\text { Loss of clusters } \quad \text { Unclear risk } & \begin{array}{l}\text { No mention of loss of clusters, although did not present the single control } \\ \text { slum that was not matched. }\end{array}\end{array}$

\begin{tabular}{|c|c|c|}
\hline Incorrect analysis & High risk & $\begin{array}{l}\text { Although reported on analysis using cluster as individuals, did not present da- } \\
\text { ta and quote unadjusted data as final. }\end{array}$ \\
\hline
\end{tabular}

\section{Strina 2012 BRA}

\begin{tabular}{ll}
\hline Methods & Case-control study (clinic-based) \\
\hline Participants & $\begin{array}{l}\text { Cases: children (aged }<10 \text { years) presenting with diarrhoea as a main complaint in } 5 \text { health facilities of } \\
\text { Salvador and tested positive for rotavirus in stool sample, } \mathrm{n}=390,39.0 \%<12 \text { months, } 43.3 \% \text { girls. } \\
\text { Controls: children without diarrhoea selected from children attending the same health facilities, at } \\
\text { well-baby consultations or because of other health problems not related to diarrhoea, such as or- } \\
\text { thopaedic procedures or evaluation before a surgical operation. Controls were frequency matched to } \\
\text { cases by age and health insurance, } \mathrm{n}=1674,31.2 \%<12 \text { months, } 47.5 \% \text { girls. }\end{array}$ \\
\hline Interventions & $\begin{array}{l}\text { Risk factor of interest: } \\
\text { inadequate disposal of excreta of children } \leq 2 \text { years old vs adequate (no definition) }\end{array}$ \\
\hline Outcomes & Rotavirus diarrhoea: children with diarrhoea who tested positive for rotavirus in stool \\
\hline Notes & Location: urban, 5 health facilities, Salvador, Brazil \\
Length of recruitment: 21 months (November 2002 to August 2004) \\
Publication status: journal
\end{tabular}

\section{Risk of bias}

\begin{tabular}{lll}
\hline Bias & Authors' judgement & Support for judgement \\
\hline $\begin{array}{l}\text { Random sequence genera- } \\
\text { tion (selection bias) }\end{array}$ & Unclear risk & NA \\
\hline $\begin{array}{l}\text { Allocation concealment } \\
\text { (selection bias) }\end{array}$ & Unclear risk & NA \\
\hline $\begin{array}{l}\text { Blinding of participants } \\
\text { and personnel (perfor- } \\
\text { mance bias) } \\
\text { All outcomes }\end{array}$ & Unclear risk & NA \\
\hline $\begin{array}{l}\text { Blinding of outcome as- } \\
\text { sessment (detection bias) } \\
\text { All outcomes }\end{array}$ & Unclear risk & NA \\
\hline $\begin{array}{l}\text { Incomplete outcome data } \\
\text { (attrition bias) }\end{array}$ & Unclear risk & NA \\
\hline
\end{tabular}


Strina 2012 BRA (Continued)

All outcomes

Selective reporting (re- Unclear risk NA

porting bias)

NA

\begin{tabular}{|c|c|c|}
\hline Other bias & Unclear risk & NA \\
\hline $\begin{array}{l}\text { Similarity of baseline out- } \\
\text { come measurements }\end{array}$ & Unclear risk & NA \\
\hline $\begin{array}{l}\text { Similarity of baseline char- } \\
\text { acteristics }\end{array}$ & Unclear risk & NA \\
\hline $\begin{array}{l}\text { Adequate allocation of in- } \\
\text { tervention concealment } \\
\text { during the study }\end{array}$ & Unclear risk & NA \\
\hline $\begin{array}{l}\text { Adequate protection } \\
\text { against contamination }\end{array}$ & Unclear risk & NA \\
\hline $\begin{array}{l}\text { Confounders adequate- } \\
\text { ly adjusted for in analy- } \\
\text { sis/design }\end{array}$ & Unclear risk & NA \\
\hline Recruitment bias & Unclear risk & NA \\
\hline Baseline imbalance & Unclear risk & NA \\
\hline Loss of clusters & Unclear risk & NA \\
\hline Incorrect analysis & Unclear risk & NA \\
\hline
\end{tabular}

Traoré 1994a BUR

\begin{tabular}{ll}
\hline Methods & Case-control study \\
\hline Participants & $\begin{array}{l}\text { Cases: children aged } \leq 36 \text { months, resident in Bobo-Dioulasso and admitted to hospital at Sanou Souro } \\
\text { Hospital during the period of the study, with symptoms which included diarrhoea or dysentery, or } \\
\text { both, as reported by the mother, } \mathrm{n}=757 \text { (1056 cases in total but } 28 \% \text { LTFU), 49\%<12 months, } 45 \% \\
\text { girls }\end{array}$ \\
& Hospital controls: any child aged $\leq 36$ months, resident in Bobo-Dioulasso and admitted to hospital at \\
& Sanou Souro Hospital during the period of the study without symptoms of diarrhoea or dysentery, $\mathrm{n}=$ \\
& 631 (72\% follow-up), $40 \%<12$ months, $46 \%$ girls \\
& Neighbourhood controls: these were neighbours of children admitted to hospital with symptoms of di- \\
& arrhoea or dysentery, or both, matched for age group, $\mathrm{n}=1405,47 \%<12$ months, 53\% girls
\end{tabular}

\begin{tabular}{ll}
\hline Interventions & Risk factors of interest: \\
& - disposing of children faeces elsewhere vs latrines \\
& - stools visible in yard (not used in the review)
\end{tabular}

Outcomes Diarrhoea: as reported by mother and examined by a doctor; dysentery: bloody or mucoid stools


Traoré 1994a BUR (Continued)

Length of recruitment: 2.5 months (15 January 1990 to 31 March 1991)

Publication status: journal

\section{Risk of bias}

\begin{tabular}{lll}
\hline Bias & Authors' judgement \\
\hline $\begin{array}{l}\text { Random sequence genera- } \\
\text { tion (selection bias) }\end{array}$ & Unclear risk \\
\hline $\begin{array}{l}\text { Allocation concealment } \\
\text { (selection bias) }\end{array}$ & Unclear risk \\
\hline $\begin{array}{l}\text { Blinding of participants } \\
\text { and personnel (perfor- } \\
\text { mance bias) } \\
\text { All outcomes }\end{array}$ & Unclear risk \\
\hline
\end{tabular}

\begin{tabular}{|c|c|c|}
\hline $\begin{array}{l}\text { Blinding of outcome as- } \\
\text { sessment (detection bias) } \\
\text { All outcomes }\end{array}$ & Unclear risk & NA \\
\hline $\begin{array}{l}\text { Incomplete outcome data } \\
\text { (attrition bias) } \\
\text { All outcomes }\end{array}$ & Unclear risk & NA \\
\hline $\begin{array}{l}\text { Selective reporting (re- } \\
\text { porting bias) }\end{array}$ & Unclear risk & NA \\
\hline Other bias & Unclear risk & NA \\
\hline $\begin{array}{l}\text { Similarity of baseline out- } \\
\text { come measurements }\end{array}$ & Unclear risk & NA \\
\hline $\begin{array}{l}\text { Similarity of baseline char- } \\
\text { acteristics }\end{array}$ & Unclear risk & NA \\
\hline $\begin{array}{l}\text { Adequate allocation of in- } \\
\text { tervention concealment } \\
\text { during the study }\end{array}$ & Unclear risk & NA \\
\hline $\begin{array}{l}\text { Adequate protection } \\
\text { against contamination }\end{array}$ & Unclear risk & NA \\
\hline $\begin{array}{l}\text { Confounders adequate- } \\
\text { ly adjusted for in analy- } \\
\text { sis/design }\end{array}$ & Unclear risk & NA \\
\hline Recruitment bias & Unclear risk & NA \\
\hline Baseline imbalance & Unclear risk & NA \\
\hline Loss of clusters & Unclear risk & NA \\
\hline Incorrect analysis & Unclear risk & NA \\
\hline
\end{tabular}


Traoré 1994b BUR

\begin{tabular}{|c|c|}
\hline Methods & Case-control study \\
\hline \multirow[t]{3}{*}{ Participants } & $\begin{array}{l}\text { Cases: children aged } \leq 36 \text { months, resident in Bobo-Dioulasso and admitted to hospital at Sanou Souro } \\
\text { Hospital during the period of the study, with symptoms which included diarrhoea or dysentery, or } \\
\text { both, as reported by the mother, } n=757 \text { ( } 1056 \text { cases in total but } 28 \% \text { LTFU), } 49 \%<12 \text { months, } 45 \% \\
\text { girls. }\end{array}$ \\
\hline & $\begin{array}{l}\text { Hospital controls: any child aged } \leq 36 \text { months, resident in Bobo-Dioulasso and admitted to hospital at } \\
\text { Sanou Souro Hospital during the period of the study without symptoms of diarrhoea or dysentery, } n= \\
631 \text { ( } 72 \% \text { follow-up), } 40 \%<12 \text { months, } 46 \% \text { girls. }\end{array}$ \\
\hline & $\begin{array}{l}\text { Neighbourhood controls: these were neighbours of children admitted to hospital with symptoms of di- } \\
\text { arrhoea or dysentery, or both, matched for age group, } n=1405,47 \%<12 \text { months, } 53 \% \text { girls. }\end{array}$ \\
\hline \multirow[t]{2}{*}{ Interventions } & Risk factors of interest: \\
\hline & - defecation elsewhere vs in potty/latrine \\
\hline Outcomes & Diarrhoea: as reported by mother and examined by a doctor; dysentery: bloody or mucoid stools \\
\hline \multirow[t]{3}{*}{ Notes } & Location: urban Bobo-Dioulasso, Burkina Faso \\
\hline & Length of recruitment: 2.5 months (15 January 1990 to 31 March 1991) \\
\hline & Publication status: journal \\
\hline
\end{tabular}

\section{Risk of bias}

\begin{tabular}{|c|c|c|}
\hline Bias & Authors' judgement & Support for judgement \\
\hline $\begin{array}{l}\text { Random sequence genera- } \\
\text { tion (selection bias) }\end{array}$ & Unclear risk & NA \\
\hline $\begin{array}{l}\text { Allocation concealment } \\
\text { (selection bias) }\end{array}$ & Unclear risk & NA \\
\hline $\begin{array}{l}\text { Blinding of participants } \\
\text { and personnel (perfor- } \\
\text { mance bias) } \\
\text { All outcomes }\end{array}$ & Unclear risk & NA \\
\hline $\begin{array}{l}\text { Blinding of outcome as- } \\
\text { sessment (detection bias) } \\
\text { All outcomes }\end{array}$ & Unclear risk & NA \\
\hline $\begin{array}{l}\text { Incomplete outcome data } \\
\text { (attrition bias) } \\
\text { All outcomes }\end{array}$ & Unclear risk & NA \\
\hline $\begin{array}{l}\text { Selective reporting (re- } \\
\text { porting bias) }\end{array}$ & Unclear risk & NA \\
\hline Other bias & Unclear risk & NA \\
\hline $\begin{array}{l}\text { Similarity of baseline out- } \\
\text { come measurements }\end{array}$ & Unclear risk & NA \\
\hline
\end{tabular}


Traoré 1994b BUR (Continued)

Similarity of baseline char- Unclear risk NA
acteristics

\begin{tabular}{lll}
\hline $\begin{array}{l}\text { Adequate allocation of in- } \\
\text { tervention concealment } \\
\text { during the study }\end{array}$ & Unclear risk \\
\hline $\begin{array}{l}\text { Adequate protection } \\
\text { against contamination }\end{array}$ & Unclear risk & NA \\
\hline $\begin{array}{l}\text { Confounders adequate- } \\
\text { ly adjusted for in analy- } \\
\text { sis/design }\end{array}$ & Unclear risk & NA \\
\hline $\begin{array}{l}\text { Recruitment bias } \\
\text { Baseline imbalance }\end{array}$ & Unclear risk & NA \\
\hline Loss of clusters & Unclear risk & NA \\
\hline \begin{tabular}{l} 
Incorrect analysis \\
\hline
\end{tabular} & Unclear risk & NA \\
\hline
\end{tabular}

\section{Waterkeyn 2005 ZIM}

\begin{tabular}{|c|c|}
\hline Methods & Controlled cross-sectional study \\
\hline \multirow[t]{2}{*}{ Participants } & Number: 908 respondents \\
\hline & $\begin{array}{l}\text { Inclusion criteria: intervention survey respondents had to be members of health clubs, control group } \\
\text { respondents came from areas with no health clubs matched with regard to demography, cultural prac- } \\
\text { tices, levels of sanitation and water coverage. }\end{array}$ \\
\hline \multirow[t]{2}{*}{ Interventions } & $\begin{array}{l}\text { Intervention ( } 382 \text { respondents from Makoni and } 354 \text { from Tsholotsho): CHCs - structured weekly } \\
\text { course of participatory health education classes. The training materials used for health promotion con- } \\
\text { sisted of } 14 \text { sets of illustrated cards. The different topics were reflected in a 'membership card' which } \\
\text { provided an outline of the syllabus: } 1 \text {. mapping of village, } 2 \text { disease identification, } 3 \text {. balanced diet, } 4 \text {. } \\
\text { nutrition plans, } 5 \text {. Diarrhoea, } 6 \text {. salt sugar solution, } 7 \text {. home hygiene, } 8 \text {. water sources, } 9 \text {. drinking wa- } \\
\text { ter, 10. water storage, } 11 \text {. hand washing, } 12 \text {. bilharzia, } 13 \text {. skin and eye diseases, } 14 \text {. worms, 15. sanita- } \\
\text { tion ladder, } 16 \text {. sanitation story, } 17 \text {. malaria, } 18 \text {. respiratory diseases, } 19 \text {. tuberculosis, and 20. AIDs and } \\
\text { STDs. Within the hygiene lesson cover: disposal of toddler's faeces in a latrine. }\end{array}$ \\
\hline & Control (113 respondents from Makoni and 59 from Tsholotsho): no CHCs \\
\hline Outcomes & 20 observable indicators of behaviour change including child faeces in yard \\
\hline \multirow[t]{3}{*}{ Notes } & $\begin{array}{l}\text { Location: rural wards in Makoni ( } 21 \text { intervention wards) and Tsholotsho districts ( } 3 \text { intervention wards), } \\
\text { Zimbabwe }\end{array}$ \\
\hline & Length of study: 7 months (August 2000 to March 2001) \\
\hline & Publication status: journal \\
\hline
\end{tabular}

\section{Risk of bias}


Waterkeyn 2005 ZIM (Continued)

Random sequence genera- High risk Intervention not randomly allocated.
tion (selection bias)

\begin{tabular}{l}
$\begin{array}{l}\text { Allocation concealment } \\
\text { (selection bias) }\end{array}$ High risk Allocation not concealed. \\
\hline
\end{tabular}

Blinding of participants Unclear risk NA
and personnel (perfor-
mance bias)
All outcomes

\begin{tabular}{lll}
\hline $\begin{array}{l}\text { Blinding of outcome as- } \\
\text { sessment (detection bias) }\end{array}$ & Unclear risk & NA \\
$\begin{array}{ll}\text { All outcomes } \\
\text { Incomplete outcome data }\end{array}$ & Low risk & It seemed they observed hygiene indicators in all HHs. \\
\hline $\begin{array}{l}\text { (attrition bias) } \\
\text { All outcomes }\end{array}$ &
\end{tabular}

(attrition bias)

All outcomes

\begin{tabular}{|c|c|c|}
\hline $\begin{array}{l}\text { Selective reporting (re- } \\
\text { porting bias) }\end{array}$ & Low risk & Behaviours prespecified were reported. \\
\hline Other bias & Unclear risk & - \\
\hline $\begin{array}{l}\text { Similarity of baseline out- } \\
\text { come measurements }\end{array}$ & Unclear risk & NA, not relevant to design. \\
\hline $\begin{array}{l}\text { Similarity of baseline char- } \\
\text { acteristics }\end{array}$ & Unclear risk & NA, not relevant to design. \\
\hline $\begin{array}{l}\text { Adequate allocation of in- } \\
\text { tervention concealment } \\
\text { during the study }\end{array}$ & High risk & No blinding. \\
\hline $\begin{array}{l}\text { Adequate protection } \\
\text { against contamination }\end{array}$ & Low risk & $\begin{array}{l}\text { Control areas were "far removed from health clubs areas (typically } 30-50 \mathrm{~km} \\
\text { away)." }\end{array}$ \\
\hline $\begin{array}{l}\text { Confounders adequate- } \\
\text { ly adjusted for in analy- } \\
\text { sis/design }\end{array}$ & High risk & No adjustments for any confounders. \\
\hline Recruitment bias & Unclear risk & NA \\
\hline Baseline imbalance & Unclear risk & NA \\
\hline Loss of clusters & Unclear risk & NA \\
\hline Incorrect analysis & Unclear risk & NA \\
\hline
\end{tabular}

\section{Wijewardene 1992 SRI}

\begin{tabular}{ll}
\hline Methods & Case-control study (community-based) \\
\hline Participants & $\begin{array}{l}\text { Cases: families with } 1 \text { child aged }<5 \text { years having acute diarrhoea in previous } 6 \text { months (identified } \\
\text { through community visits), } \mathrm{n}=100 .\end{array}$
\end{tabular}


Wijewardene 1992 SRI (Continued)

Controls: families with $\geq 1$ child aged $<5$ years that did not have a single episode of diarrhoea during the previous 6 months, matched for age of child, occupation, and ethnic group of father, $n=100$.

Interventions

Risk factor of interest:

- not disposing of children's faeces in latrine vs disposing of it in latrine.

Outcomes $\quad$ Acute diarrhoea for children aged $>1$ years: $\geq 3$ loose stools in 24 hours for $\leq 7$ days

Notes
Length of recruitment: no details
Publication status: journal

\section{Risk of bias}

\begin{tabular}{ll}
\hline Bias & Authors' judgement \\
\hline $\begin{array}{l}\text { Random sequence genera- } \\
\text { tion (selection bias) }\end{array}$ & Unclear risk \\
\hline $\begin{array}{l}\text { Allocation concealment } \\
\text { (selection bias) }\end{array}$ & Unclear risk \\
\hline $\begin{array}{l}\text { Blinding of participants } \\
\text { and personnel (perfor- } \\
\text { mance bias) } \\
\text { All outcomes }\end{array}$ & Unclear risk \\
\hline
\end{tabular}

\begin{tabular}{|c|c|c|}
\hline $\begin{array}{l}\text { Blinding of outcome as- } \\
\text { sessment (detection bias) } \\
\text { All outcomes }\end{array}$ & Unclear risk & NA \\
\hline $\begin{array}{l}\text { Incomplete outcome data } \\
\text { (attrition bias) } \\
\text { All outcomes }\end{array}$ & Unclear risk & NA \\
\hline $\begin{array}{l}\text { Selective reporting (re- } \\
\text { porting bias) }\end{array}$ & Unclear risk & NA \\
\hline Other bias & Unclear risk & NA \\
\hline $\begin{array}{l}\text { Similarity of baseline out- } \\
\text { come measurements }\end{array}$ & Unclear risk & NA \\
\hline $\begin{array}{l}\text { Similarity of baseline char- } \\
\text { acteristics }\end{array}$ & Unclear risk & NA \\
\hline $\begin{array}{l}\text { Adequate allocation of in- } \\
\text { tervention concealment } \\
\text { during the study }\end{array}$ & Unclear risk & NA \\
\hline $\begin{array}{l}\text { Adequate protection } \\
\text { against contamination }\end{array}$ & Unclear risk & NA \\
\hline $\begin{array}{l}\text { Confounders adequate- } \\
\text { ly adjusted for in analy- } \\
\text { sis/design }\end{array}$ & Unclear risk & NA \\
\hline
\end{tabular}


Wijewardene 1992 SRI (Continued)

\begin{tabular}{lll} 
Recruitment bias & Unclear risk & NA \\
\hline Baseline imbalance & Unclear risk & NA \\
\hline Loss of clusters & Unclear risk & NA \\
\hline Incorrect analysis & Unclear risk & \\
\hline
\end{tabular}

\section{Yeager 2002 PER}

\begin{tabular}{ll}
\hline Methods & Cluster RCT \\
\hline Participants & Number: 722 HHs (postintervention) \\
& Inclusion criteria: HH had to have an eligible child (aged 15-47 months) \\
\hline Interventions & $\begin{array}{l}\text { Intervention (4 clusters): hygiene promotion for potty use and keeping the home environment free } \\
\text { from faeces. The intervention was delivered through routine health services, and using video presenta- } \\
\text { tions, leaflets including } 4 \text { steps to potty training and counselling by health staff during consultations. } \\
\text { Control (4 clusters): no intervention }\end{array}$ \\
\hline Outcomes & $\begin{array}{l}\text { Observed behaviours: use of potties, defecation behaviour of children, hygiene behaviours afterwards, } \\
\text { disposal behaviour of faeces }\end{array}$ \\
\hline Notes & $\begin{array}{l}\text { Location: San Juan de Lurigancho district, Lima, Peru } \\
\text { Length of study: } 17 \text { months (October 1996 to March 1998) } \\
\text { Publication status: journal }\end{array}$
\end{tabular}

\section{Risk of bias}

\begin{tabular}{lll}
\hline Bias & Authors' judgement & Support for judgement \\
\hline $\begin{array}{l}\text { Random sequence genera- } \\
\text { tion (selection bias) }\end{array}$ & Unclear risk & $\begin{array}{l}\text { Not described. } \\
\text { Quote: "One of these groups was then selected at random as the intervention } \\
\text { group." }\end{array}$ \\
\hline $\begin{array}{l}\text { Allocation concealment } \\
\text { (selection bias) }\end{array}$ & Unclear risk & Not described. \\
\hline $\begin{array}{l}\text { Blinding of participants } \\
\text { and personnel (perfor- } \\
\text { mance bias) } \\
\text { All outcomes }\end{array}$ & High risk & No blinding. \\
\hline $\begin{array}{l}\text { Blinding of outcome as- } \\
\text { sessment (detection bias) } \\
\text { All outcomes }\end{array}$ & High risk & No blinding. \\
\hline $\begin{array}{l}\text { Incomplete outcome data } \\
\text { (attrition bias) } \\
\text { All outcomes }\end{array}$ & Unclear risk & No details of non-response. \\
\hline
\end{tabular}


Yeager 2002 PER (Continued)

Selective reporting (re- Low risk $\quad$ Report on main outcomes.
porting bias)

\begin{tabular}{|c|c|c|}
\hline Other bias & Unclear risk & - \\
\hline $\begin{array}{l}\text { Similarity of baseline out- } \\
\text { come measurements }\end{array}$ & Unclear risk & NA \\
\hline $\begin{array}{l}\text { Similarity of baseline char- } \\
\text { acteristics }\end{array}$ & Unclear risk & NA \\
\hline $\begin{array}{l}\text { Adequate allocation of in- } \\
\text { tervention concealment } \\
\text { during the study }\end{array}$ & Unclear risk & NA \\
\hline $\begin{array}{l}\text { Adequate protection } \\
\text { against contamination }\end{array}$ & Unclear risk & NA \\
\hline $\begin{array}{l}\text { Confounders adequate- } \\
\text { ly adjusted for in analy- } \\
\text { sis/design }\end{array}$ & Unclear risk & NA \\
\hline Recruitment bias & High risk & $\begin{array}{l}\text { For end of study data collection, field workers would have known allocation of } \\
\text { cluster. }\end{array}$ \\
\hline Baseline imbalance & Low risk & The implementers had matched the zones. \\
\hline Loss of clusters & Unclear risk & No loss of clusters reported. \\
\hline Incorrect analysis & High risk & No statistical calculations. \\
\hline
\end{tabular}

ARI: acute respiratory infection; BMI: body mass index; CBA: controlled before-and-after; CBEHPP: Community-Based Environmental Health Promotion Programme; CHC: community health club; CLTS: community-led total sanitation; FDCH: family daycare home; HC: health centre; HEP: health extension package; HH: household; HWWS: handwashing with soap; IYCF: infant and young child feeding; LAZ: lengthfor-age Z score; LDC: licensed daycare centre; LNS: lipid-based nutrient supplement; LTFU: lost to follow-up; MUAC: mid-upper-arm-circumference; n: number of participants; NA: not applicable; ODF: open defecation-free; OTP: Outpatient Therapeutic feeding Program; ORS: oral rehydration solution; PEM: protein-energy malnutrition; PHAST: Participatory Hygiene and Sanitation Transformation; RCT: randomized controlled trial; SAM: severe acute malnutrition; SD: standard deviation; SHEWA-B: Sanitation Hygiene Education and Water Supply in Bangladesh; STH: soil-transmitted helminth; TSSM: Total Sanitation and Sanitation Marketing; UMOH: Uganda Ministry of Health; WASEP: Water and Sanitation Extension Programme; WASH: water, sanitation, and hygiene; WAZ: weight-for-age Z score; WHO: World Health Organization; WHZ: weight-for-height Z score; WVG: Women's Health Volunteer Group.

Characteristics of excluded studies [ordered by study ID]

\begin{tabular}{ll}
\hline Study & Reason for exclusion \\
\hline Assefa 2010 & Study design not eligible. \\
\hline Babu 2015 & No control group. \\
\hline Ban 2015 & Intervention not specific to child sanitation. \\
\hline Blum 1990 & $\begin{array}{l}\text { Unclear whether child faeces disposal or use of latrines by children was included in the interven- } \\
\text { tion. }\end{array}$ \\
\hline
\end{tabular}




\begin{tabular}{|c|c|}
\hline Study & Reason for exclusion \\
\hline Boehm 2016 & No relevant outcomes. \\
\hline Bohnert 2016 & Primary school-based intervention. \\
\hline Carnell 2014 & Outcomes not eligible. \\
\hline Clarke 2016 & Intervention did not seem to include child sanitation. \\
\hline Clasen 2015 & $\begin{array}{l}\text { In the intervention there was no messaging done on child faeces disposal or toilet use behaviour } \\
\text { change. }\end{array}$ \\
\hline Ditai 2016 & Intervention not eligible, only included alcohol hand rub. \\
\hline Dumba 2013 & $\begin{array}{l}\text { Unclear whether child faeces disposal or use of latrines by children was included in the interven- } \\
\text { tion. }\end{array}$ \\
\hline Erismann 2017 & $\begin{array}{l}\text { School-based intervention (children aged } \geq 8 \text { years) so no focus on sanitation for children aged }<5 \\
\text { years. }\end{array}$ \\
\hline Francis 2016 & Intervention not eligible, water filter with no child sanitation component. \\
\hline Freeman 2015 & $\begin{array}{l}\text { In the intervention there was no messaging done on child faeces disposal or toilet use behaviour } \\
\text { change. }\end{array}$ \\
\hline Galiani 2016 & Intervention not eligible, handwashing education only, no child sanitation component. \\
\hline Garn 2016 & Intervention not eligible: WASH in primary schools. \\
\hline Gelaye 2014 & No control and intervention was not eligible: primary school intervention. \\
\hline Gorter 1998 & Study design and intervention not eligible. \\
\hline Greenland 2016 & $\begin{array}{l}\text { Intervention not eligible, it only included the toilets and service. No specific behaviour change mes- } \\
\text { saging. }\end{array}$ \\
\hline Gungoren 2007 & $\begin{array}{l}\text { Unclear whether child faeces disposal or use of latrines by children was included in the interven- } \\
\text { tion. }\end{array}$ \\
\hline Hartinger 2016 & Intervention not eligible, no child sanitation component. \\
\hline Hunter 2004 & $\begin{array}{l}\text { Risk factor was contact with toileting child or changing nappy (yes vs no) not about the disposal of } \\
\text { the faeces and where the faeces end up. }\end{array}$ \\
\hline Hürlimann 2018 & Unclear whether the intervention included child faeces disposal messaging. Author did not reply. \\
\hline IOB/UNICEF 2011 & $\begin{array}{l}\text { Insufficient detail provided on whether child faeces disposal or use of latrines by children was in- } \\
\text { cluded in the intervention whether there was a control group. }\end{array}$ \\
\hline Islam 2018 & Study design not eligible (no control), baseline data from the WASH-B study. \\
\hline JDC/IHI 2012 & Unclear whether child faeces disposal was included in the intervention. \\
\hline Kaatano 2015 & $\begin{array}{l}\text { Study design not eligible (before and after study without a control) and unclear whether the inter- } \\
\text { vention included child sanitation. }\end{array}$ \\
\hline
\end{tabular}




\begin{tabular}{|c|c|}
\hline Study & Reason for exclusion \\
\hline Lamichhane 2018 & Study design not eligible. Analysis of data from the Nepal Demographic Health Survey 2011. \\
\hline Law 2016 & $\begin{array}{l}\text { Intervention not eligible: a psychological intervention aimed at improving toilet behaviour of chil- } \\
\text { dren (aged 4-7 years) with faecal incontinence. }\end{array}$ \\
\hline Liu 2017 & $\begin{array}{l}\text { Unclear whether the intervention included messaging on child faeces disposal. The author did not } \\
\text { reply. }\end{array}$ \\
\hline Messou 1997 & $\begin{array}{l}\text { Unclear whether child faeces disposal or use of latrines by children was included in the interven- } \\
\text { tion. }\end{array}$ \\
\hline Nerkar 2015 & $\begin{array}{l}\text { Intervention not eligible: focused on toilet construction and watershed management. No focus on } \\
\text { child faeces management. }\end{array}$ \\
\hline Njuguna 2016 & Risk factor was hand washing after child faeces disposal, not child faeces disposal itself. \\
\hline Olayo 2014 & Intervention and outcomes not eligible. \\
\hline Park 2018 & Unclear whether intervention and outcomes were eligible. Author did not reply. \\
\hline Raso 2018 & Unclear whether intervention included child faeces disposal messaging. Author did not reply. \\
\hline Reese 2017 & $\begin{array}{l}\text { Intervention not eligible. Exchange with author confirmed that the intervention did not include } \\
\text { specific messaging about child faeces disposal. }\end{array}$ \\
\hline Sarkar 2014 & Risk factor was not specific to child sanitation, it was use of the latrine by all household members. \\
\hline Slayton 2016 & Intervention not eligible, antimicrobial hand towel with no child sanitation component. \\
\hline Taha 2000 & Intervention and outcome not eligible. \\
\hline Trinies 2016 & Intervention not eligible: WASH intervention based in primary school. \\
\hline Yeasmin 2017 & Intervention, study design, and outcomes not eligible. \\
\hline Yentur 2015 & Risk factor not eligible. \\
\hline Zomer 2015 & $\begin{array}{l}\text { Intervention not eligible: focused on hand hygiene only, including after nappy changing but noth- } \\
\text { ing about faeces disposal. }\end{array}$ \\
\hline
\end{tabular}

WASH: water, sanitation, and hygiene.

Characteristics of ongoing studies [ordered by study ID]

\section{ACTRN12613000523707}

Trial name or title

Methods

Participants

Interventions
The effectiveness and acceptability of the 'BALatrine': a culturally acceptable latrine intervention in resource limited environments

\section{Cluster RCT}

\section{Estimated: 4000}

The intervention is a household latrine (BALatrine) plus health education/promotion on hygiene and sanitation. The BALatrine is a simple squat latrine. 
ACTRN12613000523707 (Continued)

Primary outcome
- STH prevalence
Secondary outcome
- Improved health knowledge and hygiene and sanitation behaviour

\begin{tabular}{ll}
\hline Starting date & September 2016 \\
\hline Contact information & Prof Donald Stewart, Griffith University, South Brisbane \\
\hline Notes & Location: Wonosobo, Central Java, Indonesia \\
& Trial registration number: ACTRN12613000523707 \\
\hline
\end{tabular}

\section{ISRCTN10419317}

\begin{tabular}{|c|c|}
\hline Trial name or title & $\begin{array}{l}\text { The impact of improved sanitation on the diarrhoeal reduction of under-five children in Democrat- } \\
\text { ic Republic of Congo }\end{array}$ \\
\hline Methods & Cluster-RCT \\
\hline Participants & All children in estimated 720 households \\
\hline Interventions & $\begin{array}{l}\text { Intervention: sanitation campaign to increase latrine coverage using CLTS principle and borehole } \\
\text { drilling. Child faeces disposal messaging is included in the CLTS triggering. } \\
\text { Control: borehole drilling }\end{array}$ \\
\hline Outcomes & $\begin{array}{l}\text { Primary outcome } \\
\text { - Diarrhoeal incidence of children aged }<5 \text { years (cases/child }{ }^{*} \text { weeks) } \\
\text { Secondary outcomes } \\
\text { - Uptake of improved latrine }(\%) \\
\text { - Utilization of improved latrine }(\%)\end{array}$ \\
\hline Starting date & December 2014 \\
\hline Contact information & Dr Seungman Cha, Korea International Cooperation Agency \\
\hline Notes & $\begin{array}{l}\text { Trial registration number: ISRCTN10419317 } \\
\text { Location: Bandundu province (Democratic Republic of Congo) }\end{array}$ \\
\hline
\end{tabular}

\section{ISRCTN16961836}

Trial name or title

Efficacy of a behavioural intervention based on food consumption, nutritional state and micronutrient deficiency in under five children, Angola

\begin{tabular}{ll}
\hline Methods & RCT \\
\hline Participants & All children living in participating hamlets aged $<36$ months old and their primary caregivers.
\end{tabular}


ISRCTN16961836 (Continued)

Estimated: 2182

Interventions
Nutrition arm: participants receive 12 personalized home-based counselling visits divided in blocks of 3 monthly visits after baseline and each follow-up time point $(6,12$, and 18 months). These visits involve the delivering of 11 key recommendations and messages for promoting infant and young children optimal feeding practices regarding breastfeeding, complementary feeding (dietary diversity, meal frequency, and quantity of food), responsive feeding, feeding during and after illness; hygiene and food safety. Participants also attend 4 community group meetings at baseline, 6, 12, and 18 months, which focus on the key messages along theoretical and practical sessions.

WASH arm: participants receive 12 personalized home-based counselling visits divided in blocks of 3 monthly visits after baseline and each follow-up time point (6,12, and 18 months). These visits involve the delivering of 11 key recommendations and messages for promoting optimal parental hygiene and health practices regarding infant personal hygiene, hand washing (supplies, techniques, critical moments), safe drinking water (treatment, collection, storage), house surrounding environment, safely disposal of faeces, and malaria prevention. Participants also attend 4 community group meetings at baseline, 6,12 , and 18 months, which focus on the key messages along theoretical and practical sessions.

Control arm: no educational package between assessments.

\section{Outcomes}

\section{Primary outcomes}

- Linear growth of children aged $<5$ years is assessed by mean change in length-for-age Z-scores from baseline to $6,12,18$, and 24 months

- Serum micronutrient status of children aged $<5$ years is assessed by mean change of vitamin $A$, vitamin D, vitamin E, vitamin B12, folic acid, iron, zinc from baseline to $6,12,18$, and 24 months

\section{Secondary outcomes}

- Improvement of weight-for-length is assessed through weight-for-age $Z$ scores from baseline to $6,12,18$, and 24 months

- Energy and macronutrient intake from complementary foods measured using an interviewer administered 24-hour-dietary recall at baseline, 6, 12, 18, and 24 months

- Occurrence of parasitological infection (malaria and helminths) measured using blood testing, faeces, and urine analysis at baseline 6, 12, 18, and 24 months

\begin{tabular}{ll}
\hline Starting date & November 2014 \\
\hline Contact information & Miguel Brito \\
& Rua Direita do Caxito Hospital Geral do Bengo - Caxito, Província do Bengo Angola \\
\hline Notes & Trial registration number: ISRCTN16961836 \\
& Location: Angola \\
\hline
\end{tabular}

\section{NCT02754583}

Trial name or title

Methods

SWIFT: Sanitation, Water, and Instruction in Face-washing for Trachoma

Cluster-RCT

Participants Estimated: 220,000

Interventions

A series of 3 cluster-RCTs to assess several alternative strategies for trachoma control in communities that have been treated with many years of mass azithromycin distributions. 
The first trial ('WUHA') compares communities that receive a comprehensive WASH package (including promotion to households that the faeces of children aged $<5$ years should be deposited in a latrine) to those that receive no intervention.

The second trial ('TAITU-A') compares communities randomized to targeted antibiotic treatment vs those randomized to mass antibiotics for trachoma.

The third trial ('TAITU-B') compares communities randomized to targeted antibiotics vs those randomized to delayed antibiotics.

\section{Outcomes}

Primary outcomes

- Village-specific ocular chlamydia among children aged 0-5 years over time (first trial: WUHA) at 12, 24, and 36 months

- Ocular chlamydia among children aged 8-12 years (second trial: TAITU-A) at 24 months

- Incident ocular chlamydia in children aged 0-5 year (third trial: TAITU-B) at 24 months

- Trial-based cost-effectiveness of intervention (intervention costs per percent of chlamydia reduction) at 24 months for TAITU, 36 months for WUHA

Secondary outcomes

- Quantitative polymerase chain reaction chlamydia load at 12,24 , and 36 months

- Follicular trachoma scores; age-stratified (0-5 years, 6-9 years, $\geq 10$ years for WUHA; 0-5 years, 812 years for TAITU) at 12, 24, and 36 months

- Inflammatory trachoma scores; age-stratified (0-5 years, 6-9 years, $\geq 10$ years for WUHA; 0-5 years, 8-12 years for TAITU) at 12, 24, and 36 months

- Ocular chlamydia; age-stratified (0-5 years, 6-9 years, $\geq 10$ years for WUHA; 0-5 years, 8-12 years for TAITU) at 12, 24, and 36 months

- Nasopharyngeal pneumococcal macrolide resistance at 12, 24, and 36 months

- Proportion of the population with clean faces at the village level at 12, 24, and 36 months

- Childhood growth (height) at 12, 24, and 36 months

- Childhood growth (weight) at 12, 24, and 36 months

- STH prevalence at 12,24 , and 36 months

- STH density at 12,24 , and 36 months

- Prevalence of chlamydia and other antigen positivity from serological tests at 12,24 , and 36 months

- Prevalence of stool-based antigen (diarrhoeal pathogens, STH) positivity from serological tests at 12, 24, and 36 months

- Ancillary study: intestinal microbiome from rectal sample, using 165 rRNA deep sequencing or next-generation sequencing, or both, at 12 months

- Ancillary study: sensitivity and specificity of detecting STH using rectal swabs with logistic mixedeffects at 12 months

\begin{tabular}{ll}
\hline Starting date & November 2015 \\
\hline Contact information & Jeremy D Keenan - University of California San Francisco Proctor Foundation \\
\hline Notes & Location: Ethiopia \\
& Trial registration number: NCT02754583 \\
\hline
\end{tabular}

CLTS: community-led total sanitation; RCT: randomized controlled trial; rRNA: ribosomal ribonucleic acid; STH: soil-transmitted helminth; WASH: water, sanitation, and hygiene.

\section{DATA AND ANALYSES}


Comparison 1. Education and hygiene promotion interventions versus control

\begin{tabular}{|c|c|c|c|c|}
\hline Outcome or subgroup title & $\begin{array}{l}\text { No. of } \\
\text { studies }\end{array}$ & $\begin{array}{l}\text { No. of } \\
\text { partici- } \\
\text { pants }\end{array}$ & Statistical method & Effect size \\
\hline $\begin{array}{l}1 \text { Diarrhoea prevalence - randomized con- } \\
\text { trolled trials (RCTs) }\end{array}$ & 2 & & Risk Ratio (Random, 95\% Cl) & $0.93[0.84,1.04]$ \\
\hline 2 Diarrhoea incidence - RCTs & 2 & & Rate Ratio (Random, 95\% Cl) & $0.71[0.59,0.86]$ \\
\hline $\begin{array}{l}3 \text { Diarrhoea prevalence - controlled cohort } \\
\text { studies: SHEWA-B versus control }\end{array}$ & 2 & & Risk Ratio (Random, 95\% Cl) & $0.91[0.64,1.28]$ \\
\hline $\begin{array}{l}4 \text { Diarrhoea prevalence - controlled cross- } \\
\text { sectional: HEP model households versus non- } \\
\text { model }\end{array}$ & 2 & & Odds Ratio (Random, 95\% Cl) & $0.26[0.16,0.42]$ \\
\hline $\begin{array}{l}5 \text { Anthropometry - RCTs: height-for-age Z } \\
\text { score (HAZ) }\end{array}$ & 2 & & $\begin{array}{l}\text { Mean Difference (Random, 95\% } \\
\mathrm{Cl} \text { ) }\end{array}$ & $0.05[-0.07,0.17]$ \\
\hline $\begin{array}{l}6 \text { Behaviour change - RCTs: latrine use by } \\
\text { children }\end{array}$ & 2 & & Risk Ratio (Random, 95\% Cl) & $1.69[0.26,11.04]$ \\
\hline $\begin{array}{l}7 \text { Behaviour change - RCTs: potty use by chil- } \\
\text { dren }\end{array}$ & 2 & & Risk Ratio (Random, 95\% Cl) & $1.37[0.57,3.30]$ \\
\hline $\begin{array}{l}8 \text { Behaviour change - RCTs: safe disposal of } \\
\text { child faeces }\end{array}$ & 2 & & Risk Ratio (Random, 95\% Cl) & $1.01[0.93,1.08]$ \\
\hline $\begin{array}{l}9 \text { Behaviour change - RCTs: appropriate dis- } \\
\text { posal of child faeces }\end{array}$ & 1 & & Risk Difference (Random, 95\% Cl) & $-0.01[-0.06,0.03]$ \\
\hline $\begin{array}{l}10 \text { Behaviour change - RCTs: faeces not ob- } \\
\text { served in yard/ } \mathrm{HH}\end{array}$ & 2 & & Risk Ratio (Random, 95\% Cl) & $1.09[0.61,1.94]$ \\
\hline $\begin{array}{l}11 \text { Behaviour change - RCTs: faeces in com- } \\
\text { pound }\end{array}$ & 1 & & Risk Difference (Random, 95\% Cl) & $0.00[-0.02,0.02]$ \\
\hline $\begin{array}{l}12 \text { Behaviour change - controlled cohort } \\
\text { studies: safe vs unsafe child faeces disposal }\end{array}$ & 2 & & Risk Ratio (Random, 95\% Cl) & $1.10[0.72,1.67]$ \\
\hline $\begin{array}{l}13 \text { Behaviour change - controlled cross-sec- } \\
\text { tional studies: safe vs unsafe child faeces dis- } \\
\text { posal }\end{array}$ & 3 & & Risk Ratio (Random, 95\% Cl) & Subtotals only \\
\hline 13.1 BRAC & 1 & & Risk Ratio (Random, 95\% Cl) & $4.25[1.91,9.46]$ \\
\hline 13.2 HEP & 2 & & Risk Ratio (Random, 95\% Cl) & $1.36[0.98,1.89]$ \\
\hline
\end{tabular}


Analysis 1.1. Comparison 1 Education and hygiene promotion interventions versus control, Outcome 1 Diarrhoea prevalence - randomized controlled trials (RCTs).

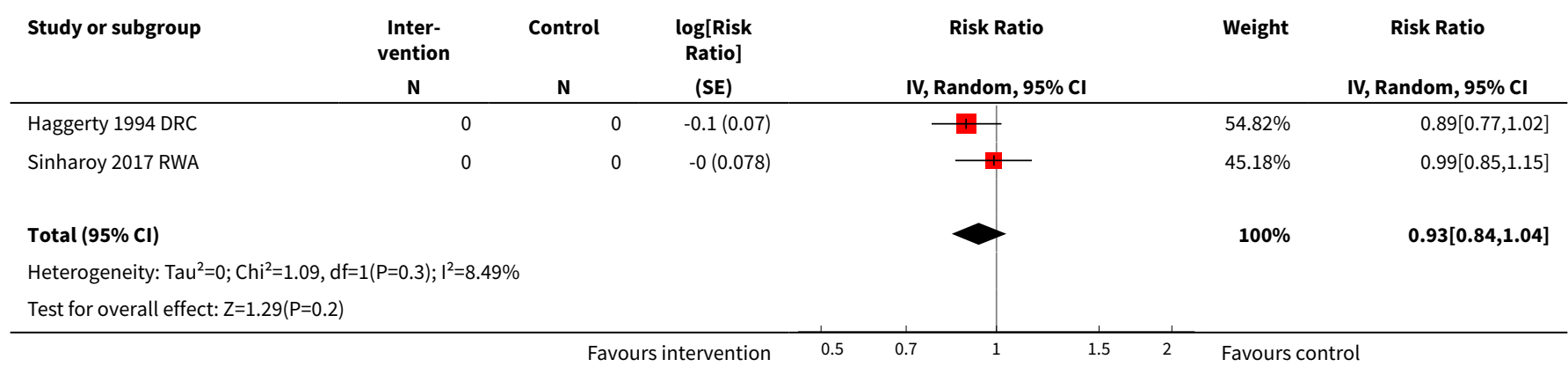

Analysis 1.2. Comparison 1 Education and hygiene promotion interventions versus control, Outcome 2 Diarrhoea incidence - RCTs.

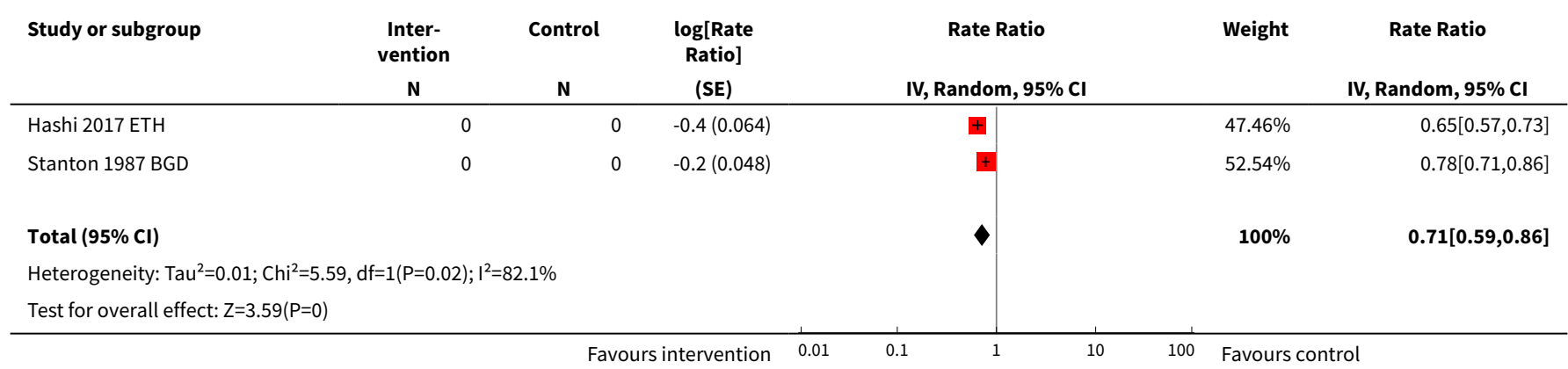

Analysis 1.3. Comparison 1 Education and hygiene promotion interventions versus control, Outcome 3 Diarrhoea prevalence - controlled cohort studies: SHEWA-B versus control.

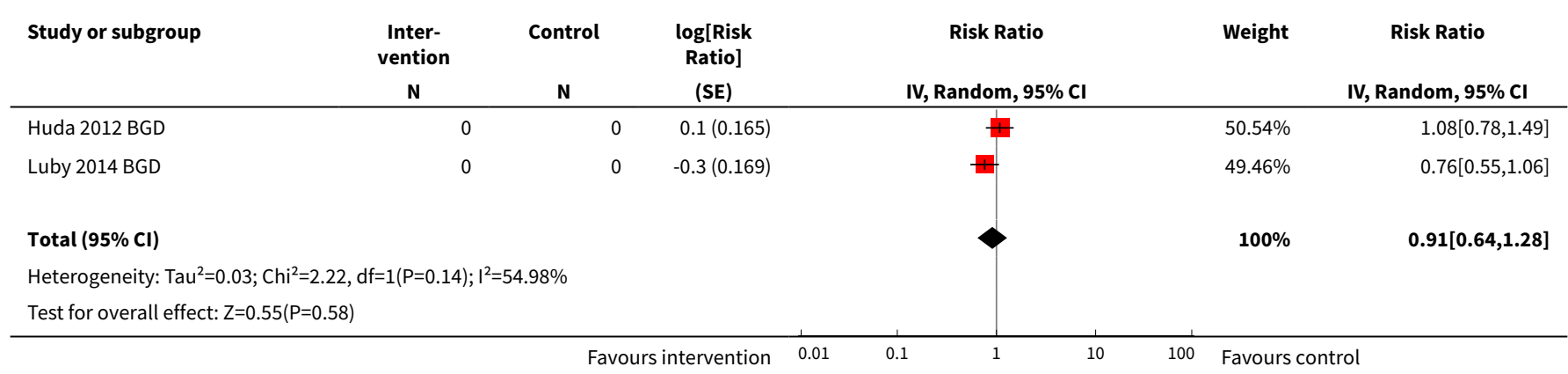


Analysis 1.4. Comparison 1 Education and hygiene promotion interventions versus control, Outcome 4 Diarrhoea prevalence - controlled cross-sectional: HEP model households versus non-model.

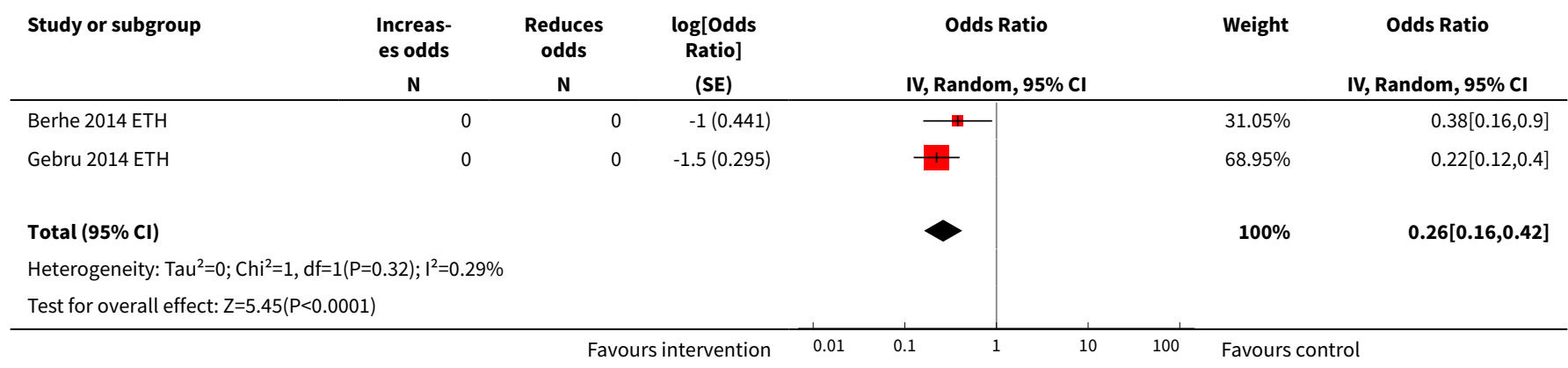

Analysis 1.5. Comparison 1 Education and hygiene promotion interventions versus control, Outcome 5 Anthropometry - RCTs: height-for-age Z score (HAZ).

\begin{tabular}{|c|c|c|c|c|c|c|}
\hline Study or subgroup & $\begin{array}{c}\text { Inter- } \\
\text { vention } \\
\mathbf{N}\end{array}$ & $\begin{array}{l}\text { Control } \\
\text { N }\end{array}$ & $\begin{array}{l}\text { Mean Dif- } \\
\text { ference } \\
\text { (SE) }\end{array}$ & $\begin{array}{c}\text { Mean Difference } \\
\text { IV, Random, } 95 \% \mathrm{CI}\end{array}$ & Weight & $\begin{array}{c}\text { Mean Difference } \\
\text { IV, Random, 95\% CI }\end{array}$ \\
\hline Nair 2017 IND & 0 & 0 & $0.1(0.06)$ & 1 & $54.79 \%$ & $0.11[-0.01,0.23]$ \\
\hline Sinharoy 2017 RWA & 0 & 0 & $-0(0.072)$ & $\longrightarrow$ & $45.21 \%$ & $-0.02[-0.16,0.12]$ \\
\hline Total $(95 \% \mathrm{CI})$ & & & & & $100 \%$ & $0.05[-0.07,0.17]$ \\
\hline Test for overall effect & & & & & & \\
\hline
\end{tabular}

Analysis 1.6. Comparison 1 Education and hygiene promotion interventions versus control, Outcome 6 Behaviour change - RCTs: latrine use by children.

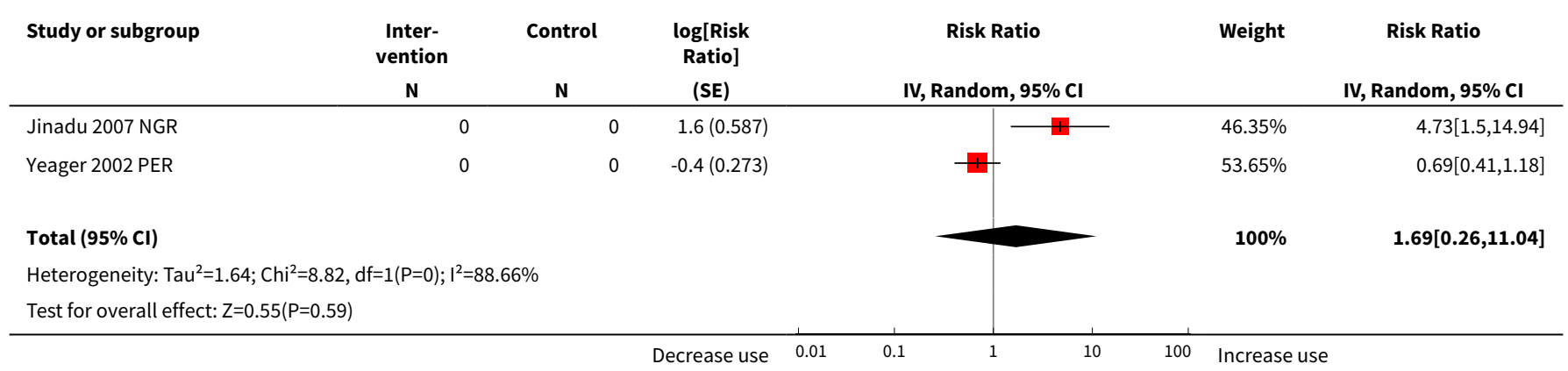


Analysis 1.7. Comparison 1 Education and hygiene promotion interventions versus control, Outcome 7 Behaviour change - RCTs: potty use by children.

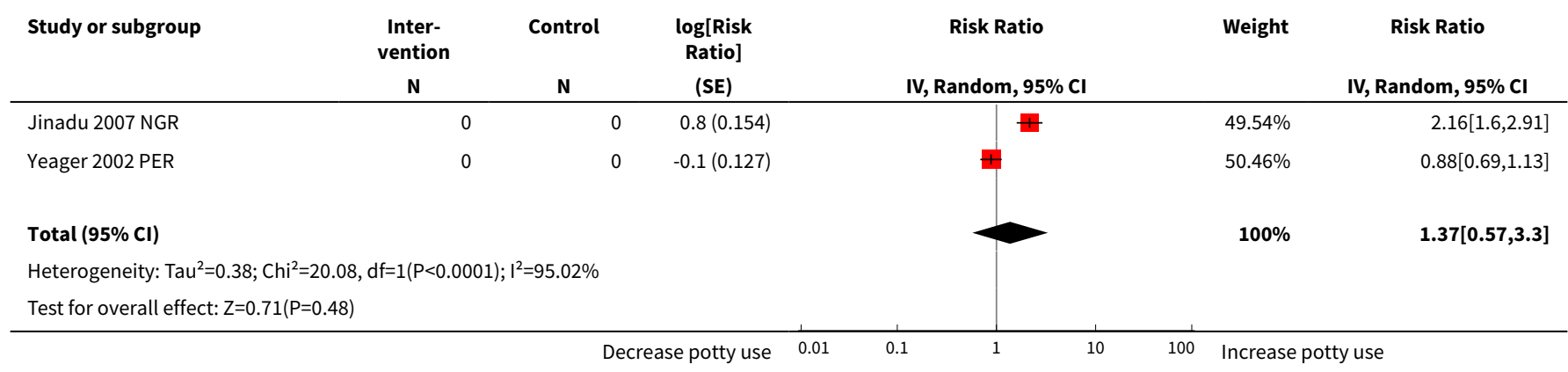

Analysis 1.8. Comparison 1 Education and hygiene promotion interventions versus control, Outcome 8 Behaviour change - RCTs: safe disposal of child faeces.

\begin{tabular}{|c|c|c|c|c|c|c|}
\hline Study or subgroup & $\begin{array}{c}\text { Inter- } \\
\text { vention } \\
\mathbf{N}\end{array}$ & $\begin{array}{c}\text { Control } \\
\text { N }\end{array}$ & $\begin{array}{c}\text { log[Risk } \\
\text { Ratio] } \\
\text { (SE) }\end{array}$ & $\begin{array}{c}\text { Risk Ratio } \\
\text { IV, Random, } 95 \% \mathrm{CI}\end{array}$ & Weight & $\begin{array}{c}\text { Risk Ratio } \\
\text { IV, Random, } 95 \% \mathrm{CI}\end{array}$ \\
\hline Sarrassat 2018 BUR & 0 & 0 & $-0(0.051)$ & +1 & $55.49 \%$ & $1[0.9,1.1]$ \\
\hline Yeager 2002 PER & 0 & 0 & $0(0.057)$ & 1 & $44.51 \%$ & $1.02[0.91,1.14]$ \\
\hline Total $(95 \% \mathrm{Cl})$ & & & & & $100 \%$ & $1.01[0.93,1.08]$ \\
\hline Test for overall effect & & & & & & \\
\hline
\end{tabular}

Analysis 1.9. Comparison 1 Education and hygiene promotion interventions versus control, Outcome 9 Behaviour change - RCTs: appropriate disposal of child faeces.

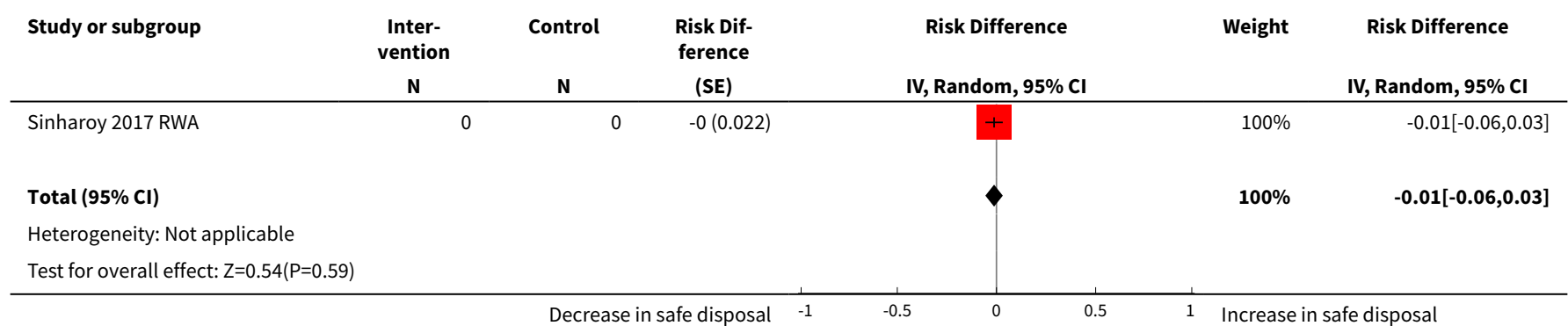

Analysis 1.10. Comparison 1 Education and hygiene promotion interventions versus control, Outcome 10 Behaviour change - RCTs: faeces not observed in yard/ HH.

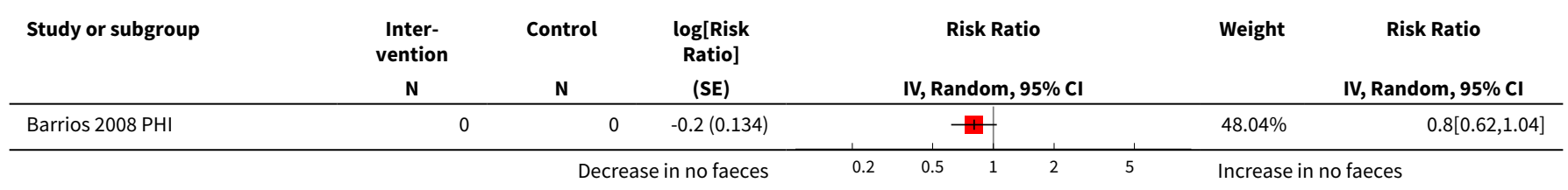




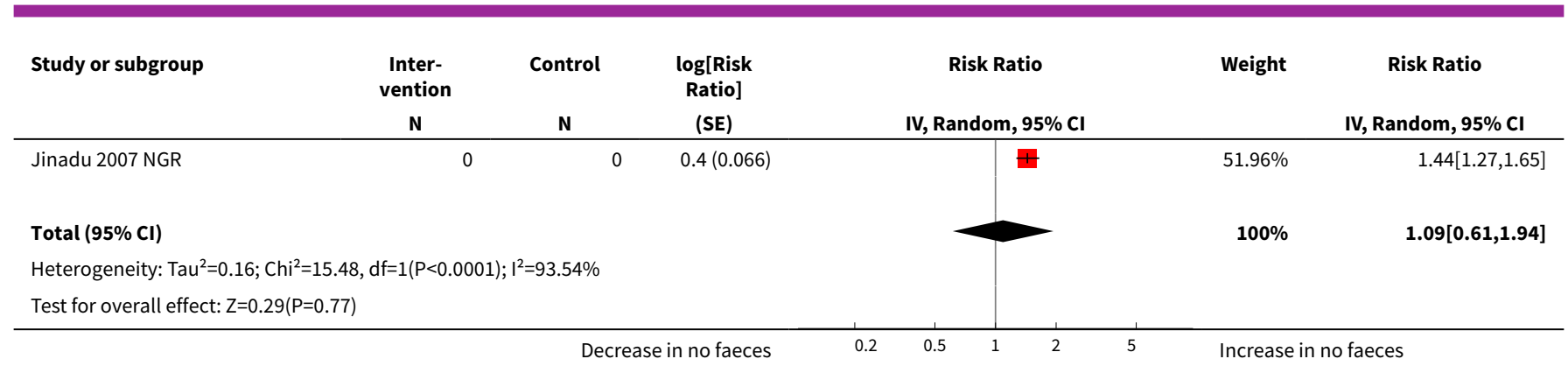

Analysis 1.11. Comparison 1 Education and hygiene promotion interventions versus control, Outcome 11 Behaviour change - RCTs: faeces in compound.

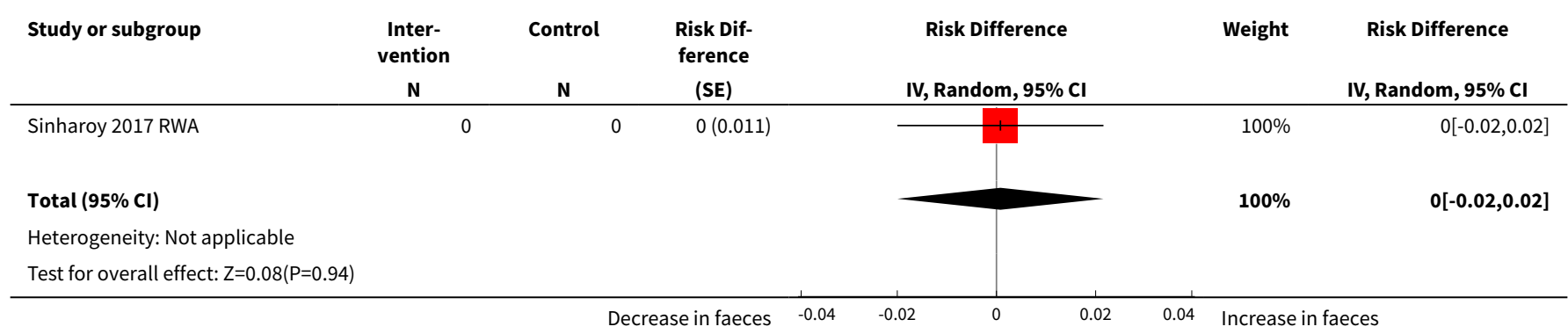

Analysis 1.12. Comparison 1 Education and hygiene promotion interventions versus control, Outcome 12 Behaviour change - controlled cohort studies: safe vs unsafe child faeces disposal.

\begin{tabular}{|c|c|c|c|c|c|c|}
\hline Study or subgroup & $\begin{array}{c}\text { Inter- } \\
\text { vention } \\
\mathbf{N}\end{array}$ & Control & $\begin{array}{c}\text { log[Risk } \\
\text { Ratio] } \\
\text { (SE) }\end{array}$ & IV, Random, 95\% CI & Weight & IV, Random, 95\% CI \\
\hline Huda 2012 BGD & 0 & 0 & $0.1(0.288)$ & - & $55.87 \%$ & $1.06[0.61,1.87]$ \\
\hline Luby 2014 BGD & 0 & 0 & $0.1(0.324)$ & 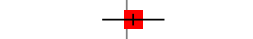 & $44.13 \%$ & $1.14[0.61,2.16]$ \\
\hline Total $(95 \% \mathrm{Cl})$ & & & & & $100 \%$ & $1.1[0.72,1.67]$ \\
\hline \multicolumn{7}{|c|}{ Test for overall effect: $Z=0.43(P=0.66)$} \\
\hline
\end{tabular}

Analysis 1.13. Comparison 1 Education and hygiene promotion interventions versus control, Outcome 13 Behaviour change - controlled cross-sectional studies: safe vs unsafe child faeces disposal.

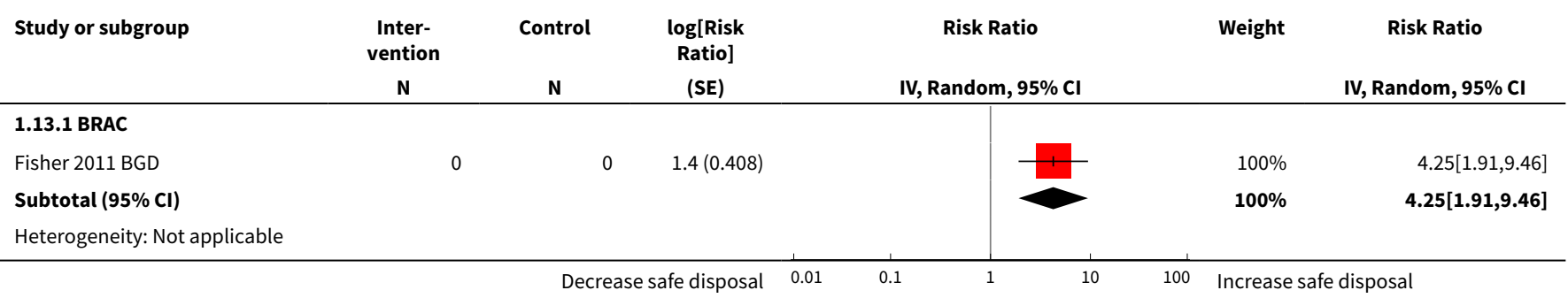




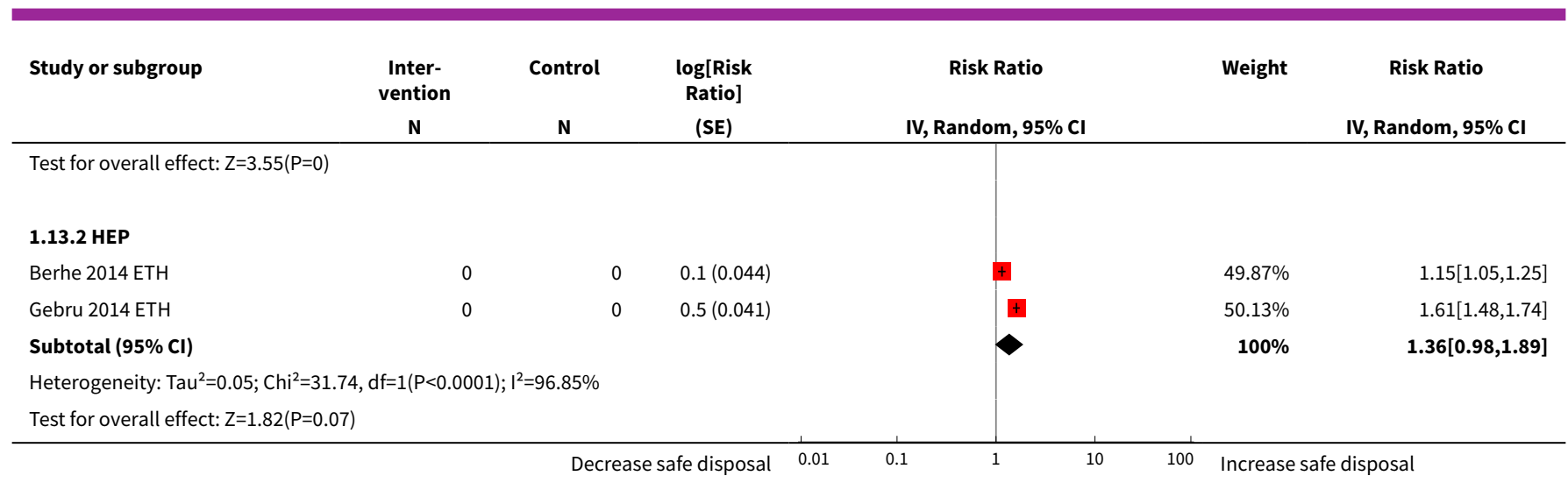

\section{Comparison 2. CLTS interventions plus adaptations}

\begin{tabular}{|c|c|c|c|c|}
\hline Outcome or subgroup title & $\begin{array}{l}\text { No. of } \\
\text { studies }\end{array}$ & $\begin{array}{l}\text { No. of } \\
\text { partici- } \\
\text { pants }\end{array}$ & Statistical method & Effect size \\
\hline $\begin{array}{l}1 \text { Diarrhoea prevalence - randomized } \\
\text { controlled trials (RCTs) }\end{array}$ & 4 & & Risk Ratio (Random, 95\% Cl) & $0.92[0.79,1.07]$ \\
\hline 2 Any helminth prevalence - RCTs & 2 & & Risk Ratio (Random, 95\% Cl) & $1.03[0.64,1.65]$ \\
\hline 3 Ascaris lumbricoides prevalence - RCTs & 2 & & Risk Ratio (Random, 95\% Cl) & $1.01[0.60,1.71]$ \\
\hline 4 Dysentery - RCTs & 2 & & Risk Ratio (Random, 95\% Cl) & $0.69[0.35,1.34]$ \\
\hline $\begin{array}{l}5 \text { Anthropometry: height-for-age Z score } \\
\text { (HAZ) - RCTs }\end{array}$ & 4 & & Mean Difference (Random, 95\% Cl) & $0.06[-0.07,0.19]$ \\
\hline $\begin{array}{l}6 \text { Anthropometry: weight-for-age Z score } \\
\text { (WAZ) - RCTs }\end{array}$ & 4 & & Mean Difference (Random, 95\% Cl) & $0.04[-0.04,0.11]$ \\
\hline $\begin{array}{l}7 \text { Behaviour change - RCTs: no open defe- } \\
\text { cation by children aged }<5 \text { years }\end{array}$ & 3 & & Risk Ratio (Random, 95\% Cl) & $1.79[0.80,4.03]$ \\
\hline $\begin{array}{l}8 \text { Behaviour change - RCTs: safe disposal } \\
\text { of child faeces }\end{array}$ & 3 & & Risk Ratio (Random, 95\% Cl) & $1.29[1.02,1.64]$ \\
\hline $\begin{array}{l}9 \text { Behaviour change - RCTs: potty use by } \\
\text { children }\end{array}$ & 1 & & Risk Ratio (Random, 95\% Cl) & $3.28[2.90,3.71]$ \\
\hline
\end{tabular}

Analysis 2.1. Comparison 2 CLTS interventions plus adaptations, Outcome 1 Diarrhoea prevalence - randomized controlled trials (RCTs).

\begin{tabular}{|c|c|c|c|c|c|c|}
\hline Study or subgroup & $\begin{array}{c}\text { Inter- } \\
\text { vention } \\
\mathbf{N} \\
\end{array}$ & $\begin{array}{l}\text { Control } \\
\text { N }\end{array}$ & $\begin{array}{c}\text { log[Risk } \\
\text { Ratio] } \\
\text { (SE) } \\
\end{array}$ & $\begin{array}{c}\text { Risk Ratio } \\
\text { IV, Random, } 95 \% \mathrm{CI} \\
\end{array}$ & Weight & $\begin{array}{c}\text { Risk Ratio } \\
\text { IV, Random, } 95 \% \text { CI }\end{array}$ \\
\hline Briceño 2015 TAN & 0 & 0 & $-0(0.144)$ & $\longrightarrow$ & $29.18 \%$ & $0.96[0.72,1.27]$ \\
\hline Cameron 2013 INA & 0 & 0 & $-0.5(0.342)$ & - & $5.21 \%$ & $0.64[0.33,1.24]$ \\
\hline
\end{tabular}




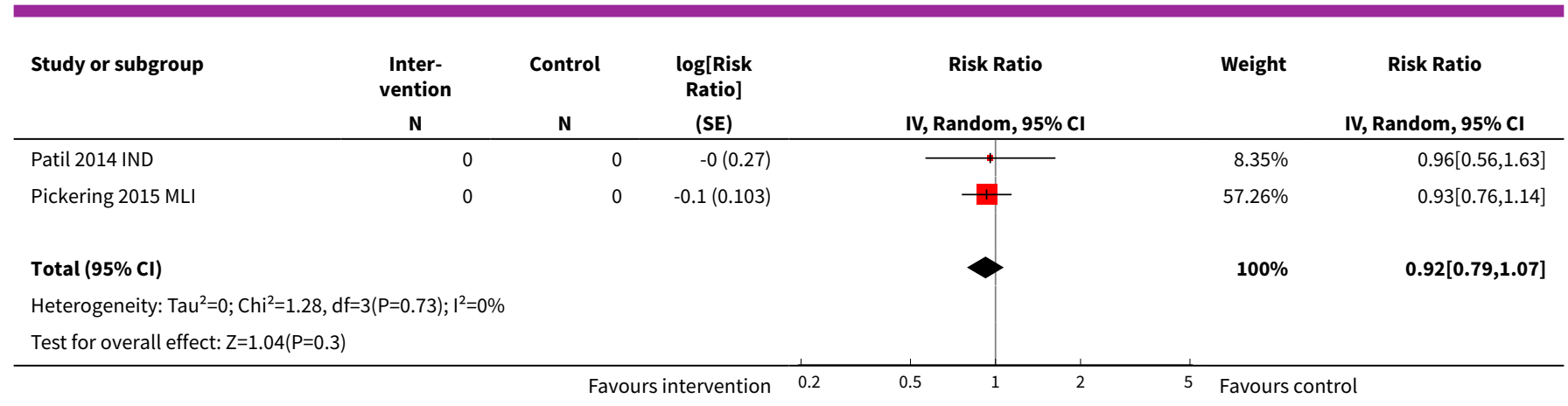

Analysis 2.2. Comparison 2 CLTS interventions plus adaptations, Outcome 2 Any helminth prevalence - RCTs.

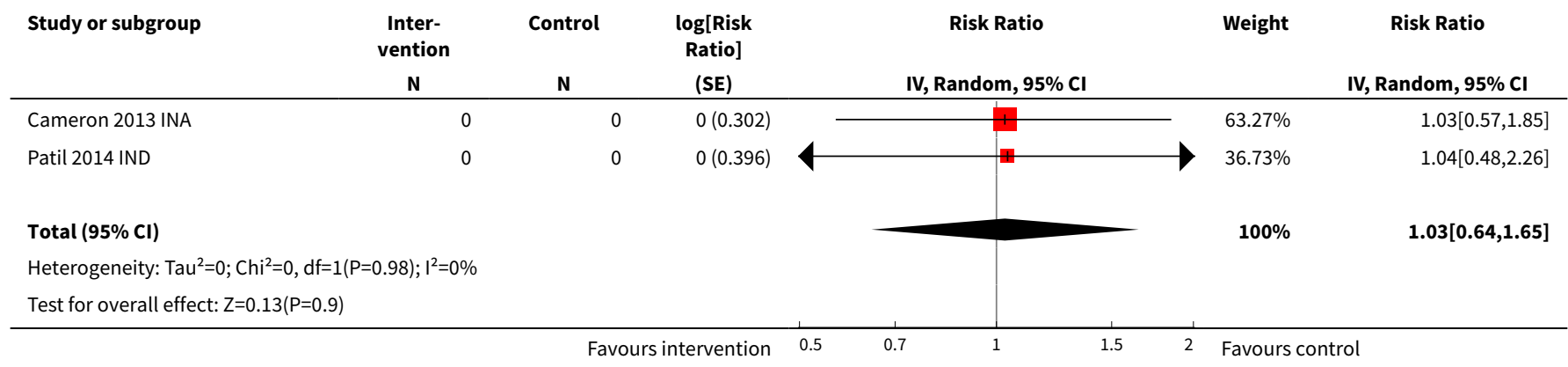

Analysis 2.3. Comparison 2 CLTS interventions plus adaptations, Outcome 3 Ascaris lumbricoides prevalence - RCTs.

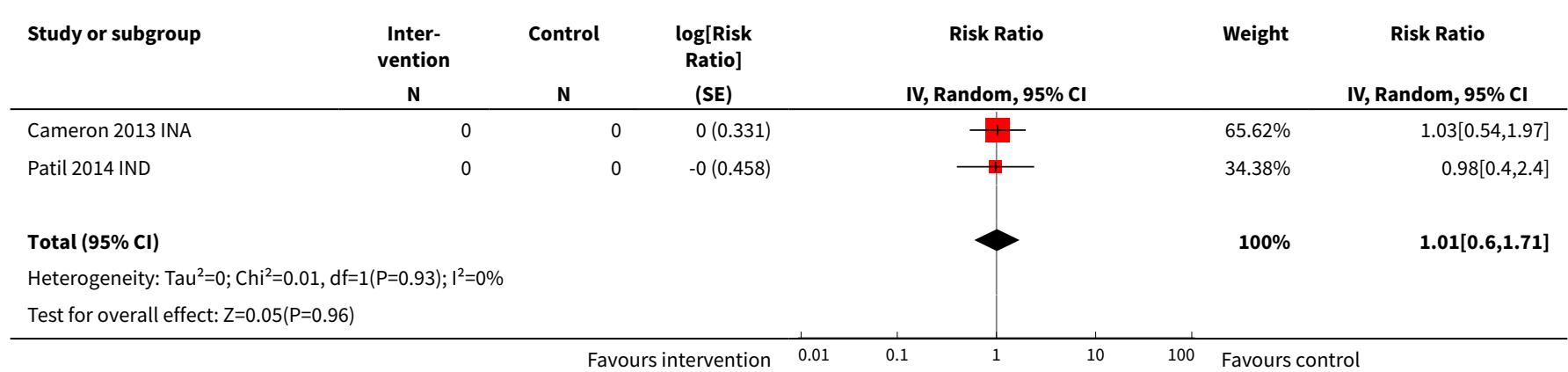

Analysis 2.4. Comparison 2 CLTS interventions plus adaptations, Outcome 4 Dysentery - RCTs.

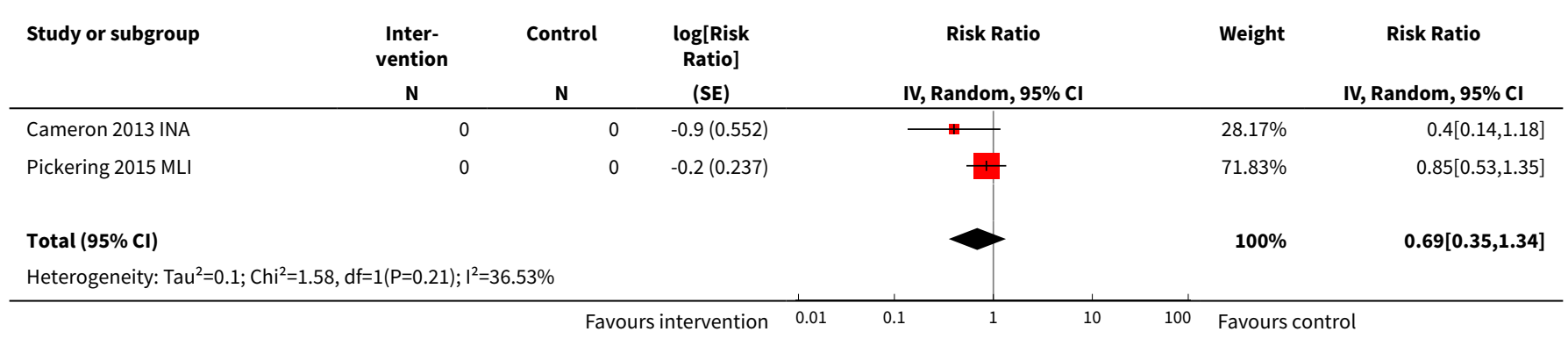




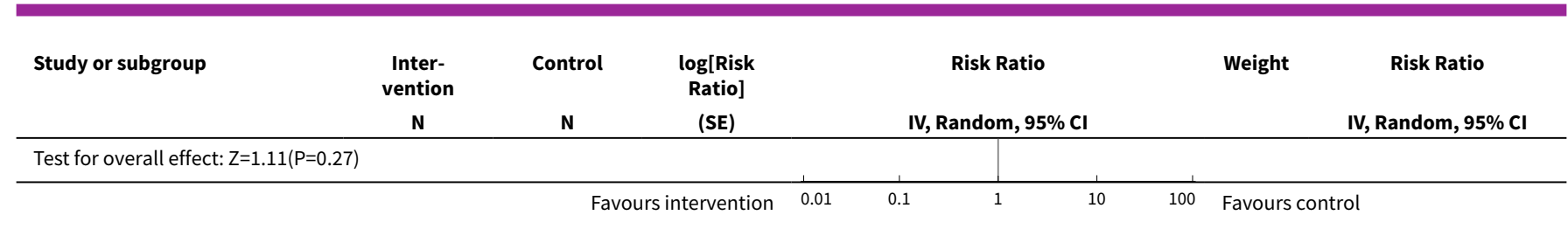

Analysis 2.5. Comparison 2 CLTS interventions plus adaptations, Outcome 5 Anthropometry: height-for-age Z score (HAZ) - RCTs.

\begin{tabular}{|c|c|c|c|c|c|c|}
\hline Study or subgroup & $\begin{array}{c}\text { Inter- } \\
\text { vention } \\
\mathbf{N}\end{array}$ & Control & $\begin{array}{l}\text { Mean Dif- } \\
\text { ference } \\
\text { (SE) }\end{array}$ & $\begin{array}{c}\text { Mean Difference } \\
\text { IV, Random, } 95 \% \mathrm{CI}\end{array}$ & Weight & $\begin{array}{c}\text { Mean Difference } \\
\text { IV, Random, } 95 \% \mathrm{CI}\end{array}$ \\
\hline Briceño 2015 TAN & 0 & 0 & $0(0.087)$ & $\longrightarrow$ & $31.7 \%$ & $0.01[-0.16,0.18]$ \\
\hline Cameron 2013 INA & 0 & 0 & $0(0)$ & & & Not estimable \\
\hline Patil 2014 IND & 0 & 0 & $-0(0.094)$ & $\longrightarrow$ & $29.18 \%$ & $-0.04[-0.22,0.14]$ \\
\hline Pickering 2015 MLI & 0 & 0 & $0.2(0.068)$ & —- & $39.12 \%$ & $0.17[0.04,0.31]$ \\
\hline Total $(95 \% \mathrm{Cl})$ & & & & & $100 \%$ & $0.06[-0.07,0.19]$ \\
\hline \multicolumn{7}{|c|}{ Heterogeneity: $\mathrm{Tau}^{2}=0.01 ; \mathrm{Chi}^{2}=4.16, \mathrm{df}=2(\mathrm{P}=0.12) ; \mathrm{I}^{2}=51.94 \%$} \\
\hline \multicolumn{7}{|c|}{ Test for overall effect: $Z=0.87(P=0.38)$} \\
\hline
\end{tabular}

Analysis 2.6. Comparison 2 CLTS interventions plus adaptations, Outcome 6 Anthropometry: weight-for-age Z score (WAZ) - RCTs.

\begin{tabular}{|c|c|c|c|c|c|c|}
\hline Study or subgroup & $\begin{array}{c}\text { Inter- } \\
\text { vention } \\
\mathbf{N} \\
\end{array}$ & Control & $\begin{array}{l}\text { Mean Dif- } \\
\text { ference } \\
\text { (SE) }\end{array}$ & $\begin{array}{c}\text { Mean Difference } \\
\text { IV, Random, } 95 \% \mathrm{Cl}\end{array}$ & Weight & IV, Random, 95\% CI \\
\hline Briceño 2015 TAN & 0 & 0 & $-0(0.068)$ & $\longrightarrow$ & $33.57 \%$ & $-0.03[-0.16,0.11]$ \\
\hline Cameron 2013 INA & 0 & 0 & $0(0)$ & & & Not estimable \\
\hline Patil 2014 IND & 0 & 0 & $0(0.087)$ & + & $20.85 \%$ & $0.03[-0.14,0.2]$ \\
\hline Pickering 2015 MLI & 0 & 0 & $0.1(0.059)$ & + & $45.58 \%$ & $0.09[-0.03,0.2]$ \\
\hline Total $(95 \% \mathrm{CI})$ & & & & & $100 \%$ & $0.04[-0.04,0.11]$ \\
\hline \multicolumn{7}{|c|}{ Heterogeneity: $\operatorname{Tau}^{2}=0 ; \mathrm{Chi}^{2}=1.69, \mathrm{df}=2(\mathrm{P}=0.43) ; \mathrm{I}^{2}=0 \%$} \\
\hline Test for overall effect & & & & & & \\
\hline
\end{tabular}

Analysis 2.7. Comparison 2 CLTS interventions plus adaptations, Outcome 7 Behaviour change - RCTs: no open defecation by children aged $<5$ years.

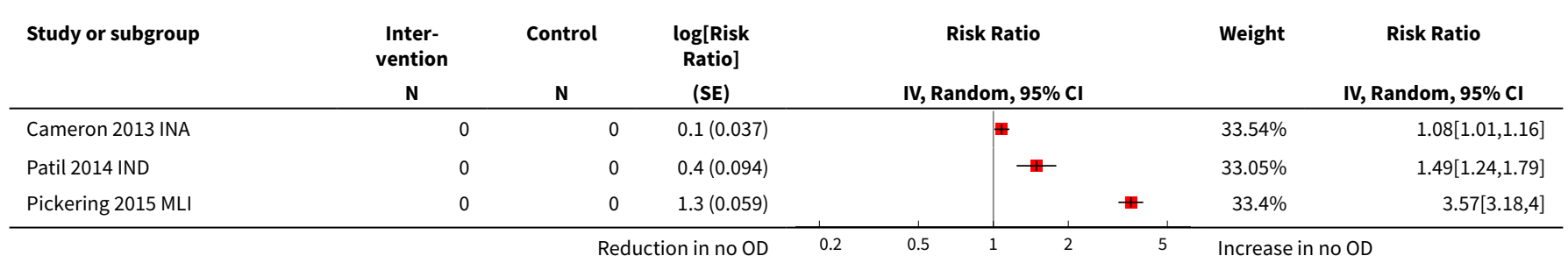




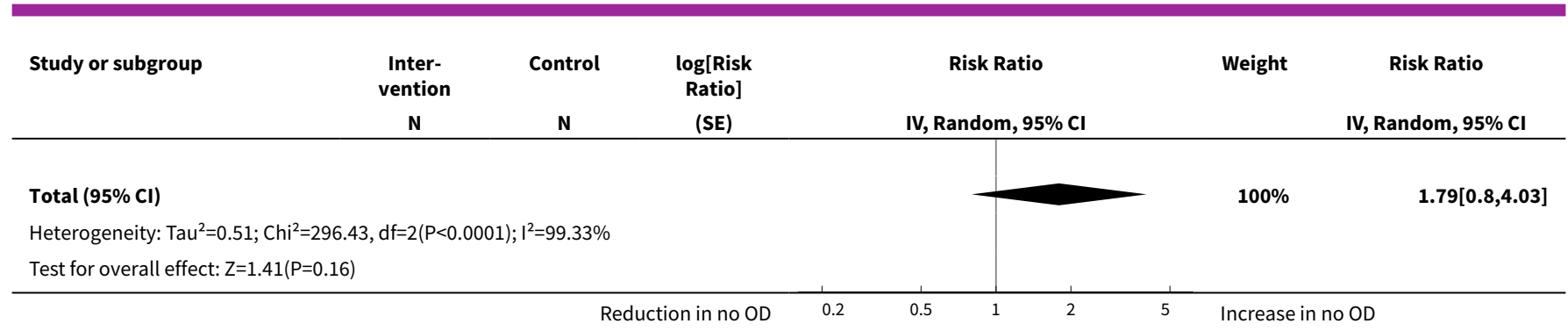

Analysis 2.8. Comparison 2 CLTS interventions plus adaptations, Outcome 8 Behaviour change - RCTs: safe disposal of child faeces.

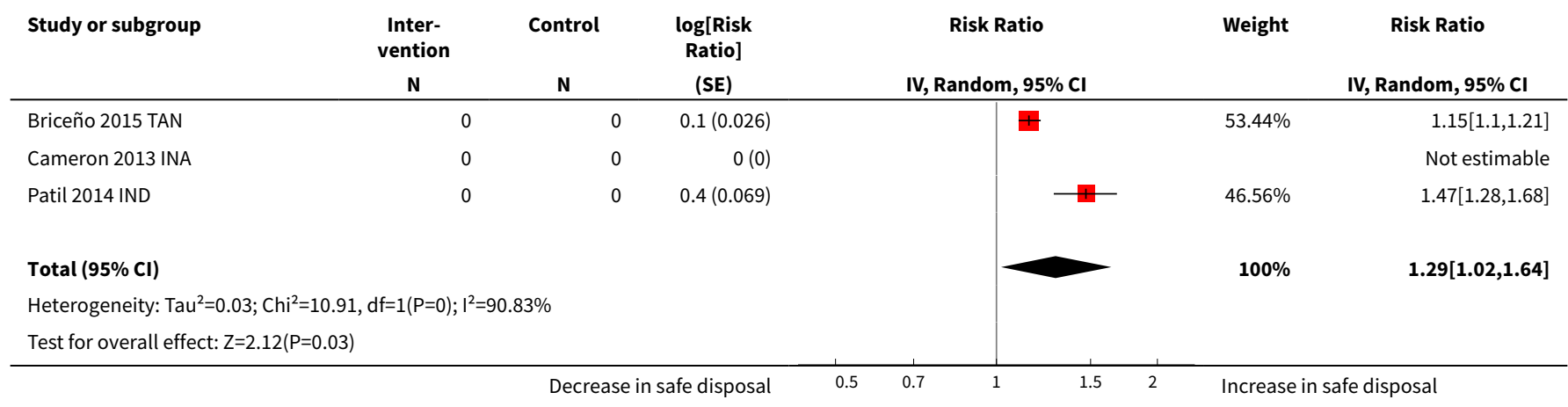

Analysis 2.9. Comparison 2 CLTS interventions plus adaptations, Outcome 9 Behaviour change - RCTs: potty use by children.

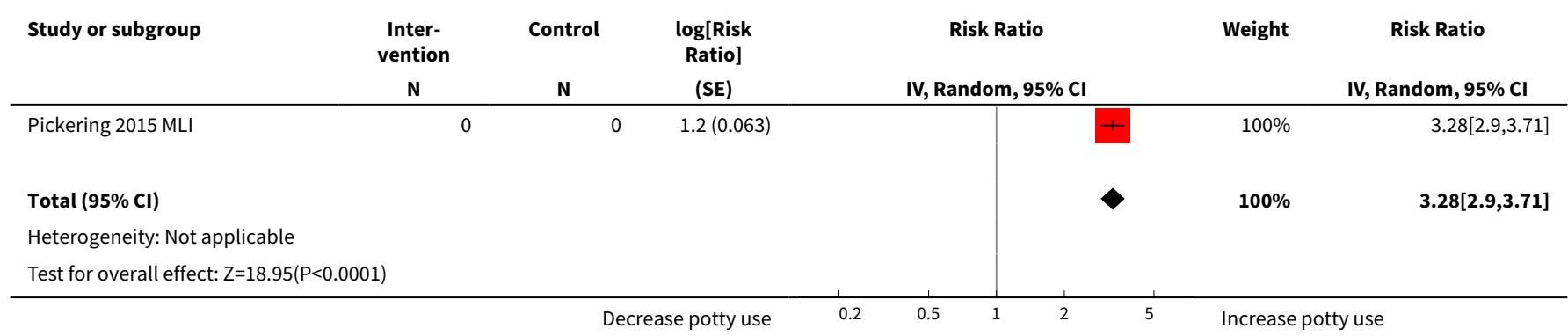

\section{Comparison 3. Sanitation hardware and behaviour change interventions}

\begin{tabular}{lllll}
\hline Outcome or subgroup title & $\begin{array}{l}\text { No. of } \\
\text { studies }\end{array}$ & $\begin{array}{l}\text { No. of } \\
\text { partici- } \\
\text { pants }\end{array}$ & Statistical method & Effect size \\
\hline $\begin{array}{l}1 \text { Diarrhoea prevalence - randomized con- } \\
\text { trolled trials (RCTs) }\end{array}$ & 2 & Risk Ratio (Random, 95\% Cl) & $0.79[0.49,1.26]$ \\
\hline $\begin{array}{l}2 \text { Anthropometry: height-for-age Z score } \\
\text { (HAZ) - RCTs }\end{array}$ & 2 & Mean Difference (Random, 95\% Cl) & $-0.04[-0.12,0.04]$ \\
\hline
\end{tabular}




\begin{tabular}{lllll}
\hline Outcome or subgroup title & $\begin{array}{l}\text { No. of } \\
\text { studies }\end{array}$ & $\begin{array}{l}\text { No. of } \\
\text { partici- } \\
\text { pants }\end{array}$ & Statistical method & Effect size \\
\hline $\begin{array}{l}3 \text { Anthropometry: weight-for-age Z score } \\
\text { (WAZ) - RCTs }\end{array}$ & 2 & Mean Difference (Random, 95\% Cl) & $-0.04[-0.11,0.04]$ \\
\hline $\begin{array}{l}4 \text { Behaviour change - RCTs: safe disposal of } \\
\text { child faeces }\end{array}$ & 2 & Risk Ratio (Random, 95\% Cl) & $3.22[2.16,4.79]$ \\
\hline $\begin{array}{l}5 \text { Behaviour change - RCTs: appropriate dis- } \\
\text { posal of child faeces }\end{array}$ & 2 & Risk Difference (Random, 95\% Cl) & $0.32[-0.04,0.68]$ \\
\hline $\begin{array}{l}6 \text { Behaviour change - RCTs: potty use by } \\
\text { children }\end{array}$ & 1 & Risk Ratio (Random, 95\% Cl) & $1.69[1.08,2.65]$ \\
\hline $\begin{array}{l}7 \text { Behaviour change - RCTs: faeces in com- } \\
\text { pound }\end{array}$ & 3 & Risk Difference (Random, 95\% Cl) & $-0.08[-0.13,-0.03]$ \\
\hline
\end{tabular}

Analysis 3.1. Comparison 3 Sanitation hardware and behaviour change interventions, Outcome 1 Diarrhoea prevalence - randomized controlled trials (RCTs).

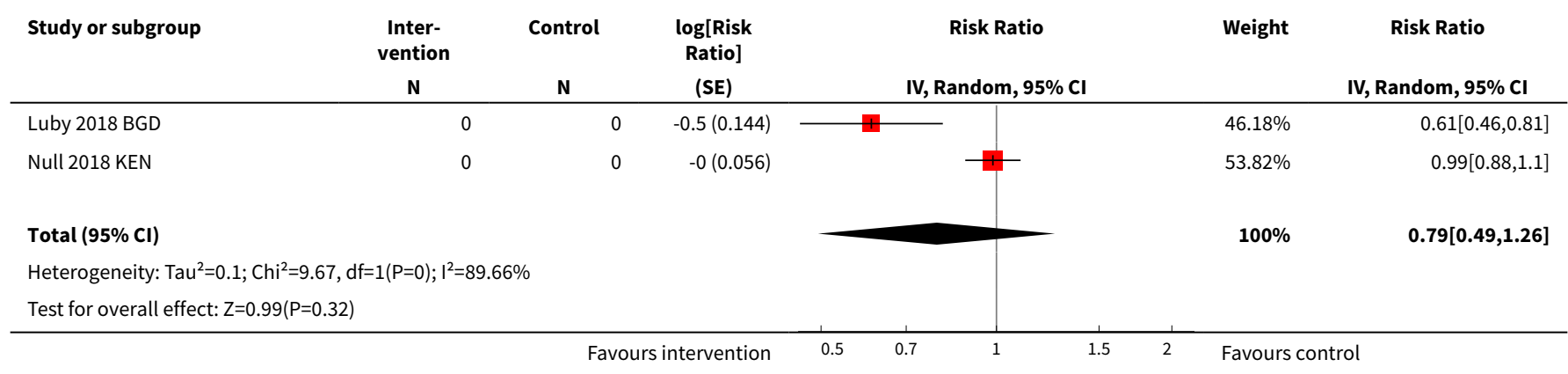

Analysis 3.2. Comparison 3 Sanitation hardware and behaviour change interventions, Outcome 2 Anthropometry: height-for-age Z score (HAZ) - RCTs.

\begin{tabular}{|c|c|c|c|c|c|c|}
\hline Study or subgroup & $\begin{array}{c}\text { Inter- } \\
\text { vention } \\
\mathbf{N}\end{array}$ & Control & $\begin{array}{l}\text { Mean Dif- } \\
\text { ference } \\
\text { (SE) }\end{array}$ & IV, Random, $95 \% \mathrm{CI}$ & Weight & IV, Random, 95\% CI \\
\hline Luby 2018 BGD & 0 & 0 & $-0(0.058)$ & 十 & $51.27 \%$ & $-0.02[-0.14,0.09]$ \\
\hline Null 2018 KEN & 0 & 0 & $-0.1(0.06)$ & $-1-$ & $48.73 \%$ & $-0.06[-0.18,0.05]$ \\
\hline Total $(95 \% \mathrm{CI})$ & & & & & $100 \%$ & $-0.04[-0.12,0.04]$ \\
\hline \multicolumn{7}{|c|}{ Heterogeneity: $\mathrm{Tau}^{2}=0 ; \mathrm{Chi}^{2}=0.24, \mathrm{df}=1(\mathrm{P}=0.62) ; \mathrm{I}^{2}=0 \%$} \\
\hline \multicolumn{7}{|c|}{ Test for overall effect: $\mathrm{Z}=1.01(\mathrm{P}=0.31)$} \\
\hline
\end{tabular}


Analysis 3.3. Comparison 3 Sanitation hardware and behaviour change interventions, Outcome 3 Anthropometry: weight-for-age Z score (WAZ) - RCTs.

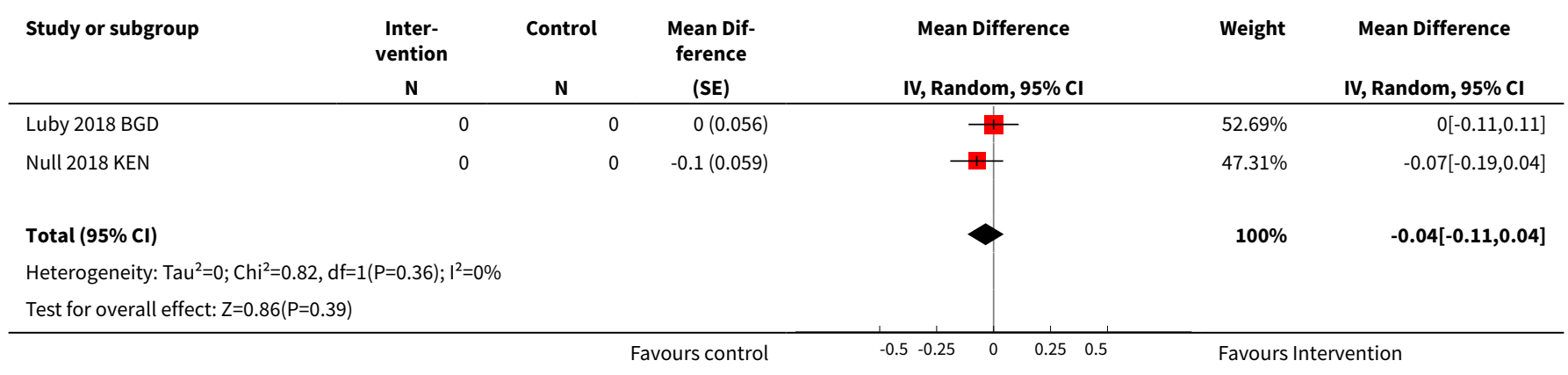

Analysis 3.4. Comparison 3 Sanitation hardware and behaviour change interventions, Outcome 4 Behaviour change - RCTs: safe disposal of child faeces.

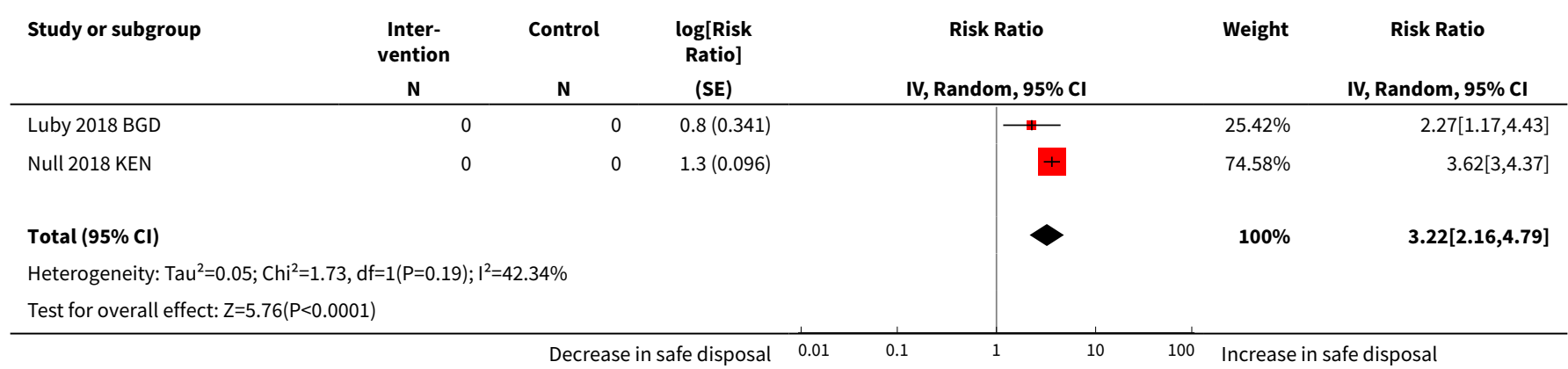

Analysis 3.5. Comparison 3 Sanitation hardware and behaviour change interventions, Outcome 5 Behaviour change - RCTs: appropriate disposal of child faeces.

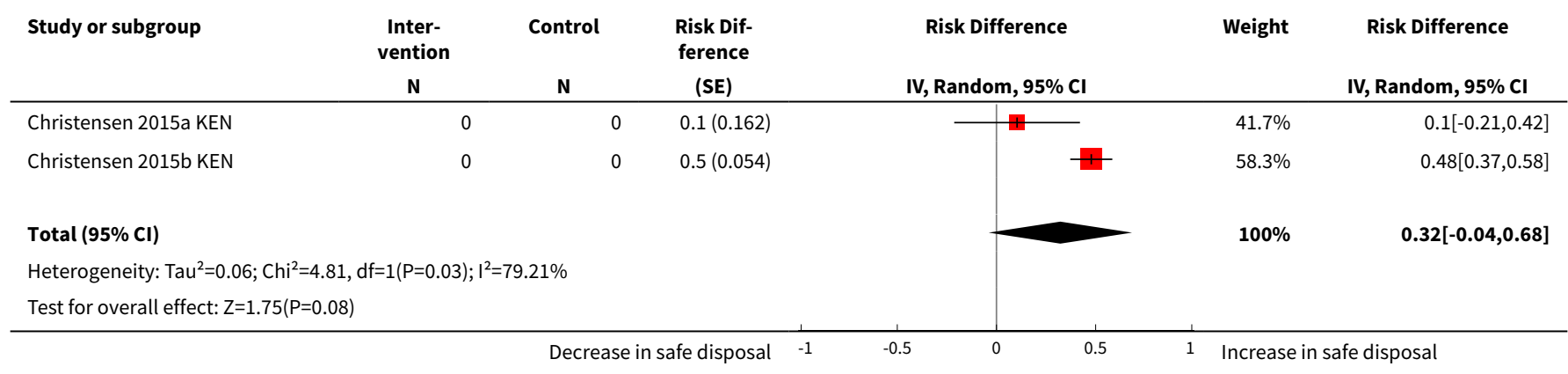


Analysis 3.6. Comparison 3 Sanitation hardware and behaviour change interventions, Outcome 6 Behaviour change - RCTs: potty use by children.

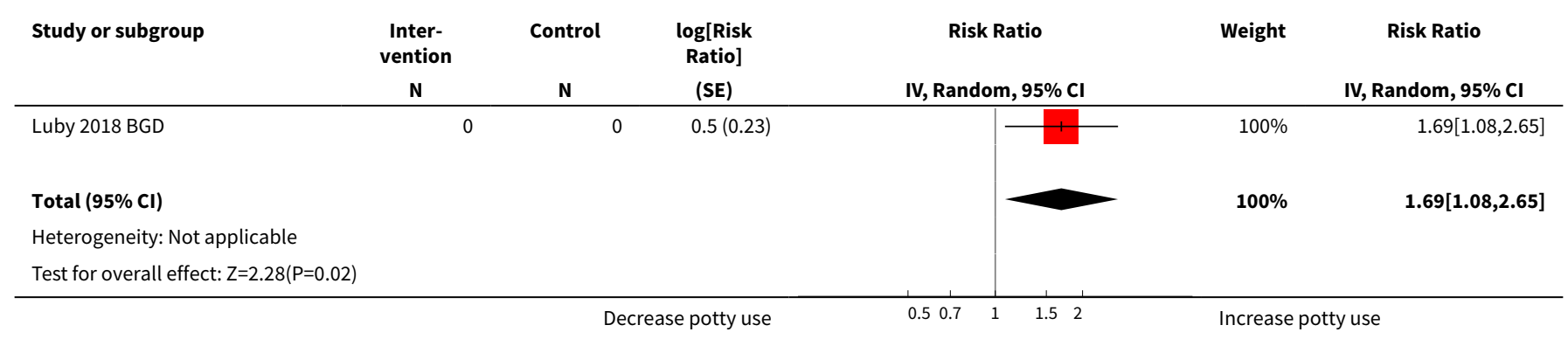

Analysis 3.7. Comparison 3 Sanitation hardware and behaviour change interventions, Outcome 7 Behaviour change - RCTs: faeces in compound.

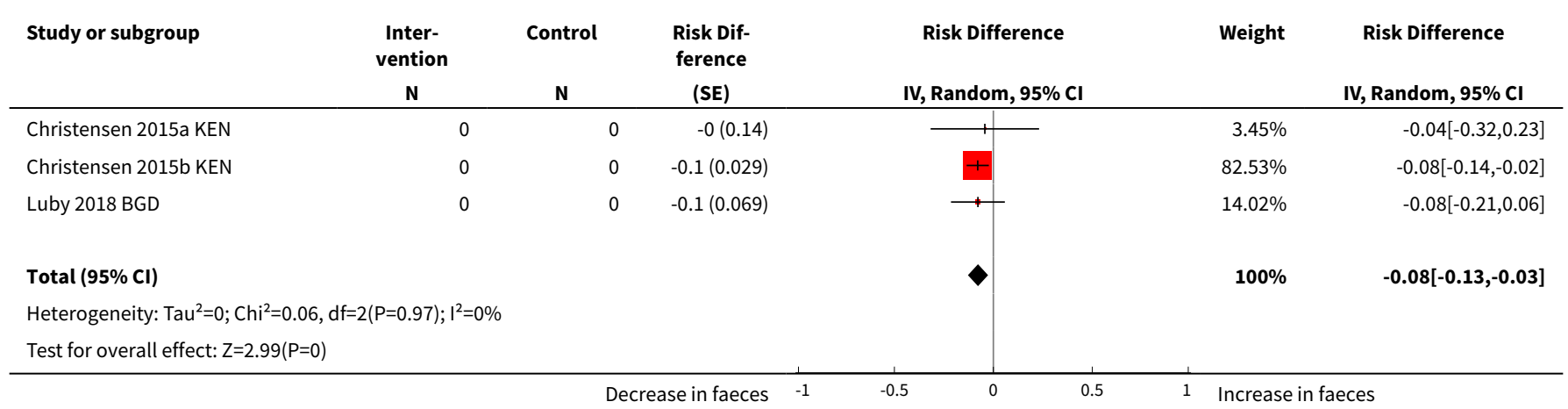

\section{Comparison 4. WASH hardware and education interventions}

\begin{tabular}{lllll}
\hline Outcome or subgroup title & $\begin{array}{l}\text { No. of } \\
\text { studies }\end{array}$ & $\begin{array}{l}\text { No. of } \\
\text { partici- } \\
\text { pants }\end{array}$ & Statistical method & Effect size \\
\hline $\begin{array}{l}1 \text { Diarrhoea prevalence - randomized controlled } \\
\text { trials }\end{array}$ & 1 & Risk Ratio (Random, 95\% Cl) & Subtotals only \\
\hline $\begin{array}{l}2 \text { Diarrhoea incidence - controlled before-and-af- } \\
\text { ter studies }\end{array}$ & 2 & Rate Ratio (Random, 95\% Cl) & $0.77[0.71,0.84]$ \\
\hline
\end{tabular}

Analysis 4.1. Comparison 4 WASH hardware and education interventions, Outcome 1 Diarrhoea prevalence - randomized controlled trials.

\begin{tabular}{|c|c|c|c|c|c|c|}
\hline Study or subgroup & $\begin{array}{c}\text { Inter- } \\
\text { vention } \\
\mathbf{N}\end{array}$ & Control & $\begin{array}{c}\log [\text { Risk } \\
\text { Ratio] } \\
\text { (SE) }\end{array}$ & IV, Random, 95\% CI & Weight & ndom, 95\% Cl \\
\hline Humphrey 2019 ZIM & 0 & 0 & $0.1(0.107)$ & + & $0 \%$ & $1.15[0.93,1.41]$ \\
\hline
\end{tabular}


Analysis 4.2. Comparison 4 WASH hardware and education interventions, Outcome 2 Diarrhoea incidence - controlled before-and-after studies.

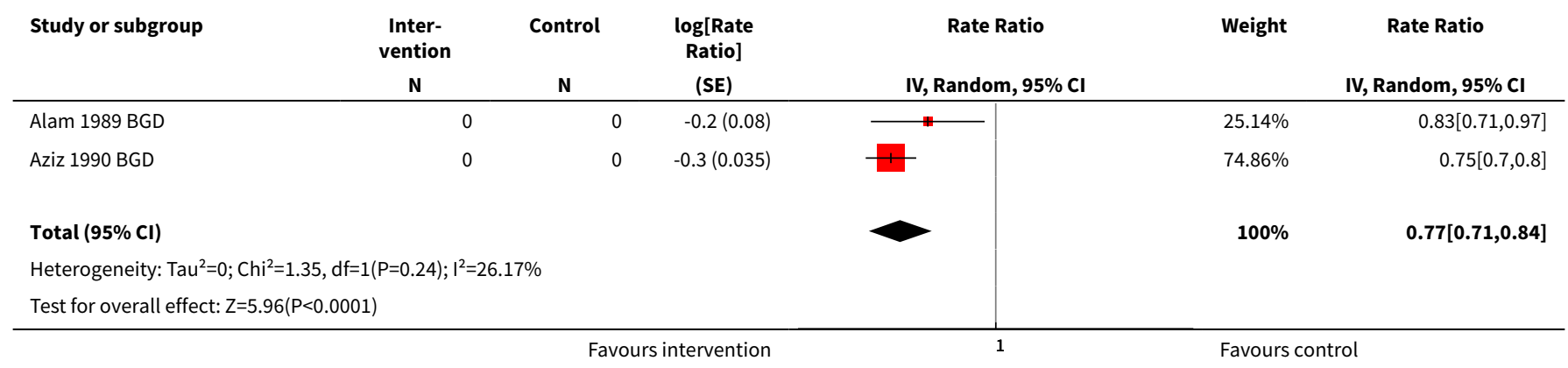

Comparison 5. Case-control studies: disposal of child faeces in latrine versus elsewhere

\begin{tabular}{lllll}
\hline Outcome or subgroup title & $\begin{array}{l}\text { No. of } \\
\text { studies }\end{array}$ & $\begin{array}{l}\text { No. of } \\
\text { partici- } \\
\text { pants }\end{array}$ & Statistical method & Effect size \\
\hline $\begin{array}{l}1 \text { Diarrhoea (including se- } \\
\text { vere and cholera): sub- } \\
\text { grouped by age group }\end{array}$ & 23 & & Odds Ratio (Random, 95\% Cl) & Subtotals only \\
\hline
\end{tabular}

\begin{tabular}{|c|c|c|c|}
\hline 1.1 All ages & 23 & Odds Ratio (Random, $95 \% \mathrm{Cl}$ ) & $0.73[0.62,0.85]$ \\
\hline 1.2 Aged $\leq 5$ years & 20 & Odds Ratio (Random, $95 \% \mathrm{Cl}$ ) & $0.72[0.61,0.85]$ \\
\hline $\begin{array}{l}2 \text { Diarrhoea in all ages (in- } \\
\text { cluding severe and cholera): } \\
\text { subgrouped by country in- } \\
\text { come level }\end{array}$ & 23 & Odds Ratio (Random, 95\% Cl) & $0.73[0.62,0.85]$ \\
\hline 2.1 Low & 9 & Odds Ratio (Random, 95\% Cl) & $0.62[0.40,0.96]$ \\
\hline 2.2 Lower middle & 10 & Odds Ratio (Random, $95 \% \mathrm{Cl}$ ) & $0.82[0.70,0.96]$ \\
\hline 2.3 Upper middle & 3 & Odds Ratio (Random, $95 \% \mathrm{Cl}$ ) & $0.75[0.60,0.94]$ \\
\hline 2.4 High & 1 & Odds Ratio (Random, 95\% Cl) & $0.68[0.48,0.97]$ \\
\hline $\begin{array}{l}3 \text { Diarrhoea in all ages (in- } \\
\text { cluding severe and cholera): } \\
\text { subgrouped by type of diar- } \\
\text { rhoea }\end{array}$ & 23 & Odds Ratio (Random, 95\% Cl) & $0.73[0.62,0.85]$ \\
\hline 3.1 Persistent diarrhoea & 1 & Odds Ratio (Random, 95\% Cl) & $0.53[0.17,1.68]$ \\
\hline $\begin{array}{l}3.2 \text { Moderate-to-severe di- } \\
\text { arrhoea }\end{array}$ & 7 & Odds Ratio (Random, 95\% Cl) & $0.96[0.83,1.11]$ \\
\hline $\begin{array}{l}\text { 3.3 Acute (possibly) bloody } \\
\text { diarrhoea }\end{array}$ & 4 & Odds Ratio (Random, $95 \% \mathrm{Cl}$ ) & $0.67[0.56,0.81]$ \\
\hline
\end{tabular}




\begin{tabular}{|c|c|c|c|c|}
\hline Outcome or subgroup title & $\begin{array}{l}\text { No. of } \\
\text { studies }\end{array}$ & $\begin{array}{l}\text { No. of } \\
\text { partici- } \\
\text { pants }\end{array}$ & Statistical method & Effect size \\
\hline 3.4 Acute watery diarrhoea & 6 & & Odds Ratio (Random, 95\% Cl) & $0.63[0.38,1.05]$ \\
\hline 3.5 No case definition & 5 & & Odds Ratio (Random, 95\% Cl) & $0.54[0.39,0.75]$ \\
\hline $\begin{array}{l}4 \text { Diarrhoea in all ages (in- } \\
\text { cluding severe and cholera): } \\
\text { subgrouped by study quali- } \\
\text { ty }\end{array}$ & 23 & & Odds Ratio (Random, 95\% Cl) & $0.73[0.62,0.85]$ \\
\hline 4.14 stars & 7 & & Odds Ratio (Random, 95\% Cl) & $0.96[0.83,1.11]$ \\
\hline 4.25 stars & 7 & & Odds Ratio (Random, 95\% Cl) & $0.65[0.52,0.82]$ \\
\hline 4.36 stars & 4 & & Odds Ratio (Random, 95\% Cl) & $0.60[0.35,1.05]$ \\
\hline $4.4 \geq 7$ stars & 5 & & Odds Ratio (Random, 95\% Cl) & $0.66[0.51,0.84]$ \\
\hline $\begin{array}{l}5 \text { Diarrhoea in all ages (in- } \\
\text { cluding severe and cholera): } \\
\text { subgrouped by setting }\end{array}$ & 23 & & Odds Ratio (Random, 95\% Cl) & $0.73[0.62,0.85]$ \\
\hline 5.1 Rural & 10 & & Odds Ratio (Random, 95\% Cl) & $0.65[0.49,0.87]$ \\
\hline 5.2 Urban & 10 & & Odds Ratio (Random, 95\% Cl) & $0.74[0.61,0.90]$ \\
\hline $\begin{array}{l}5.3 \text { Periurban/urban and } \\
\text { rural }\end{array}$ & 3 & & Odds Ratio (Random, 95\% Cl) & $0.98[0.70,1.38]$ \\
\hline $\begin{array}{l}6 \text { Diarrhoea in all ages (in- } \\
\text { cluding severe and cholera): } \\
\text { subgrouped by method of } \\
\text { data collection }\end{array}$ & 23 & & Odds Ratio (Random, 95\% Cl) & $0.73[0.62,0.85]$ \\
\hline 6.1 Questionnaire & 19 & & Odds Ratio (Random, 95\% Cl) & $0.75[0.64,0.89]$ \\
\hline 6.2 Observation & 2 & & Odds Ratio (Random, 95\% Cl) & $0.48[0.29,0.79]$ \\
\hline 6.3 Unclear & 2 & & Odds Ratio (Random, 95\% Cl) & $0.67[0.48,0.94]$ \\
\hline
\end{tabular}

\section{Analysis 5.1. Comparison 5 Case-control studies: disposal of child faeces in latrine versus elsewhere, Outcome 1 Diarrhoea (including severe and cholera): subgrouped by age group.}

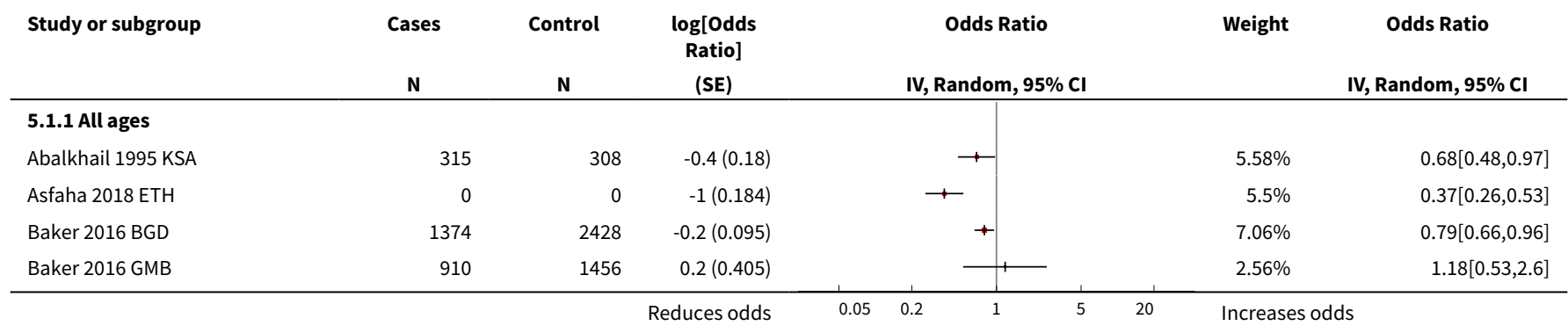




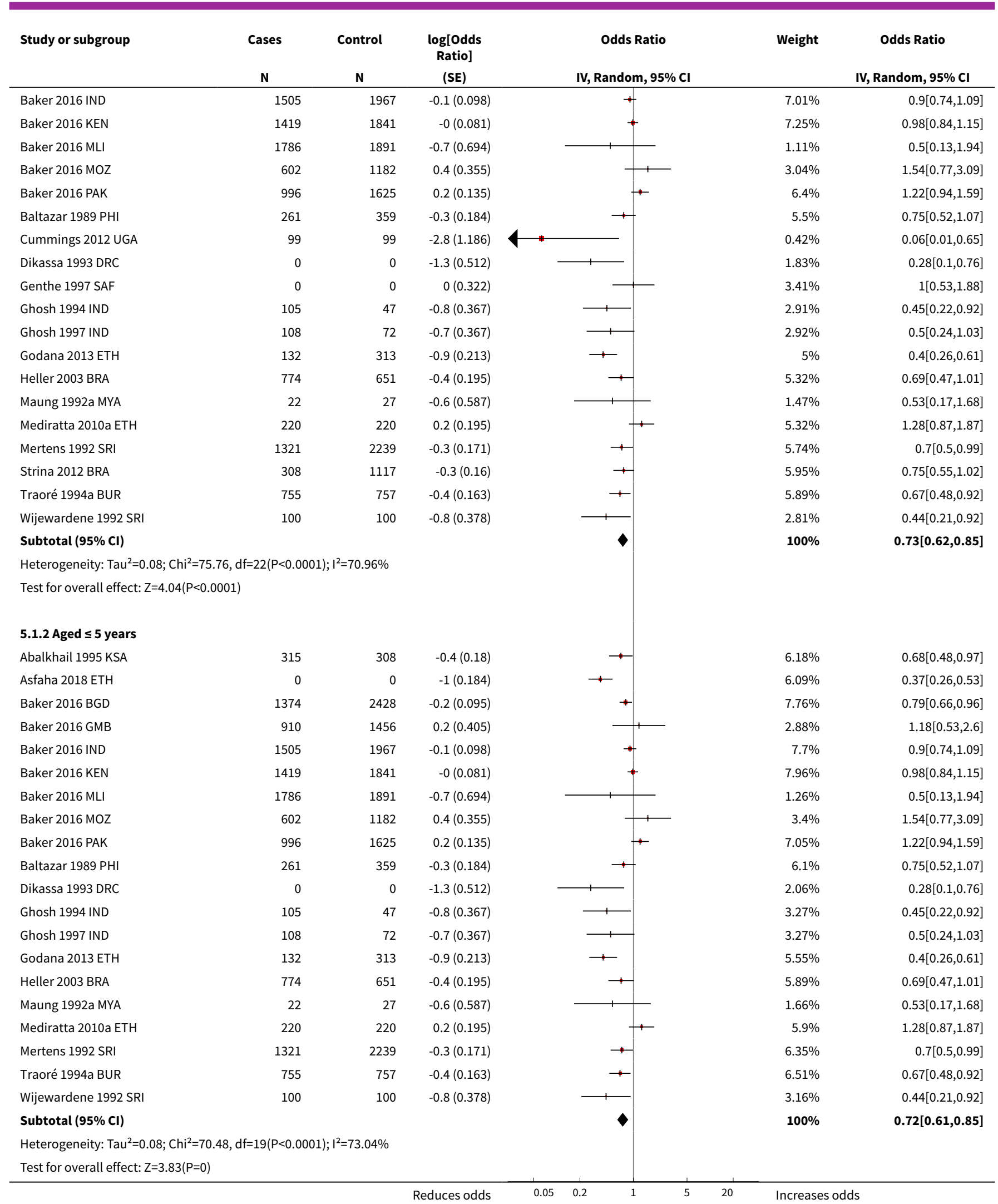


Analysis 5.2. Comparison 5 Case-control studies: disposal of child faeces in latrine versus elsewhere, Outcome 2 Diarrhoea in all ages (including severe and cholera): subgrouped by country income level.

\begin{tabular}{|c|c|c|c|c|c|c|}
\hline Study or subgroup & $\mathbf{N}$ & Control & $\begin{array}{c}\text { log[Odds } \\
\text { Ratio] } \\
\text { (SE) }\end{array}$ & IV, Random, 95\% CI & Weight & IV, Random, 95\% CI \\
\hline 5.2.1 Low & & & & & & \\
\hline Asfaha 2018 ETH & 0 & 0 & $-1(0.184)$ & - & $5.5 \%$ & $0.37[0.26,0.53]$ \\
\hline Baker 2016 GMB & 910 & 1456 & $0.2(0.405)$ & 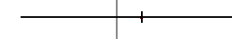 & $2.56 \%$ & $1.18[0.53,2.6]$ \\
\hline Baker 2016 MLI & 1786 & 1891 & $-0.7(0.694)$ & & $1.11 \%$ & $0.5[0.13,1.94]$ \\
\hline Baker 2016 MOZ & 602 & 1182 & $0.4(0.355)$ & & $3.04 \%$ & $1.54[0.77,3.09]$ \\
\hline Cummings 2012 UGA & 99 & 99 & $-2.8(1.186)$ & & $0.42 \%$ & $0.06[0.01,0.65]$ \\
\hline Dikassa 1993 DRC & 0 & 0 & $-1.3(0.512)$ & & $1.83 \%$ & $0.28[0.1,0.76]$ \\
\hline Godana 2013 ETH & 132 & 313 & $-0.9(0.213)$ & & $5 \%$ & $0.4[0.26,0.61]$ \\
\hline Mediratta 2010a ETH & 220 & 220 & $0.2(0.195)$ & 1 & $5.32 \%$ & $1.28[0.87,1.87]$ \\
\hline Traoré 1994a BUR & 755 & 757 & $-0.4(0.163)$ & $\longrightarrow$ & $5.89 \%$ & $0.67[0.48,0.92]$ \\
\hline Subtotal $(95 \% \mathrm{CI})$ & & & & & $30.67 \%$ & $0.62[0.4,0.96]$ \\
\hline Test for overall effect: 2 & & & & & & \\
\hline 5.2.2 Lower middle & & & & & & \\
\hline Baker 2016 BGD & 1374 & 2428 & $-0.2(0.095)$ & $\div$ & $7.06 \%$ & $0.79[0.66,0.96]$ \\
\hline Baker 2016 IND & 1505 & 1967 & $-0.1(0.098)$ & $\rightarrow$ & $7.01 \%$ & $0.9[0.74,1.09]$ \\
\hline Baker 2016 KEN & 1419 & 1841 & $-0(0.081)$ & $\rightarrow$ & $7.25 \%$ & $0.98[0.84,1.15]$ \\
\hline Baker 2016 PAK & 996 & 1625 & $0.2(0.135)$ & 4 & $6.4 \%$ & $1.22[0.94,1.59]$ \\
\hline Baltazar 1989 PHI & 261 & 359 & $-0.3(0.184)$ & & $5.5 \%$ & $0.75[0.52,1.07]$ \\
\hline Ghosh 1994 IND & 105 & 47 & $-0.8(0.367)$ & & $2.91 \%$ & $0.45[0.22,0.92]$ \\
\hline Ghosh 1997 IND & 108 & 72 & $-0.7(0.367)$ & & $2.92 \%$ & $0.5[0.24,1.03]$ \\
\hline Maung 1992a MYA & 22 & 27 & $-0.6(0.587)$ & & $1.47 \%$ & $0.53[0.17,1.68]$ \\
\hline Mertens 1992 SRI & 1321 & 2239 & $-0.3(0.171)$ & & $5.74 \%$ & $0.7[0.5,0.99]$ \\
\hline Wijewardene 1992 SRI & 100 & 100 & $-0.8(0.378)$ & & $2.81 \%$ & $0.44[0.21,0.92]$ \\
\hline Test for overall effect: 2 & & & & & & \\
\hline 5.2.3 Upper middle & & & & & & \\
\hline Genthe 1997 SAF & 0 & 0 & $0(0.322)$ & & $3.41 \%$ & $1[0.53,1.88]$ \\
\hline Heller 2003 BRA & 774 & 651 & $-0.4(0.195)$ & & $5.32 \%$ & $0.69[0.47,1.01]$ \\
\hline Strina 2012 BRA & 308 & 1117 & $-0.3(0.16)$ & & $5.95 \%$ & $0.75[0.55,1.02]$ \\
\hline Subtotal $(95 \% \mathrm{Cl})$ & & & & & $14.67 \%$ & $0.75[0.6,0.94]$ \\
\hline Heterogeneity: $\operatorname{Tau}^{2}=0$ & $P=0.61) ; I^{2}=$ & & & & & \\
\hline Test for overall effect: & & & & & & \\
\hline 5.2.4 High & & & & & & \\
\hline Abalkhail 1995 KSA & 315 & 308 & $-0.4(0.18)$ & & $5.58 \%$ & $0.68[0.48,0.97]$ \\
\hline Subtotal $(95 \% \mathrm{CI})$ & & & & & $5.58 \%$ & $0.68[0.48,0.97]$ \\
\hline Heterogeneity: $\operatorname{Tau}^{2}=0$ & $0.0001) ; I^{2}=1$ & & & & & \\
\hline Test for overall effect: 2 & & & & & & \\
\hline Total $(95 \% \mathrm{Cl})$ & & & & & $100 \%$ & $0.73[0.62,0.85]$ \\
\hline Heterogeneity: $\operatorname{Tau}^{2}=0$ & $\mathrm{f}=22(\mathrm{P}<0.0 \mathrm{C}$ & $I^{2}=70.96 \%$ & & & & \\
\hline Test for overall effect: 2 & & & & & & \\
\hline Test for subgroup diffe & $\mathrm{df}=1(\mathrm{P}=0.5$ & $=0 \%$ & & & & \\
\hline
\end{tabular}




\section{Analysis 5.3. Comparison 5 Case-control studies: disposal of child faeces in latrine versus elsewhere, Outcome 3 Diarrhoea in all ages (including severe and cholera): subgrouped by type of diarrhoea.}

\begin{tabular}{|c|c|c|c|}
\hline Study or subgroup & Cases & Control & $\begin{array}{c}\text { log[Odds } \\
\text { Ratio] } \\
\text { (SE) }\end{array}$ \\
\hline \multicolumn{4}{|c|}{ 5.3.1 Persistent diarrhoea } \\
\hline Maung 1992a MYA & 22 & 27 & $-0.6(0.587)$ \\
\hline \multicolumn{4}{|l|}{ Subtotal $(95 \% \mathrm{Cl})$} \\
\hline \multicolumn{4}{|c|}{ Heterogeneity: Not applicable } \\
\hline \multicolumn{4}{|c|}{ Test for overall effect: $\mathrm{Z}=1.08(\mathrm{P}=0.28)$} \\
\hline \multicolumn{4}{|c|}{ 5.3.2 Moderate-to-severe diarrhoea } \\
\hline Baker 2016 BGD & 1374 & 2428 & $-0.2(0.095)$ \\
\hline Baker 2016 GMB & 910 & 1456 & $0.2(0.405)$ \\
\hline Baker 2016 IND & 1505 & 1967 & $-0.1(0.098)$ \\
\hline Baker 2016 KEN & 1419 & 1841 & $-0(0.081)$ \\
\hline Baker 2016 MLI & 1786 & 1891 & $-0.7(0.694)$ \\
\hline Baker 2016 MOZ & 602 & 1182 & $0.4(0.355)$ \\
\hline Baker 2016 PAK & 996 & 1625 & $0.2(0.135)$ \\
\hline
\end{tabular}

Subtotal $(95 \% \mathrm{Cl})$

Heterogeneity: $\mathrm{Tau}^{2}=0.01 ; \mathrm{Chi}^{2}=10.47, \mathrm{df}=6(\mathrm{P}=0.11) ; \mathrm{I}^{2}=42.71 \%$

Test for overall effect: $\mathrm{Z}=0.51(\mathrm{P}=0.61)$

\subsubsection{Acute (possibly) bloody diarrhoea}

Abalkhail 1995 KSA

$\begin{array}{rrr}315 & 308 & -0.4(0.18) \\ 108 & 72 & -0.7(0.367) \\ 1321 & 2239 & -0.3(0.171) \\ 755 & 757 & -0.4(0.163)\end{array}$

Ghosh 1997 IND

$-0.4(0.163)$

Traoré 1994a BUR

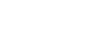

Subtotal $(95 \% \mathrm{Cl})$

Heterogeneity: $\mathrm{Tau}^{2}=0 ; \mathrm{Chi}^{2}=0.72, \mathrm{df}=3(\mathrm{P}=0.87) ; \mathrm{I}^{2}=0 \%$

Test for overall effect: $Z=4.2(P<0.0001)$

\subsubsection{Acute watery diarrhoea}

Asfaha 2018 ETH

Cummings 2012 UGA

Genthe 1997 SAF

Mediratta 2010a ETH

Strina 2012 BRA

Wijewardene 1992 SRI

Subtotal $(95 \% \mathrm{CI})$

Heterogeneity: $\operatorname{Tau}^{2}=0.28 ; \mathrm{Chi}^{2}=28.42, \mathrm{df}=5(\mathrm{P}<0.0001) ; \mathrm{I}^{2}=82.41 \%$

Test for overall effect: $Z=1.78(P=0.08)$

\subsubsection{No case definition}

Baltazar $1989 \mathrm{PHI}$

Dikassa 1993 DRC

Ghosh 1994 IND

Godana 2013 ETH

Heller 2003 BRA

$\begin{array}{rrr}0 & 0 & -1(0.184) \\ 99 & 99 & -2.8(1.186) \\ 0 & 0 & 0(0.322) \\ 220 & 220 & 0.2(0.195) \\ 308 & 1117 & -0.3(0.16) \\ 100 & 100 & -0.8(0.378)\end{array}$

$$
\begin{array}{r}
-1(0.184) \\
-2.8(1.186) \\
0(0.322) \\
0.2(0.195) \\
-0.3(0.16) \\
-0.8(0.378)
\end{array}
$$

Odds Ratio

Weight

Odds Ratio

V, Random, 95\% Cl

IV, Random, 95\% CI

$1.47 \%$

0.53[0.17,1.68]

$1.47 \%$

$0.53[0.17,1.68]$

\begin{tabular}{rrr} 
& & \\
$1.47 \%$ & $0.53[0.17,1.68]$ \\
\hline
\end{tabular}

Subtotal $(95 \% \mathrm{CI})$

Heterogeneity: $\mathrm{Tau}^{2}=0.06 ; \mathrm{Chi}^{2}=8.17, \mathrm{df}=4(\mathrm{P}=0.09) ; \mathrm{I}^{2}=51.03 \%$

$\begin{array}{rr}359 & -0.3(0.184) \\ 0 & -1.3(0.512) \\ 47 & -0.8(0.367) \\ 313 & -0.9(0.213) \\ 651 & -0.4(0.195)\end{array}$

$\begin{array}{rr}5.58 \% & 0.68[0.48,0.97] \\ 2.92 \% & 0.5[0.24,1.03] \\ 5.74 \% & 0.7[0.5,0.99] \\ 5.89 \% & 0.67[0.48,0.92] \\ \mathbf{2 0 . 1 3} \% & \mathbf{0 . 6 7}[\mathbf{0 . 5 6}, \mathbf{0 . 8 1}]\end{array}$

$0.79[0.66,0.96]$

$1.18[0.53,2.6]$

$0.9[0.74,1.09]$

$0.98[0.84,1.15]$

$0.5[0.13,1.94]$

$1.54[0.77,3.09]$

1.22[0.94,1.59]

$0.96[0.83,1.11]$

$0.67[0.56,0.81]$

$0.37[0.26,0.53]$

$0.06[0.01,0.65]$

$1[0.53,1.88]$

$1.28[0.87,1.87]$

$0.75[0.55,1.02]$

$0.44[0.21,0.92]$

$0.63[0.38,1.05]$

$0.75[0.52,1.07]$

$0.28[0.1,0.76]$

$0.45[0.22,0.92]$

$0.4[0.26,0.61]$

$0.69[0.47,1.01]$

$0.54[0.39,0.75]$ 


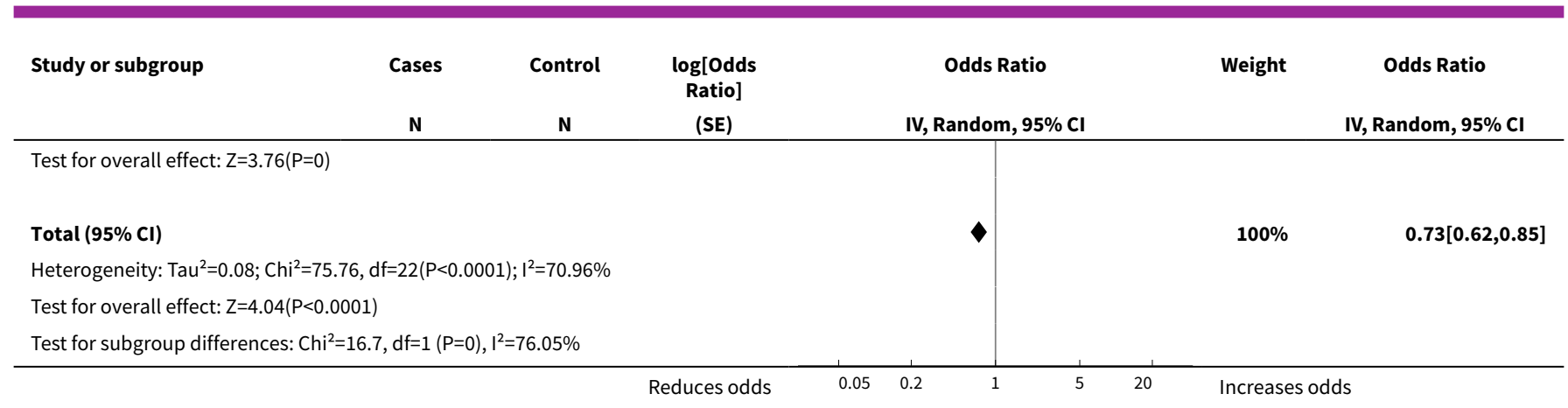

\section{Analysis 5.4. Comparison 5 Case-control studies: disposal of child faeces in latrine versus elsewhere, Outcome 4 Diarrhoea in all ages (including severe and cholera): subgrouped by study quality.}

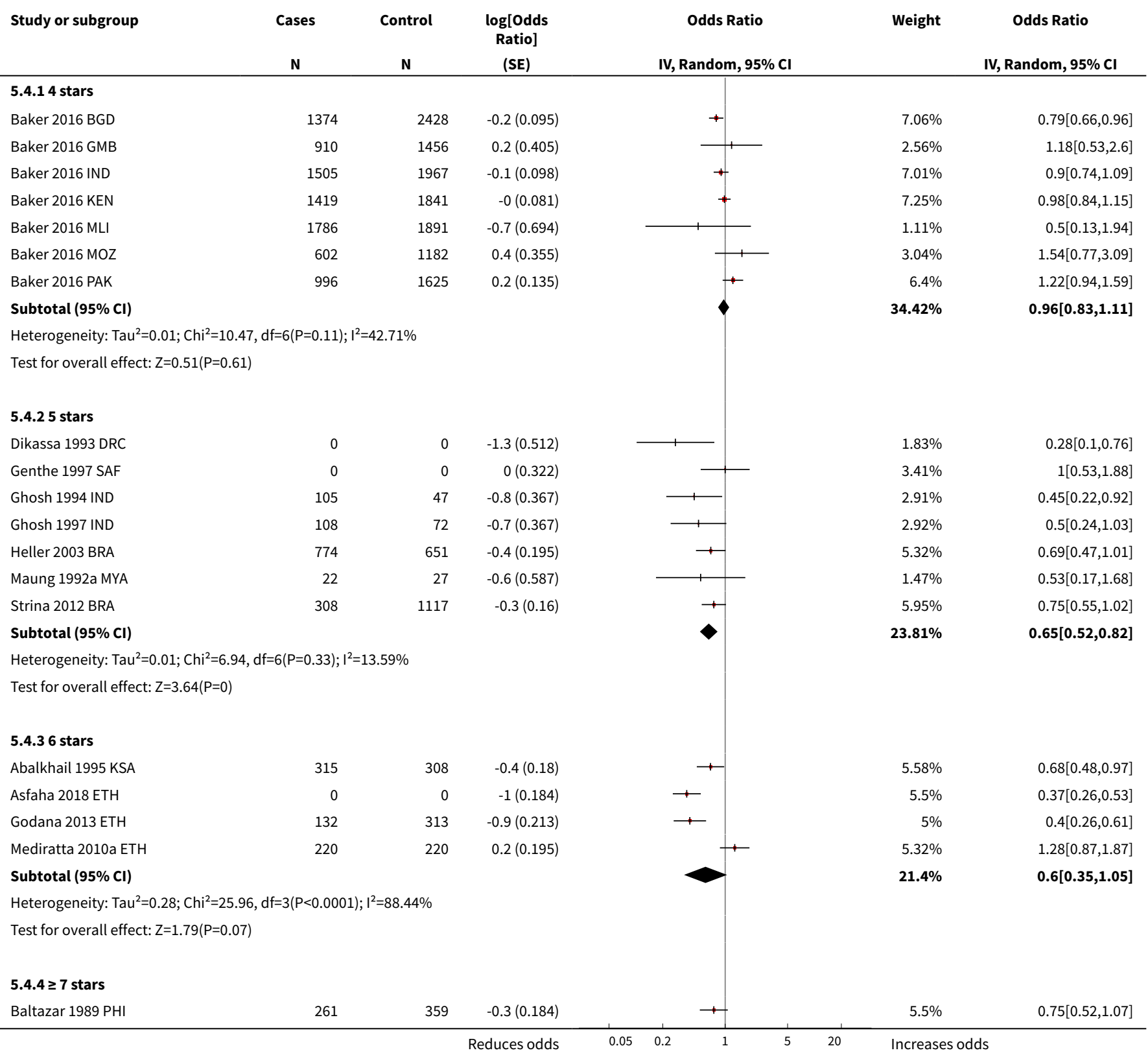




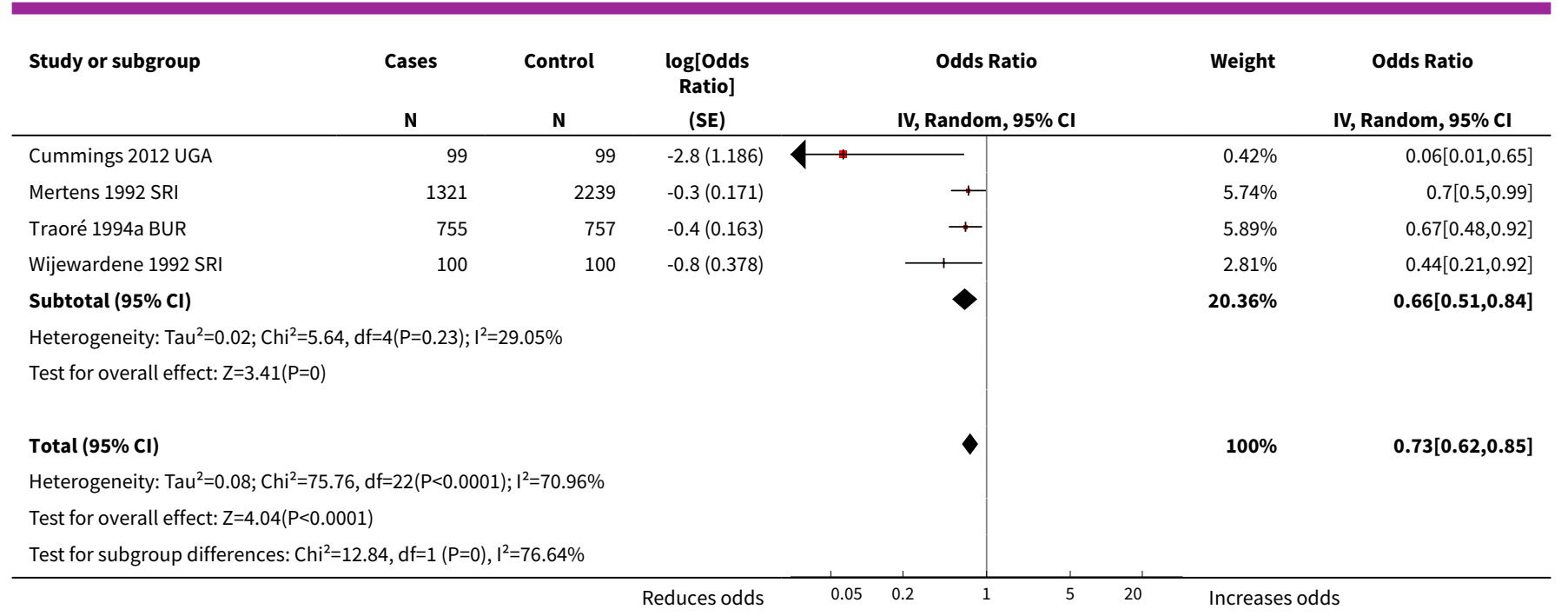

Analysis 5.5. Comparison 5 Case-control studies: disposal of child faeces in latrine versus elsewhere, Outcome 5 Diarrhoea in all ages (including severe and cholera): subgrouped by setting.

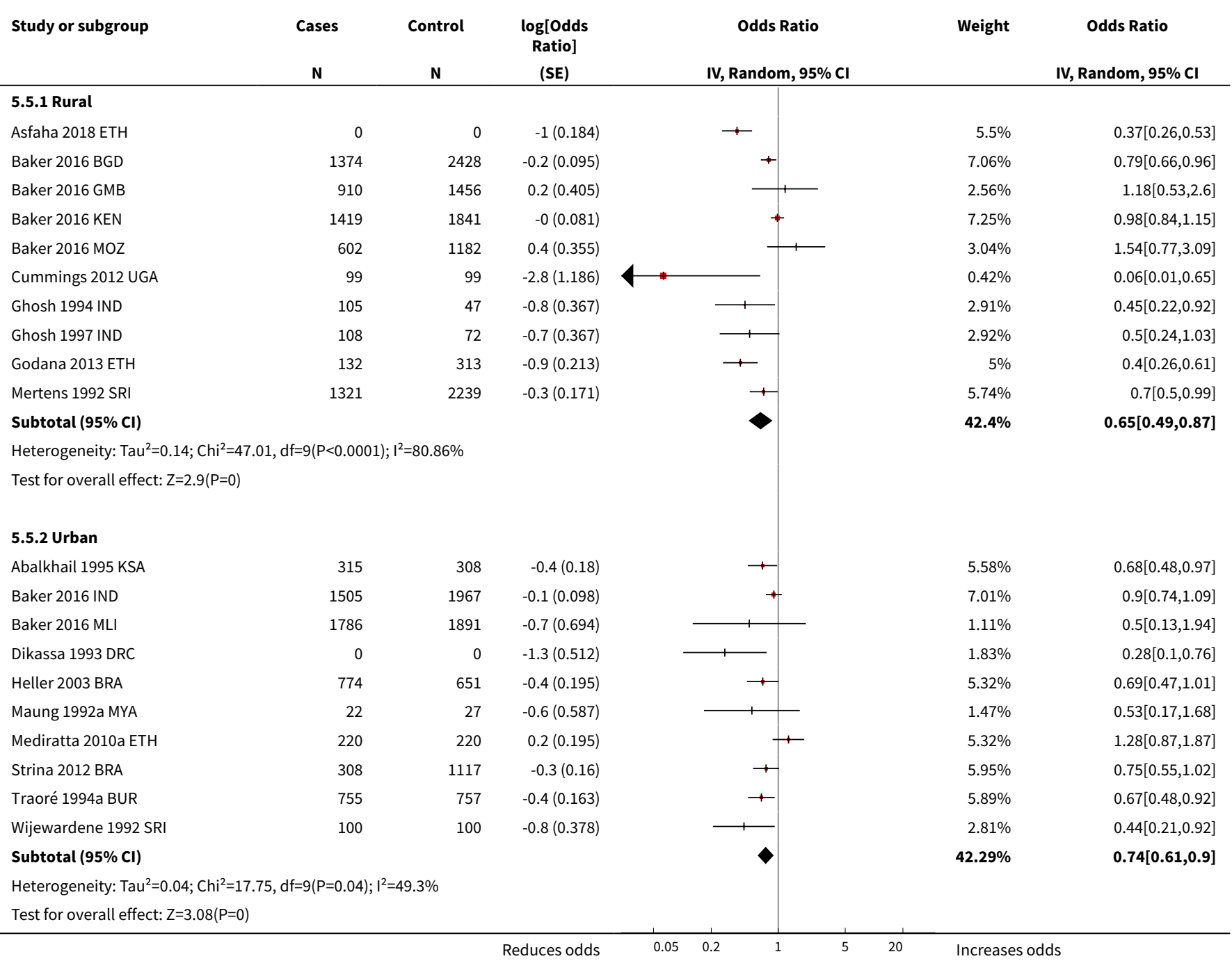




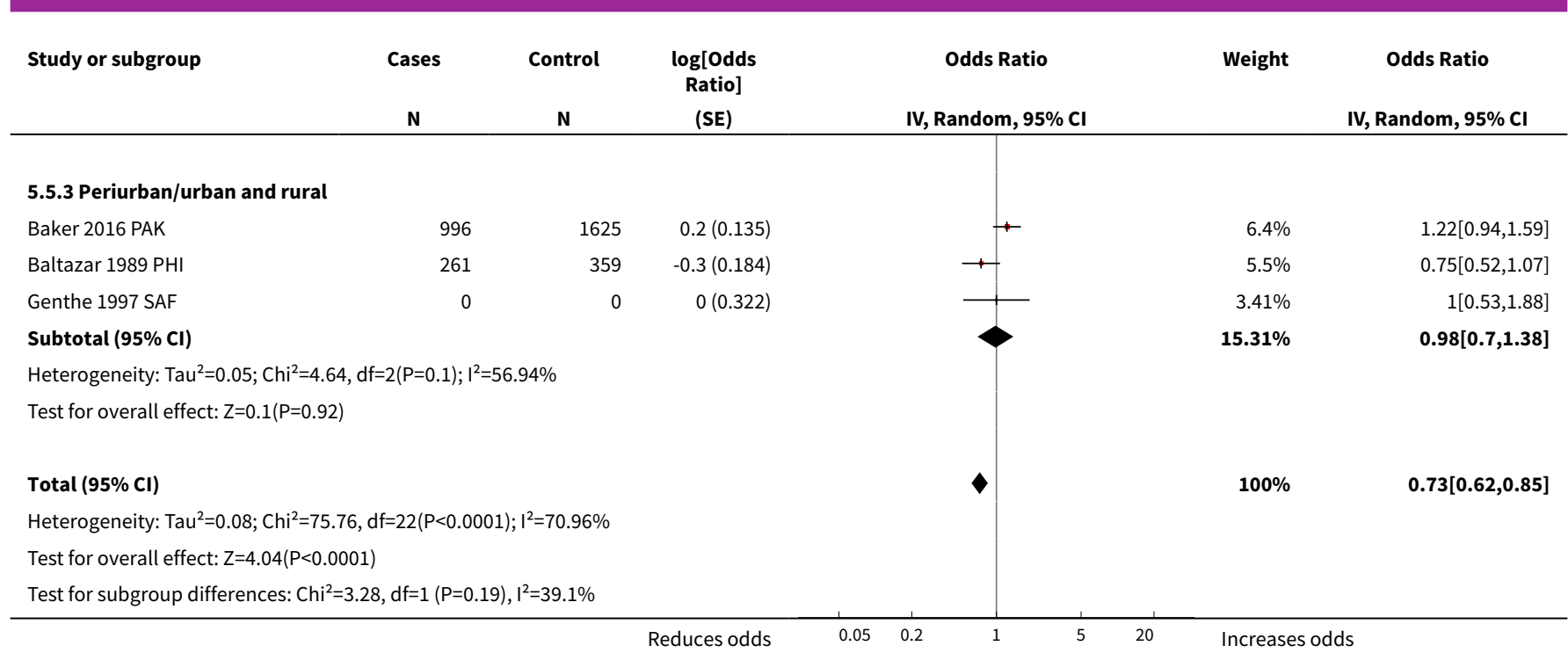

\section{Analysis 5.6. Comparison 5 Case-control studies: disposal of child faeces in latrine versus elsewhere, Outcome 6 Diarrhoea in all ages (including severe and cholera): subgrouped by method of data collection.}

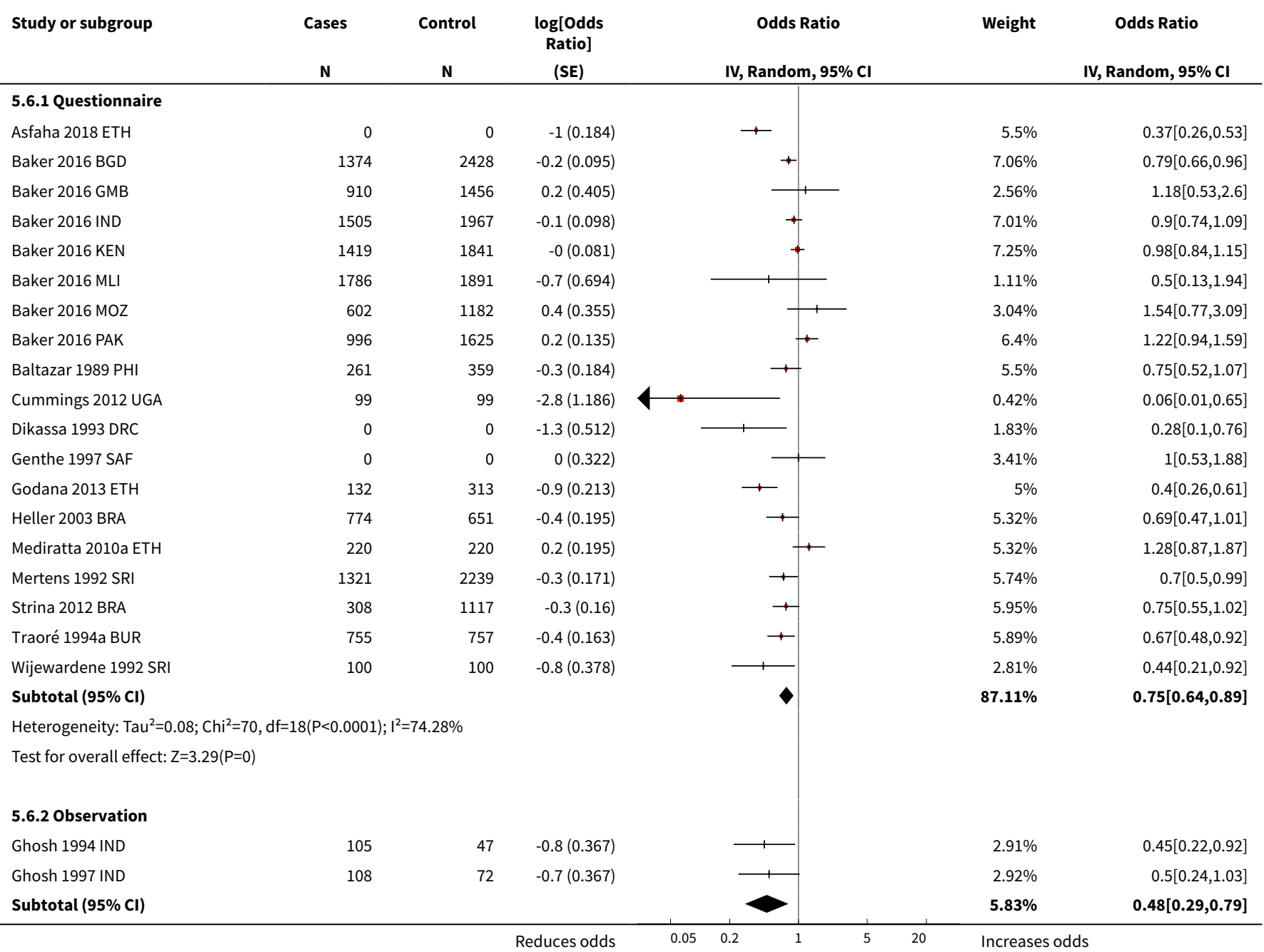




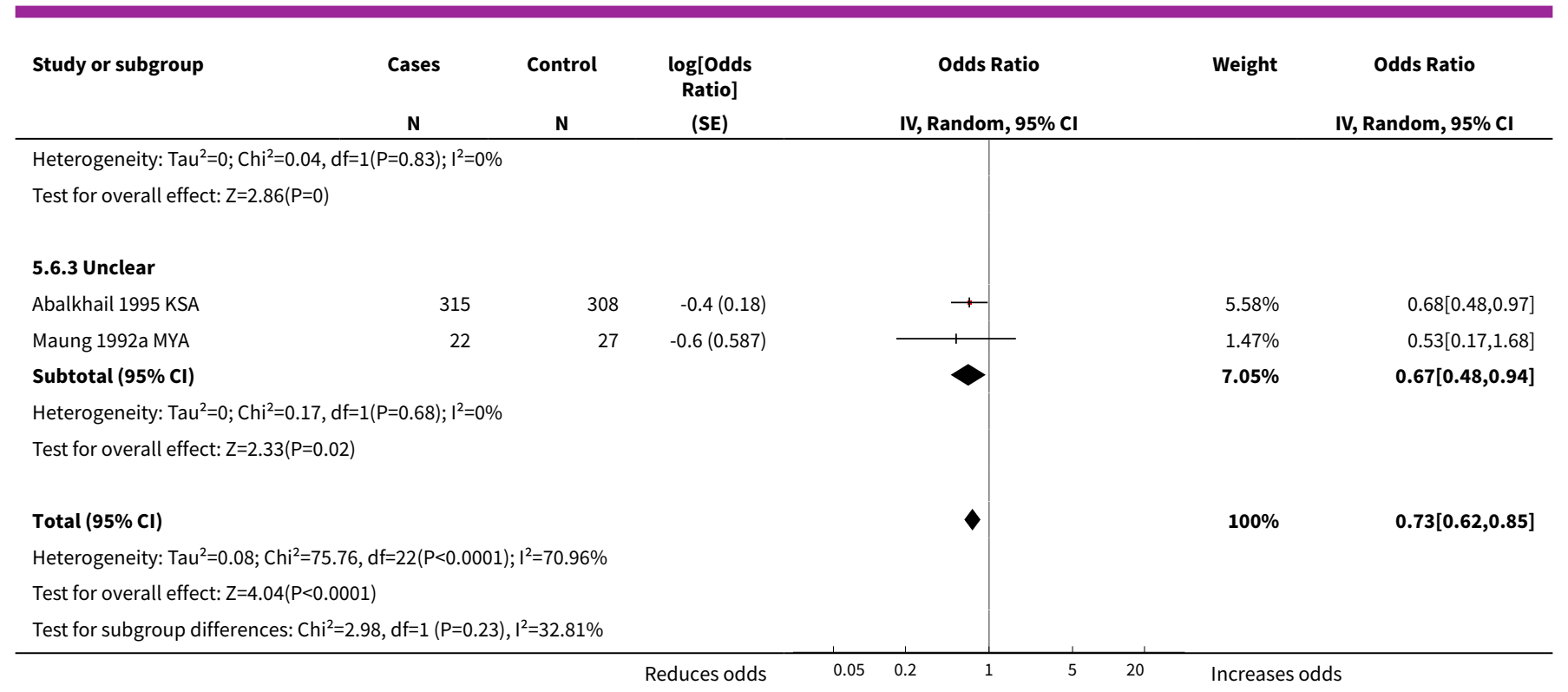

\section{Comparison 6. Case-control studies: defecation of children in latrine versus elsewhere}

\begin{tabular}{|c|c|c|c|c|}
\hline $\begin{array}{l}\text { Outcome or subgroup ti- } \\
\text { tle }\end{array}$ & $\begin{array}{l}\text { No. of } \\
\text { studies }\end{array}$ & $\begin{array}{l}\text { No. of } \\
\text { partici- } \\
\text { pants }\end{array}$ & Statistical method & Effect size \\
\hline $\begin{array}{l}1 \text { Diarrhoea: case-control } \\
\text { studies: subgrouped by } \\
\text { age group }\end{array}$ & 7 & & Odds Ratio (Random, 95\% Cl) & Subtotals only \\
\hline 1.1 All ages & 7 & & Odds Ratio (Random, 95\% Cl) & $0.54[0.33,0.90]$ \\
\hline 1.2 Aged $\leq 5$ years & 5 & & Odds Ratio (Random, 95\% Cl) & $0.54[0.28,1.07]$ \\
\hline $\begin{array}{l}2 \text { Diarrhoea in all ages: } \\
\text { case-control studies: sub- } \\
\text { grouped by country in- } \\
\text { come level }\end{array}$ & 7 & & Odds Ratio (Random, 95\% Cl) & $0.54[0.33,0.90]$ \\
\hline 2.1 Low & 3 & & Odds Ratio (Random, 95\% Cl) & $0.61[0.23,1.60]$ \\
\hline 2.2 Lower middle & 2 & & Odds Ratio (Random, 95\% Cl) & $0.23[0.11,0.48]$ \\
\hline 2.3 Upper middle & 2 & & Odds Ratio (Random, 95\% Cl) & $0.78[0.53,1.14]$ \\
\hline $\begin{array}{l}3 \text { Diarrhoea in all ages: } \\
\text { case-control studies: sub- } \\
\text { grouped by type of diar- } \\
\text { rhoea }\end{array}$ & 7 & & Odds Ratio (Random, 95\% Cl) & $0.54[0.33,0.90]$ \\
\hline 3.1 Other & 1 & & Odds Ratio (Random, 95\% Cl) & $0.14[0.03,0.52]$ \\
\hline 3.2 Persistent diarrhoea & 1 & & Odds Ratio (Random, 95\% Cl) & $0.27[0.12,0.60]$ \\
\hline
\end{tabular}




\begin{tabular}{|c|c|c|c|c|}
\hline $\begin{array}{l}\text { Outcome or subgroup ti- } \\
\text { tle }\end{array}$ & $\begin{array}{l}\text { No. of } \\
\text { studies }\end{array}$ & $\begin{array}{l}\text { No. of } \\
\text { partici- } \\
\text { pants }\end{array}$ & Statistical method & Effect size \\
\hline $\begin{array}{l}\text { 3.3 Acute (possibly) bloody } \\
\text { diarrhoea }\end{array}$ & 2 & & Odds Ratio (Random, 95\% Cl) & $0.85[0.65,1.12]$ \\
\hline 3.4 Acute watery diarrhoea & 3 & & Odds Ratio (Random, 95\% Cl) & $0.58[0.20,1.65]$ \\
\hline $\begin{array}{l}4 \text { Diarrhoea in all ages: } \\
\text { case-control studies: sub- } \\
\text { grouped by study quality }\end{array}$ & 7 & & Odds Ratio (Random, 95\% Cl) & $0.54[0.33,0.90]$ \\
\hline 4.14 stars & 2 & & Odds Ratio (Random, 95\% Cl) & $0.13[0.05,0.37]$ \\
\hline 4.25 stars & 1 & & Odds Ratio (Random, 95\% Cl) & $0.27[0.12,0.60]$ \\
\hline 4.36 stars & 3 & & Odds Ratio (Random, 95\% Cl) & $0.82[0.57,1.17]$ \\
\hline 4.47 stars & 1 & & Odds Ratio (Random, 95\% Cl) & $0.91[0.64,1.29]$ \\
\hline $\begin{array}{l}5 \text { Diarrhoea in all ages: } \\
\text { case-control studies: sub- } \\
\text { grouped by setting }\end{array}$ & 7 & & Odds Ratio (Random, 95\% Cl) & $0.54[0.33,0.90]$ \\
\hline 5.1 Rural & 1 & & Odds Ratio (Random, 95\% Cl) & $0.75[0.29,1.93]$ \\
\hline 5.2 Semi-urban & 1 & & Odds Ratio (Random, 95\% Cl) & $0.79[0.52,1.19]$ \\
\hline 5.3 Urban & 5 & & Odds Ratio (Random, 95\% Cl) & $0.40[0.17,0.94]$ \\
\hline $\begin{array}{l}6 \text { Diarrhoea in all ages: } \\
\text { case-control studies: sub- } \\
\text { grouped by method of da- } \\
\text { ta collection }\end{array}$ & 7 & & Odds Ratio (Random, 95\% Cl) & $0.54[0.33,0.90]$ \\
\hline 6.1 Questionnaire & 5 & & Odds Ratio (Random, 95\% Cl) & $0.75[0.50,1.13]$ \\
\hline 6.2 Observation & 1 & & Odds Ratio (Random, 95\% Cl) & $0.13[0.02,0.66]$ \\
\hline 6.3 Unclear & 1 & & Odds Ratio (Random, 95\% Cl) & $0.27[0.12,0.60]$ \\
\hline
\end{tabular}

Analysis 6.1. Comparison 6 Case-control studies: defecation of children in latrine versus elsewhere, Outcome 1 Diarrhoea: case-control studies: subgrouped by age group.

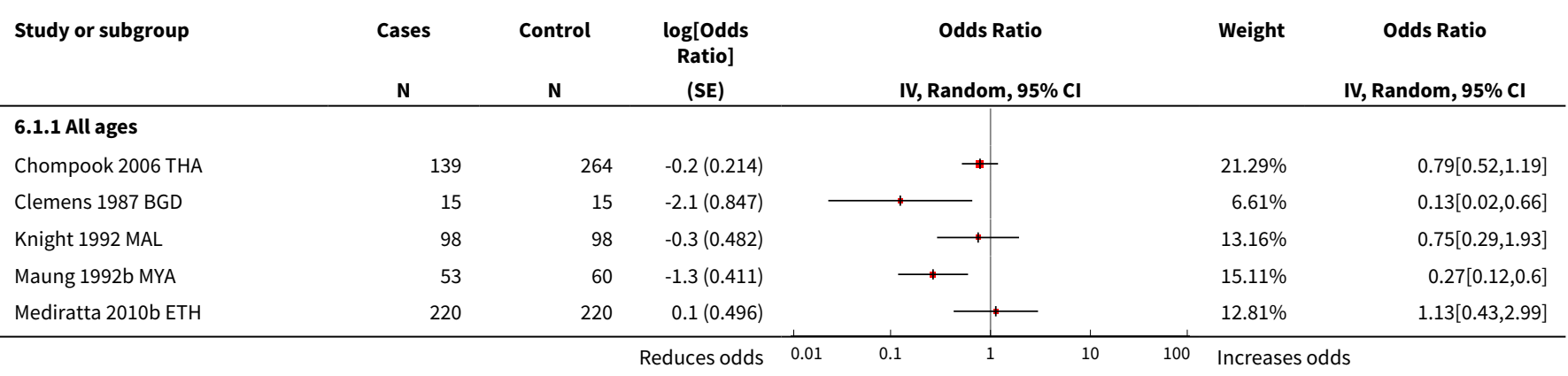




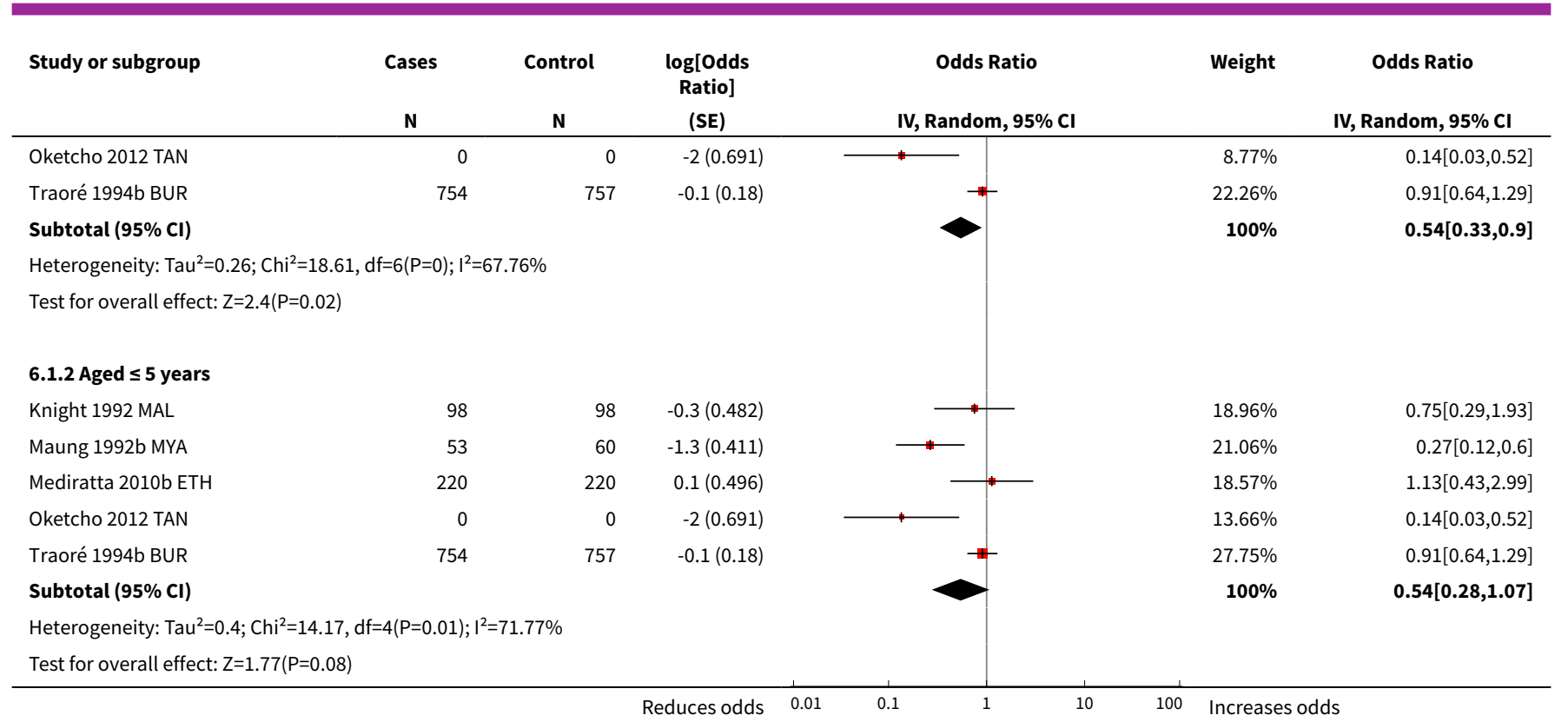

\section{Analysis 6.2. Comparison 6 Case-control studies: defecation of children in latrine versus elsewhere, Outcome 2 Diarrhoea in all ages: case-control studies: subgrouped by country income level.}

\begin{tabular}{|c|c|c|c|}
\hline \multirow[t]{2}{*}{ Study or subgroup } & Cases & Control & $\begin{array}{c}\text { log[Odds } \\
\text { Ratio] }\end{array}$ \\
\hline & $\mathbf{N}$ & $\mathbf{N}$ & (SE) \\
\hline \multicolumn{4}{|l|}{ 6.2.1 Low } \\
\hline Mediratta 2010b ETH & 220 & 220 & $0.1(0.496)$ \\
\hline Oketcho 2012 TAN & 0 & 0 & $-2(0.691)$ \\
\hline Traoré 1994b BUR & 754 & 757 & $-0.1(0.18)$ \\
\hline
\end{tabular}

Subtotal $(95 \% \mathrm{CI})$

Heterogeneity: $\mathrm{Tau}^{2}=0.51 ; \mathrm{Chi}^{2}=7.51, \mathrm{df}=2(\mathrm{P}=0.02) ; \mathrm{I}^{2}=73.36 \%$

Test for overall effect: $\mathrm{Z}=1(\mathrm{P}=0.32)$

\subsubsection{Lower middle}

Clemens 1987 BGD

Maung 1992b MYA

\section{5}

Subtotal $(95 \% \mathrm{Cl})$

Heterogeneity: $\mathrm{Tau}^{2}=0 ; \mathrm{Chi}^{2}=0.64, \mathrm{df}=1(\mathrm{P}=0.42) ; \mathrm{I}^{2}=0 \%$

Test for overall effect: $Z=3.97(P<0.0001)$

\subsubsection{Upper middle}

Chompook 2006 THA

Knight $1992 \mathrm{MAL}$

139

98

264

$-0.2(0.214)$

Subtotal $(95 \% \mathrm{Cl})$

Heterogeneity: $\mathrm{Tau}^{2}=0 ; \mathrm{Chi}^{2}=0.01, \mathrm{df}=1(\mathrm{P}=0.93) ; \mathrm{I}^{2}=0 \%$

Test for overall effect: $Z=1.27(P=0.2)$

Total $(95 \% \mathrm{Cl})$

Heterogeneity: $\operatorname{Tau}^{2}=0.26 ; \mathrm{Chi}^{2}=18.61, \mathrm{df}=6(\mathrm{P}=0) ; \mathrm{I}^{2}=67.76 \%$

Test for overall effect: $Z=2.4(P=0.02)$

Test for subgroup differences: $\mathrm{Chi}^{2}=8.52, \mathrm{df}=1(\mathrm{P}=0.01), \mathrm{I}^{2}=76.51 \%$
Weight Odds Ratio

IV, Random, $95 \% \mathrm{CI}$

IV, Random, 95\% CI

$\begin{array}{rrr} & & \\ & 12.81 \% & 1.13[0.43,2.99] \\ & 8.77 \% & 0.14[0.03,0.52] \\ & 22.26 \% & 0.91[0.64,1.29] \\ & \mathbf{4 3 . 8 4 \%} & \mathbf{0 . 6 1 [ 0 . 2 3 , 1 . 6}]\end{array}$


Analysis 6.3. Comparison 6 Case-control studies: defecation of children in latrine versus elsewhere, Outcome 3 Diarrhoea in all ages: case-control studies: subgrouped by type of diarrhoea.

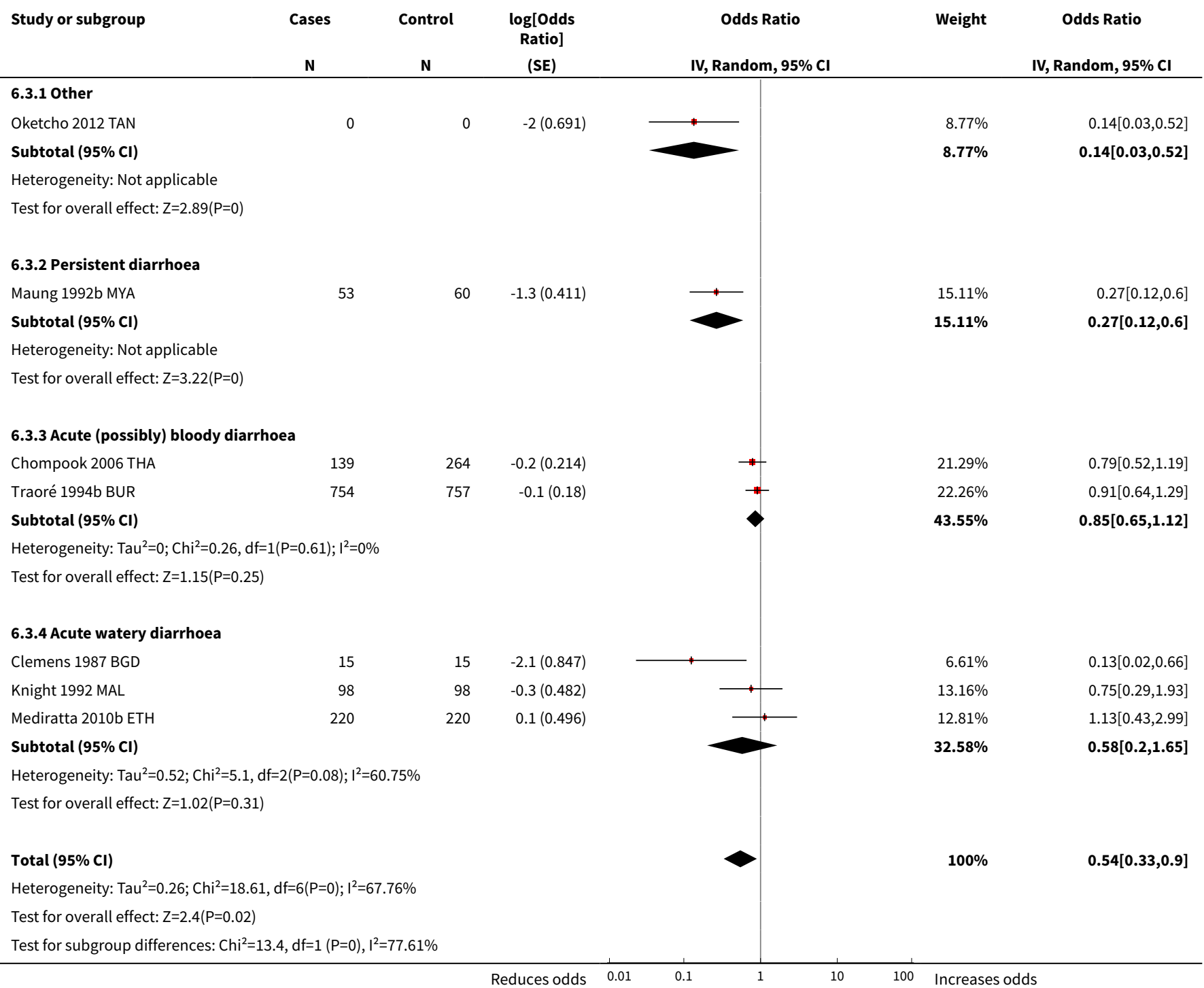

Analysis 6.4. Comparison 6 Case-control studies: defecation of children in latrine versus elsewhere, Outcome 4 Diarrhoea in all ages: case-control studies: subgrouped by study quality.

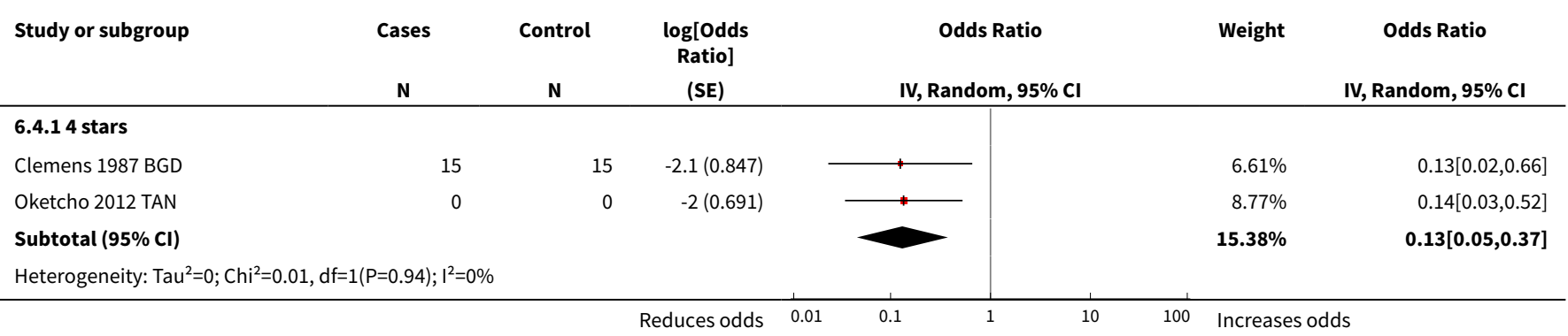




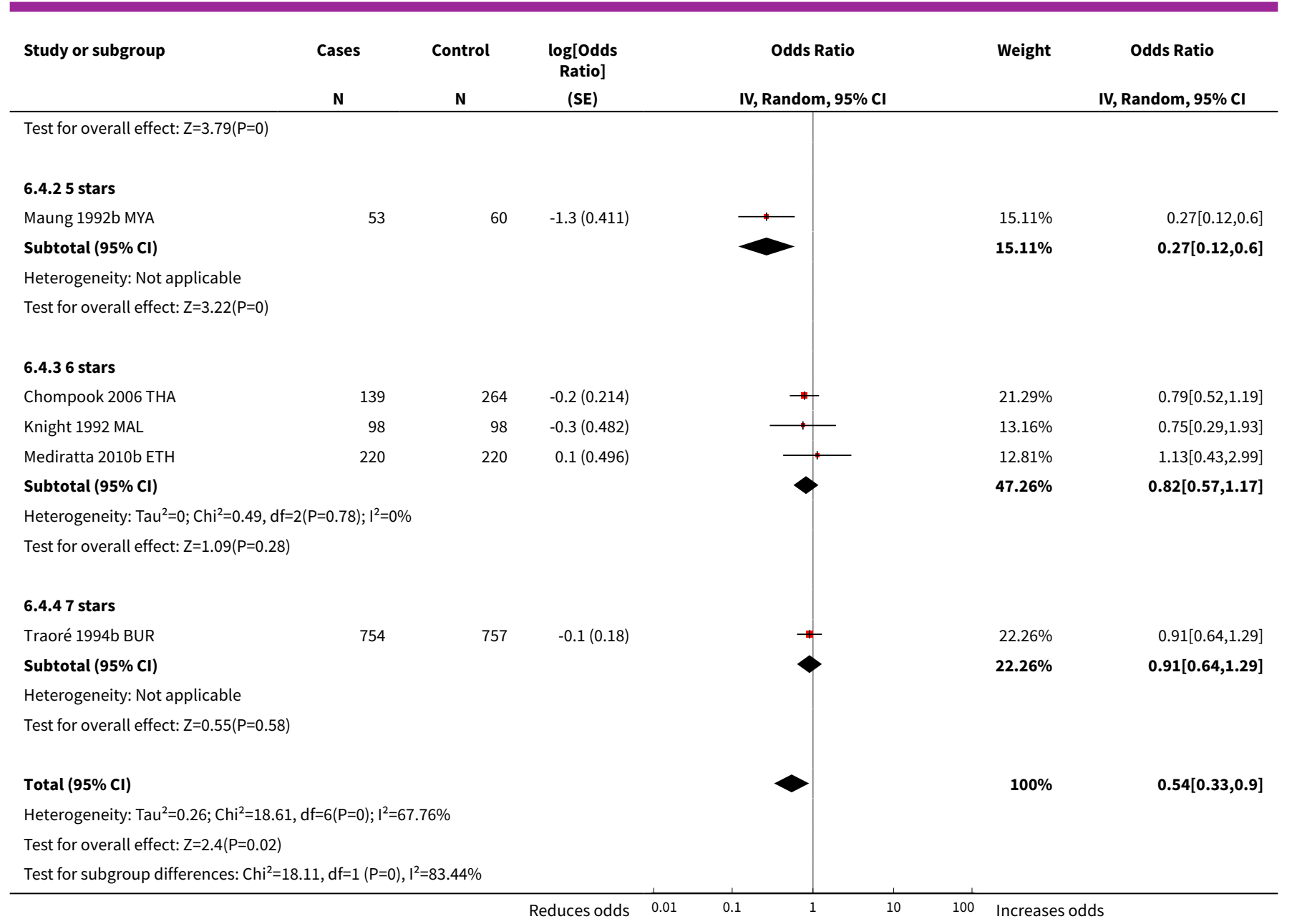

Analysis 6.5. Comparison 6 Case-control studies: defecation of children in latrine versus
elsewhere, Outcome 5 Diarrhoea in all ages: case-control studies: subgrouped by setting.

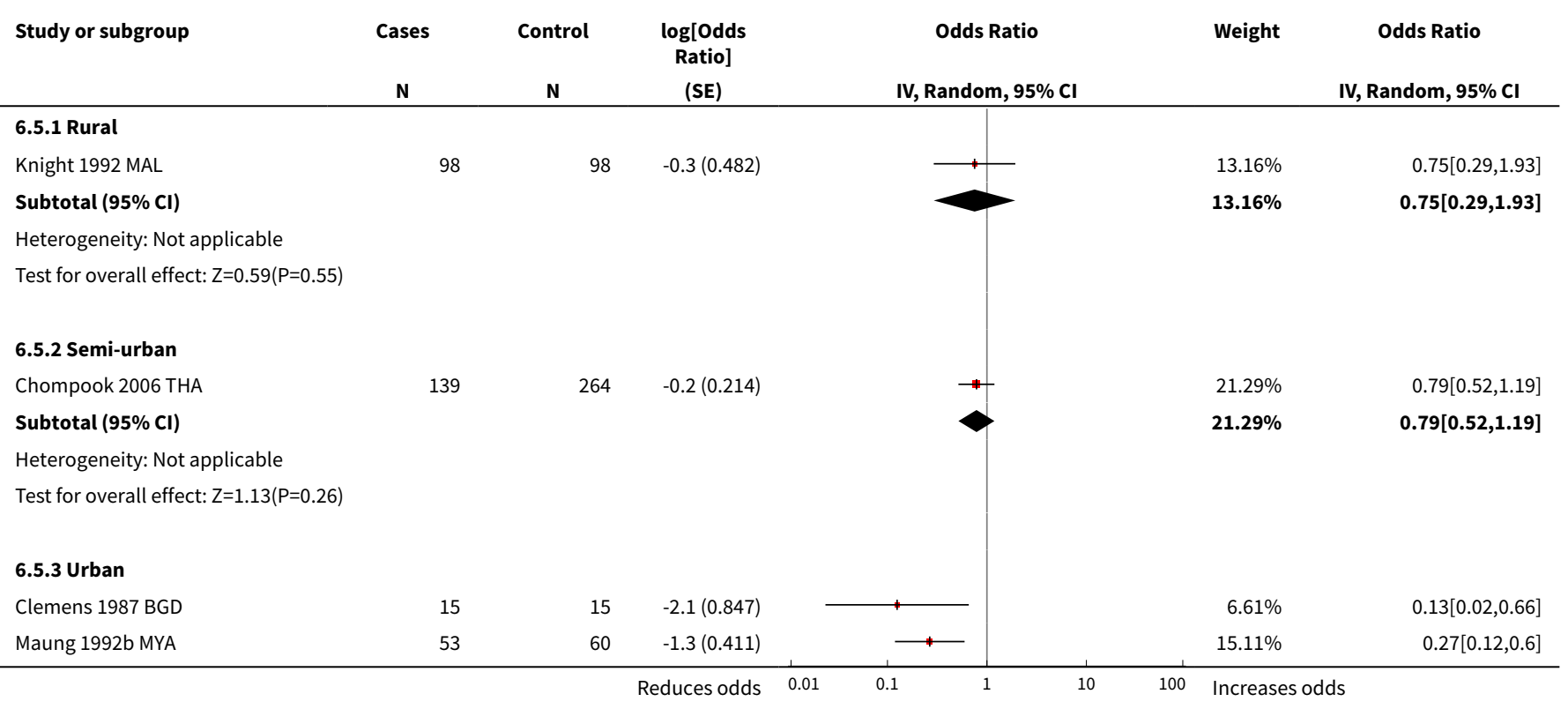




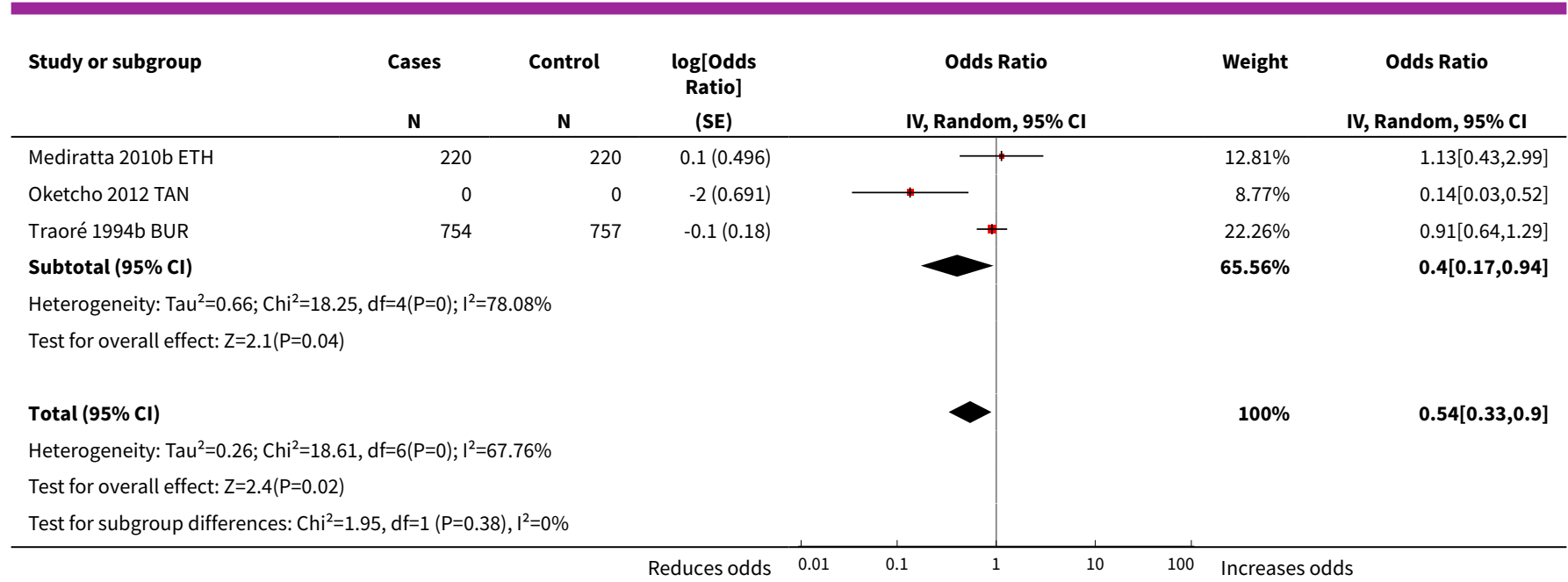

\section{Analysis 6.6. Comparison 6 Case-control studies: defecation of children in latrine versus elsewhere, Outcome 6 Diarrhoea in all ages: case-control studies: subgrouped by method of data collection.}

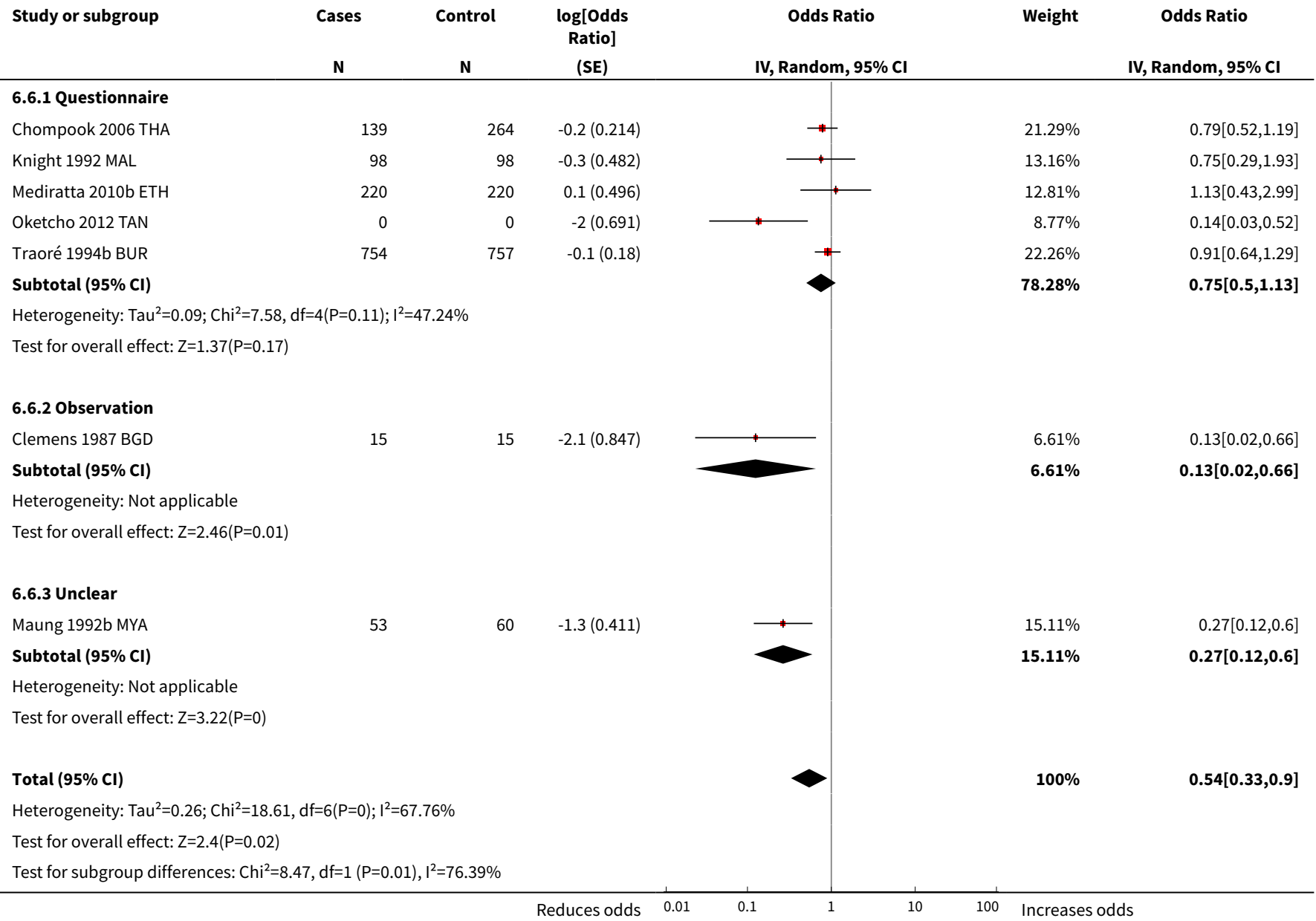


ADDITIONAL TABLES

Table 1. Summary of intervention categories

\begin{tabular}{lll}
\hline Intervention category & $\begin{array}{l}\text { Child faeces compo- } \\
\text { nent of intervention }\end{array}$ & Other intervention components \\
\hline Education and hygiene promotion interventions & Software only & None or limited hardware \\
\hline $\begin{array}{l}\text { Community-led total sanitation interventions + adap- } \\
\text { tations }\end{array}$ & Software only & Software only - focus on ending open defecation \\
\hline $\begin{array}{l}\text { Sanitation hardware and behaviour change interven- } \\
\text { tions }\end{array}$ & $\begin{array}{l}\text { Software + hardware } \\
\text { (potties/scoops) }\end{array}$ & Software + hardware (sanitation only) \\
\hline $\begin{array}{l}\text { WASH hardware and education/behaviour change in- } \\
\text { terventions }\end{array}$ & Software only & $\begin{array}{l}\text { Software + hardware (e.g. hand pumps, latrines, } \\
\text { water treatment solution, soap, handwashing facil- } \\
\text { ities, protected infant play space) }\end{array}$ \\
\hline $\begin{array}{l}\text { Daycare centre-based hygiene hardware and educa- } \\
\text { tion interventions }\end{array}$ & Software + hardware & Software + hardware \\
\hline
\end{tabular}

WASH: water, sanitation, and hygiene.

Table 2. Summary of the study designs, settings, and outcome measures of the education and hygiene promotion interventions (Continued)

\begin{tabular}{|c|c|c|c|c|c|}
\hline \multirow{2}{*}{$\begin{array}{l}\text { Study } \\
\text { (setting) }\end{array}$} & \multirow[t]{2}{*}{ Summary of the intervention } & \multicolumn{4}{|c|}{ Outcomes used in review $a$} \\
\hline & & Diarrhoea & $\begin{array}{l}\text { Anthro- } \\
\text { pometry }\end{array}$ & $\begin{array}{l}\text { Behav- } \\
\text { iour } \\
\text { change }\end{array}$ & Other \\
\hline
\end{tabular}

\section{RCTS}

Altmann

2018 TCD

(not spec-

ified)
Intervention in health centres to children admitted for OTP. The intervention group received routine OTP services (as the control group) plus WASH kit and promotion, which included messaging to bury children's stool. The household WASH kit given at admission contained a safe drinking water storage container with a lid, water disinfection consumables (180 chlorine tablets), 12 bars of soap for hand washing, a plastic cup with handle (to be reserved for the child to facilitate safe drinking water practice), and a laminated leaflet with pictures representing the main hygiene messages. They also received a promotion session on the kit use at each weekly visit to the health centre and 2 extra home visits for assessing and reinforcing adherence. Promotion at health centre included key messages on:

- a protected space for children to play;

- washing the child with soap;

- cleaning and rapid burial of children's stools;

- hand washing at key times;

- safe storage of water;

- exclusive breastfeeding of children aged < 6 months; and

- water treatment and food hygiene.

\begin{tabular}{|c|c|c|c|}
\hline $\begin{array}{l}\text { Longitudi- } \\
\text { nal preva- } \\
\text { lence } \\
\text { (narra- }\end{array}$ & $\begin{array}{l}\text { Recovery } \\
\text { rate from } \\
\text { SAM (nar- } \\
\text { ratively) }\end{array}$ & - & $\begin{array}{l}\text { Death rate } \\
\text { (narra- } \\
\text { tively) }\end{array}$ \\
\hline
\end{tabular}

tively) 
Table 2. Summary of the study designs, settings, and outcome measures of the education and hygiene promotion interventions (Continued)

The household WASH kit was designed to last for 3 months ( 2 months during treatment in the OTP and 1 month after the end of the treatment).

$\begin{array}{ll}\text { Barrios } & \text { Hygiene promotion programme that focused on improving hand } \\ 2008 \mathrm{PHI} & \text { washing and stool disposal behaviours. Delivered by midwives } \\ \text { (rural) } & \text { and health workers in small group meetings and in home visits. } \\ & \text { For the disposal of child faeces, caretakers were encouraged to } \\ & \text { use toilets (any type) as the final site of faeces disposal. When a } \\ & \text { toilet was not available, burying faeces } \geq 10 \text { m away from water } \\ & \text { sources and living areas was discussed. The main message was } \\ & \text { the sanitary disposal of faeces, regardless of where a child defe- } \\ & \text { cated. }\end{array}$

Haggerty Education intervention to improve personal and domestic hy-
1994 DRC giene behaviours including: disposal of animal faeces, hand (rural) washing before meal preparation and after defecation/washing hands and buttocks of young children after defecation, disposal of children's faeces (emphasized digging or improving pit latrines). The messages were delivered by female community volunteers in village-wide meetings and small group discussions.

\begin{tabular}{|c|c|c|c|}
\hline $\begin{array}{l}\text { 7-day re- } \\
\text { call }\end{array}$ & - & - & - \\
\hline $\begin{array}{l}\geq 1 \\
\text { episodes } \\
\text { of diar- } \\
\text { rhoea at } \\
\text { any time } \\
\text { during the } \\
\text { surveil- } \\
\text { lance pe- } \\
\text { riod. }\end{array}$ & & & \\
\hline $\begin{array}{l}\text { Incidence } \\
\text { (2-week } \\
\text { recall) }\end{array}$ & - & - & - \\
\hline
\end{tabular}

$\begin{array}{llll}\text { Hashi } & \text { Health education and provision of soap (white bars). The health } & \text { Incidence } & -\end{array}-$
and use, safe waste disposal) and demonstration of hand washing with soap.

Messages to dispose of children's waste properly were delivered via demonstrations and instructions. The messages were to dispose of children's waste properly in the waste disposal site (in a waste container at the corner/back of the house) as opposed to the garbage (uncollected waste) and in a latrine (if they had 1) but never in the open field, garbage, or around utensils and kitchen.

\begin{tabular}{|c|c|c|c|c|c|}
\hline $\begin{array}{l}\text { Jinadu } \\
2007 \text { NGR } \\
\text { (rural) }\end{array}$ & $\begin{array}{l}\text { Educational intervention programme to promote the hygienic } \\
\text { disposal of children's faeces: } \\
\text { - educating mothers about the hygienic use of chamber pots for } \\
\text { the disposal of children faeces; } \\
\text { - discouraging children from defecation around households; } \\
\text { - educating the heads of households about the construction and } \\
\text { use of cheap, affordable, ventilated improved latrines by mem- } \\
\text { bers of the communities; } \\
\text { - educating mothers to wash hands with soap and water after } \\
\text { going to toilet and after cleaning up children's faeces. }\end{array}$ & - & - & $\begin{array}{l}\text { Potty use } \\
\text { Faeces } \\
\text { observed } \\
\text { in the } \\
\text { yard/ } \\
\text { house } \\
\text { Latrine } \\
\text { use by } \\
\text { children }\end{array}$ & - \\
\hline $\begin{array}{l}\text { Nair } 2017 \\
\text { IND (rural) }\end{array}$ & $\begin{array}{l}\text { Intervention involving community-based female worker (Su- } \\
\text { poshan Karyakarta, or SPK) carrying home visits with individ- } \\
\text { ual families and participatory meetings with groups of women, } \\
\text { to improve health and nutrition in the first } 1000 \text { days of life. The } \\
\text { training to prepare SPK to home visits included: advising care- } \\
\text { givers to place the child's faeces in a pit latrine, or if no latrines }\end{array}$ & - & $\begin{array}{l}\text { LAZ at } 18 \\
\text { months } \\
\text { Other } \\
\text { anthro- } \\
\text { pometry }\end{array}$ & - & $\begin{array}{l}\text { Mortali- } \\
\text { ty (narra- } \\
\text { tively) }\end{array}$ \\
\hline
\end{tabular}


Table 2. Summary of the study designs, settings, and outcome measures of the education and hygiene promotion interventions (Continued)

are available (the case for $>90 \%$ of households in the trial areas), to bury them in a shallow hole away from their living area and any waterway rather than disposing of them in the open field or

measures

(WHZ,

WAZ, the household compound.
MUAC, stunting, wasting, underweight) (narratively)

\section{Sarrassat \\ Mass radio campaign targeted at women of reproductive age and} 2018 BUR (rural) caregivers of children aged $<5$ years, on 17 childcare behaviours, including safe child faeces disposal. The radio campaign included short spots (1 minute' duration, broadcast approximately 10 times per day) and interactive long-format programmes ( 2 hours' duration, broadcast 5 days per week, followed by phone-ins to allow listeners to comment). All materials were produced in the predominant local languages of each intervention cluster. Behaviours covered by spots changed weekly. The long-format programme covered 2 behaviours a day and changed daily. Safe child stool disposal was covered in 3 weeks of spots and 94 longformat modules. The recommendation was for all faeces (including the faeces of babies and small children) to be disposed of after defecation in a hygienic way. Either by using latrines or by using pots for young children or burying the stools outside the house/compound.

\section{Safe dis- All-cause posal of post- last chil- neonatal dren's mortali- stools ty in chil- dren aged $<5$ years (narra- tively)}

All-cause mortality in children aged $<5$ years (narratively)

\footnotetext{
Sinharoy Community-Based Environmental Health Promotion Pro2017 RWA gramme, which used the community health club approach to (rural) promote healthy practices. The study evaluated 2 versions of the programme: a lite (8 education sessions) and classic ( 20 sessions). Education sessions include: personal hygiene, handwashing, diarrhoea, water sources, safe storage of drinking water, treatment of drinking water, and sanitation.

Both lite and classic interventions included messages on child sanitation under the topic of sanitation ("zero open defecation"). The participants were recommended the following:

- children should defecate into a chamber pot;

- children's faeces should be buried if there was no latrine (cat sanitation) - but always emphasized throwing the faeces in the latrine;

- never let dogs or pigs eat children's faeces.
}

\begin{tabular}{|c|c|c|c|c|c|}
\hline $\begin{array}{l}\text { Stanton } \\
1987 \text { BGD } \\
\text { (urban) }\end{array}$ & $\begin{array}{l}\text { Educational intervention emphasizing } 3 \text { messages: } \\
\text { - proper hand washing before food preparation; } \\
\text { - defecation away from the house and in a proper site; } \\
\text { - suitable disposal of waste and faeces. } \\
\text { The intervention was delivered in the community over } 8 \text { weeks } \\
\text { through small group discussions, larger demonstrations, com- } \\
\text { munity wide planning and action meeting, posters, games, pic- } \\
\text { torial stories, and flexi flans (flannel board with movable charac- } \\
\text { ters). }\end{array}$ & $\begin{array}{l}\text { Incidence } \\
\text { (2-week } \\
\text { recall) }\end{array}$ & $\begin{array}{l}\text { Weight for } \\
\text { age } \\
\text { Height for } \\
\text { age }\end{array}$ & $\begin{array}{l}\text { Open } \\
\text { defeca- } \\
\text { tion of } \\
\text { children } \\
\text { aged }<5 \\
\text { years }\end{array}$ & $\begin{array}{l}\text { Mortali- } \\
\text { ty (narra- } \\
\text { tively) }\end{array}$ \\
\hline
\end{tabular}


Table 2. Summary of the study designs, settings, and outcome measures of the education and hygiene promotion interventions (Continued)

Yeager

2002 PER

(urban)
Hygiene promotion for potty use and keeping the home environment free from faeces. The intervention was delivered through routine health services, and using video presentations, leaflets including 4 steps to potty training and counselling by health staff during consultations
Potty use -

Latrine

use by

children

Safe dis-

posal of

child fae-

ces

\section{CBA studies}

Ahmed Participatory behaviour change intervention "Porichchhanna Ji1993 BGD bon" (clean life). The campaign was developed in partnership (rural) with the community. The intervention involved teaching the germ theory of disease then encouraging mothers to identify their problems and to find solutions through group participation and discussion. The interventions developed were:

- theme l: ground sanitation - keeping babies from touching and eating disease-causing matter on the dirt surface of the compound:

* sweep the baby's play area 4 times a day.

* use a dirt thrower (similar to a flat garden trowel provided by the project at USD 0.30 ) to immediately remove the baby's or animal faeces from the compound surface, so that the crawling baby could not be contaminated by faeces from the ground.

* construct a faeces pit to dispose of faeces and other filthy matter from the compound. The faeces pit was about 2 feet deep, with a narrow neck.

* wash babies in a particular place after defecation so that germ-contaminated water did not spread everywhere.

* keep crawling babies in a playpen (locally constructed, provided by the project at a cost of USD 1.0) instead of permitting them to crawl in the dirt;

- theme II: personal hygiene - reducing the transmission of germs from defecation and other personal hygiene behaviours (hand washing with ashes or soap, anal cleaning, clean baby after defecation, cut nails, clean rag to dry hands, clean baby rug/mat)

- theme III: food hygiene - reducing the transmission of germs during supplementary and bottle feeding (do not use any feeding bottle if possible, clean bottle, prepare small amount, use tube well water for drinking and baby food, wash hands before eating, cover food, do not eat leftovers, store plates and pans upside down, cover water pitchers).
Trends in

daily di-

arrhoea

preva-

lence

(narra-

tively)
Weight for -

age (nar-

ratively) 
Table 2. Summary of the study designs, settings, and outcome measures of the education and hygiene promotion interventions (Continued)

into hygienic latrines" using household visits, courtyard meetings and different activities for example hygiene fairs, village theatre, and group discussions in tea stalls. Promoters used flip charts and flash cards.
Luby 2014
BGD (rur-
Improved SHEWA-B. Changes in the intervention included a mass al and ur- media campaign including radio spots across 6 regional chan-
ban) nels from November 2011 to February 2012 encouraging HWWS before food, after defecation, and after cleaning a child and video spots on 5 television stations (November-February 2012) encouraging HWWS, using sanitary latrines for defecation, dis- carding child faeces, and keeping latrines clean to reduce bad smells and flies. A second series of videos encouraged testing tube-wells for arsenic and using arsenic free water for cooking and drinking. The intervention target population also expanded to include urban households.

$\begin{array}{lll}\begin{array}{l}\text { Diarrhoea } \\ \text { preva- }\end{array} & \text { HAZ } & \begin{array}{l}\text { Safe dis- } \\ \text { posal of } \\ \text { child fae- } \\ \text { lence (2- } \\ \text { day recall) }\end{array} \\ & \text { WAZ } & \begin{array}{l}\text { ces (ob- } \\ \text { served) }\end{array} \\ & \begin{array}{l}\text { (narra- } \\ \text { tively) }\end{array}\end{array}$

\section{Controlled cross-sectional studies}

$\begin{array}{ll}\begin{array}{l}\text { Berhe } \\ \text { 2014 ETH }\end{array} & \text { The HEP is implemented by full-time female health extension } \\ \text { (rural) } & \text { workers who provide training to households. The packages in- } \\ & \text { clude interventions in } 4 \text { main categories: family health services, } \\ \text { infectious disease prevention and control, hygiene and envi- } \\ \text { ronmental sanitation, and health education and communica- } \\ \text { tion. The maternal and child health package (in the family health } \\ \text { services category) includes safe child stool disposal (the stool } \\ \text { should be cleaned and disposed in a pit latrine, or shall be cov- } \\ \text { ered with a leaf or paper and be buried) (HEP 2003). }\end{array}$

\begin{tabular}{ll}
\hline Fisher & BRAC hygiene education intervention, trained field workers pro- \\
2011 BGD & vide WASH education to separate clusters of men, women, ado- \\
(rural) & $\begin{array}{l}\text { lescents, and children at least once every } 3 \text { months. The educa- } \\
\text { tion uses pictorial flip chart with a total of } 39 \text { messages covering }\end{array}$ \\
& multiple aspects of cleanliness, clean water, and sanitation. Vil- \\
& lagers are also encouraged to learn the '19 Messages to Remem- \\
& ber', concerning hand washing, sanitation (includes child faeces \\
& disposal in latrine), and safe water.
\end{tabular}

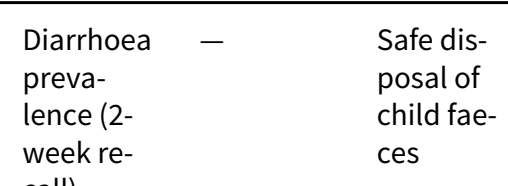

call)

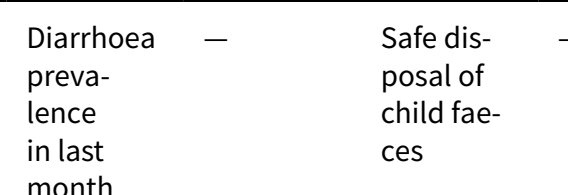

(anth

(narra-

tively)

Gebru HEP intervention, as in Berhe 2014 ETH.
2014 ETH
(rural)

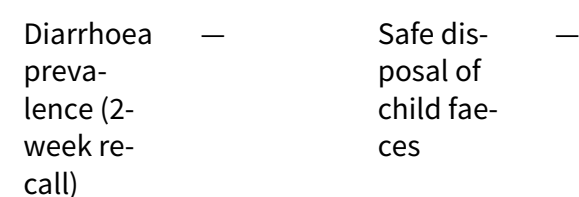

\begin{tabular}{|c|c|c|c|}
\hline $\begin{array}{l}\text { Mathew } \\
2004 \text { ZIM } \\
\text { (rural) }\end{array}$ & $\begin{array}{l}\text { Community health clubs - structured weekly course of partici- } \\
\text { patory health education classes. } 15 \text { health topics covered using } \\
\text { PHAST techniques, within the hygiene lesson covering disposal } \\
\text { of toddler's faeces in a latrine. }\end{array}$ & - & $\begin{array}{l}\text { Percent- } \\
\text { age of } \\
\text { children } \\
\text { (aged < } \\
5 \text { years) } \\
\text { present at } \\
\text { the time } \\
\text { of obser- } \\
\text { vations } \\
\text { not using } \\
\text { a latrine } \\
\text { (narra- } \\
\text { tively) }\end{array}$ \\
\hline
\end{tabular}


Table 2. Summary of the study designs, settings, and outcome measures of the education and hygiene promotion interventions (Continued)

Oguro WVGs were established by organizing women and training them 2016 MYA using a participatory approach. The activities of the WVGs after 3 (rural) years of being established included:

- educating pregnant women and mothers regarding the necessity of health checks and immunizations and helping them attend these appointments;

- early detection of abnormal signs and symptoms during the perinatal period;

- managing the family planning fund, which allowed women who could not afford contraception to borrow money at no interest;

- providing first aid to injured people (e.g. for injuries that were sustained during agricultural work); and

- educating women regarding appropriate sanitation and malaria prevention.

The WVG encouraged latrine use by children aged $<5$ years to villagers as part of a programme to promote sanitation education and promoted appropriate disposal (flushing in a latrine) of child faeces.

\begin{tabular}{|c|c|c|c|c|}
\hline $\begin{array}{l}\text { Wa- } \\
\text { terkeyn } \\
2005 \text { ZIM } \\
\text { (rural) }\end{array}$ & CHC intervention, as Mathew 2004 ZIM. & - & - & $\begin{array}{l}\text { Observed } \\
\text { child fae- } \\
\text { ces in the } \\
\text { yard (nar- } \\
\text { ratively) }\end{array}$ \\
\hline
\end{tabular}

CBA: controlled before-and-after; HAZ: height-for-age Z score; HEP: Health Extension Package; HWWS: handwashing with soap; PHAST: Participatory Hygiene and Sanitation Transformation; OTP: Outpatient Therapeutic feeding Program; LAZ: length-for-age Z score; MUAC: mid-upper-arm-circumference; RCT: randomized controlled trial; SAM: severe acute malnutrition; SHEWA-B: Sanitation Hygiene Education and Water Supply in Bangladesh; WASH: water, sanitation, and hygiene; WAZ: weight-for-age Z score; WHZ: weight-for-height Z score; WVG: Women's Health Volunteer Group.

aNone of the education and hygiene promotion interventions measured soil-transmitted helminth outcomes.

Table 3. Summary of the study designs, settings, and outcome measures of the CLTS interventions plus

\begin{tabular}{|c|c|c|c|c|c|c|}
\hline \multirow{2}{*}{$\begin{array}{l}\text { adaptations } \\
\text { (setting) }\end{array}$} & \multirow{2}{*}{$\begin{array}{l}\text { (Continued) } \\
\text { Summary of intervention }\end{array}$} & \multicolumn{5}{|c|}{ Outcomes used in review } \\
\hline & & Diarrhoea & STH & $\begin{array}{l}\text { Anthro- } \\
\text { pometry }\end{array}$ & $\begin{array}{l}\text { Behav- } \\
\text { iour } \\
\text { change }\end{array}$ & Other \\
\hline \multicolumn{7}{|l|}{ RCTs } \\
\hline $\begin{array}{l}\text { Briceño } \\
2015 \text { TANa } \\
\text { (rural) }\end{array}$ & $\begin{array}{l}\text { TSSM uses CLTS (triggering of community to in- } \\
\text { crease demand for improved sanitation and pro- } \\
\text { mote open defecation-free communities) and sani- } \\
\text { tation marketing to increase demand for improved } \\
\text { sanitation. Also strengthens the supply of sanita- } \\
\text { tion goods and services to local markets to make } \\
\text { these products more affordable and accessible. } \\
\text { Sanitation marketing messages concentrated on } \\
\text { positive aspirational messages rather than shame } \\
\text { tactics. No subsidies were used. }\end{array}$ & $\begin{array}{l}\text { Preva- } \\
\text { lence, } 7 \text { - } \\
\text { and } 14- \\
\text { day recall }\end{array}$ & - & $\begin{array}{l}\text { WAZ } \\
\text { HAZ }\end{array}$ & $\begin{array}{l}\text { Safe child } \\
\text { faeces dis- } \\
\text { posal }\end{array}$ & $\begin{array}{l}\text { Mortali- } \\
\text { ty (narra- } \\
\text { tively) }\end{array}$ \\
\hline
\end{tabular}


Table 3. Summary of the study designs, settings, and outcome measures of the CLTS interventions plus

\begin{tabular}{|c|c|c|c|c|c|}
\hline $\begin{array}{l}\text { adaptation } \\
2013 \text { INA } \\
\text { (rural) }\end{array}$ & $\begin{array}{l}\text { SESTM which includes CLTS to stop open defecation, } \\
\text { social sanitation marketing to increase availability } \\
\text { of products and services, and strengthening the en- } \\
\text { abling environment at policy and institutional lev- } \\
\text { els. }\end{array}$ & $\begin{array}{l}\text { Preva- } \\
\text { lence (2-, } \\
7-\text {, and 14- } \\
\text { day recall) }\end{array}$ & $\begin{array}{l}\text { Ascaris, } \\
\text { Trichuris, } \\
\text { and hook- } \\
\text { worm in- } \\
\text { fections }\end{array}$ & $\begin{array}{l}\text { WAZ } \\
\text { HAZ (nar- } \\
\text { ratively) }\end{array}$ & $\begin{array}{l}\text { Open } \\
\text { defeca- } \\
\text { tion of } \\
\text { children } \\
\text { aged }<5 \\
\text { years }\end{array}$ \\
\hline
\end{tabular}

Safe dis-

posal of

child fae-

ces

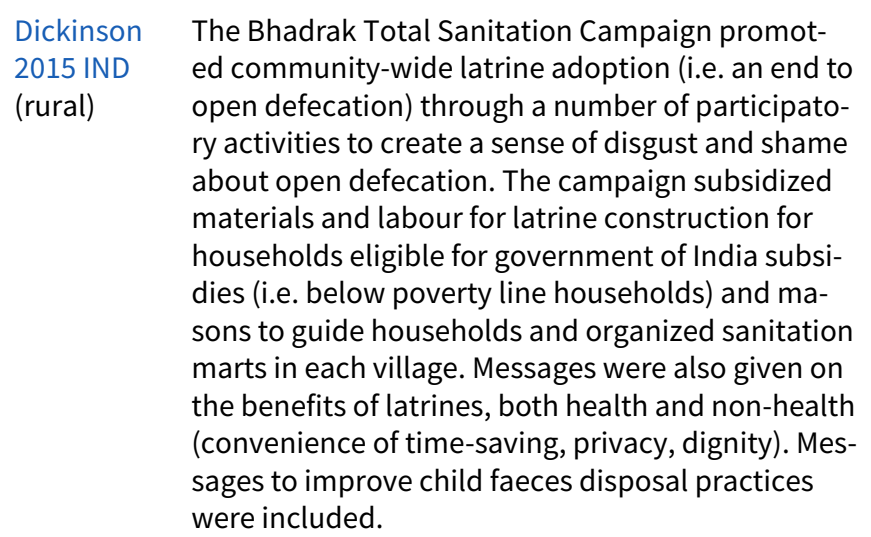

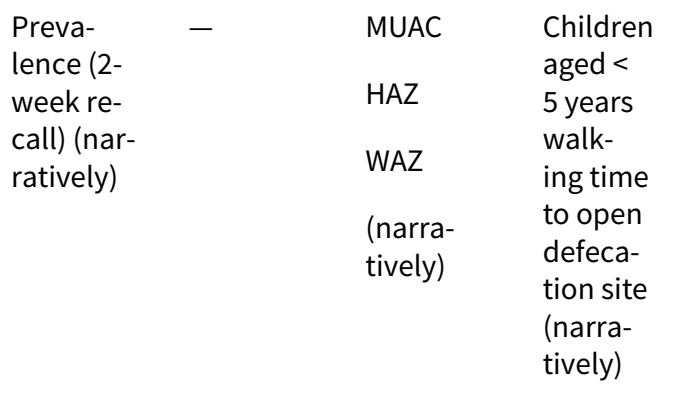

Patil 2014 India Total Sanitation Campaign (subsidies and

IND (rural) promotion of individual household latrines) and Nirmal Vatika (additional subsidies) and support from Water and Sanitation Program through the TSSM project, which included creation of enabling environment + capacity building to implement CLTS-based behaviour change methods.

\begin{tabular}{|c|c|c|c|c|}
\hline $\begin{array}{l}\text { Preva- } \\
\text { lence ( } 7 \text { - } \\
\text { day recall) }\end{array}$ & $\begin{array}{l}\text { Any } \\
\text { helminth } \\
\text { A lumbri- } \\
\text { coides }\end{array}$ & $\begin{array}{l}\text { WAZ } \\
\text { HAZ }\end{array}$ & $\begin{array}{l}\text { No open } \\
\text { defeca- } \\
\text { tion of } \\
\text { children } \\
\text { aged < } 5 \\
\text { years } \\
\text { Safe dis- } \\
\text { posal of } \\
\text { child fae- } \\
\text { ces }\end{array}$ & $\begin{array}{l}\text { Presence } \\
\text { of patho- } \\
\text { genic mi- } \\
\text { crobes in } \\
\text { stool as- } \\
\text { says (nar- } \\
\text { ratively) }\end{array}$ \\
\hline $\begin{array}{l}\text { Preva- } \\
\text { lence (2- } \\
\text { day or 2- } \\
\text { week re- } \\
\text { call) }\end{array}$ & - & $\begin{array}{l}\text { HAZ } \\
\text { WAZ }\end{array}$ & $\begin{array}{l}\text { Potty use } \\
\text { Open } \\
\text { defeca- } \\
\text { tion by } \\
\text { children } \\
\text { aged < } 5 \\
\text { years }\end{array}$ & $\begin{array}{l}\text { Dysentery } \\
\text { Mortali- } \\
\text { ty (narra- } \\
\text { tively) }\end{array}$ \\
\hline
\end{tabular}

\section{Controlled cross-sectional studies}

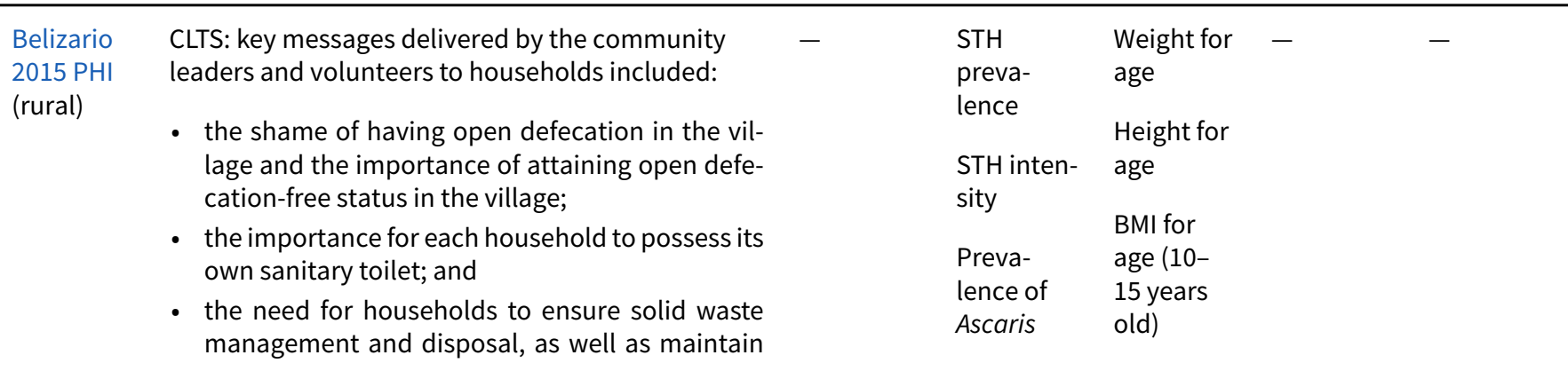


Table 3. Summary of the study designs, settings, and outcome measures of the CLTS interventions plus

adaptations (Consianitery conditions in animal facilities in the backyard (e.g. pig pens).

The criteria for declaring open defecation-free status included the following:

- no signs of open defecation were observed during transect walks and household visits;

- $100 \%$ of households possessed sanitary toilets;

- enactment of local legislation at the village level supporting CLTS activities; and

- implementation of other local government activities that supported the maintenance of ODF status (e.g. village "clean and green" programme).

Messages about child faeces disposal and use of toilets by children were included during the CLTS activities in the villages.
Intensity
of Ascaris
Preva-
lence of
Trichuris
Inten-
sity of
Trichuris
Preva-
lence of
hook-
worm
Intensity
of hook-
worm
(narra-
tively)

(narra-

tively)

BMI: body mass index; CLTS: community-led total sanitation; HAZ: height-for-age Z score; MUAC: mid-upper-arm-circumference; RCT: randomized controlled trial; STH: soil-transmitted helminth; TSSM: Total Sanitation and Sanitation Marketing; WAZ: weight-for-age Z score. a Briceño 2015 TAN had two intervention arms: TSSM and TSSM plus hand washing with soap. In all analyses we use the results for the TSSM arm only.

Table 4. Summary of the study designs, settings, and outcome measures of the sanitation hardware and behaviour change interventions (Continued)

\begin{tabular}{|c|c|c|c|c|c|}
\hline \multirow{2}{*}{$\begin{array}{l}\text { Study } \\
\text { (setting) }\end{array}$} & \multirow[t]{2}{*}{ Summary of intervention } & \multicolumn{4}{|c|}{ Outcomes used in review } \\
\hline & & Diarrhoea & $\begin{array}{l}\text { Anthro- } \\
\text { pometry }\end{array}$ & $\begin{array}{l}\text { Behav- } \\
\text { iour } \\
\text { change }\end{array}$ & Other \\
\hline \multicolumn{6}{|l|}{ RCTs } \\
\hline $\begin{array}{l}\text { Caruso } \\
2019 \text { IND } \\
\text { (rural) }\end{array}$ & $\begin{array}{l}\text { "Sundara Grama", a multilevel behaviour change intervention } \\
\text { that included the following activities. } \\
\text { - Community-level activities: } \\
\text { * a Palla, a folk dance performance common in Odisha, that } \\
\text { communicated messages about latrine use, health, child } \\
\text { faeces disposal, and the importance of overall village clean- } \\
\text { liness; } \\
\text { * a transect walk that went around the village and marked } \\
\text { piles of faeces with coloured powder; } \\
\text { * a community meeting to discuss the village state and create } \\
\text { a plan for its cleanliness; } \\
\text { * the recognition of households whose members all use the } \\
\text { latrine all the time, with a banner hung in front of their } \\
\text { house; } \\
\text { * a village map painting of all households, with special recog- } \\
\text { nition of those using the latrines at all times and a descrip- } \\
\text { tion of the community action plan decided in the meeting. }\end{array}$ & - & - & $\begin{array}{l}\text { Safe child } \\
\text { faeces dis- } \\
\text { posal }\end{array}$ & - \\
\hline
\end{tabular}


Table 4. Summary of the study designs, settings, and outcome measures of the sanitation hardware and behaviour change interventions (Continued)

- Household-level activities:

* a targeted visit for latrine owners, reiterated messages from the other activities and elicited commitment from the household members to use the latrine to keep the village clean and beautiful;

* latrine repairs were carried out to provide minor repairs to those latrines that were not functional and to doors to all latrines that did not have 1 or had 1 that was broken.

- A mother's group meeting for mothers and caregivers of children aged $<5$ years, regardless of their household latrine status to provide action knowledge and hardware to enable the safe disposal of child faeces.

\section{Chris- \\ Pilot of the WASH-B study in Kenya. Study included 2 pilot RCTs,}

tensen

$2015 a$

KEN;

Chris-

tensen

2015b

KEN (rur-

al) 1 testing individual arms (water, sanitation, and hygiene) (Christensen 2015a KEN) and the other the combination of the 3 (WASH), the combination plus nutrition (WASH + ) and nutrition alone (Christensen 2015b KEN). In the analyses, we include results for the sanitation only arm and WASH arm.

The sanitation arm included hardware: compounds received a faeces disposal sani-scooper tool similar to a dustpan with a metal paddle ( 1 for each household in the compound), a plastic child potty ( 1 for each household in the compound with a child aged < 3 years), and improvements to their existing latrine (consisting of a plastic latrine slab with a built-in drop-hole cover if the latrine floor was not concrete and simple mud walls, roof, and door if not present) or construction of a new latrine if they had none. In addition there were monthly household visits for behaviour change communication. The sanitation intervention's primary behaviour change messages emphasized preventing faecal contamination of the environment and safe removal of faeces (human and animal) from the environment facilitated by the potty, sani-scooper, and latrine. It also focused on contamination pathways, behaviours that could lead to exposure, and motivators and barriers of the targeted behaviours.
Luby 2018 WASH Benefits study consisting of 6 intervention arms: water BGD (rur- quality, sanitation, hygiene, combined WASH, nutrition, and al) combine WASH + nutrition. In the review, we used results for the sanitation arm.

Sanitation arm consisted of: provision of free child potties, saniscoop hoes to remove faeces from household environments, and latrine upgrades or construction if did not have 1. Local promoters encouraged mothers to teach their children to use the potties, to safely dispose of faeces in latrines, and to regularly remove animal and human faeces from the compound.

\begin{tabular}{|c|c|c|c|}
\hline $\begin{array}{l}\text { Preva- } \\
\text { lence (7- } \\
\text { day recall) }\end{array}$ & $\begin{array}{l}\text { LAZ } \\
\text { WAZ } \\
\text { Other } \\
\text { anthro- } \\
\text { pometry } \\
\text { measures } \\
\text { (narra- } \\
\text { tively) }\end{array}$ & $\begin{array}{l}\text { From } \\
\text { Parez } \\
\text { 2018: } \\
\text { - potty } \\
\text { use } \\
\text { - safe } \\
\text { dispos- } \\
\text { al of } \\
\text { child } \\
\text { faeces } \\
\text { - faeces } \\
\text { in com- } \\
\text { pound }\end{array}$ & $\begin{array}{l}\text { Mortali- } \\
\text { ty (narra- } \\
\text { tively) }\end{array}$ \\
\hline $\begin{array}{l}\text { Preva- } \\
\text { lence (7- } \\
\text { day recall) }\end{array}$ & $\begin{array}{l}\text { LAZ } \\
\text { WAZ } \\
\text { Other } \\
\text { anthro- } \\
\text { pometry }\end{array}$ & $\begin{array}{l}\text { Safe dis- } \\
\text { posal of } \\
\text { child fae- } \\
\text { ces }\end{array}$ & $\begin{array}{l}\text { Mortali- } \\
\text { ty (narra- } \\
\text { tively) }\end{array}$ \\
\hline
\end{tabular}

Safe child -
faeces dis-
posal
Faeces
observed
in the
com-
pound


Table 4. Summary of the study designs, settings, and outcome measures of the sanitation hardware and behaviour change interventions (Continued)

trine upgrades or construction if did not have 1. Local promoters visited study compounds to deliver behaviour change messages on the use of latrines for defecation and the removal of human and animal faeces from the compound. measures

(narra-

tively)

RCT: randomized controlled trial; WASH-B: Water, Sanitation, and Hygiene - Benefits.

Table 5. Summary of the study designs, settings, and outcome measures of the WASH hardware and education/ behaviour change interventions (Continued)

\begin{tabular}{|c|c|c|c|c|c|c|}
\hline \multirow{2}{*}{$\begin{array}{l}\text { Study } \\
\text { (setting) }\end{array}$} & \multirow[t]{2}{*}{ Summary of intervention } & \multicolumn{5}{|c|}{ Outcomes used in review } \\
\hline & & Diarrhoea & STH & $\begin{array}{l}\text { Anthro- } \\
\text { pometry }\end{array}$ & $\begin{array}{l}\text { Behav- } \\
\text { iour } \\
\text { change }\end{array}$ & Other \\
\hline \multicolumn{7}{|l|}{ RCTs } \\
\hline $\begin{array}{l}\text { Humphrey } \\
2019 \text { ZIM } \\
\text { (rural) }\end{array}$ & $\begin{array}{l}3 \text { intervention arms. Only included the WASH vs } \\
\text { non-WASH results in review. } \\
\text { - WASH: standard of care messages plus informa- } \\
\text { tion about safe disposal of faeces in a latrine, } \\
\text { handwashing with soap at key times, protection } \\
\text { of infants from geophagia and ingestion of an- } \\
\text { imal faeces, chlorination of drinking water (es- } \\
\text { pecially for infants), and hygienic preparation } \\
\text { of complementary food. Provision of house- } \\
\text { hold ventilated improved pit latrines, chlorine } \\
\text { for water treatment, } 2 \text { handwashing facilities, } \\
\text { soap, and a plastic mat and play space for in- } \\
\text { fants. } \\
\text { - IYCF } \\
\text { - WASH and IYCF combined }\end{array}$ & $\begin{array}{l}\text { Mean } \\
\text { preva- } \\
\text { lence of } \\
\text { diarrhoea } \\
\text { (7-day re- } \\
\text { call) }\end{array}$ & - & $\begin{array}{l}\text { Mean LAZ } \\
\text { score } \\
\text { Mean WAZ } \\
\text { scores } \\
\text { Weight-for- } \\
\text { length Z } \\
\text { scores } \\
\text { MUAC- } \\
\text { for-age Z } \\
\text { scores } \\
\text { Head cir- } \\
\text { cumfer- } \\
\text { ence-for- } \\
\text { age Z } \\
\text { scores } \\
\text { Stunting } \\
\text { (LAZ score } \\
<-2 \text { ) } \\
\text { Severely } \\
\text { stunted } \\
\text { (LAZ score } \\
<-3 \text { ) } \\
\text { Under- } \\
\text { weight } \\
\text { (WAZ } \\
\text { scores <-2) } \\
\text { Wasted } \\
\text { (weight- } \\
\text { for-height Z } \\
\text { scores <-2) } \\
\text { (narrative- } \\
\text { ly) }\end{array}$ & $\begin{array}{l}\text { Dispose } \\
\text { of wa- } \\
\text { ter from } \\
\text { clean- } \\
\text { ing infant } \\
\text { nappies } \\
\text { with fae- } \\
\text { ces in a la- } \\
\text { trine (nar- } \\
\text { ratively) }\end{array}$ & $\begin{array}{l}\text { Cumula- } \\
\text { tive mor- } \\
\text { tality (nar- } \\
\text { ratively) }\end{array}$ \\
\hline
\end{tabular}


Table 5. Summary of the study designs, settings, and outcome measures of the WASH hardware and education/ behaviour change interventions (Continued)

\section{CBA studies}

\begin{abstract}
Alam 1989 Hand pumps were provided with a ratio of 4-6 BGD (rur- households ( 3 times more than control) + health al) tent and exclusive use of hand pump water, improvement of water handling and storage practices, disposal of child's faeces soon after defecation, washing hands before handling food, and rubbing hands in ash or using soap after defecation).
\end{abstract}

Diarrhoea
incidence

(7-day re-

call)

Aziz 1990
BGD (rur-
$\begin{array}{ll}\text { al) } & \text { erage) + free maintenance, } 92 \% \text { of households re- } \\ \text { ceived a double-pit water-sealed latrine, hygiene } \\ \text { education emphasising exclusive use of the pump }\end{array}$

water for all personal and domestic use, and the
need for all members of the household, including
young children to use the latrines.

\begin{tabular}{|c|c|c|c|}
\hline $\begin{array}{l}\text { Diarrhoea } \\
\text { incidence } \\
\text { (7-day re- } \\
\text { call) }\end{array}$ & - & $\begin{array}{l}\text { Anthro- } \\
\text { pometry } \\
\text { (weight } \\
\text { for age, } \\
\text { height for } \\
\text { age, weight } \\
\text { for height) } \\
\text { (narrative- } \\
\text { ly) }\end{array}$ & $\begin{array}{l}\text { Dysen- } \\
\text { tery, per- } \\
\text { sistent di- } \\
\text { arrhoea } \\
\text { (narra- } \\
\text { tively) }\end{array}$ \\
\hline
\end{tabular}

$\begin{array}{lll}\text { Park 2016 } & \begin{array}{l}\text { Budi's Amphibious Latrine (BALatrines) (sim- } \\ \text { INA (rural) } \\ \text { ple squat latrines with a septic tank or pit) were } \\ \text { constructed and all residents were given health } \\ \text { education regarding hygiene, sanitation, and } \\ \text { prevention of STH infections. The health educa- } \\ \text { tion included many messages about preventing } \\ \text { soil-transmitted helminthiases. For mothers of } \\ \text { small children, the messages included not dis- } \\ \text { posing of used nappies in the garden, bushes, } \\ \begin{array}{l}\text { or waterways. Children were also told that they } \\ \text { should stay away from any faeces they might find } \\ \text { around their home, and that they should report } \\ \text { any symptoms (diarrhoea, fever, etc.) to a parent } \\ \text { or teacher. }\end{array}\end{array} \quad \begin{array}{l}\text { STH infec- } \\ \text { tion (pres- } \\ \text { ence of } \\ \text { helminth } \\ \text { eggs in } \\ \text { stool (nar- } \\ \text { ratively) }\end{array} \\ \end{array}$

\section{Controlled cohort studies}

Hoq 2016 Community-based health project + WASH-focused BGD (peri- activities.

urban)
WASH-focused activities included:

- construction of 119 community-managed, deep-tube wells and 1280 household pour-flush twin pit latrines;

- monthly neighbourhood WASH committee meetings;

- toilet maintenance promotion;

- monthly children's club meetings for hygiene: included messages about child faeces disposal;

- mother's group meetings: 20 mothers in a session, monthly basis. Discussion point-hygienic latrine, use, operation and maintenance of latrine, safe drinking water, child faeces disposal, hand washing, etc.

Weight for
age (un-
derweight
defined as
WAZ $<-2$ )
MUAC
(acute mal-
nutrition
defined as
MUAC $<125$
mm)

(narrative-

ly) 
Table 5. Summary of the study designs, settings, and outcome measures of the WASH hardware and education/ behaviour change interventions (Continued)

Child faeces disposal messages (included in both intervention and control but this was done in more detail in the children's clubs and mother's group meetings):

- throw the child faeces in the latrine immediately after defecation;

- use handy tool (shovel, etc.) to collect and dispose the faeces. Keep the tool clean;

- encourage the children and start practicing defecation in the latrine instead of defecating in yard;

- "child faeces are more harmful than the adult" as the mothers believe that children faeces are less harmful;

- wash hands after dispose of child faeces.

CBA: controlled before-and-after; IYCF: infant and young child feeding; LAZ: length-for-age Z score; MUAC: mid-upper-arm-circumference; RCT: randomized controlled trial; STH: soil-transmitted helminth; WASH: water, sanitation, and hygiene; WAZ: weight-for-age Z score.

Table 6. Summary of the study designs, settings, and outcome measures of the daycare centre-based hygiene hardware and education interventions (Continued)

\begin{tabular}{|c|c|c|c|c|c|c|}
\hline \multirow{2}{*}{$\begin{array}{l}\text { Study } \\
\text { (setting) }\end{array}$} & \multirow[t]{2}{*}{ Summary of intervention } & \multicolumn{5}{|c|}{ Outcomes used in review } \\
\hline & & Diarrhoea & STH & $\begin{array}{l}\text { Anthro- } \\
\text { pometry }\end{array}$ & $\begin{array}{l}\text { Behav- } \\
\text { iour } \\
\text { change }\end{array}$ & Other \\
\hline $\begin{array}{l}\text { Butz } 1990 \\
\text { USA (Ur- } \\
\text { ban (day- } \\
\text { care cen- } \\
\text { tres)) }\end{array}$ & $\begin{array}{l}\text { Instruction to daycare providers on modes of trans- } \\
\text { mission of pathogens, instructions of handwash- } \\
\text { ing, and use of vinyl gloves and disposable nappy } \\
\text { changing pads at each nappy change. Providers } \\
\text { were instructed to dispose of gloves, disposable } \\
\text { pads, and nappies in plastic bags and given sup- } \\
\text { plies (gloves, nappy changing pads, hand rinse so- } \\
\text { lution). }\end{array}$ & $\begin{array}{l}\text { Preva- } \\
\text { lence } \\
\text { (narra- } \\
\text { tively). } \\
\text { Symp- } \\
\text { toms } \\
\text { recorded } \\
\text { daily }\end{array}$ & - & - & - & - \\
\hline $\begin{array}{l}\text { Kotch } \\
2007 \text { USA } \\
\text { (Rural } \\
\text { and urban } \\
\text { (daycare } \\
\text { centres)) }\end{array}$ & $\begin{array}{l}\text { Staff in childcare centres were trained using the } \\
\text { 'Keep It Clean' training module to improve and } \\
\text { standardize the hand-washing, sanitation, nappy } \\
\text { changing, and food-preparation procedures. Nap- } \\
\text { py changing, hand-washing, and food-prepara- } \\
\text { tion equipment with impermeable, seamless sur- } \\
\text { facing for food preparation, nappy changing, and } \\
\text { hand washing were provided. In addition, automat- } \\
\text { ic faucets and foot-activated, roll-out waste bins for } \\
\text { nappy disposal were provided. }\end{array}$ & $\begin{array}{l}\text { Diarrhoea } \\
\text { incidence } \\
\text { (2-week } \\
\text { recall) } \\
\text { (narra- } \\
\text { tively) }\end{array}$ & - & - & - & - \\
\hline
\end{tabular}

STH: soil-transmitted helminth. 


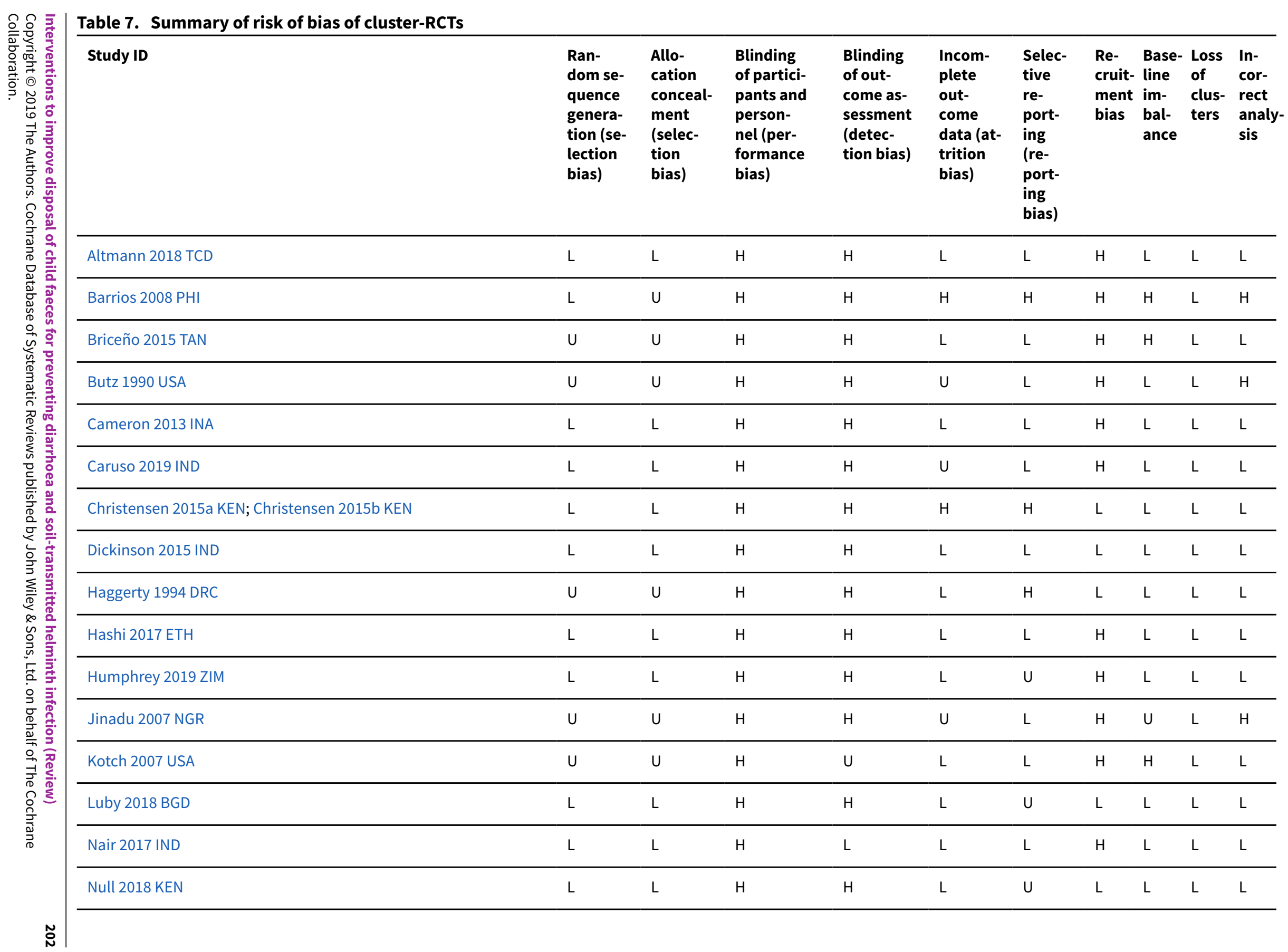




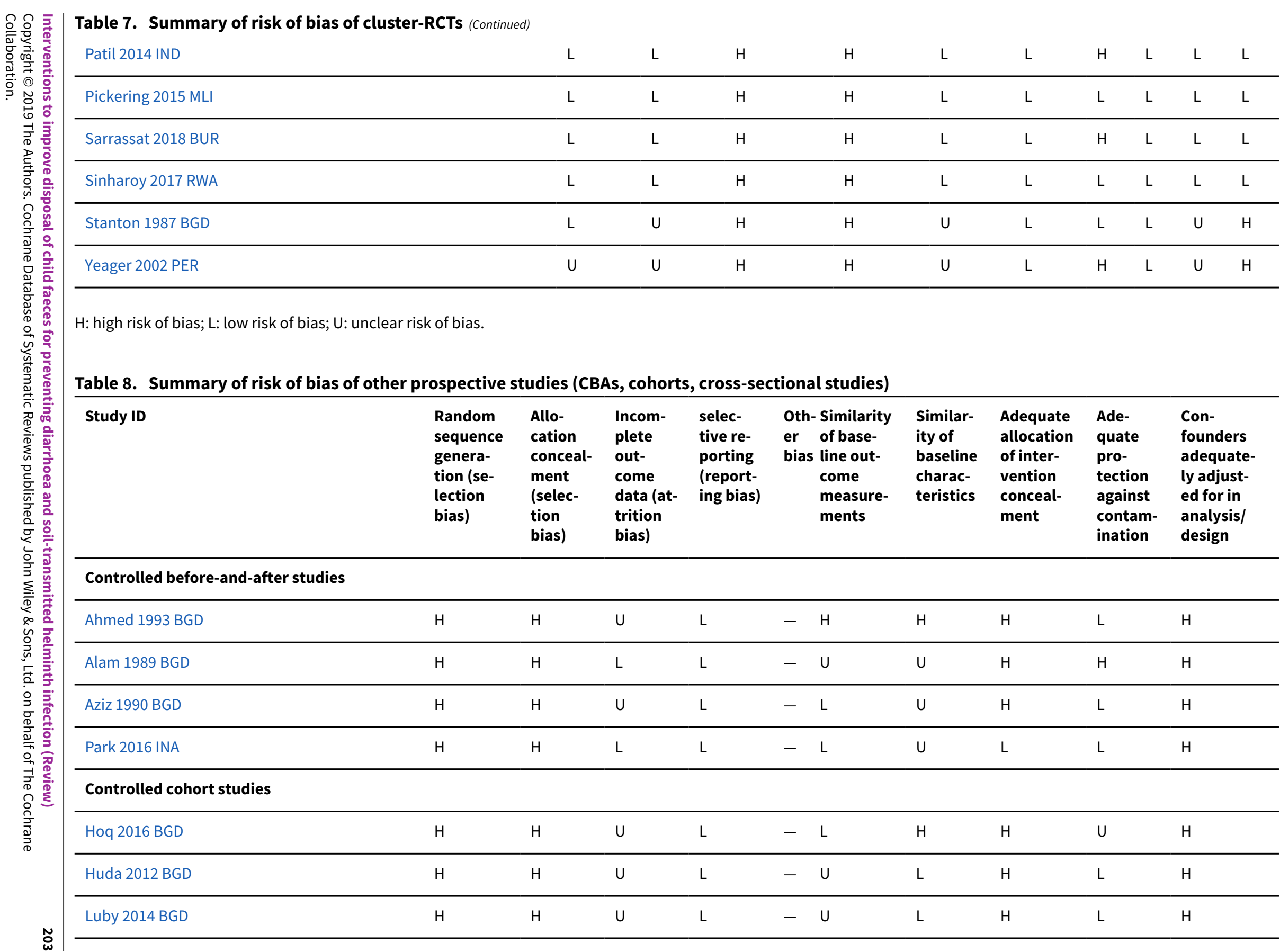




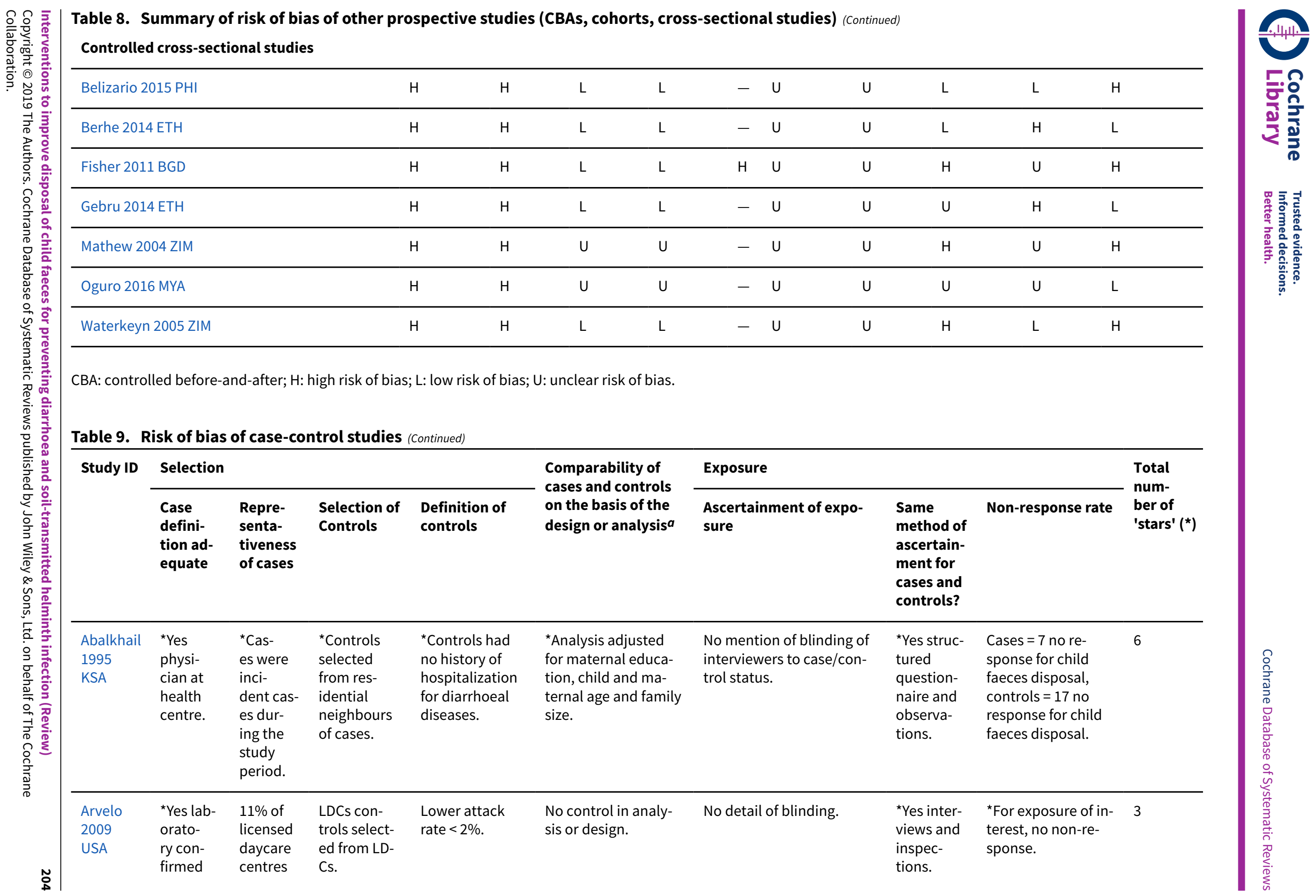




\begin{tabular}{|c|c|c|c|c|c|c|c|c|c|}
\hline 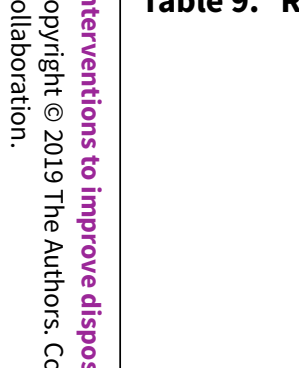 & $\begin{array}{l}\text { then cal- } \\
\text { culated } \\
\text { attack } \\
\text { rate. }\end{array}$ & $\begin{array}{l}\text { (LDCs) } \\
\text { did not } \\
\text { partici- } \\
\text { pate in } \\
\text { investi- } \\
\text { gation - } \\
\text { no rea- } \\
\text { son de- } \\
\text { scribed. }\end{array}$ & & & & & & & \\
\hline 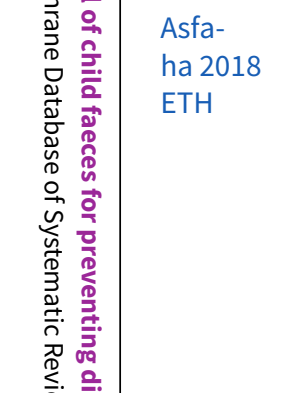 & $\begin{array}{l}\text { Self-re- } \\
\text { ports }\end{array}$ & $\begin{array}{l}{ }^{\star} \text { Ran- } \\
\text { dom } \\
\text { sam- } \\
\text { ple of } \\
200 \text { cas- } \\
\text { es from } \\
250 .\end{array}$ & $\begin{array}{l}{ }^{*} \text { Communi- } \\
\text { ty controls, } \\
\text { random- } \\
\text { ly selected } \\
\text { from poten- } \\
\text { tial controls. }\end{array}$ & $\begin{array}{l}{ }^{*} \text { Without diar- } \\
\text { rhoea in the } \\
\text { preceding of } 2 \\
\text { weeks. }\end{array}$ & $\begin{array}{l}\text { No control for con- } \\
\text { founders in analysis } \\
\text { of risk factor of inter- } \\
\text { est (child faeces dis- } \\
\text { posal). }\end{array}$ & $\begin{array}{l}\text { *"Both data collectors and } \\
\text { supervisors did not partic- } \\
\text { ipate in the survey; were } \\
\text { unaware of diarrhoeal dis- } \\
\text { ease status of the study } \\
\text { groups and were provid- } \\
\text { ed only the identification } \\
\text { numbers of households } \\
\text { and name and age of the } \\
\text { child." }\end{array}$ & ${ }^{*}$ Yes & $\begin{array}{l}\text { *Same for both } \\
\text { groups. }\end{array}$ & 6 \\
\hline $\begin{array}{l}\text { Baker } \\
2016 \\
\text { BGD; } \\
\text { Baker } \\
2016 \\
\text { GMB; } \\
\text { Baker } \\
2016 \\
\text { IND; } \\
\text { Baker } \\
2016 \\
\text { KEN; } \\
\text { Baker } \\
2016 \\
\text { MLI; Bak- } \\
\text { er 2016 } \\
\text { MOZ; } \\
\text { Baker } \\
\text { 2016 PAK }\end{array}$ & $\begin{array}{l}{ }^{\star} \text { Yes, } \\
\text { GEMS } \\
\text { clinician. }\end{array}$ & $\begin{array}{l}\text { "Each } \\
\text { site re- } \\
\text { stricted } \\
\text { enrol- } \\
\text { ment to } \\
\text { about } \\
\text { the first } \\
\text { nine el- } \\
\text { igible } \\
\text { cases } \\
\text { per age } \\
\text { stratum } \\
\text { per fort- } \\
\text { night to } \\
\text { maintain } \\
\text { a man- } \\
\text { ageable } \\
\text { work } \\
\text { flow } \\
\text { through- } \\
\text { out the } \\
\text { study." }\end{array}$ & $\begin{array}{l}{ }^{*} \text { Communi- } \\
\text { ty controls }\end{array}$ & $\begin{array}{l}\text { *"No diarrhoea } \\
\text { in the previous } \\
7 \text { days." }\end{array}$ & $\begin{array}{l}{ }^{*} \text { Matched for age and } \\
\text { adjusted for wealth. }\end{array}$ & $\begin{array}{l}\text { "Case enrolment inter- } \\
\text { views took place at the } \\
\text { SHC whereas control care- } \\
\text { takers were interviewed at } \\
\text { home," so assume knew } \\
\text { status }\end{array}$ & $\begin{array}{l}\text { No, ini- } \\
\text { tial inter- } \\
\text { view was } \\
\text { in health } \\
\text { centre for } \\
\text { cases and } \\
\text { in home } \\
\text { for con- } \\
\text { trols, al- } \\
\text { though at } \\
60 \text { days, } \\
\text { also did } \\
\text { a house- } \\
\text { hold visit } \\
\text { to both }\end{array}$ & $\begin{array}{l}\text { Unclear what the } \\
\text { non-response rate } \\
\text { for child faeces dis- } \\
\text { posal was }\end{array}$ & 4 \\
\hline
\end{tabular}




\begin{tabular}{|c|c|c|c|c|c|c|c|c|c|c|}
\hline 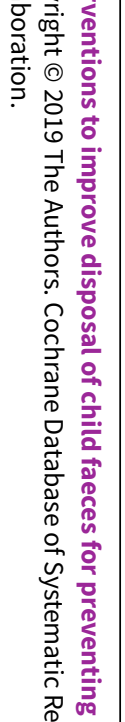 & $\begin{array}{l}\text { Baltazar } \\
1989 \mathrm{PHI}\end{array}$ & $\begin{array}{l}{ }^{\star} \text { Cas- } \\
\text { es were } \\
\text { brought } \\
\text { to the } \\
\text { clinic. }\end{array}$ & $\begin{array}{l}\text { *"All } \\
\text { cases } \\
\text { seen at } \\
\text { the clin- } \\
\text { ics on a } \\
\text { "morbid- } \\
\text { ity day" } \\
\text { during } \\
\text { the re- } \\
\text { cruit- } \\
\text { ment pe- } \\
\text { riod that } \\
\text { satisfied } \\
\text { this de- } \\
\text { finition } \\
\text { were in- } \\
\text { cluded } \\
\text { in the } \\
\text { study. }\end{array}$ & $\begin{array}{l}\text { "Chil- } \\
\text { dren aged } \\
<2 \text { years } \\
\text { who were } \\
\text { brought to } \\
\text { the clinic } \\
\text { because of } \\
\text { an acute } \\
\text { respiratory } \\
\text { infection." }\end{array}$ & $\begin{array}{l}{ }^{*} \text { Had not had } \\
\text { diarrhoea } \\
\text { during the pre- } \\
\text { vious } 24 \text { hours. }\end{array}$ & $\begin{array}{l}{ }^{*} \text { Adjusted for toi- } \\
\text { let facilities) and } \\
\text { *water supply, sex, } \\
\text { education of head } \\
\text { of household and } \\
\text { mother, feeding } \\
\text { practices, level of } \\
\text { health service uti- } \\
\text { lization, number of } \\
\text { children under } 5 \text { in } \\
\text { household. }\end{array}$ & $\begin{array}{l}\text { Did not specify if they } \\
\text { were blinded to status of } \\
\text { case/control }\end{array}$ & $\begin{array}{l}\text { *Yes, } \\
\text { structured } \\
\text { question- } \\
\text { naire. }\end{array}$ & $\begin{array}{l}{ }^{\star} \text { No missing values } \\
\text { for child faeces dis- } \\
\text { posal. }\end{array}$ & 7 \\
\hline 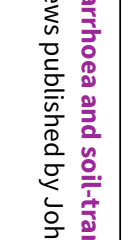 & $\begin{array}{l}\text { Bassal } \\
2016 \text { ISR }\end{array}$ & $\begin{array}{l}{ }^{*} \text { Stool } \\
\text { samples }\end{array}$ & $\begin{array}{l}{ }^{\star} \text { Ran- } \\
\text { domly } \\
\text { selected } \\
\text { from all } \\
\text { eligible } \\
\text { cases. }\end{array}$ & $\begin{array}{l}{ }^{*} \text { Communi- } \\
\text { ty controls, } \\
\text { identified } \\
\text { from Israeli } \\
\text { population } \\
\text { register. }\end{array}$ & $\begin{array}{l}{ }^{*} \text { No diarrhoea } \\
\text { in past } 2 \text { weeks. }\end{array}$ & $\begin{array}{l}{ }^{\star} \text { Controls for age and } \\
\text { number of children } \\
\text { in household. }\end{array}$ & $\begin{array}{l}\text { Structured interview, no } \\
\text { mention of blinding. }\end{array}$ & $\begin{array}{l}\text { *Yes, } \\
\text { structured } \\
\text { interview. }\end{array}$ & $\begin{array}{l}\text { *Same for both } \\
\text { groups (response } \\
\text { rate was } 86.3 \% \text { for } \\
\text { cases and } 85 \% \text { for } \\
\text { controls). }\end{array}$ & 7 \\
\hline 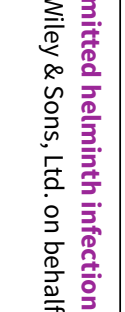 & $\begin{array}{l}\text { Chiang } \\
2005 \\
\text { TWN }\end{array}$ & $\begin{array}{l}{ }^{\star} \text { Cas- } \\
\text { es were } \\
\text { con- } \\
\text { firmed } \\
\text { by lab- } \\
\text { oratory } \\
\text { test. }\end{array}$ & $\begin{array}{l}\text { No de- } \\
\text { tails on } \\
\text { how the } \\
\text { cases } \\
\text { were se- } \\
\text { lected. }\end{array}$ & $\begin{array}{l}\text { Hospi- } \\
\text { tal/clinic } \\
\text { control }\end{array}$ & $\begin{array}{l}{ }^{\star} \text { Children who } \\
\text { went to the } \\
\text { clinics for vac- } \\
\text { cination and } \\
\text { showing no } \\
\text { symptoms of } \\
\text { diarrhoea or } \\
\text { fever. }\end{array}$ & *Matched for age. & $\begin{array}{l}\text { Did not specify if they } \\
\text { were blinded to status of } \\
\text { case/control. }\end{array}$ & $\begin{array}{l}{ }^{\star} \text { Yes, se- } \\
\text { mi-struc- } \\
\text { tured } \\
\text { question- } \\
\text { naire. }\end{array}$ & $\begin{array}{l}\text { No details on miss- } \\
\text { ing values for child } \\
\text { defecation variable }\end{array}$ & 4 \\
\hline 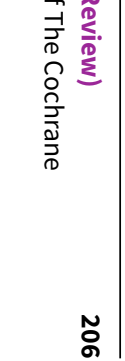 & $\begin{array}{l}\text { Chom- } \\
\text { pook } \\
2006 \\
\text { THA }{ }^{1}\end{array}$ & $\begin{array}{l}\text { *Yes lab- } \\
\text { oratory } \\
\text { diagno- } \\
\text { sis. }\end{array}$ & $\begin{array}{l}\text { "All } \\
\text { shigel- } \\
\text { losis cas- } \\
\text { es ascer- } \\
\text { tained } \\
\text { from the } \\
\text { popula- } \\
\text { tion-based } \\
\text { surveil- }\end{array}$ & $\begin{array}{l}{ }^{*} \text { Communi- } \\
\text { ty control. } \\
\text { "For each } \\
\text { case en- } \\
\text { rolled, two } \\
\text { matched } \\
\text { controls } \\
\text { were ran- } \\
\text { domly se- }\end{array}$ & $\begin{array}{l}\text { *"Individuals } \\
\text { free from diar- } \\
\text { rhoea or dysen- } \\
\text { tery during the } \\
\text { four weeks pri- } \\
\text { or to recruit- } \\
\text { ment were el- } \\
\text { igible to par- } \\
\text { ticipate in the }\end{array}$ & $\begin{array}{l}\text { *Study controls for } \\
\text { age in design. }\end{array}$ & $\begin{array}{l}\text { "Un-blinded status of the } \\
\text { investigator visiting the } \\
\text { households and conduct- } \\
\text { ing interviews to the case/ } \\
\text { control status of the par- } \\
\text { ticipant." }\end{array}$ & $\begin{array}{l}\text { *Yes, ques- } \\
\text { tionnaire } \\
\text { and obser- } \\
\text { vations. }\end{array}$ & $\begin{array}{l}{ }^{*} \text { No missing values } \\
\text { for child defecation } \\
\text { variable. }\end{array}$ & 6 \\
\hline
\end{tabular}




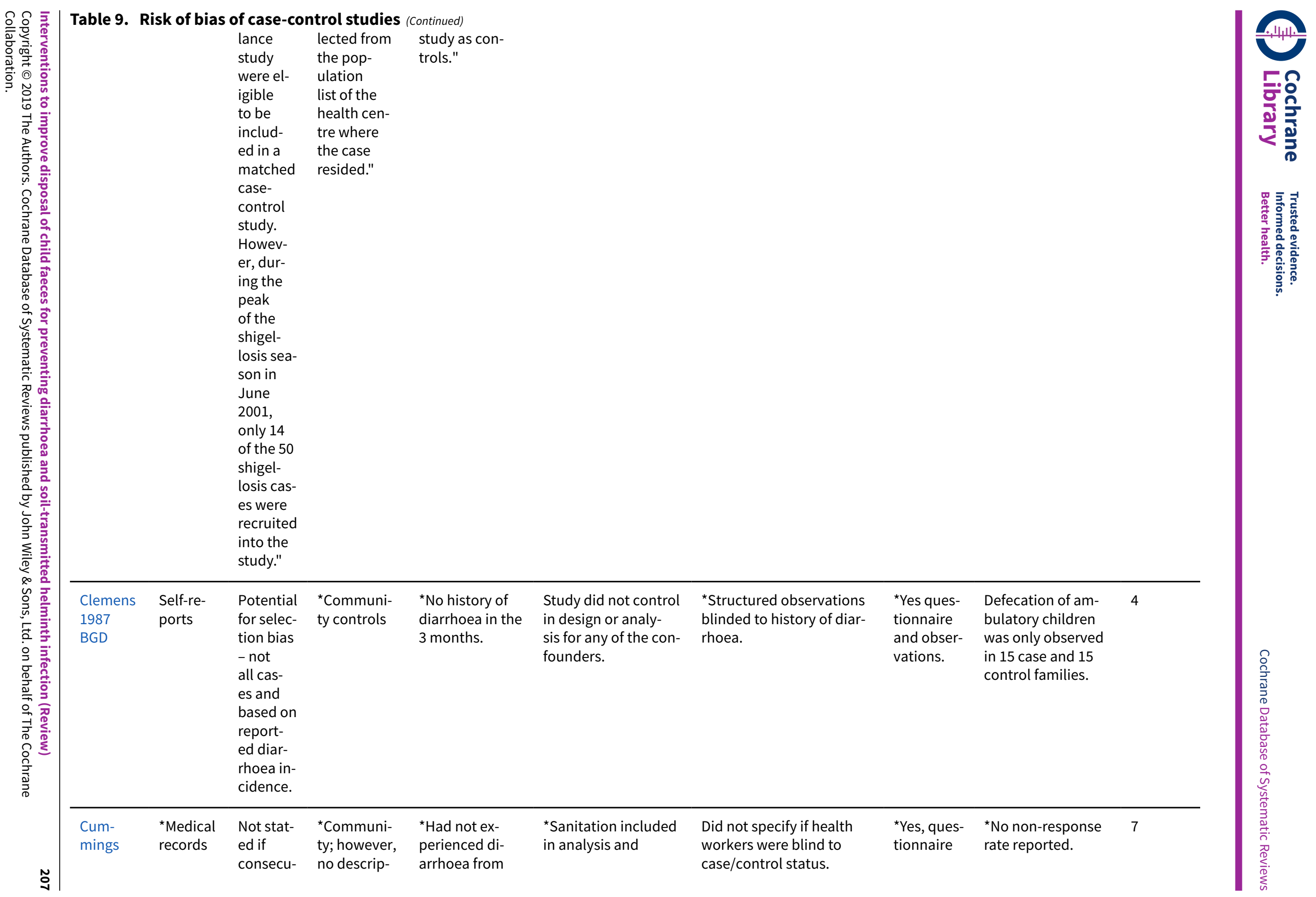




\begin{tabular}{|c|c|c|c|c|c|c|c|c|c|c|c|}
\hline 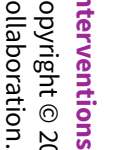 & $\begin{array}{l}2012 \\
\text { UGA }\end{array}$ & & $\begin{array}{l}\text { tive/rep- } \\
\text { resenta- } \\
\text { tive }\end{array}$ & $\begin{array}{l}\text { tion on how } \\
\text { they select- } \\
\text { ed them. }\end{array}$ & $\begin{array}{l}\text { April to time of } \\
\text { investigation. }\end{array}$ & $\begin{array}{l}{ }^{\star} \text { Controls for age, } \\
\text { gender, and water } \\
\text { treatment practices. }\end{array}$ & & & & & 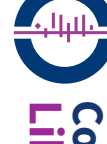 \\
\hline 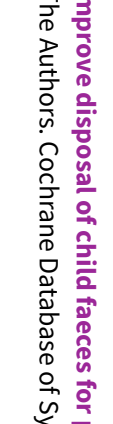 & $\begin{array}{l}\text { Daniels } \\
1990 \text { LES }\end{array}$ & $\begin{array}{l}{ }^{\star} \text { Hospi- } \\
\text { tal nurse }\end{array}$ & $\begin{array}{l}{ }^{\star} \text { Con- } \\
\text { secutive } \\
\text { cases re- } \\
\text { cruited. }\end{array}$ & $\begin{array}{l}\text { Hospital } \\
\text { controls } \\
\text { with ARI/ } \\
\text { trauma. }\end{array}$ & $\begin{array}{l}{ }^{*} \text { No diarrhoea } \\
\text { at recruitment }\end{array}$ & $\begin{array}{l}\text { *Sanitation is main } \\
\text { exposure, } \\
\text { *Age, education of } \\
\text { mother }\end{array}$ & $\begin{array}{l}\text { Interview not blinded as } \\
\text { nurse did interview at hos- } \\
\text { pital }\end{array}$ & $\begin{array}{l}\text { *Yes, ques- } \\
\text { tionnaire } \\
\text { and for } \\
\text { subsam- } \\
\text { ple second } \\
\text { interview } \\
\text { at home } \\
\text { with ob- } \\
\text { servations } \\
\text { of facili- } \\
\text { ties. }\end{array}$ & $\begin{array}{l}\text { No information on } \\
\text { non-response. }\end{array}$ & 6 & 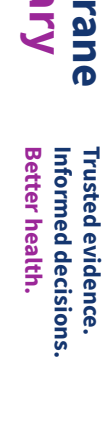 \\
\hline 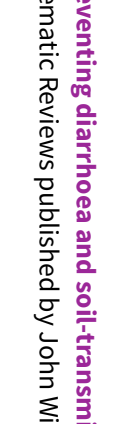 & $\begin{array}{l}\text { Dikas- } \\
\text { sa } 1993 \\
\text { DRC }\end{array}$ & $\begin{array}{l}\text { *Hospi- } \\
\text { tal }\end{array}$ & $\begin{array}{l}\text { Incident } \\
\text { case } \\
\text { identi- } \\
\text { fied at } \\
\text { hospital } \\
\text { - no de- } \\
\text { tails on } \\
\text { whether } \\
\text { it was } \\
\text { consecu- } \\
\text { tive. }\end{array}$ & $\begin{array}{l}{ }^{*} \text { Communi- } \\
\text { ty; howev- } \\
\text { er, not de- } \\
\text { scribed how } \\
\text { they select- } \\
\text { ed them. }\end{array}$ & $\begin{array}{l}{ }^{\star} \text { No history of } \\
\text { hospitalization } \\
\text { for diarrhoea }\end{array}$ & $\begin{array}{l}\text { No statistical dif- } \\
\text { ference in sanita- } \\
\text { tion but it was not } \\
\text { matched for in de- } \\
\text { sign. } \\
\text { *Matches for educa- } \\
\text { tion in analysis and } \\
\text { age of child in de- } \\
\text { sign. }\end{array}$ & $\begin{array}{l}\text { Not specified that inter- } \\
\text { viewers were blinded to } \\
\text { case/control status. }\end{array}$ & $\begin{array}{l}\text { *Yes, } \\
\text { structured } \\
\text { interview } \\
\text { and obser- } \\
\text { vations. }\end{array}$ & $\begin{array}{l}\text { Numbers not de- } \\
\text { scribed - no infor- } \\
\text { mation about non- } \\
\text { respondents }\end{array}$ & 5 & \\
\hline 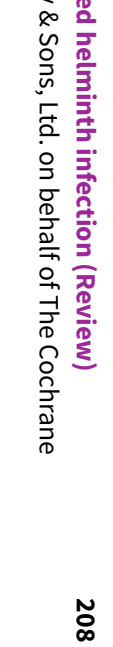 & $\begin{array}{l}\text { Genthe } \\
1997 \text { SAF }\end{array}$ & $\begin{array}{l}{ }^{\star} \text { Yes hos- } \\
\text { pital } \\
\text { staff. }\end{array}$ & $\begin{array}{l}\text { No detail } \\
\text { on how } \\
\text { "a sam- } \\
\text { ple was } \\
\text { drawn } \\
\text { from } \\
\text { pre- } \\
\text { school } \\
\text { children } \\
\text { who } \\
\text { were } \\
\text { brought } \\
\text { to the } \\
\text { day hos- } \\
\text { pitals } \\
\text { with }\end{array}$ & $\begin{array}{l}{ }^{*} \text { Communi- } \\
\text { ty controls }\end{array}$ & $\begin{array}{l}\text { *"Children who } \\
\text { suffered from } \\
\text { diarrhoea dur- } \\
\text { ing the preced- } \\
\text { ing } 14 \text { days at } \\
\text { the time of the } \\
\text { visit were ex- } \\
\text { cluded as con- } \\
\text { trols." }\end{array}$ & $\begin{array}{l}\text { *Study controlled for } \\
\text { age in design. }\end{array}$ & $\begin{array}{l}\text { "It was not possible to } \\
\text { blind the interviewer to } \\
\text { the disease status of the } \\
\text { child under study." }\end{array}$ & $\begin{array}{l}\text { *Yes, inter- } \\
\text { views and } \\
\text { spot check } \\
\text { observa- } \\
\text { tions. }\end{array}$ & $\begin{array}{l}\text { Non-respondents } \\
\text { not described. }\end{array}$ & 5 & 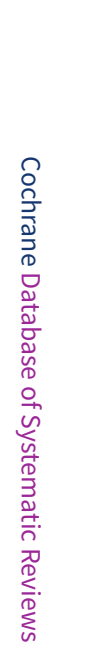 \\
\hline
\end{tabular}




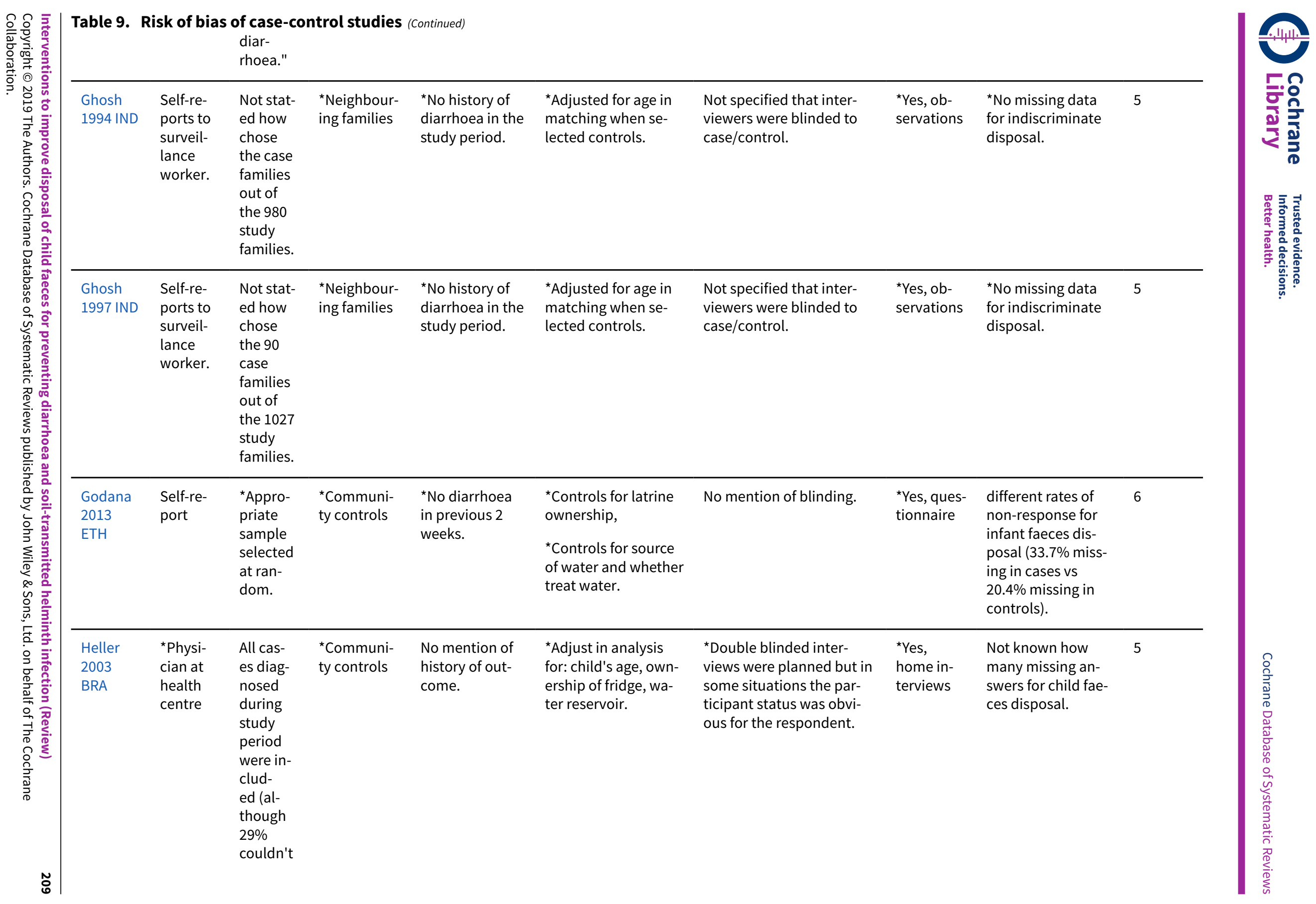




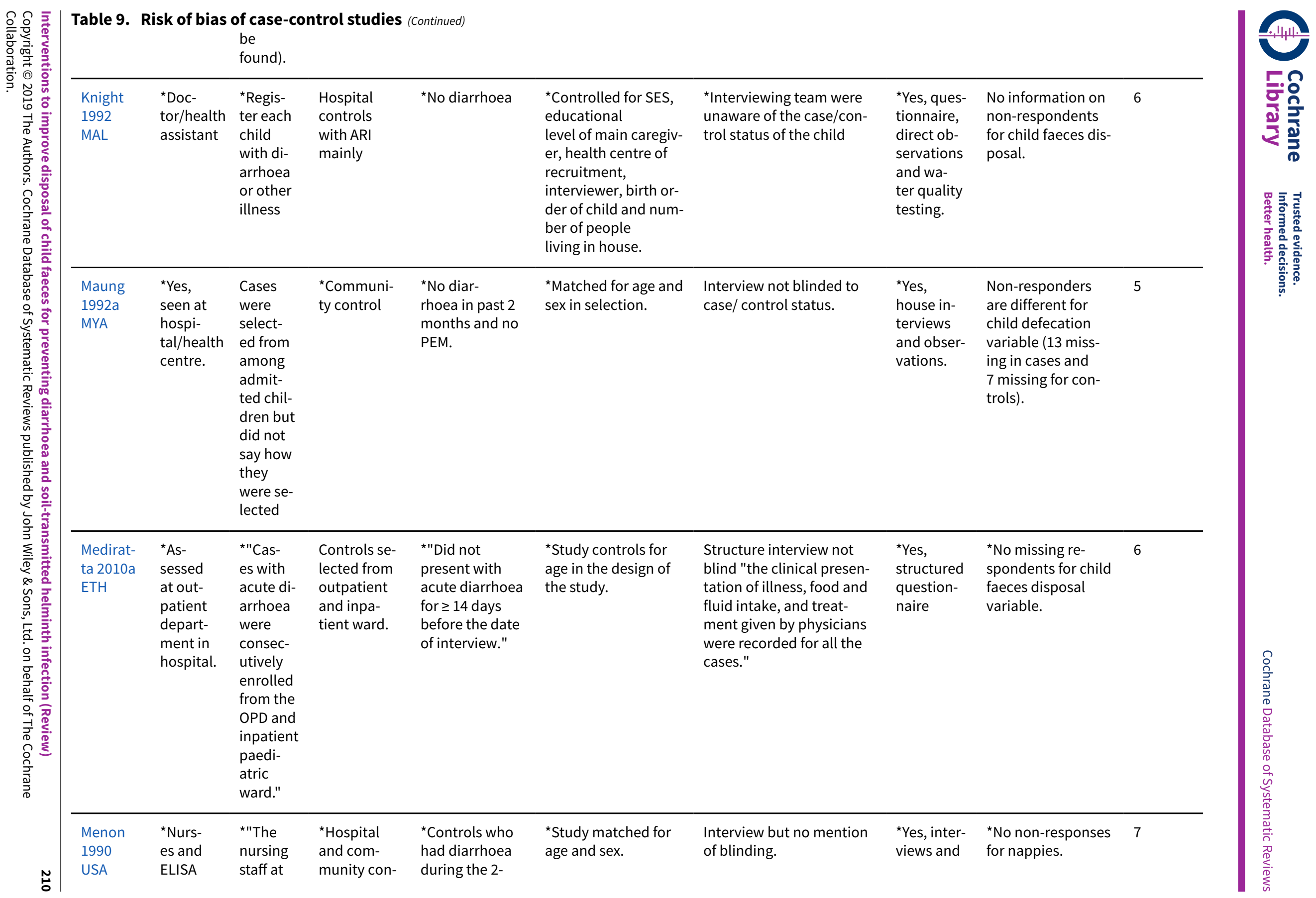




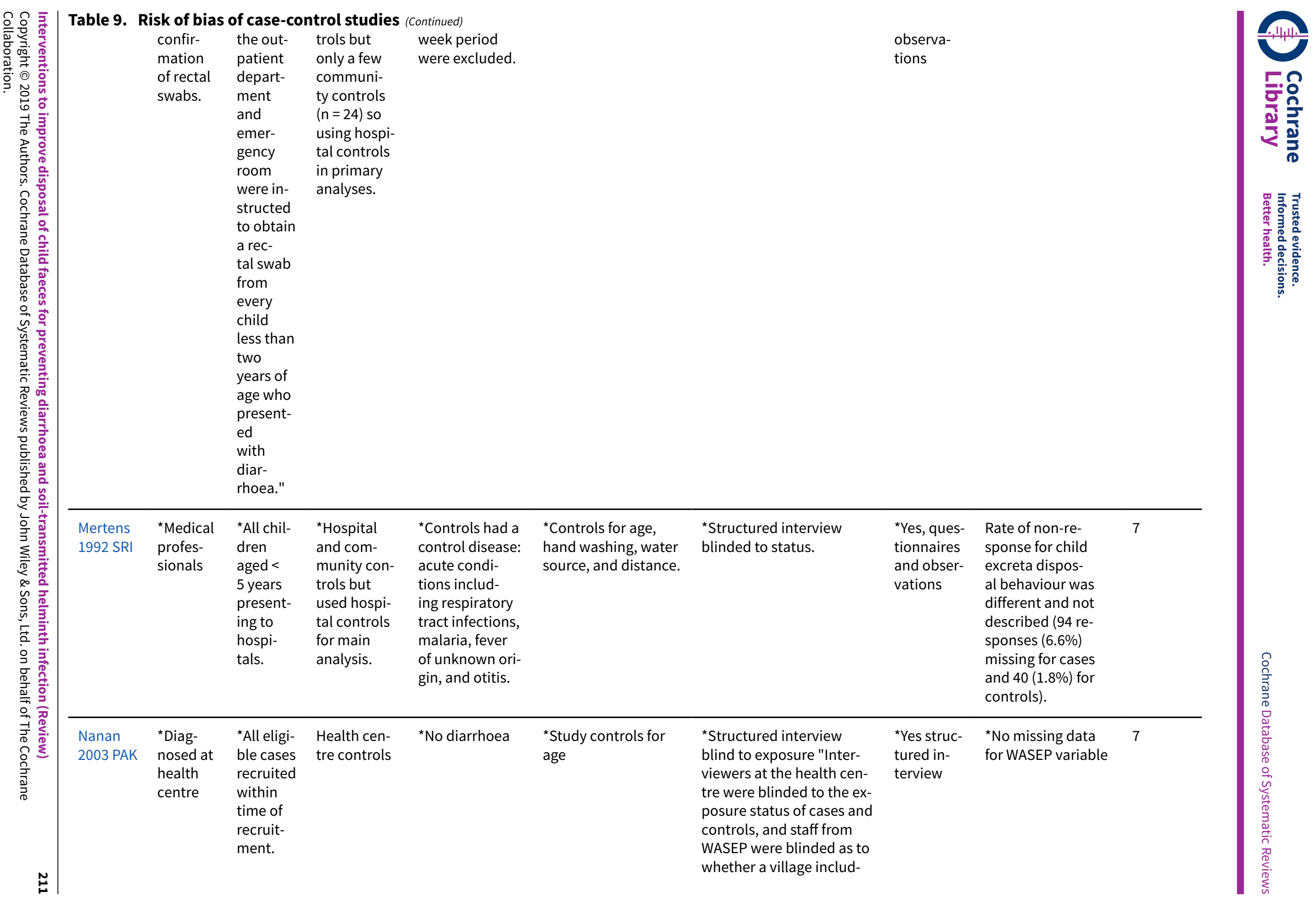




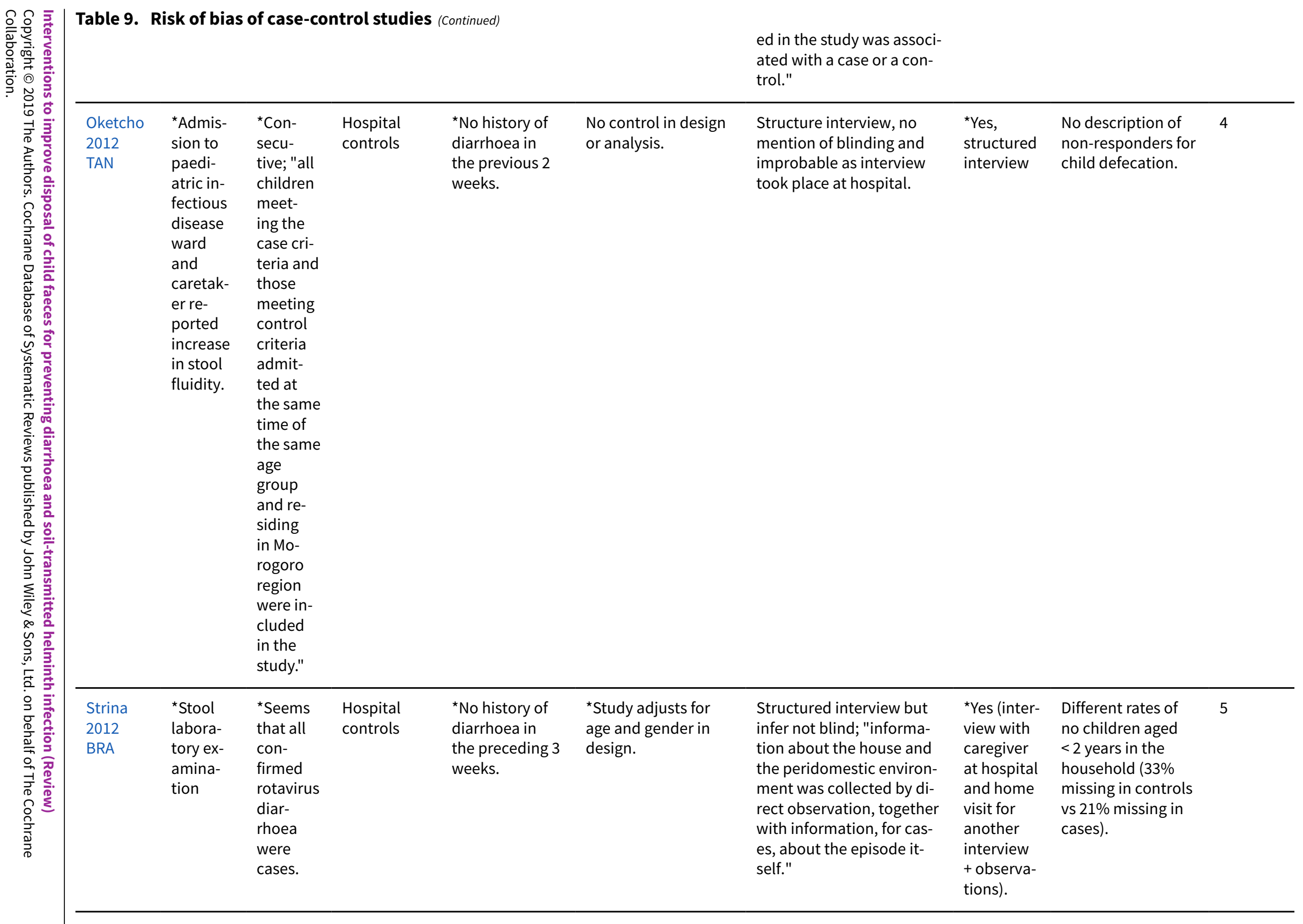




\begin{tabular}{|c|c|c|c|c|c|c|c|c|c|}
\hline 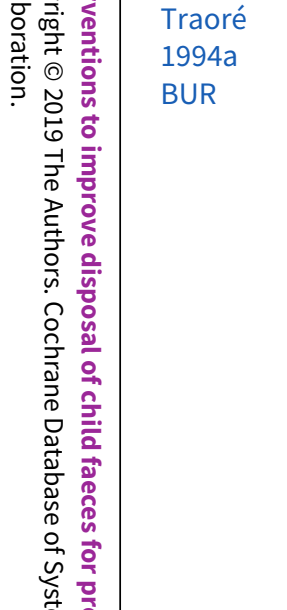 & $\begin{array}{l}\text { *Yes } \\
\text { caregiv- } \\
\text { er and } \\
\text { doctor }\end{array}$ & $\begin{array}{l}\text { All cas- } \\
\text { es pre- } \\
\text { senting } \\
\text { to hospi- } \\
\text { tal with } \\
\text { diar- } \\
\text { rhoea or } \\
\text { dysen- } \\
\text { tery or } \\
\text { both } \\
\text { but 28\% } \\
\text { could } \\
\text { not be } \\
\text { found } \\
\text { for inter- } \\
\text { view. }\end{array}$ & $\begin{array}{l}{ }^{*} \text { Communi- } \\
\text { ty and hos- } \\
\text { pital con- } \\
\text { trols. } \\
\text { Main analy- } \\
\text { sis using } \\
\text { community } \\
\text { control. }\end{array}$ & $\begin{array}{l}{ }^{\star} \text { Not admit- } \\
\text { ted to hospital } \\
\text { and for those at } \\
\text { hospital no di- } \\
\text { arrhoea/ dysen- } \\
\text { tery. }\end{array}$ & $\begin{array}{l}{ }^{*} \text { Controls for age, } \\
\text { water source, SES } \\
\text { (radio ownership), } \\
\text { and household size. }\end{array}$ & $\begin{array}{l}\text { *Interviewers were not } \\
\text { blind to whether child had } \\
\text { been to hospital but were } \\
\text { blind to whether had diar- } \\
\text { rhoea/not. }\end{array}$ & $\begin{array}{l}\text { *Yes, ques- } \\
\text { tionnaire } \\
\text { and spot } \\
\text { checks }\end{array}$ & $\begin{array}{l}\text { *In cases only } 2 \text { an- } \\
\text { swers missing for } \\
\text { disposal }(0.3 \%) \text { and } \\
3 \text { missing for defe- } \\
\text { cation }(0.4 \%) \text { and } \\
0 \text { missing for com- } \\
\text { munity controls. }\end{array}$ & 7 \\
\hline 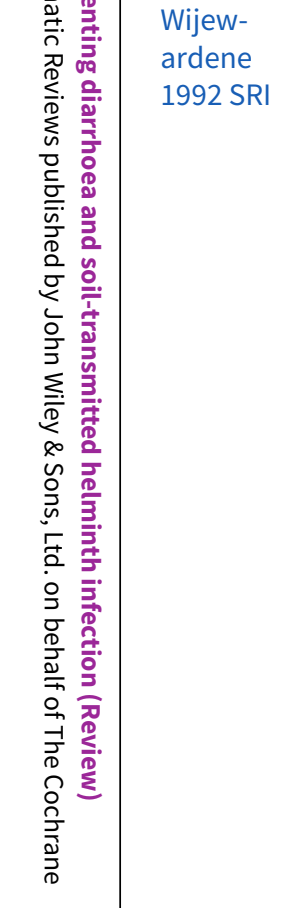 & $\begin{array}{l}{ }^{*} \text { Yes } \\
\text { (stated } \\
\text { in the } \\
\text { limita- } \\
\text { tions } \\
\text { that all } \\
\text { children } \\
\text { were } \\
\text { clinical- } \\
\text { ly exam- } \\
\text { ined and } \\
\text { cross- } \\
\text { checked } \\
\text { for re- } \\
\text { cent } \\
\text { visits } \\
\text { to doc- } \\
\text { tor, and } \\
\text { child } \\
\text { welfare } \\
\text { cards } \\
\text { available } \\
\text { were ex- } \\
\text { amined). }\end{array}$ & $\begin{array}{l}\text { *"The } \\
\text { first hun- } \\
\text { dred } \\
\text { consecu- } \\
\text { tive fam- } \\
\text { ilies with } \\
\text { children } \\
\text { aged < } \\
5 \text { years } \\
\text { with an } \\
\text { acute } \\
\text { episode } \\
\text { of diar- } \\
\text { rhoea." }\end{array}$ & $\begin{array}{l}{ }^{*} \text { Communi- } \\
\text { ty cases and } \\
\text { controls }\end{array}$ & $\begin{array}{l}{ }^{*} \text { No diarrhoea } \\
\text { episode in last } 6 \\
\text { months. }\end{array}$ & $\begin{array}{l}{ }^{*} \text { Controls for use of } \\
\text { shared/public la- } \\
\text { trines vs private and } \\
{ }^{\star} \text { Controlled for age } \\
\text { in matching and } \\
\text { other relevant con- } \\
\text { founders in regres- } \\
\text { sion. }\end{array}$ & No mention of blinding & $\begin{array}{l}\text { *Yes, ques- } \\
\text { tionnaire } \\
\text { and obser- } \\
\text { vations of } \\
\text { the facili- } \\
\text { ties }\end{array}$ & $\begin{array}{l}{ }^{\star} \text { From table } 1 \text {, ap- } \\
\text { peared there were } \\
\text { no missing values } \\
\text { for child faeces dis- } \\
\text { posal. }\end{array}$ & 8 \\
\hline
\end{tabular}

ARI: acute respiratory infection; ELISA: enzyme-linked immunosorbent assay; GEMS: Global Enteric Multicenter Study; LDC: licensed daycare centre; n: number; OPD: outpatient $\stackrel{N}{\omega}$ department; PEM: protein-energy malnutrition; SES: socioeconomic status; SHC: sentinel health centre; WASEP: Water and Sanitation Extension Programme. 
aRisk factors listed in the column are those relevant to the review (prespecified in the protocol). For a full list of confounders adjusted for in the analysis, see Table 10 and Table 11. ${ }^{1}$ The thesis reported that child faeces disposal was insignificant but no data were presented.

*These are the 'stars' given based on the Newcastle-Ottawa quality assessment scale. Total number of stars are provided in the appropriate column. 
Table 10. Case-control studies: disposal elsewhere versus latrine

\begin{tabular}{|c|c|c|c|c|c|}
\hline $\begin{array}{l}\text { Study ID } \\
\text { (setting) }\end{array}$ & $\begin{array}{l}\text { Age } \\
\text { group } \\
\text { (years) }\end{array}$ & Outcome & Specific comparison & $\begin{array}{l}\text { Measure } \\
\text { of effect } \\
(95 \% \mathrm{CI})\end{array}$ & What was it adjusted for? \\
\hline $\begin{array}{l}\text { Abalkhail } \\
1995 \text { KSA } \\
\text { (urban) }\end{array}$ & $<3$ & Diarrhoea & $\begin{array}{l}\text { Disposal of child faeces elsewhere vs in } \\
\text { latrine. }\end{array}$ & $\begin{array}{l}\text { OR (adj): } \\
1.46(1.03 \\
\text { to } 2.08)\end{array}$ & $\begin{array}{l}\text { Paternal education, child and ma- } \\
\text { ternal age and family size }\end{array}$ \\
\hline $\begin{array}{l}\text { Asfaha } \\
2018 \text { ETH } \\
\text { (rural) }\end{array}$ & $<5$ & Diarrhoea & $\begin{array}{l}\text { Unsafe vs safe child stool disposal (no } \\
\text { definition of safe/unsafe). }\end{array}$ & $\begin{array}{l}\text { OR: } 2.69 \\
(1.88 \text { to } \\
3.87)\end{array}$ & - \\
\hline $\begin{array}{l}\text { Baker } \\
2016 \text { BGD } \\
\text { (rural) }\end{array}$ & $<5$ & MSD & $\begin{array}{l}\text { Disposal of child faeces in the open vs } \\
\text { in any type of latrine with a pit or sew- } \\
\text { er. Hanging latrines and bucket latrines } \\
\text { were considered open disposal. }\end{array}$ & $\begin{array}{l}\text { OR (adj): } \\
1.26(1.05 \\
\text { to } 1.52)\end{array}$ & $\begin{array}{l}\text { Adjusted for wealth quintiles and } \\
\text { the presence of both parents in the } \\
\text { home. }\end{array}$ \\
\hline $\begin{array}{l}\text { Baker } \\
2016 \text { GMB } \\
\text { (rural) }\end{array}$ & $<5$ & MSD & $\begin{array}{l}\text { Disposal of child faeces in the open vs in } \\
\text { any type of latrine with pit/sewer. }\end{array}$ & $\begin{array}{l}\text { OR }(\text { adj): } \\
0.85(0.38 \\
\text { to } 1.88)\end{array}$ & $\begin{array}{l}\text { Adjusted for wealth quintiles and } \\
\text { the presence of both parents in the } \\
\text { home. }\end{array}$ \\
\hline $\begin{array}{l}\text { Baker } \\
2016 \text { IND } \\
\text { (urban) }\end{array}$ & $<5$ & MSD & $\begin{array}{l}\text { Disposal of child faeces in the open vs in } \\
\text { any type of latrine with pit/sewer. }\end{array}$ & $\begin{array}{l}\text { OR (adj): } \\
1.11(0.92 \\
\text { to } 1.35)\end{array}$ & $\begin{array}{l}\text { Adjusted for wealth quintiles and } \\
\text { the presence of both parents in the } \\
\text { home. }\end{array}$ \\
\hline $\begin{array}{l}\text { Baker } \\
2016 \text { KEN } \\
\text { (rural) }\end{array}$ & $<5$ & MSD & $\begin{array}{l}\text { Disposal of child faeces in the open vs in } \\
\text { any type of latrine with pit/sewer. }\end{array}$ & $\begin{array}{l}\text { OR (adj): } \\
1.02(0.87 \\
\text { to } 1.2)\end{array}$ & $\begin{array}{l}\text { Adjusted for wealth quintiles and } \\
\text { the presence of both parents in the } \\
\text { home. }\end{array}$ \\
\hline $\begin{array}{l}\text { Baker } \\
2016 \mathrm{MLI} \\
\text { (urban) }\end{array}$ & $<5$ & MSD & $\begin{array}{l}\text { Disposal of child faeces in the open vs in } \\
\text { any type of latrine with pit/sewer. }\end{array}$ & $\begin{array}{l}\text { OR (adj): } \\
2.01(0.51 \\
\text { to } 7.82)\end{array}$ & $\begin{array}{l}\text { Adjusted for wealth quintiles and } \\
\text { the presence of both parents in the } \\
\text { home. }\end{array}$ \\
\hline $\begin{array}{l}\text { Baker } \\
2016 \mathrm{MOZ} \\
\text { (rural) }\end{array}$ & $<5$ & MSD & $\begin{array}{l}\text { Disposal of child faeces in the open vs in } \\
\text { any type of latrine with pit/sewer. }\end{array}$ & $\begin{array}{l}\text { OR (adj): } \\
0.65(0.32 \\
\text { to } 1.3)\end{array}$ & $\begin{array}{l}\text { Adjusted for wealth quintiles and } \\
\text { the presence of both parents in the } \\
\text { home. }\end{array}$ \\
\hline $\begin{array}{l}\text { Baker } \\
2016 \text { PAK } \\
\text { (periur- } \\
\text { ban) }\end{array}$ & $<5$ & MSD & $\begin{array}{l}\text { Disposal of child faeces in the open vs in } \\
\text { any type of latrine with pit/sewer. }\end{array}$ & $\begin{array}{l}\text { OR (adj): } \\
0.82(0.63 \\
\text { to } 1.07)\end{array}$ & $\begin{array}{l}\text { Adjusted for wealth quintiles and } \\
\text { the presence of both parents in the } \\
\text { home. }\end{array}$ \\
\hline $\begin{array}{l}\text { Baltazar } \\
1989 \mathrm{PHI} \\
\text { (urban } \\
\text { and rural) }\end{array}$ & $<2$ & Diarrhoea & $\begin{array}{l}\text { Unsanitary vs sanitary disposal of child } \\
\text { faeces (sanitary = child defecated in a } \\
\text { nappy and faeces thrown away in wash- } \\
\text { ing, child used chamber pot/piece of pa- } \\
\text { per and faecal matter was thrown in the } \\
\text { toilet or child used the toilet). }\end{array}$ & $\begin{array}{l}\text { OR (adj): } \\
1.34(0.93 \\
\text { to } 1.92)\end{array}$ & $\begin{array}{l}\text { Water supply, toilet facilities, sex, } \\
\text { education of head of household } \\
\text { and mother, feeding practices, } \\
\text { level of health service utilization, } \\
\text { number of children aged < } 5 \text { years } \\
\text { in household. }\end{array}$ \\
\hline $\begin{array}{l}\text { Cum- } \\
\text { mings } \\
2012 \\
\text { UGAa (rur- } \\
\text { al) }\end{array}$ & $>10$ & Cholera & $\begin{array}{l}\text { Not disposing of child faeces in latrine } \\
\text { vs using latrine to dispose of faeces in } \\
\text { cases vs control. }\end{array}$ & $\begin{array}{l}\text { OR (adj): } \\
15.76 \\
(1.54 \text { to } \\
161.25)\end{array}$ & $\begin{array}{l}\text { Reside in household with anoth- } \\
\text { er case, did not use chlorine tablet } \\
\text { to disinfect water, ate roadside } \\
\text { food, girls, age group (10-17 years } \\
\text { old), no latrine in household, did } \\
\text { not wash hands after defecation, } \\
\text { did not store water in sealed con- }\end{array}$ \\
\hline
\end{tabular}


Table 10. Case-control studies: disposal elsewhere versus latrine (Continued)

tainer, eats mostly cold meals, and drinks local alcoholic beverage.

\begin{tabular}{|c|c|c|c|c|c|}
\hline $\begin{array}{l}\text { Dikassa } \\
1993 \text { DRC } \\
\text { (urban) }\end{array}$ & $<3$ & Diarrhoea & $\begin{array}{l}\text { Not disposing of child faeces in latrine } \\
\text { vs using latrine to dispose of faeces. }\end{array}$ & $\begin{array}{l}\text { OR (adj): } \\
3.61 \text { (1.32 } \\
\text { to } 9.85)\end{array}$ & $\begin{array}{l}\text { Garbage disposal, caretaker hy- } \\
\text { giene, maternal education }\end{array}$ \\
\hline $\begin{array}{l}\text { Genthe } \\
1997 \text { SAF } \\
\text { (urban/ } \\
\text { periur- } \\
\text { ban) }\end{array}$ & $\begin{array}{l}\text { Preschool } \\
\text { children }\end{array}$ & Diarrhoea & $\begin{array}{l}\text { Open disposal of stools vs disposal into } \\
\text { any form of sanitation. }\end{array}$ & $\begin{array}{l}\text { OR: } 1 \\
(0.53 \text { to } \\
1.88)\end{array}$ & - \\
\hline
\end{tabular}

\begin{tabular}{|c|c|c|c|c|c|}
\hline $\begin{array}{l}\text { Ghosh } \\
1994 \text { IND } \\
\text { (rural) }\end{array}$ & $<3$ & $\begin{array}{l}\text { Diarrhoea } \\
\text { case fami- } \\
\text { lies }\end{array}$ & Indiscriminate disposal of child stools. & $\begin{array}{l}\text { OR: } 2.22 \\
(1.08 \text { to } \\
4.56)\end{array}$ & - \\
\hline $\begin{array}{l}\text { Ghosh } \\
1997 \text { IND } \\
\text { (rural) }\end{array}$ & $<4$ & $\begin{array}{l}\text { Diarrhoea } \\
\text { case fami- } \\
\text { lies }\end{array}$ & Indiscriminate disposal of child stools. & $\begin{array}{l}\text { OR (adj): } \\
1.99(0.97 \\
\text { to } 4.08)\end{array}$ & $\begin{array}{l}\text { Bottle feeding, cleaning feeding } \\
\text { container without soap, using } \\
\text { pond water for cleaning feeding } \\
\text { container, storing drinking water } \\
\text { in wide mouthed vessel (bucket). }\end{array}$ \\
\hline
\end{tabular}

\begin{tabular}{|c|c|c|c|c|}
\hline $\begin{array}{l}\text { Godana } \\
2013 \text { ETHb } \\
\text { (rural) }\end{array}$ & $<5$ & $\begin{array}{l}\text { Acute di- } \\
\text { arrhoea }\end{array}$ & $\begin{array}{l}\text { Child faeces disposal elsewhere vs in la- } \\
\text { trine. }\end{array}$ & $\begin{array}{l}\text { OR (calc): } \\
2.49(1.64 \\
\text { to } 3.77)\end{array}$ \\
\hline
\end{tabular}

\begin{tabular}{|c|c|c|c|c|c|}
\hline $\begin{array}{l}\text { Heller } \\
2003 \text { BRA } \\
\text { (urban) }\end{array}$ & $<5$ & Diarrhoea & $\begin{array}{l}\text { Faeces disposal from swaddle else- } \\
\text { where vs in toilet/latrine. }\end{array}$ & $\begin{array}{l}\text { OR (adj): } \\
1.45(0.99 \\
\text { to } 2.12)\end{array}$ & $\begin{array}{l}\text { Fruit and green hygiene, mother's } \\
\text { religion, superficial presence of } \\
\text { wastewater in street, refuse stor- } \\
\text { age, domestic reservoir ( } 2 \text { cat- } \\
\text { egories), child's age, refuse dis- } \\
\text { posal, number of children, near } \\
\text { stream existence, own a fridge, } \\
\text { cockroach presence, flooding in } \\
\text { lot, mosquito presence, refuse col- } \\
\text { lection frequency, domestic wa- } \\
\text { ter reservoir ( } 3 \text { categories), fae- } \\
\text { ces disposal from swaddle (no } \\
\text { swaddle use vs latrine/toilet) + in- } \\
\text { teraction terms for wastewater } \\
\text { in street* refuse storage, domes- } \\
\text { tic reservoir (no storage vs cov- } \\
\text { ered + clean)*cockroach, domes- } \\
\text { tic reservoir (vessel storage vs } \\
\text { covered+clean)* cockroach, do- } \\
\text { mestic water storage ( } 3 \text { differ- } \\
\text { ent categories)* cockroach, cock- } \\
\text { roach }{ }^{\star} \text { mosquito. }\end{array}$ \\
\hline
\end{tabular}

\begin{tabular}{|c|c|c|c|c|c|}
\hline $\begin{array}{l}\text { Maung } \\
1992 a \\
\text { MYA (ur- } \\
\text { ban) }\end{array}$ & $\begin{array}{l}1-59 \\
\text { months }\end{array}$ & $\begin{array}{l}\text { Persistent } \\
\text { diarrhoea } \\
+ \text { PEM }\end{array}$ & $\begin{array}{l}\text { Faeces were disposed of around house } \\
\text { vs latrine. }\end{array}$ & $\begin{array}{l}\text { OR: } 1.88 \\
(0.6 \text { to } \\
5.96)\end{array}$ & - \\
\hline $\begin{array}{l}\text { Mediratta } \\
\text { 2010a ETH } \\
\text { (urban) }\end{array}$ & $<5$ & $\begin{array}{l}\text { Acute di- } \\
\text { arrhoea }\end{array}$ & $\begin{array}{l}\text { Disposal of stool elsewhere (garbage, } \\
\text { buried, left on ground) vs in latrine. }\end{array}$ & $\begin{array}{l}\text { OR: } 0.78 \\
(0.53 \text { to } \\
1.15)\end{array}$ & - \\
\hline
\end{tabular}


Table 10. Case-control studies: disposal elsewhere versus latrine (Continued)

\begin{tabular}{|c|c|c|c|c|c|}
\hline $\begin{array}{l}\text { Mertens } \\
1992 \text { SRI } \\
\text { (rural) }\end{array}$ & $<5$ & diarrhoea & $\begin{array}{l}\text { Unsanitary vs sanitary disposal of }<5 \\
\text { child stools. Unsanitary stool disposal = } \\
\text { stools passed, or disposed of, in or out } \\
\text { of the yard without being later (within } 1 \\
\text { day) disposed of in a latrine or in a cov- } \\
\text { ered rubbish pit. Proper = stools passed } \\
\text { in a potty } \\
\text { and later disposed of in a latrine or in a }\end{array}$ & $\begin{array}{l}\text { OR (adj): } \\
1.42(1.01 \\
\text { to } 1.98)\end{array}$ & $\begin{array}{l}\text { Child's age, recruitment clinic, the } \\
\text { distance from the home to the clin- } \\
\text { ic, handwashing } \\
\text { before a meal, water quantity, oc- } \\
\text { cupation of the head of the house- } \\
\text { hold, main type of water source } \\
\text { used, and distance to the water } \\
\text { source. }\end{array}$ \\
\hline
\end{tabular}

\begin{tabular}{|c|c|c|c|}
\hline $\begin{array}{l}\text { Strina } \\
2012 \text { BRA } \\
\text { (urban) }\end{array}$ & $<10$ & $\begin{array}{l}\text { Rotavirus } \\
\text { diarrhoea }\end{array}$ & $\begin{array}{l}\text { Inadequate vs adequate disposal of exc- } \\
\text { reta of children aged } \leq 2 \text { years (no defin- } \\
\text { ition). }\end{array}$ \\
\hline
\end{tabular}

\begin{tabular}{lll}
\hline Traoré & $<3$ & Diar- $\quad$ Disposal elsewhere vs in latrines. \\
1994a & rhoea/dysen- \\
BUR (ur- & tery & OR \\
ban) & & to 2
\end{tabular}

OR (adj): Age and gender
$1.34(0.98$
to 1.83$)$

OR (adj): Age, mother's religion, father's oc1.5 (1.09 cupation, source of drinking wa-

to 2.06) ter, possession of a radio-cassette, whether the child was reported to eat soil, whether the mother practised "lavements" (anal purging) on the child, number of people in the household.

\begin{tabular}{|c|c|c|c|c|c|}
\hline $\begin{array}{l}\text { Wijewar- } \\
\text { dene } 1992 \\
\text { SRI (ur- } \\
\text { ban) }\end{array}$ & $<5$ & $\begin{array}{l}\text { Acute di- } \\
\text { arrhoea }\end{array}$ & $\begin{array}{l}\text { Children's faeces not disposed in latrine } \\
\text { in cases vs controls. }\end{array}$ & $\begin{array}{l}\text { OR (adj): } \\
2.28 \text { (1.09 } \\
\text { to } 4.78)\end{array}$ & $\begin{array}{l}\text { Household size, source of water, } \\
\text { disposal of garbage, adult defe- } \\
\text { cation site, mother's education, } \\
\text { mother's lack of knowledge re- } \\
\text { garding infectivity of diarrhoea, } \\
\text { mother's lack of knowledge of } \\
\text { mode of spread of diarrhoea, fami- } \\
\text { lies that keep cooked food, feeding } \\
\text { bottle and children's drinking cups } \\
\text { uncovered. }\end{array}$ \\
\hline
\end{tabular}

adj: adjusted; calc: calculated crude value; Cl: confidence interval; MSD: Moderate-to-severe diarrhoea; OR: odds ratio; PEM: protein-energy malnutrition.

aCummings 2012 UGA reported a $\mathrm{Cl}$ of 1.54 to 161.25 ; however, the closest we could enter was 161.26.

bCalculated a crude OR as could not obtain as narrow Cls as what was reported in the paper.

Table 11. Case-control studies: defecation elsewhere versus in latrine

\begin{tabular}{|c|c|c|c|c|c|}
\hline $\begin{array}{l}\text { Study ID (set- } \\
\text { ting) }\end{array}$ & $\begin{array}{l}\text { Age } \\
\text { group }\end{array}$ & Outcome & Specific comparison & $\begin{array}{l}\text { Measure of } \\
\text { effect }(95 \% \\
\text { CI) }\end{array}$ & What was it adjusted for? \\
\hline $\begin{array}{l}\text { Chompook } \\
2006 \text { THA (Se- } \\
\text { mi-urban) }\end{array}$ & All & $\begin{array}{l}\text { Shigel- } \\
\text { losis }\end{array}$ & $\begin{array}{l}\text { Child not/sometimes using la- } \\
\text { trine vs using latrine. }\end{array}$ & $\begin{array}{l}\text { OR } 1.27(0.84 \\
\text { to } 1.93)\end{array}$ & - \\
\hline $\begin{array}{l}\text { Clemens } 1987 \\
\text { BGD (urban) }\end{array}$ & $<6$ years & $\begin{array}{l}\text { Diar- } \\
\text { rhoea } \geq \\
1.7 \text { times } \\
\text { rate ex- } \\
\text { pected }\end{array}$ & $\begin{array}{l}\text { Open defecation in the family } \\
\text { living area rather than latrine } \\
\text { or some other specially des- } \\
\text { ignated place in cases vs con- } \\
\text { trols. }\end{array}$ & $\begin{array}{l}\text { OR } 8(1.52 \text { to } \\
42.04)\end{array}$ & - \\
\hline $\begin{array}{l}\text { Knight } 1992 \\
\text { MAL (rural) }\end{array}$ & $\begin{array}{l}4 \text { to } 59 \\
\text { months }\end{array}$ & Diarrhoea & $\begin{array}{l}\text { Indiscriminate defecation of } \\
\text { child (not in latrine or nappy) } \\
\text { vs defecation in nappy/latrine. }\end{array}$ & $\begin{array}{l}\text { OR (adj) } 1.33 \\
(0.52 \text { to } 3.42)\end{array}$ & $\begin{array}{l}\text { SES, educational level of main care- } \\
\text { giver, health centre of recruitment, }\end{array}$ \\
\hline
\end{tabular}


Table 11. Case-control studies: defecation elsewhere versus in latrine

(Continued)

interviewer, birth order of child and number of people living in house.

\begin{tabular}{|c|c|c|c|c|c|}
\hline $\begin{array}{l}\text { Maung 1992b } \\
\text { MYA (urban) }\end{array}$ & $\begin{array}{l}1 \text { to } 59 \\
\text { months }\end{array}$ & $\begin{array}{l}\text { Persistent } \\
\text { diarrhoea } \\
+ \text { PEM }\end{array}$ & $\begin{array}{l}\text { Child defecated on the floor vs } \\
\text { in pot/latrine. }\end{array}$ & $\begin{array}{l}\text { OR } 3.76(1.68 \\
\text { to } 8.42)\end{array}$ & - \\
\hline $\begin{array}{l}\text { Mediratta } \\
\text { 2010b ETH } \\
\text { (urban) }\end{array}$ & $<5$ & $\begin{array}{l}\text { Acute di- } \\
\text { arrhoea }\end{array}$ & $\begin{array}{l}\text { Defecation elsewhere vs in la- } \\
\text { trines. }\end{array}$ & $\begin{array}{l}\text { OR } 0.88(0.33 \\
\text { to } 2.34)\end{array}$ & - \\
\hline $\begin{array}{l}\text { Oketcho } 2012 \\
\text { TAN (urban) }\end{array}$ & $\begin{array}{l}6 \text { to } 60 \\
\text { months }\end{array}$ & Diarrhoea & $\begin{array}{l}\text { Use of latrine by children vs } \\
\text { defecation elsewhere. }\end{array}$ & $\begin{array}{l}\text { OR } 7.38(1.91 \\
\text { to } 28.58)\end{array}$ & - \\
\hline $\begin{array}{l}\text { Traoré 1994b } \\
\text { BUR (urban) }\end{array}$ & $<3$ & $\begin{array}{l}\text { Diar- } \\
\text { rhoea / } \\
\text { dysentery }\end{array}$ & $\begin{array}{l}\text { Defecation elsewhere vs in } \\
\text { pots/latrines. }\end{array}$ & $\begin{array}{l}\text { OR (adj) } 1.1 \\
\text { (0.78 to } 1.57)\end{array}$ & $\begin{array}{l}\text { Age, mother's religion, father's oc- } \\
\text { cupation, source of drinking wa- } \\
\text { ter, possession of a radio-cassette, } \\
\text { whether the child was reported to } \\
\text { eat soil, whether the mother prac- } \\
\text { tised "lavements" (anal purging) on } \\
\text { the child, number of people in the } \\
\text { household. }\end{array}$ \\
\hline
\end{tabular}

adj: adjusted; Cl: confidence interval; OR: odds ratio; PEM: protein-energy malnutrition; SES: socioeconomic status.

\section{AP P E N D I CES}

\section{Appendix 1. Study design definitions}

- Quasi-randomized controlled trial (quasi-RCT): a study with an experimental design where participants are allocated to different interventions using a quasi-random method, such as date of birth, alternation, and medical record number.

- Non-RCT: a study with an experimental design where participants are allocated to different interventions using a non-random method.

- Controlled before-and-after study: a study where observations are made in a control and intervention group, before and after the implementation of an intervention.

- Interrupted time series study: a study in which observations are done at multiple time points before and after an intervention (interruption). The design of the study enables researchers to see if the intervention has an effect that is greater than underlying trend over time.

- Historically controlled study: a study comparing a group of participants receiving an intervention with a similar group from the past that did not.

- Cohort study: a study that follows a defined group of people (cohort) over a period of time to examine interventions received and subsequent outcomes. A 'prospective' cohort study recruits participants before an intervention and follows them whereas a 'retrospective' cohort study recruits participants from the past using records from the past that describe the interventions received and follows them in the past using the records.

- Case-control study: a study that compares participants with a certain outcome (cases) with people from the same source population without the outcome (controls) and examines the associations between the outcome and prior exposures (e.g. receiving an intervention).

- Cross-sectional study: a study where information on past or current interventions and health outcomes are collected for a group of people at a particular time point in order to study associations between outcomes and exposure to interventions.

We adopted these definitions from the Cochrane Handbook for Systematic Reviews of Interventions (Higgins 2011c).

\section{Appendix 2. Detailed search strategy}




\section{CENTRAL}

MEDLINE

\section{Embase}

Global Health

Web of Science

LILACS

POPLINE

1 feces OR faeces OR

faecal OR fecal OR

(f?eces or f?ecal

(f?eces or f?ecal

(f?eces or f?ecal

F\$eces ORf

feces or fae

feces OR fae-

stool $^{\star}$ OR excreta*

or stool\$ or excre-

or stool\$ or excre-

or stool* or excre-

ces OR faecal

ta\$ or excrement

ta\$ or excrement

ta* or excrement

$\$$ ecal OR stool*

ces or fe-

ces OR faecal

OR excrement OR

or diarrh?ea or de-

or diarrh?ea or de-

or diarrh?ea or de-

OR excreta* OR

stool* OR exc-

fa?cation or human

rhea OR defaeca-

waste) adj3 (man-

fa?cation or human

tion OR defecation

agement or dis-

waste) adj3 (man-

OR human waste

diarrhoe

agement or dis-

fa?cation or human

excrement OR

cal or fae-

OR fecal OR

diarrh\$ea OR

cal or stool

stool $^{*}$ OR exc-

agemento

defecation OR

excre-

reta* OR ex-

diarrhea OR pos\$ or remov\$ or

pos\$ or remov\$ or

pos*or remov* or

defaecation cleansing or clean-

cleansing or clean-

human waste

ta\$ or excre-

crement OR

OR defecation

ing or washing))

cleansing or clean-

OR human

ing or washing)

ment or di-

diarrhea OR

arrhea or di- diarrhoea OR

arrhoea or defaecation

defecation OR defecation

or defaeca- OR human

tion or hu- waste

man waste

\begin{tabular}{lll}
\hline 2. & management OR & management \\
dispos ${ }^{\star}$ OR remov & \\
OR cleansing OR & OR dispos $^{\star}$ OR \\
cleaning OR wash- & remov ${ }^{\star}$ OR \\
ing & cleansing OR \\
& cleaning OR \\
& washing
\end{tabular}

sanitation or pot-

ty or potties or di-

aper\$ or nappy or

nappies or latrine\$

or toilet\$ or cloth\$

diaper\$ or swaddle

or wrap\$

sanitation or pot-

ty or potties or di-

aper\$ or nappy or

nappies or latrine\$

or toilet\$ or cloth\$

diaper\$ or swaddle

or wrap\$

sanitation or pot-

ty or potties or di-

aper* or nappy or

nappies or latrine

or toilet* or cloth ${ }^{\star}$

or diaper* or swad-

dle or wrap*

management

OR dispos*OR

remov ${ }^{\star} \mathrm{OR}$

cleansing $O R$

cleaning OR

washing

manage-

management

ment or dis- OR dispos*

pos\$ or re- $\quad$ OR remov* OR

mov\$ or

cleansing OR

cleansing or cleaning OR

cleaning or washing

washing

\begin{tabular}{|c|c|c|c|c|c|c|c|c|}
\hline 3 & 1 AND 2 & 1 AND 2 & 1 or 2 & 1 or 2 & 1 or 2 & 1 AND 2 & 1 AND 2 & 1 AND 2 \\
\hline 4 & $\begin{array}{l}\text { sanitation OR pot- } \\
\text { ty OR potties OR } \\
\text { diaper* OR nappy } \\
\text { OR nappies OR la- } \\
\text { trine* OR toilet } \\
\text { OR cloth* OR dia- } \\
\text { per* OR swaddle } \\
\text { OR wrap* }^{\star}\end{array}$ & $\begin{array}{l}\text { sanitation OR } \\
\text { potty OR pot- } \\
\text { ties OR dia- } \\
\text { per* OR nap- } \\
\text { py OR nappies } \\
\text { OR latrine*} \\
\text { OR toilet OR } \\
\text { cloth` OR dia- }^{\star} \\
\text { per }^{\star} \text { OR swad- } \\
\text { dle OR wrap*}\end{array}$ & exp Sanitation/ & $\begin{array}{l}\text { exp sanitation/or } \\
\text { exp environmental } \\
\text { sanitation/ }\end{array}$ & exp sanitation/ & $\begin{array}{l}\text { sanitation OR } \\
\text { potty OR pot- } \\
\text { ties OR diaper* } \\
\text { OR nappy OR } \\
\text { nappies OR la- } \\
\text { trine* OR toilet* } \\
\text { OR cloth OR di- } \\
\text { aper* OR swad- } \\
\text { dle OR wrap* }\end{array}$ & $\begin{array}{l}\text { child\$ or ba- } \\
\text { bies or baby } \\
\text { or infant\$ or } \\
\text { toddler\$ or } \\
\text { neonate } \$ \text { or } \\
\text { preschool or } \\
\text { pre-school }\end{array}$ & $\begin{array}{l}\text { sanitation OR } \\
\text { potty OR pot- } \\
\text { ties OR dia- } \\
\text { per* OR nap- } \\
\text { py OR nappies } \\
\text { OR latrine* } \\
\text { OR toilet* OR } \\
\text { cloth OR dia- } \\
\text { per }^{\star} \text { OR swad- } \\
\text { dle OR wrap*}\end{array}$ \\
\hline
\end{tabular}

\begin{tabular}{|c|c|c|c|c|c|c|c|c|}
\hline 5 & 3 OR 4 & 3 OR 4 & 3 or 4 & 3 or 4 & 3 or 4 & 3 OR 4 & 3 AND 4 & 3 OR 4 \\
\hline 6 & $\begin{array}{l}\text { child* OR babies } \\
\text { OR baby OR in- } \\
\text { fant* OR toddler } \\
\text { OR neonate }{ }^{\star} \text { OR }\end{array}$ & [Sanitation] & $\begin{array}{l}\text { child\$ or babies } \\
\text { or baby or infant\$ } \\
\text { or toddler\$ or } \\
\text { neonate\$ or pre? } \\
\text { school }\end{array}$ & $\begin{array}{l}\text { child\$ or babies } \\
\text { or baby or infant\$ } \\
\text { or toddler } \$ \text { or } \\
\text { neonate\$ or pre? } \\
\text { school }\end{array}$ & $\begin{array}{l}\text { child }^{\star} \text { or babies } \\
\text { or baby or infant } \\
\text { or toddler } \\
\text { neonate } \\
\text { school }\end{array}$ & $\begin{array}{l}\text { child* OR ba- } \\
\text { bies OR baby } \\
\text { OR infant* OR } \\
\text { toddler* OR } \\
\text { neonate* OR }\end{array}$ & & $\begin{array}{l}\text { Keywords: } \\
\text { sanitation OR } \\
\text { Hygiene }\end{array}$ \\
\hline
\end{tabular}

schoo

school

school

neonate* $\mathrm{OR}$ 


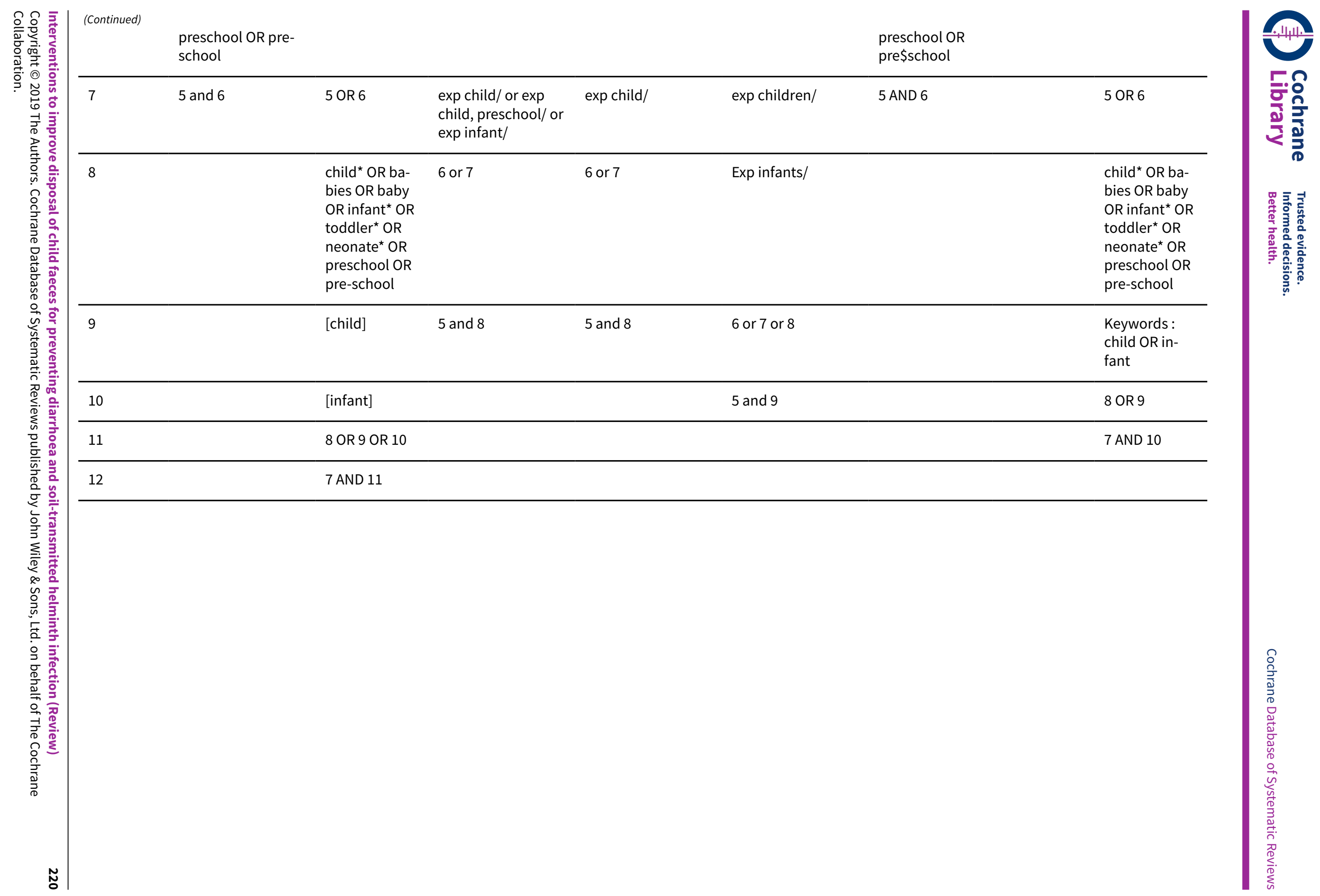


aCochrane Infectious Diseases Group Specialized Register.

\section{Appendix 3. Items for data extraction}

\section{Study data}

Person extracting data

Date of extraction

Study ID

Report ID (if different from study ID)

Reference citation

Study author details

Publication type

Publication status

Notes (e.g. questions for authors, statistical concerns)

\section{Study eligibility: (if answer no to one of the criteria, exclude)}

Type of study: RCT or NRS with control group (quasi-RCTs, non-RCTs, controlled before-and-after studies, interrupted time series studies, historically controlled studies, case-control studies, cohort studies and cross-sectional studies)

Participants: adults or children

Type of intervention: hardware or software interventions that reduce the direct or indirect contact with child (aged $<5$ years) faeces?

Type of comparison: no intervention or other intervention?

Type of outcome: diarrhoea episodes; infections with $\geq 1$ species of STHs; intensity of infection with $\geq 1$ species of STH; dysentery; severe diarrhoea; persistent diarrhoea; clinical visits for diarrhoea; presence of pathogenic microbes in stools; anthropometry; serology; other markers of infection and disease; adverse events; mortality; or behaviour change?

If excluded, reasons for exclusion

\section{Characteristics of included studies}

Country and district, state, or town

Setting (hospital, school, community, urban, or rural)

\section{Season}

\section{Design}

Description of design

Was it a multicentre study?

\section{Funding source}


(Continued)

Duration of study (start and end date of study)

Duration of participation (start of recruitment until last follow-up time point)

Ethical approval if needed

\section{Missing data and reasons}

Unit of randomization and whether the analysis adjusted for clustering if cluster design

\section{Participants}

Population demographics

Study inclusion criteria

Study exclusion criteria

Method of participant recruitment

Total number of participants recruited

Withdrawals, exclusions, loss to follow-up

\section{Age \\ Sex}

Household size

Education level

Socioeconomic level

Pre- and postintervention water quality

Sanitation type and coverage

Hygiene practices

Type of water supply and coverage

Baseline child faeces disposal sites

Prevalence of open defecation

Deworming history in the study population

Solid waste disposal practices

Animal ownership

School or preschool attendance

Shoe wearing practices 
(Continued)

\section{Intervention group}

Description of intervention

Number of participants

\section{Cointerventions?}

Who delivered the intervention?

Format and timing of delivery?

Coverage and uptake of child faeces collection and disposal practices

Compliance to intervention

\section{Control group}

Description of control

Number of participants

\section{Cointervention?}

\section{Outcomes}

\section{Case definition for health outcomes}

Measuring/diagnosis method (if self-reported include recall period)

Time points measured

Effect estimate and $95 \% \mathrm{Cl}$ and raw numbers (for NRS record adjusted and unadjusted measures with confounders adjusted for; for cluster RCT specify if effect estimate is adjusted for clustering)

List of outcomes measured in study

Key conclusions of authors

Explanations of unexpected findings

\section{Risk of bias assessment}

\section{RCTs (high, low, or unclear risk)}

Random sequence generation?

Allocation concealment?

Blinding of participants and personnel?

Blinding of outcome assessment?

\section{Incomplete outcome data?}

\section{Selective reporting?}


(Continued)

Other risks of bias?

\section{Cluster-RCTs (high, low, or unclear risk)}

Recruitment bias?

Baseline imbalance?

Loss of clusters?

Incorrect analyses?

NRS except case-control and interrupted time series (high, low, or unclear risk)

Random sequence generation?

Allocation concealment?

Baseline outcome measures similar?

Baseline characteristics similar?

Incomplete outcome data?

Adequate allocation of intervention concealment?

Adequate protection against contamination?

Selective reporting?

Other risks of bias?

Confounders adequately adjusted for in analysis or design? (describe adjustment method)

Methods to identify and measure confounders

List all confounders considered in study

\section{Interrupted time series (high, low, or unclear risk)}

Intervention independent from other changes?

Prespecified shape of the intervention?

Intervention likely to affect the data collection?

Knowledge of the allocated interventions was adequately prevented?

Incomplete outcome data?

Selective outcome reporting?

Other risk of bias?

\section{Case-control studies}


(Continued)

- Selection

Is the case definition adequate?

Representativeness of the cases

Selection of controls

Definition of controls

- Comparability

Comparability of cases and controls on the basis of the design or analysis

- Exposure

Ascertainment of exposure

Same method of ascertainment for cases and controls

Non-response rate

NRS: non-randomized study; RCT: randomized controlled trial; STH: soil-transmitted helminth.

\section{CONTRIBUTIONS OF AUTHORS}

TC and FM planned the review.

FM drafted the protocol.

FM screened titles.

FM, BT, and GC screened abstracts and full texts.

FM contacted study authors for additional information.

FM, BT, and GC extracted data.

FM entered the data and BT checked a sample.

FM drafted the review.

All authors provided comments on the review, and read and approved the final review version.

\section{DECLARATIONSOF INTEREST}

FM has no known conflicts of interest.

BT has no known conflicts of interest.

GC has no known conflicts of interest.

TC has no known conflicts of interest.

\section{SOURCES OF SUPPORT}

\section{Internal sources}

- Liverpool School of Tropical Medicine, UK. 


\section{External sources}

- Bill \& Melinda Gates Foundation, USA.

This research is supported in part by a grant from the foundation

- Department for International Development, UK.

Project number 300342-104

\section{DIFFERENCES BETWEEN PROTOCOLANDREVIEW}

In the published protocol, Majorin 2014, we prespecified that if there were a sufficient number of included studies (more than 10) we would investigate causes of heterogeneity using subgroup analysis. However, we investigated causes of heterogeneity using subgroup analysis even when there were fewer than 10 studies.

We pooled comparable studies together if there was more than one study, even when the $I^{2}$ statistic value was greater than $75 \%$.

One review author assessed the certainty of the evidence rather than two as planned in the protocol.

\section{INDEX TERMS}

\section{Medical Subject Headings (MeSH)}

*Sanitation; Controlled Before-After Studies; Diarrhea [ ${ }^{*}$ parasitology]; Feces; Helminthiasis [ ${ }^{*}$ prevention \& control] [ ${ }^{\star}$ transmission]; Helminths; Randomized Controlled Trials as Topic; Soil [*parasitology]

\section{MeSH check words}

Animals; Child; Child, Preschool; Humans; Infant 